\title{
An Artificial $\beta$-Sheet that Dimerizes Through Parallel $\beta$-Sheet Interactions
}

\author{
Sergiy Levin and James S. Nowick* \\ Department of Chemistry, University of California, Irvine, California, \\ 92697-2025
}




\section{Supporting Information Table of Contents}

General Procedures $\quad$ S4

Synthetic Scheme

Representative synthetic procedures for the synthesis of the compound 1a-1f: Artificial $\beta$-sheet 1d Amide 4d

${ }^{1} \mathrm{H}$ NMR spectrum $\left(\mathrm{CDCl}_{3}, 298 \mathrm{~K}\right)$

S7

${ }^{13} \mathrm{C}$ NMR spectrum $\left(\mathrm{CDCl}_{3}, 298 \mathrm{~K}\right)$

Amine 5d.

S8

${ }^{1} \mathrm{H}$ NMR spectrum $\left(\mathrm{CDCl}_{3}, 298 \mathrm{~K}\right)$

${ }^{13} \mathrm{C}$ NMR spectrum $\left(\mathrm{CDCl}_{3}, 298 \mathrm{~K}\right) \quad \mathrm{S} 12$

Ester 6d

$\begin{array}{ll}\mathrm{d} & \mathrm{S} 13\end{array}$

$\begin{array}{ll}{ }^{1} \mathrm{H} \text { NMR spectrum }\left(\mathrm{CDCl}_{3}, 298 \mathrm{~K}\right) & \mathrm{S} 14\end{array}$

${ }^{13} \mathrm{C}$ NMR spectrum $\left(\mathrm{CDCl}_{3}, 298 \mathrm{~K}\right) \quad \mathrm{S} 15$

Amide 7d

$\mathrm{S} 16$

$\begin{array}{ll}{ }^{1} \mathrm{H} \text { NMR spectrum }\left(\mathrm{CDCl}_{3}, 298 \mathrm{~K}\right) & \mathrm{S} 17\end{array}$

${ }^{13} \mathrm{C}$ NMR spectrum $\left(\mathrm{CDCl}_{3}, 298 \mathrm{~K}\right) \quad \mathrm{S} 18$

Salt 8d

$\begin{array}{ll}{ }^{1} \mathrm{H} \text { NMR spectrum }\left(\mathrm{CD}_{3} \mathrm{SOCD}_{3}, 298 \mathrm{~K}\right) & \text { S20 }\end{array}$

${ }^{13} \mathrm{C}$ NMR spectrum $\left(\mathrm{CD}_{3} \mathrm{SOCD}_{3}, 298 \mathrm{~K}\right) \quad \mathrm{S} 21$

Oxazolidinone 9d.

$\mathrm{S} 22$

$\begin{array}{ll}{ }^{1} \mathrm{H} \text { NMR spectrum }\left(\mathrm{CDCl}_{3}, 298 \mathrm{~K}\right) & \text { S23 }\end{array}$

${ }^{13} \mathrm{C}$ NMR spectrum $\left(\mathrm{CDCl}_{3}, 298 \mathrm{~K}\right) \quad$ S24

Salt 10d

${ }^{1} \mathrm{H}$ NMR spectrum $\left(\mathrm{CD}_{3} \mathrm{SOCD}_{3}, 298 \mathrm{~K}\right) \quad$ S26

${ }^{13} \mathrm{C}$ NMR spectrum $\left(\mathrm{CD}_{3} \mathrm{SOCD}_{3}, 298 \mathrm{~K}\right) \quad \mathrm{S} 27$

Salt 12d

${ }^{1} \mathrm{H}$ NMR spectrum $\left(\mathrm{CD}_{3} \mathrm{SOCD}_{3}, 298 \mathrm{~K}\right) \quad$ S29

Artificial $\beta$-sheet 1d

$\mathrm{S} 30$

${ }^{1} \mathrm{H}$ NMR spectrum $\left(\mathrm{CDCl}_{3}, 268 \mathrm{~K}\right) \quad$ S31

Spectroscopic data for the artificial $\beta$-sheet 1a $\quad$ S32

${ }^{1} \mathrm{H}$ NMR spectrum $\left(800 \mathrm{MHz}, 10 \mathrm{mM}, \mathrm{CDCl}_{3}, 268 \mathrm{~K}\right) \quad \mathrm{S} 33$

COSY spectrum $\left(800 \mathrm{MHz}, 10 \mathrm{mM}, \mathrm{CDCl}_{3}, 268 \mathrm{~K}\right) \quad \mathrm{S} 35$

TOCSY spectrum $\left(800 \mathrm{MHz}, 10 \mathrm{mM}, \mathrm{CDCl}_{3}, 268 \mathrm{~K}\right) \quad \mathrm{S} 42$

NOESY spectrum $\left(800 \mathrm{MHz}, 10 \mathrm{mM}, \mathrm{CDCl}_{3}, 268 \mathrm{~K}\right) \quad \mathrm{S} 49$

ROESY spectrum $\left(600 \mathrm{MHz}, 10 \mathrm{mM}, \mathrm{CDCl}_{3}, 268 \mathrm{~K}\right) \quad \mathrm{S} 63$

$\begin{array}{ll}\text { Spectroscopic data for the artificial } \beta \text {-sheet 1b } & \text { S64 }\end{array}$

${ }^{1} \mathrm{H}$ NMR spectrum $\left(800 \mathrm{MHz}, 6 \mathrm{mM}, \mathrm{CDCl}_{3}, 268 \mathrm{~K}\right) \quad \mathrm{S} 65$

COSY spectrum $\left(800 \mathrm{MHz}, 6 \mathrm{mM}, \mathrm{CDCl}_{3}, 268 \mathrm{~K}\right) \quad \mathrm{S} 67$

TOCSY spectrum $\left(800 \mathrm{MHz}, 6 \mathrm{mM}, \mathrm{CDCl}_{3}, 268 \mathrm{~K}\right) \quad \mathrm{S} 74$

NOESY spectrum $\left(800 \mathrm{MHz}, 6 \mathrm{mM}, \mathrm{CDCl}_{3}, 268 \mathrm{~K}\right) \quad \mathrm{S} 81$

$\begin{array}{ll}\text { Spectroscopic data for the artificial } \beta \text {-sheet 1c } & \text { S92 }\end{array}$

$\begin{array}{ll}{ }^{1} \mathrm{H} \text { NMR spectrum }\left(800 \mathrm{MHz}, 8 \mathrm{mM}, \mathrm{CDCl}_{3}, 268 \mathrm{~K}\right) & \mathrm{S} 93\end{array}$

COSY spectrum $\left(800 \mathrm{MHz}, 8 \mathrm{mM}, \mathrm{CDCl}_{3}, 268 \mathrm{~K}\right) \quad \mathrm{S} 95$

TOCSY spectrum $\left(800 \mathrm{MHz}, 8 \mathrm{mM}, \mathrm{CDCl}_{3}, 268 \mathrm{~K}\right) \quad \mathrm{S} 101$

NOESY spectrum $\left(800 \mathrm{MHz}, 8 \mathrm{mM}, \mathrm{CDCl}_{3}, 268 \mathrm{~K}\right) \quad \mathrm{S} 107$

$\begin{array}{ll}\text { Spectroscopic data for the artificial } \beta \text {-sheet 1d } & \text { S115 }\end{array}$

${ }^{1} \mathrm{H}$ NMR spectrum $\left(800 \mathrm{MHz}, 20 \mathrm{mM}, \mathrm{CDCl}_{3}, 268 \mathrm{~K}\right) \quad \mathrm{S} 116$

COSY spectrum $\left(800 \mathrm{MHz}, 20 \mathrm{mM}, \mathrm{CDCl}_{3}, 268 \mathrm{~K}\right) \quad \mathrm{S} 118$

TOCSY spectrum $\left(800 \mathrm{MHz}, 20 \mathrm{mM}, \mathrm{CDCl}_{3}, 268 \mathrm{~K}\right) \quad \mathrm{S} 124$ 
NOESY spectrum ( $800 \mathrm{MHz}, 20 \mathrm{mM}, \mathrm{CDCl}_{3}, 268 \mathrm{~K}$ )

Spectroscopic data for the artificial $\beta$-sheet 1e

${ }^{1} \mathrm{H}$ NMR spectrum $\left(800 \mathrm{MHz}, 1 \mathrm{mM}, \mathrm{CDCl}_{3}, 268 \mathrm{~K}\right)$

COSY spectrum $\left(800 \mathrm{MHz}, 1 \mathrm{mM}, \mathrm{CDCl}_{3}, 268 \mathrm{~K}\right)$

TOCSY spectrum $\left(800 \mathrm{MHz}, 1 \mathrm{mM}, \mathrm{CDCl}_{3}, 268 \mathrm{~K}\right)$

$\mathrm{S} 150$

NOESY spectrum $\left(800 \mathrm{MHz}, 1 \mathrm{mM}, \mathrm{CDCl}_{3}, 268 \mathrm{~K}\right)$

${ }^{1} \mathrm{H}$ NMR spectrum $\left(800 \mathrm{MHz}, 4 \mathrm{mM}, \mathrm{CDCl}_{3}, 268 \mathrm{~K}\right)$

NOESY spectrum $\left(800 \mathrm{MHz}, 4 \mathrm{mM}, \mathrm{CDCl}_{3}, 268 \mathrm{~K}\right)$ 
General Procedures. Commercial solvents and reagents were used without further purification, with the exception of THF, which was dried by filtration through alumina according to the procedure of Grubbs. ${ }^{1}$ Solution-phase reactions were monitored by thin-layer chromatography (TLC) and carried out on $250 \mu \mathrm{m}$ silica gel polyester plates on fluorescent silica gel with UV visualization. Column chromatography was performed on $40-63 \mu \mathrm{m}$ silica gel using flash chromatography. Solvents were removed by rotary evaporation. Residual solvents were removed under vacuum $(<0.01 \mathrm{mmHg})$. Precipitated and recrystallized products were dried either under vacuum $(<0.01 \mathrm{mmHg})$ or by air suction through a filter funnel. High resolution mass spectra were obtained by electrospray ionization (ESI) on a Waters Micromass LCT Premier (instrument variation $\sigma<5 \mathrm{ppm}$ ). NMR spectra were recorded using a $500 \mathrm{MHz}$ Bruker AVANCE ${ }^{\mathrm{TM}}, 600$

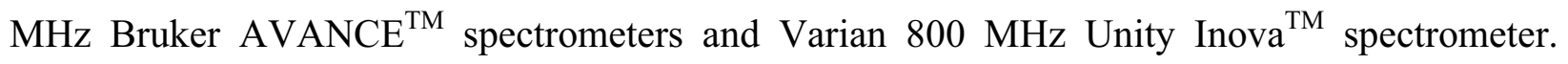
Chemical shifts are reported in parts per million (ppm) on the $\delta$ scale. ${ }^{1} \mathrm{H}$ spectra NMR were referenced with TMS $(\delta=0.00 \mathrm{ppm})$ and ${ }^{13} \mathrm{C}$ NMR NMR spectra were referenced either with TMS $(\delta=0.00 \mathrm{ppm})$ or with DMSO-d6 $(\delta=39.53 \mathrm{ppm})$.

\footnotetext{
${ }^{1}$ Pangborn, A. B.; Giardello, M. A.; Grubbs, R. H.; Rosen, R. K.; Trimmers, F. J. Organometallics 1996, 15, 15181520.
} 


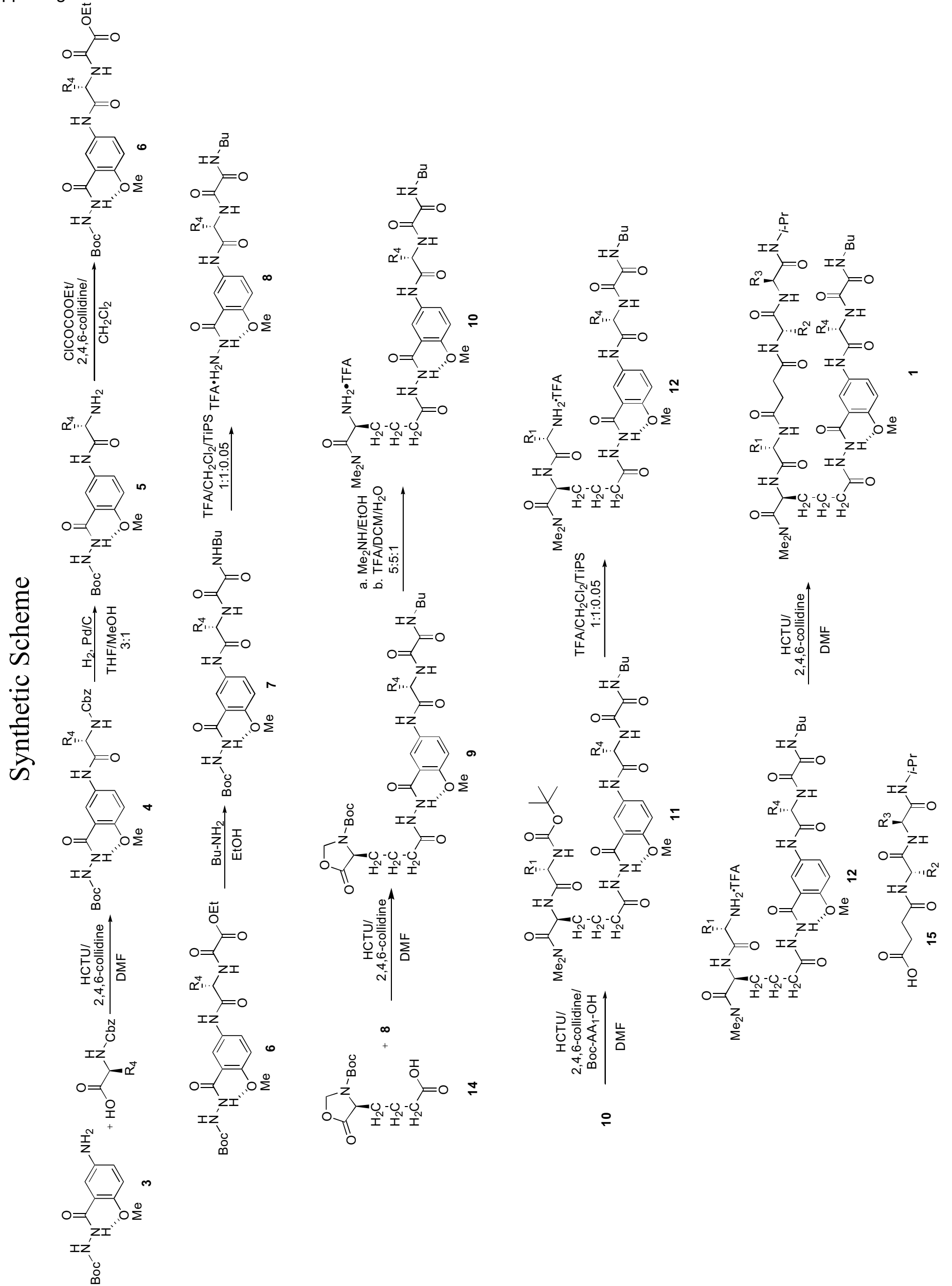




\section{Synthetic Scheme (continued)}
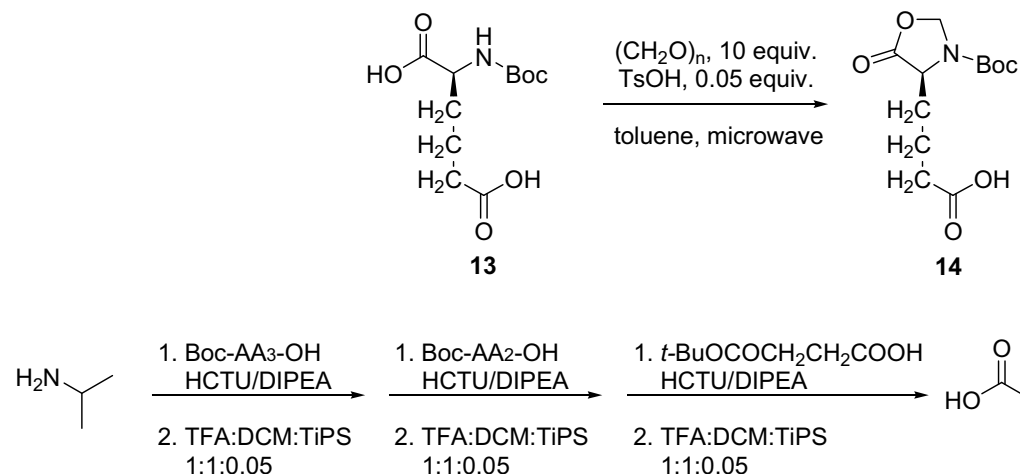
1. $t-\mathrm{BuOCOCH} \mathrm{CH}_{2} \mathrm{COOH}$ HCTU/DIPEA
2. TFA:DCM:TiPS
1:1:0.05

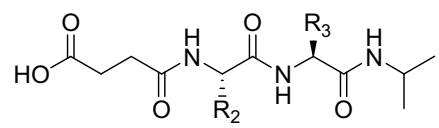

1:1:0.05 


\title{
Representative synthetic procedures for the synthesis of the
}

\author{
compound 1a-1f: Artificial $\beta$-sheet 1d.
}

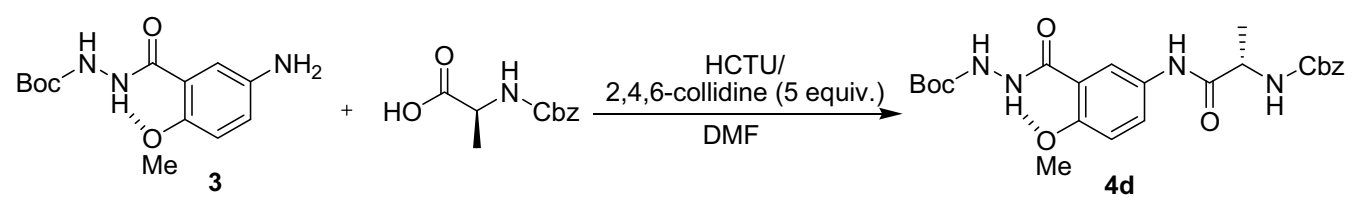

Amide 4d. A 500-mL, three-necked, round-bottomed flask equipped with a nitrogen inlet adapter, a glass stopper, a rubber septum, and a magnetic stirring bar was charged with aniline $\mathbf{3}^{2}$ (2.7 g, $9.5 \mathrm{mmol})$ and DMF (100 mL). The apparatus was evacuated and flushed with nitrogen (3 times). A solution of Cbz-Ala-OH (2.4 g, $10.8 \mathrm{mmol})$ and HCTU (4.7 g, $11.4 \mathrm{mmol})$ in DMF $(100 \mathrm{~mL})$ was added to the stirred solution through syringe, followed by $2,4,6$-collidine $(6.3 \mathrm{~mL}$, $48 \mathrm{mmol}$ ). The pale yellow solution was left under nitrogen for $24 \mathrm{~h}$, then concentrated under vacuum. The residue was dissolved in dichloromethane $(200 \mathrm{~mL})$, and the solution was washed 1 $\mathrm{M} \mathrm{HCl}(3 \times$ ca. $100 \mathrm{~mL})$, saturated aqueous $\mathrm{NaHCO}_{3}(3 \times$ ca. $100 \mathrm{~mL})$, water $(3 \times$ ca. $100 \mathrm{~mL})$, dried over $\mathrm{MgSO}_{4}$, filtered and concentrated to give $4.8 \mathrm{~g}(98 \%)$ of $\mathbf{4 d}$ as a white solid. The compound was used without further purification.

\footnotetext{
${ }^{2}$ Tsai, J. H.; Waldman, A-S.; Nowick, J. S. Bioorg. Med. Chem, 1999, 7, 29-38.
} 


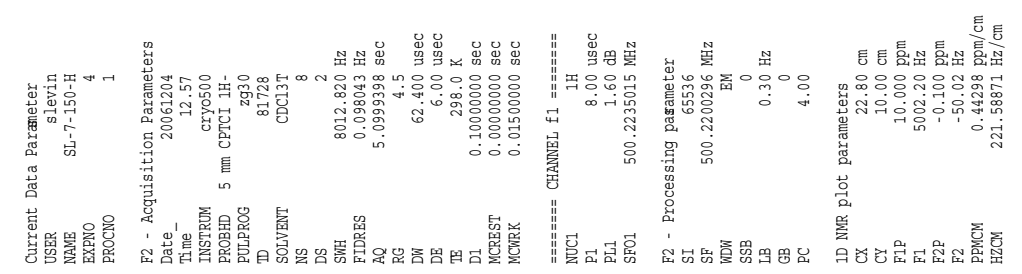

0

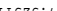

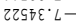

宅

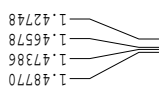

วZTรE: $L$

తु
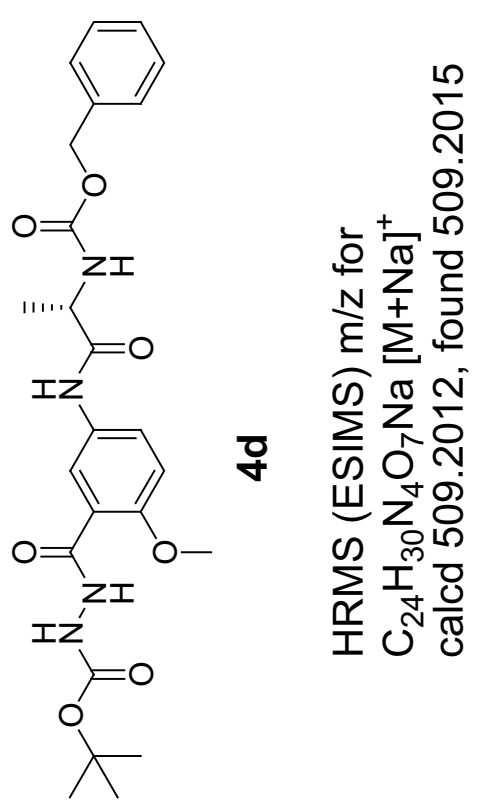

空

를
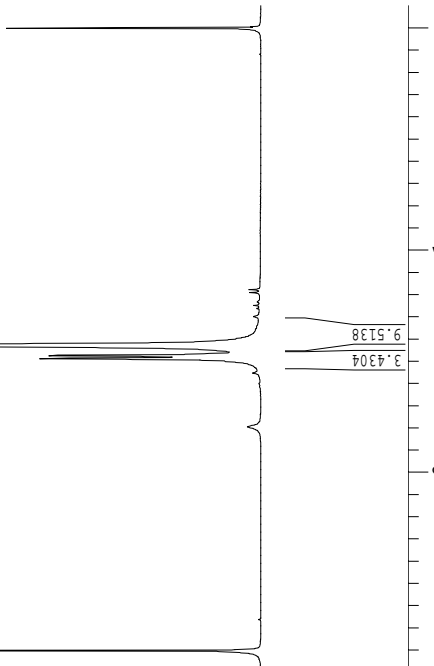

$$
\frac{1}{2}
$$

象

บ

i

กั $\int^{-10}$

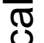$$
\text { . }
$$ 
Supporting Information

S. Levin and J. S. Nowick

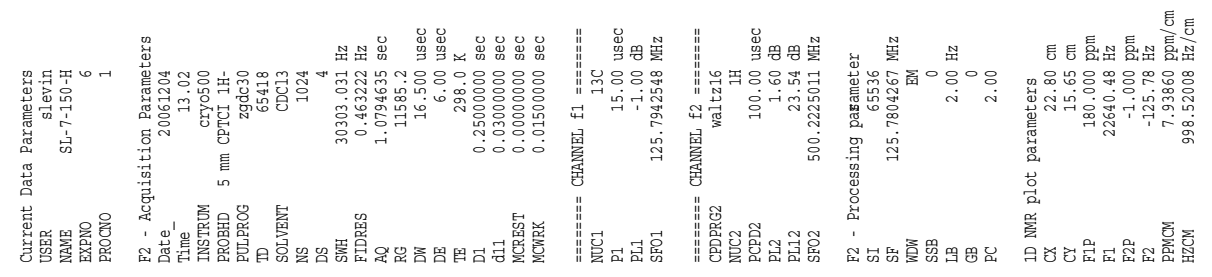

$00 \cdot 0-\square$

ว

$\varepsilon L \cdot 6 \tau$

$\nabla \tau \cdot 82$

โ9. $8 \varepsilon$

To. TS

โซ. 99

$90^{\circ} \angle 9$

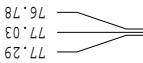

$0 \varepsilon^{\circ} \mathrm{T} 8$
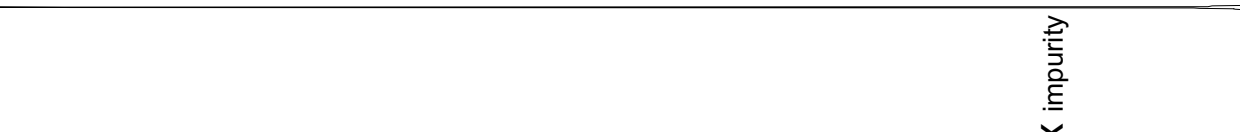

$\times$

-아

8

$66^{\circ} \mathrm{TIT}$

$00.6 \mathrm{TI}$

(2)

$\bar{\nabla} \cdot \varepsilon Z T-$
$\tau \varepsilon \cdot g Z T-$

$00.82 \mathrm{Z}$

$2 \mathrm{TI}^{\circ} \cdot 8 \mathrm{ZIT} \longrightarrow$

$\varepsilon \mp \cdot \tau \varepsilon \tau-$

ข้อ

$\varepsilon I^{\circ} 9 \varepsilon \tau-$

品

巨్

प्4

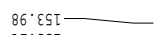

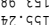

โZ.99โ-

远

감

Eโ· $99 \mathrm{-}$

$80^{\circ}$ TLI

$>$<smiles>C[C](C)C(=O)C(C)=O</smiles>

$\sum$ II

Iz

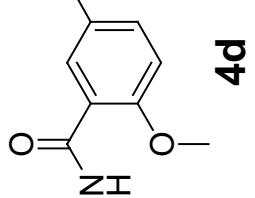

IZ

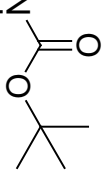

\& 


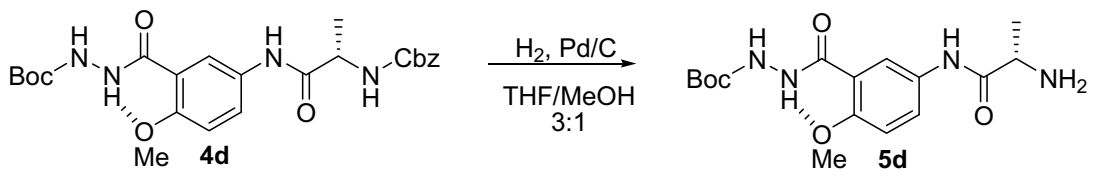

Amine 5d. A 500-mL, three-necked, round-bottomed flask equipped with a nitrogen inlet adapter, a glass stopper, a gas stopper, a gas inlet adapter fitted with a hydrogen balloon, and a magnetic stirring bar was charged with $250 \mathrm{~mL}$ of tetrahydrofuran, $100 \mathrm{~mL}$ of methanol, and amide 4d (4.6 g, $9.5 \mathrm{mmol}) .10 \% \mathrm{Pd} / \mathrm{C}(0.5 \mathrm{~g})$ was added to the reaction mixture under the nitrogen flow. The flask was evacuated and filled with hydrogen and then maintained under the hydrogen atmosphere for $4.5 \mathrm{~h}$. The reaction mixture was filtered through celite, the celite was washed with ethyl acetate $(100 \mathrm{~mL})$, and the combined solution was concentrated under vacuum yielding $3.6 \mathrm{~g}(109 \%)$ of amine $\mathbf{5 d}$ as a fluffy white solid, which was used without further purification. An analytical sample was further dried under high vacuum. 


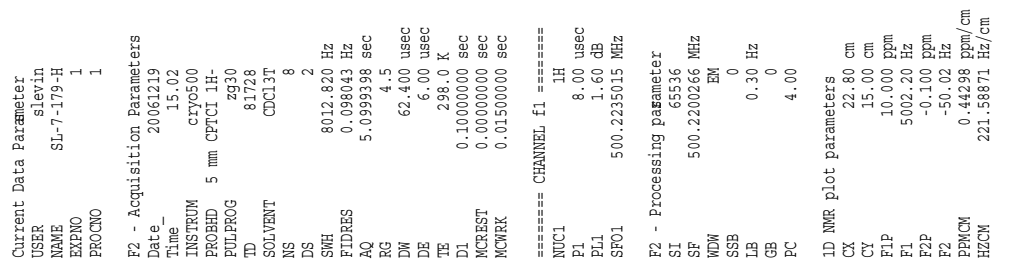

S. Levin and J. S. Nowick

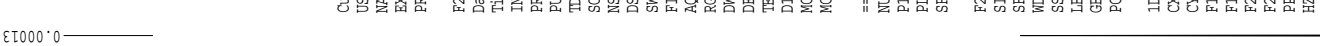

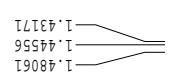

โวย $08^{\circ} \tau$

${ }_{\text {8รฐ } 26.9}$

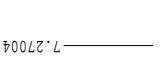

च્ర

臬

$-\rightarrow$

言

苞

क

䍃

出

事

웅
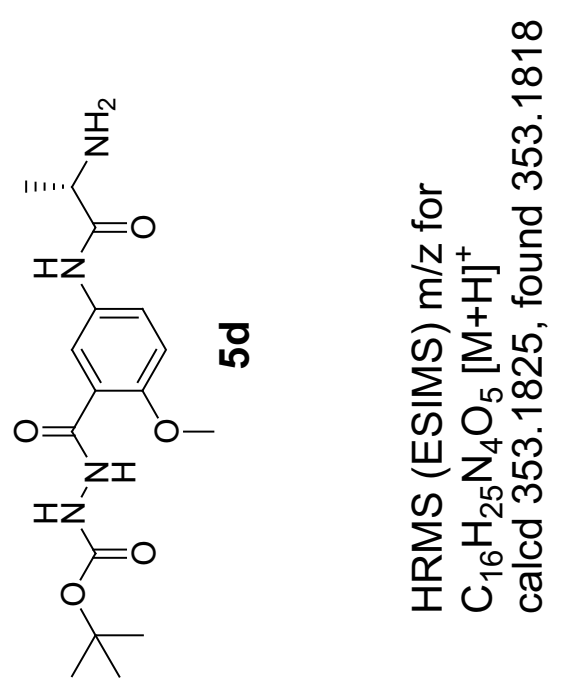


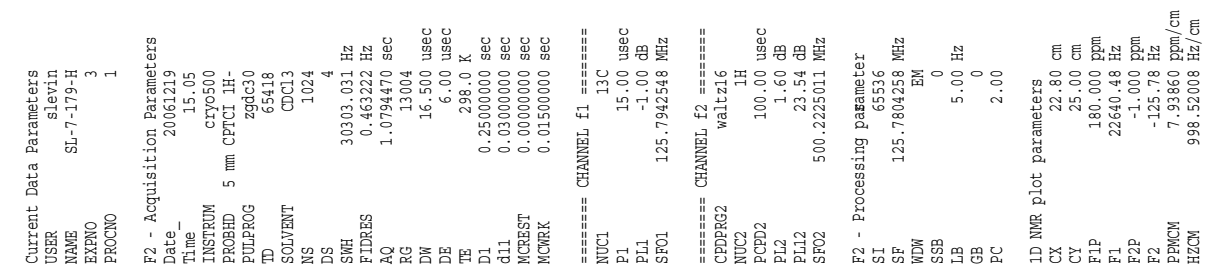

$20^{\circ} 2 \mathrm{IT}$

T0.6IT

० เ $\varepsilon ट \tau-$

$\varepsilon 0^{\circ} \cdot \mathrm{ZT}$

${ }^{\circ} 6^{\circ} \mathrm{T \varepsilon T}$

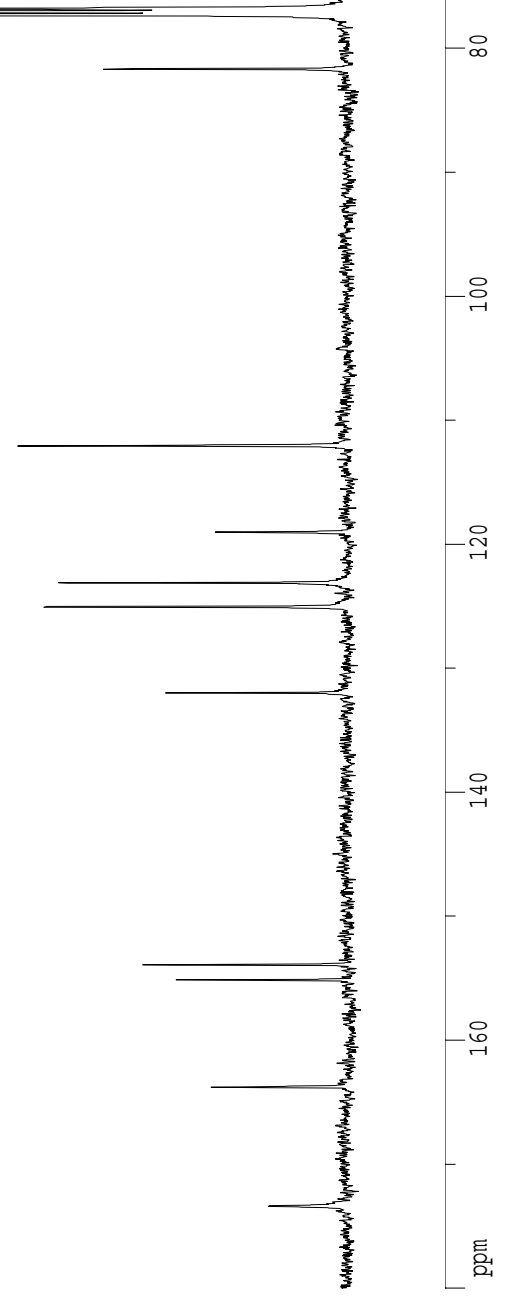




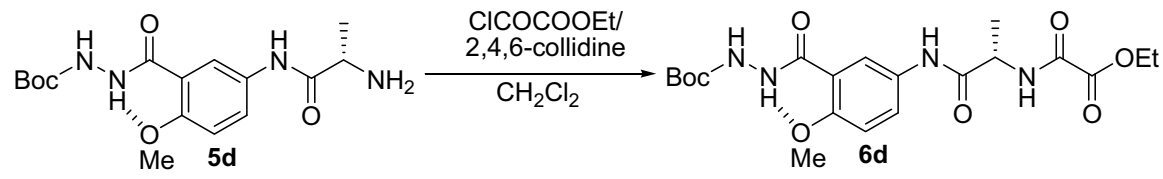

Ester 6d. A solution of the amide $5 \mathbf{d}$ (3.30 g, $9.40 \mathrm{mmol})$ and 2,4,6-collidine (2.50 mL, 19.0 $\mathrm{mmol})$ in dichloromethane $(250 \mathrm{~mL})$ was cooled to $0^{\circ} \mathrm{C}$ with an ice-bath. Ethyl chlorooxoacetate (1.05 $\mathrm{mL}, 9.40 \mathrm{mmol}$ ) was slowly added to the stirred solution over $15 \mathrm{~min}$. The solution was left stired for an extra 15 minutes and washed $1 \mathrm{M} \mathrm{HCl}$ (ca. $250 \mathrm{~mL}$ ), saturated aqueous $\mathrm{NaHCO}_{3}$ (ca. $250 \mathrm{~mL}$ ), and water (ca. $250 \mathrm{~mL}$ ). The solution was dried over $\mathrm{MgSO}_{4}$, filtered and concentrated to give $4.07 \mathrm{~g}(96 \%)$ of $\mathbf{6 d}$ as a white solid, which was used without further purification. An analytical sample was recrystallized from the ethanol. 


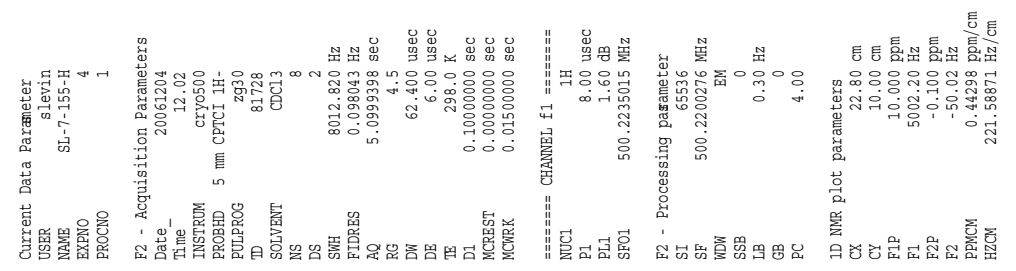

LLSLE:T—

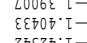

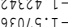

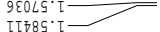

$79986^{\circ} \varepsilon-$

SOOLE. $7-$

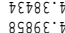

6LZTฤ.

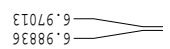

$\angle 8 L 9 Z^{\circ} L-$

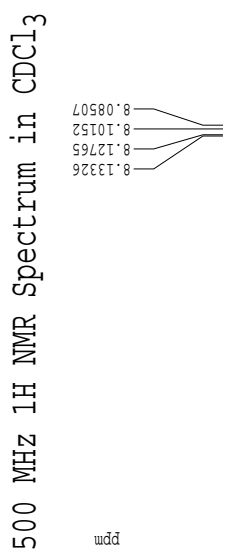

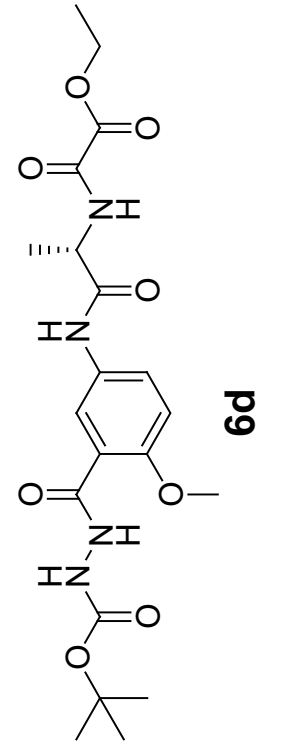

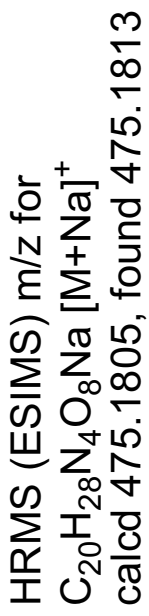

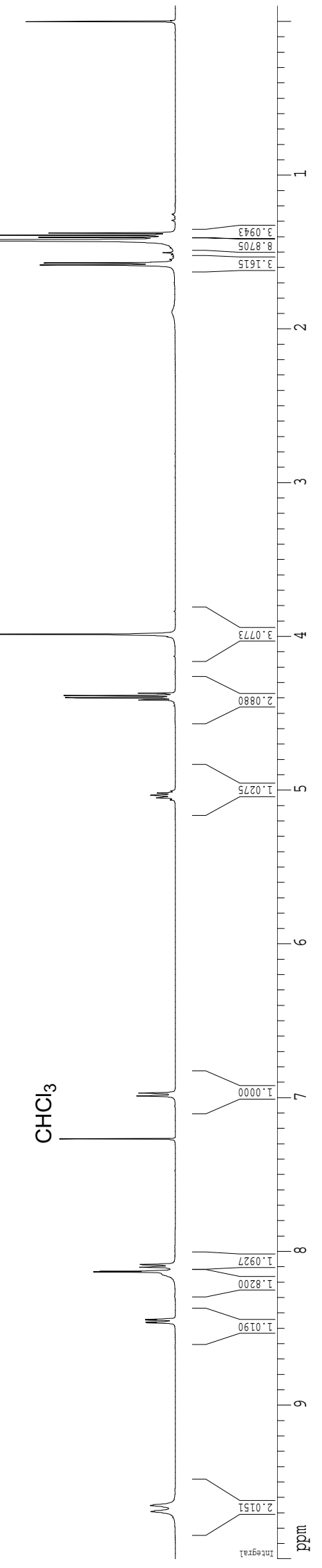




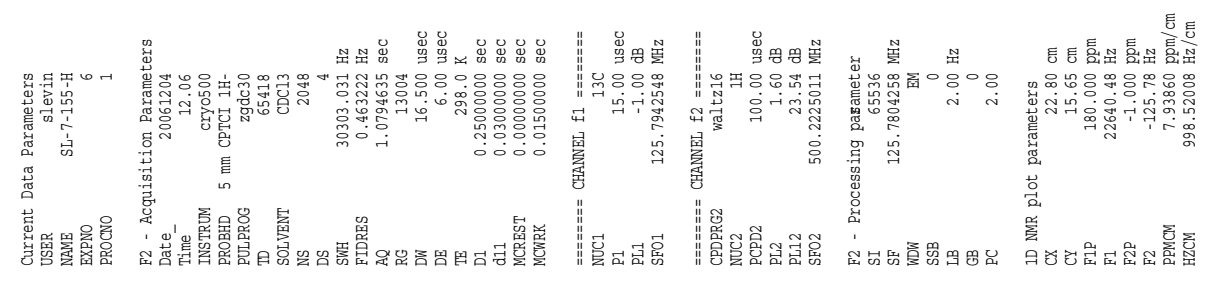

${ }^{\circ} \circ \mathrm{LT}$

It $6 \tau$

รT. 82

$L T \cdot O S$

万I. 99

ธ๋' ๕9 -

$8 L \cdot 9 L$

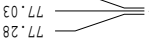

$92^{\circ} \div 8$

$\angle 0^{\circ} \mathrm{ZTI}$

$\angle 6.8 \mathrm{II}$

$\angle T \cdot \varepsilon Z \tau$

$90^{\circ} \mathrm{gZt}$

$\angle F^{\circ}$ 'ZET-

它

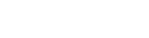

孚

罗

论

\section{$\angle 0^{\circ}$. $5 \mathrm{~T}$}

$90^{\circ}$. $5 \mathrm{~T}$
SE. $99 \mathrm{~T}$

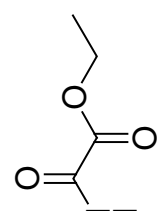

ZI

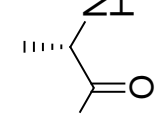

IZ

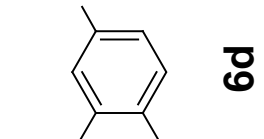

$25 \cdot 59 \tau$

$88 \cdot 691$

尝

늠 


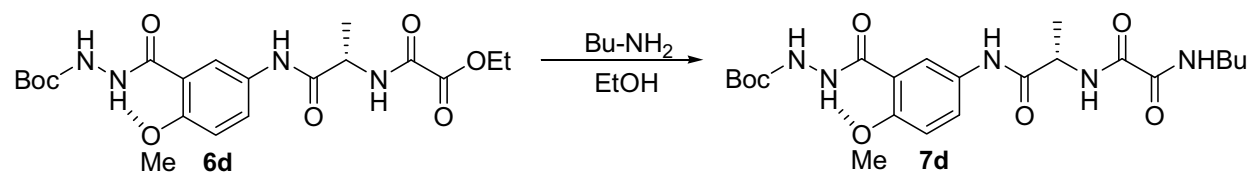

Amide 7d. Butylamine $(5.0 \mathrm{~mL}, 54 \mathrm{mmol})$ was added in one portion to a solution of the ester $\mathbf{6 d}$ $(4.07 \mathrm{~g}, 9.00 \mathrm{mmol})$ in ethanol $(150 \mathrm{~mL})$. After 10 minutes a white precipitate formed. The precipitate was filtered and dried to yield $3.70 \mathrm{~g}(86 \%)$ of amide $7 \mathbf{d}$ as a white solid. 

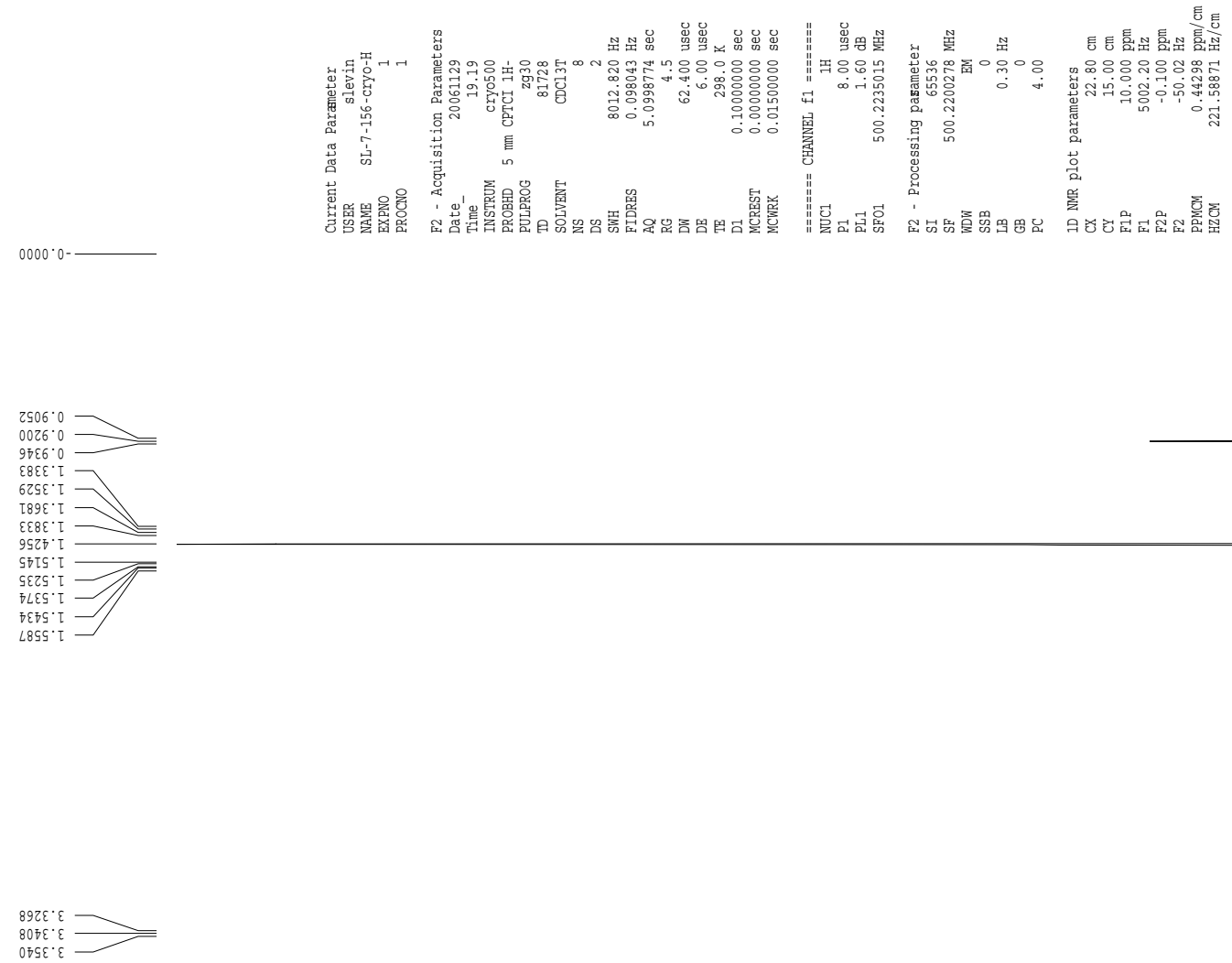

$8096^{\circ} \varepsilon$

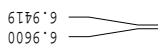

$9 L 9 C^{\circ} L$

$69 \varepsilon 9 \cdot L$

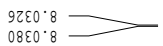

от $98: 8$

$079 \varepsilon^{\circ} 8$
$869 \varepsilon^{\circ} 8$

$09\left\llcorner\varepsilon^{\circ} 8\right.$

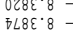

志

过

है

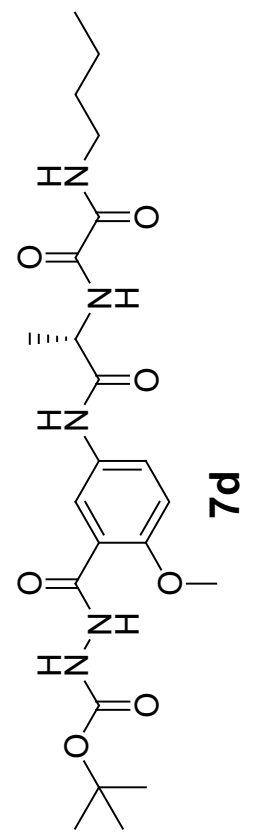

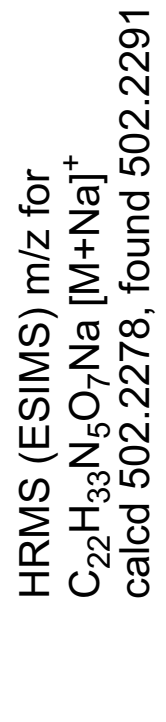

হั)

กิ

क $\infty \infty^{\circ}$

$\sum \underset{1}{\infty}$ N

世经合

(D) लำ

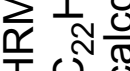

I O

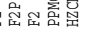


Supporting Information

S. Levin and J. S. Nowick

$00.0-$
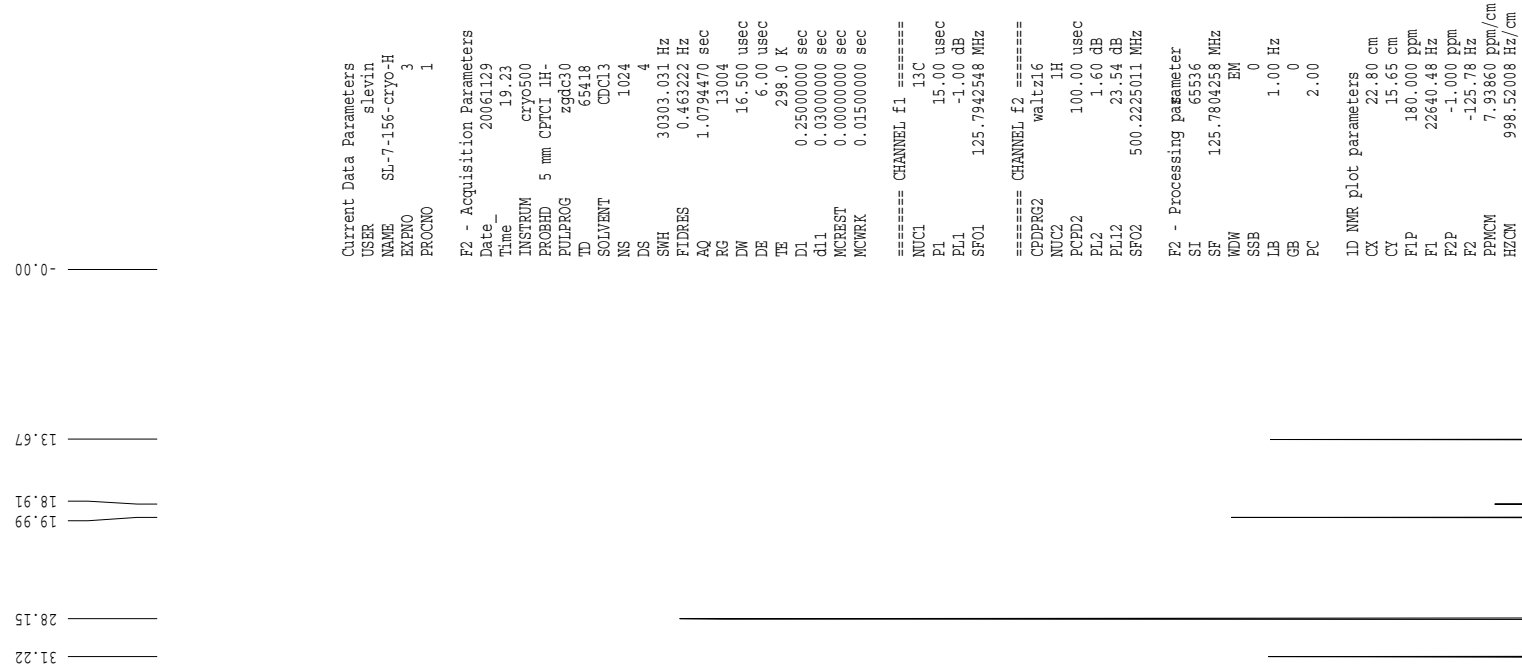

$\varepsilon \varsigma^{\circ} 6 \varepsilon-$

00.05

77.99
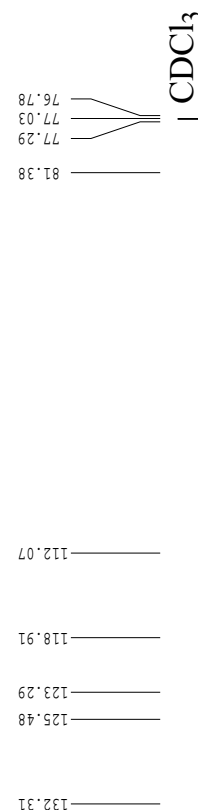

โร'วยโ-

SO .

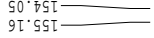

$L I \cdot 69 T$

$60^{\circ} 59 \tau$

$\angle 9 \cdot 69 \mathrm{I}$
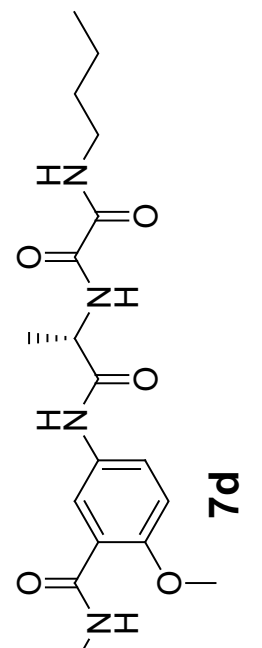

Iź
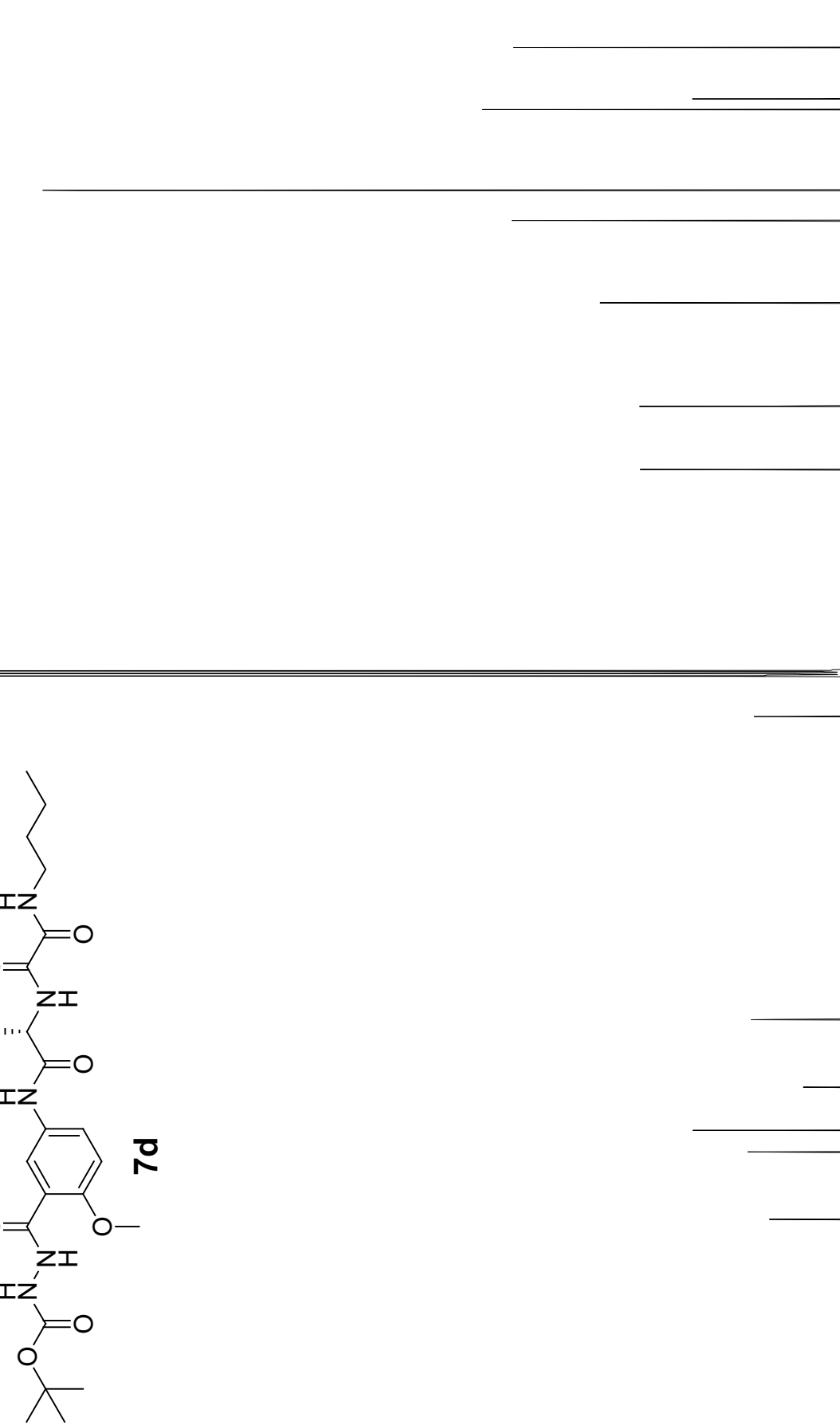


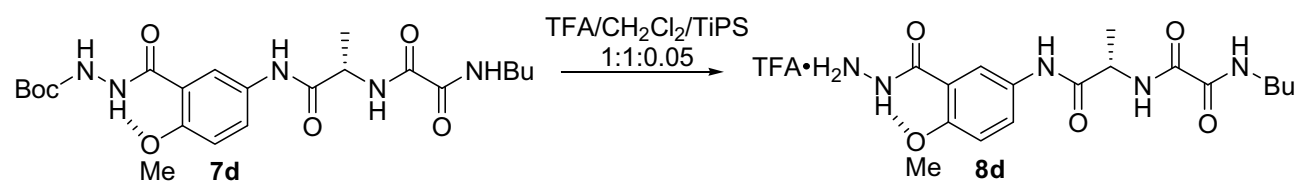

Salt 8d. Amide 7d (3.70 g, $7.37 \mathrm{mmol})$ was dissolved in a mixture of dichloromethane (100 $\mathrm{mL})$, trifluoroacetic acid $(100 \mathrm{~mL})$ and trisopropylsilane $(0.5 \mathrm{~mL})$. After $0.5 \mathrm{~h}$, the solution was concentrated and the residue triturated with the diethyl ether to yield $3.55 \mathrm{~g}(92 \%)$ of salt $\mathbf{8 d}$ as a white powder. 

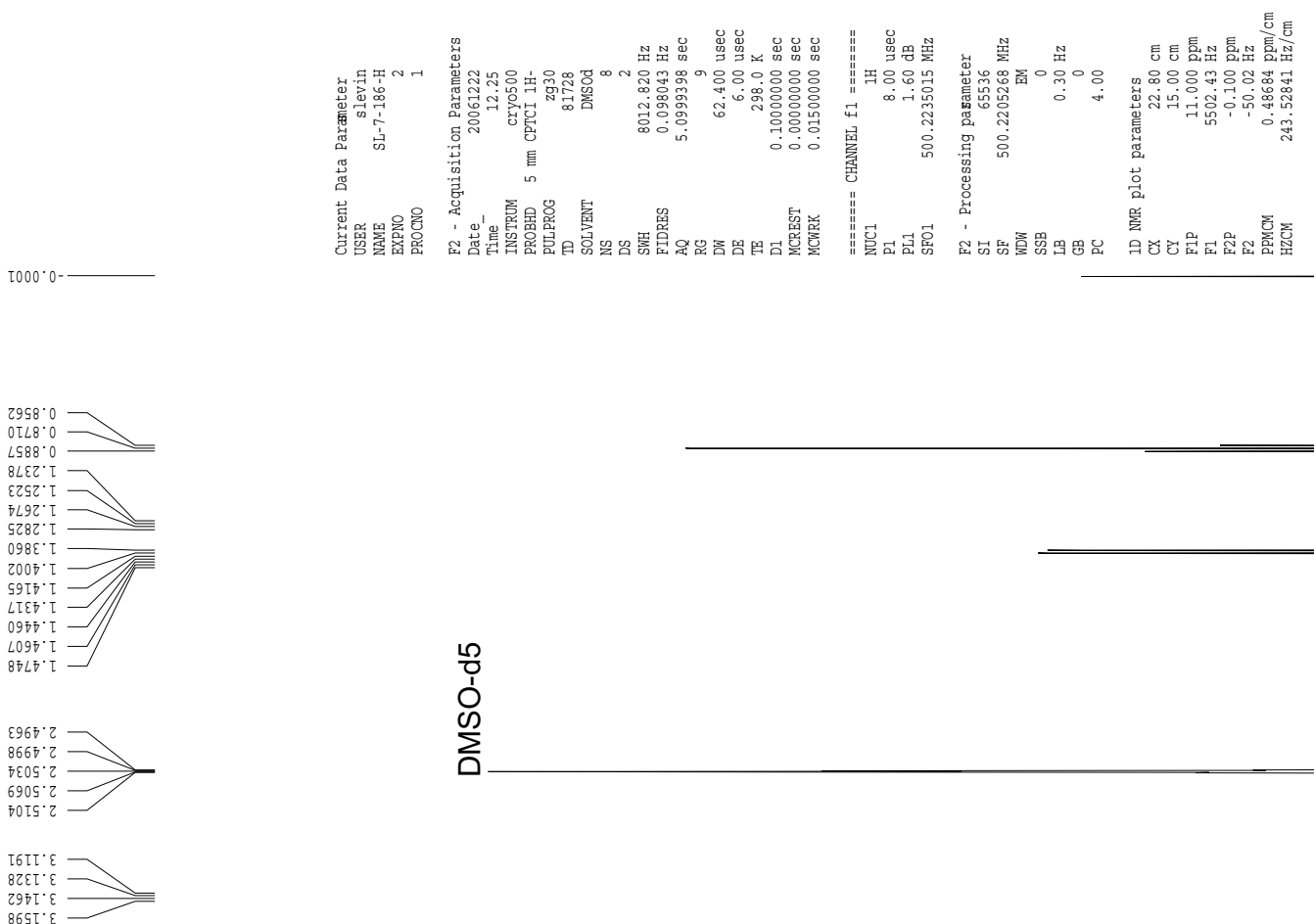

0
0
0
0
0
0

$28 L 8^{\circ} \varepsilon$

$090 \nabla^{\circ} \cdot 7$

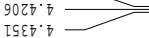

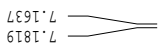

$9 L G L \cdot L-$
IEgL $L$

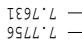

IT $8 L^{\circ} L$

$6720.8=$

ర్ర

(2)

管

FLZ9.8 -

$9279^{\circ} 8$
$\varepsilon \angle 8 L .8$

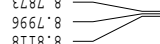

ह气

产

ठै

$815.8=$<smiles>C=C(CCCCC)C(=O)C(=O)C(=O)C(C)C(C)=O</smiles>

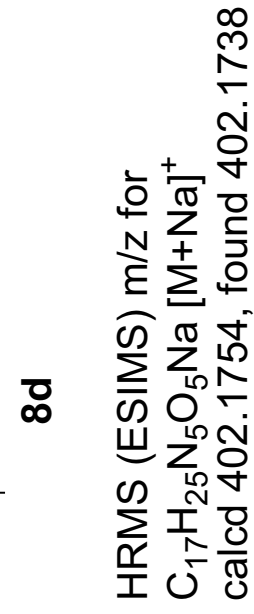

$+Z_{I}$
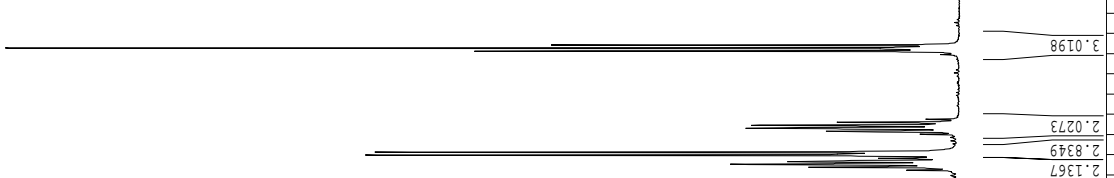

p

$\sum$

品

$\angle 9 z T^{\circ} 0 \tau$

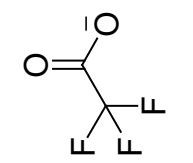

$\infty$

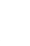

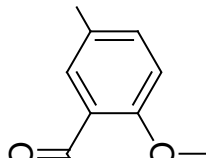

ZI

$z^{\prime}$

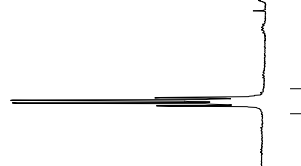

$2890^{\circ} \mathrm{Z}$

응

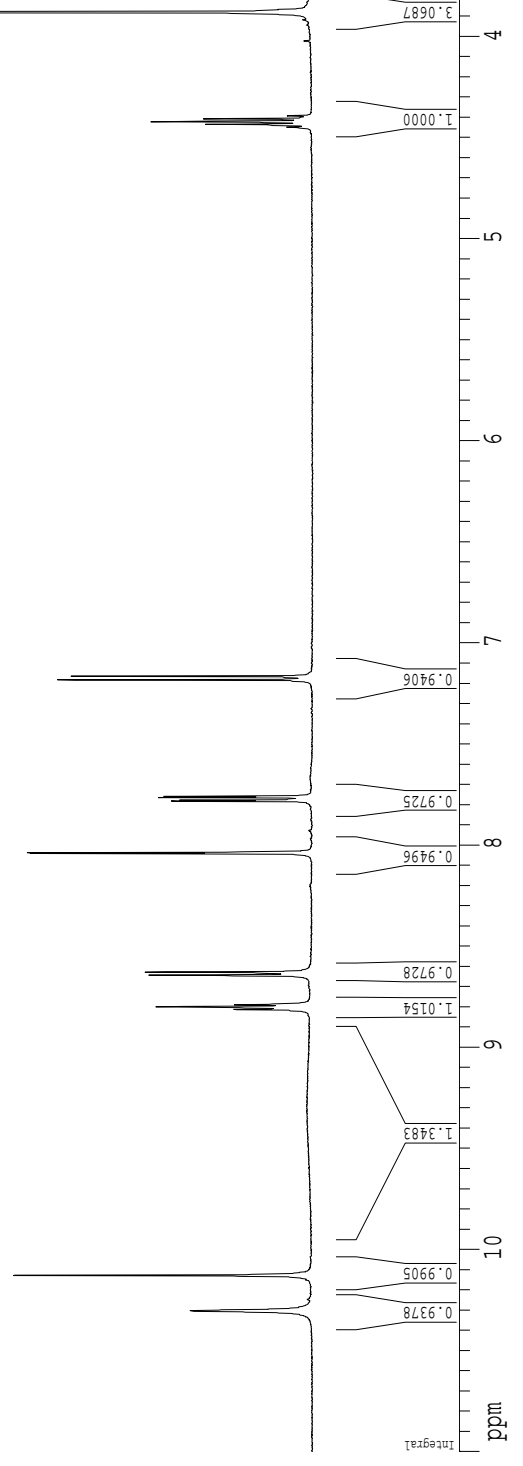



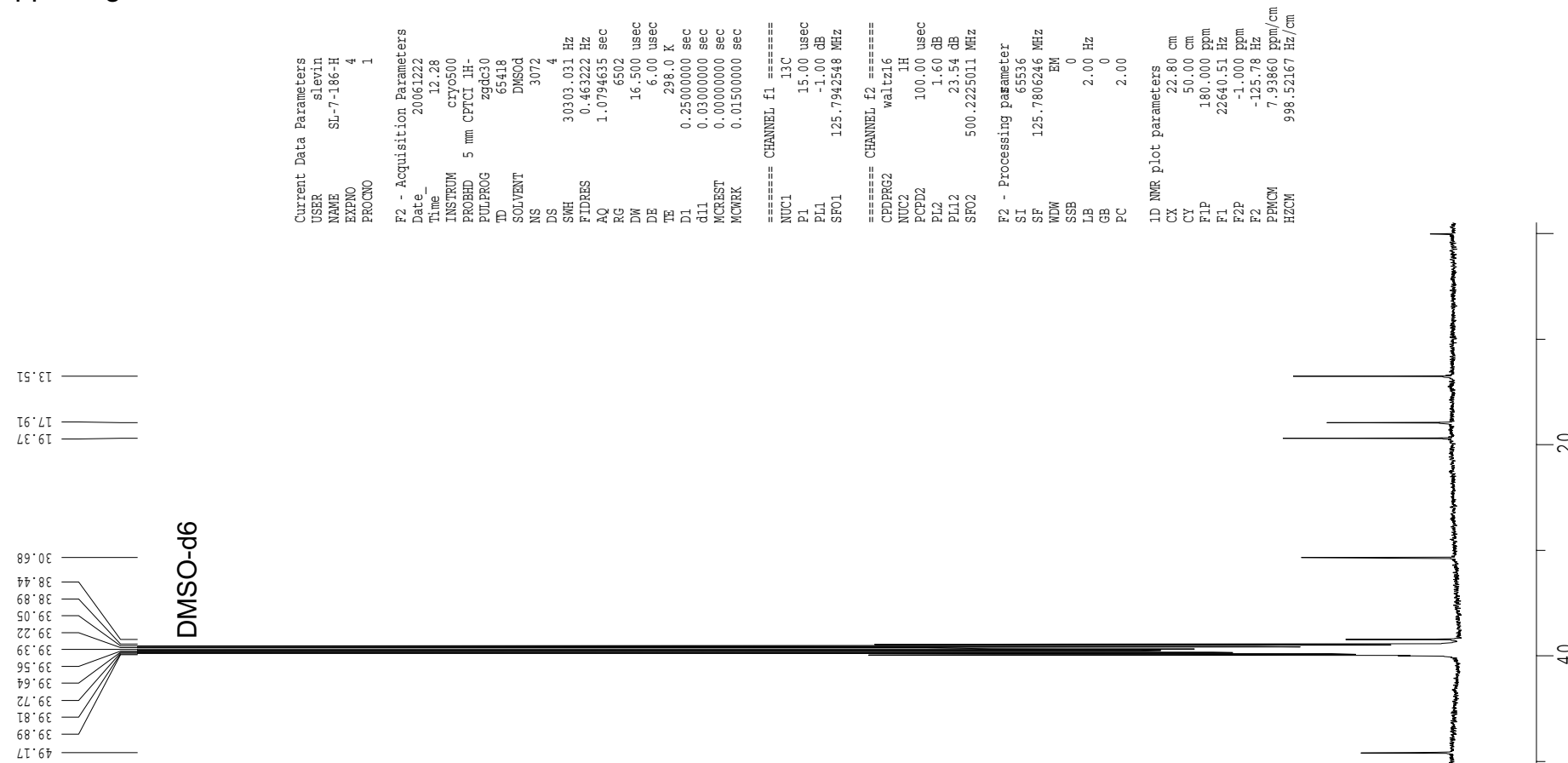

0
0
1
0
0
0

I0.99

$6 \pi^{\circ} \mathrm{ZTI}-$

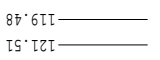

$\angle \varepsilon^{\circ} \ni Z T-$

โร'

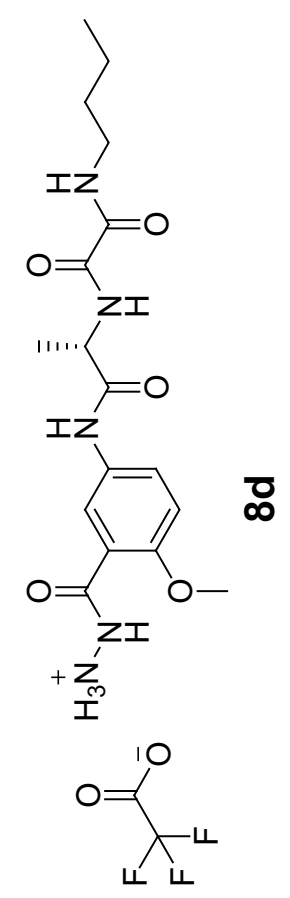




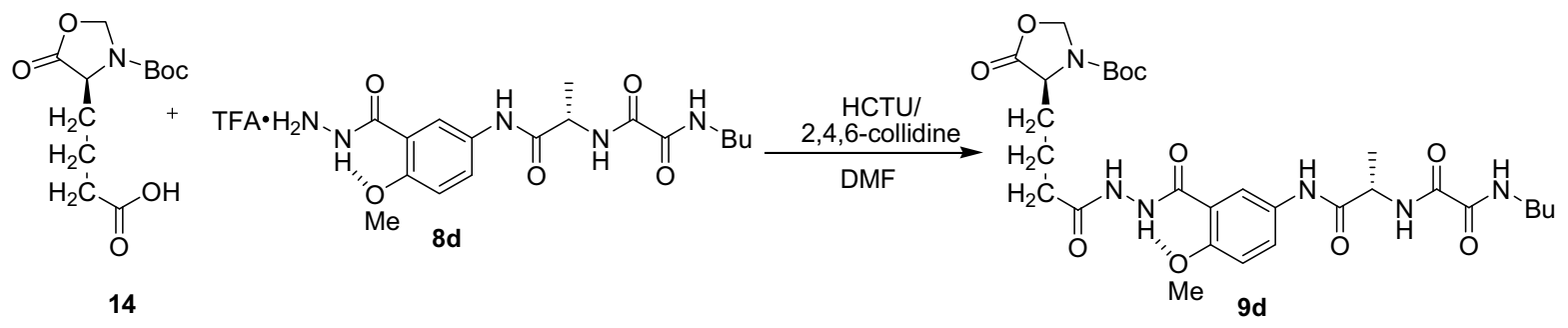

Oxazolidinone 9d. A solution of oxazolidinone $14^{3,4}(1.7 \mathrm{~g}, 6.1 \mathrm{mmol})$, HCTU $(2.8 \mathrm{~g}, 6.7$ $\mathrm{mmol})$, and 2,4,6-collidine $(2.0 \mathrm{~mL}, 15.2 \mathrm{mmol})$ in dimethylformamide $(50 \mathrm{~mL})$ was added to a solution of $8 \mathbf{d}(3.0 \mathrm{~g}, 6.1 \mathrm{mmol})$ and 2,4,6-collidine $(2.0 \mathrm{~mL}, 15 \mathrm{mmol})$ in dimethylformamide $(50 \mathrm{~mL})$. After $6 \mathrm{~h}$, the reaction mixture, was poured into $700 \mathrm{~mL}$ ice-water, and the resulting precipitate was filtered and washed with water $(200 \mathrm{~mL})$. Upon drying, $3.20 \mathrm{~g}(83 \%)$ of reasonably pure (90\% purity) compound 9d was isolated. Further purification was achieved using column chromatography (4:1 chloroform: $i-\operatorname{Pr}-\mathrm{OH})$ yielding $2.1 \mathrm{~g}(54 \%)^{5}$ of pure $9 \mathrm{~d}$ as a white solid.

\footnotetext{
${ }^{3}$ Olsen, R. K.; Ramasamy, K. J. Org. Chem. 1985, 50, 2264-2271.

${ }^{4} \mathbf{1 4}$ was synthesized according to the procedure: Tantry, S. J.; Kantaraju.; Suresh Babu, V. V. Tetrahedron Lett. 2002, 43, 9461-9462.

${ }^{5}$ The low yield is due to compound loss while trying to purify $9 \mathbf{d}$ with different solvent system.
} 


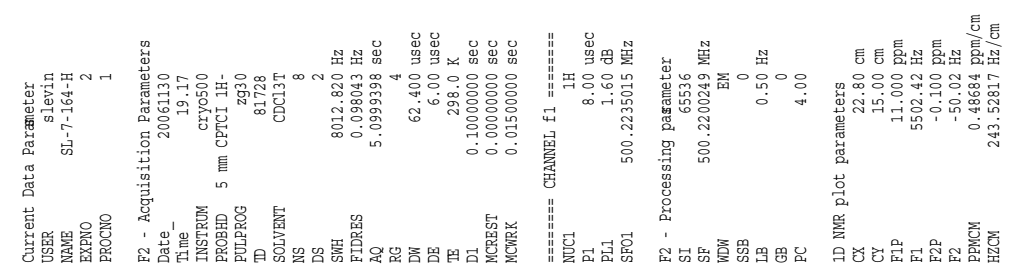

$0000 \cdot 0-$

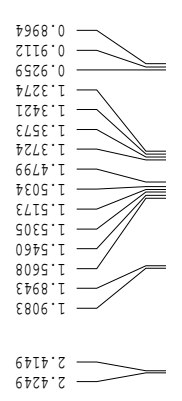

$\not 262 \cdot \varepsilon \square$
$\varepsilon 62 \varepsilon \cdot \varepsilon$
$97 \nabla \varepsilon \cdot \varepsilon$

$\varpi \mp L 6 \cdot \varepsilon-$

$2 \mp 9 L^{\circ} \succcurlyeq$

$\tau I 6 \tau \cdot s$
$0002 \cdot 9$

$\varepsilon 006.9$
$98 \mathrm{~T} 6.9$

$6 Z L Z \cdot L$

TLt8: $L$

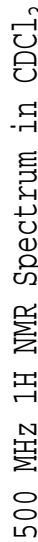

$2980^{\circ} 6$

(2)

(n)

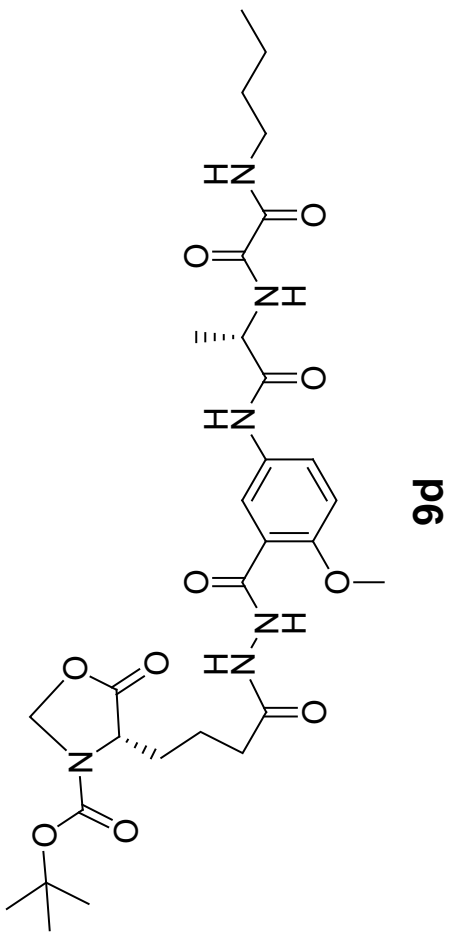

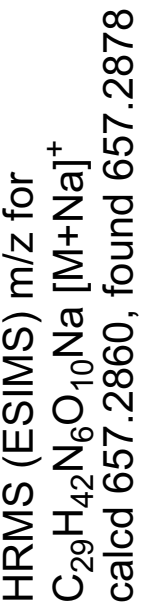

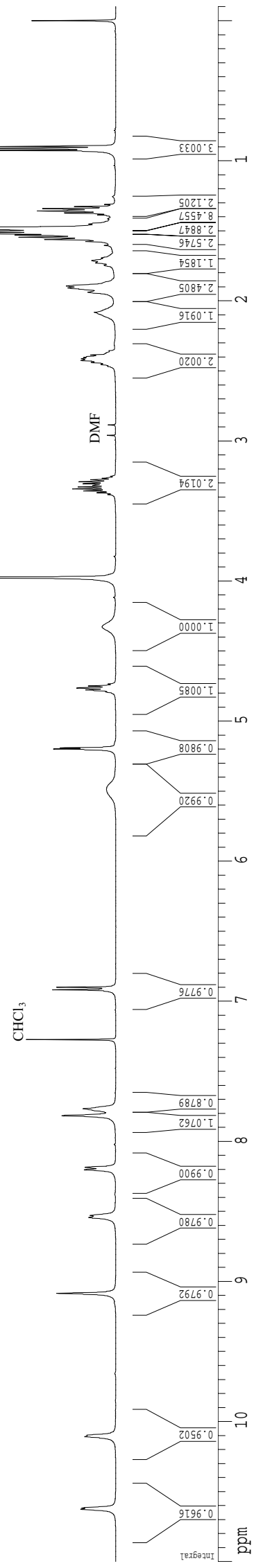


Supporting Information

S. Levin and J. S. Nowick
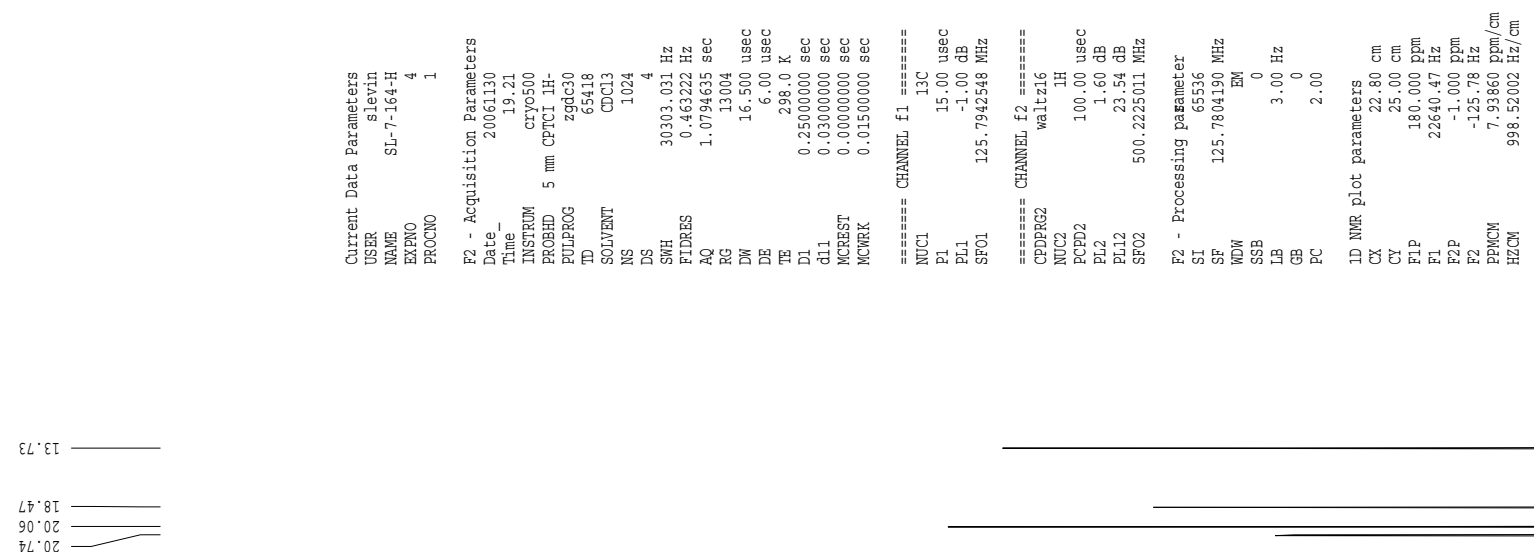

$90^{\circ} 02$
$7 L \cdot 02$

$0 \varepsilon \cdot 8 \tau$
$\emptyset \tau \cdot 0 \varepsilon$
$\varpi \tau^{\circ} \tau \varepsilon$
$0 \varepsilon \cdot \varepsilon \varepsilon$

$79 \cdot 6 \varepsilon$

60.05

$\varepsilon ૬ \cdot 9 \varsigma$

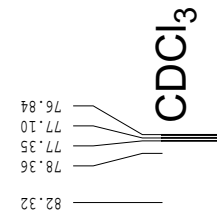

$\varepsilon \tau^{\prime} \cdot \mathrm{ZTI}$

己

-.

역

을

Оิ

ठ

车

독

要

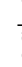

थै

m

芷

늠

$2 \pi \cdot 8 \mathrm{IT}$

I6. $2 Z \mathrm{~L}$

$96.9 Z \mathrm{-}$

To ' $\varepsilon \varepsilon โ$

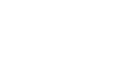

$66^{\circ} \varepsilon \varsigma \tau$

$07 \cdot 6 \mathrm{TI}$

$6 \mathrm{~T}^{\circ} 09 \mathrm{~T}-$
$80^{\circ} \mathrm{T}$

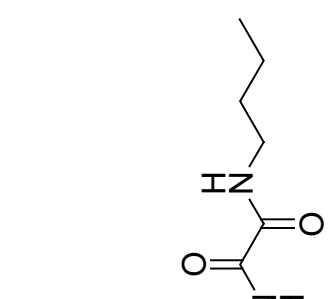

$06 \cdot 89 \tau$

$06.89 \mathrm{I}$
$7 L \cdot 69 \mathrm{~L}$

IF. $\varepsilon L I$
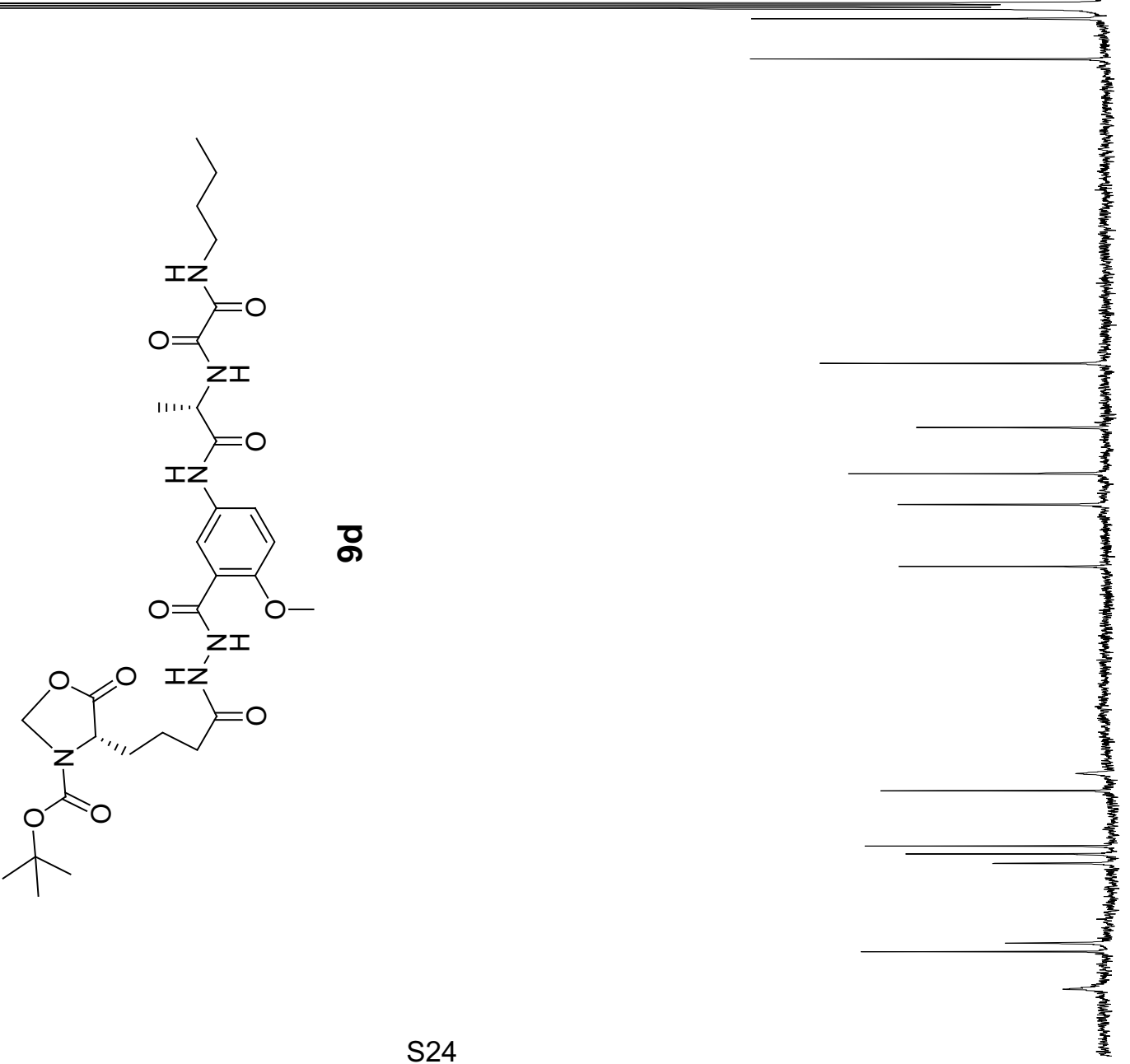


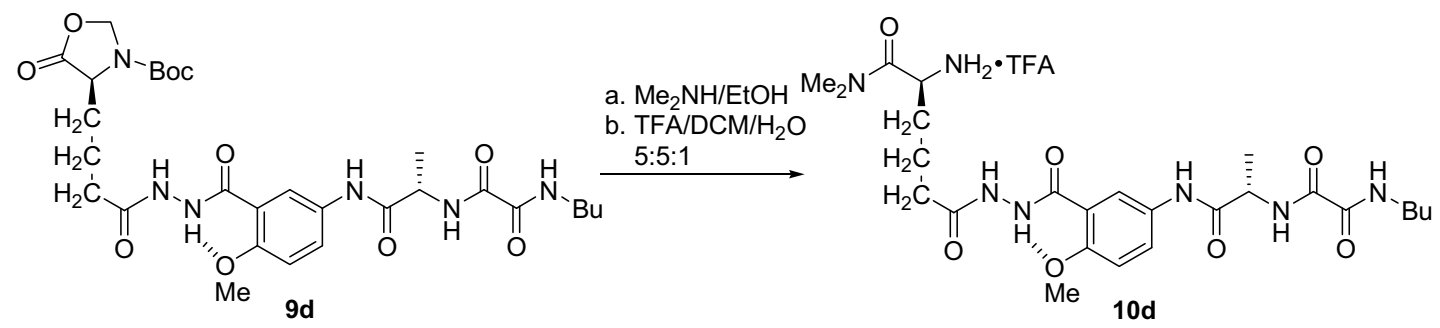

Salt 10d. A 5.6 M solution of dimethylamine in ethanol (15 mL, $84 \mathrm{mmol})$ was added to a stirred mixture of 9d (1.5 g, $2.2 \mathrm{mmol})$ and ethanol $(100 \mathrm{~mL})$. The mixture was warmed to form a homogeneous solution, and the solution was stirred for $0.5 \mathrm{~h}$ and then concentrated under vacuum. The white residue was dissolved in a deprotection cocktail (100 $\mathrm{mL}, 5: 5: 1$ TFA:DCM: $\mathrm{H}_{2} \mathrm{O}$ ) and the resulting solution was left to react for $1 \mathrm{~h}$. The solution was concentrated under vacuum and the residue was triturated with diethyl ether to afford $1.59 \mathrm{~g}$ $(101 \%)$ of $10 d$ as a white powder, which was used in the next step without purification. An analytical sample was purified by RP-HPLC $\left(\mathrm{C}_{18}\right.$ column, gradient $20 \% \mathrm{CH}_{3} \mathrm{CN}-90 \% \mathrm{H}_{2} \mathrm{O}$ to $\left.90 \% \mathrm{CH}_{3} \mathrm{CN}-10 \% \mathrm{H}_{2} \mathrm{O}\right)$. 

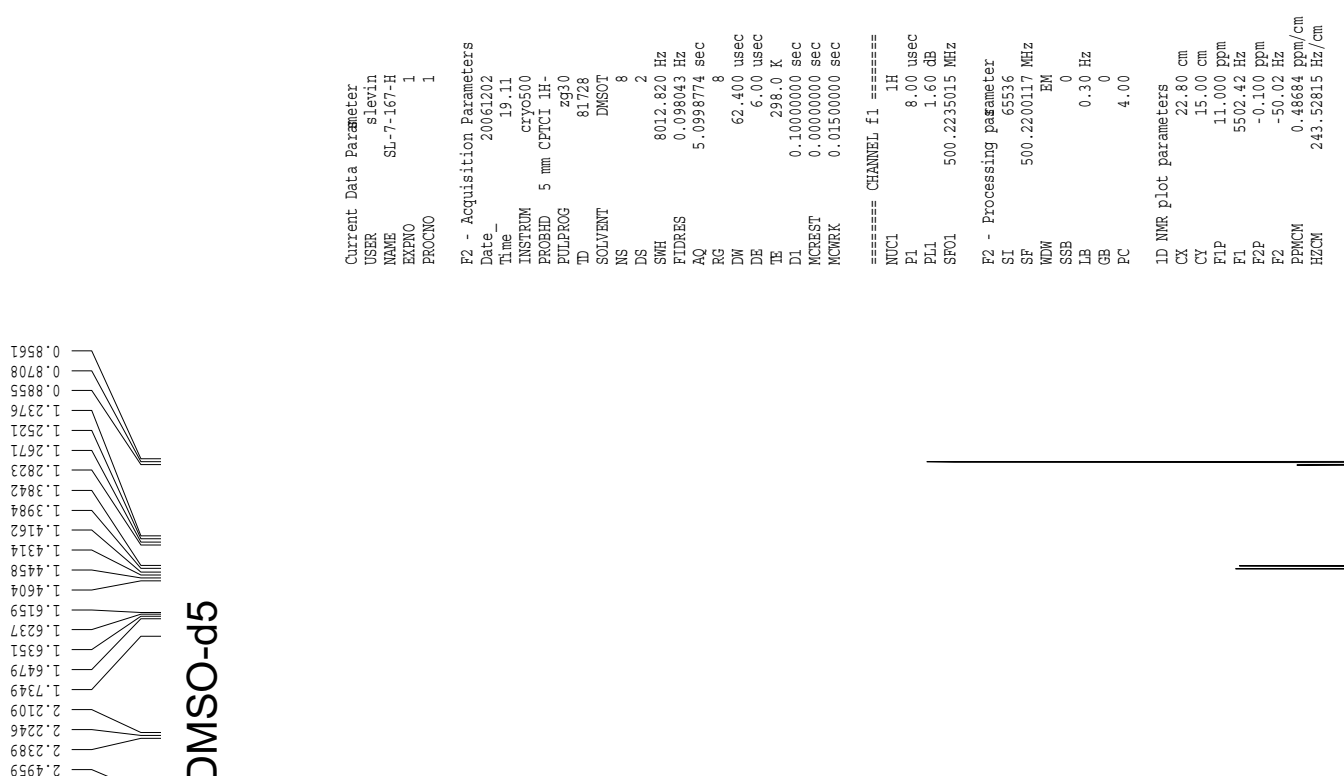

$6960^{\circ} \cdot 2$
$7667^{\circ} \mathrm{r}$

$00^{\circ} 09^{\circ} z$

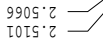

$\square \mp \angle 8^{\circ} z$

โ506:

$\varepsilon 900^{\circ} \varepsilon$

28IT. $\varepsilon$

$\varepsilon \operatorname{lit}^{\circ} \cdot \varepsilon=$

$9978 \cdot \varepsilon=$

2907

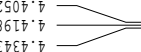

10
0
0
0
0

${ }_{89 S T \cdot L L}=$

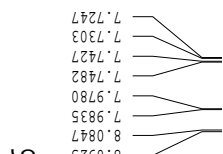

0 07т9.8 $2629 \cdot 8 \longrightarrow$ T68L:8

†T08.8
$9 \varepsilon \tau 8.8$

巨్ర

号

ชి

$\sum_{7566.6} \longrightarrow$

$9200^{\circ} \cdot 0$

可

عOSZ 0 工 $\longrightarrow$

愛

음
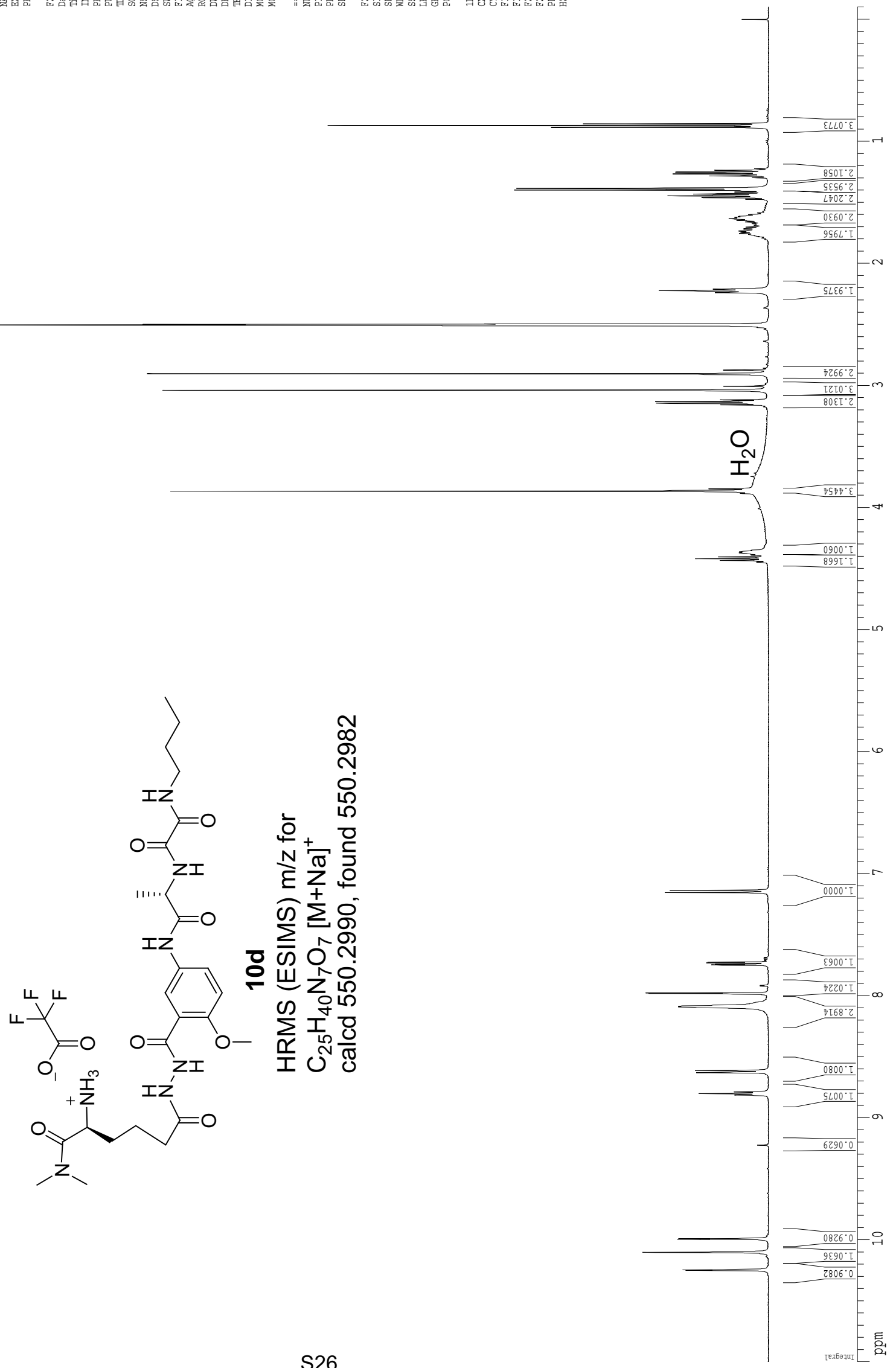

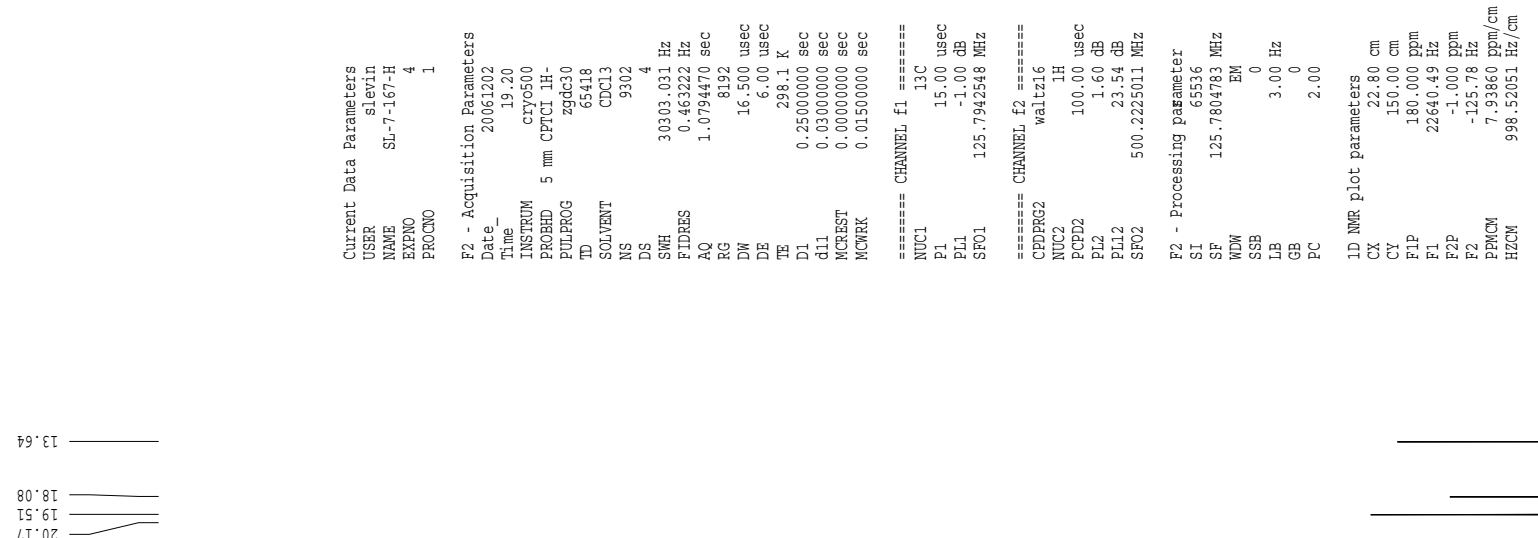

${ }_{L \tau}^{\operatorname{TS} .02}=$

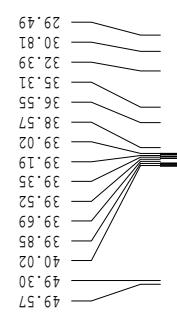

IZ.99

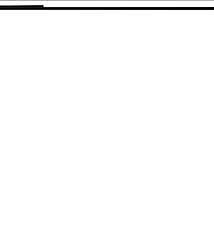

$29^{\prime} \cdot 2 \pi$
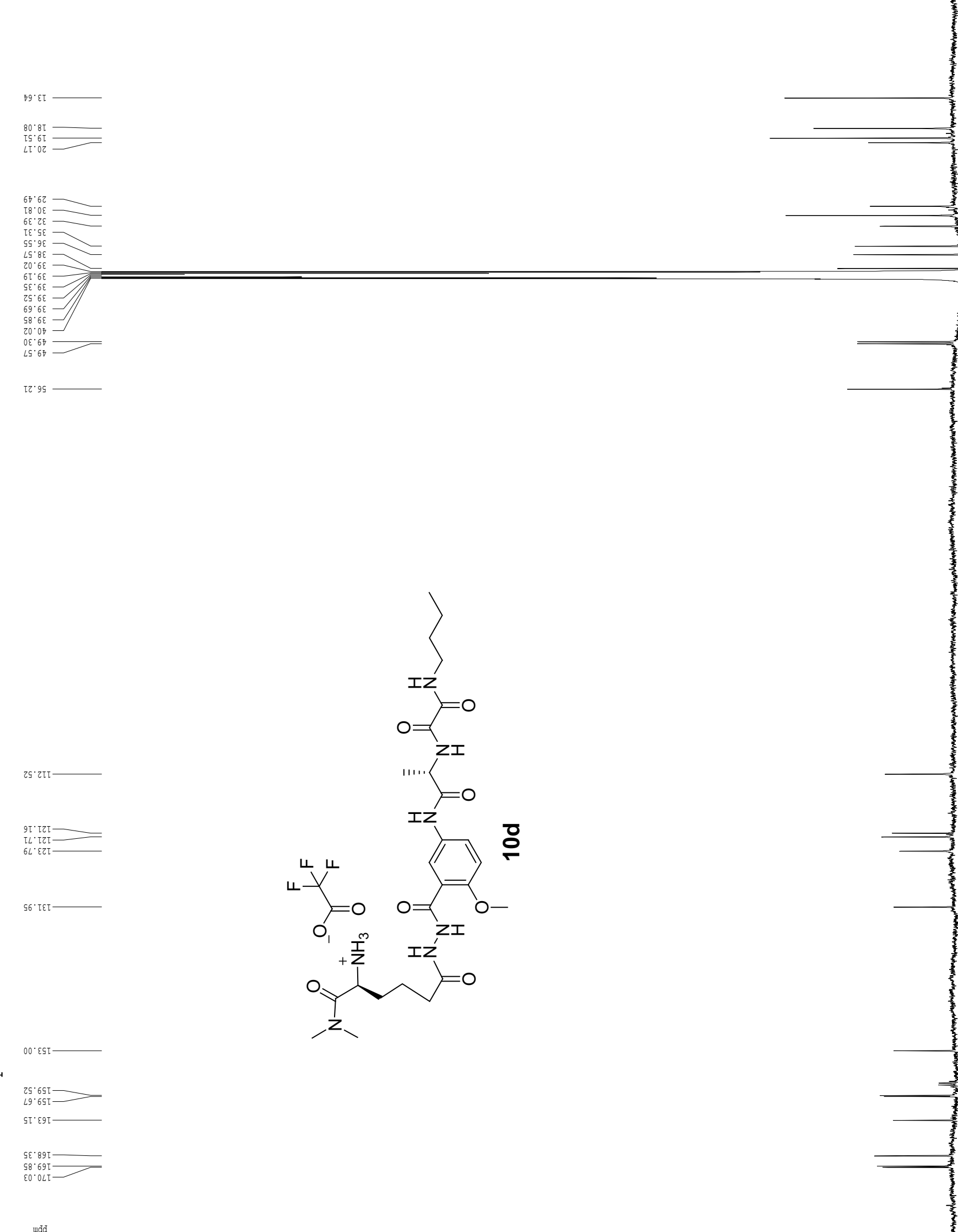
<smiles>CCCC(NC(C)=O)C(=O)NNC(=O)C(=O)NCC(=O)Nc1ccc(OC)c(C(=O)NC(C)C(=O)NC(C)(C)C)c1</smiles>
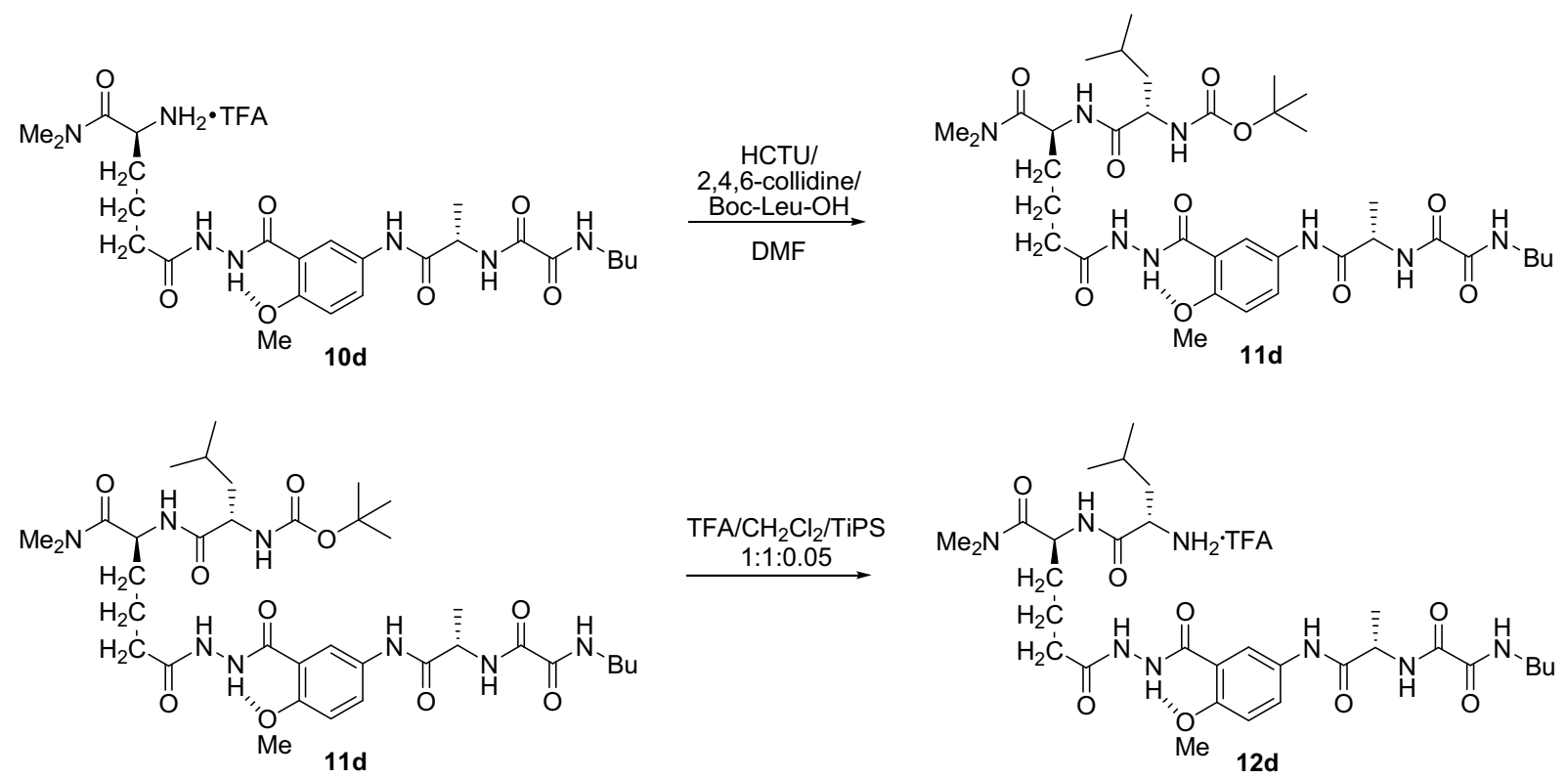

Salt 12d. A solution of 10d (0.52 g, $0.80 \mathrm{mmol})$, HCTU (0.39 g, $0.94 \mathrm{mmol})$, and 2,4,6-collidine $(1.0 \mathrm{~mL}, 7.6 \mathrm{mmol})$ in DMF $(50 \mathrm{~mL})$ was stirred at room temperature for $1.5 \mathrm{~h}$ and then concentrated under vacuum to form a brown oil. The oil was triturated with water and dried under vacuum. Purification by column chromatography (9:1 chloroform-isopropanol) yielded $0.33 \mathrm{~g}(55 \%)$ of $11 \mathrm{~d}$ as a white solid, which was deprotected for $0.5 \mathrm{~h}$ with cleavage cocktail (100 mL, 1:1:0.05 TFA- $\mathrm{CH}_{2} \mathrm{Cl}_{2}$-triisopropylsilane). The solution was concentrated under vaccum and the residue triturated with diethyl ether to afford $0.25 \mathrm{~g}(76 \%)$ of $\mathbf{1 2 d}$ as a white solid. The compound was used in the next step without further purification. 

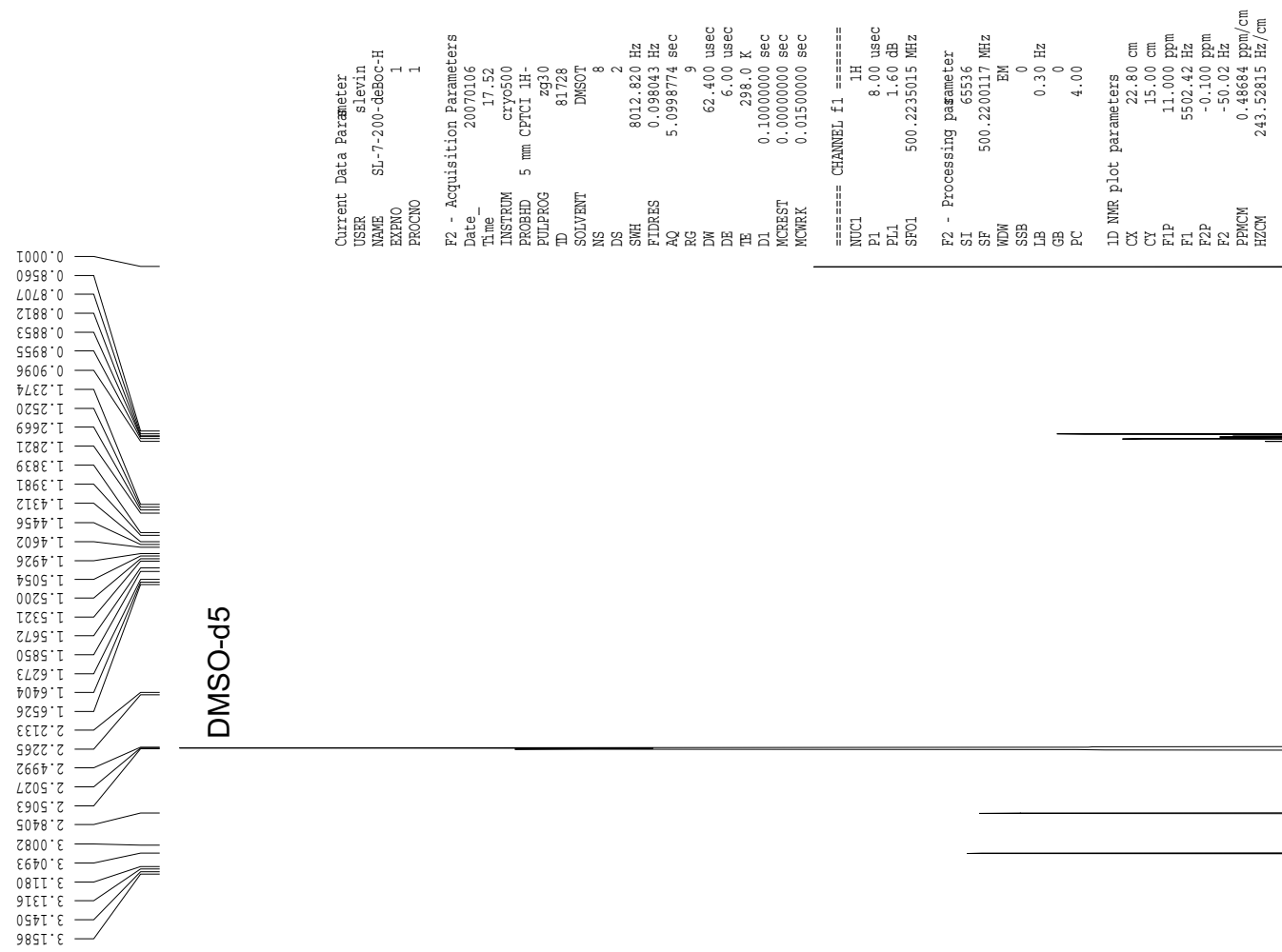

$\tau 978^{\circ} \varepsilon$
$L \mp 98^{\circ} \varepsilon$

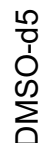
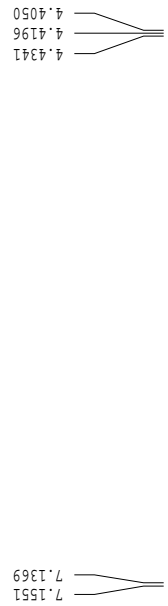

S9ZL:L

$60 \varepsilon L \cdot L$
$\nabla \varepsilon \nabla L \cdot L$

$\forall E \mp L$
$88 \pi L$

${ }_{55 L 6^{\circ} L}$

6260.8
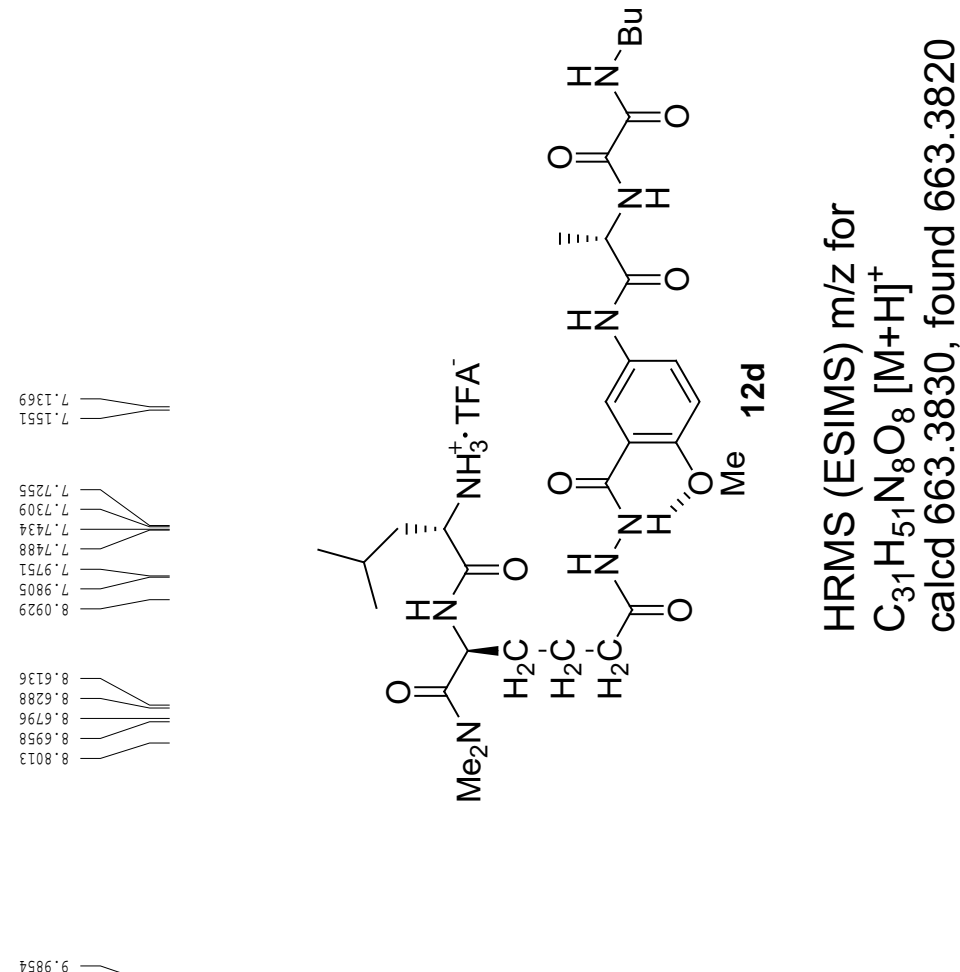

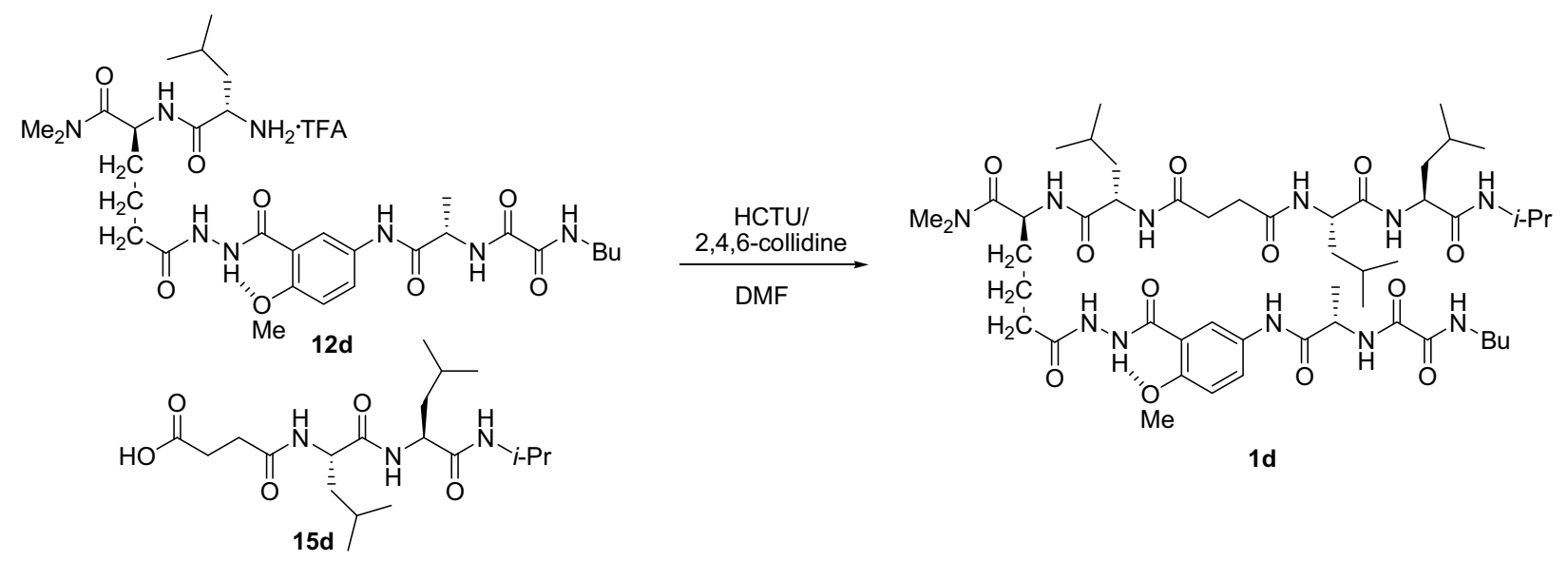

Artificial $\beta$-sheet 1d. A mixture of 12d (0.070 g, $0.090 \mathrm{mmol}), 15(0.035 \mathrm{~g}, 0.091 \mathrm{mmol})$ and 2,4,6-collidine $(0.12 \mathrm{~mL}, 0.91 \mathrm{mmol})$ in dimethylformamide $(10 \mathrm{~mL})$ was allowed to react for $3.5 \mathrm{~h}$ at room temperature. The solution was concentrated under vacuum and residue was partitioned between dichloromethane $(50 \mathrm{~mL})$ and water $(50 \mathrm{~mL})$. The water layer was washed extra dichloromethane $(50 \mathrm{~mL})$. The organic layers were combined and concentrated to give $0.060 \mathrm{~g}(64 \%)$ of $1 \mathbf{d}$ as a white powder, which was further purified by the recrystallization from acetonitrile ( 2 times), to yield $0.030 \mathrm{~g}(32 \%)$ of $\mathbf{1 d}$ as a white powder. 


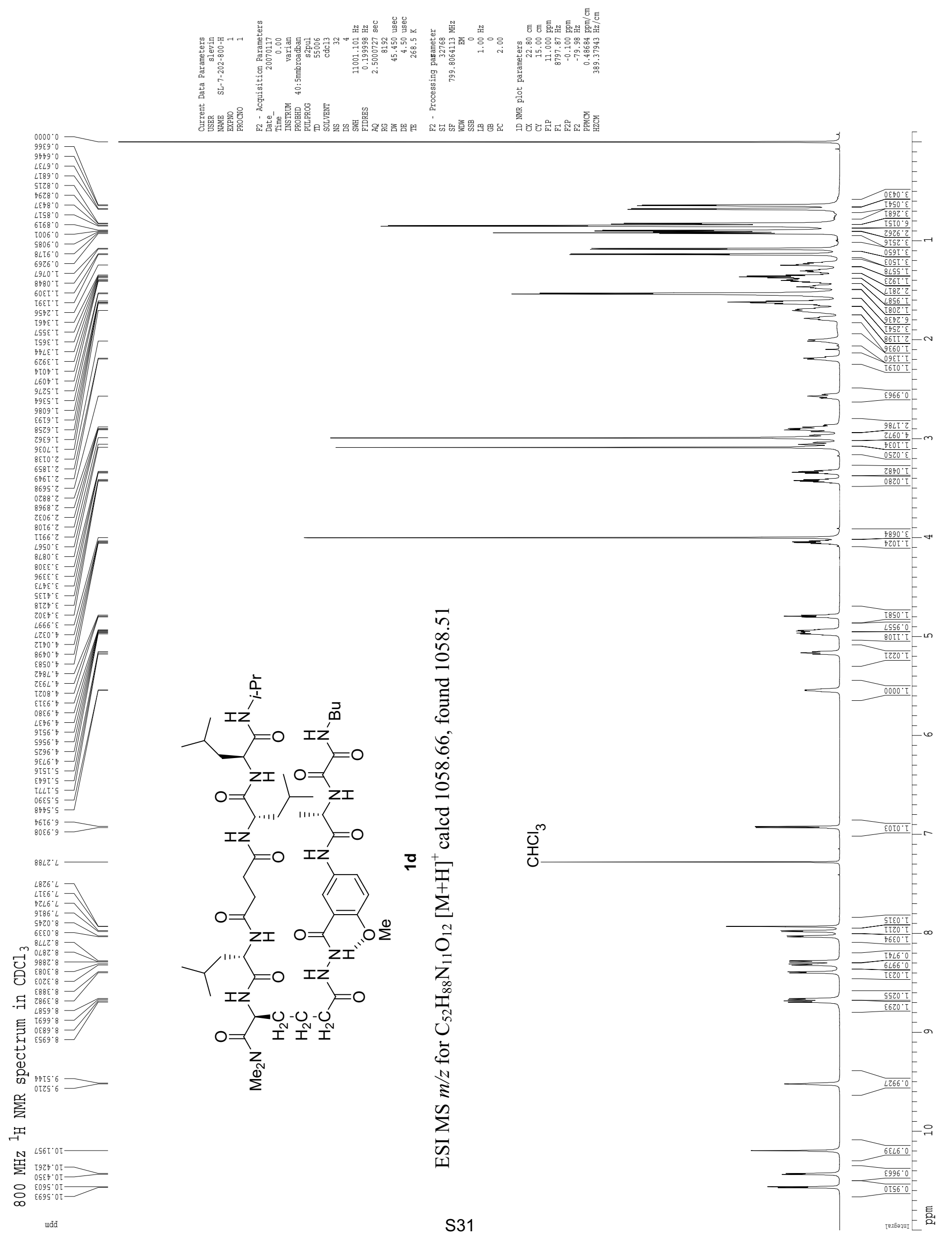




\section{Spectroscopic DATA for the compound 1a}

$\left(10 \mathrm{mM}, \mathrm{CDCl}_{3} 268 \mathrm{~K}\right)$

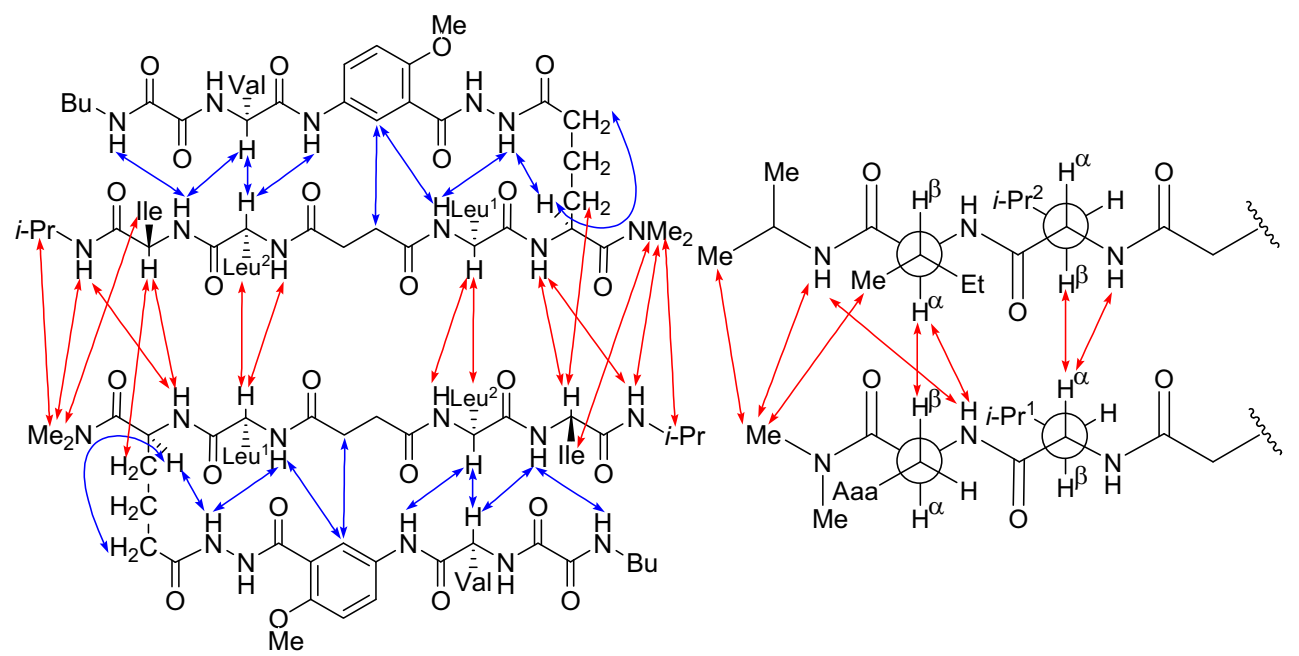

Key NOEs assigned as intermolecular are represented with red arrows

Key NOEs assigned as intramolecular are represented with blue arrows

Key data for amino acids.

\begin{tabular}{|c|c|c|c|}
\hline Amino acid & $\mathrm{H}_{\alpha}$ Shift ppm & NH Shift ppm & ${ }^{3} J_{\mathrm{NH} \alpha} \mathrm{Hz}$ \\
\hline Aaa & 5.15 & 8.57 & 8.4 \\
\hline $\mathrm{R}_{1}=\mathrm{Leu}^{1}$ & 5.51 & 8.52 & 7.7 \\
\hline $\mathrm{R}_{2}=\mathrm{Leu}^{2}$ & 5.09 & 8.24 & 9.7 \\
\hline $\mathrm{R}_{3}=\mathrm{Ile}$ & 4.73 & 8.70 & 10.1 \\
\hline $\mathrm{R}_{4}=\mathrm{Val}$ & 4.49 & 8.05 & 9.4 \\
\hline
\end{tabular}

Aminoadipic acid (Aaa) $\delta$ proton resonances.

pro-R: 2.92 ppm, t, (overlap)

pro-S: $2.18 \mathrm{ppm}, \mathrm{td},\left(J_{1}=11.5 \mathrm{~Hz} ; J_{2}=7.0 \mathrm{~Hz}\right)$

anisotropy $=0.74 \mathrm{ppm}$

Key data for other NH protons.

\begin{tabular}{|c|c|c|}
\hline Proton & NH Shift ppm & $J \mathrm{~Hz}$ \\
\hline Ar-NH & 10.29 & - \\
\hline Hydrazide Ar & 10.67 & 7.7 \\
\hline Hydrazide Aaa & 10.49 & 7.7 \\
\hline Bu-NH & 9.62 & 5.5 \\
\hline$i$-Pr-NH & 8.00 & 8.1 \\
\hline
\end{tabular}

ESI MS $m / z$ for $\mathrm{C}_{52} \mathrm{H}_{88} \mathrm{~N}_{11} \mathrm{O}_{12}[\mathrm{M}+\mathrm{H}]^{+}$calcd 1058.66, found 1058.51. 

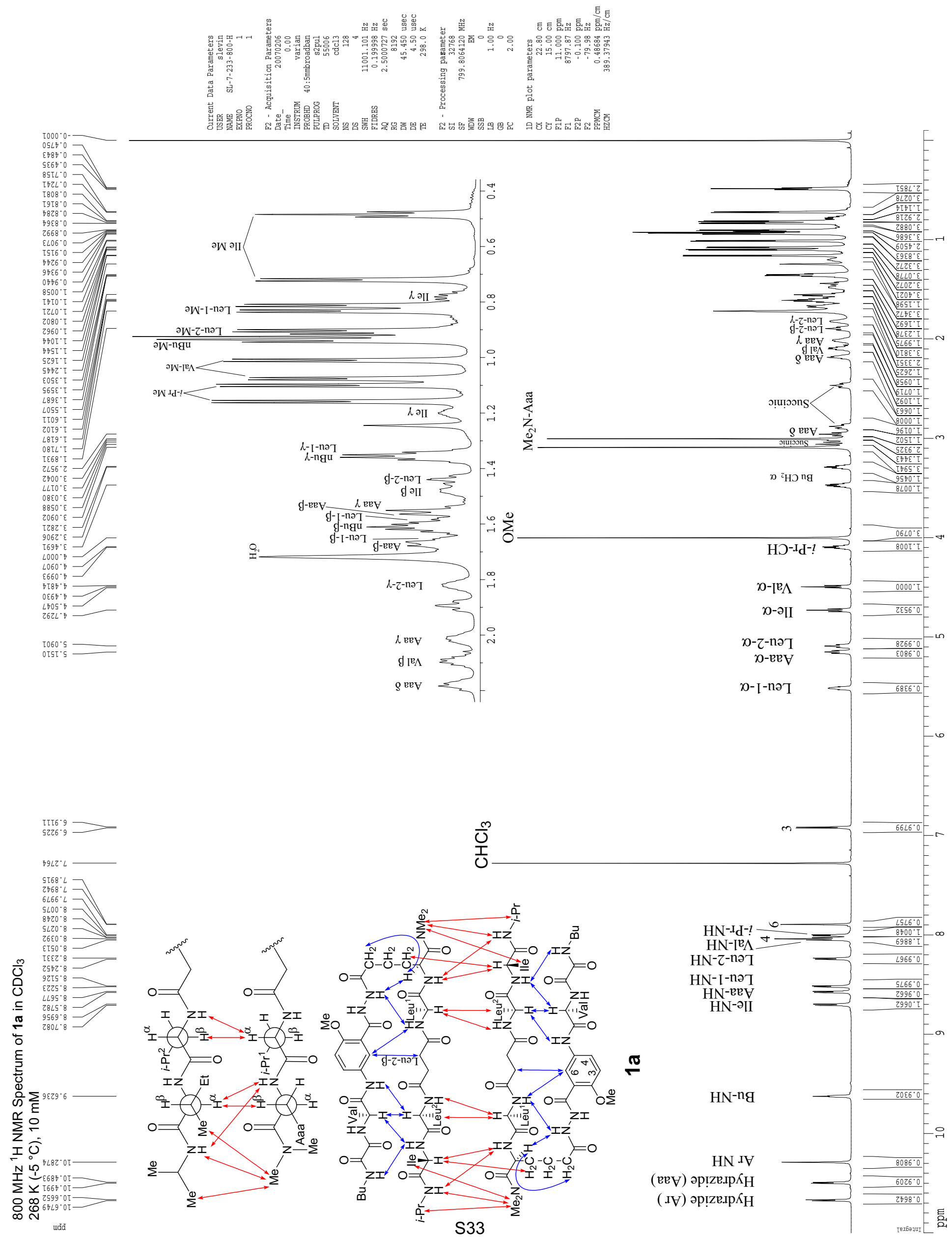


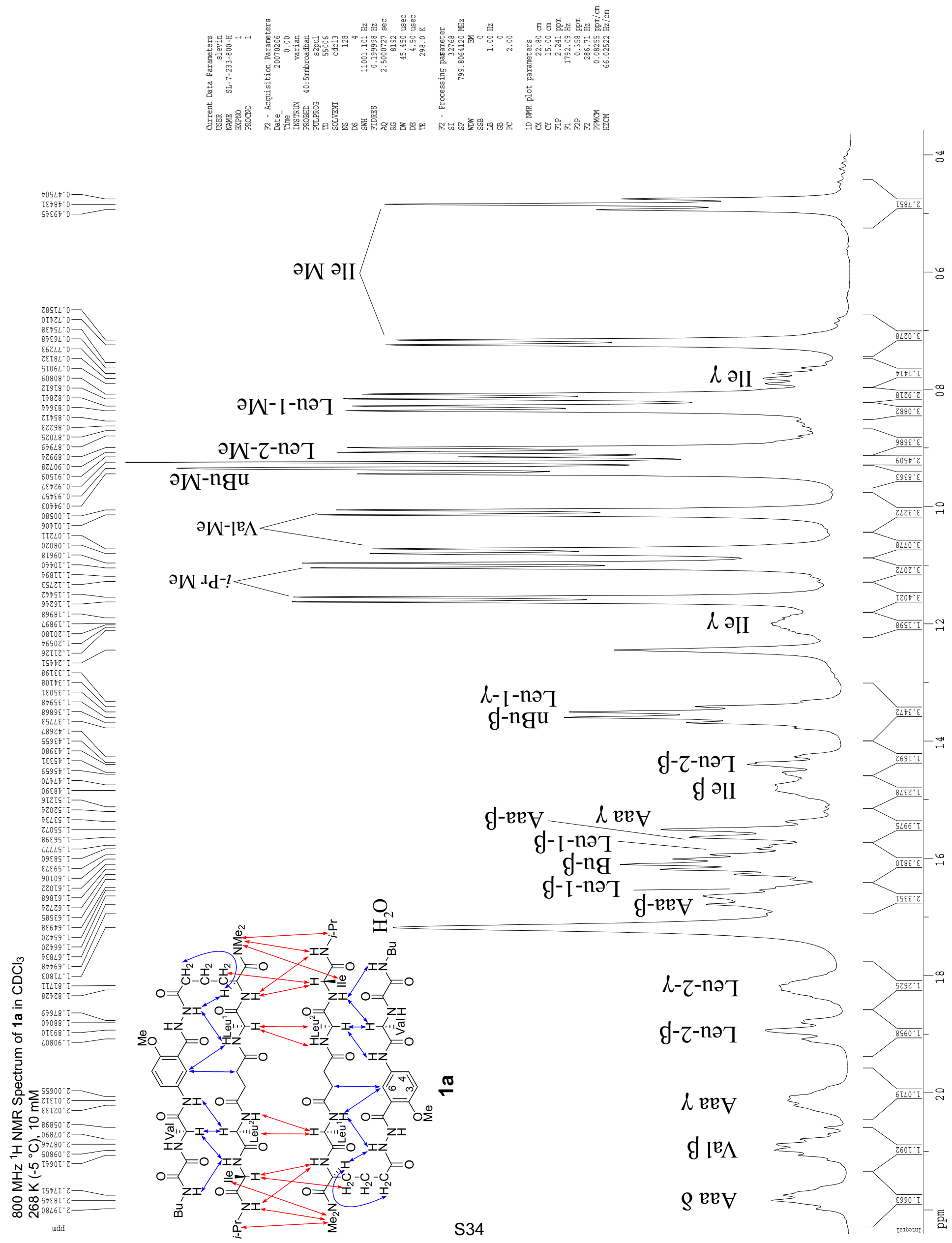




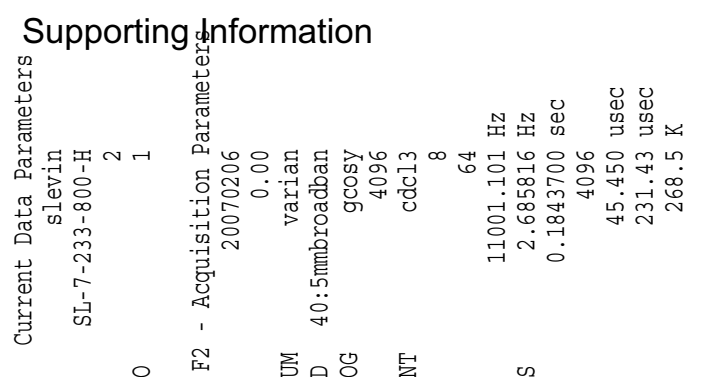

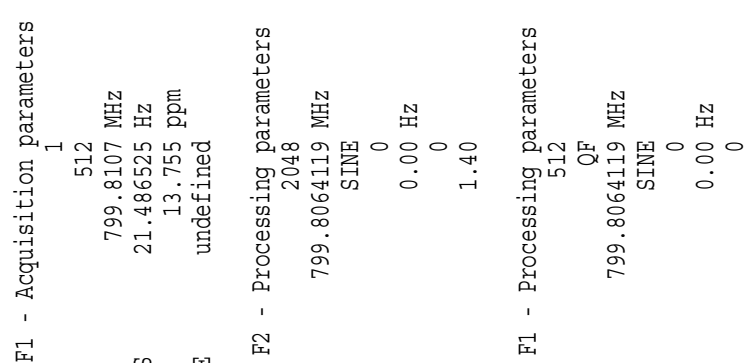

표
S. Levin and J. S. Nowick

壳

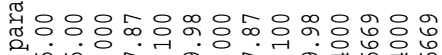

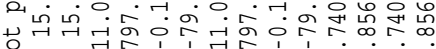
要 ลे

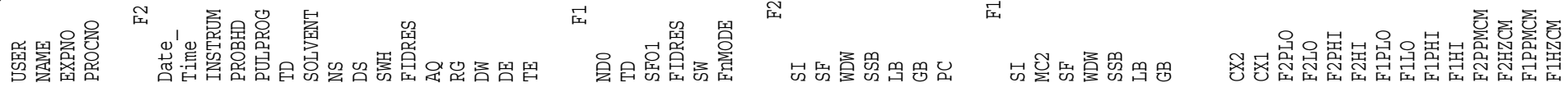

$\sim$

\&

6

우 흠

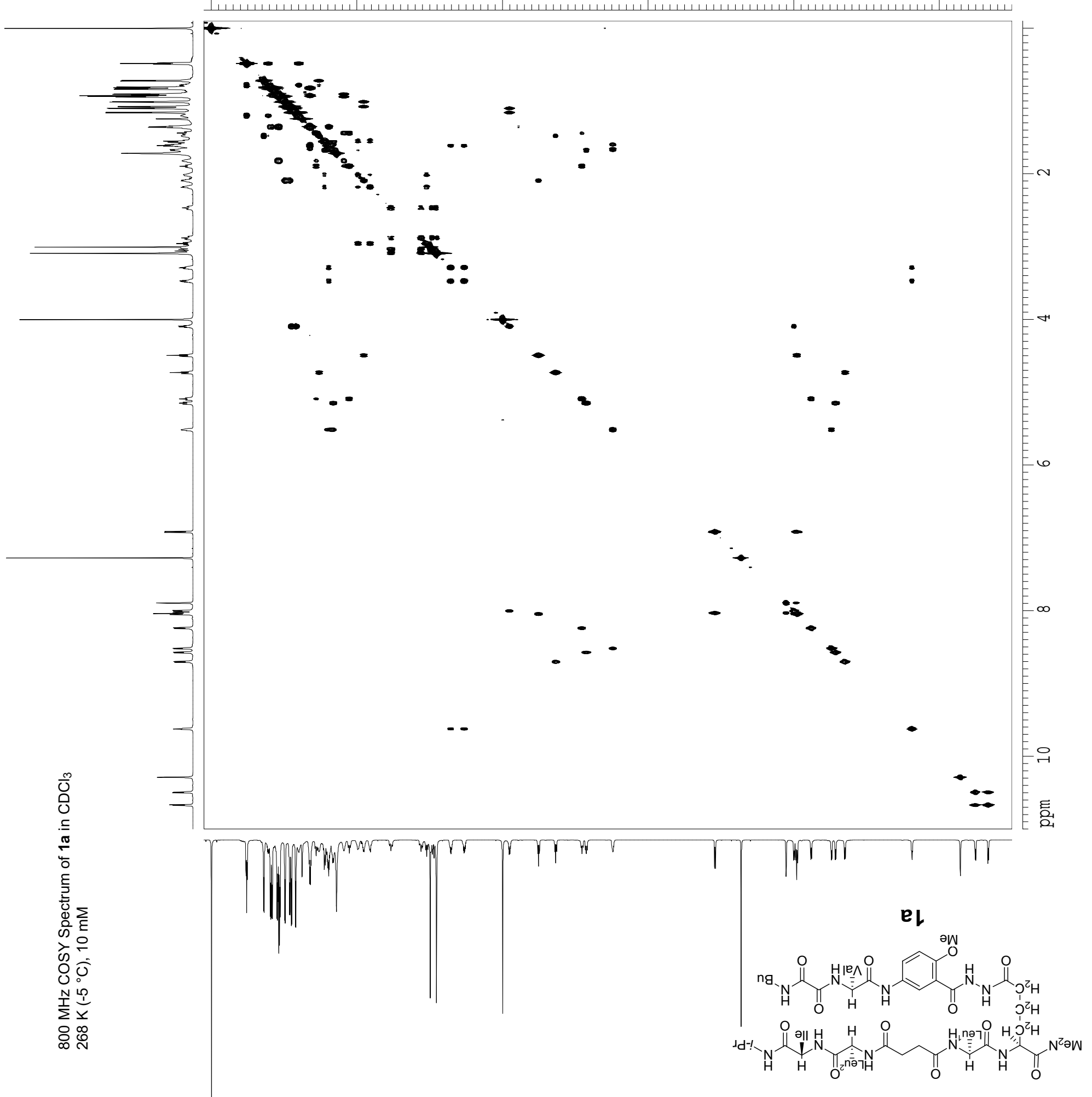


Supporting Information

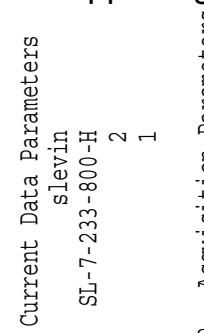

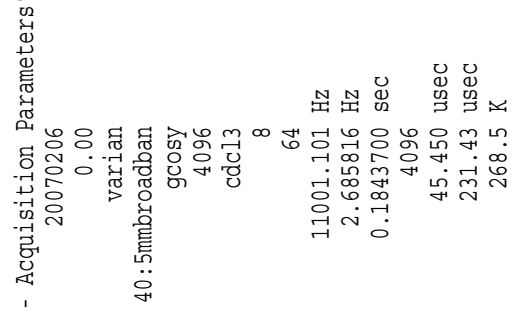

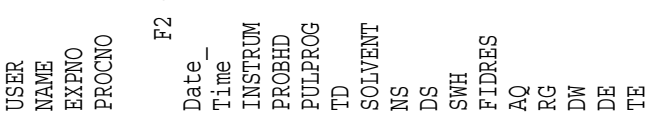

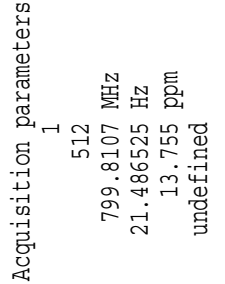

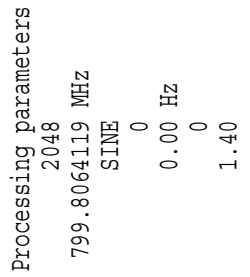

学

兵

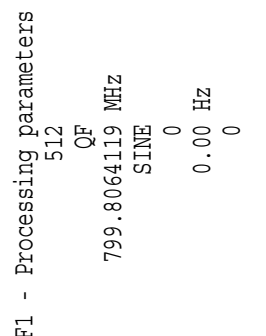

S. Levin and J. S. Nowick

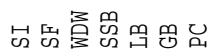

(1)

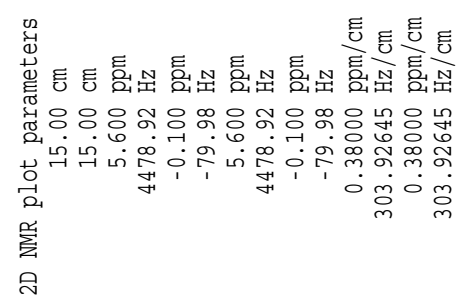

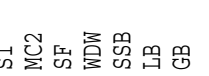

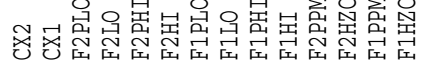

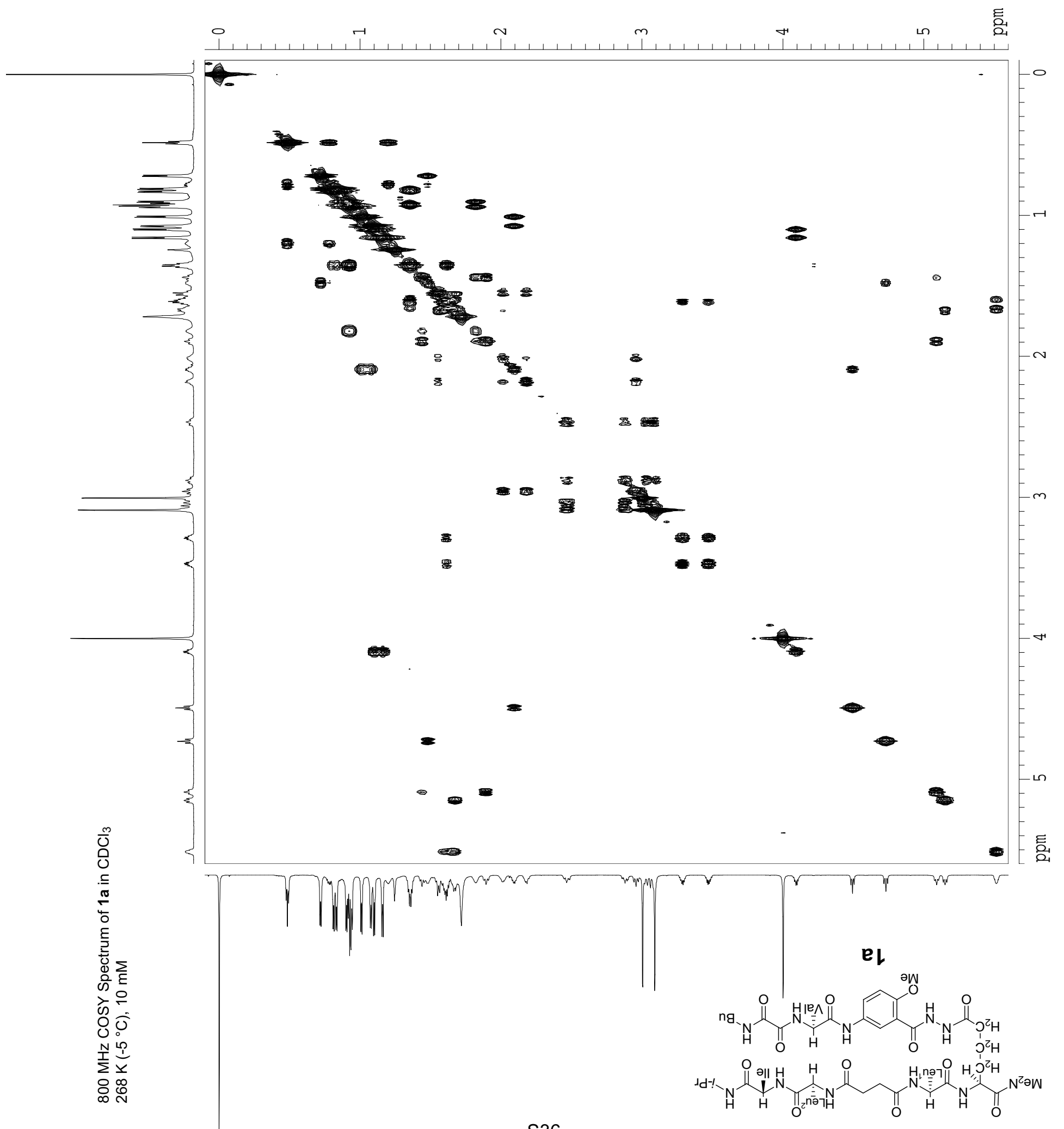


Supporting Information
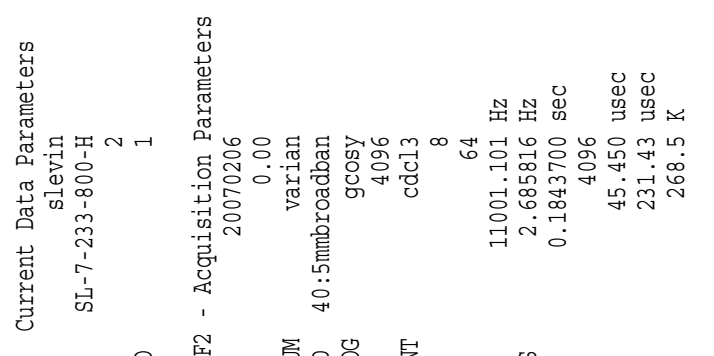

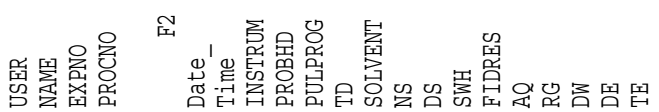
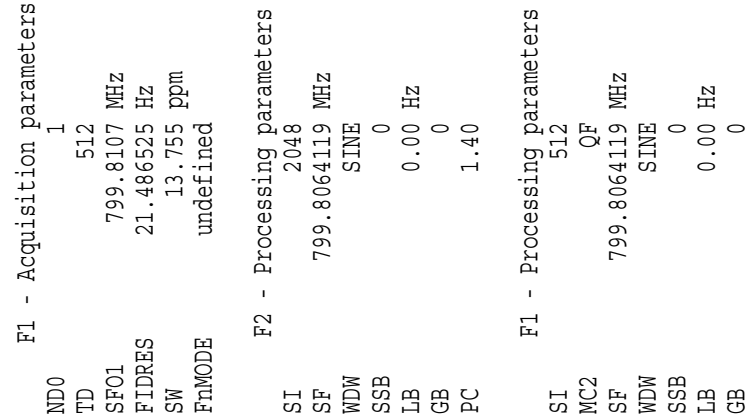

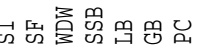

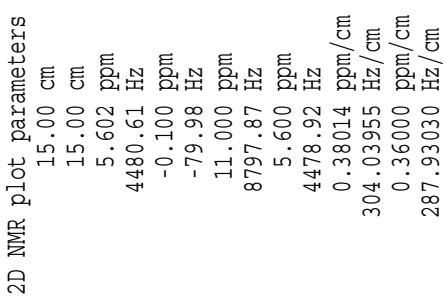

S. Levin and J. S. Nowick

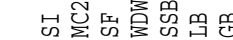

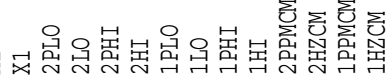

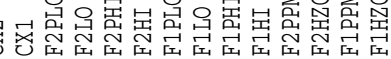

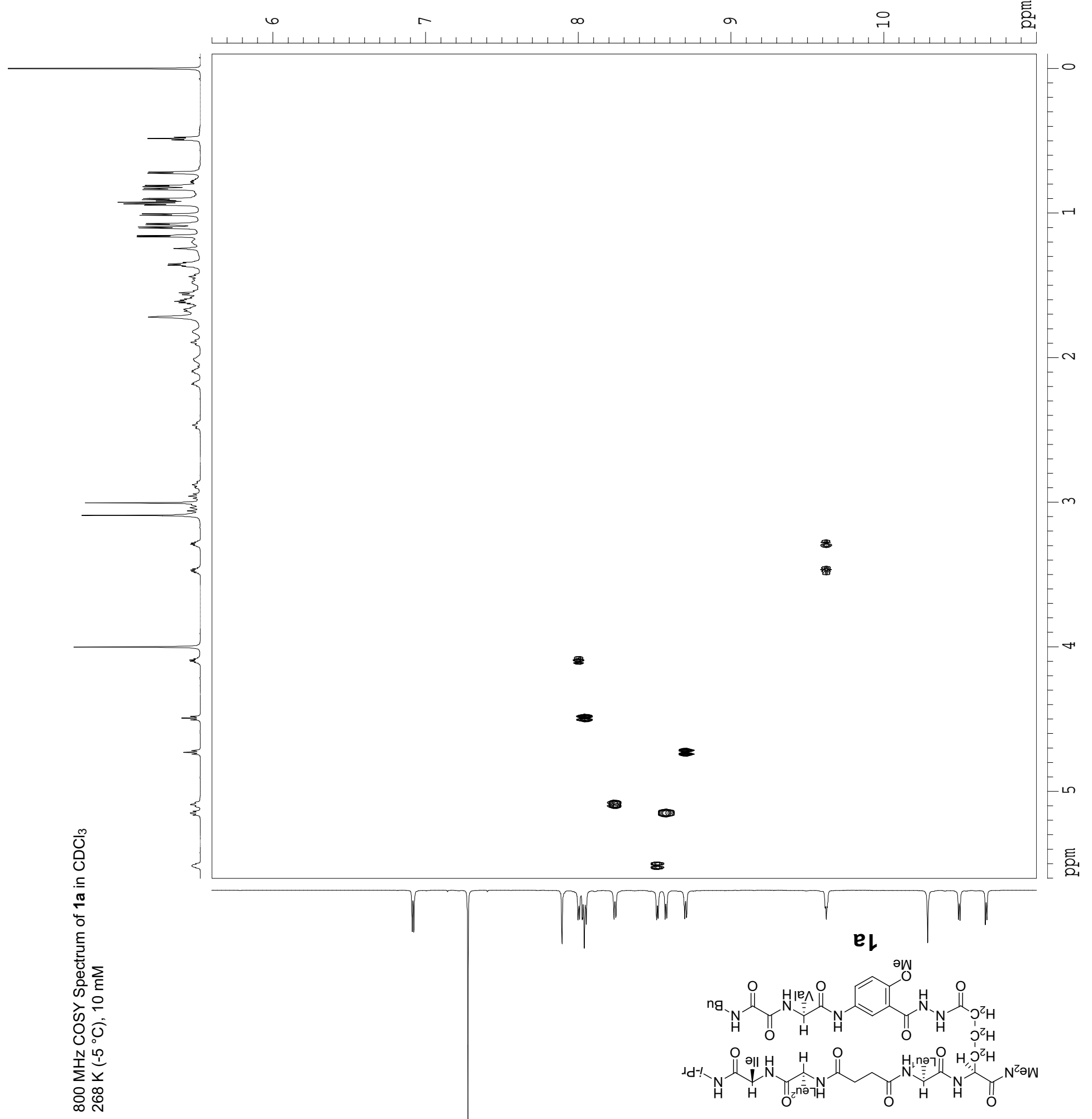


Supporting Information
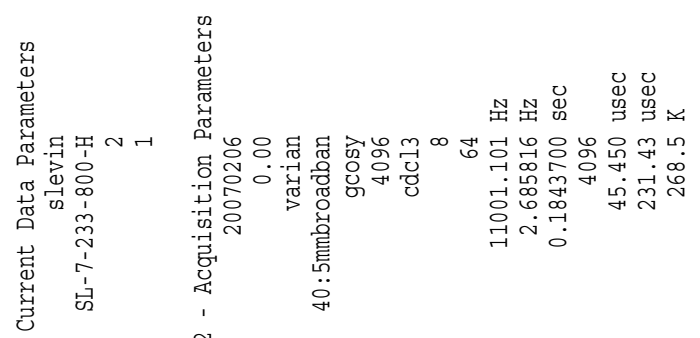

舅罢总总

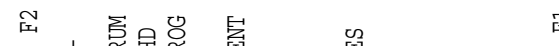

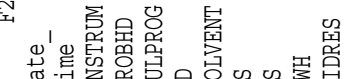

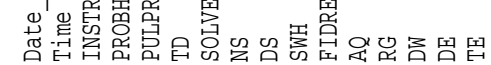

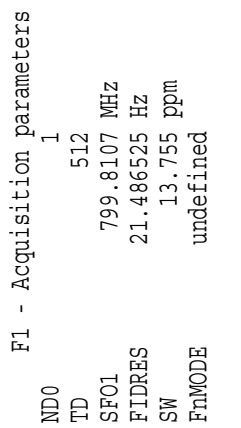

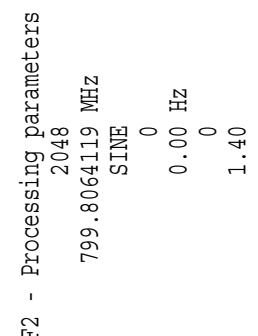

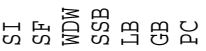

S. Levin and J. S. Nowick

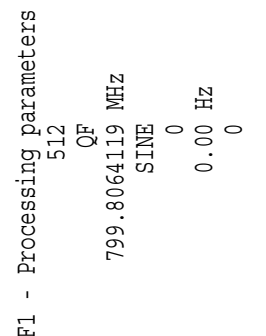

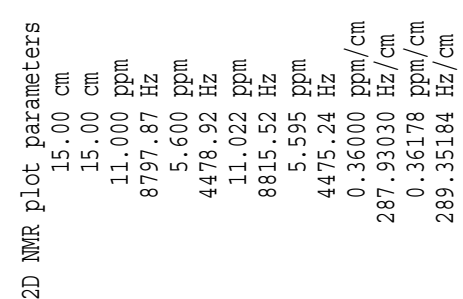

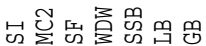

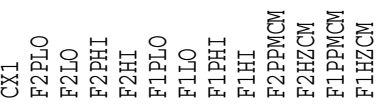

言

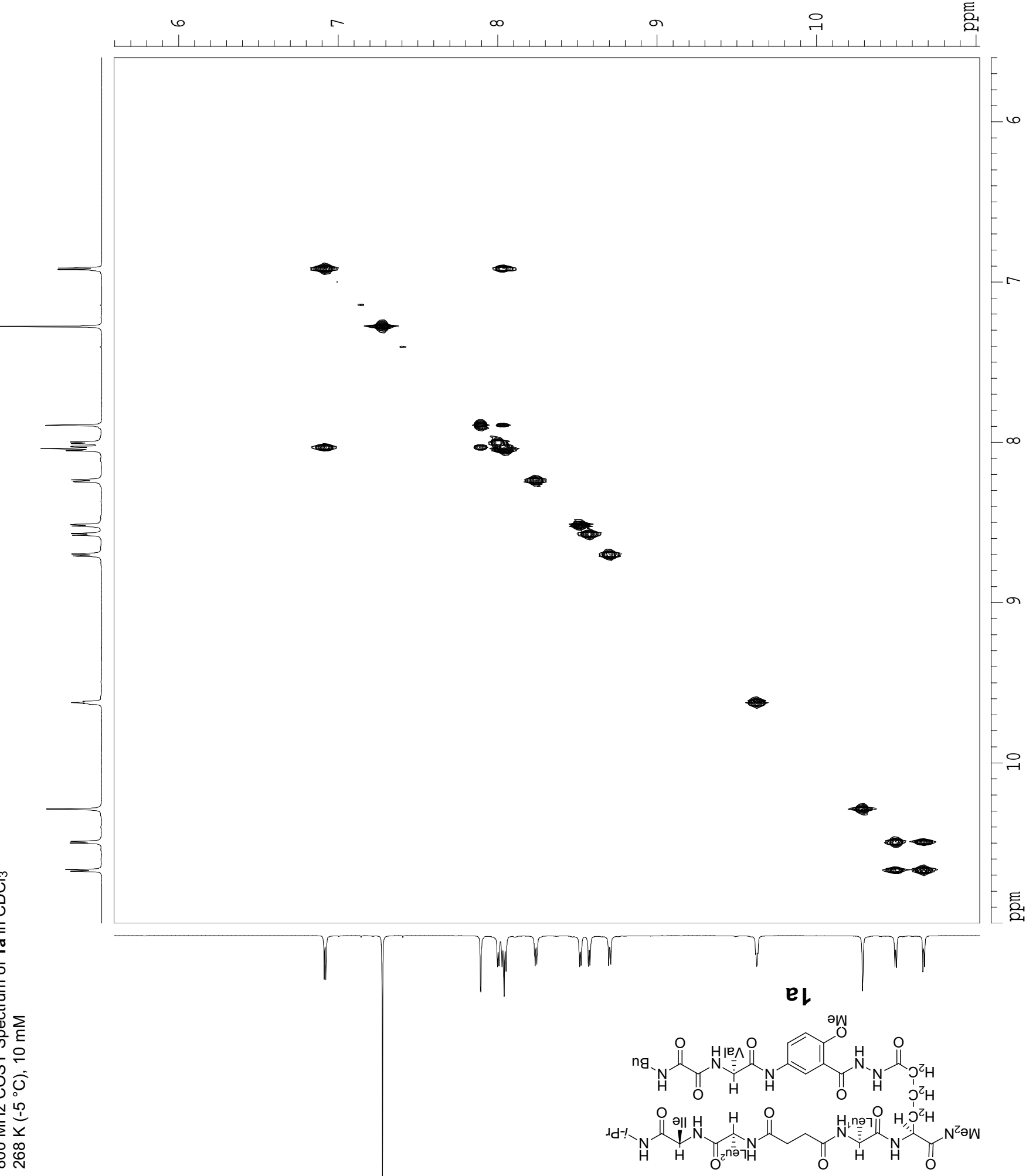


Supporting Information

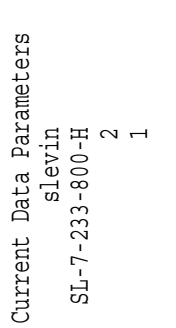

品息兑

空

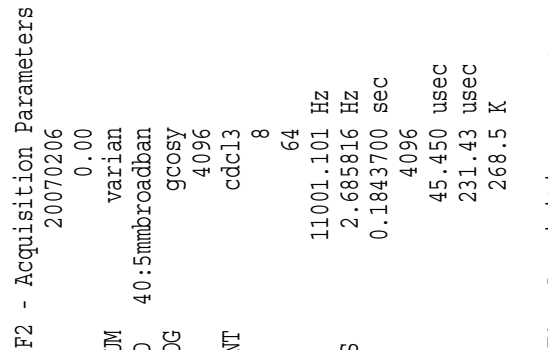

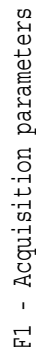

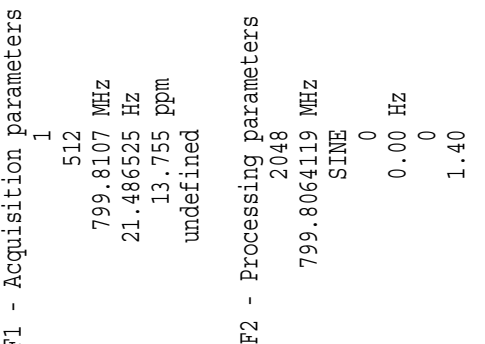

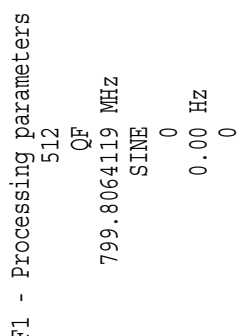

㞋
S. Levin and J. S. Nowick

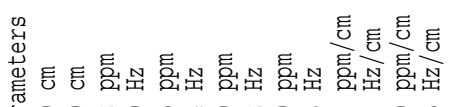

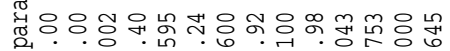

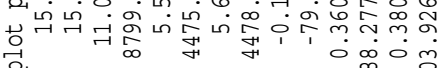
罢

ลิ

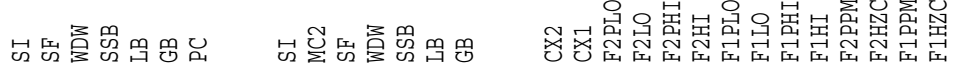

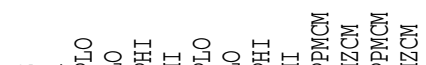

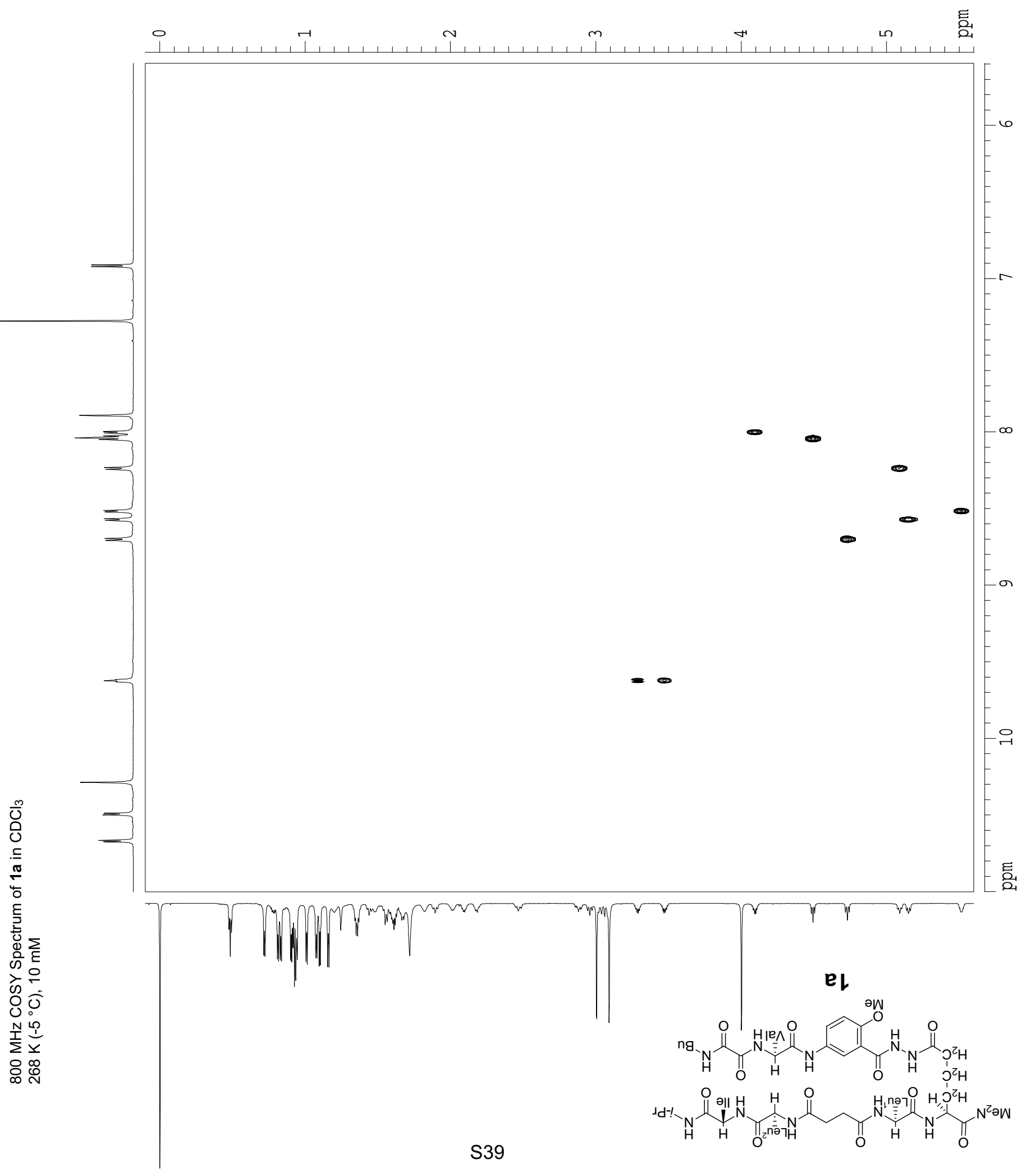


Supporting Information
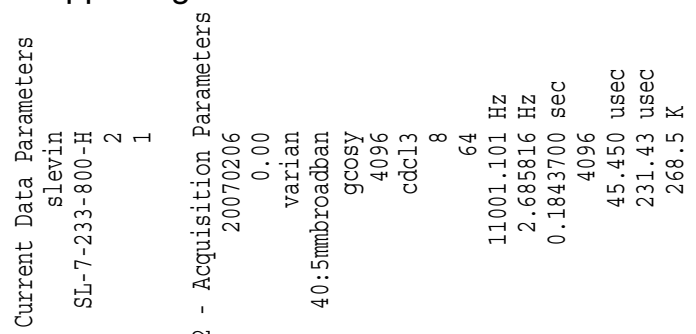

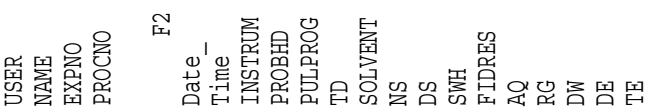

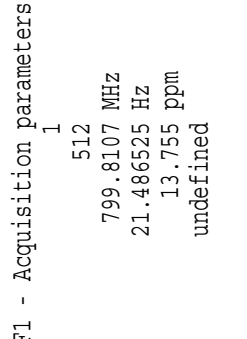

至

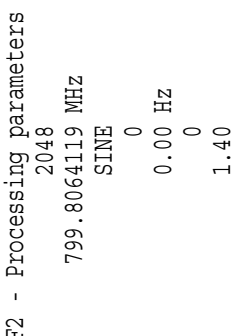

番

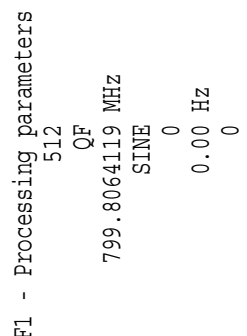

宫
S. Levin and J. S. Nowick

造

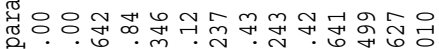

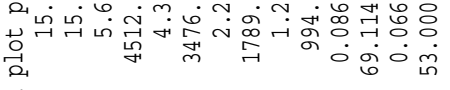
管

ลิ

을

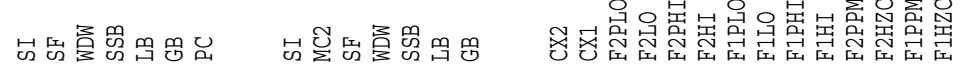

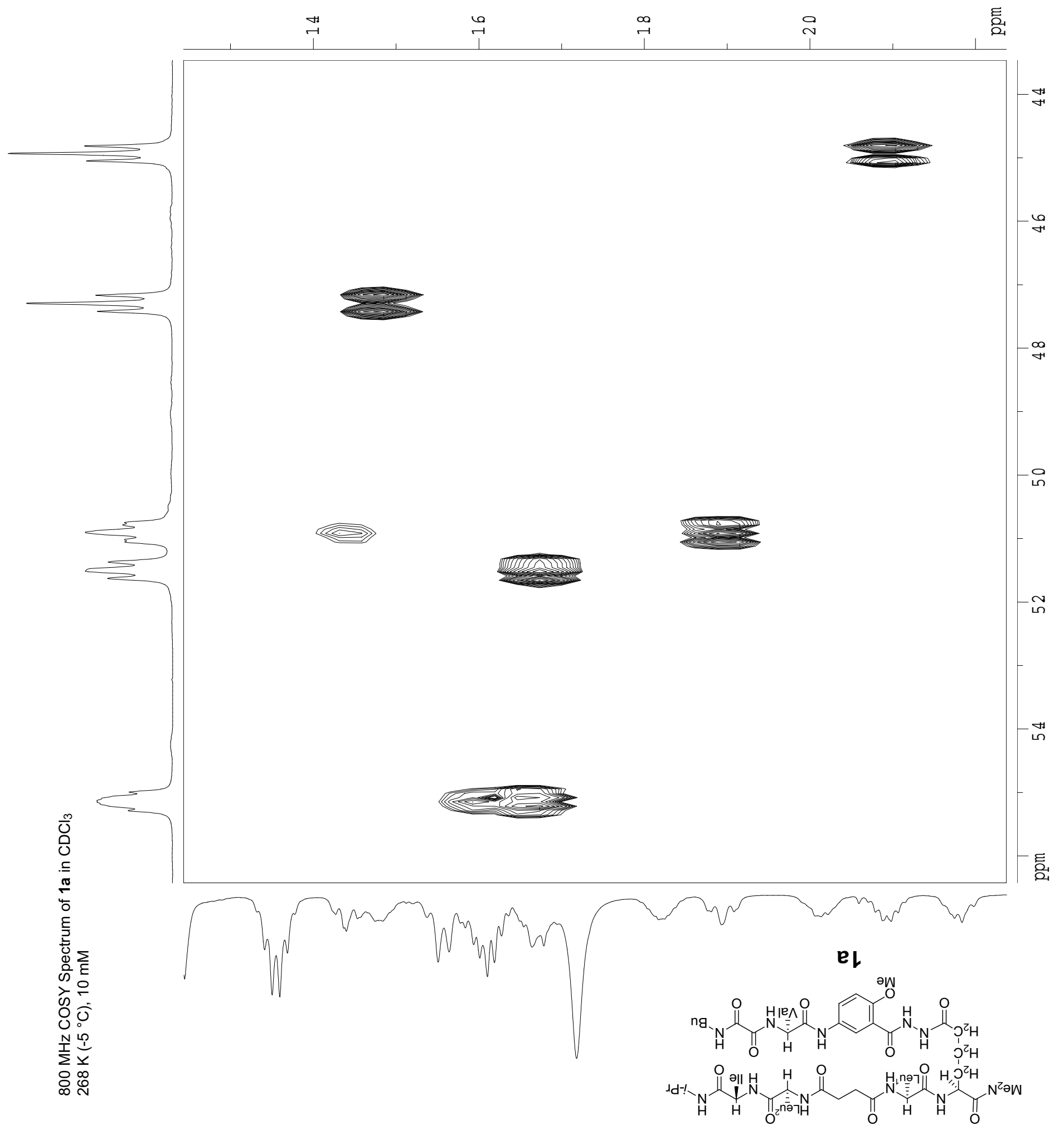



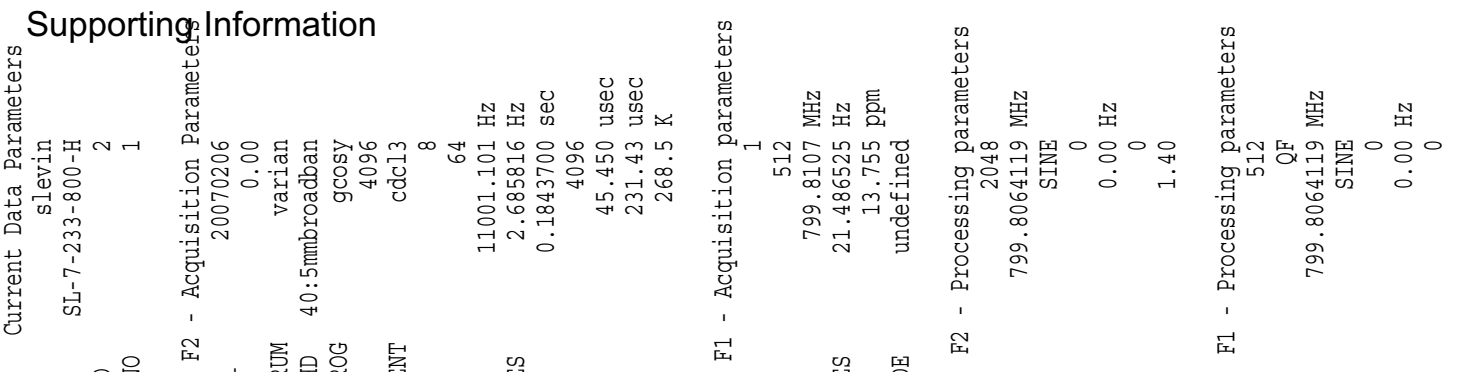

S. Levin and J. S. Nowick

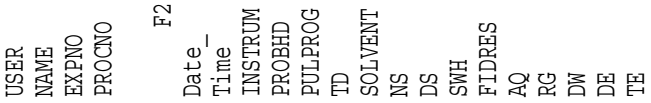

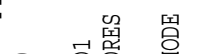

䍃鬲
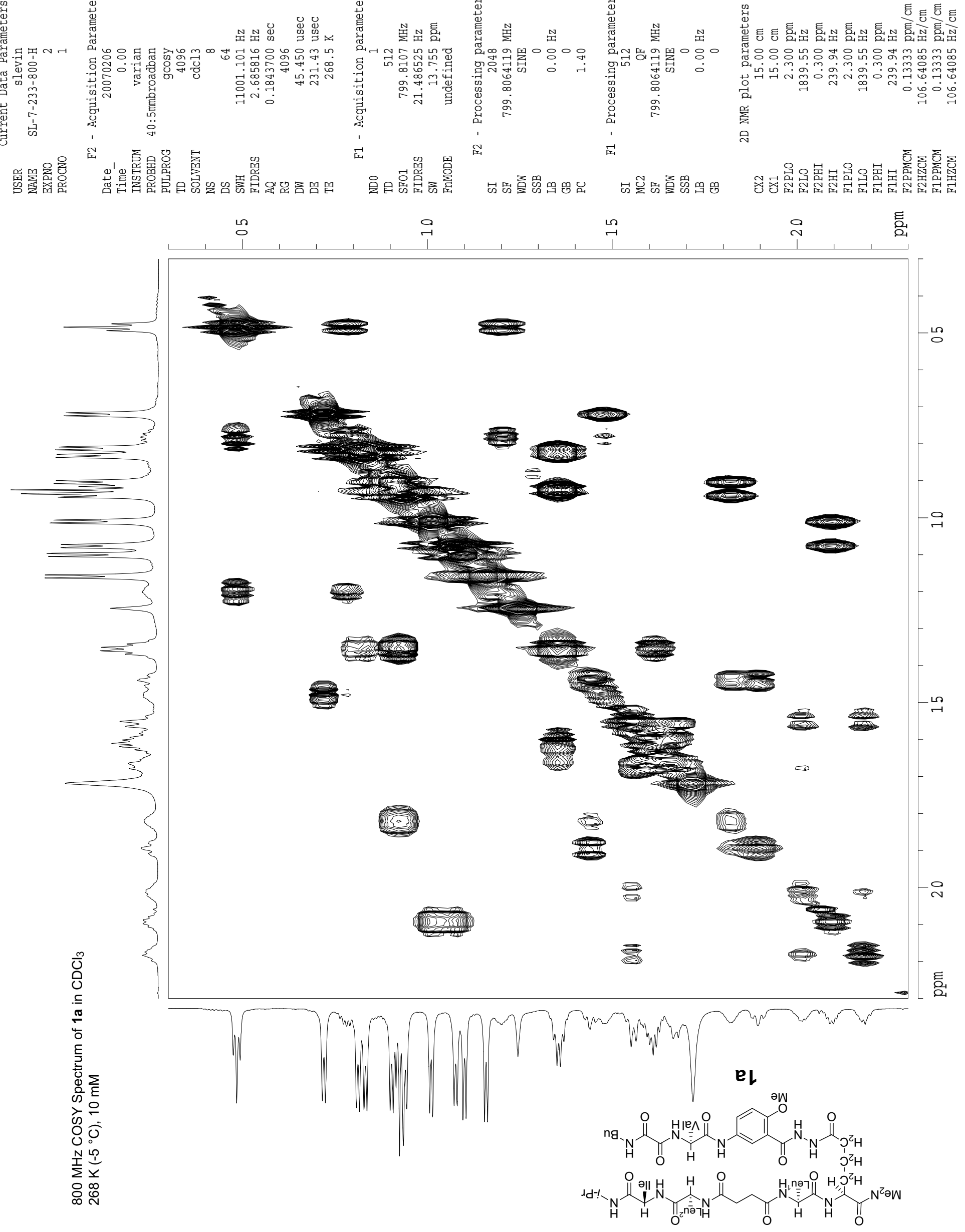

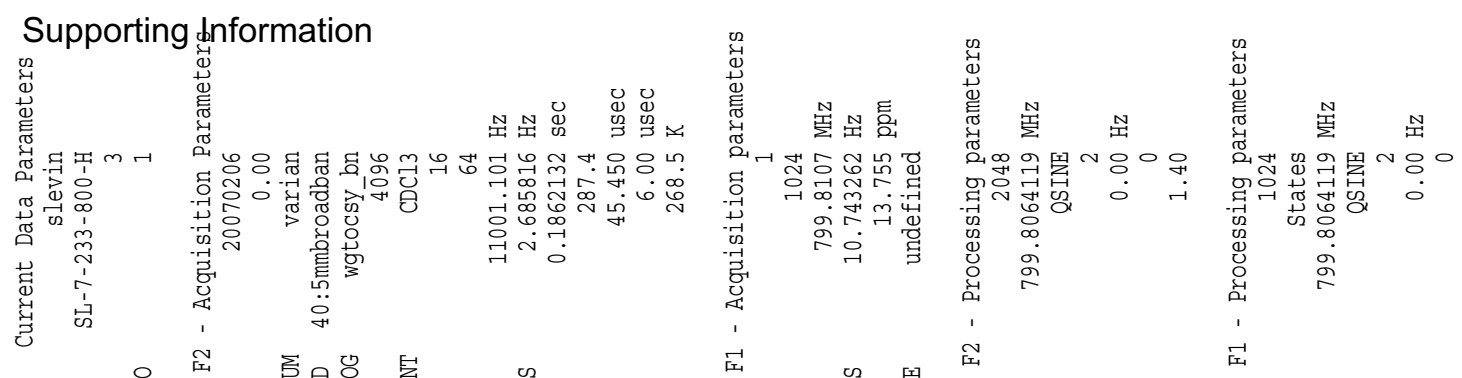

S. Levin and J. S. Nowick

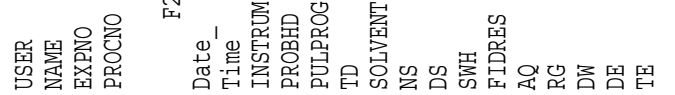

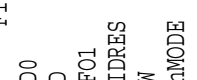

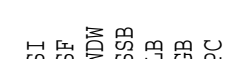

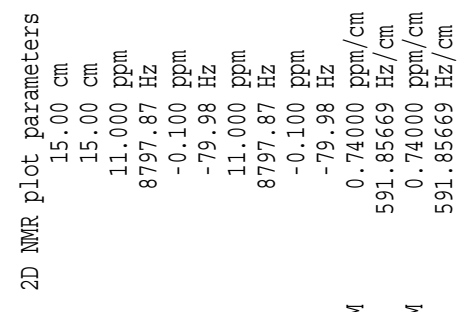

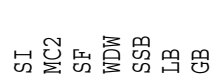
8ะ N

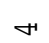
$6 \infty$ $\infty \quad \stackrel{\circ}{\circ}$ है,

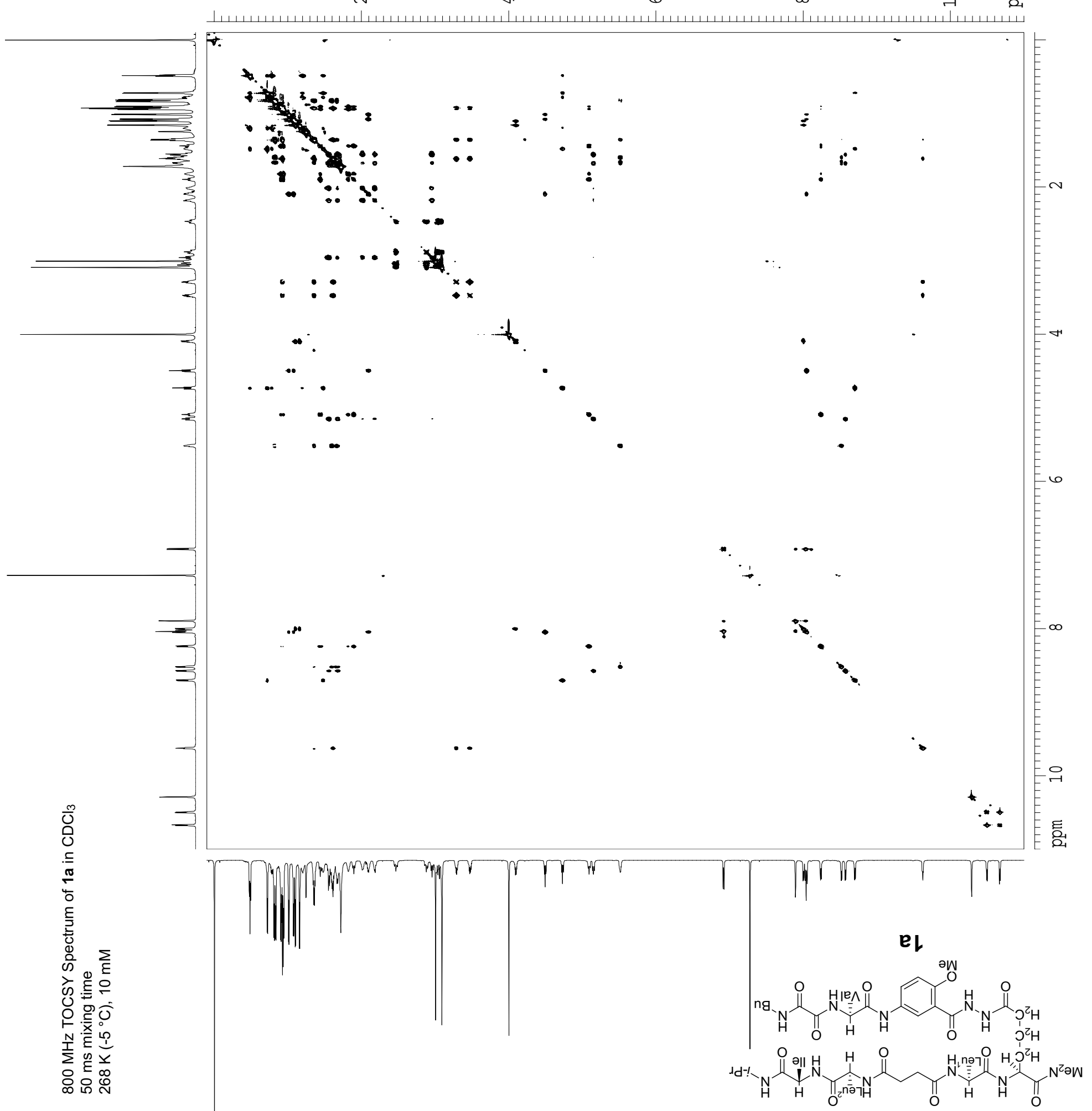


Supporting Information

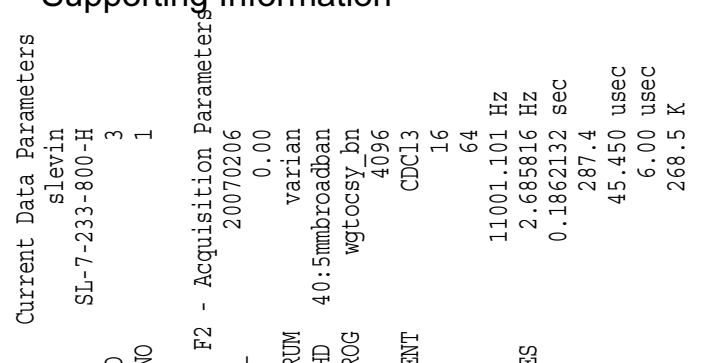

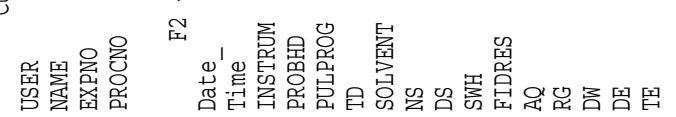
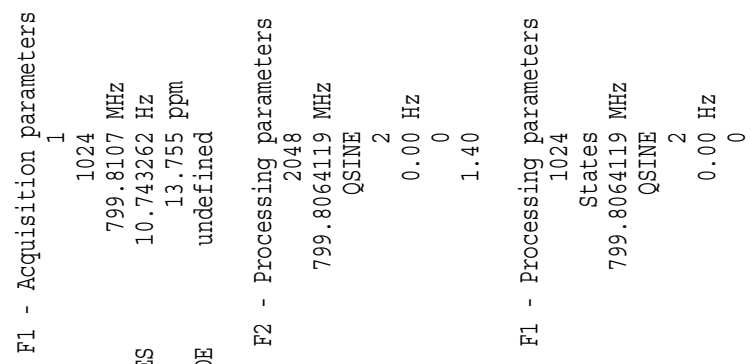

S. Levin and J. S. Nowick 焉

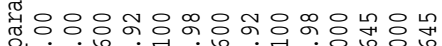
状

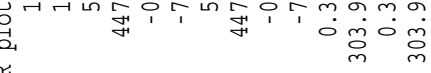
㴅 ลิ 占

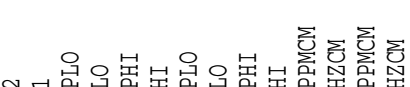

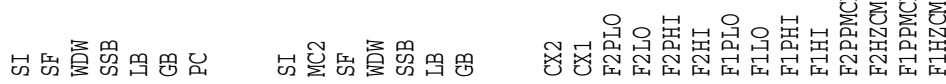

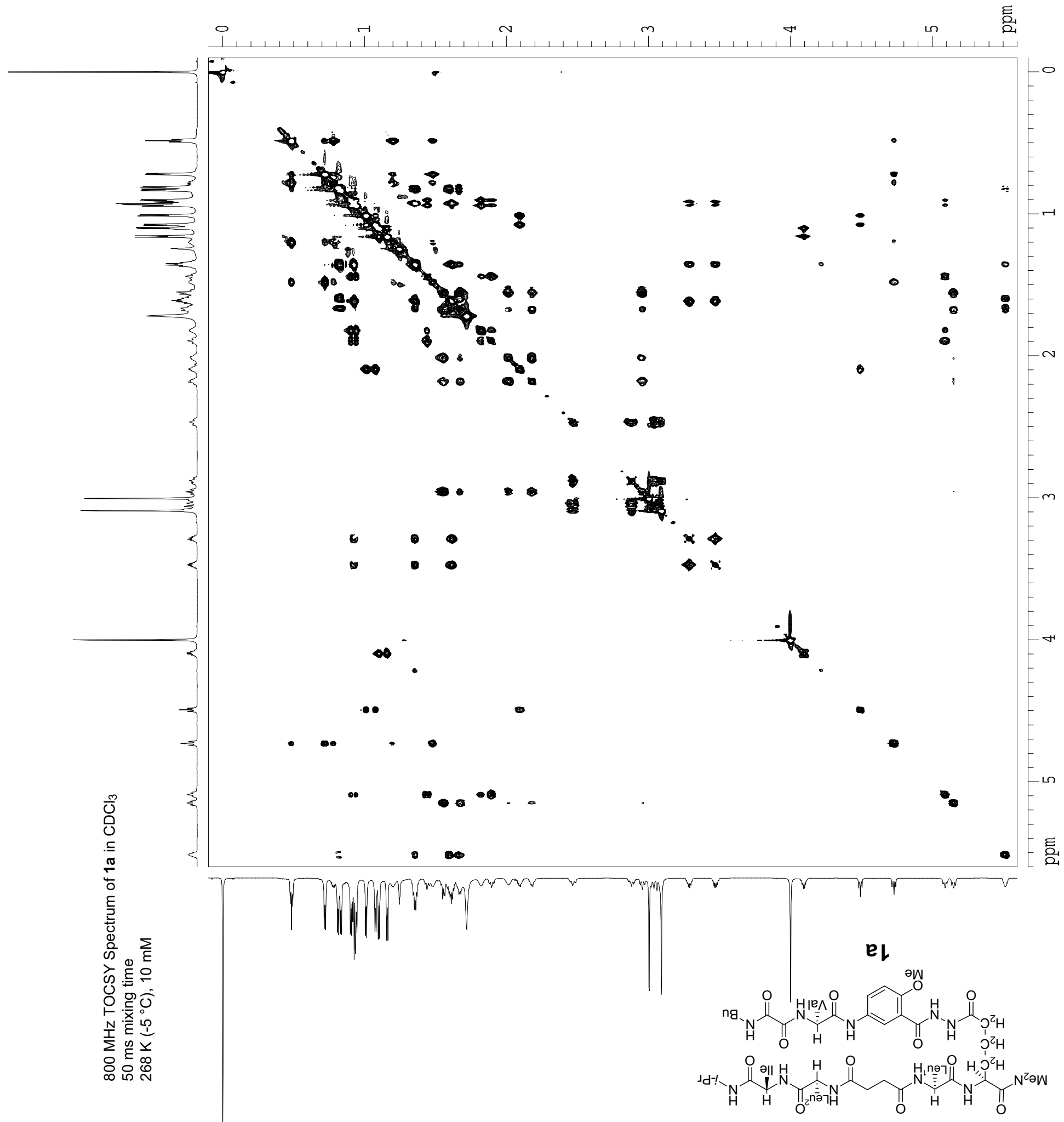




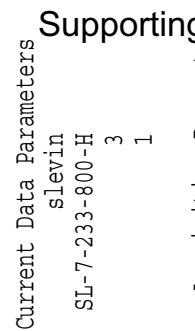

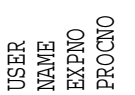

ing్ginformation

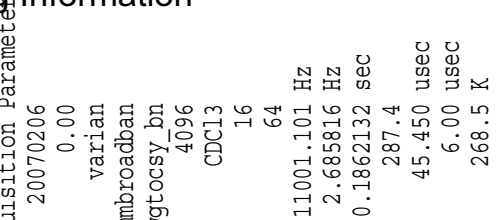

또
政

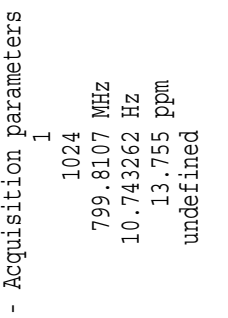

活牙留

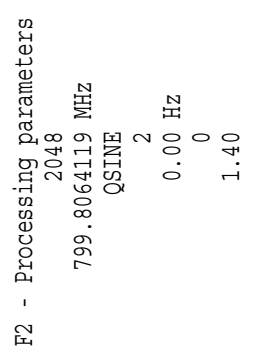

赵

0
0
0
0
0
0
0
0
0
0
7
0
0
0
0
$\vdots$
$\vdots$
1
1

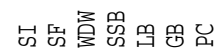

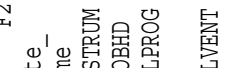

䞏

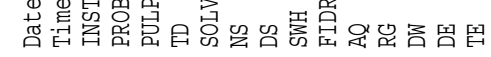

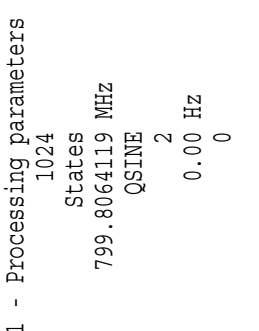

S. Levin and J. S. Nowick 进

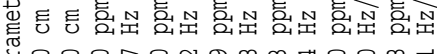

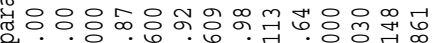

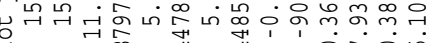
䍗

ล

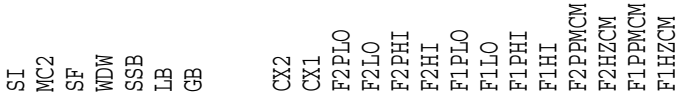

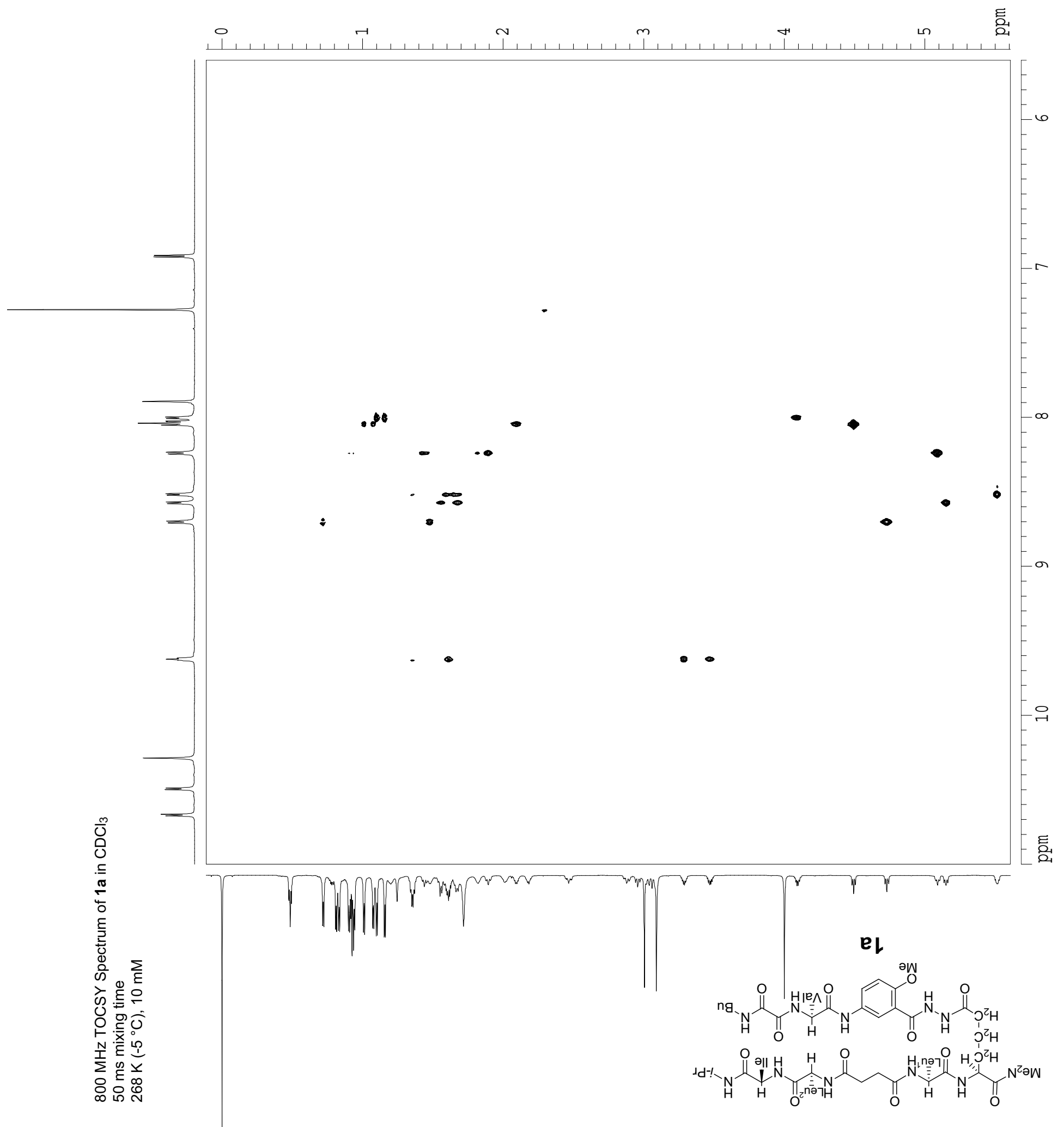




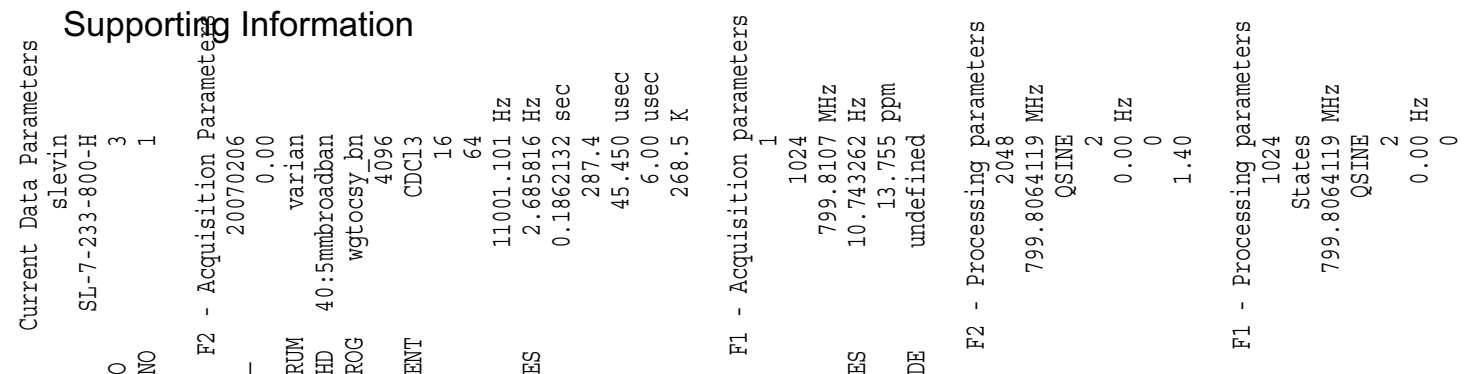

S. Levin and J. S. Nowick

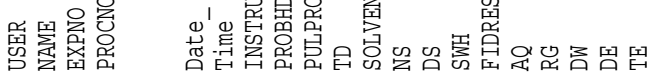

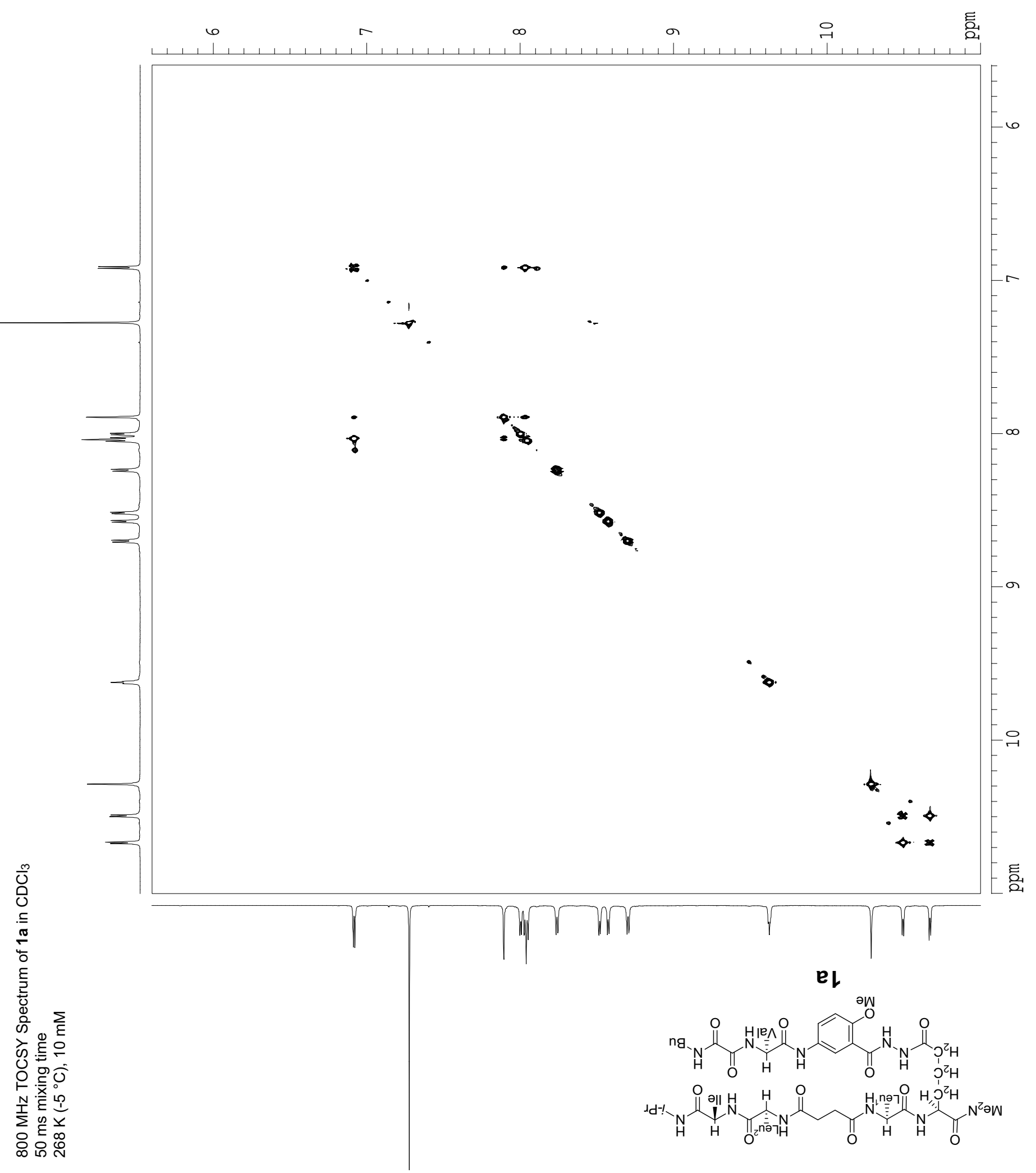


Supporting Information
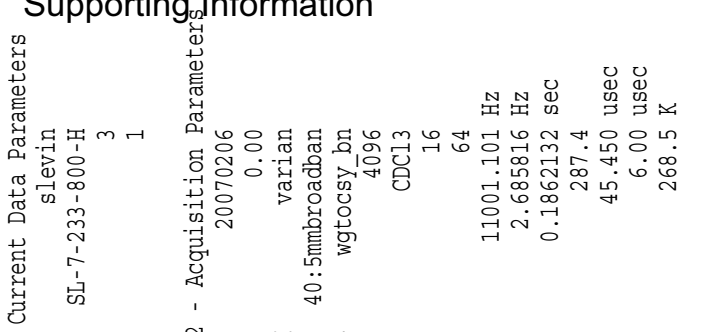

盢罢总总总
포

\section{s,}

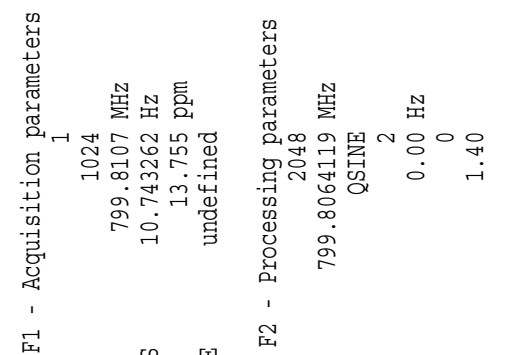

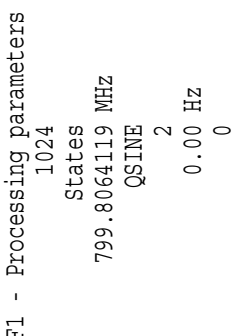

S. Levin and J. S. Nowick

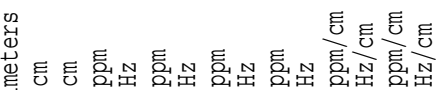

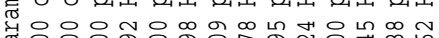

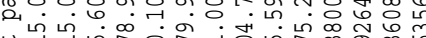

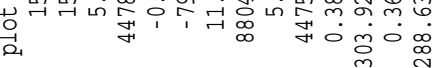
罢

(3)

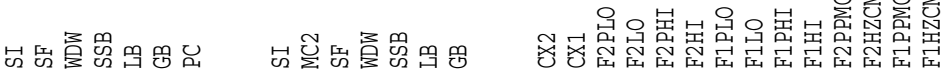

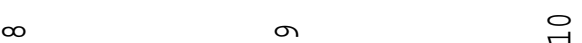

ह్

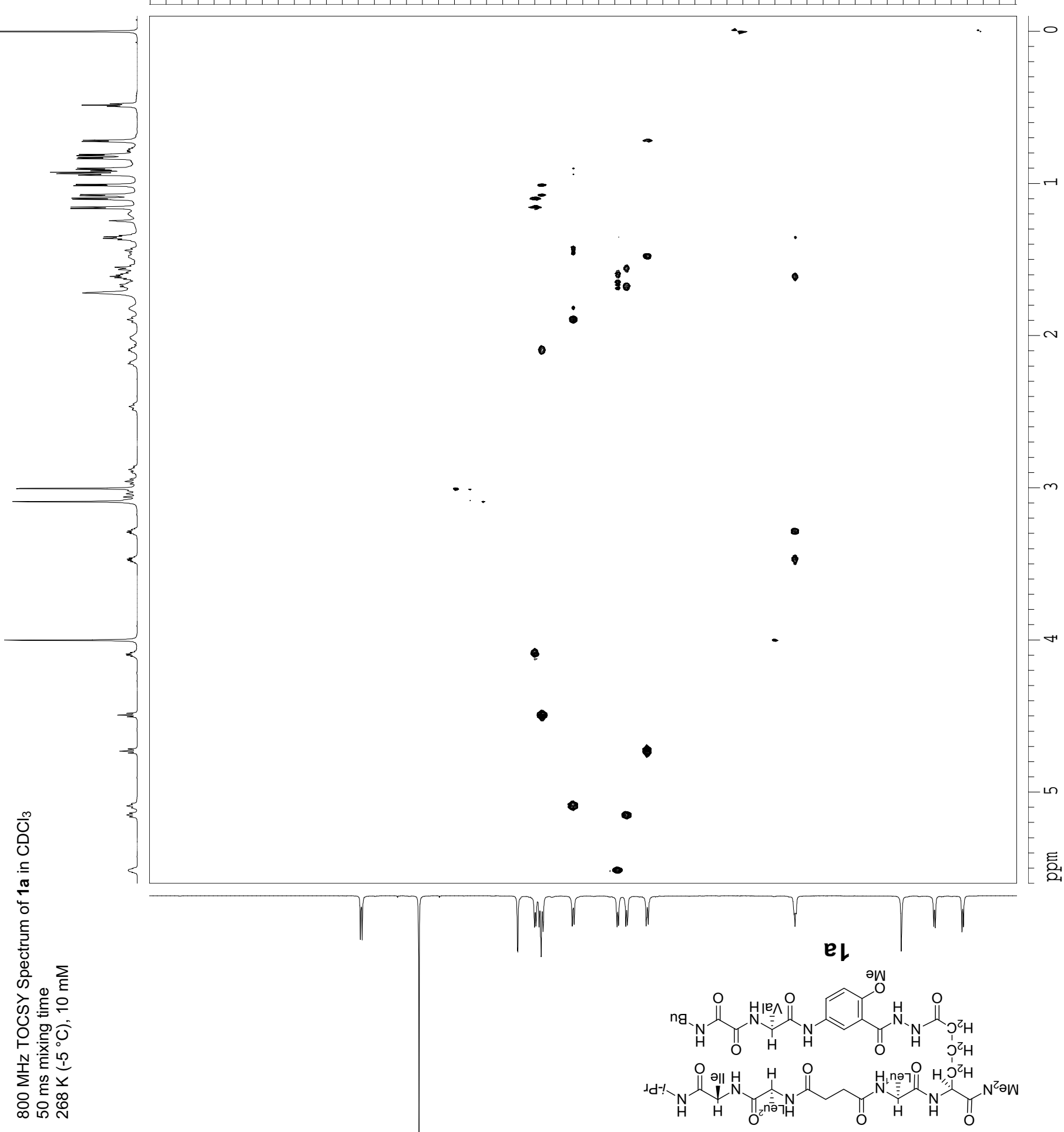



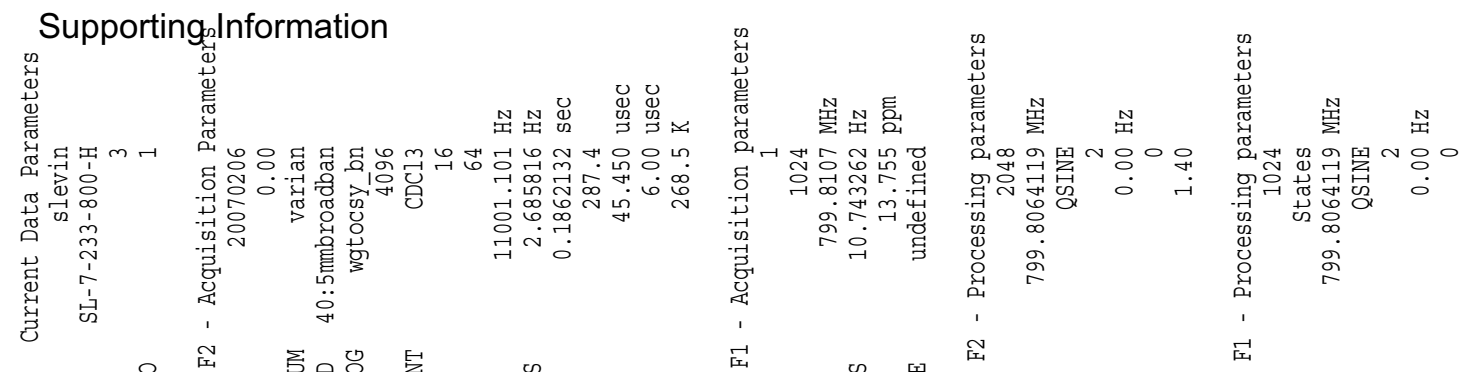

S. Levin and J. S. Nowick

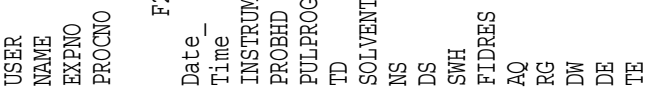

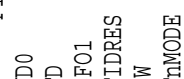

号

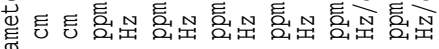

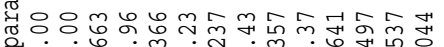

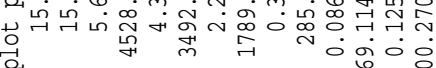
览

ลิ
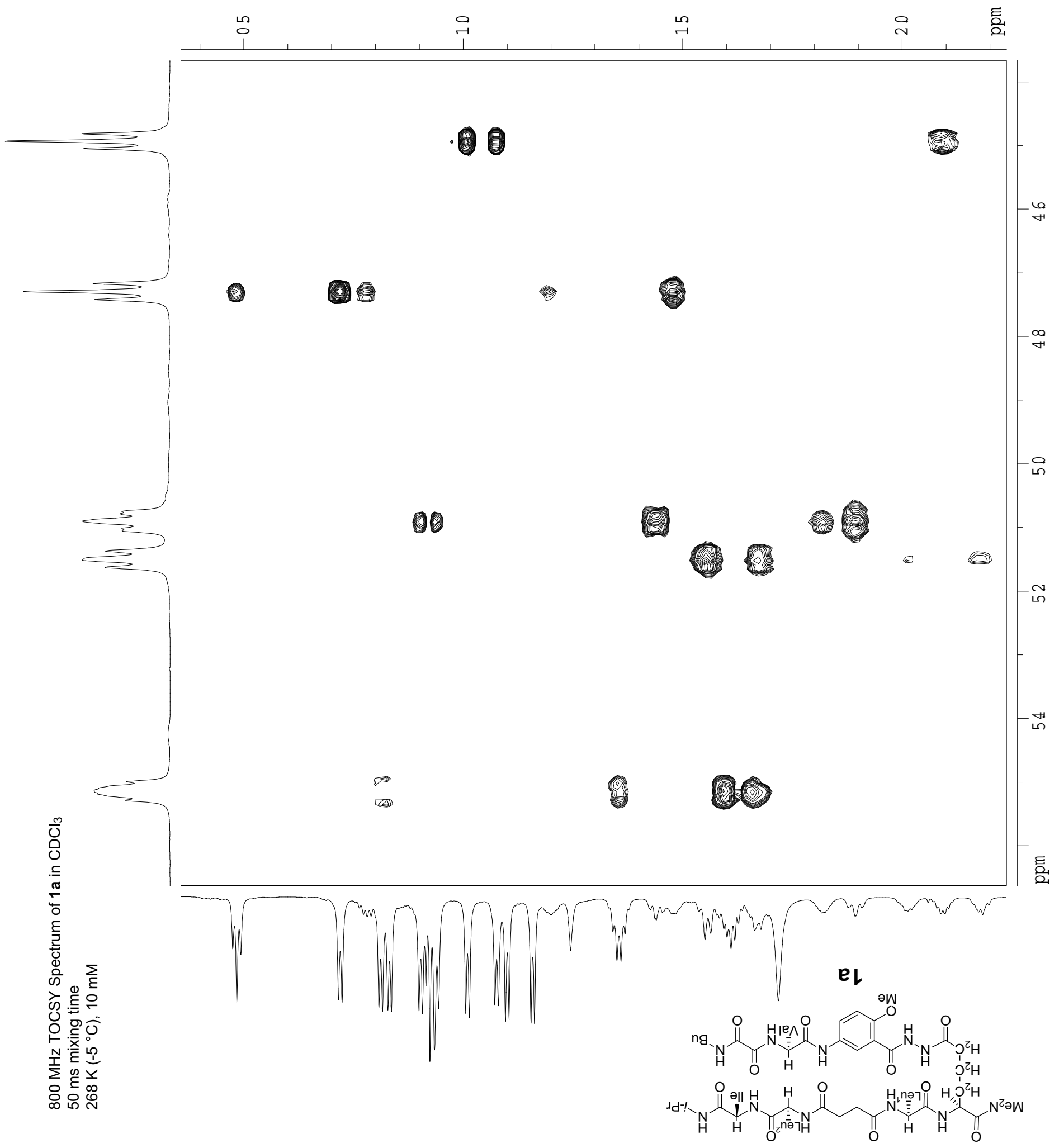

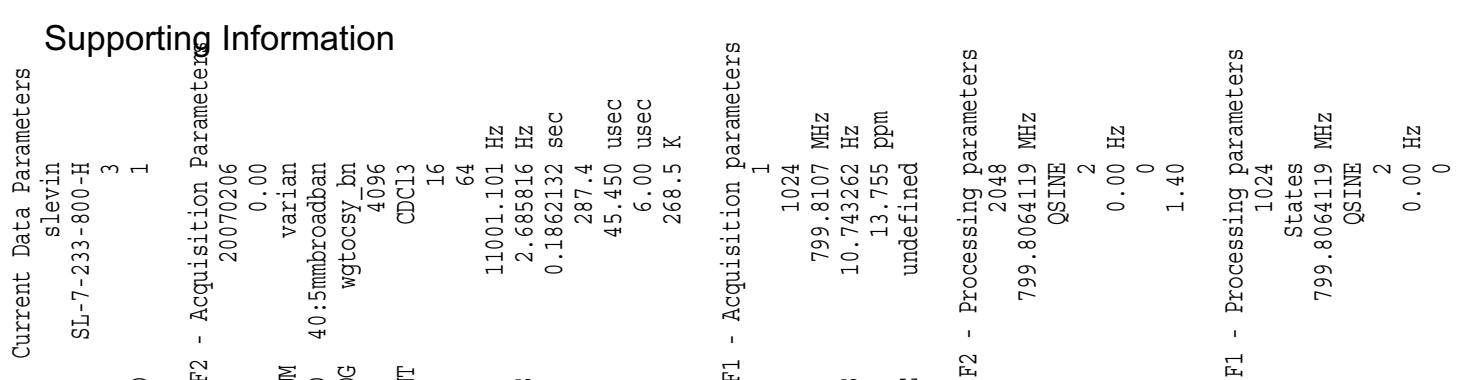

S. Levin and J. S. Nowick

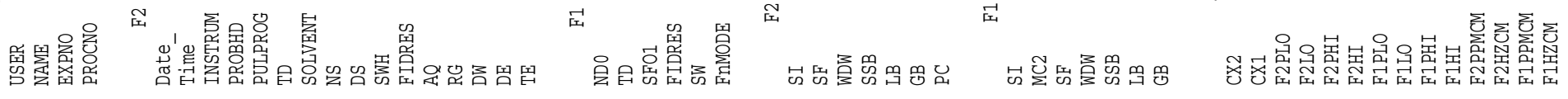

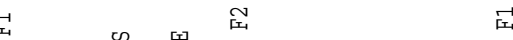

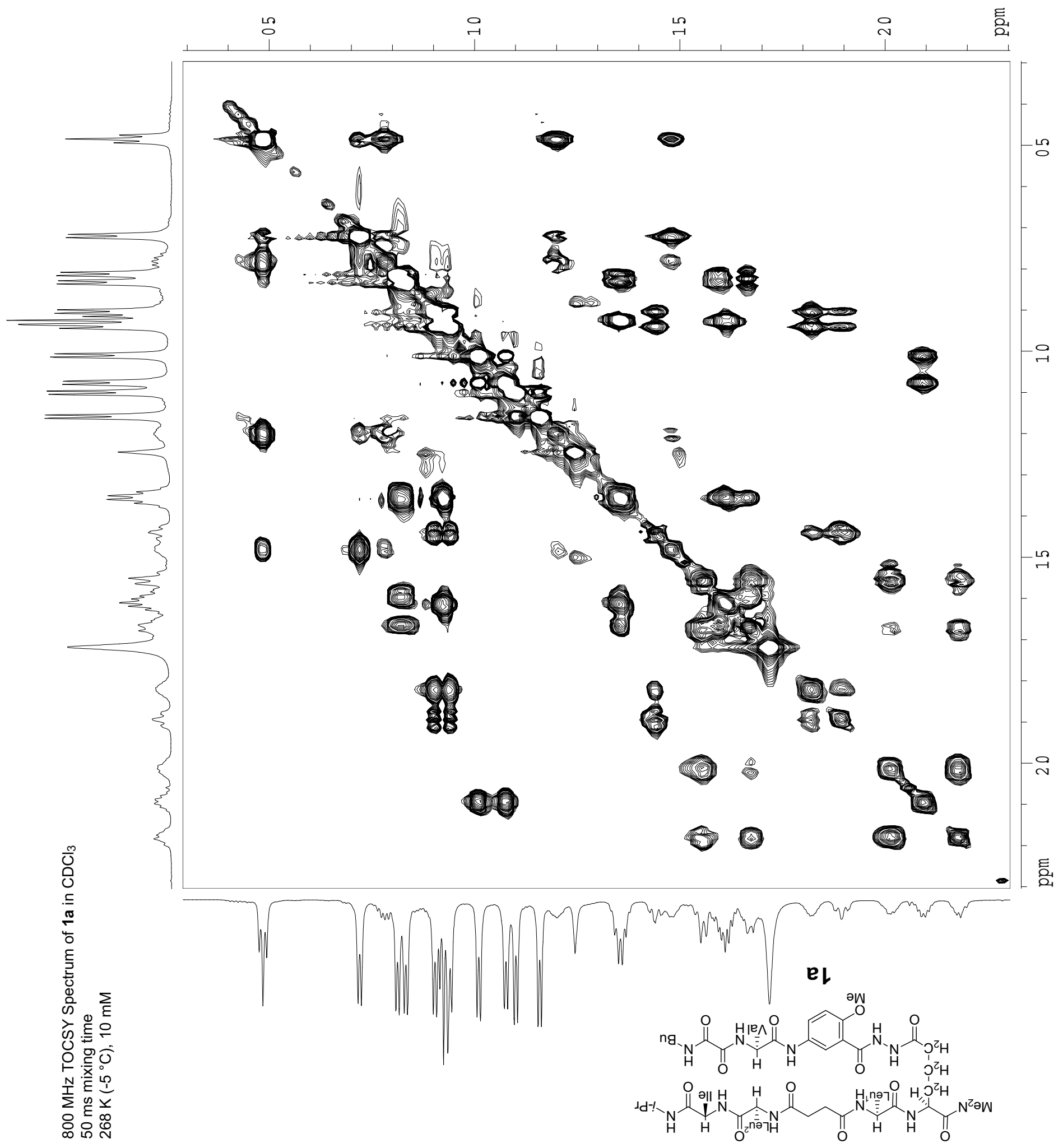


Supporting Information

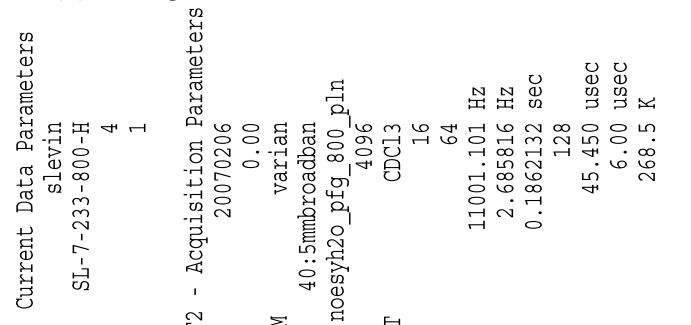

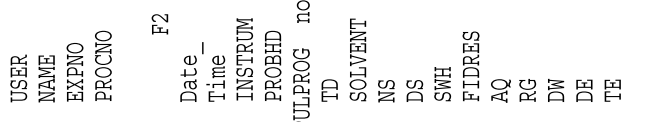

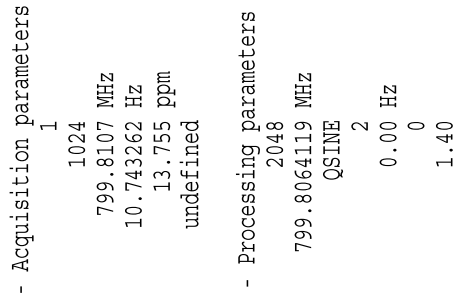

岳

S. Levin and J. S. Nowick

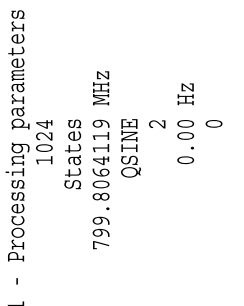

空

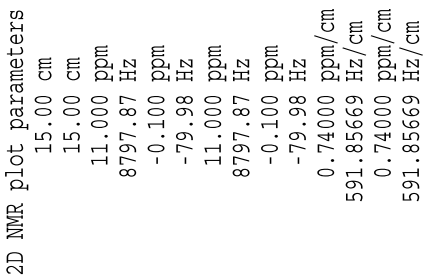

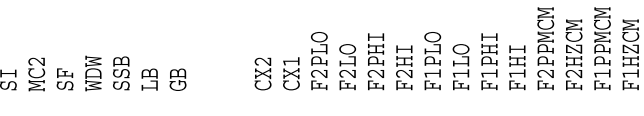

$\sim$

み

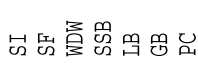

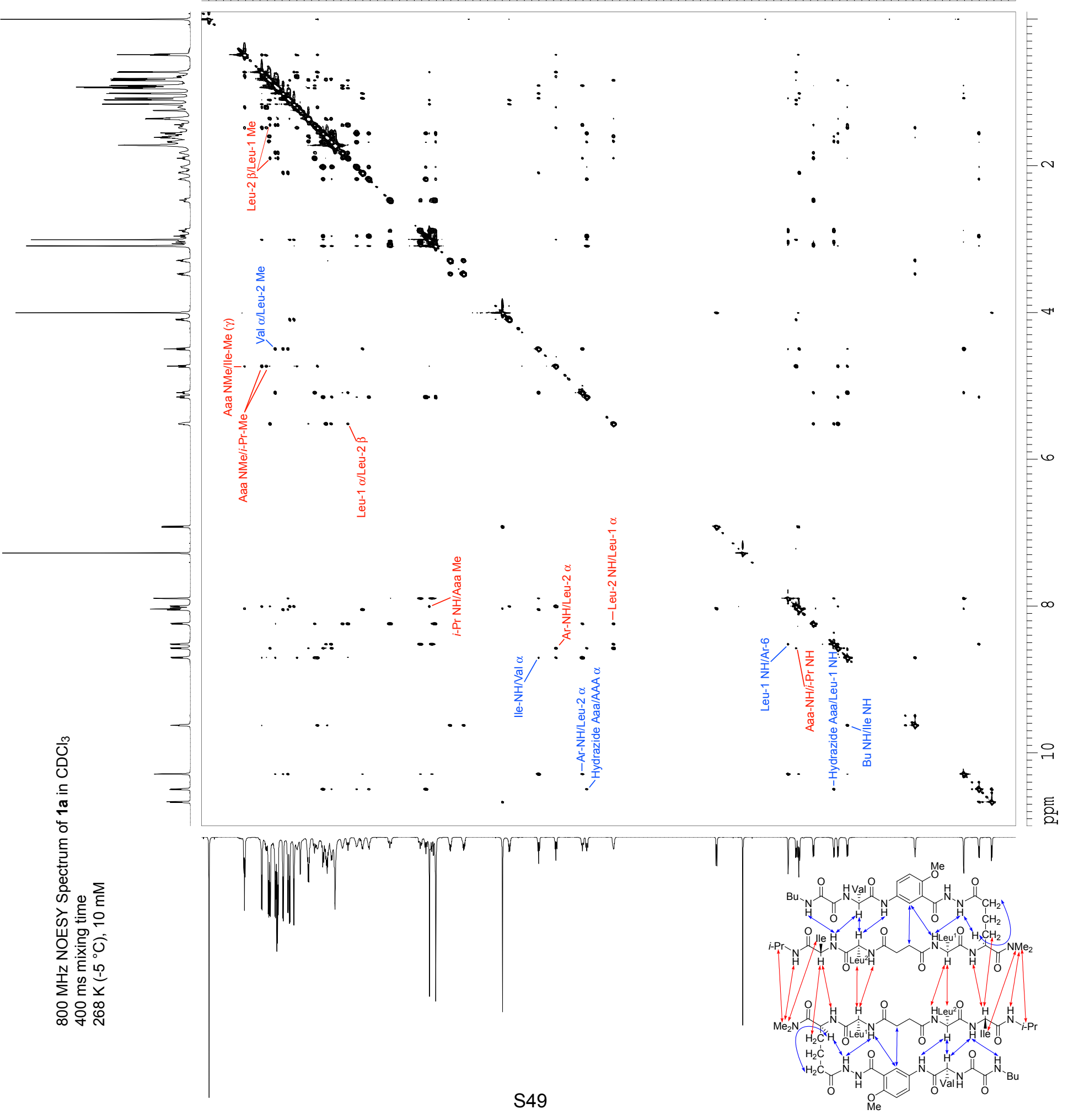


Supporting Information
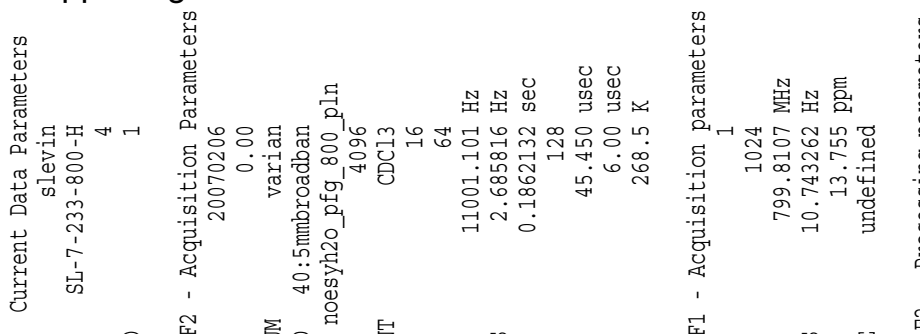

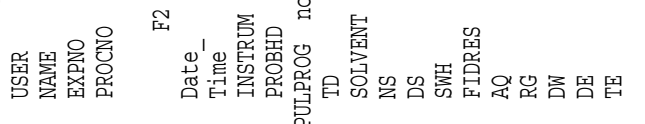

\section{"}

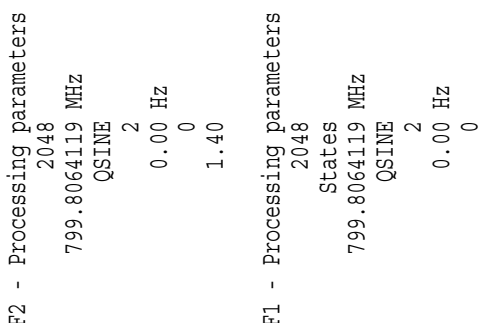

S. Levin and J. S. Nowick

官

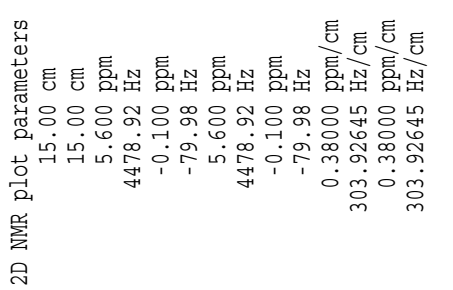

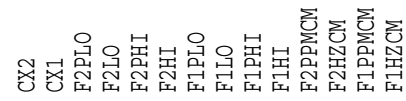

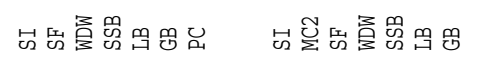

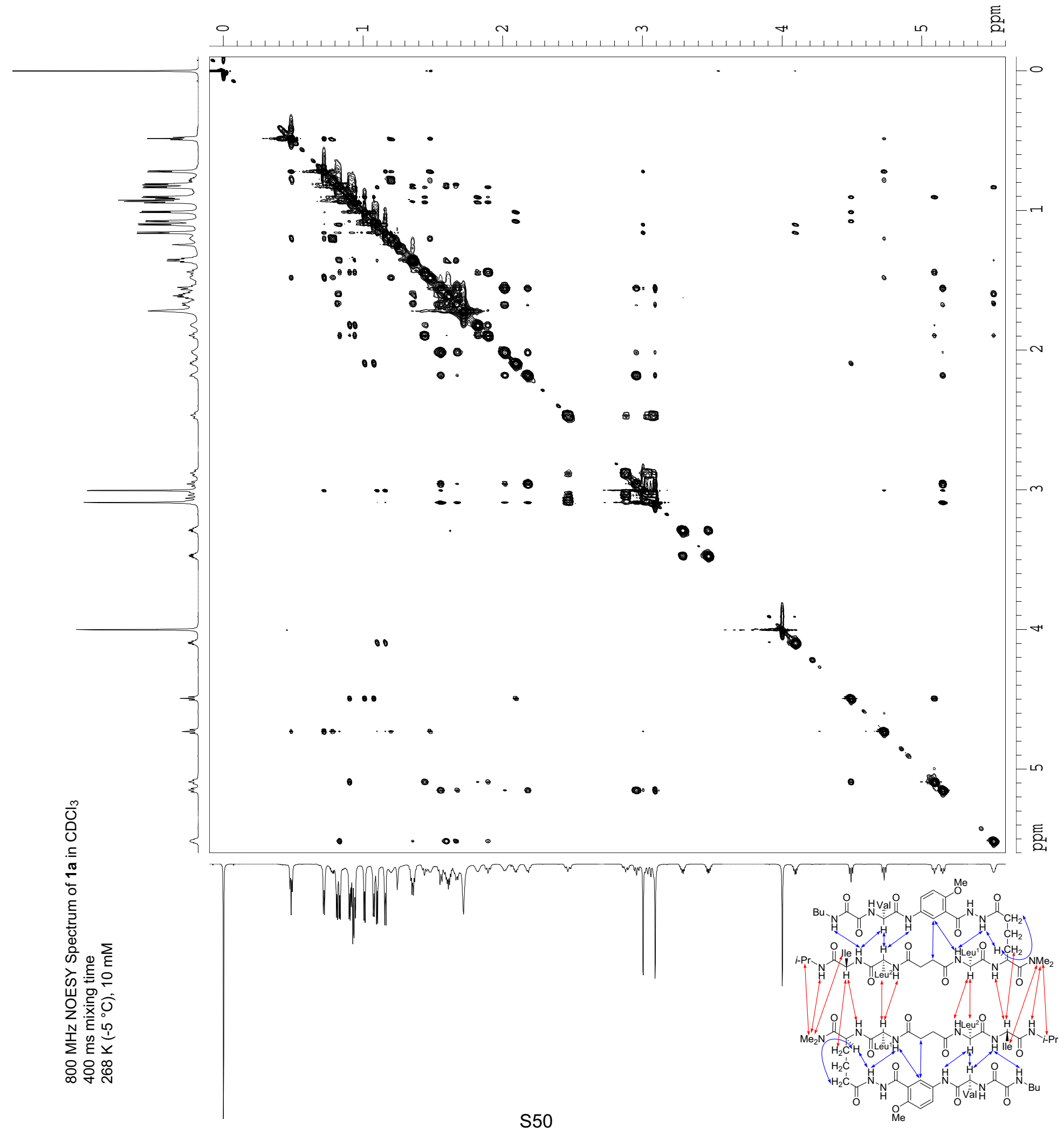



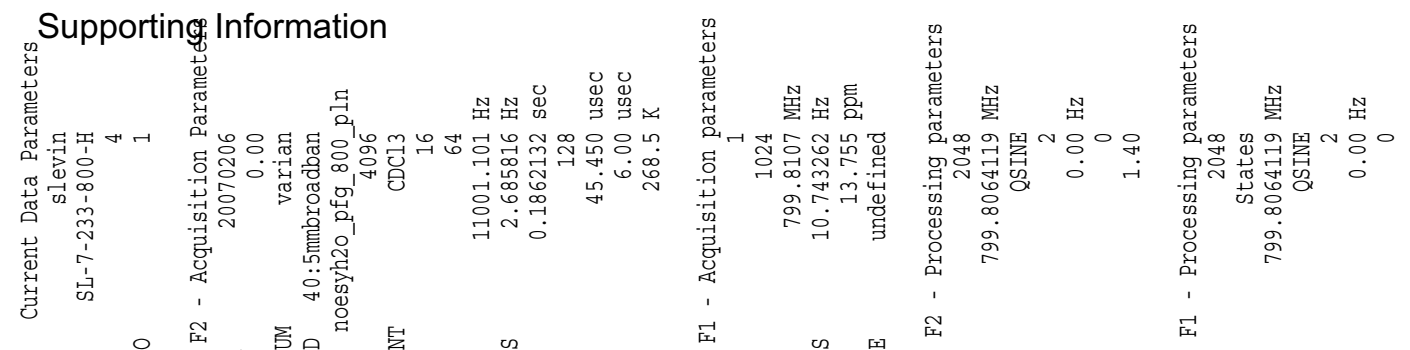

S. Levin and J. S. Nowick

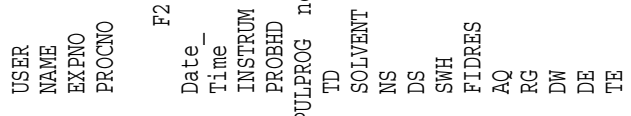

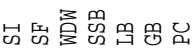

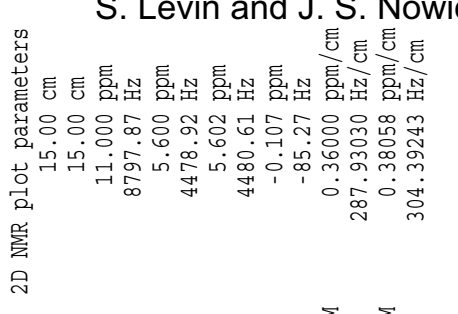

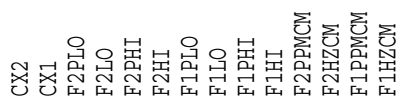

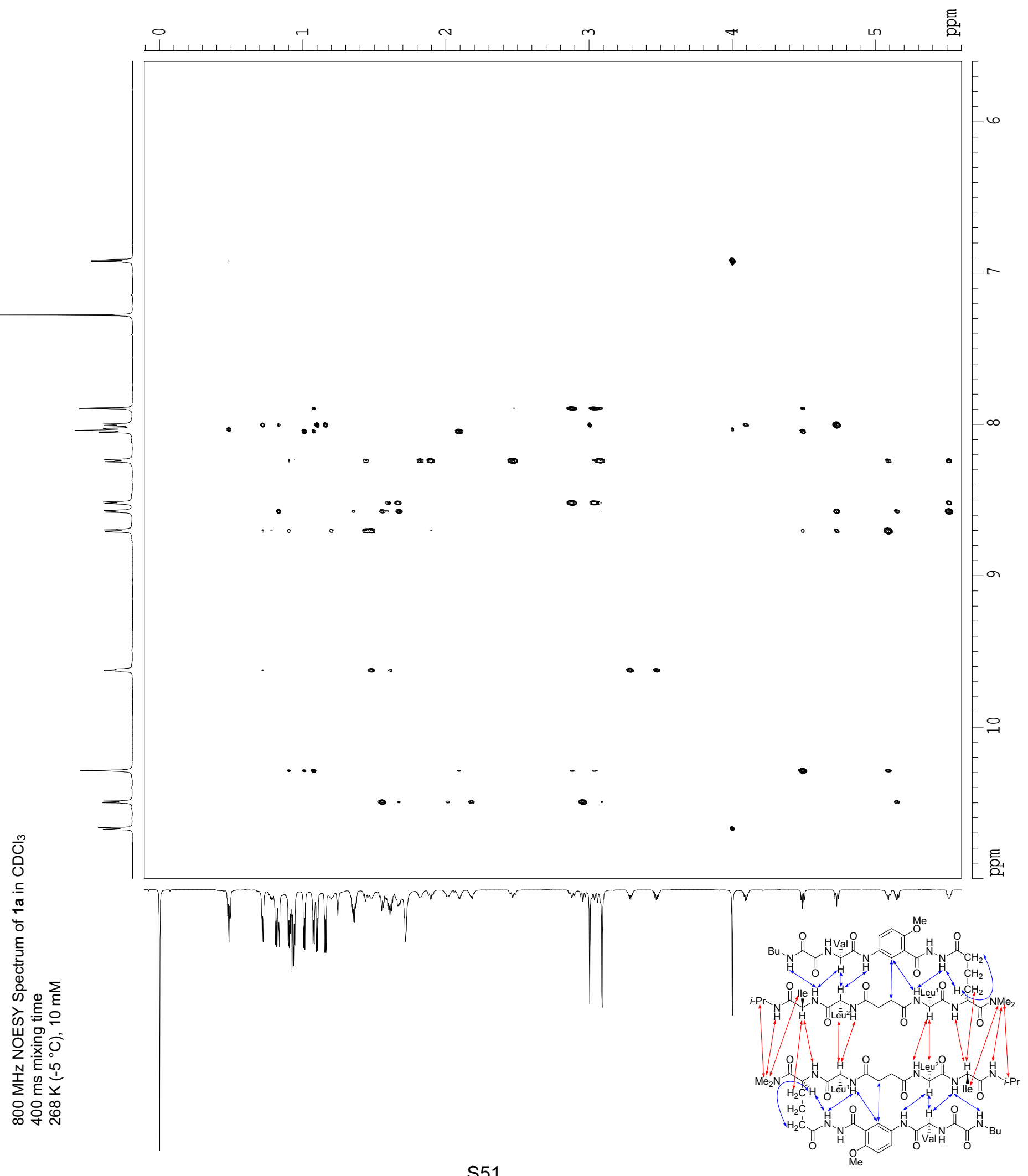


Supporting Information

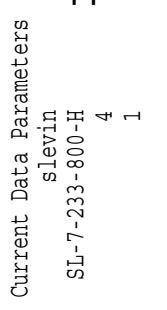

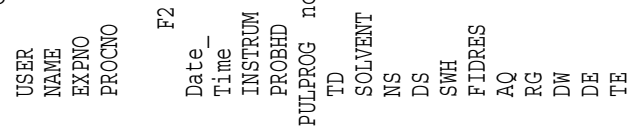
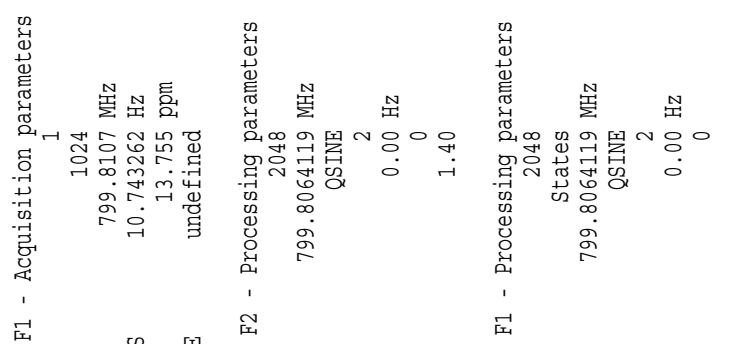

S. Levin and J. S. Nowick

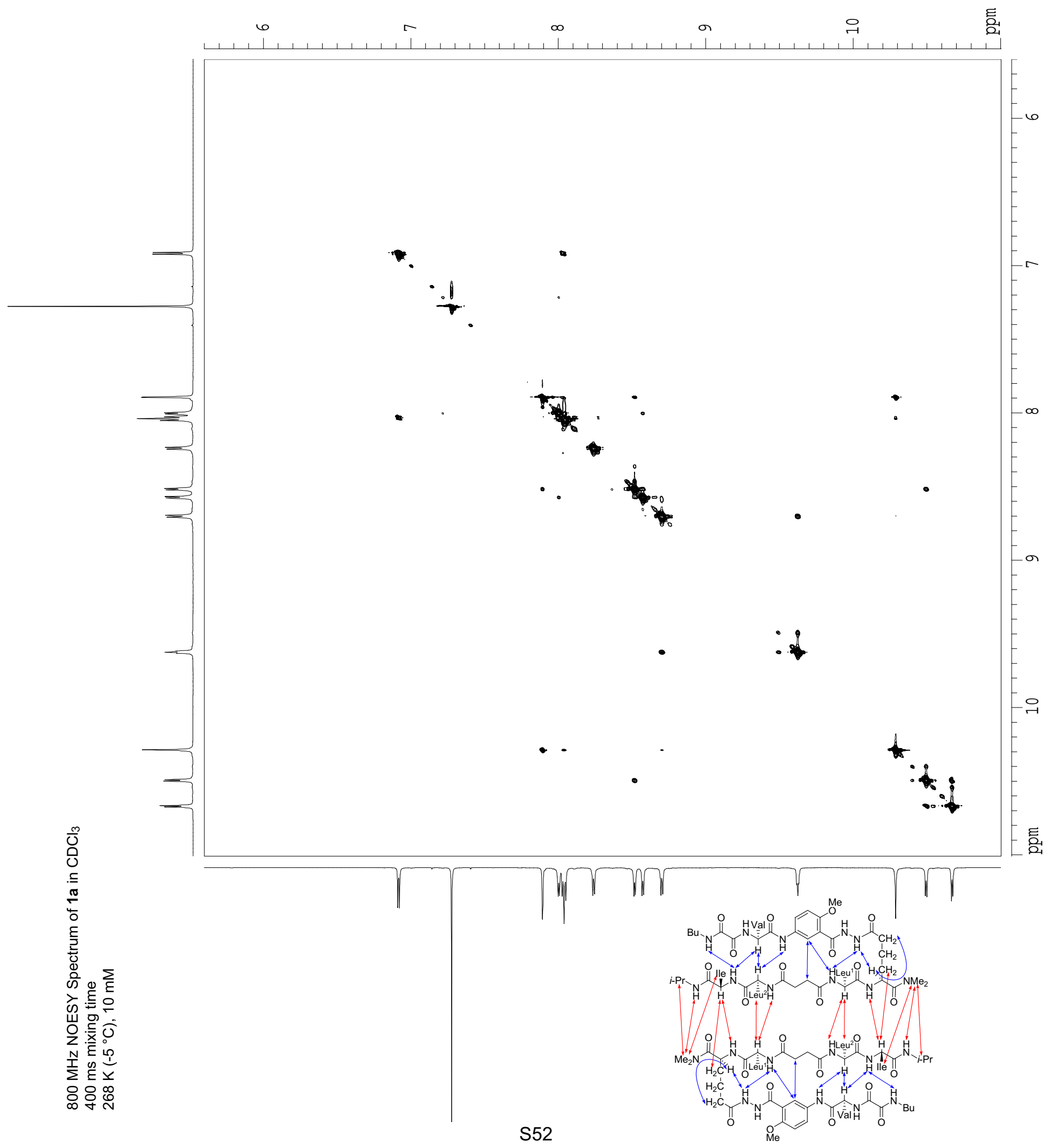


Supporting Information
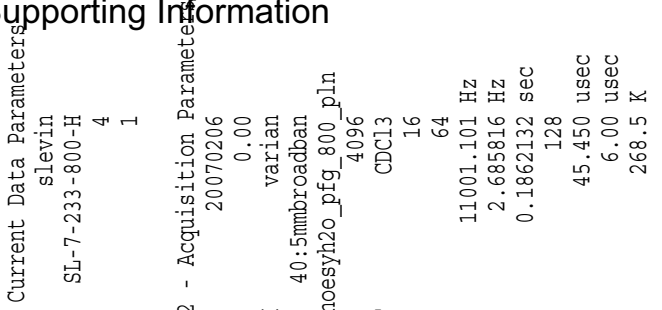

画罢离总

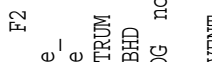

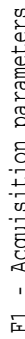

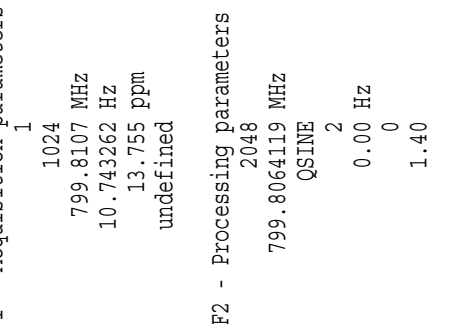

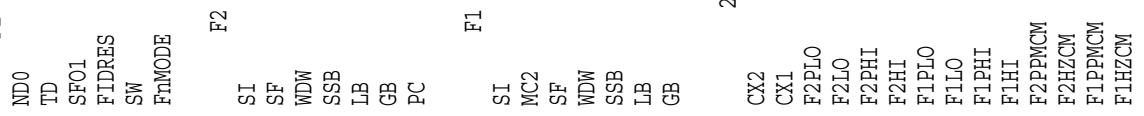

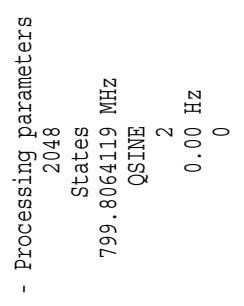

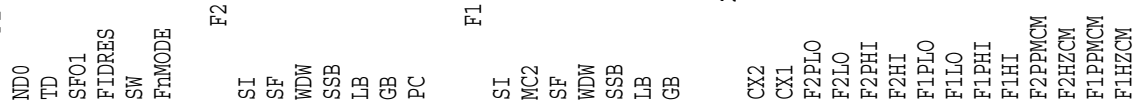

S. Levin and J. S. Nowick 离 ॠ

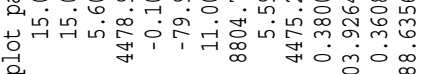
曽 ลิ

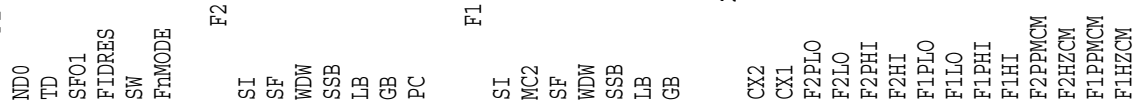

6 $\curvearrowleft \infty$ ต

言

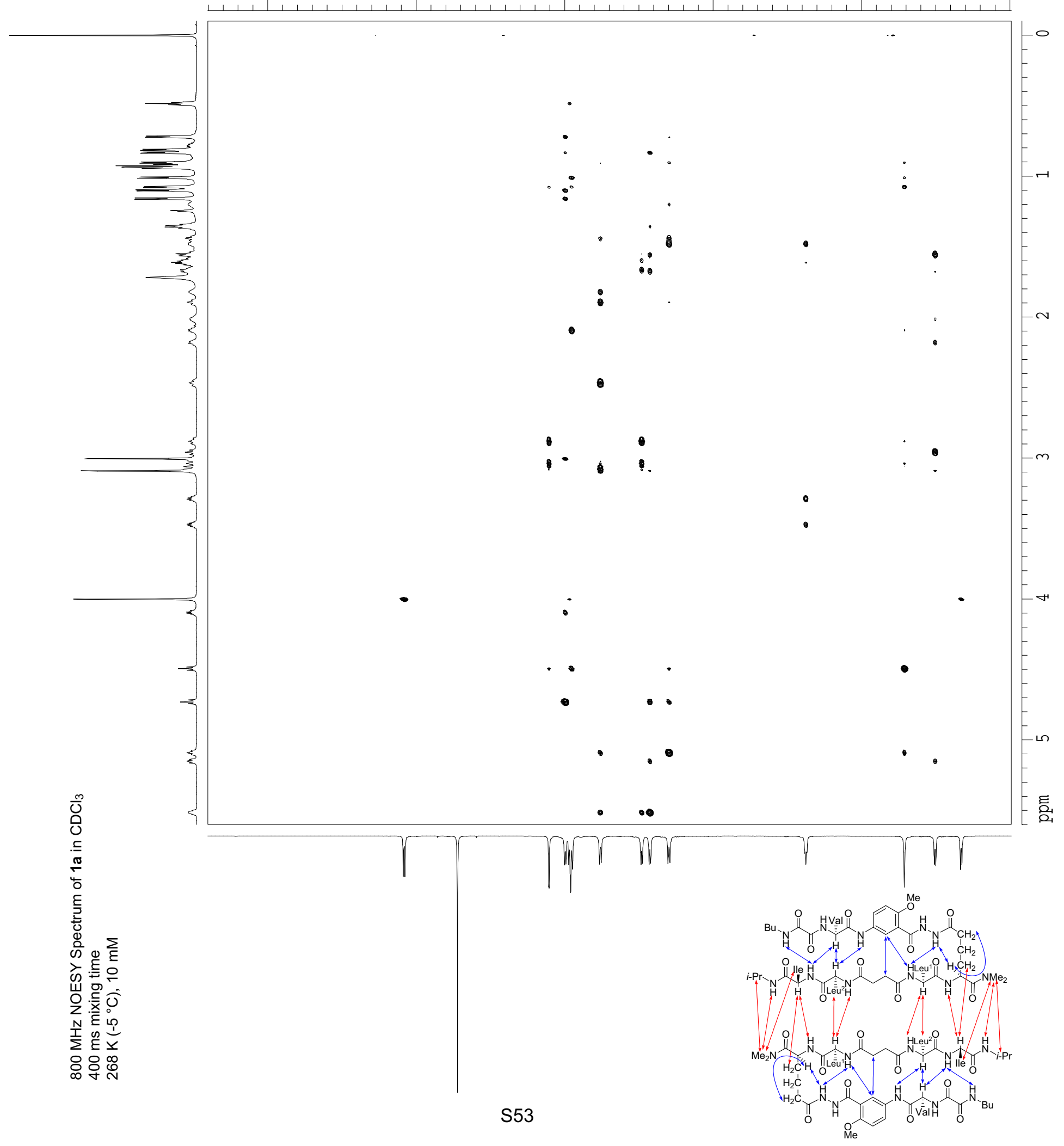


Supporting Information

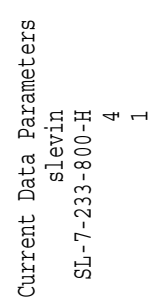

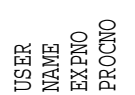

ता

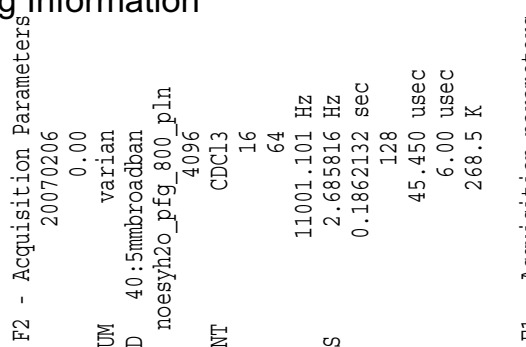

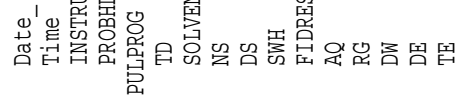

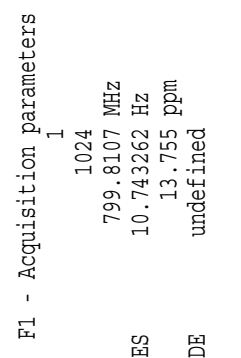

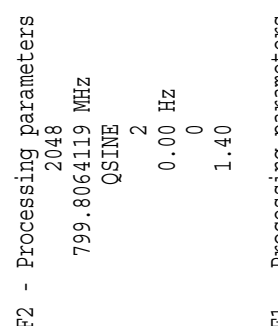

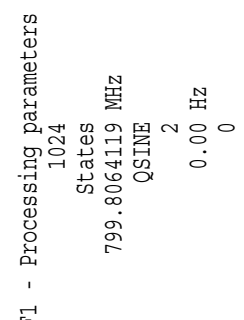

空

S. Levin and J. S. Nowick

过

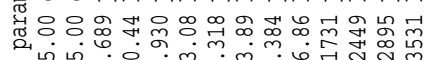

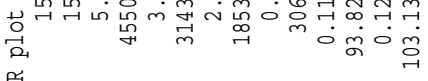
紋

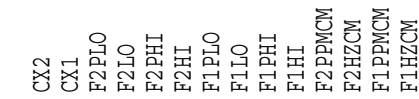

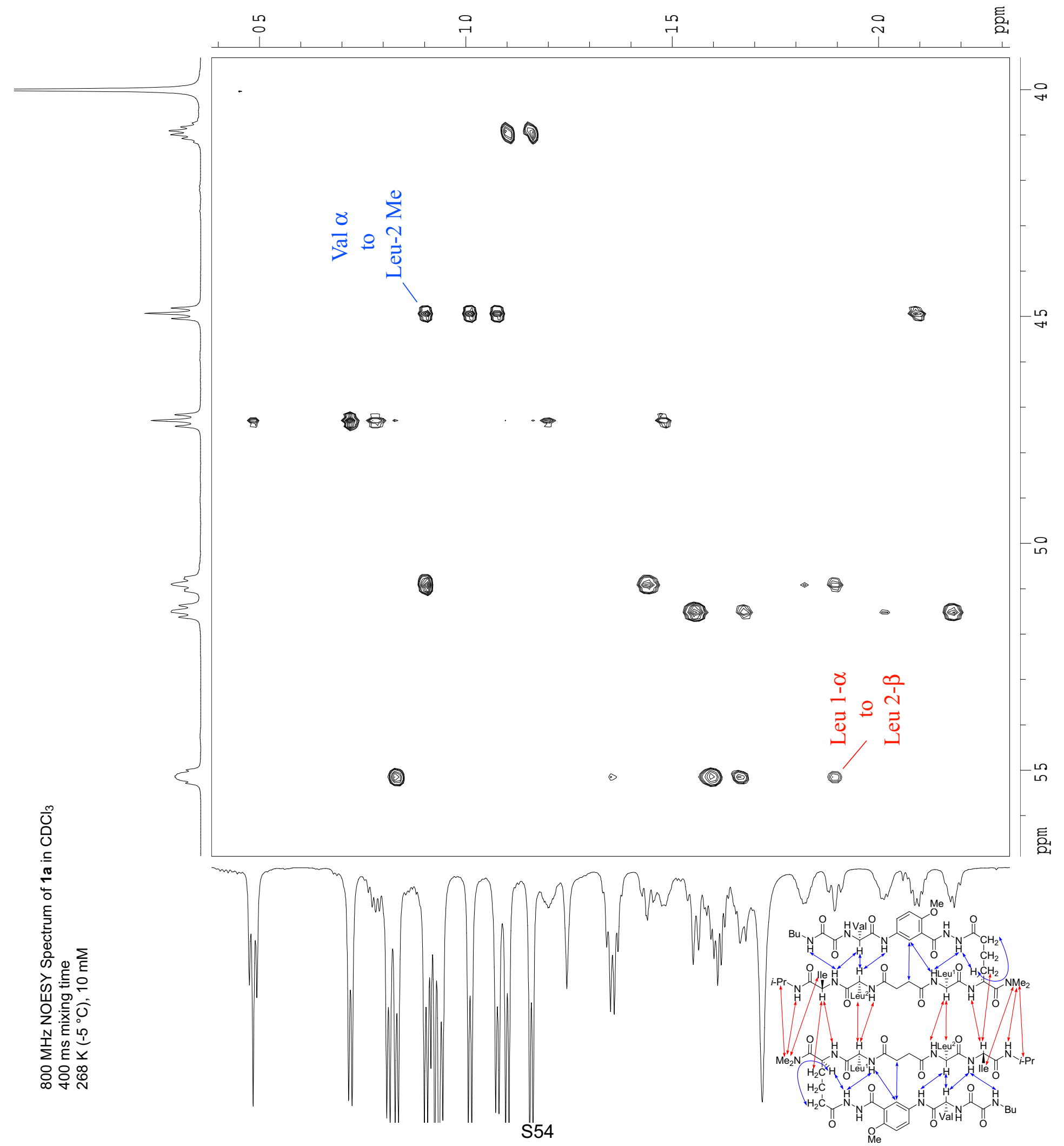




\section{Supporting dnformation}

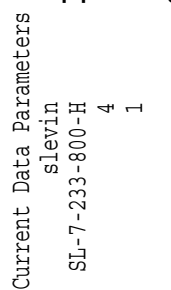

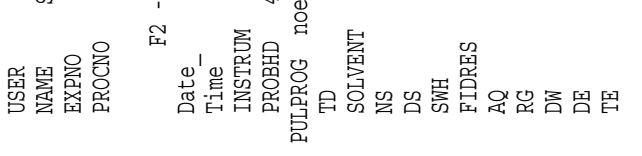
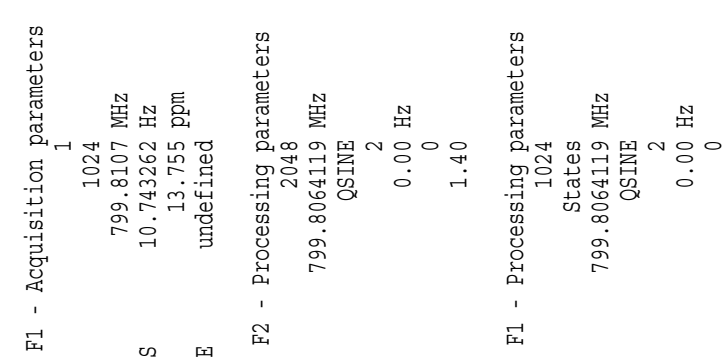

诖
S. Levin and J. S. Nowick
岂

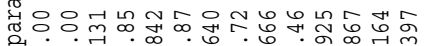

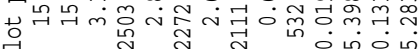
罢 จิ

(1)

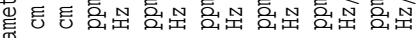

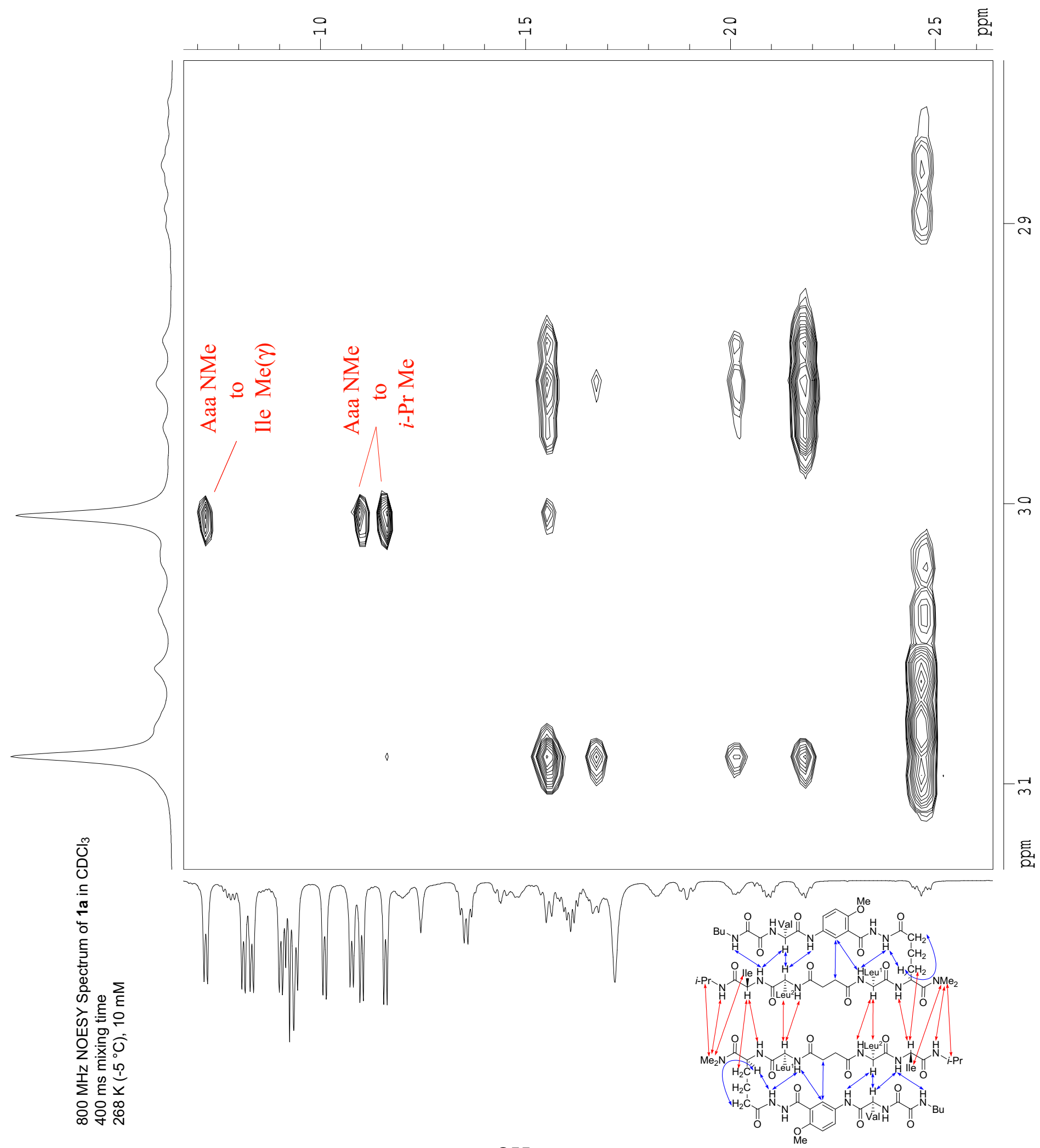




\section{Supporting Information}

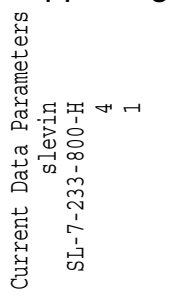

品留虽虽

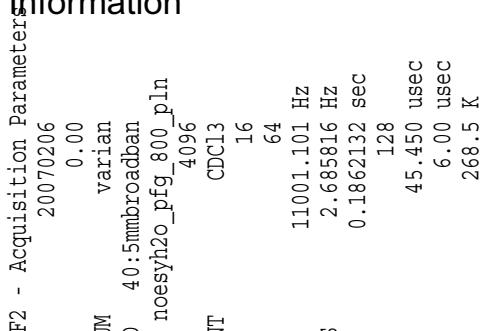

त्ञ,

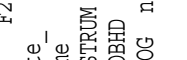

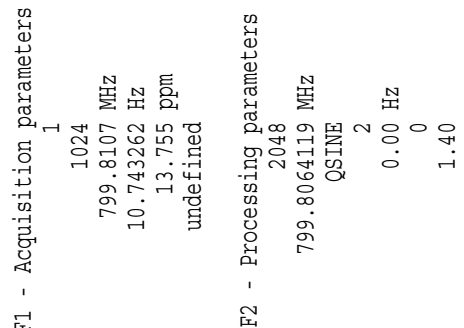
届留 㖉

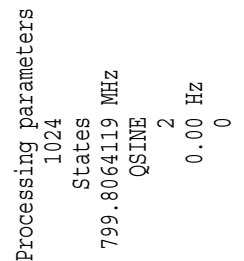

厒
S. Levin and J. S. Nowick

岂

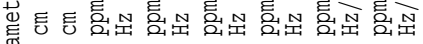

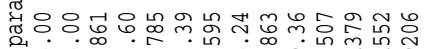

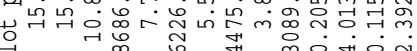
蓉 ลे

प्र

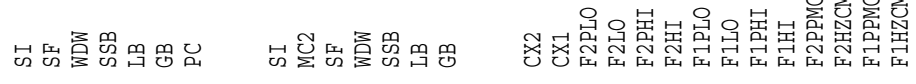

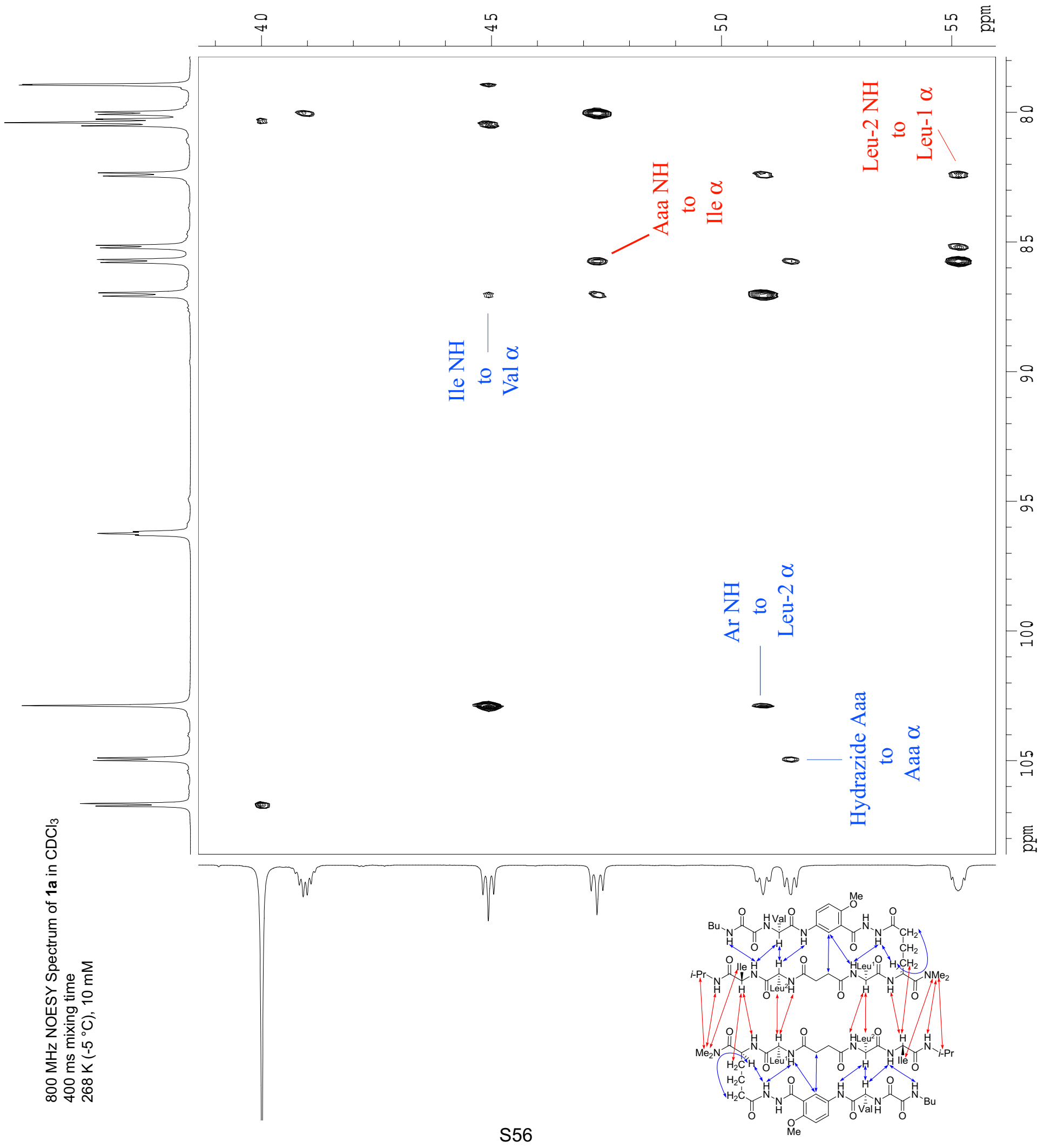


Supporting Information
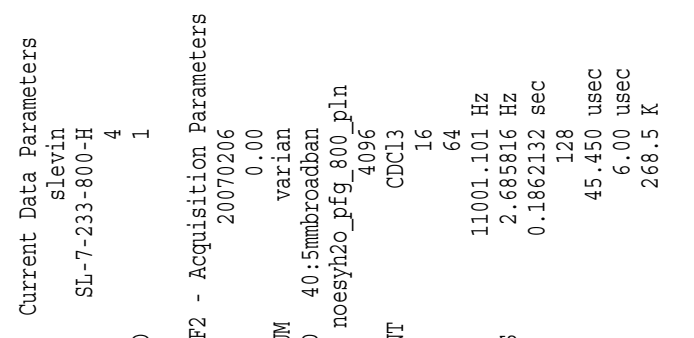

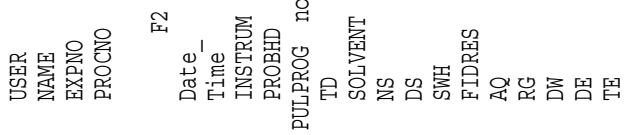

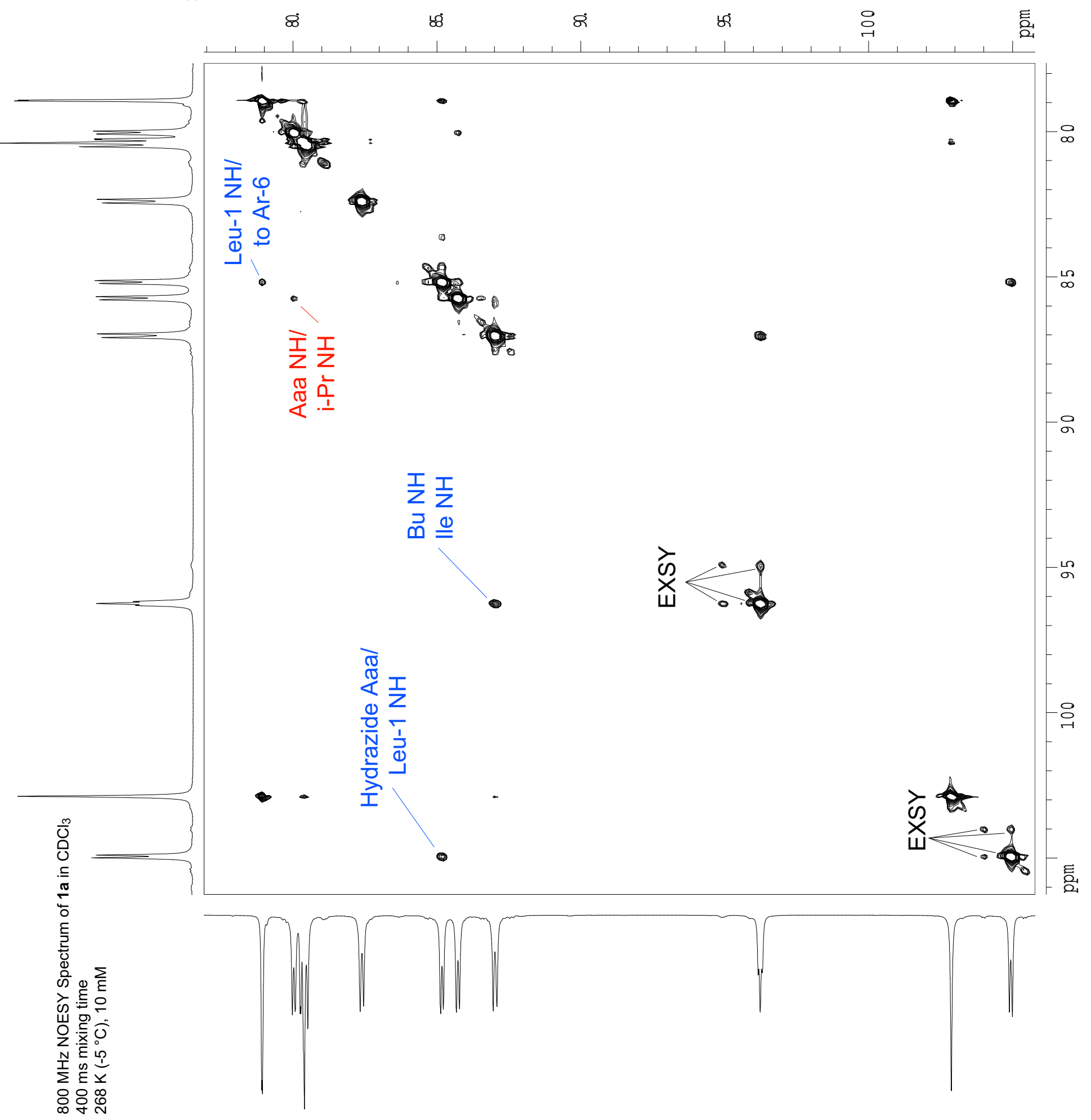




\section{Supporting Information}

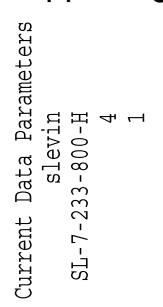

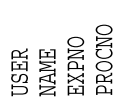

i

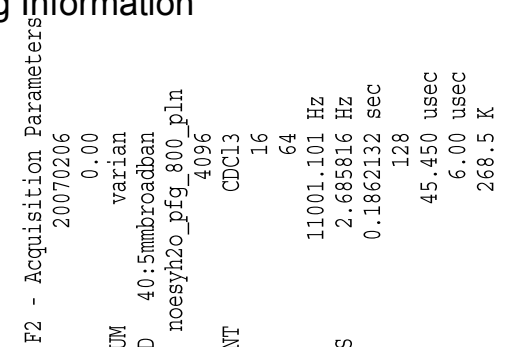

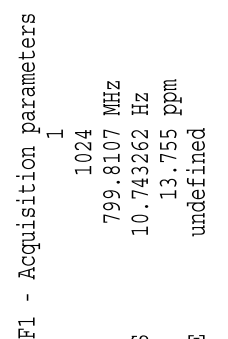

$\vec{r}$

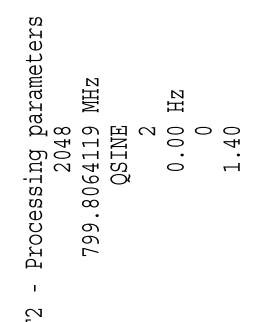

艒

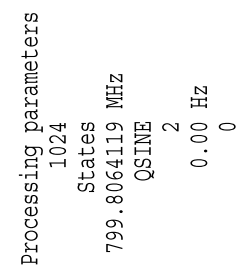

堂

S. Levin and J. S. Nowick

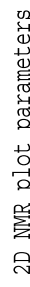

嵒

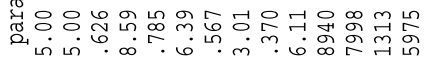
苟 崇

今

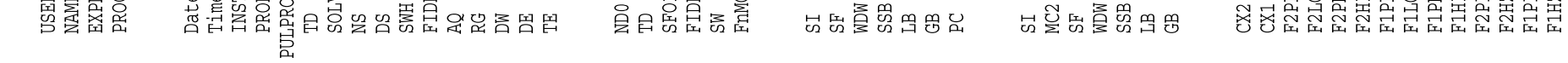

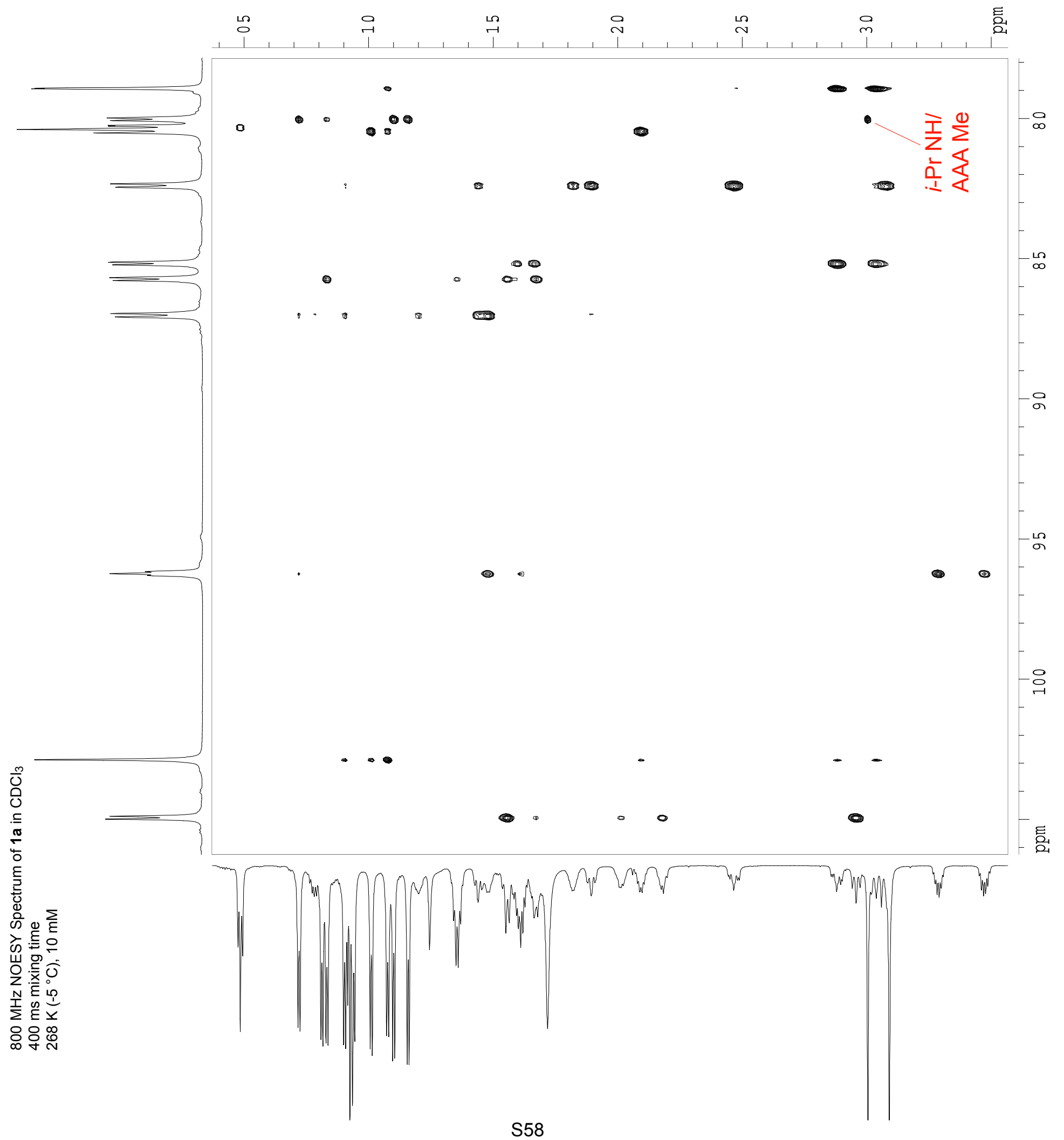



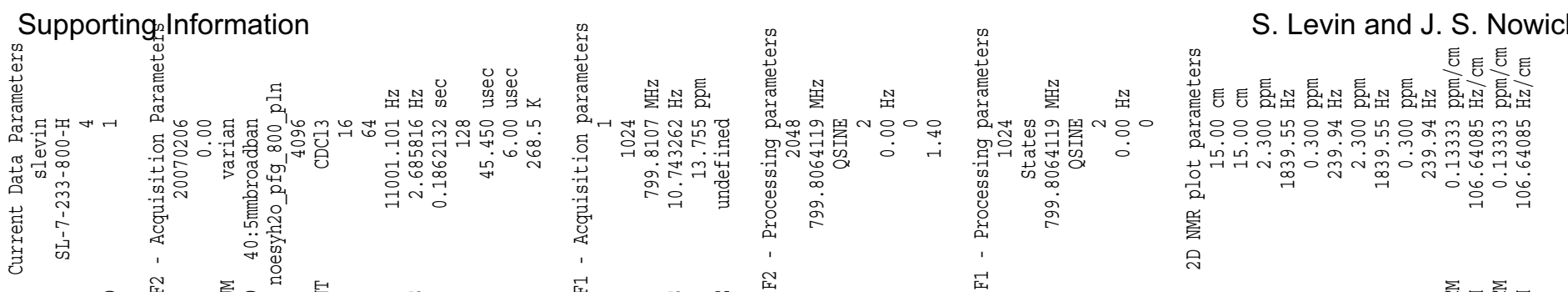

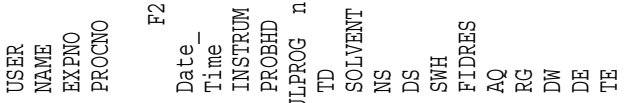

惫罢 Ge日a

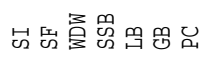

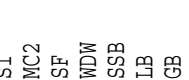

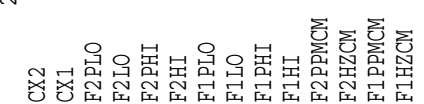

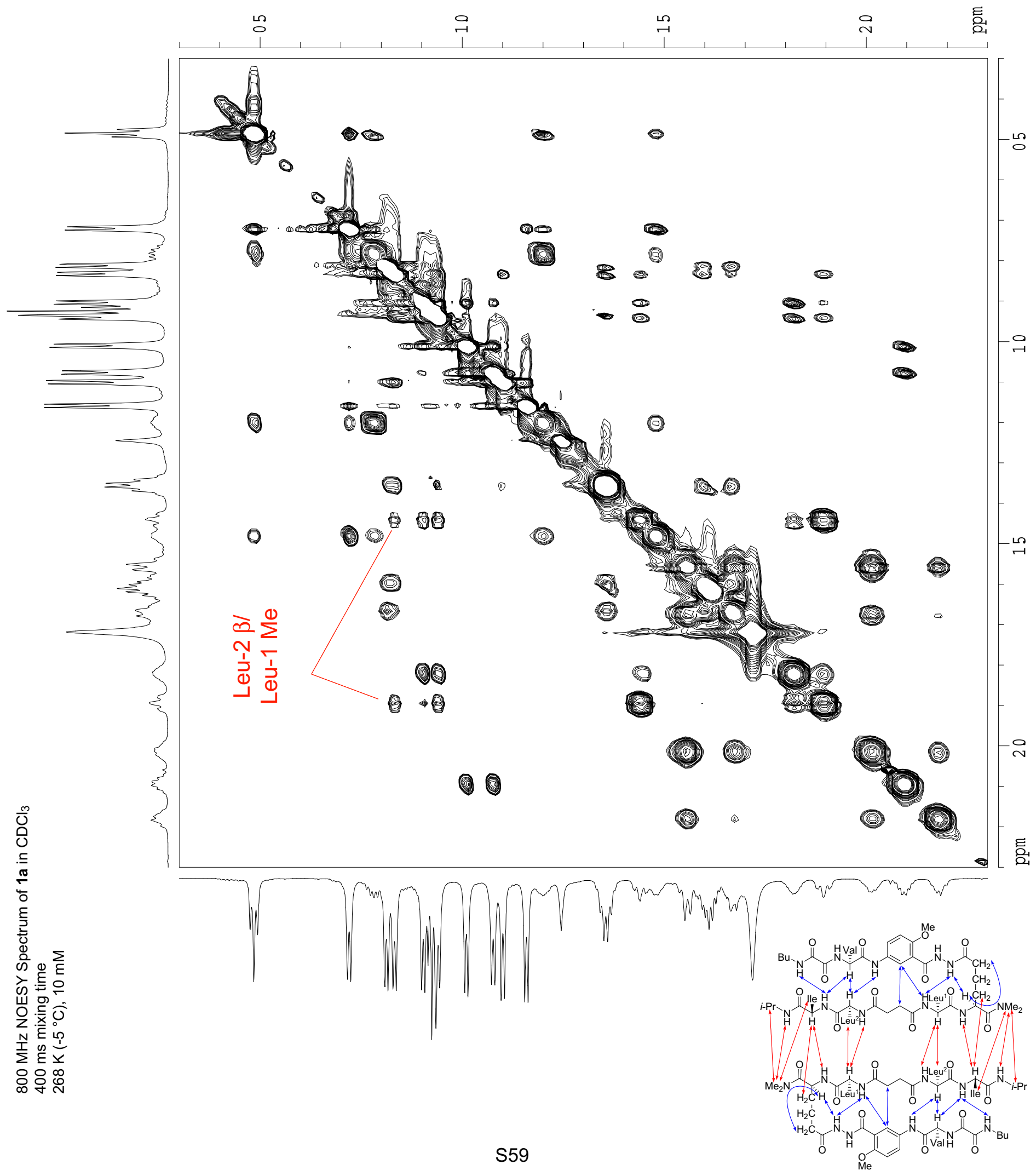


Supporting Information
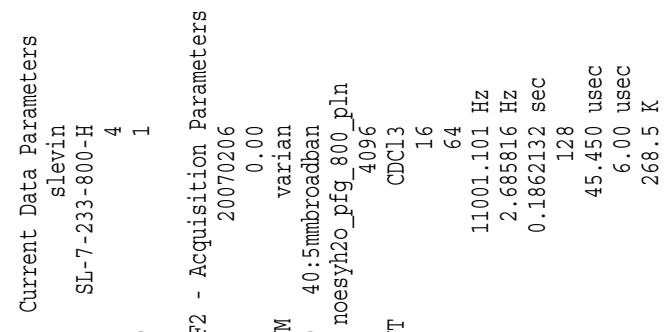

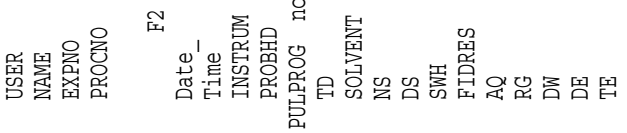

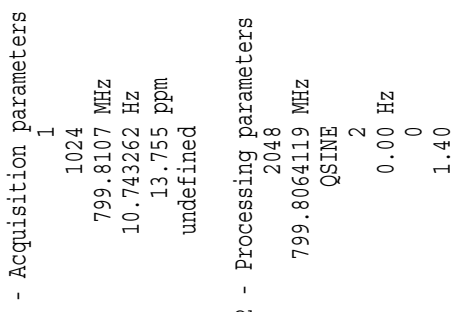

牙证

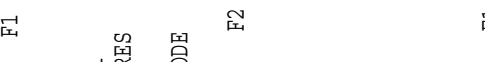

S. Levin and J. S. Nowick
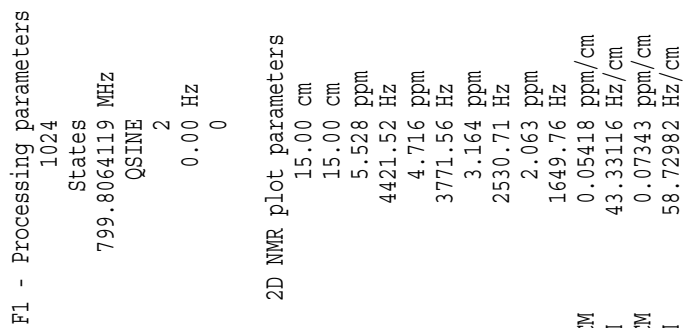

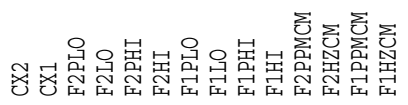

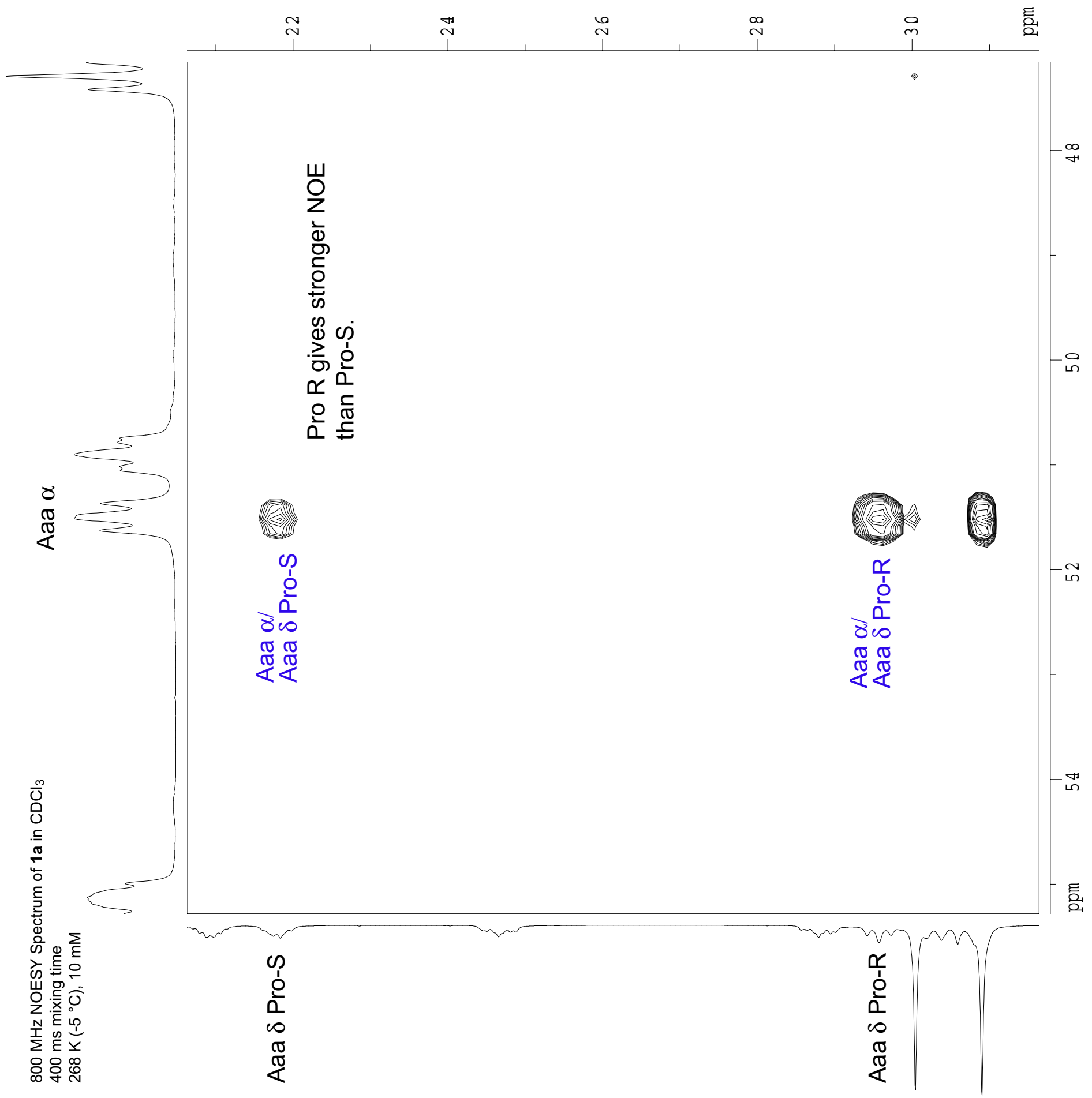


Supporting Information

S. Levin and J. S. Nowick
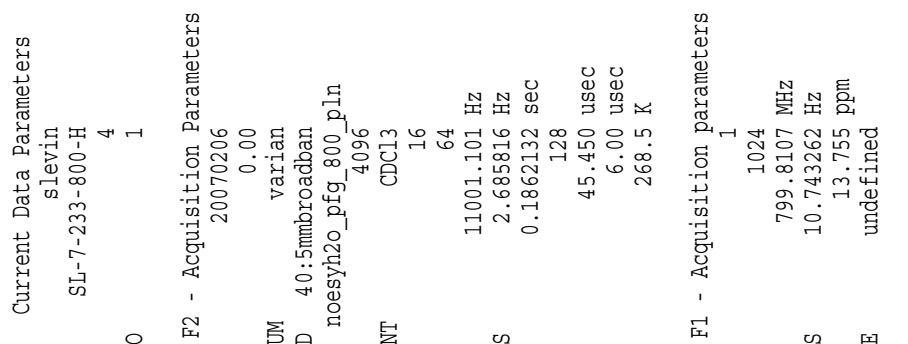

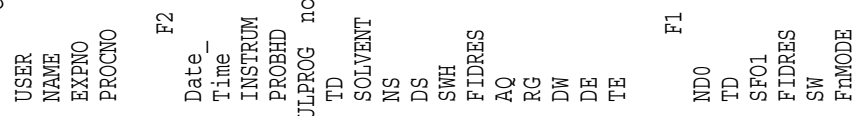
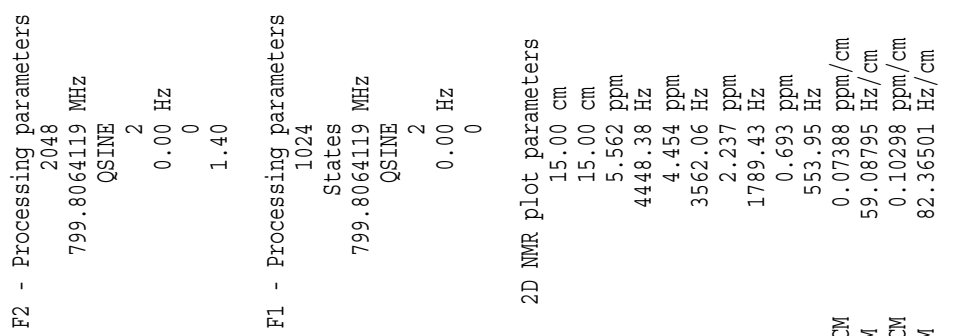

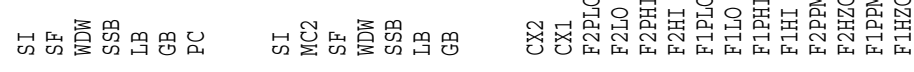

不

(2)

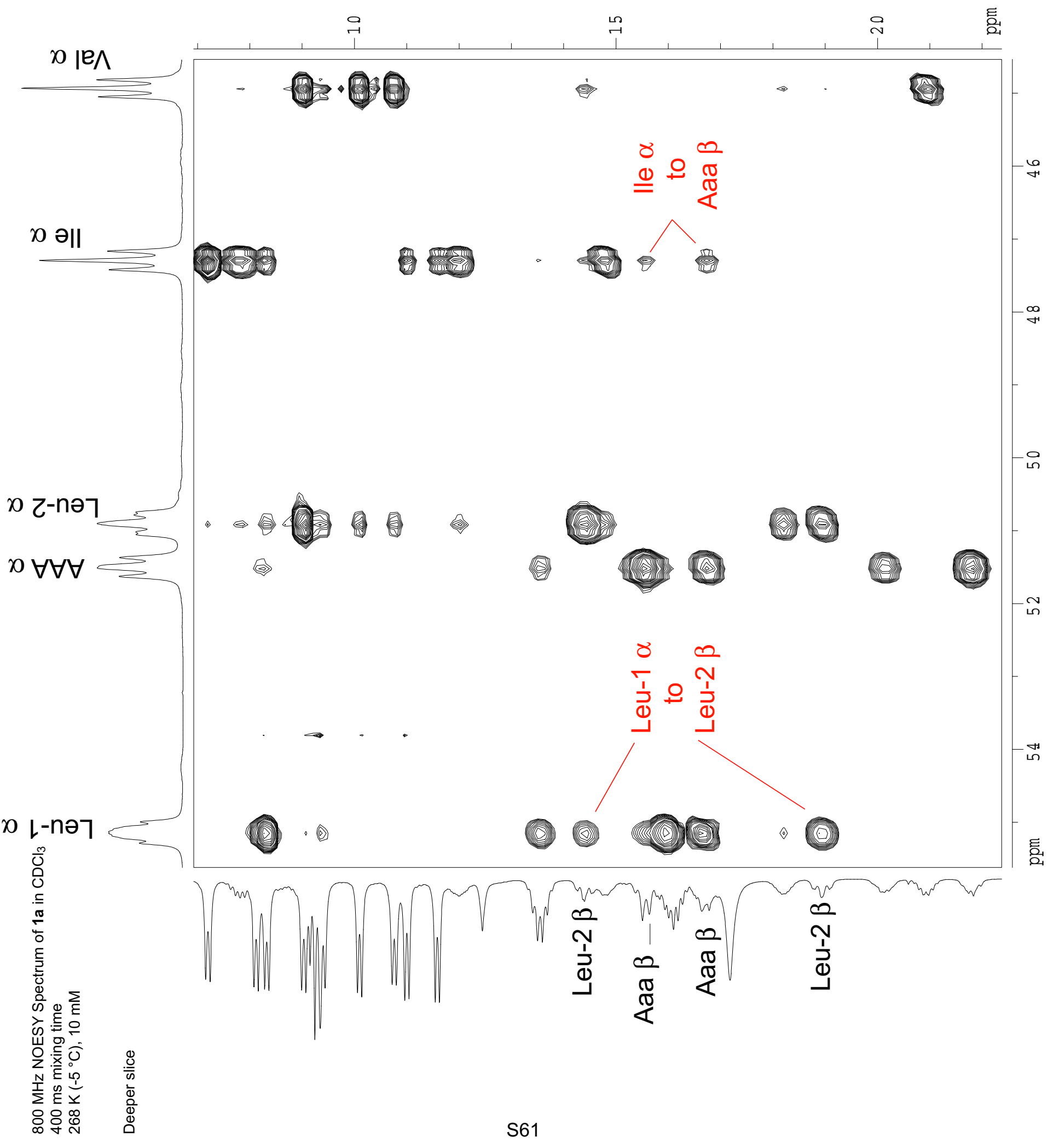


Supporting Information

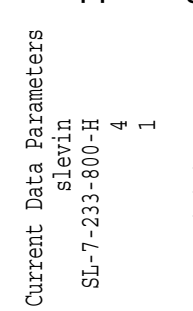

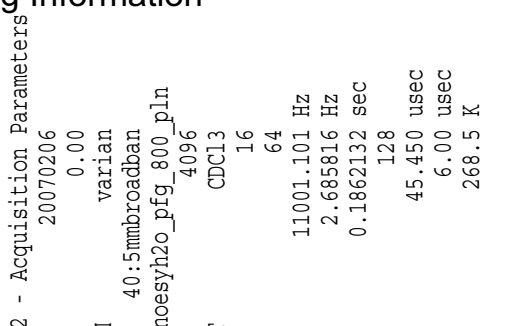

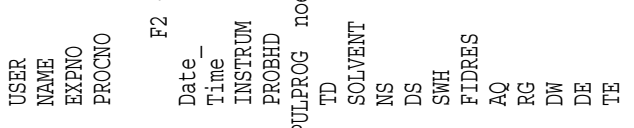

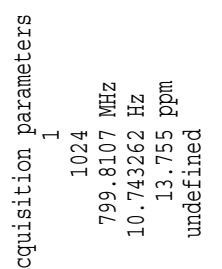

$\overrightarrow{1}$ 포

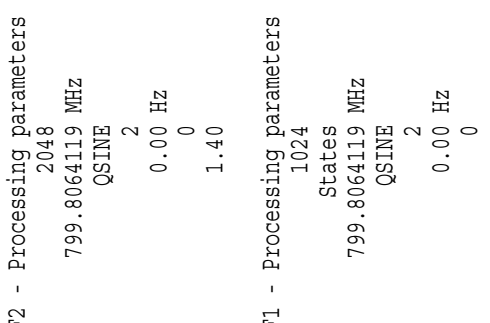

届

S. Levin and J. S. Nowick

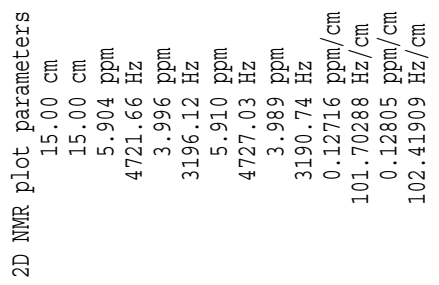

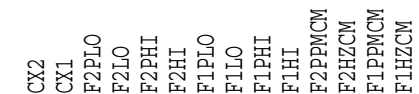

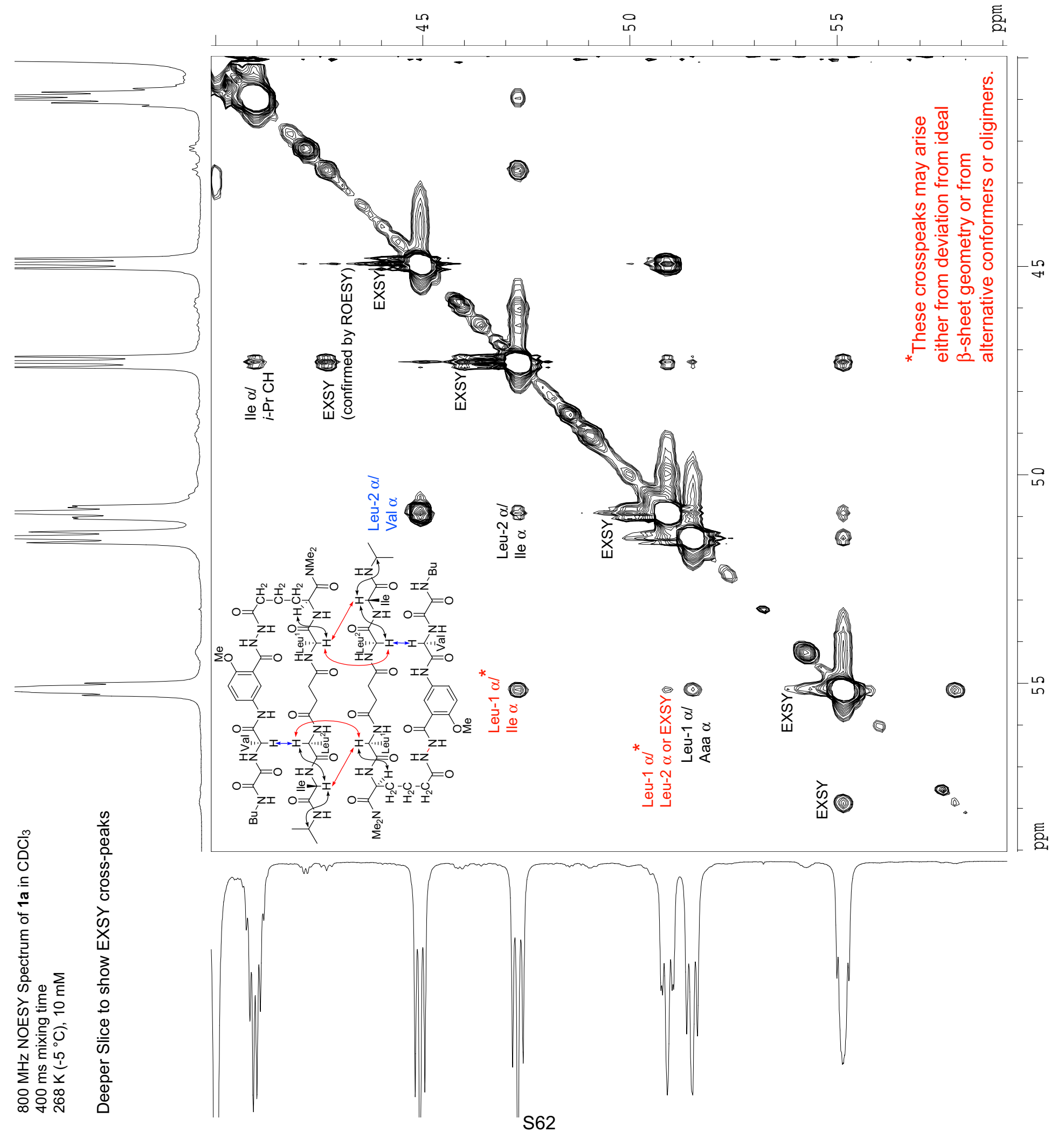


Supporting Information

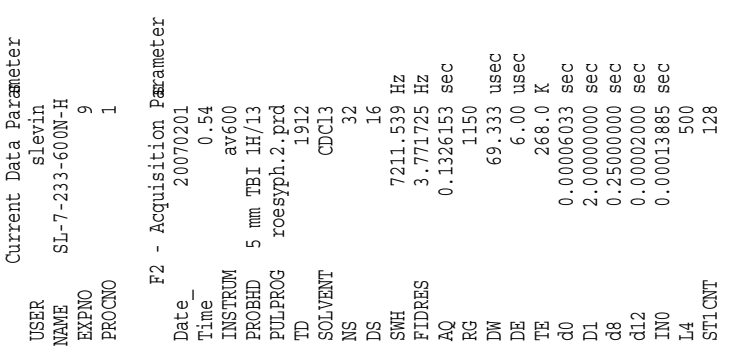

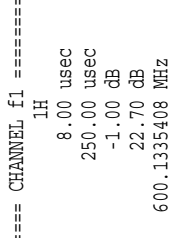

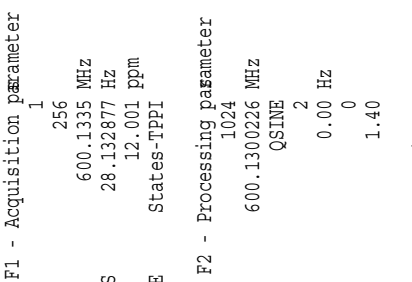

S. Levin and J. S. Nowick

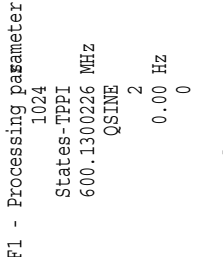

㟧

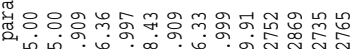
崫

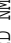

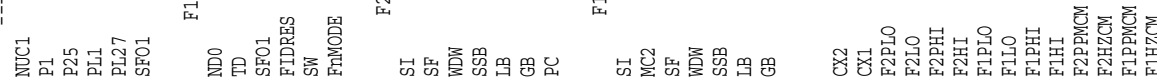

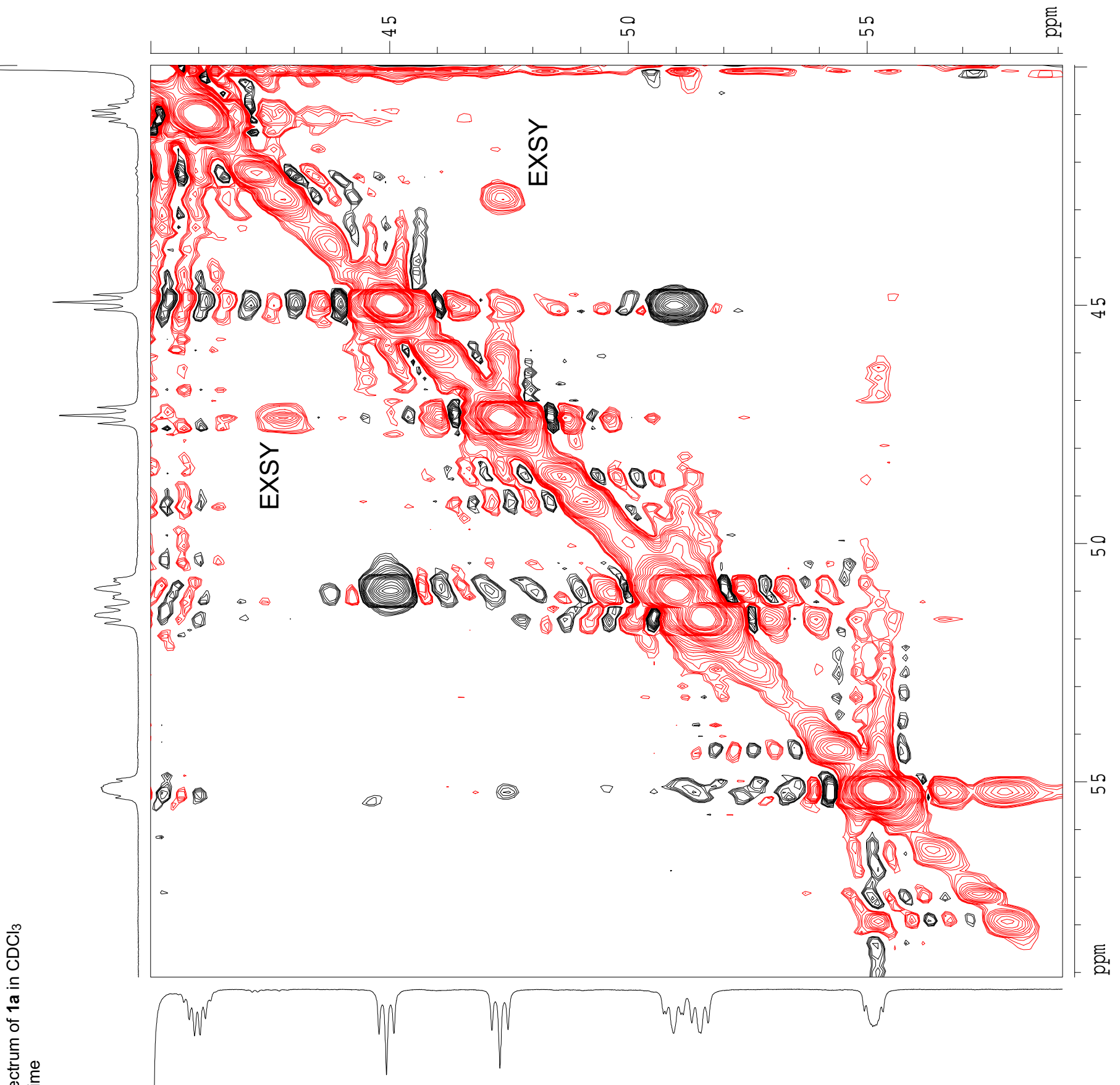




\section{Spectroscopic DATA for the compound $\mathbf{1 b}$ \\ (6 mM, $\left.\mathrm{CDCl}_{3} 268 \mathrm{~K}\right)$}
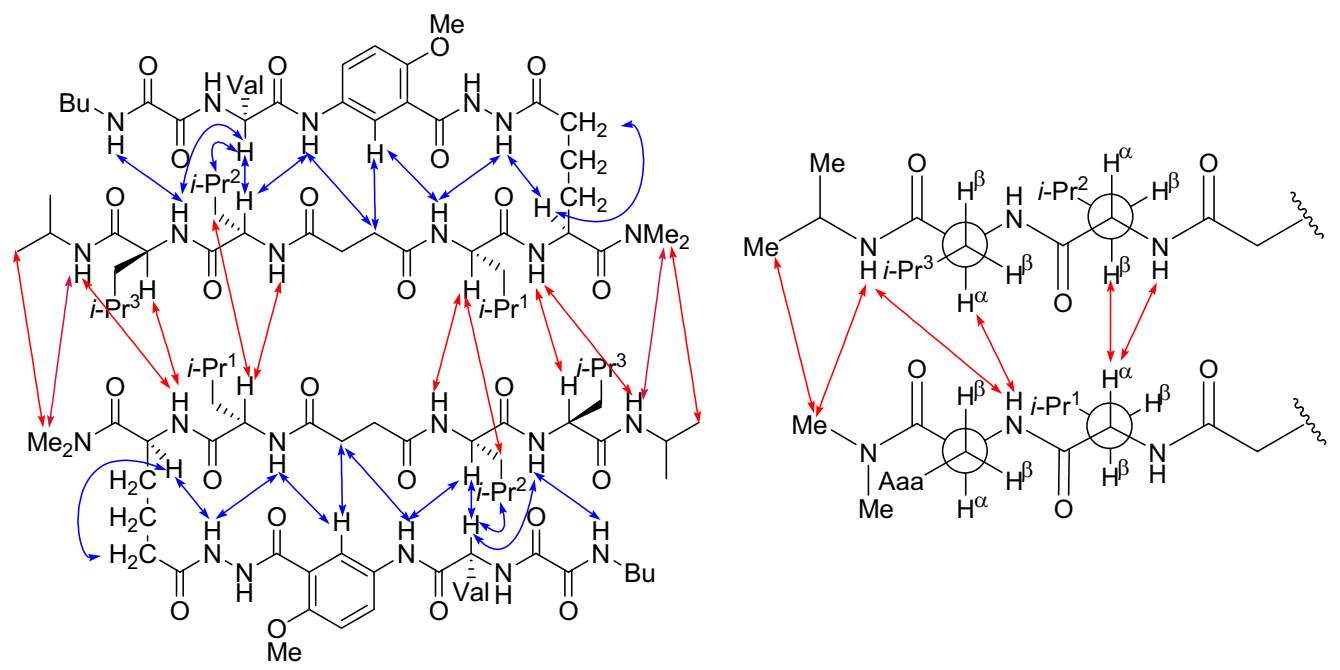

Key NOEs assigned as intermolecular are represented with red arrows

Key NOEs assigned as intramolecular are represented with blue arrows

Key data for amino acids.

\begin{tabular}{|c|c|c|c|}
\hline Amino acid & $\mathrm{H}_{\alpha}$ Shift ppm & NH Shift ppm & ${ }^{3} J_{\mathrm{NH} \alpha} \mathrm{Hz}$ \\
\hline Aaa & 5.16 & 8.67 & 9.1 \\
\hline $\mathrm{R}_{1}=\mathrm{Leu}^{1}$ & 5.53 & 8.47 & 8.0 \\
\hline $\mathrm{R}_{2}=\mathrm{Leu}^{2}$ & 5.02 & 8.25 & 9.7 \\
\hline $\mathrm{R}_{3}=\mathrm{Leu}^{3}$ & 5.01 & 8.68 & 10.4 \\
\hline $\mathrm{R}_{4}=\mathrm{Val}$ & 4.46 & 8.04 & 8.7 \\
\hline
\end{tabular}

Aminoadipic acid (Aaa) $\delta$ proton resonances.

pro-R: 2.92 ppm, t, (overlap)

pro-S: $2.18 \mathrm{ppm}, \mathrm{td},\left(J_{1}=11.5 \mathrm{~Hz} ; J_{2}=7.0 \mathrm{~Hz}\right)$

anisotropy $=0.74 \mathrm{ppm}$

Key data for other NH protons.

\begin{tabular}{|c|c|c|}
\hline Proton & NH Shift ppm & $J \mathrm{~Hz}$ \\
\hline Ar-NH & 10.28 & - \\
\hline Hydrazide Ar & 10.59 & 7.4 \\
\hline Hydrazide Aaa & 10.44 & 7.4 \\
\hline Bu-NH & 9.54 & 5.9 \\
\hline$i$-Pr-NH & 8.03 & 7.7 \\
\hline
\end{tabular}

ESI MS $m / z$ for $\mathrm{C}_{52} \mathrm{H}_{88} \mathrm{~N}_{11} \mathrm{O}_{12}[\mathrm{M}+\mathrm{H}]^{+}$calcd 1058.66, found 1058.51. 

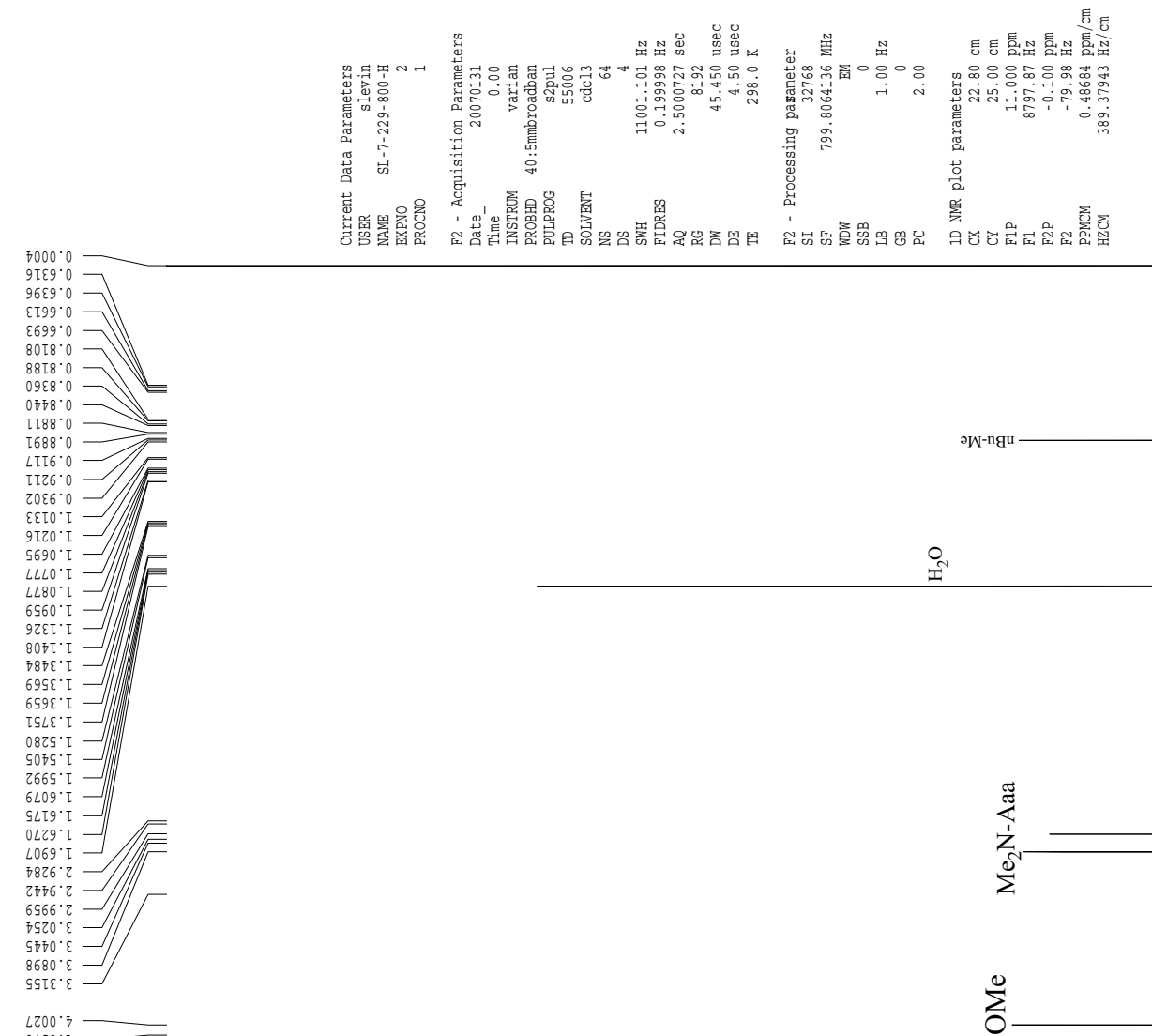

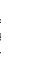

$O \angle S 0^{\circ} \mathrm{F}$

OZ97.

senes

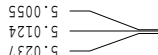
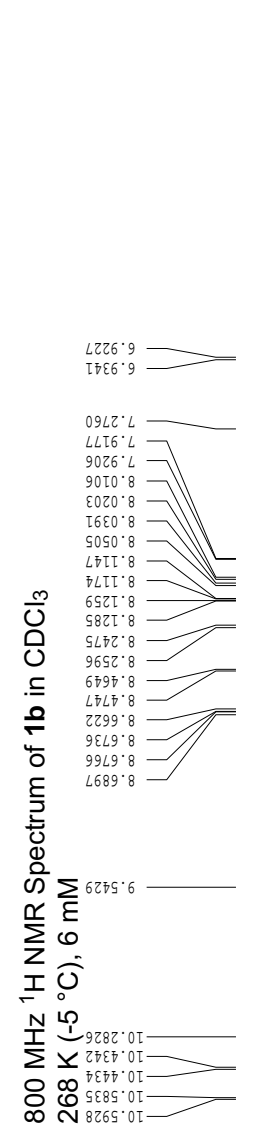

udd
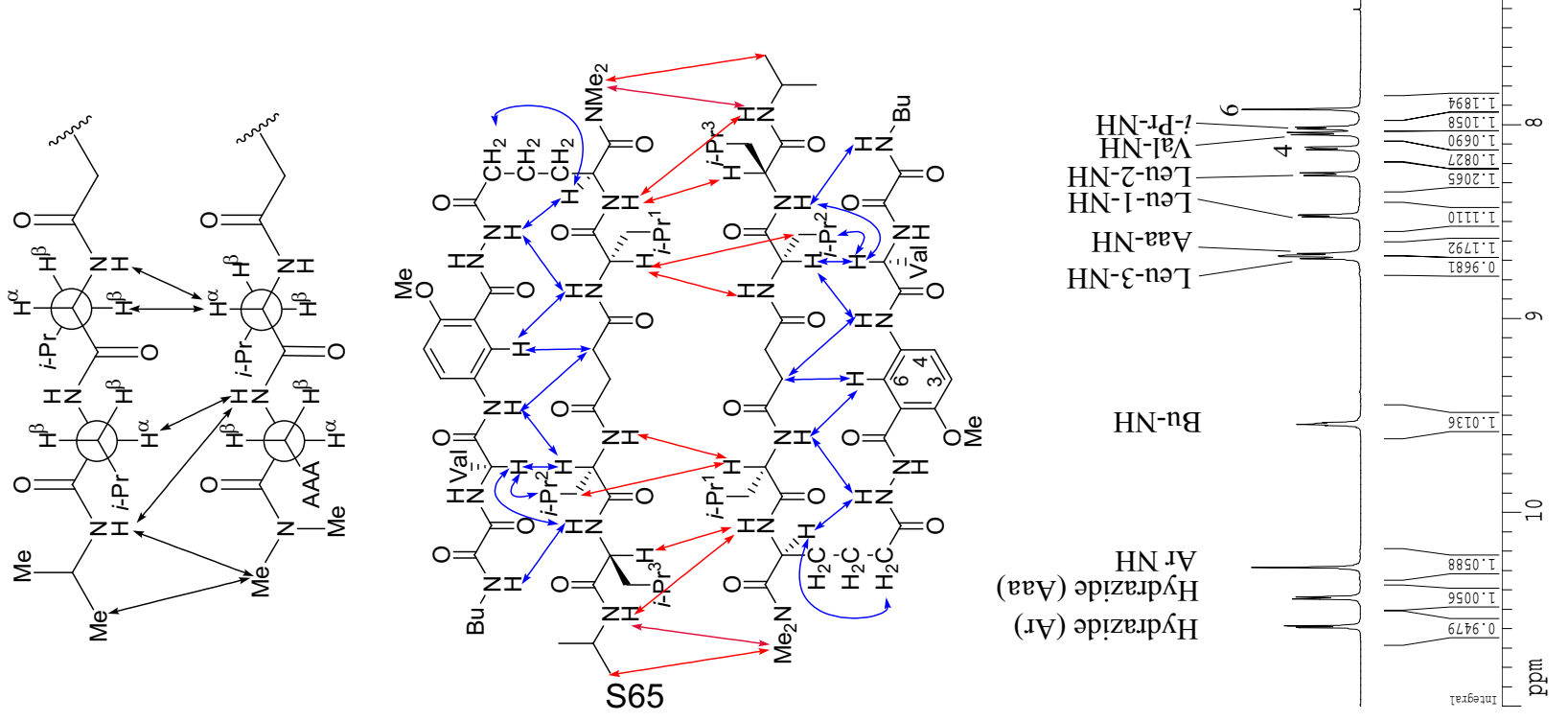

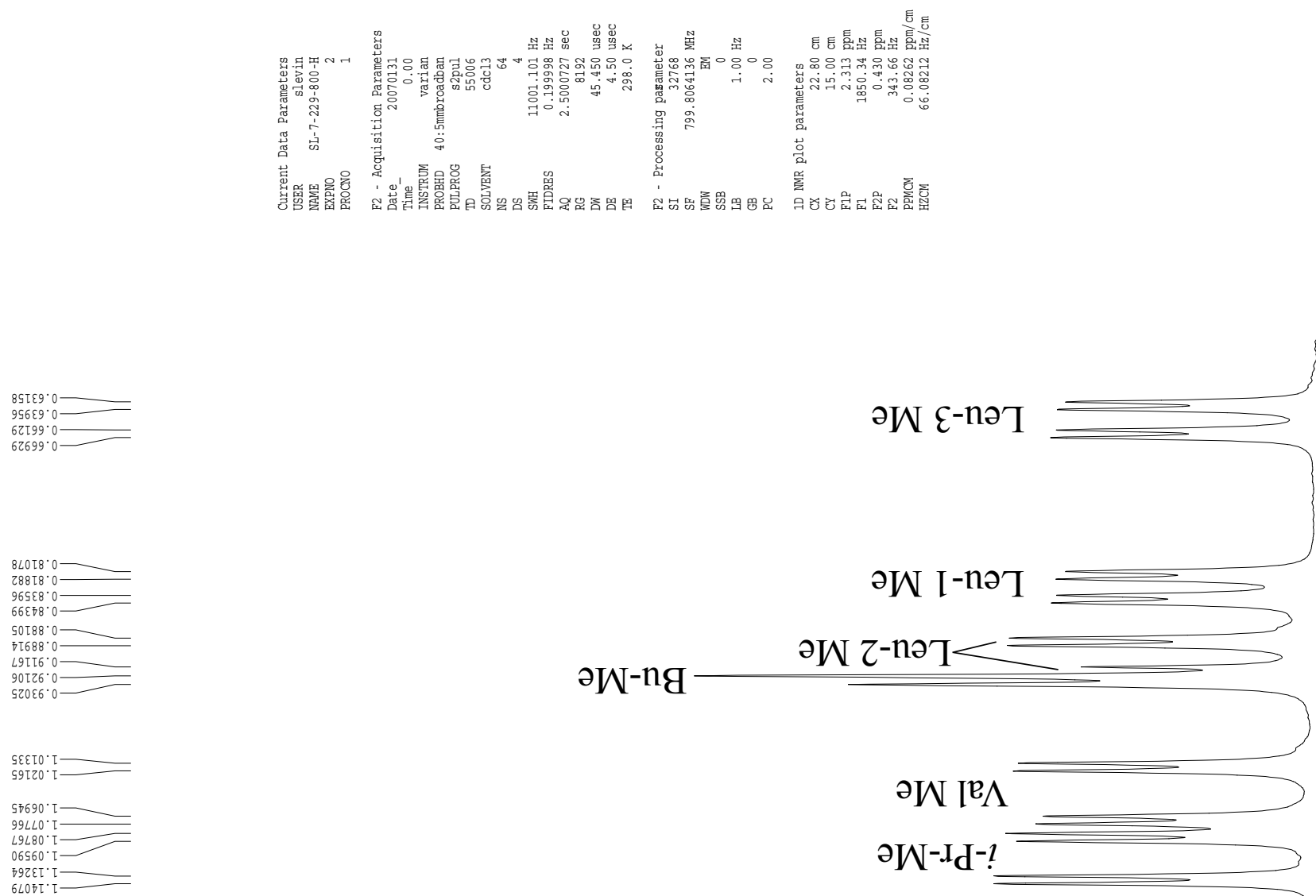

W-ng
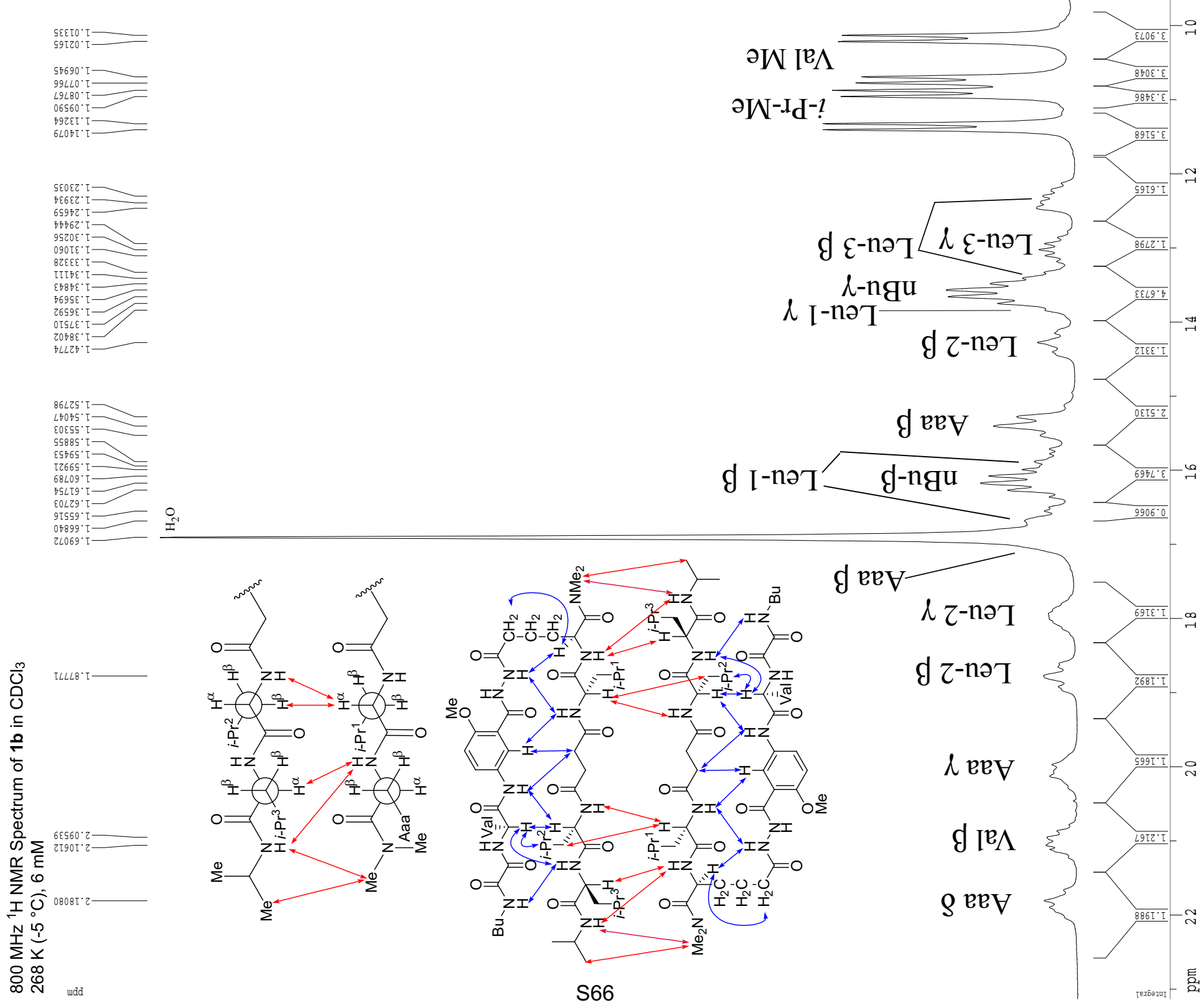

옥
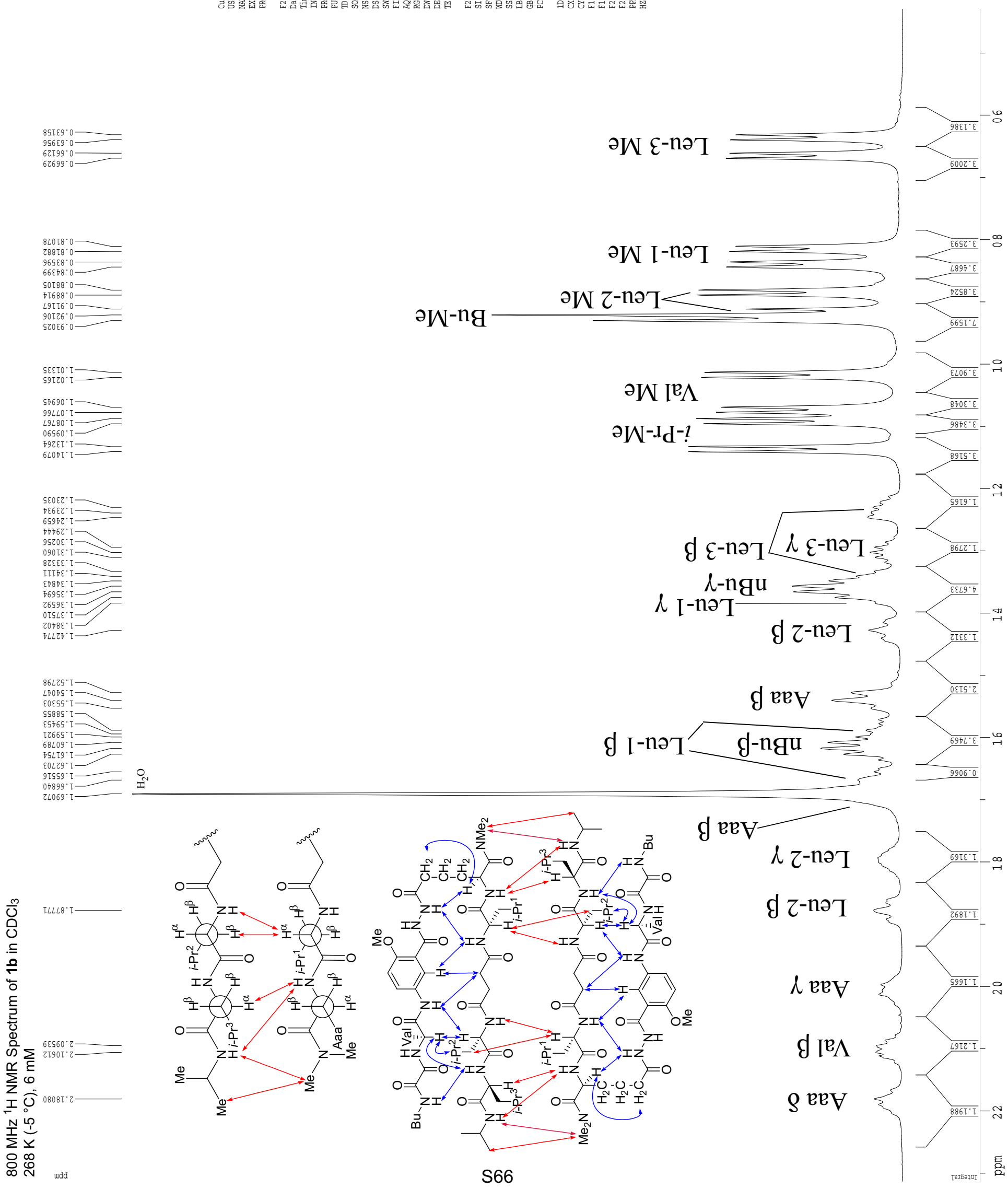


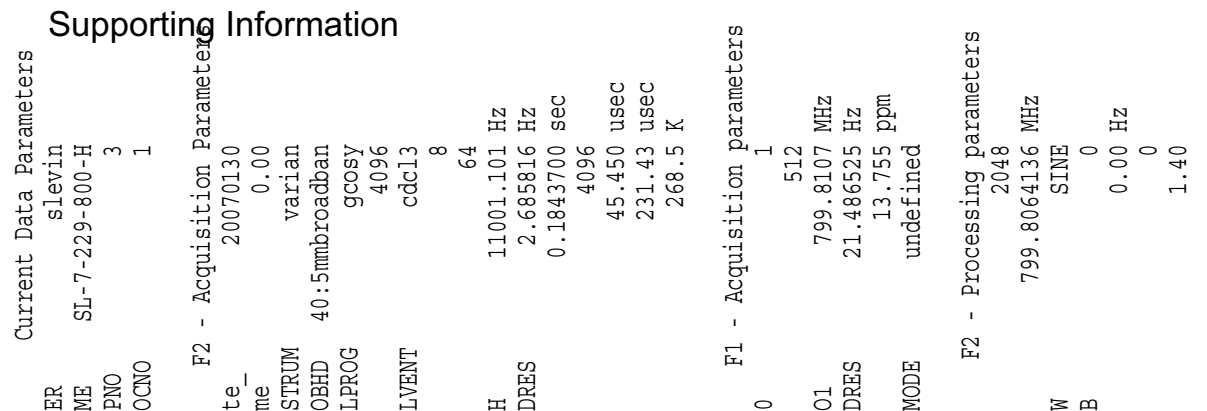

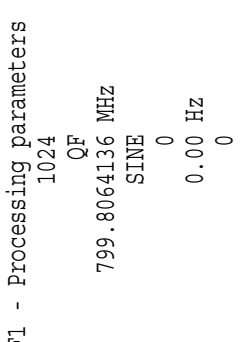

宔
S. Levin and J. S. Nowick

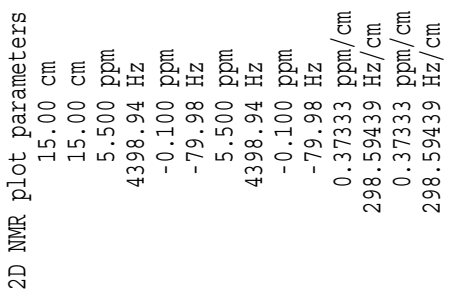

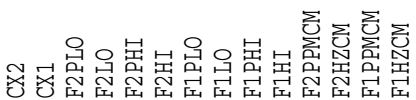

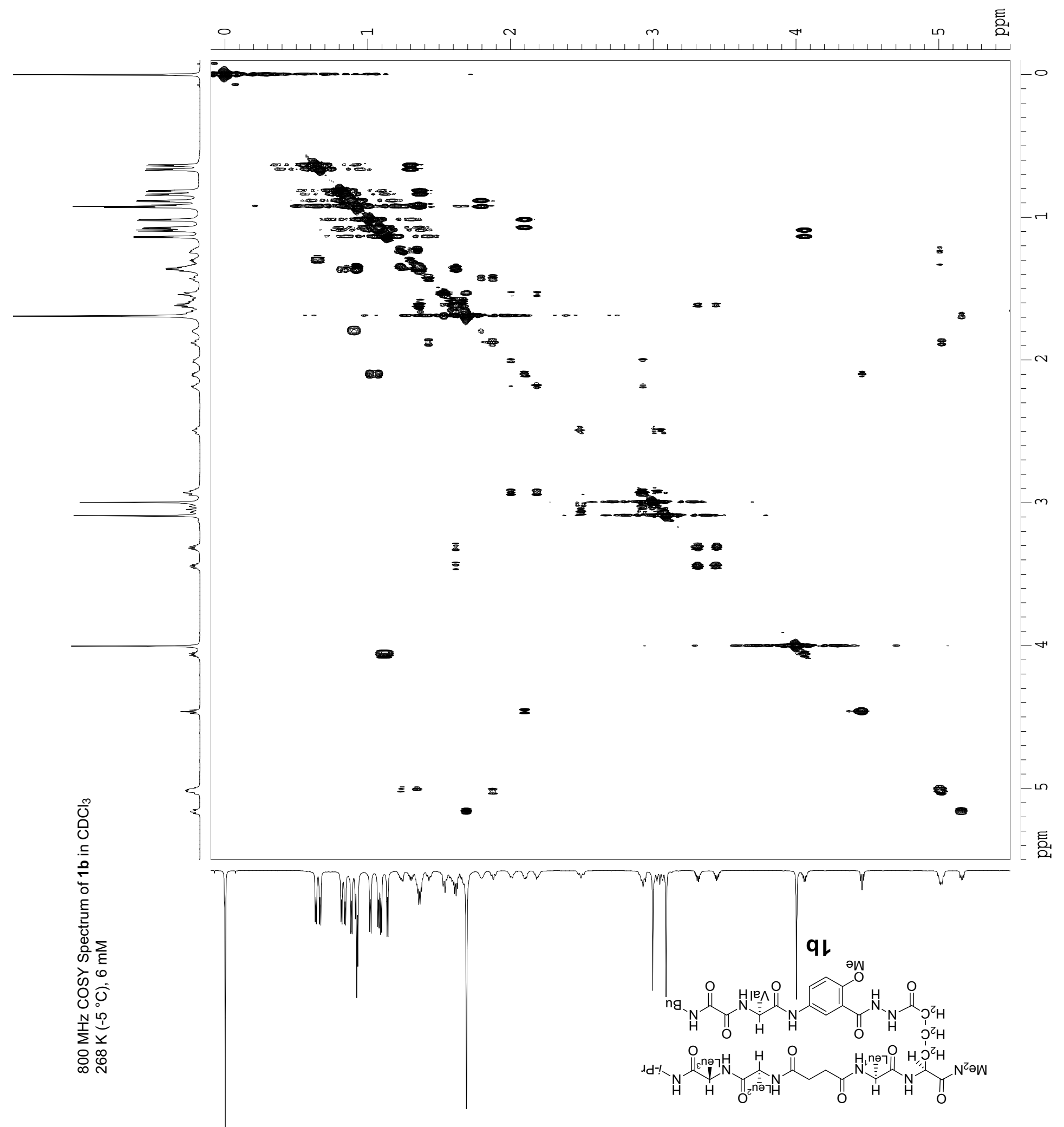



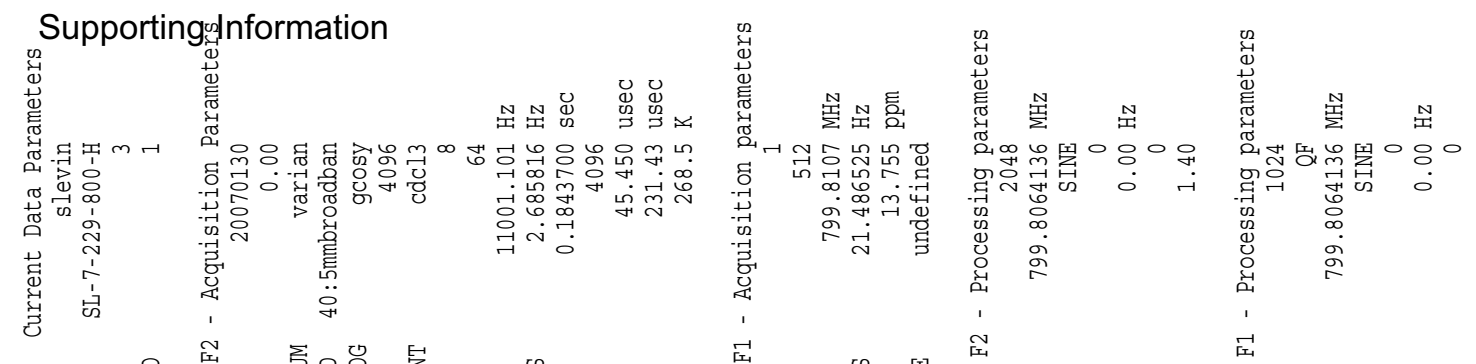

S. Levin and J. S. Nowick

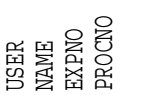
䑰 㞋 舀

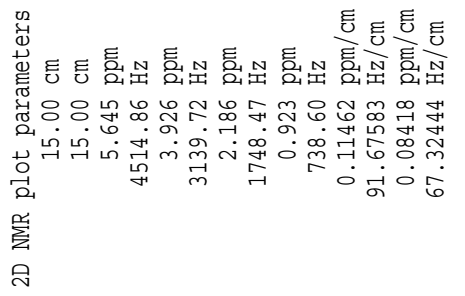

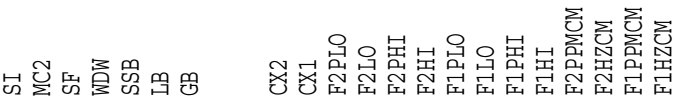

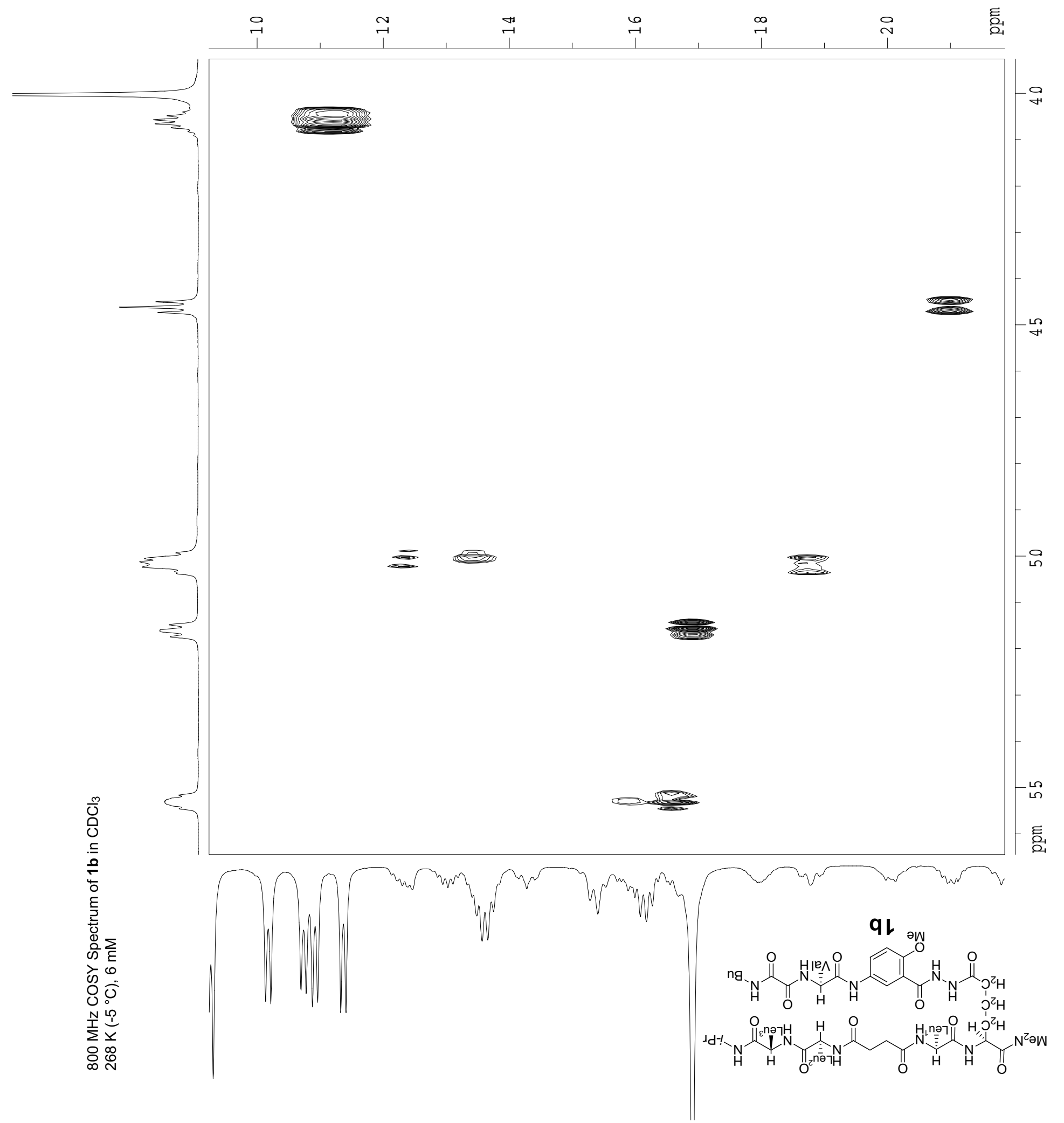




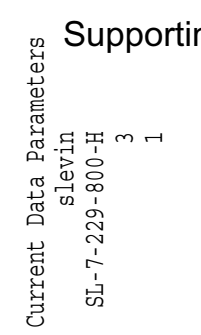

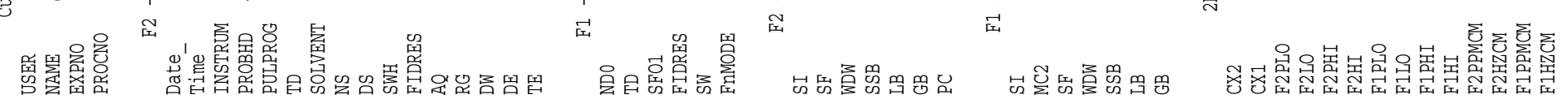

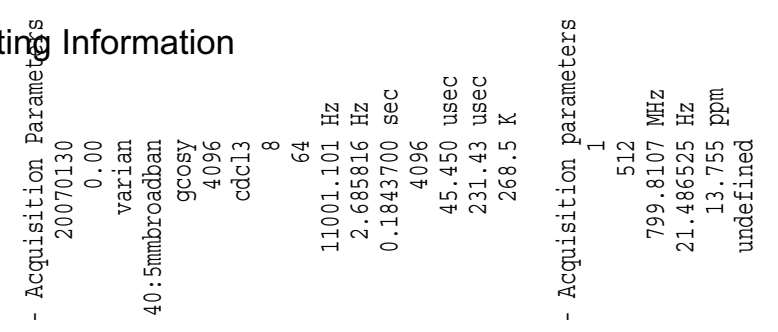

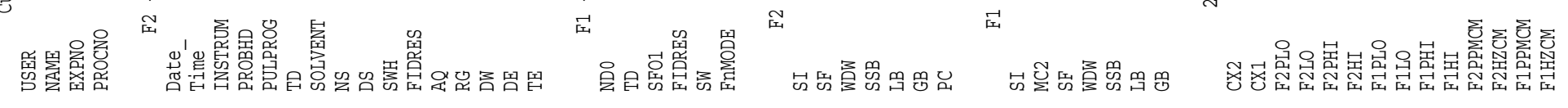

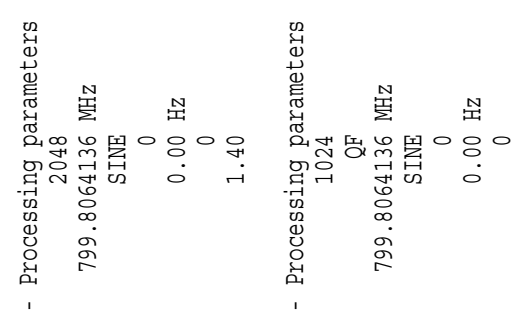

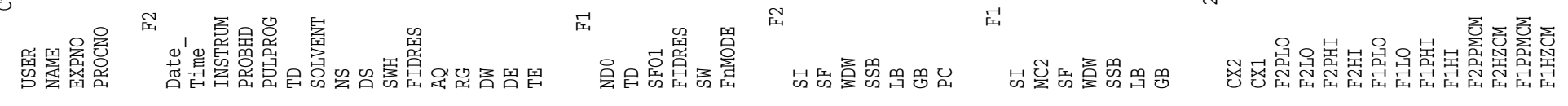

S. Levin and J. S. Nowick

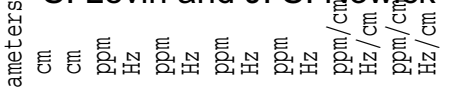

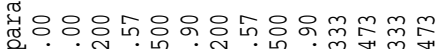

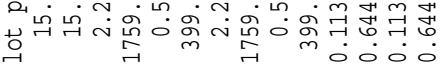
产

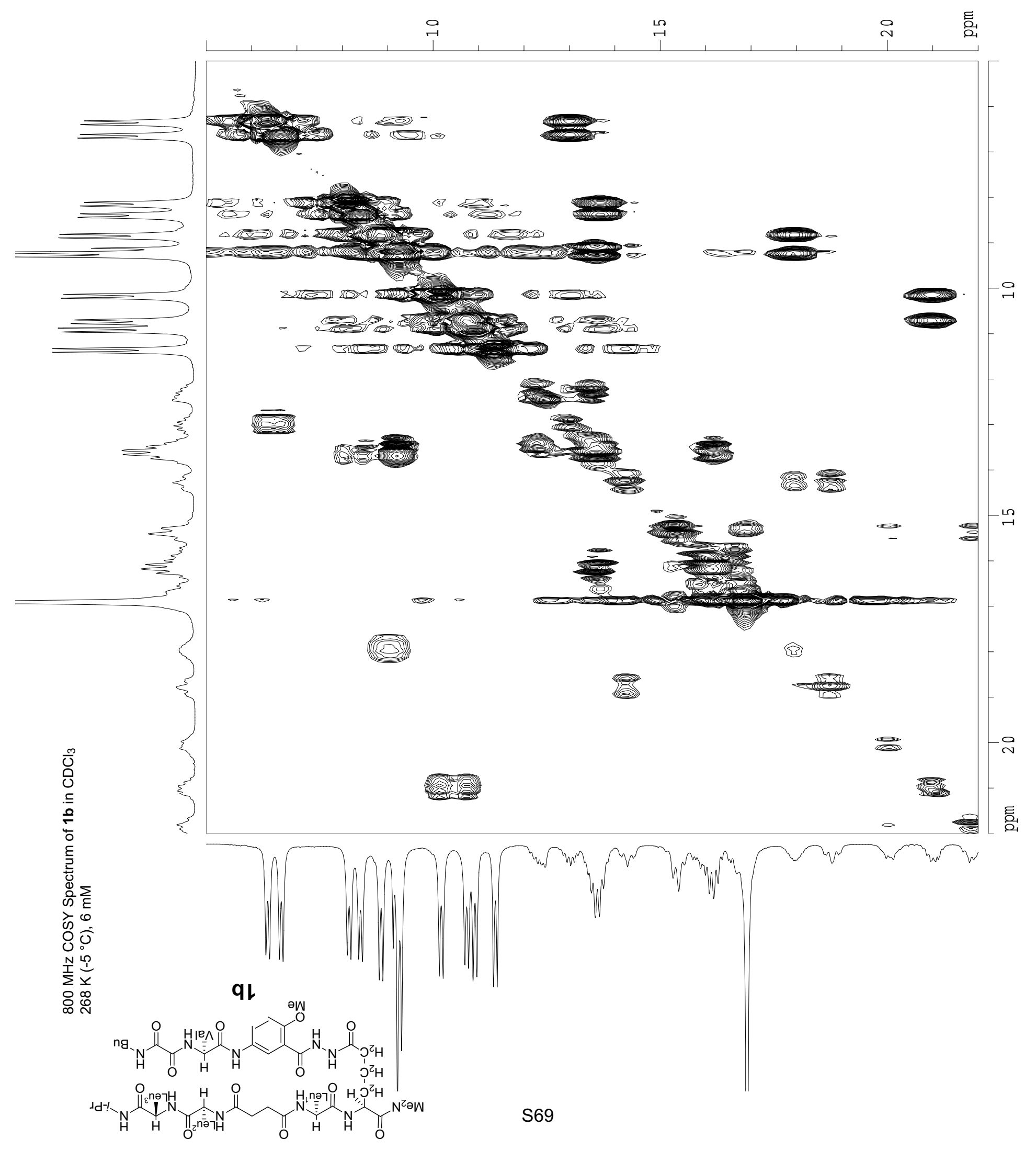




\section{Supporting Information}
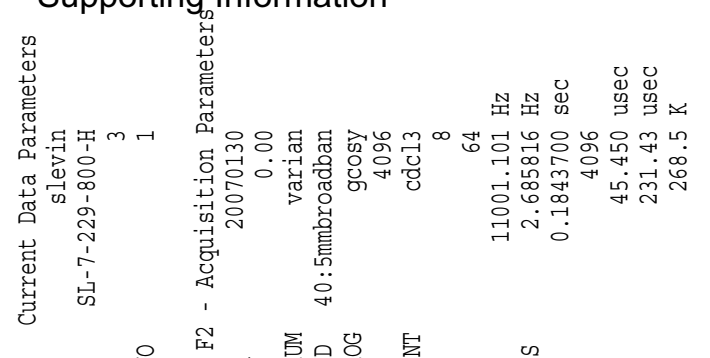

置罢总总

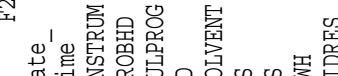

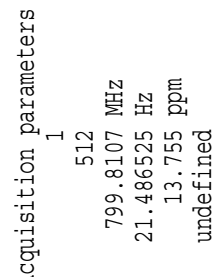

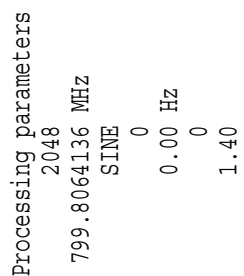

\section{展㷏}

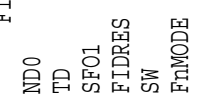

S. Levin and J. S. Nowick

ขั้

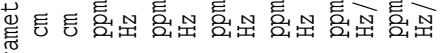

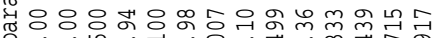
น山่ 畣 罢 ลे

宝
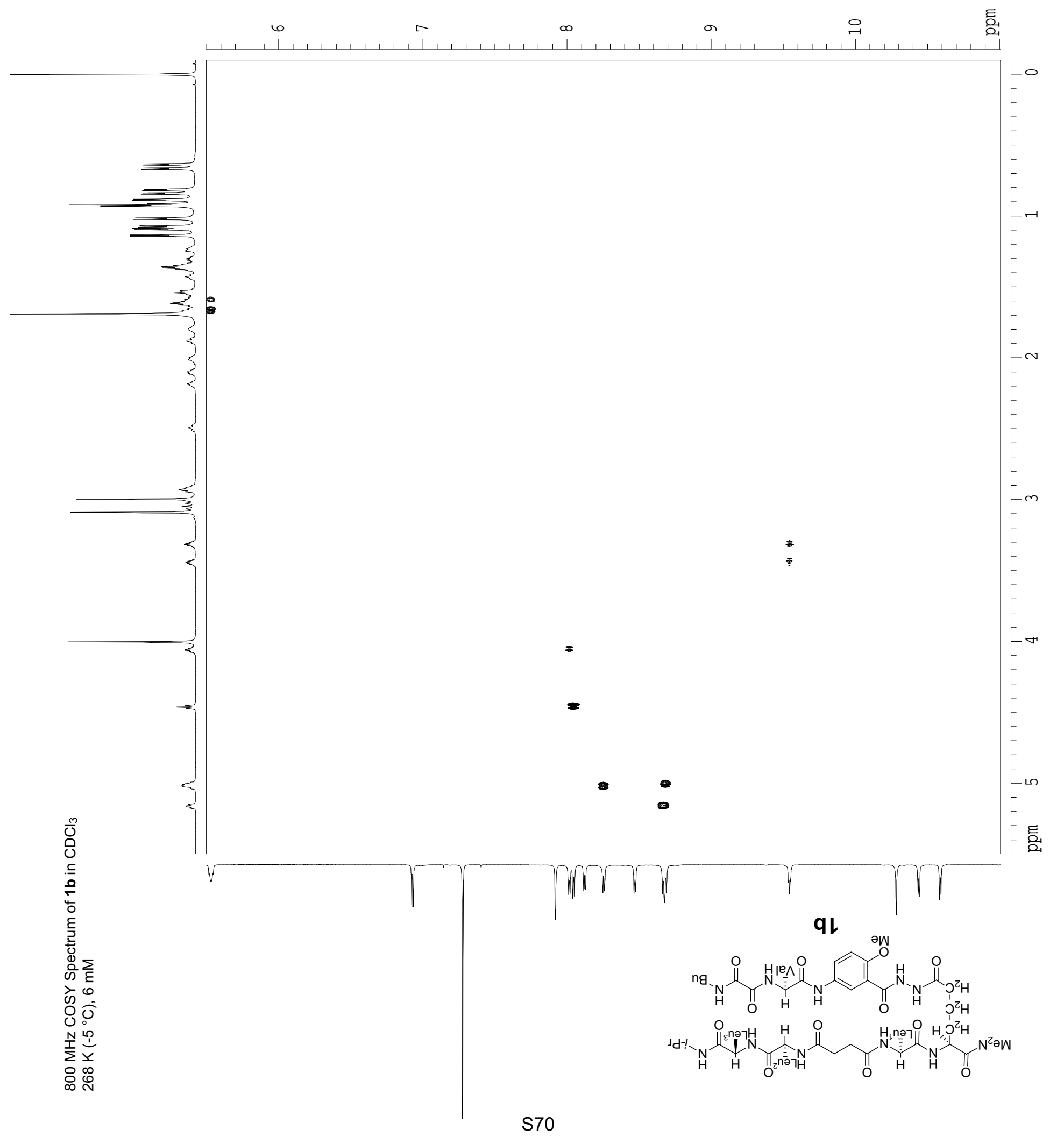
Supporting Information
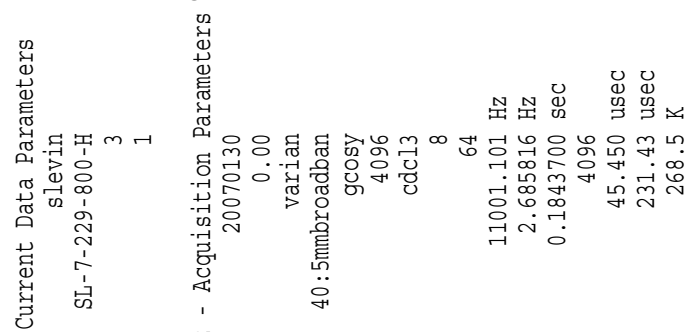

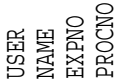

平

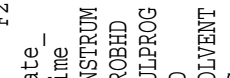

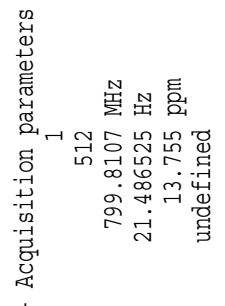

空

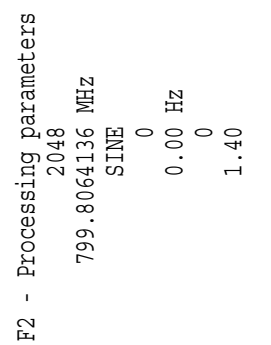

辛
S. Levin and J. S. Nowick

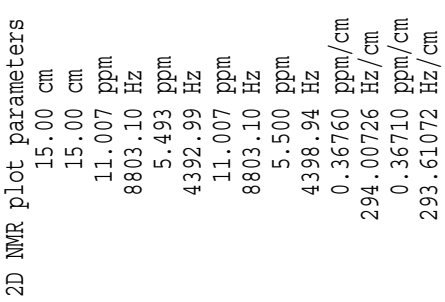

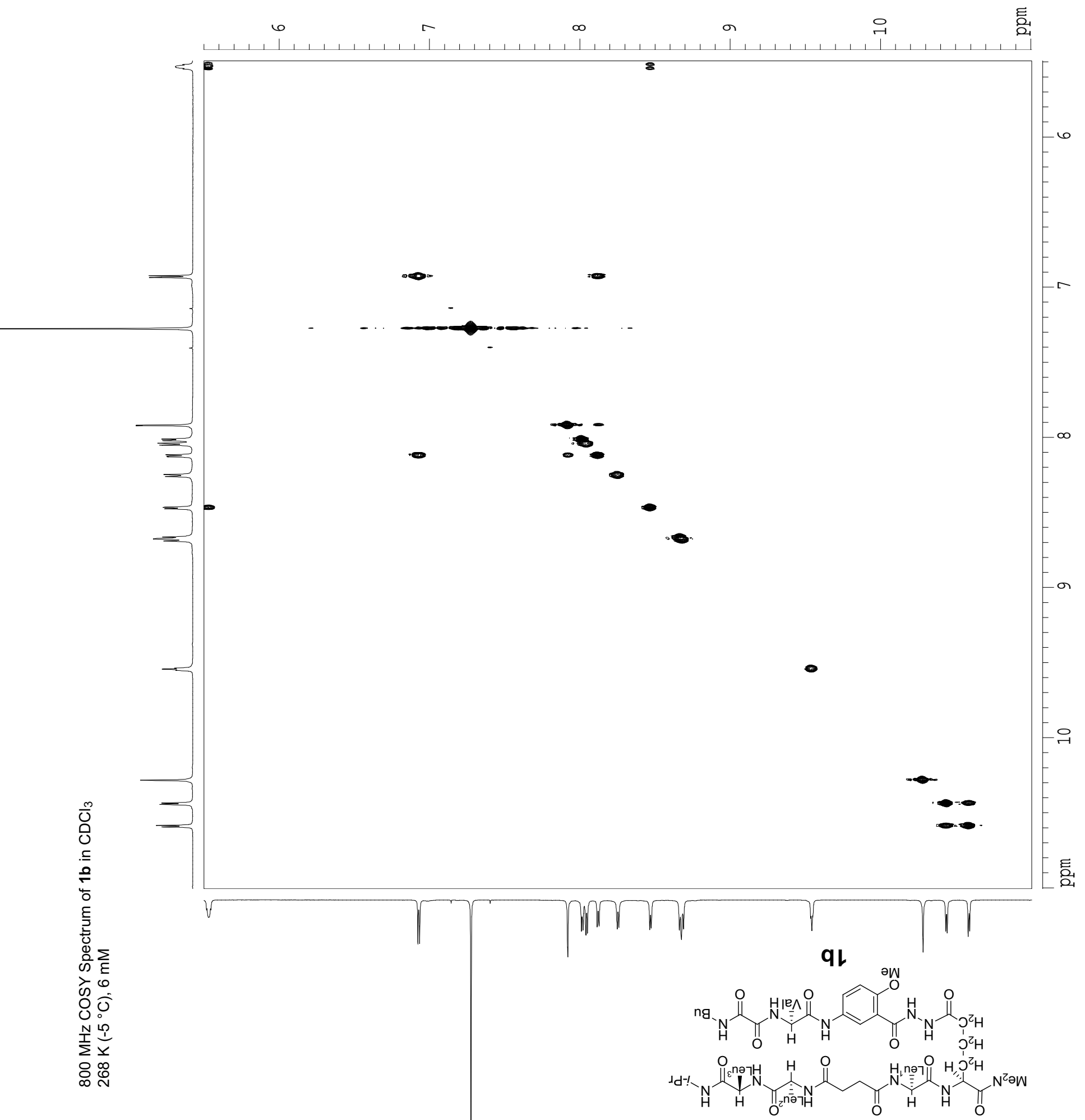




\section{Supporting Information}
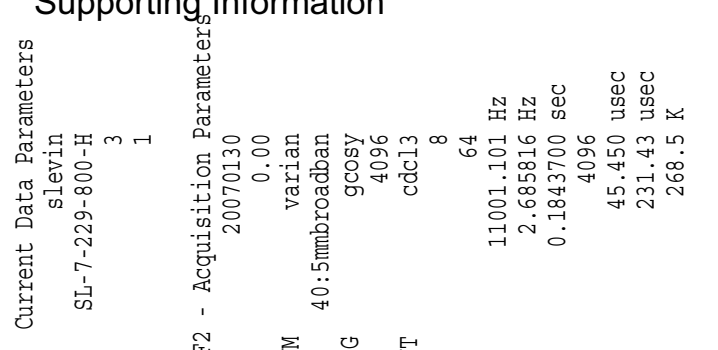

舀罢总总总

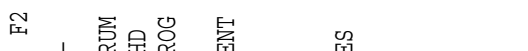

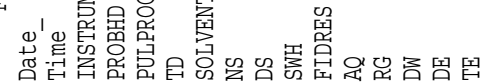

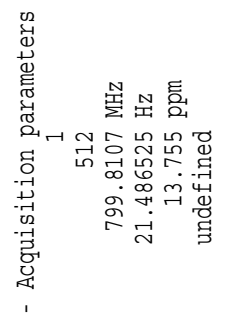

㞋

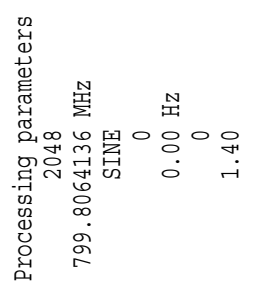

또

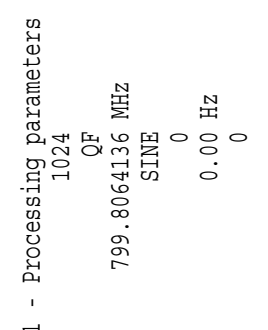

宝
S. Levin and J. S. Nowick

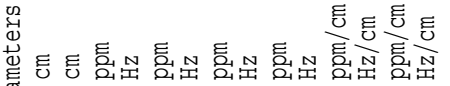

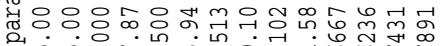

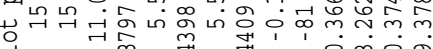
㧛

ลิ

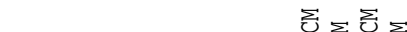
륨

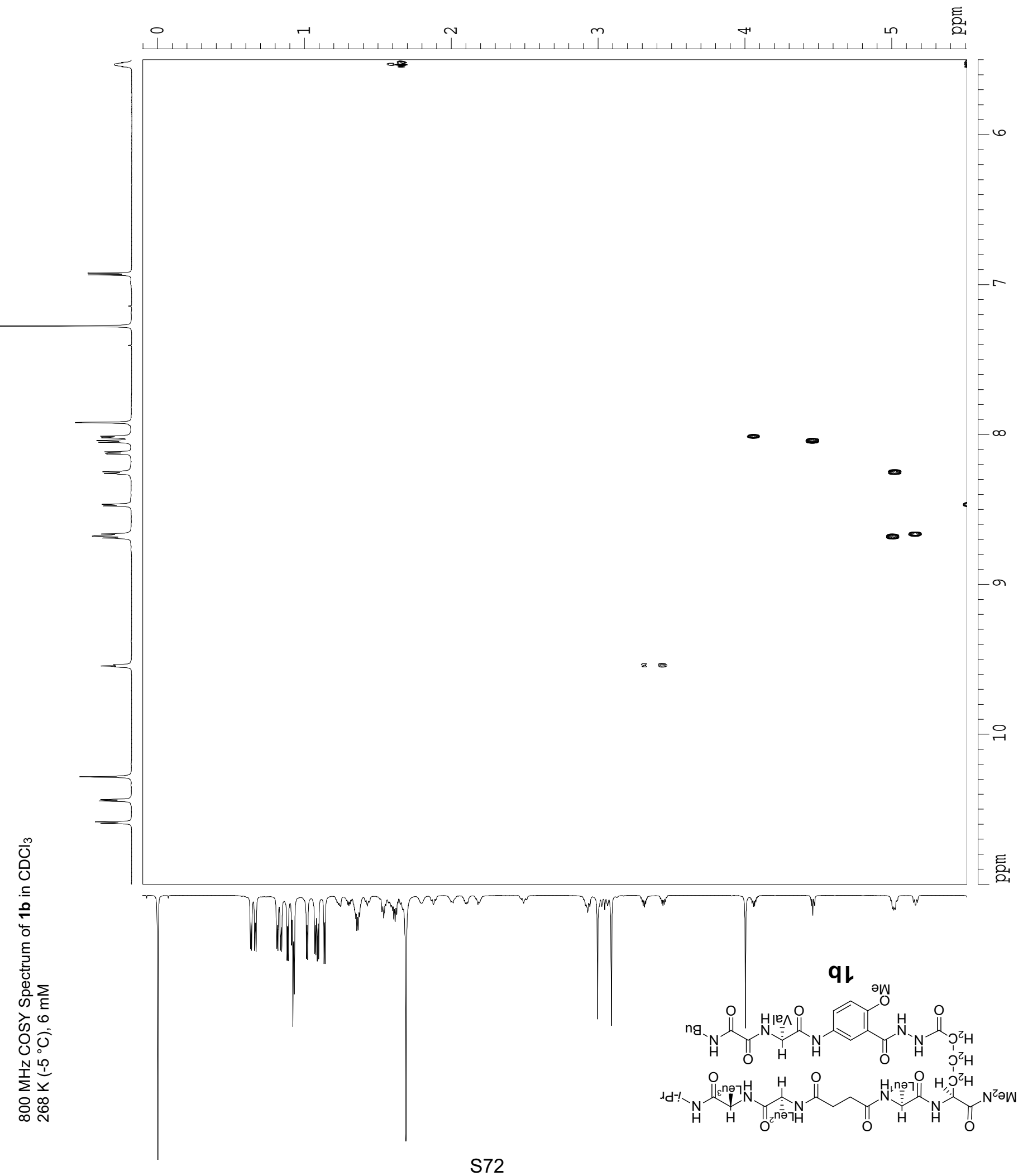




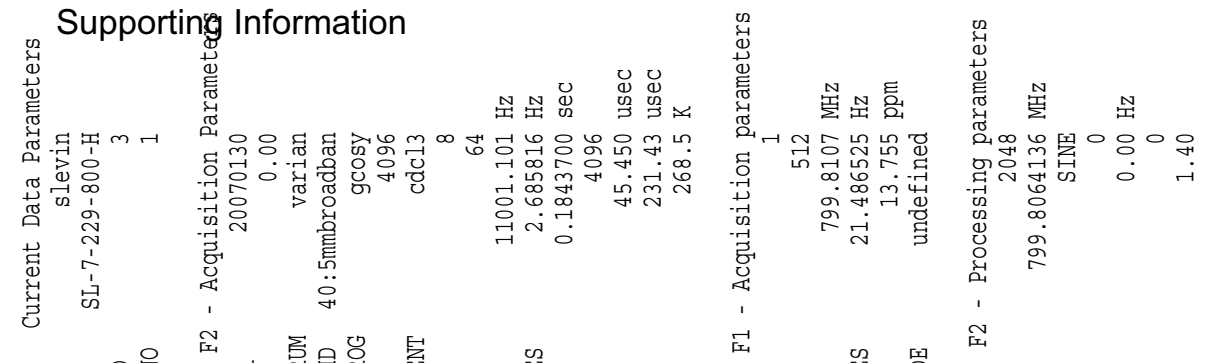

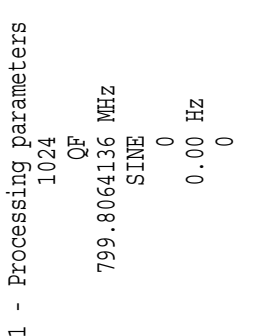

喪
S. Levin and J. S. Nowick

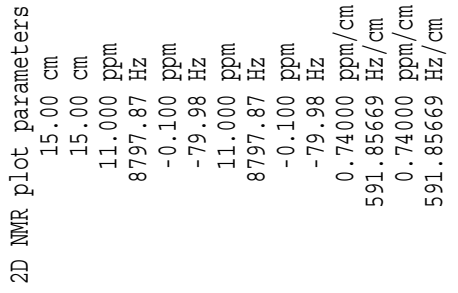

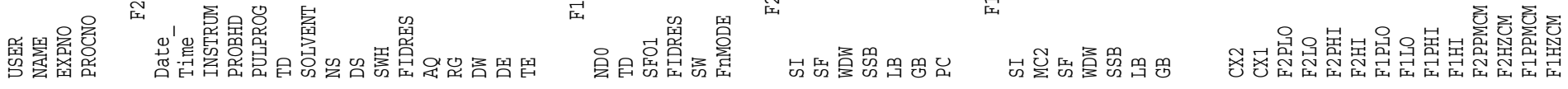

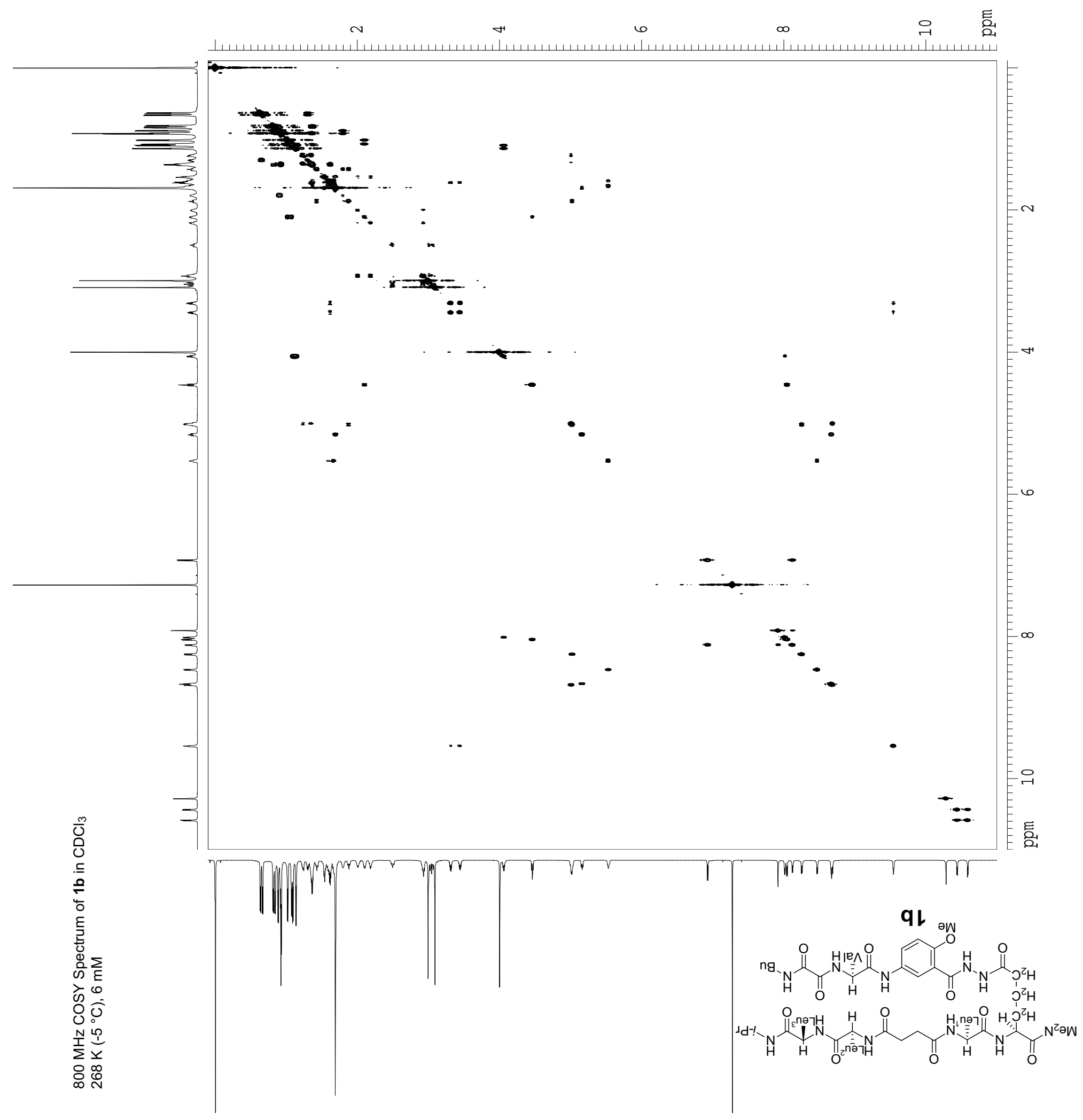


Supporting $g_{2}$ Information
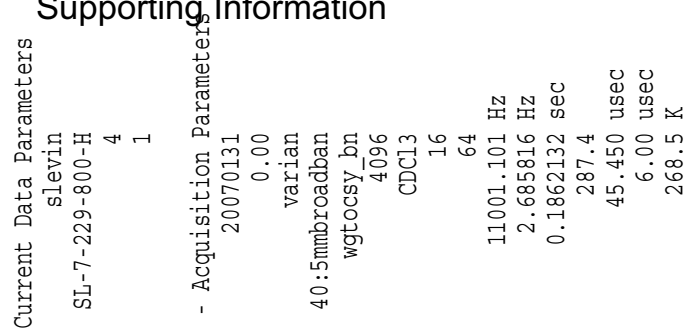

品筳虽

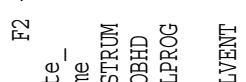

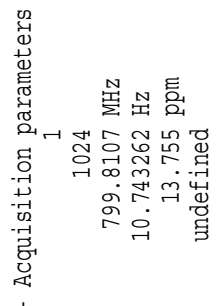

空

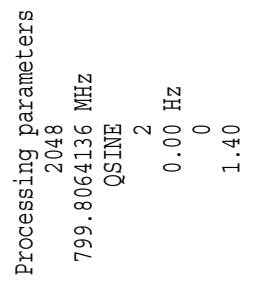

舀
S. Levin and J. S. Nowick

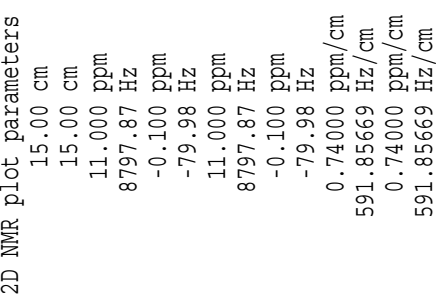

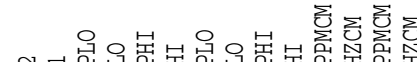

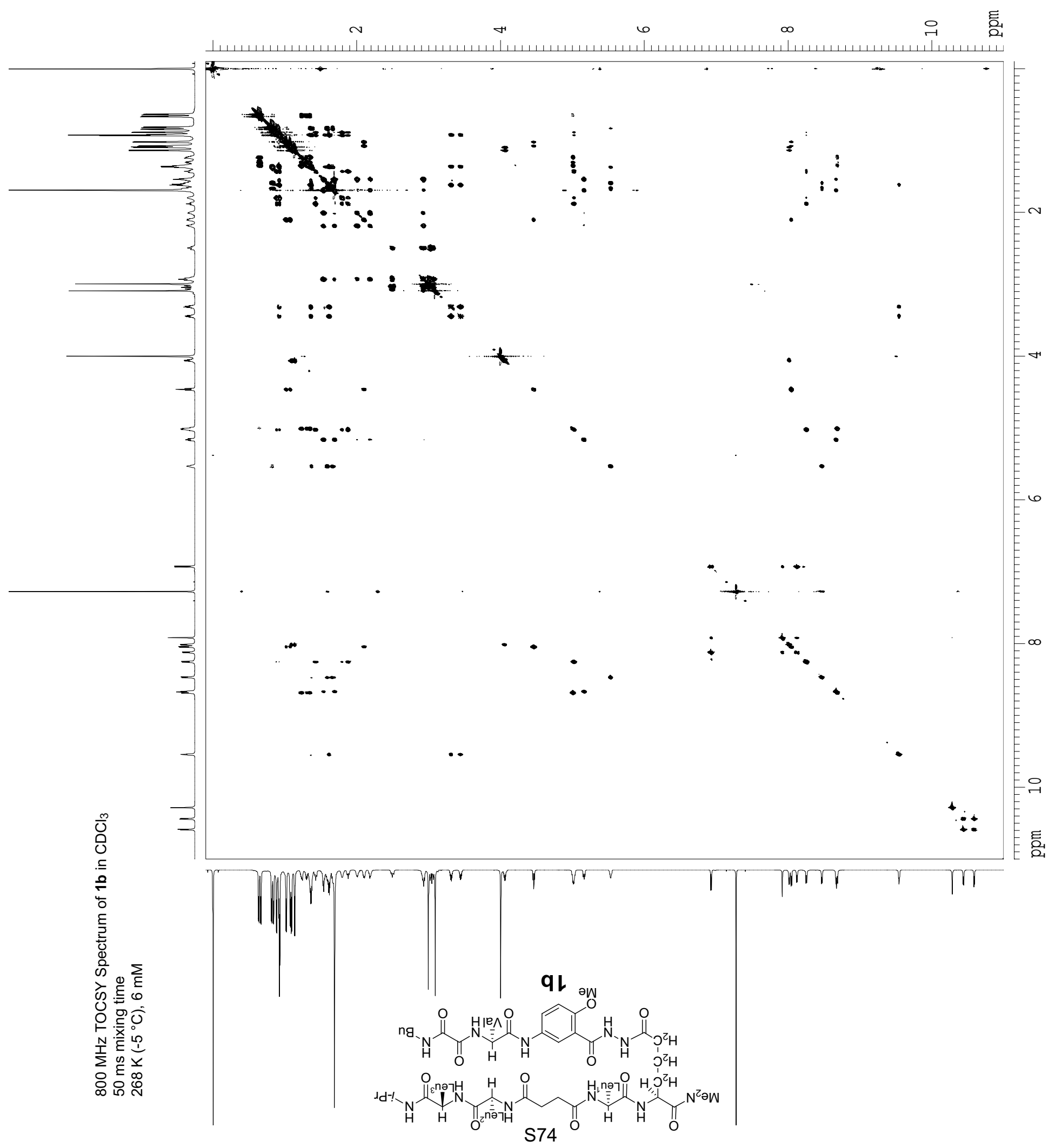


Supporting lăghormation

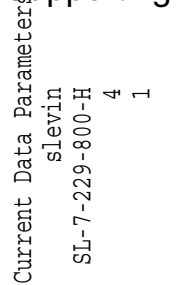

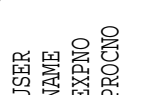

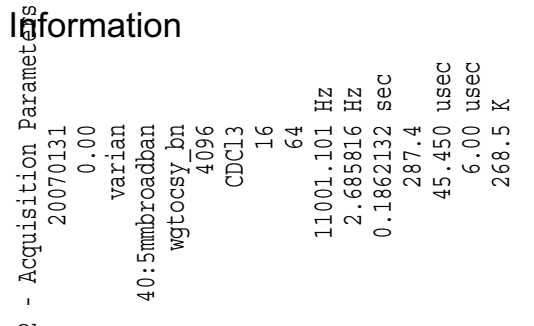

${ }^{2}$ "

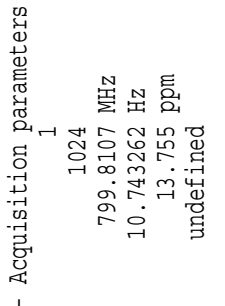

早

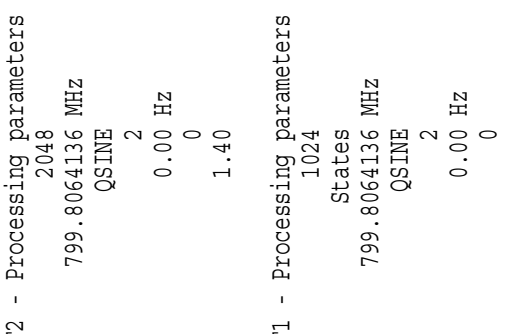

㞋

S. Levin and J. S. Nowick

嵌

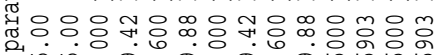
मे. 㴅

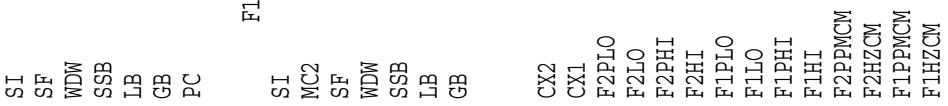

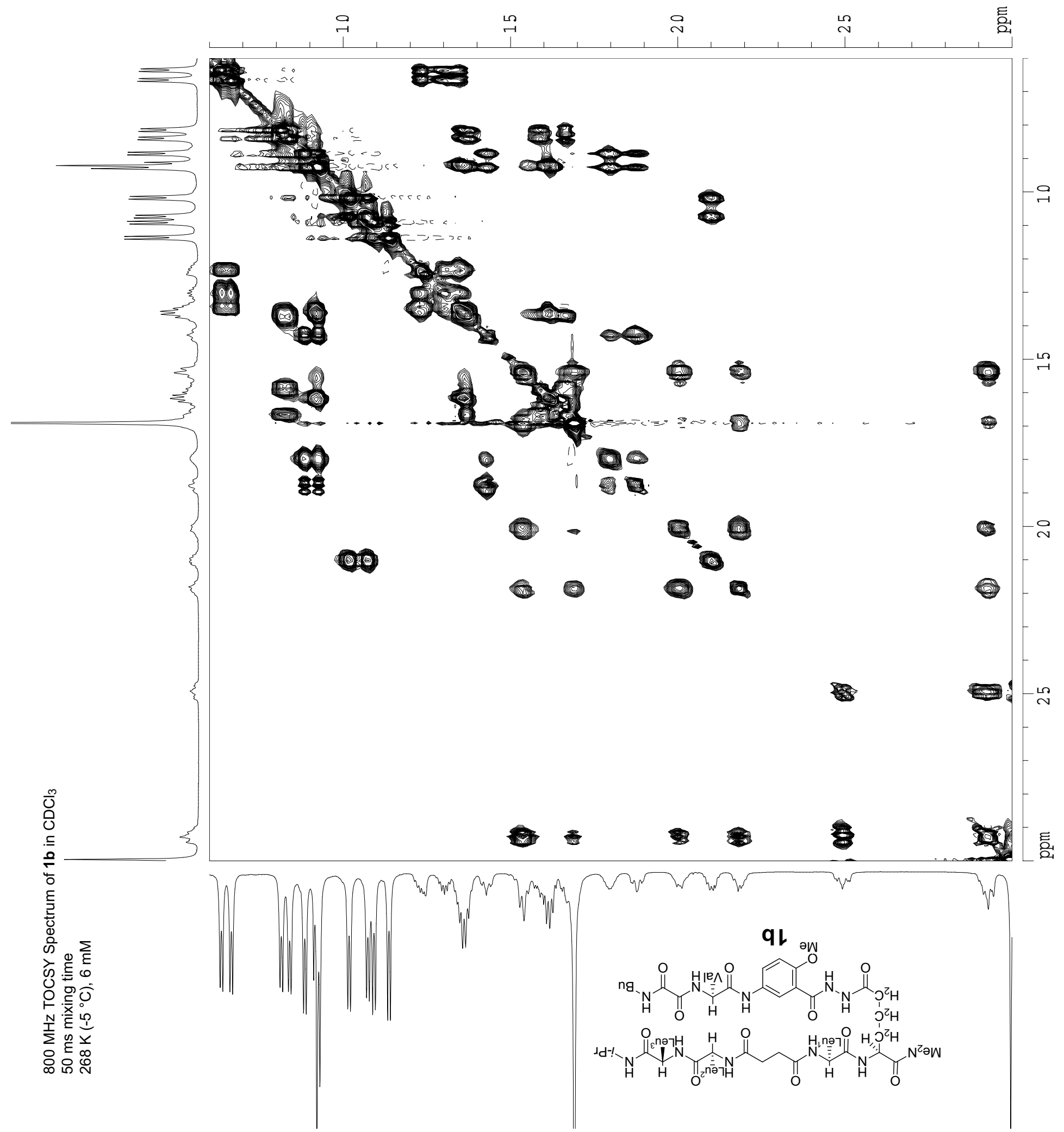



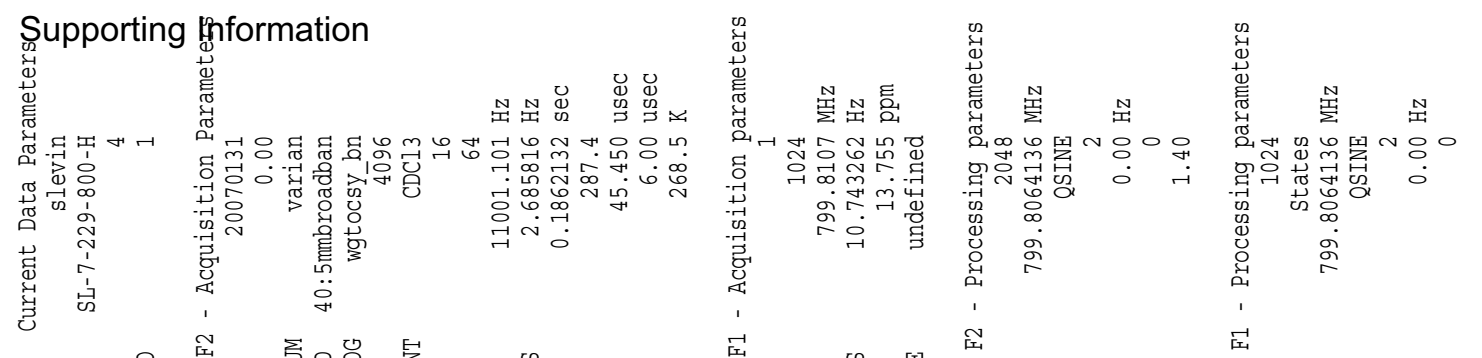

S. Levin and J. S. Nowick

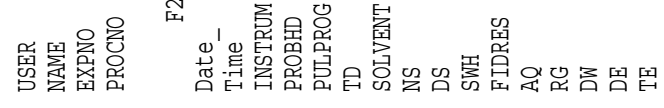

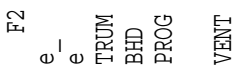

㞌留 四

空

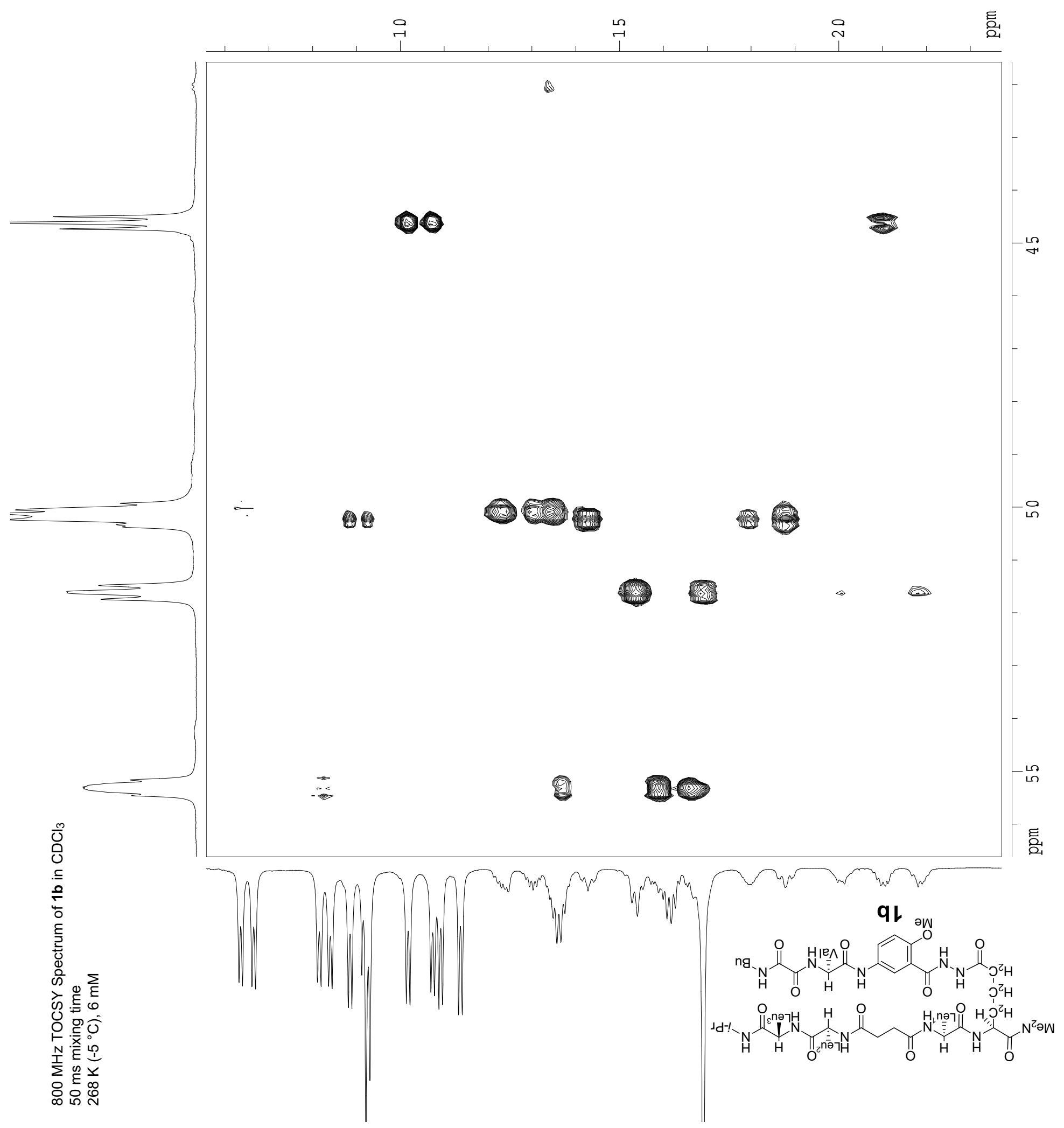


Supporting Information

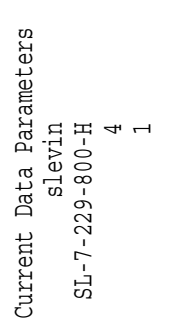

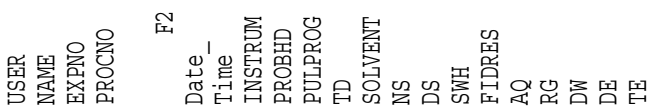

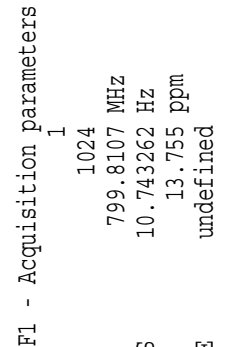

荘

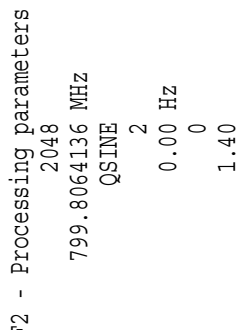

空

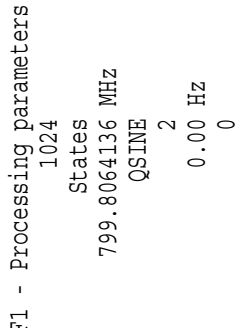

宔

S. Levin and J. S. Nowick

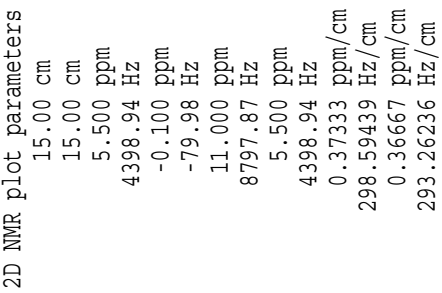

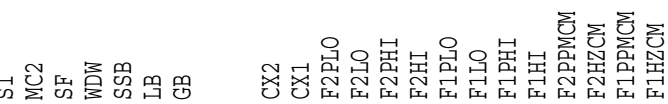

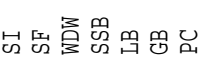

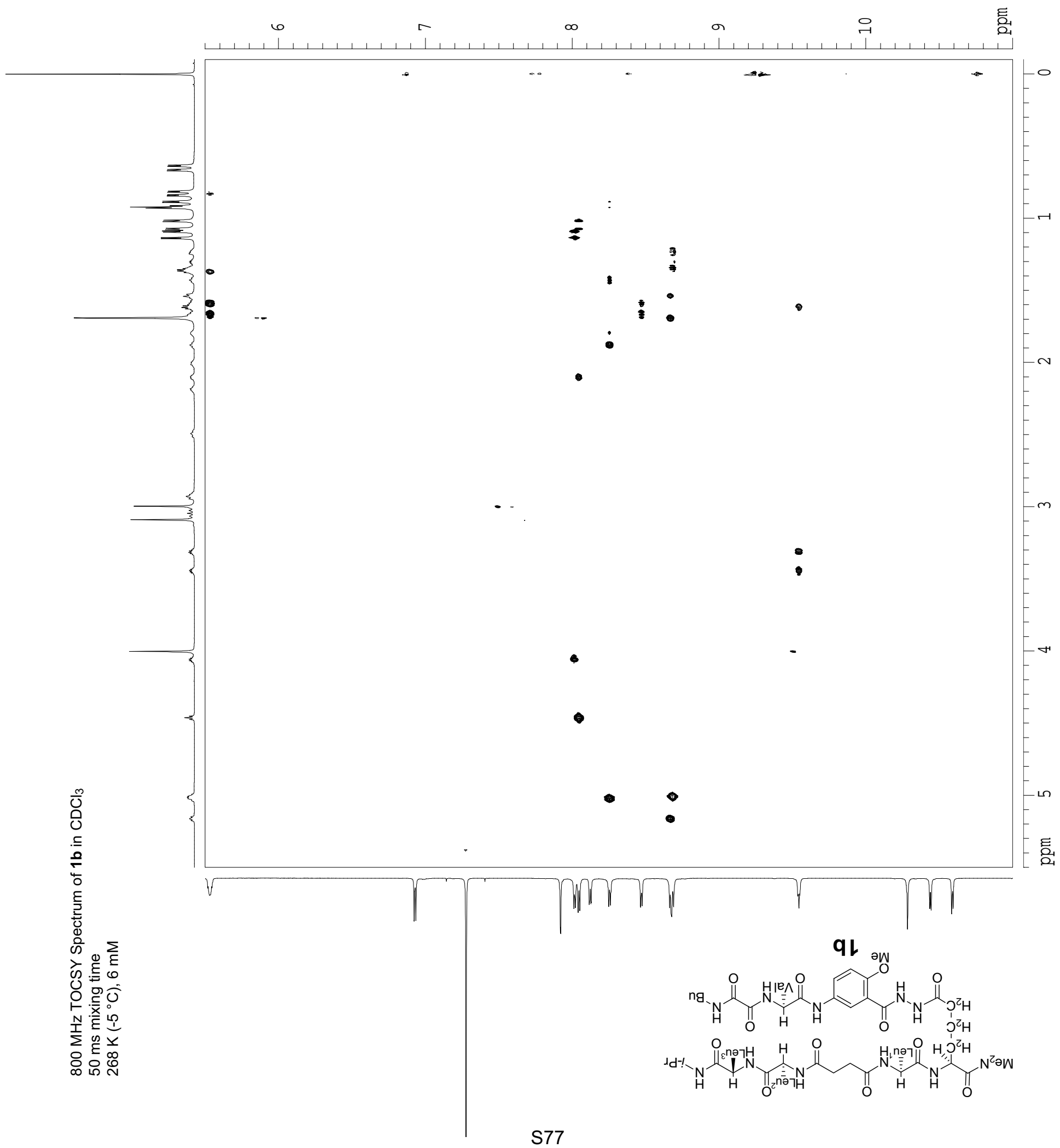




\section{Supporting Information}
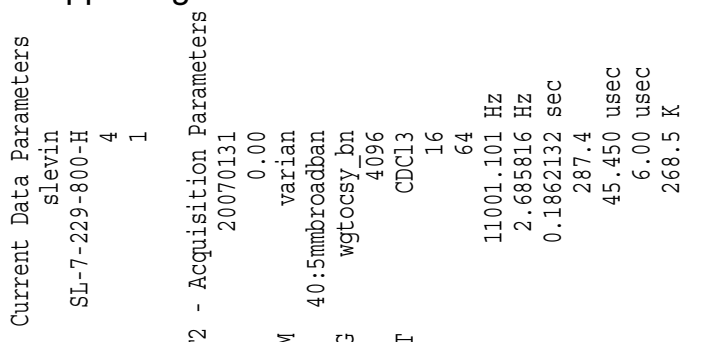

舀淟总总

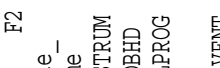

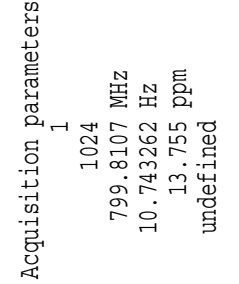

寽

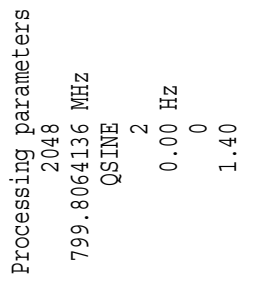

社

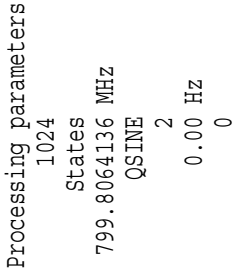

覀
S. Levin and J. S. Nowick

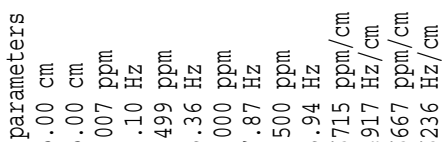

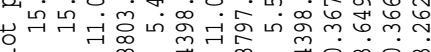
罢

ลे

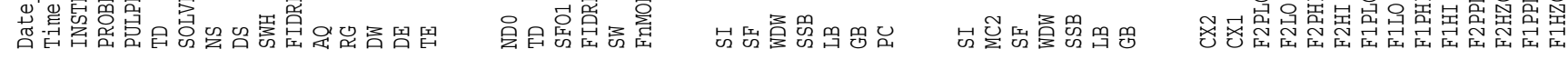

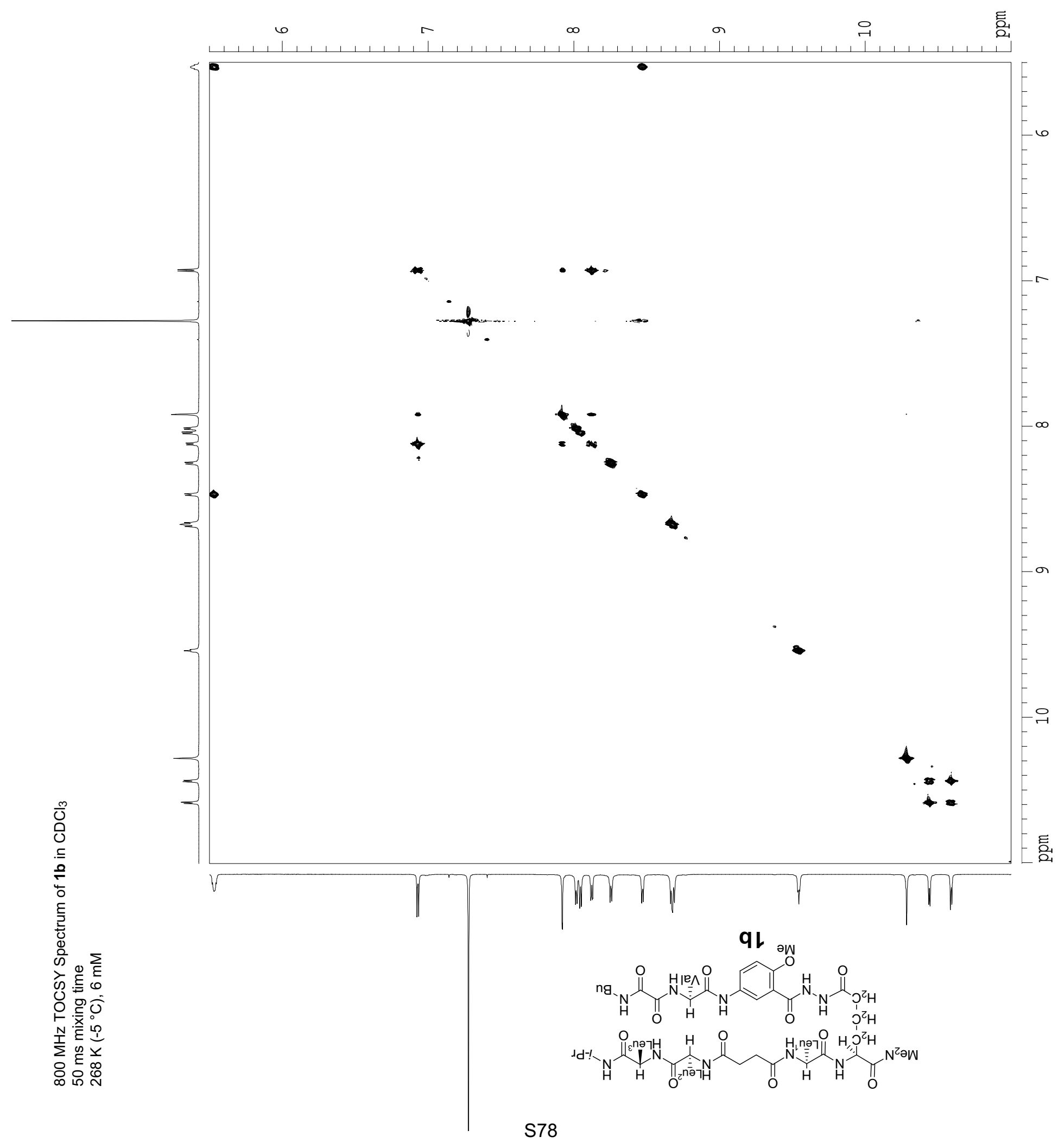




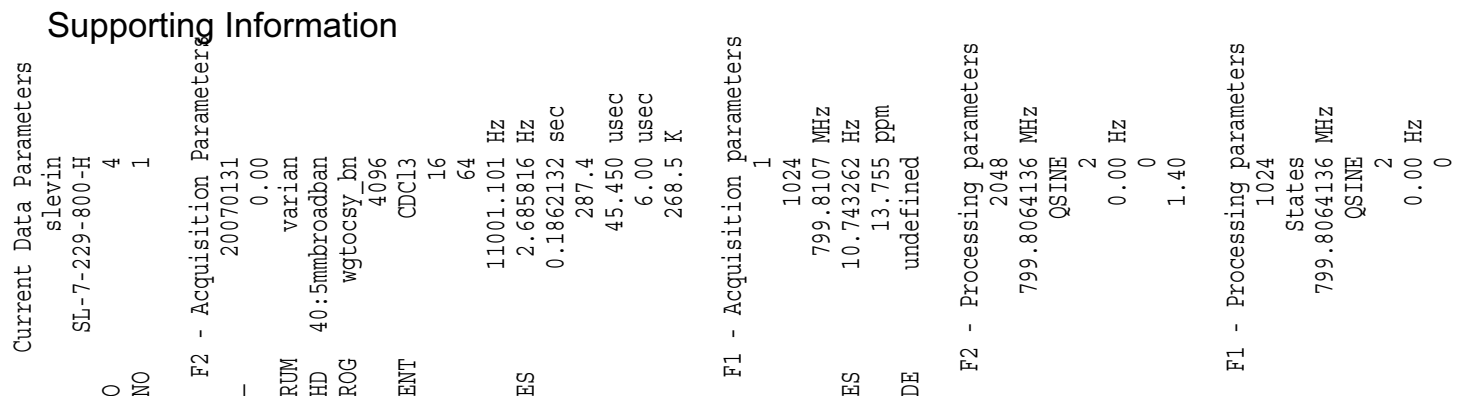

S. Levin and J. S. Nowick

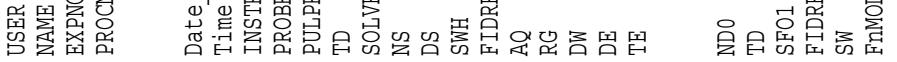

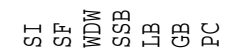

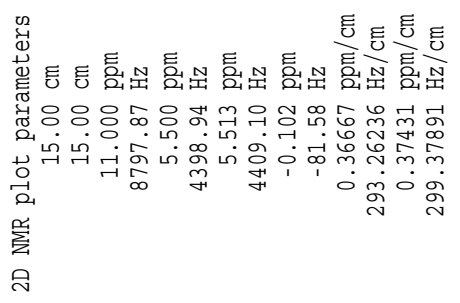

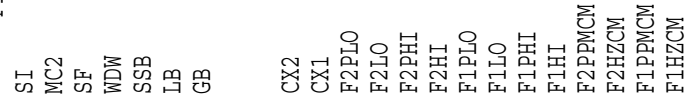

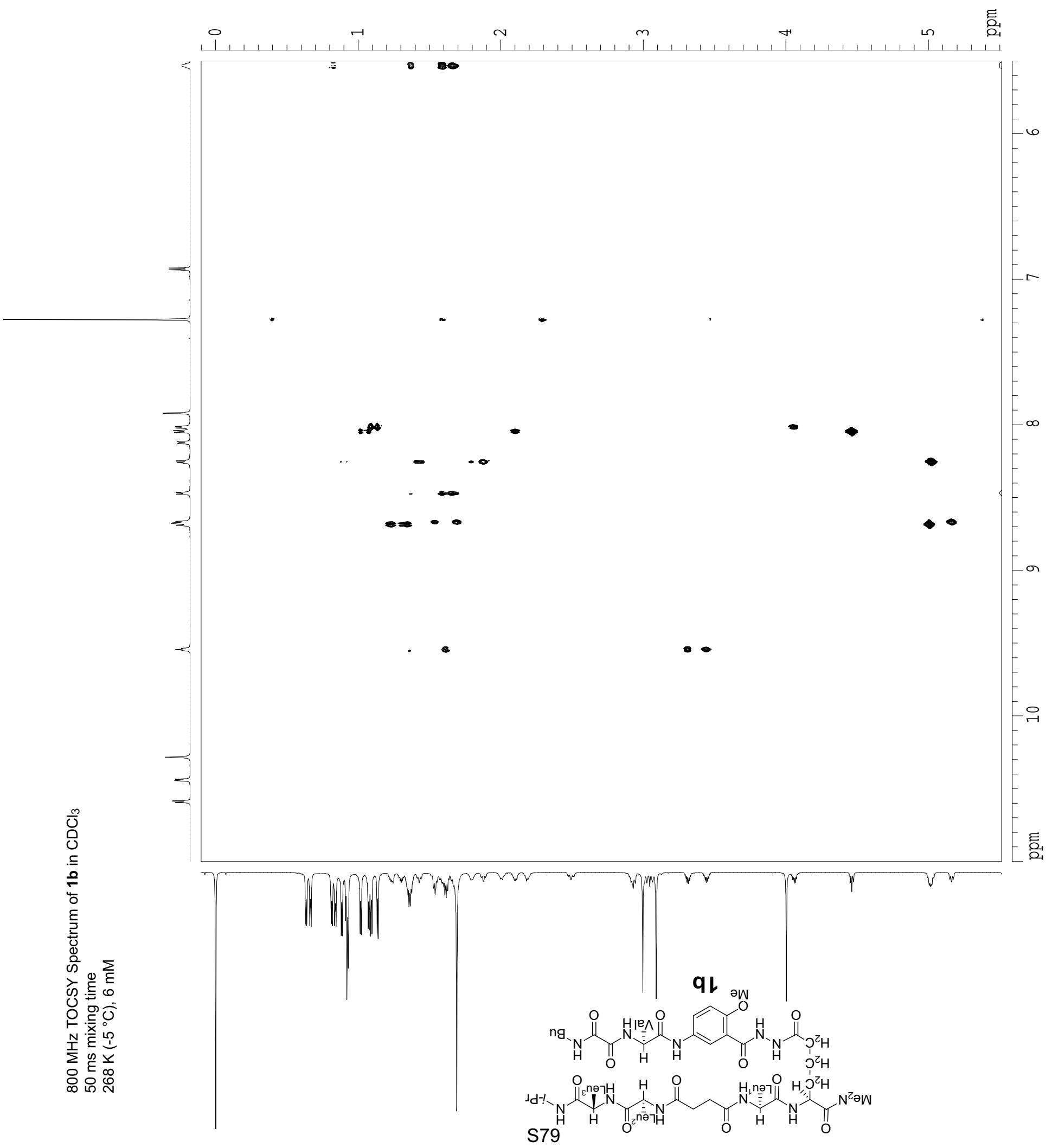



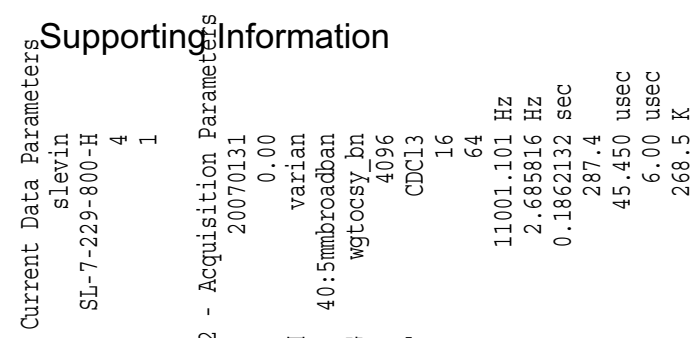

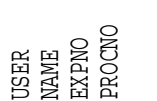

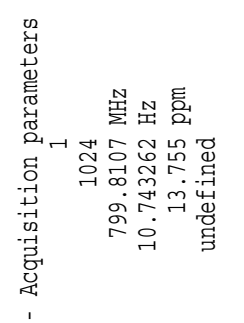

荘

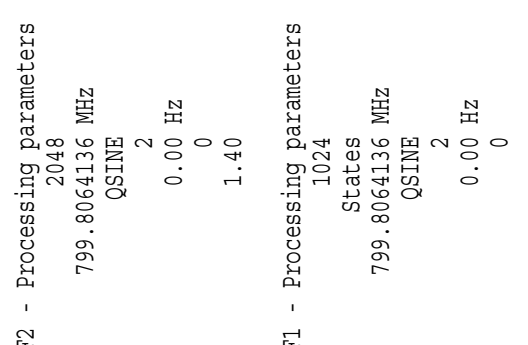

${ }_{20}$ S. Levin and J. S. Nowick 岁

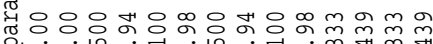
வ ம் ம 雚 爱

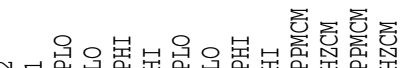

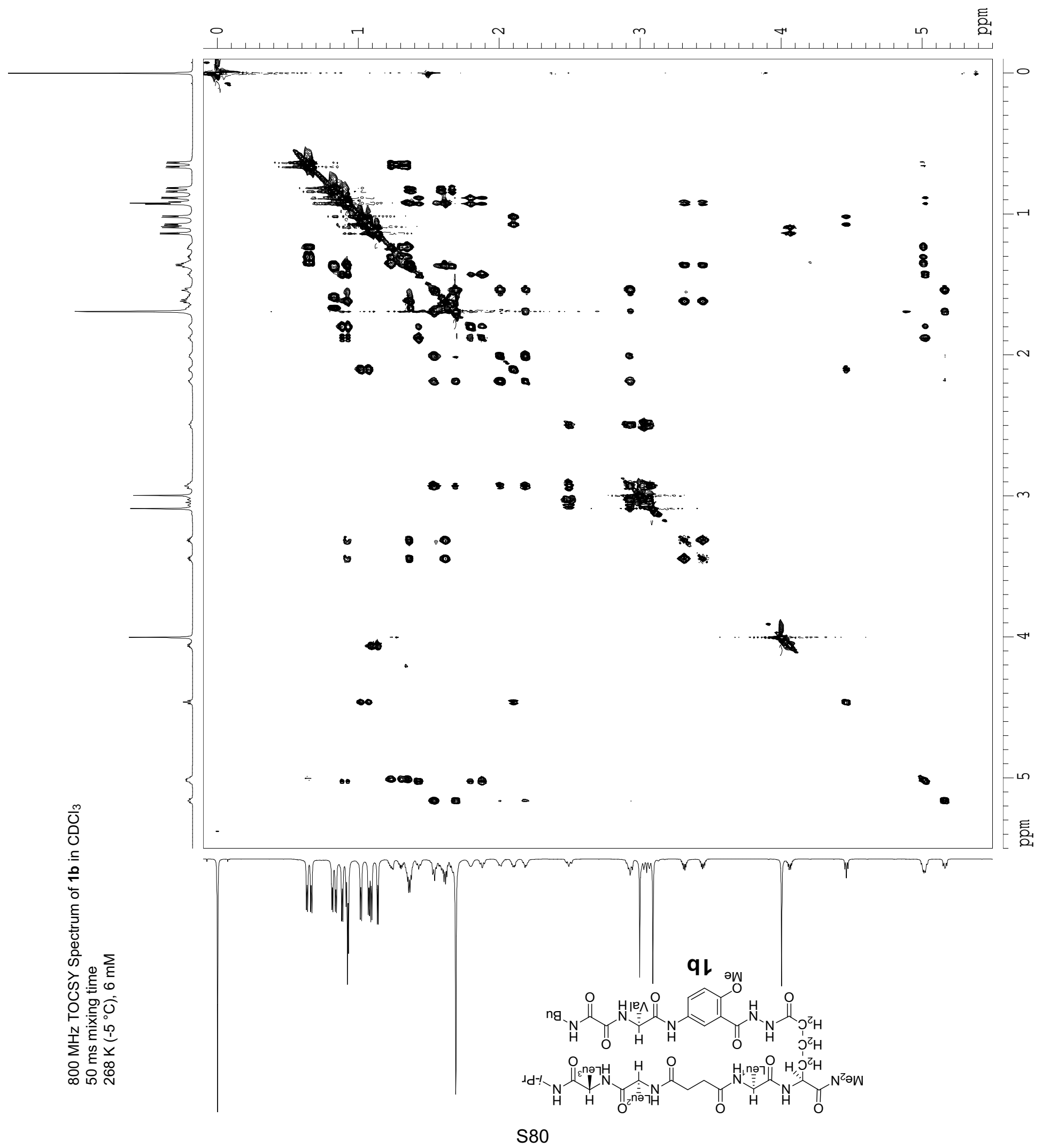




\section{Supporting}

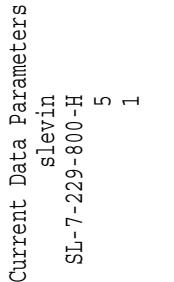

變

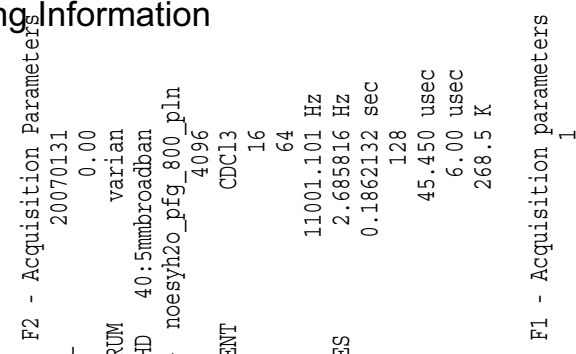

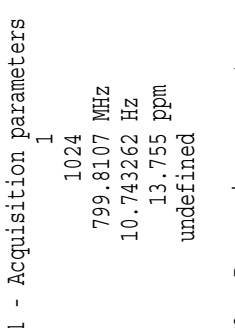

모

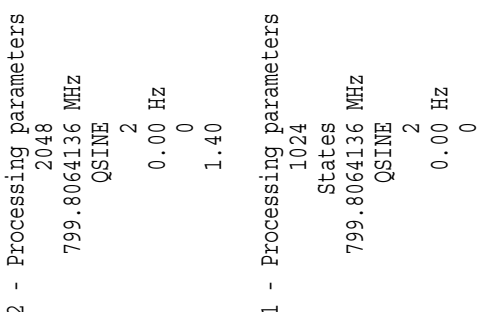

空

S. Levin and J. S. Nowick

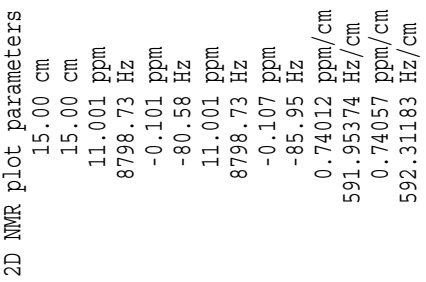

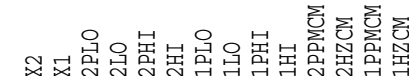

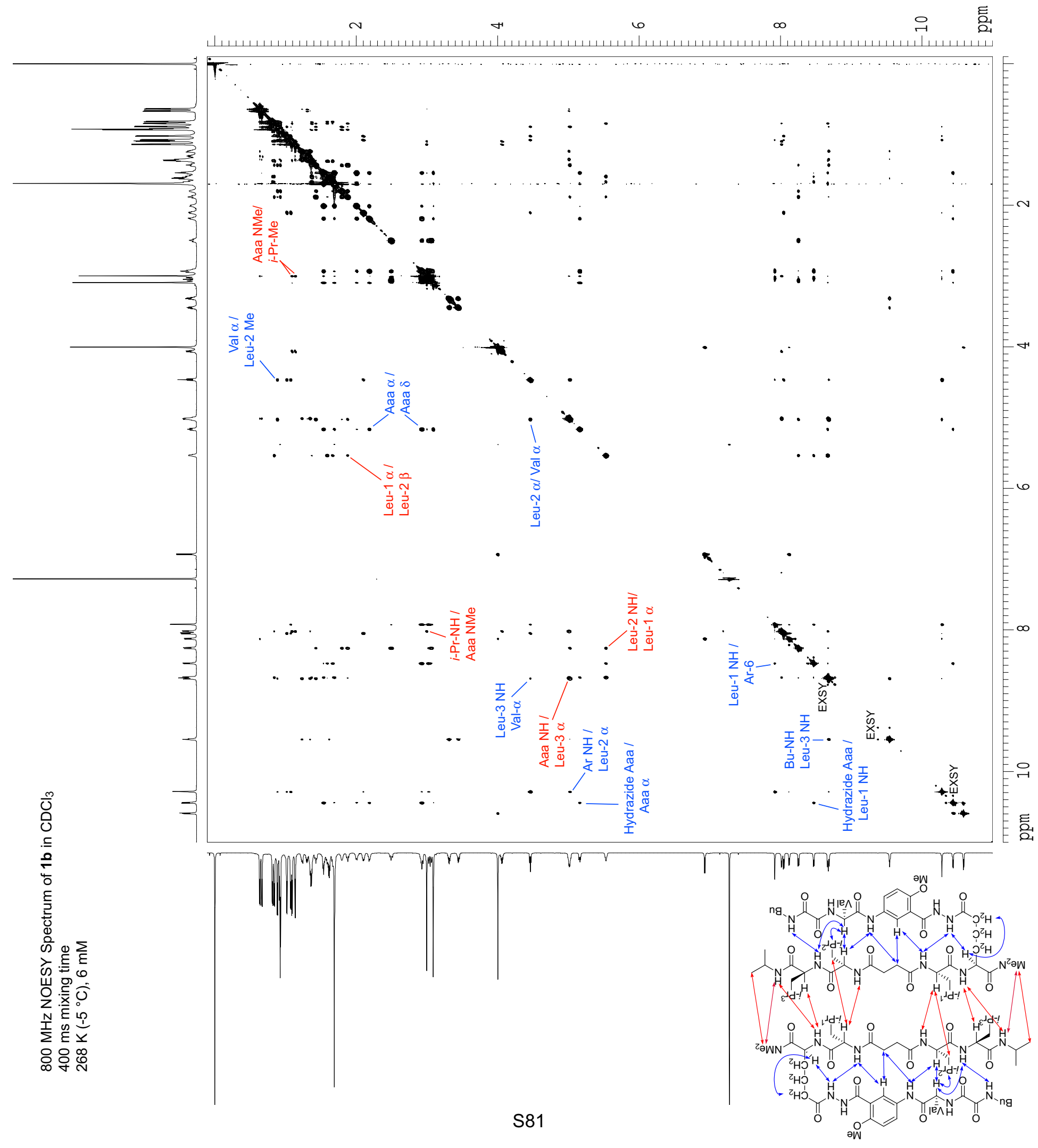



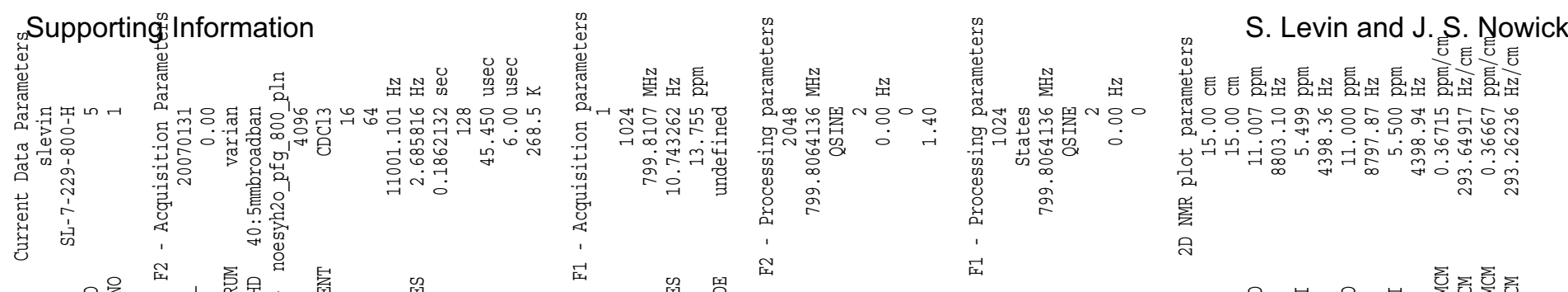

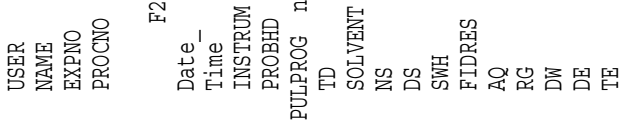

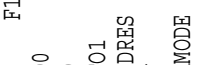

品

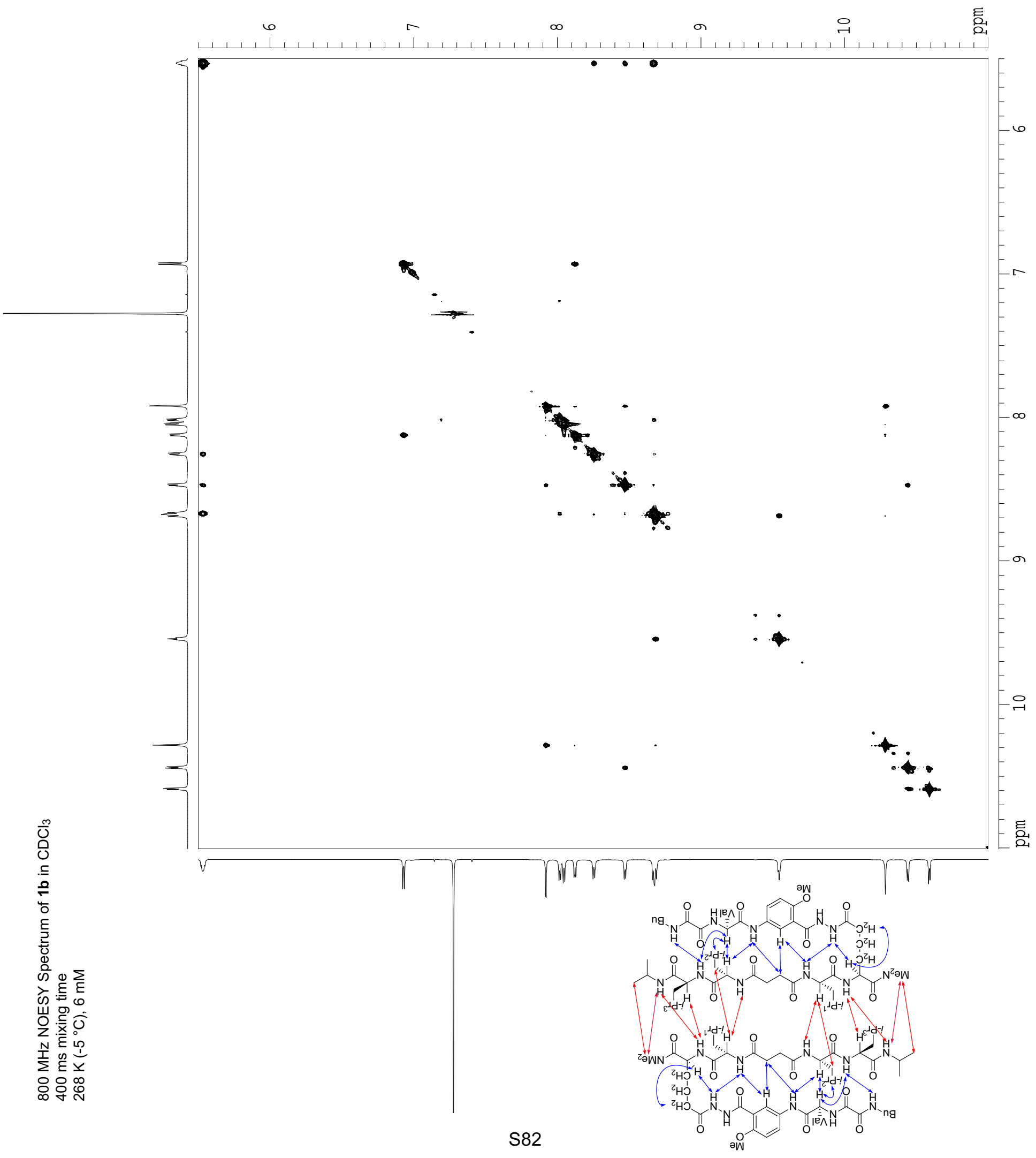




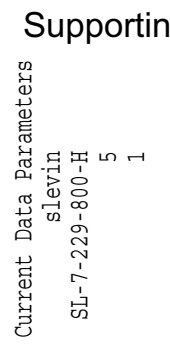

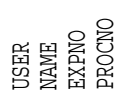

\section{$g_{0}$ Information}

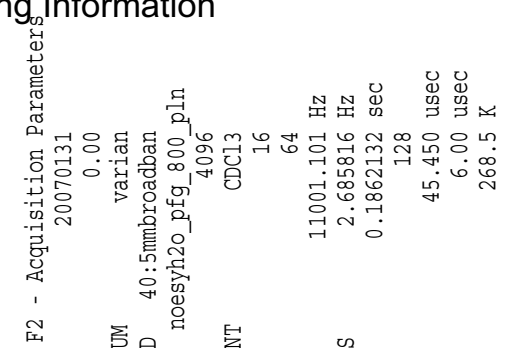

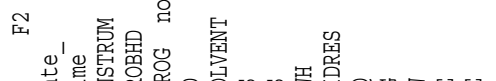

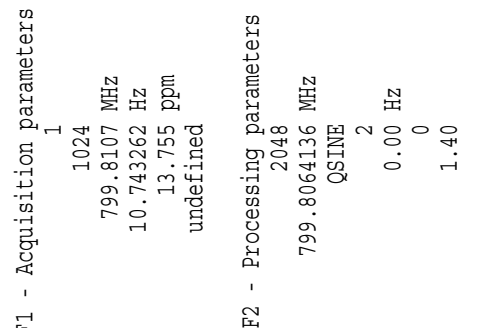

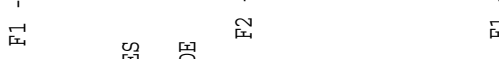

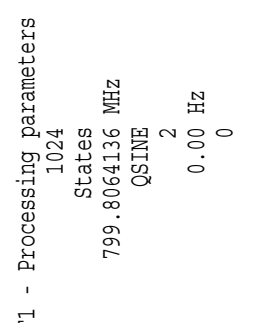

S. Levin and J. S. Nowick

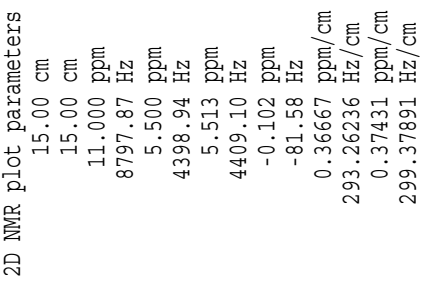

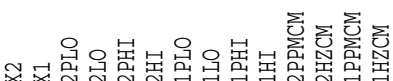

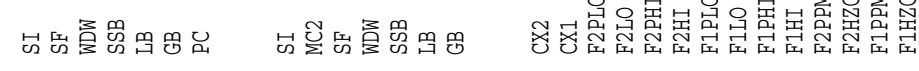

행

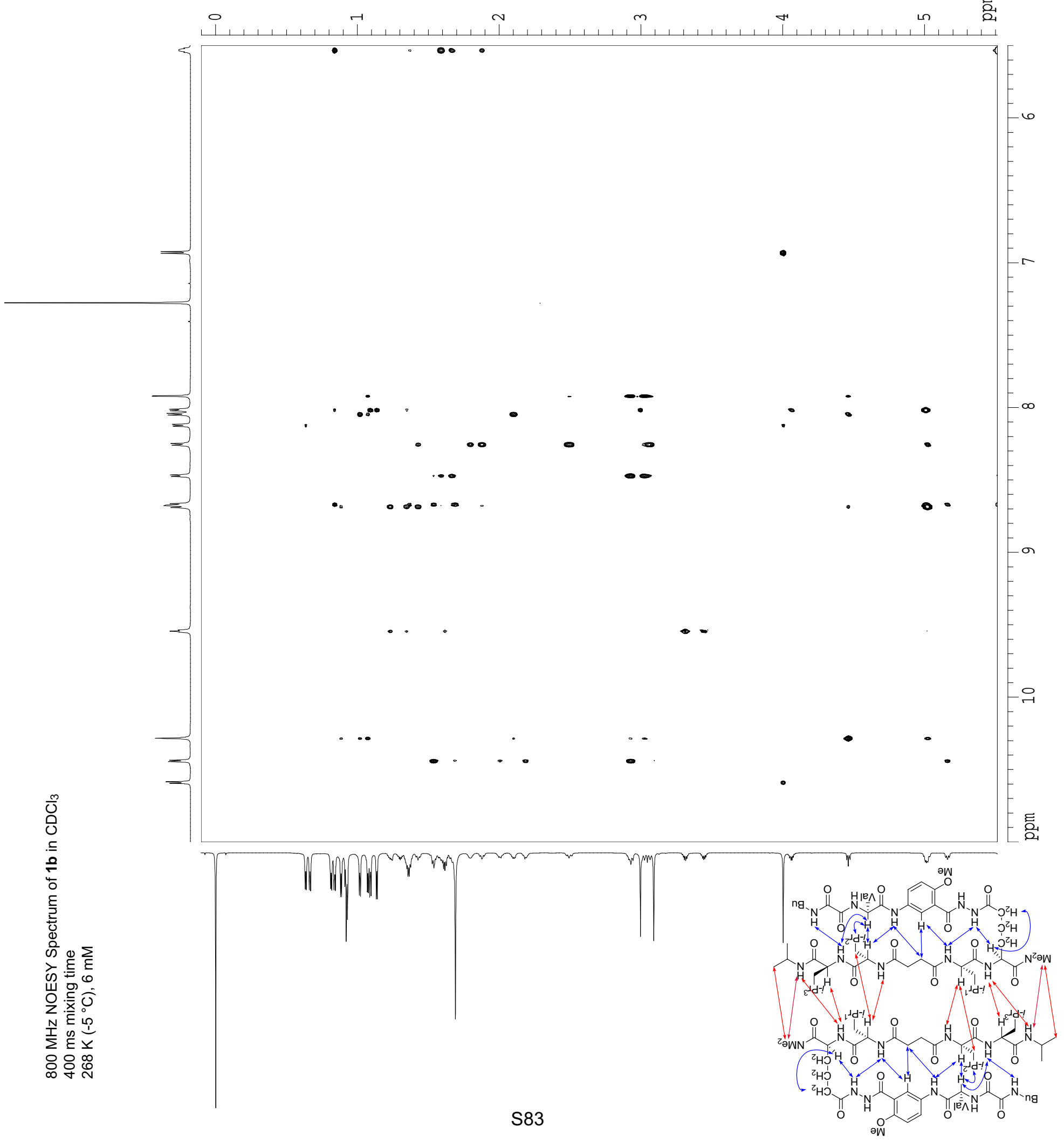


Supporting Information

S. Levin and J. S. Nowick

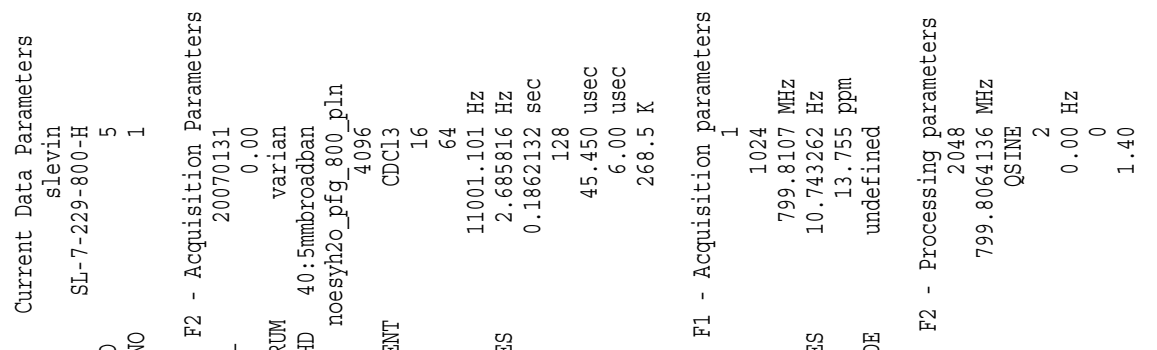

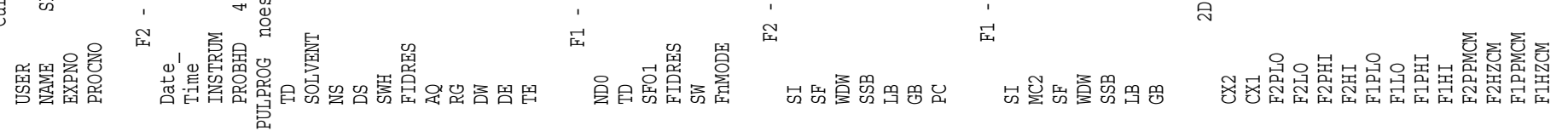
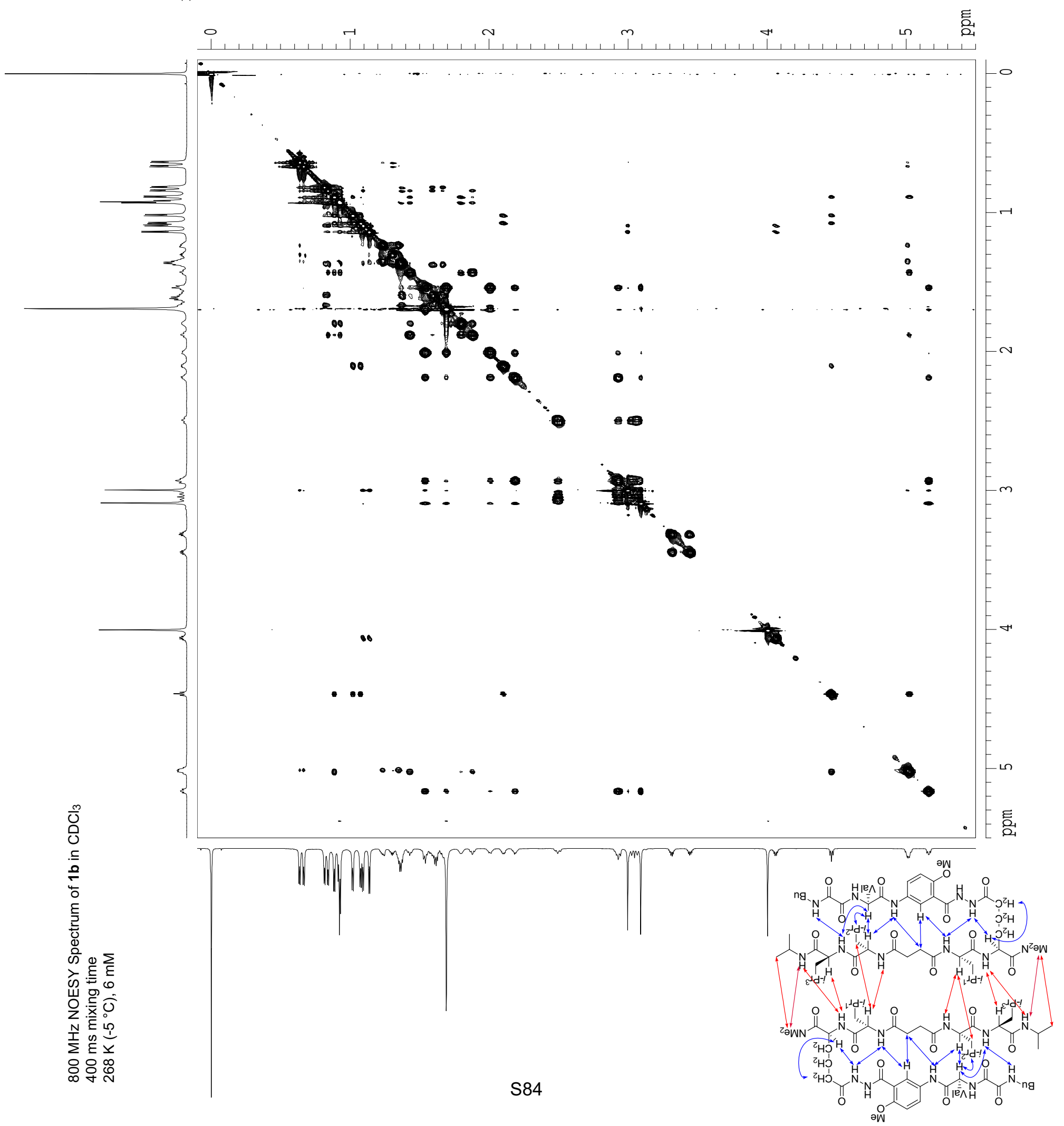

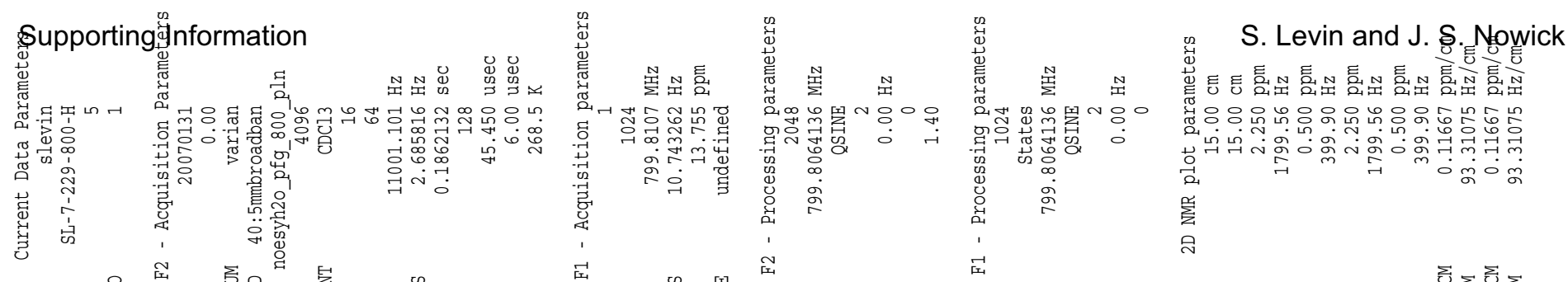

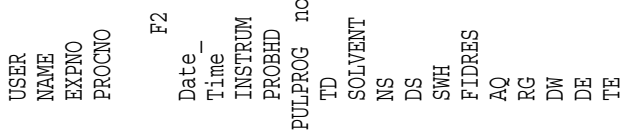

"

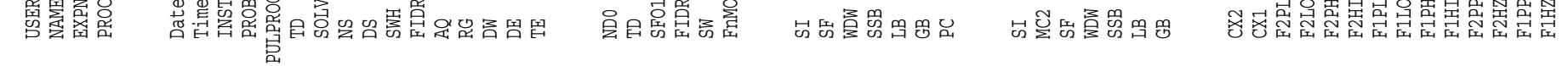

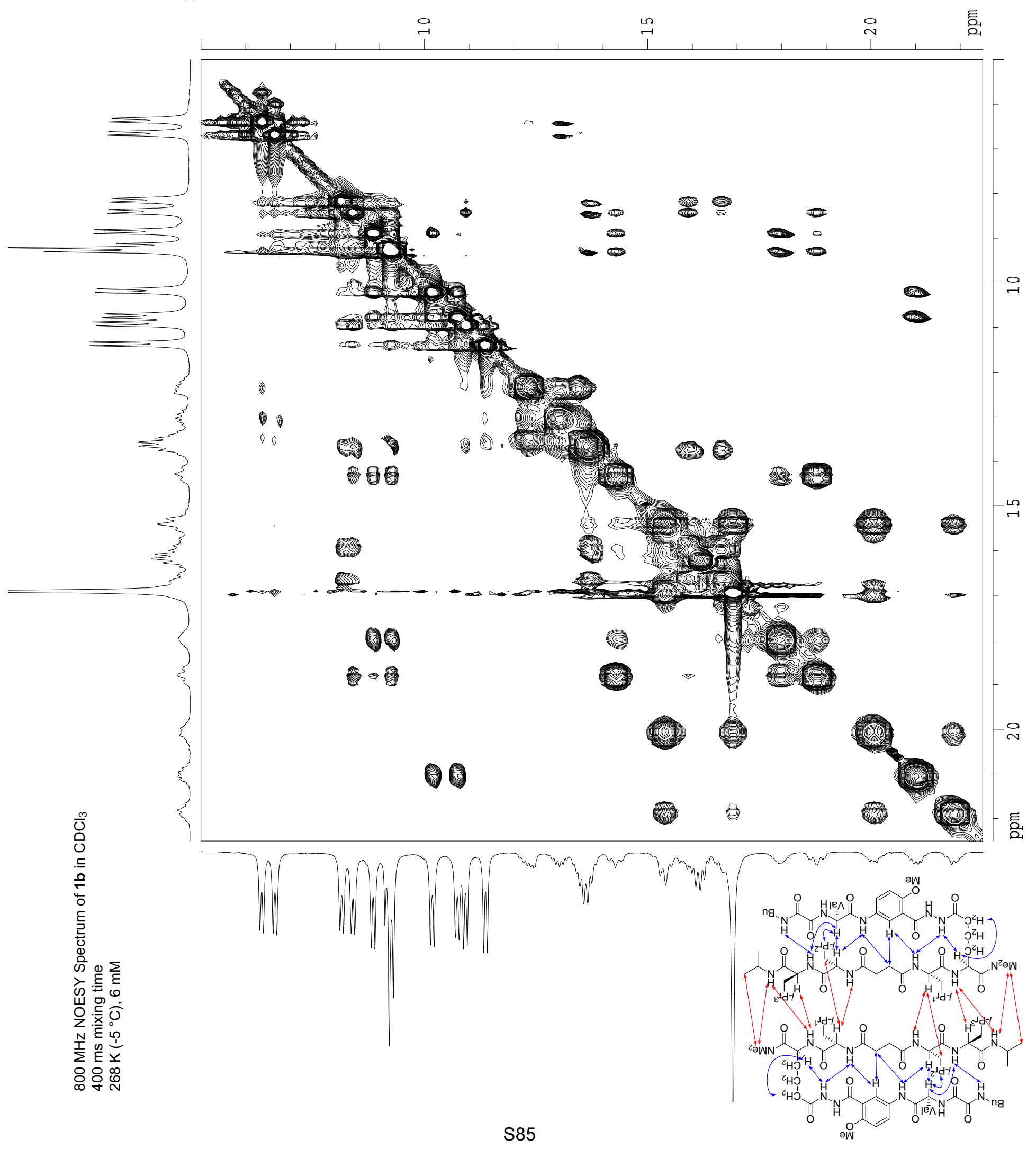



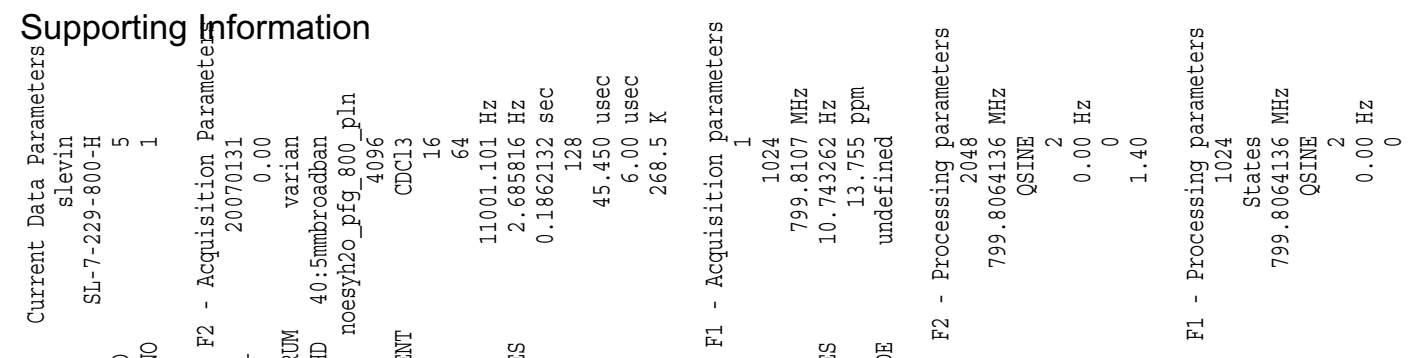

S. Levin and J. S. Nowick

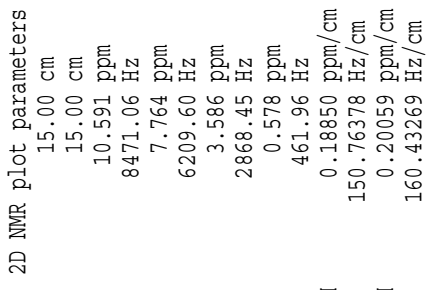

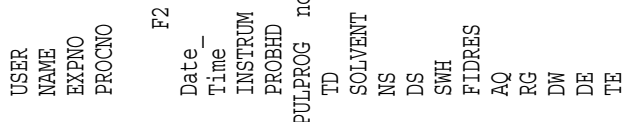

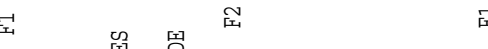

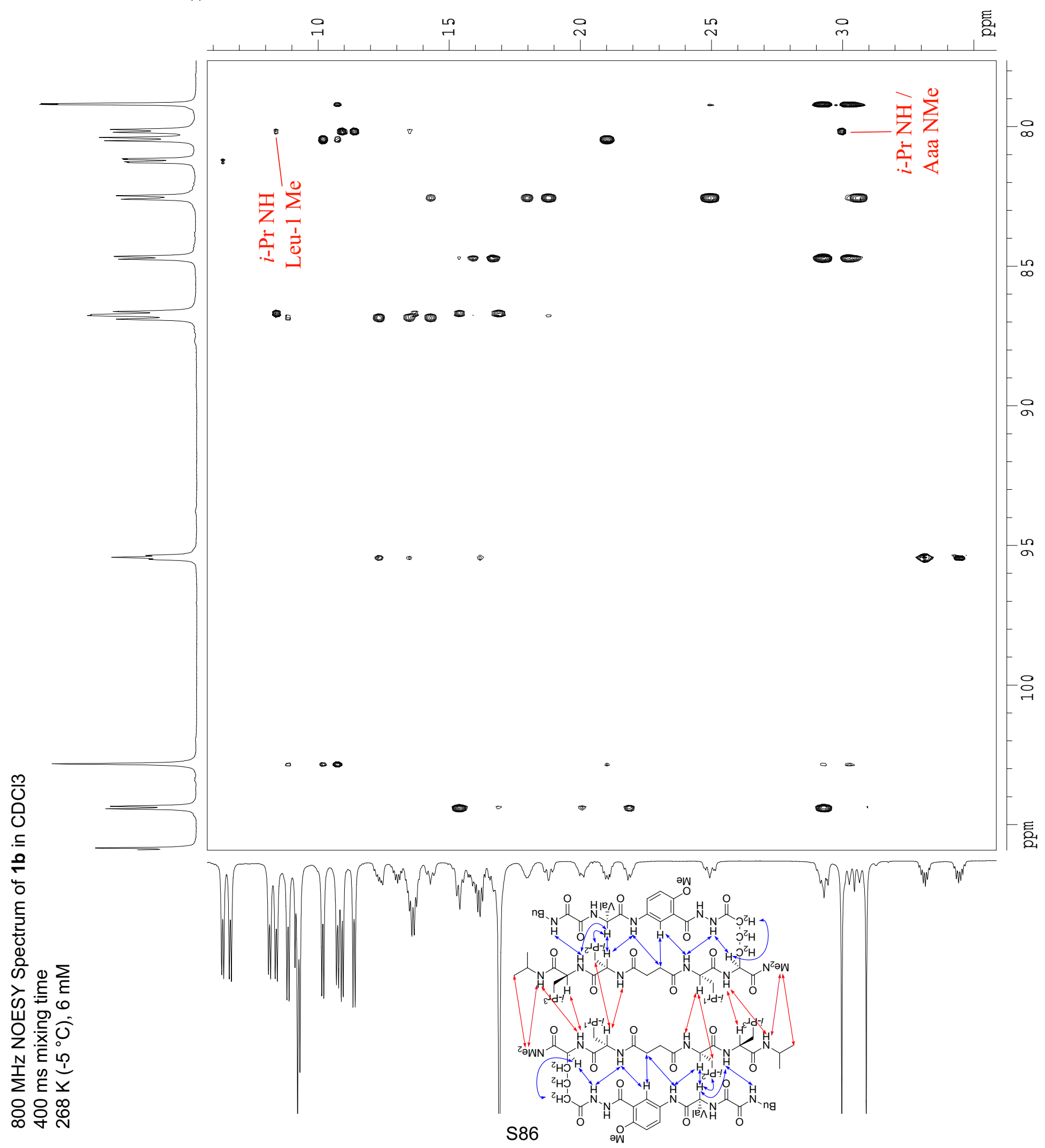




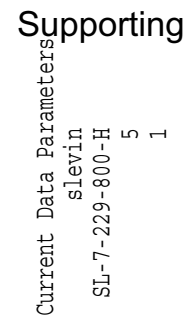

㩊罢总总总
Irgarformation

焉

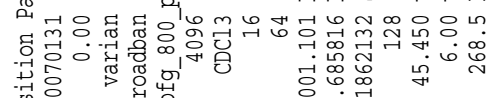

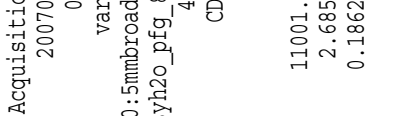
i 目

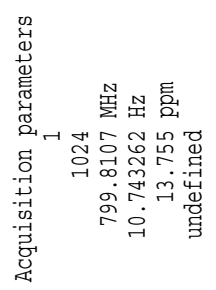

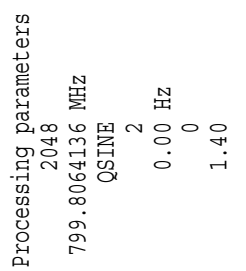
푸

S. Levin and J. S. Nowick

过 E्) हE

等

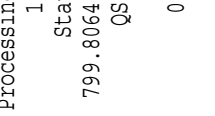

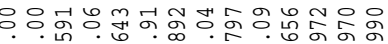

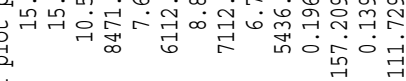

ㅇ

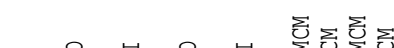

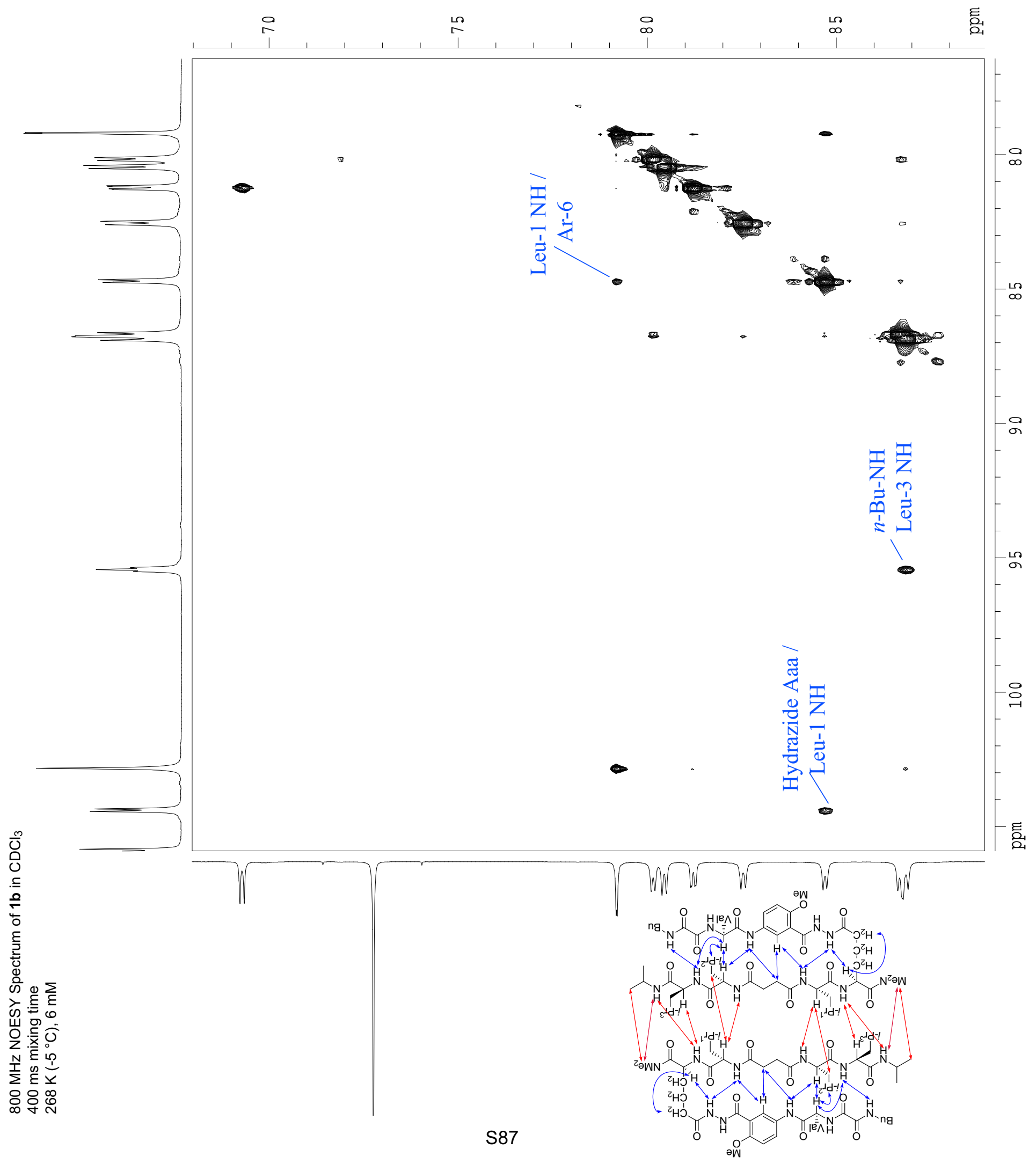




\section{Supporting Information}

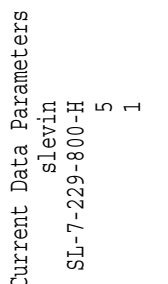

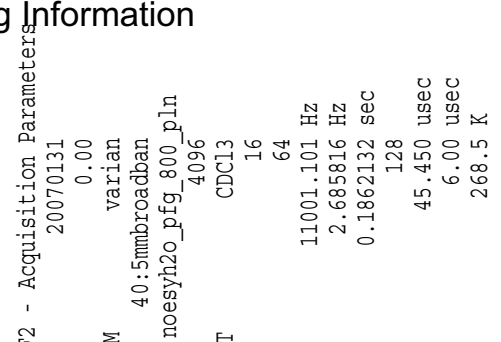

第罢总总

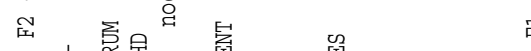

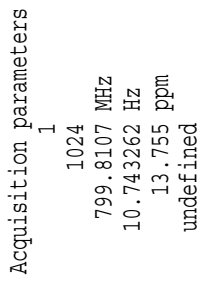

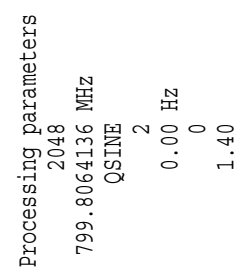

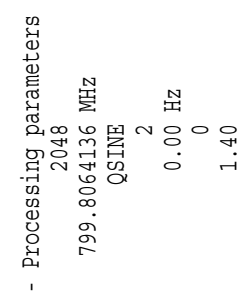

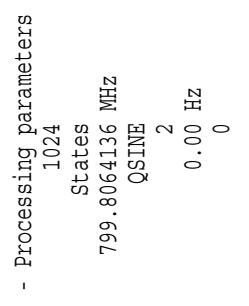

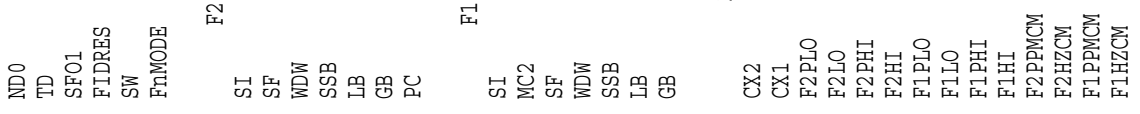

S. Levin and J. S. Nowick

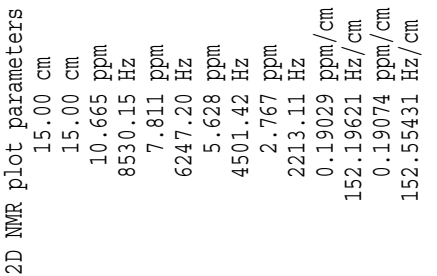

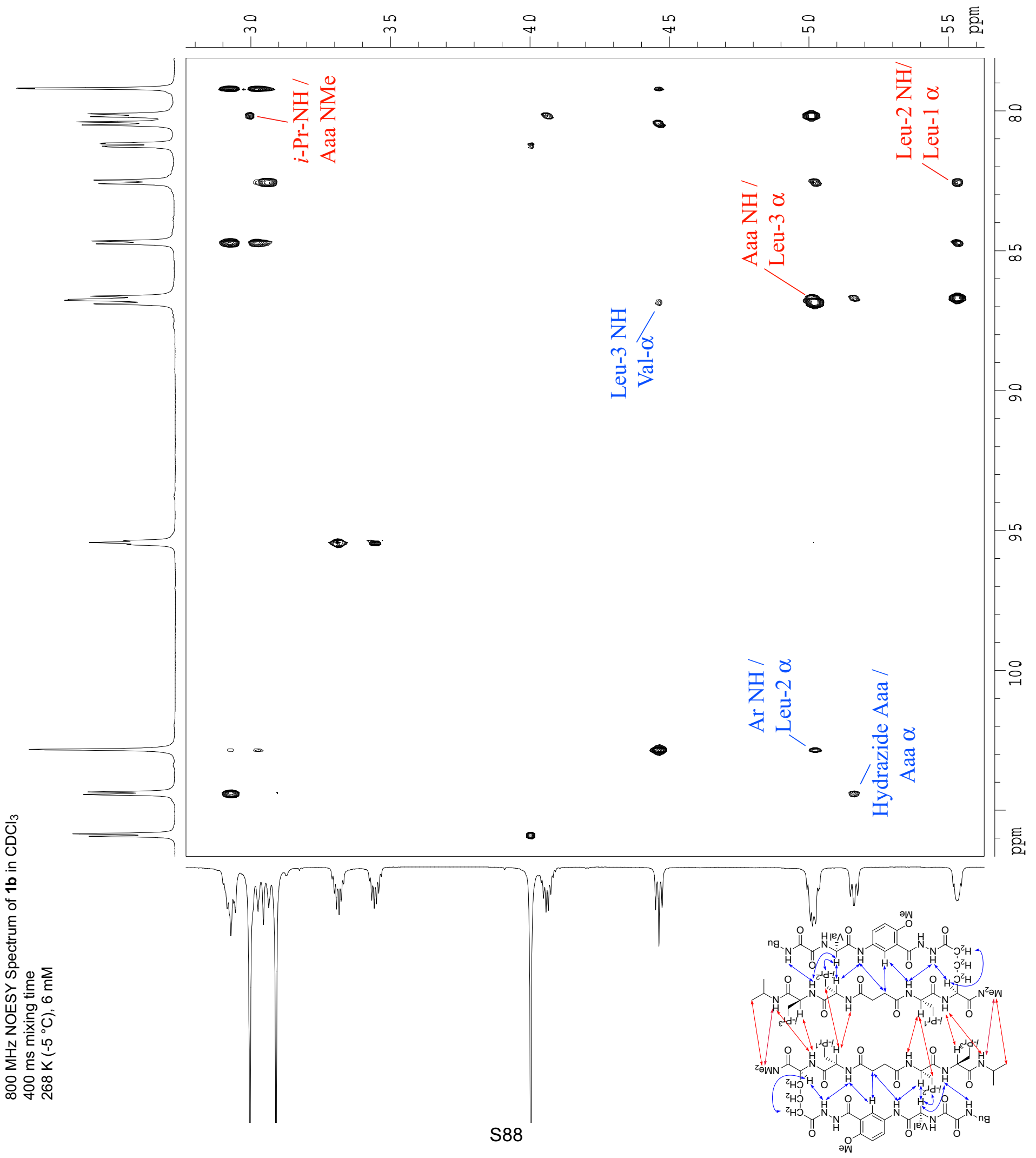


Supporting 奶formation

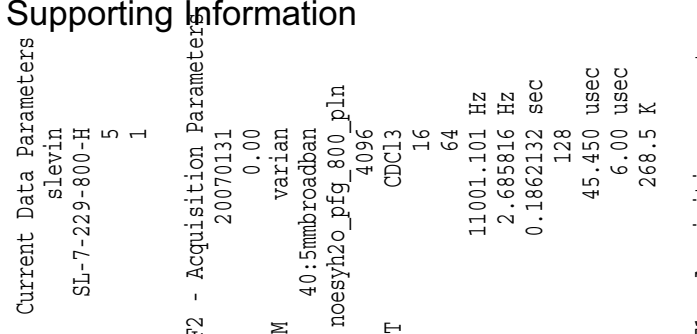

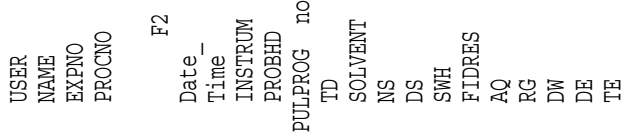

S. Levin and J. S. Nowick

嵌

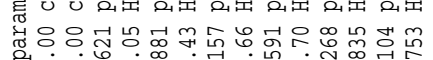

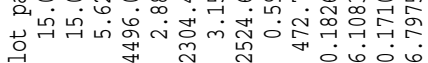

兴 ล

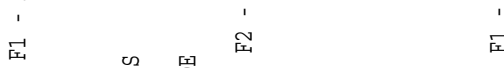

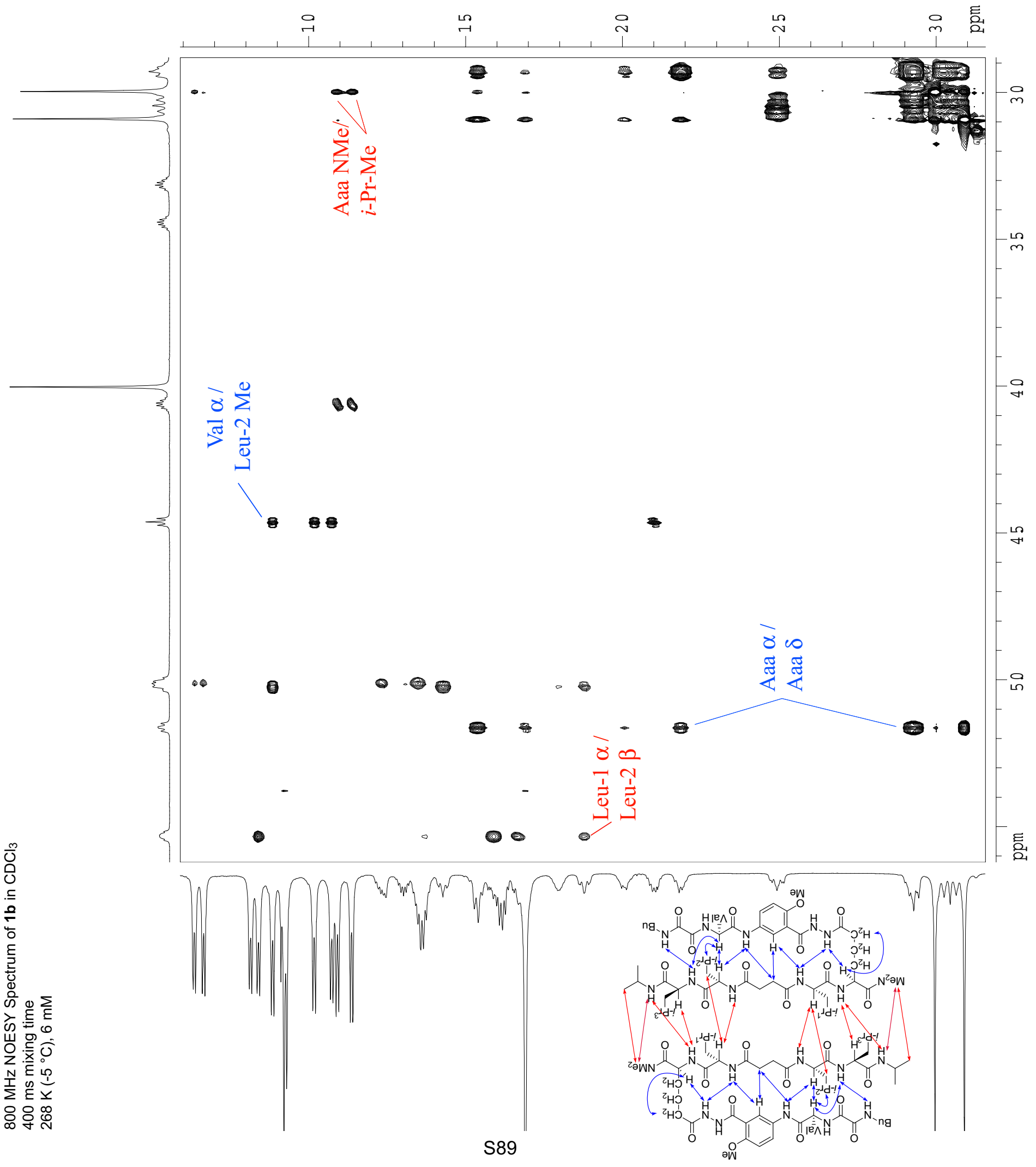




\section{Supporting Information}

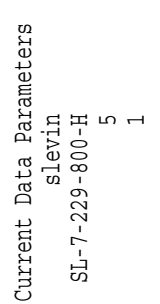

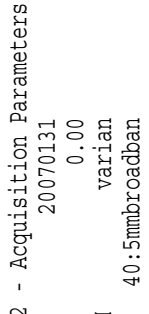

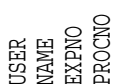

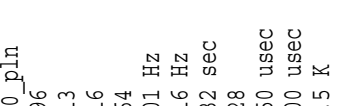

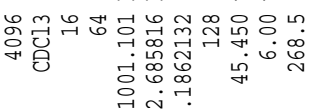
这 ㄴ.

\section{兵}

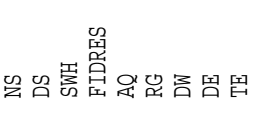

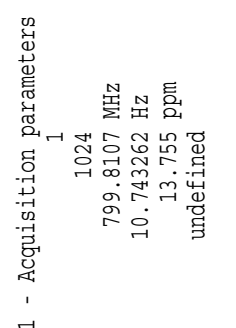

$\vec{r}$

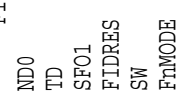

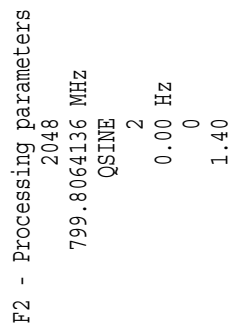

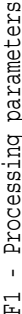

S. Levin and J. S. Nowick

造

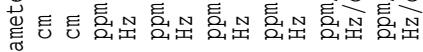

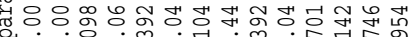

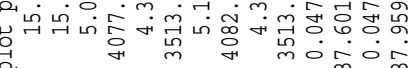
笶

ลิ

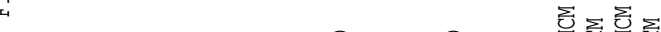

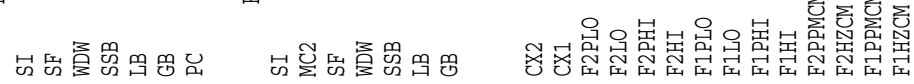

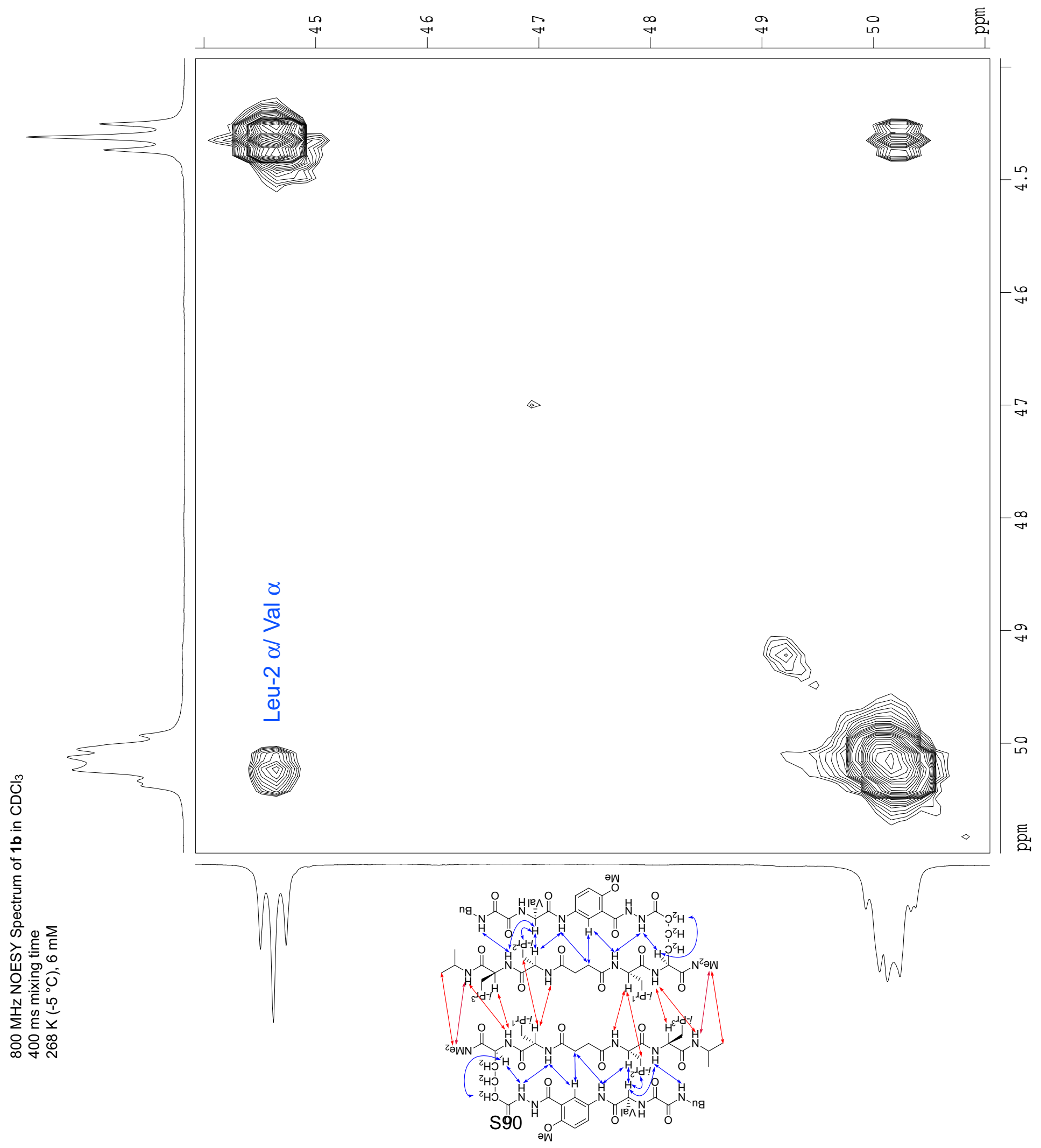




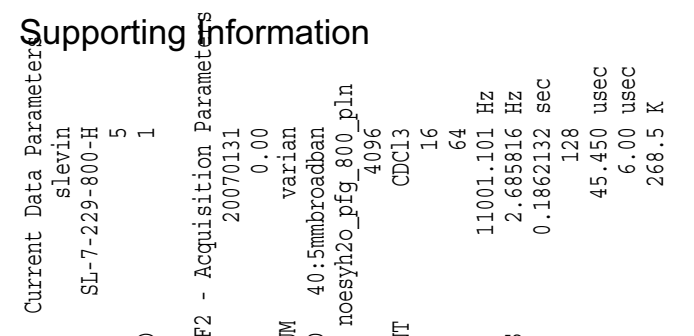

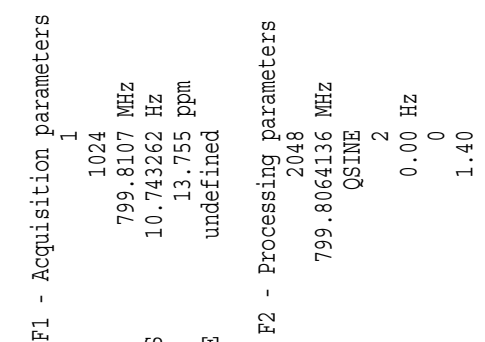

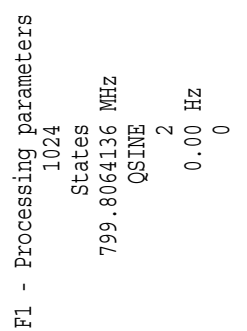

S. Levin and J. S. Nowick

号

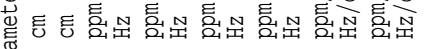

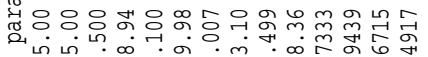

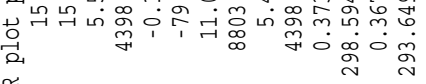

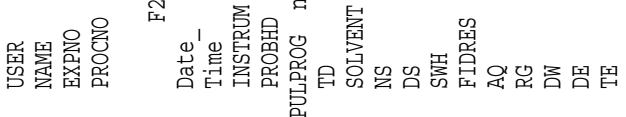

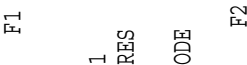

i

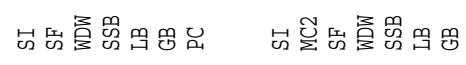

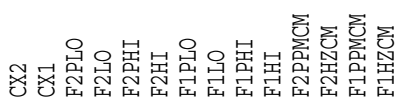

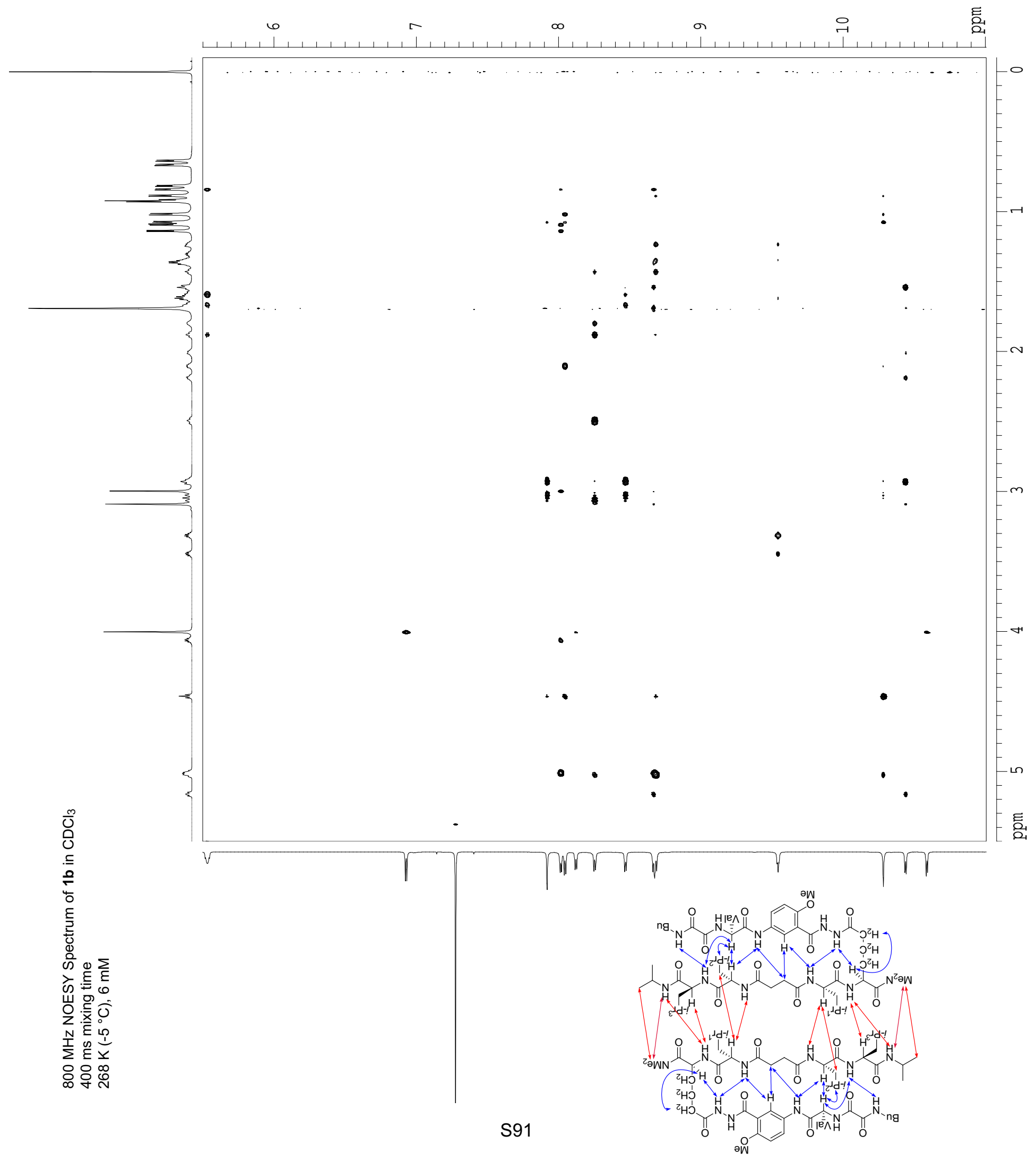




\section{Spectroscopic DATA for the compound 1c}

(8 $\left.\mathrm{mM}, \mathrm{CDCl}_{3} 268 \mathrm{~K}\right)$

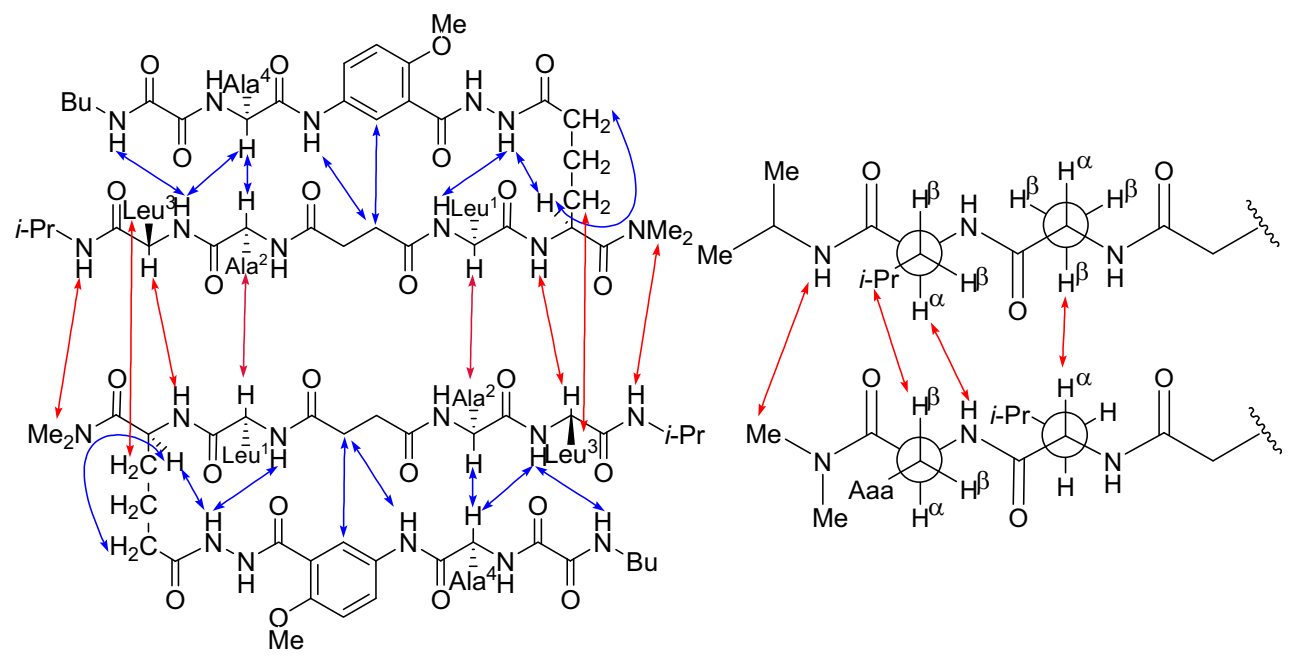

Key NOEs assigned as intermolecular are represented with red arrows

Key NOEs assigned as intramolecular are represented with blue arrows

Key data for amino acids.

\begin{tabular}{|c|c|c|c|}
\hline Amino acid & $\mathrm{H}_{\alpha}$ Shift ppm & NH Shift ppm & ${ }^{3} J_{\mathrm{NH} \alpha} \mathrm{Hz}$ \\
\hline Aaa & 5.17 & 8.37 & broad \\
\hline $\mathrm{R}_{1}=\mathrm{Leu}^{1}$ & 5.53 & 8.40 & broad \\
\hline $\mathrm{R}_{2}=\mathrm{Ala}^{2}$ & 5.06 & 8.42 & broad \\
\hline $\mathrm{R}_{3}=\mathrm{Leu}^{3}$ & 5.01 & 8.60 & 8.4 \\
\hline $\mathrm{R}_{4}=\mathrm{Ala}^{4} 1$ & 4.68 & 8.00 & broad \\
\hline
\end{tabular}

Aminoadipic acid (Aaa) $\delta$ proton resonances.

pro-R: $2.94 \mathrm{ppm}, \mathrm{t},(J=12.2 \mathrm{~Hz})$

pro-S: 2.19 ppm, td, $\left(J_{1}=11.4 \mathrm{~Hz} ; J_{2}=7.0 \mathrm{~Hz}\right)$

anisotropy $=0.75 \mathrm{ppm}$

Key data for other NH protons.

\begin{tabular}{|c|c|c|}
\hline Proton & NH Shift ppm & $J \mathrm{~Hz}$ \\
\hline Ar-NH & 10.19 & - \\
\hline Hydrazide Ar & 10.71 & broad \\
\hline Hydrazide Aaa & 10.50 & broad \\
\hline Bu-NH & 9.50 & broad \\
\hline$i$-Pr-NH & 8.00 & broad \\
\hline
\end{tabular}

ESI MS $m / z$ for $\mathrm{C}_{47} \mathrm{H}_{78} \mathrm{~N}_{11} \mathrm{O}_{12}[\mathrm{M}+\mathrm{H}]^{+}$calcd 988.58 , found 988.45 . 


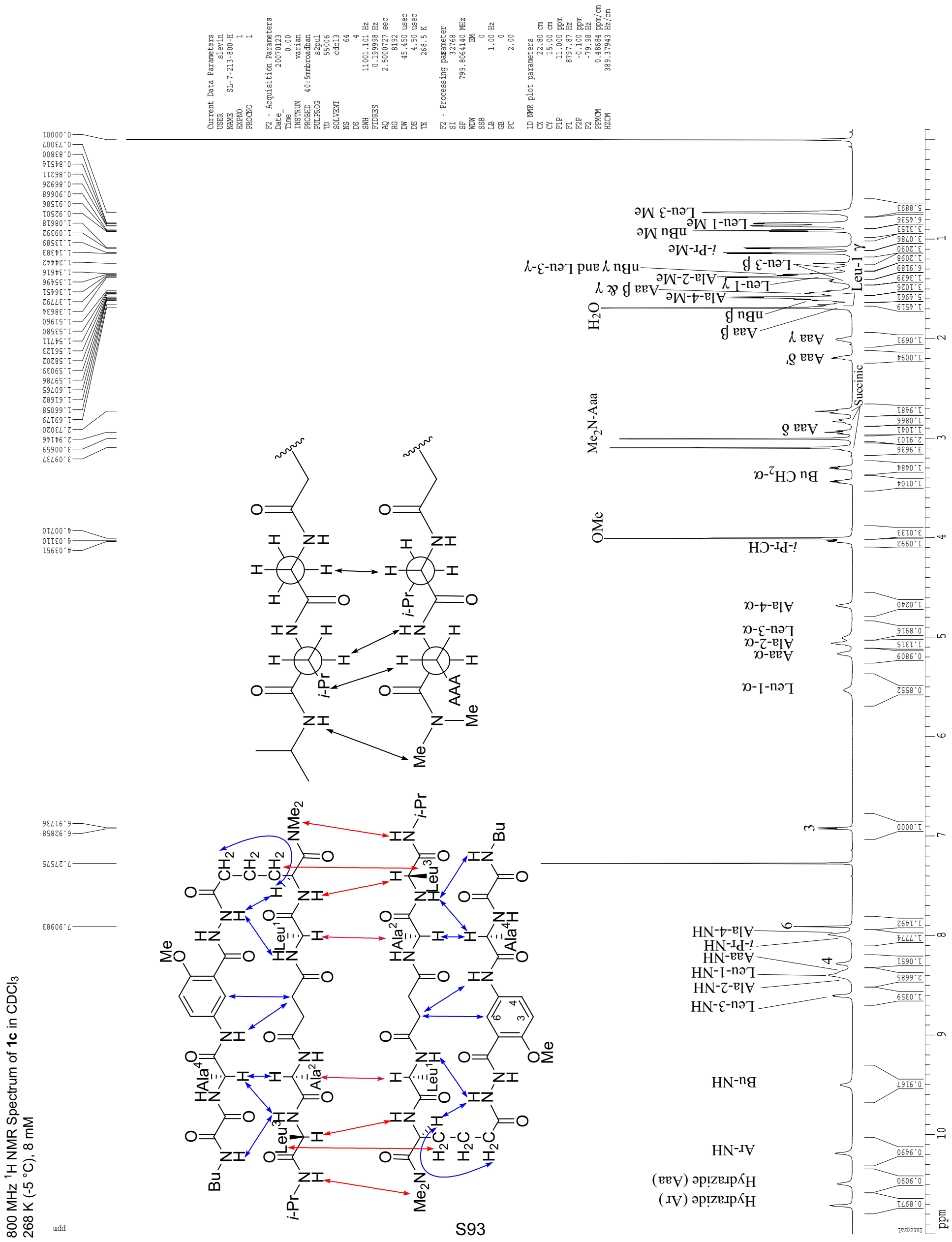




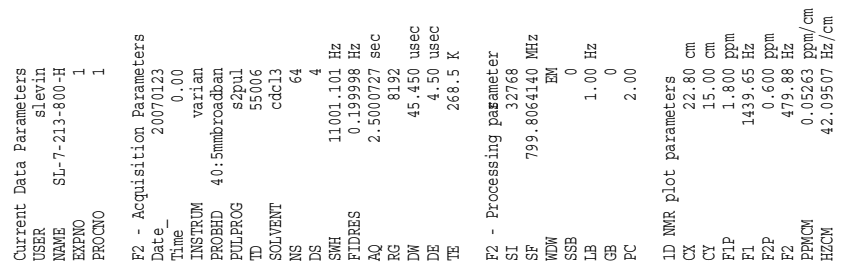

LOOEL $\cdot 0$

$008 \varepsilon 8 \cdot 0$

IIZ698.0

$89906^{\circ} 0$

$98956^{\circ} \cdot 0$

วW $\varepsilon-$-nว

$8 \mathrm{~L} 980^{\circ}$

$\partial W-\mathrm{I}_{\mathbf{d}}-?$

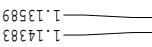

วW I-nว

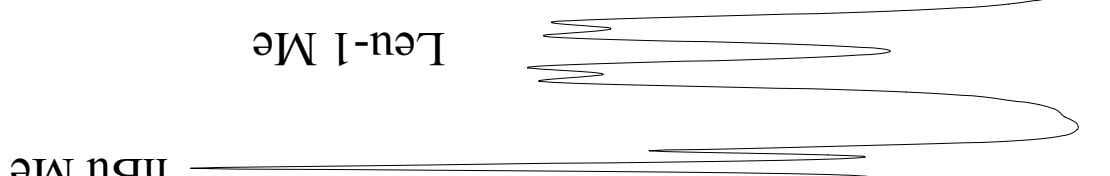

әW ngu

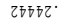

$88962^{\circ} \tau$

g $\varepsilon$-nəา

9т9ғв
$9679 \varepsilon$

Т $979 \varepsilon^{\circ} \mathrm{T}$

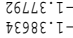

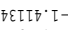

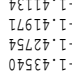

\section{คพ ngu}
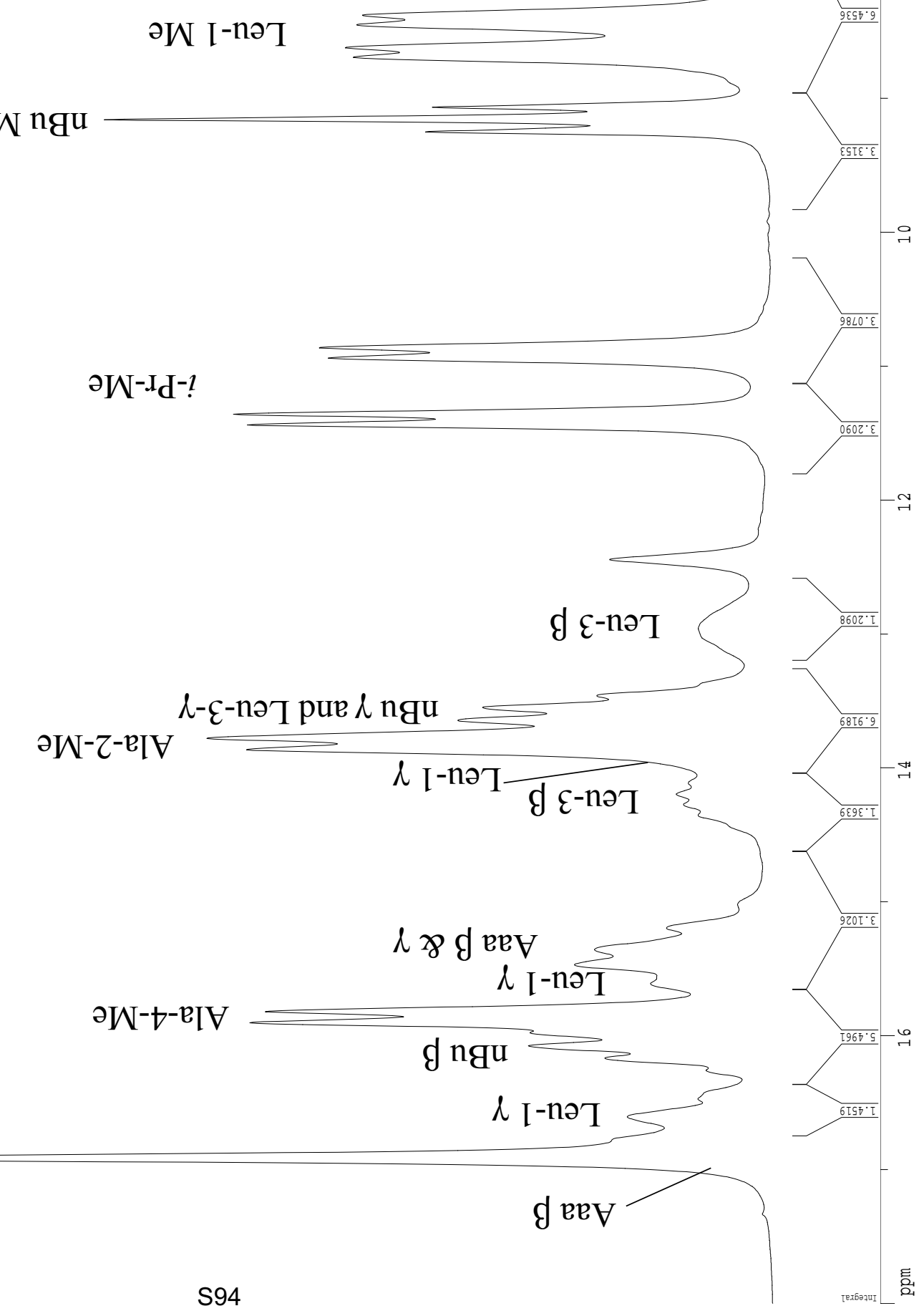

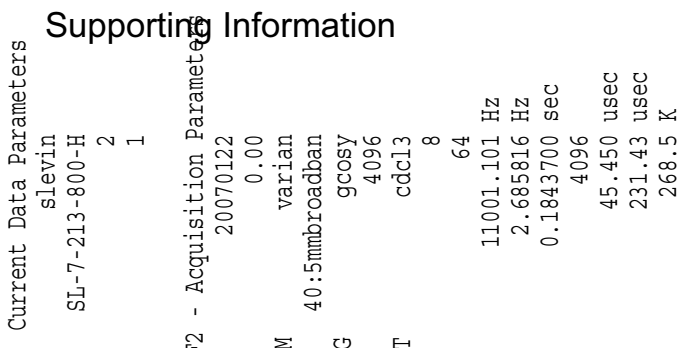

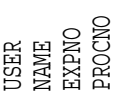
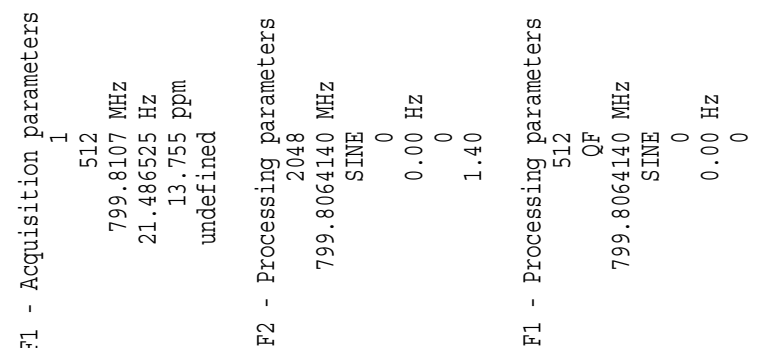

S. Levin and J. S. Nowick 号

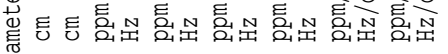

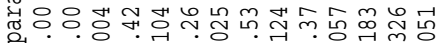

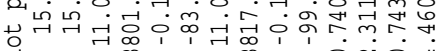
些 ลิ

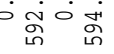

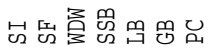

居

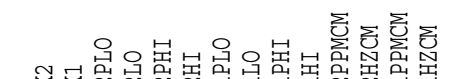
88ำ

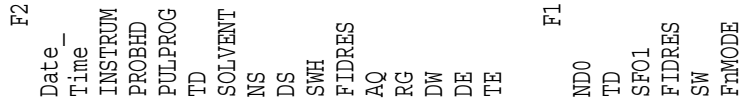

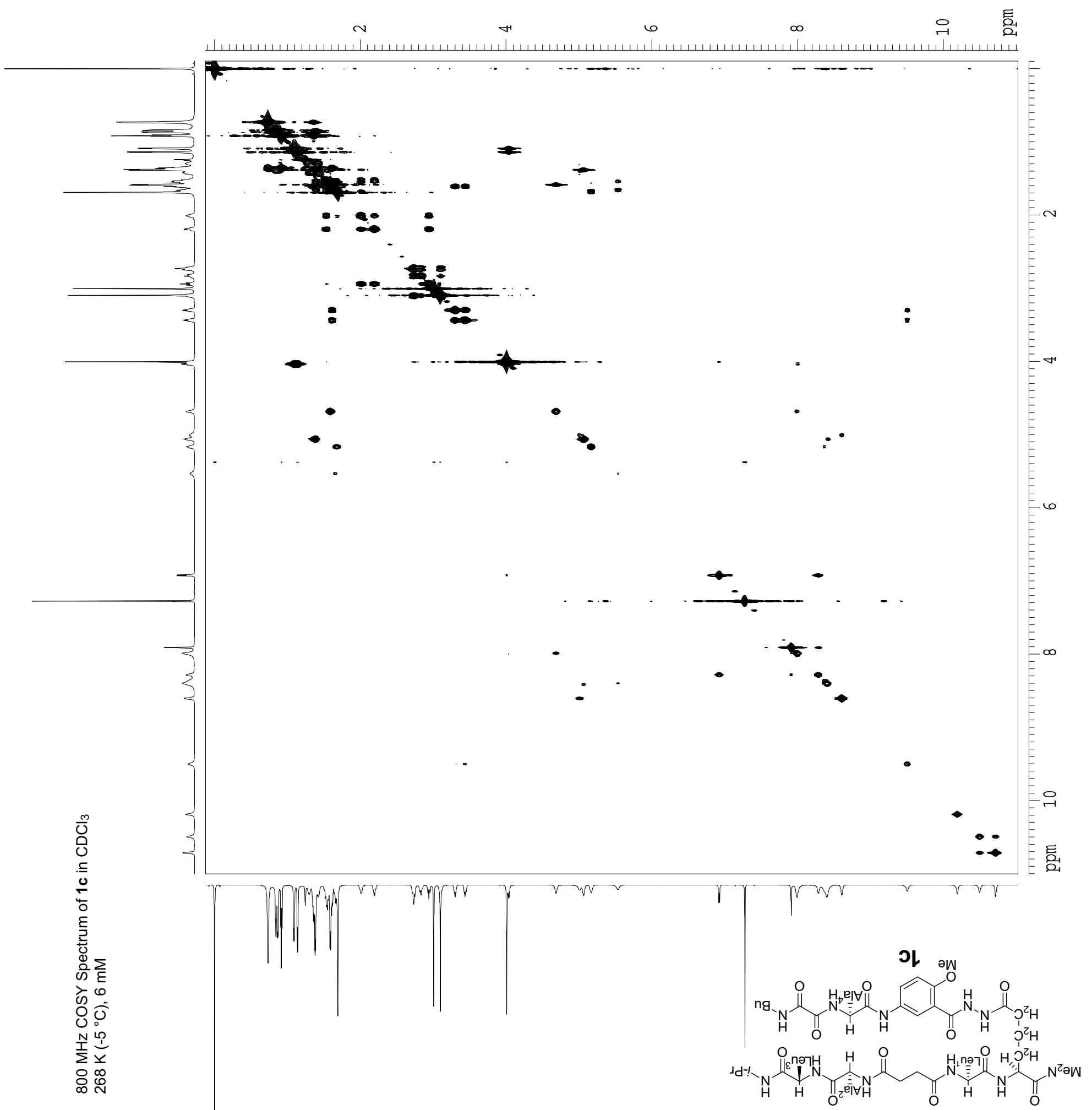


Supporting Information

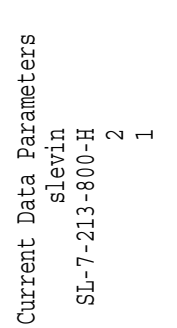

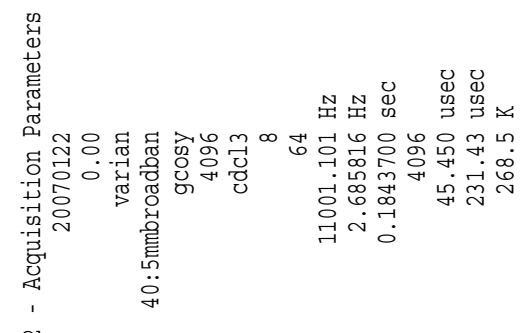

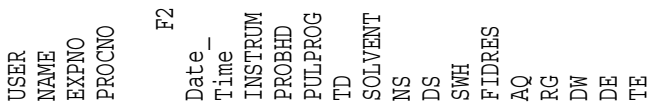

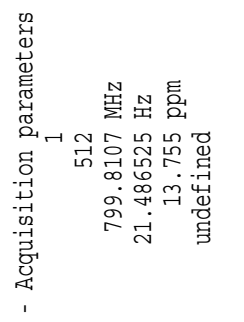

届

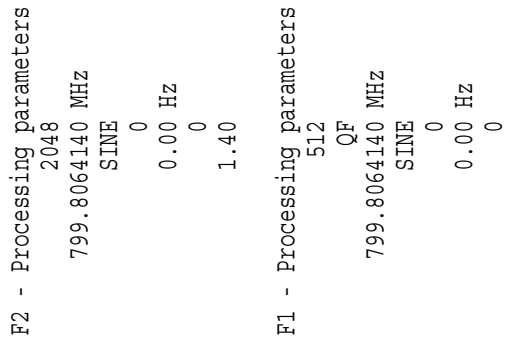

S. Levin and J. S. Nowick

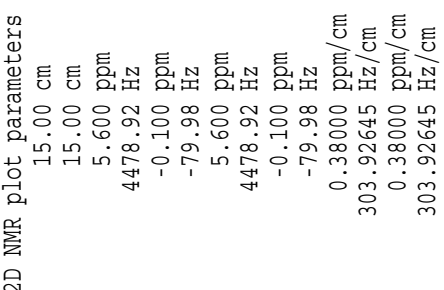

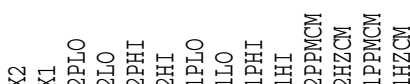

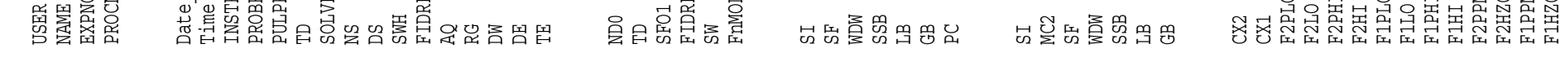

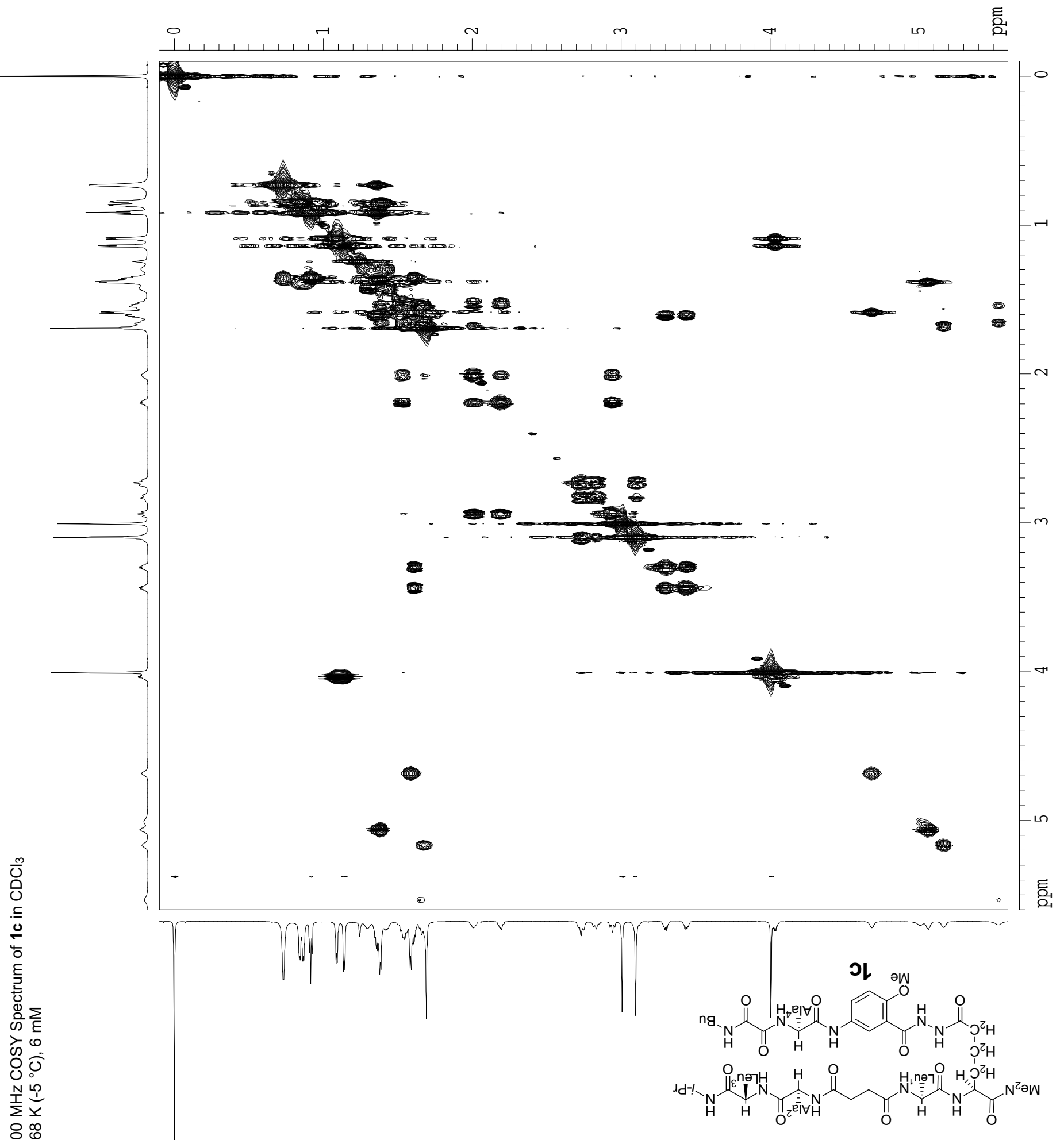


Supporting Information
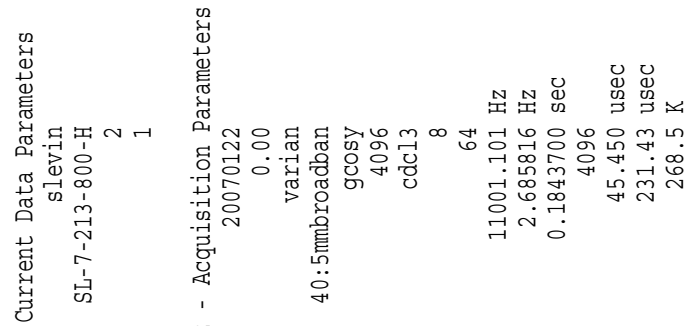

乘罢总兑
포

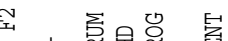

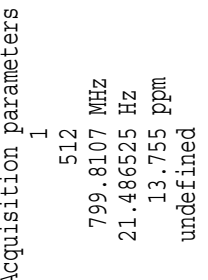

完

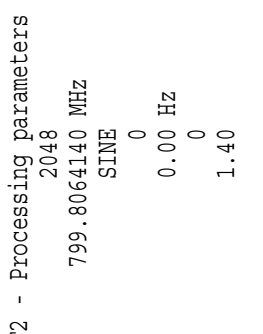

포
S. Levin and J. S. Nowick

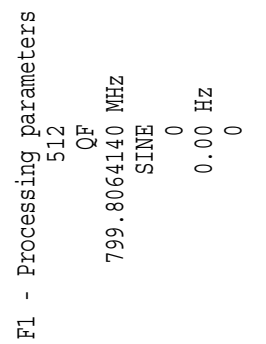

岁

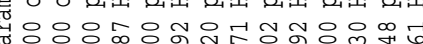

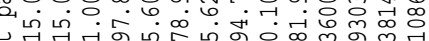
至 ลิ

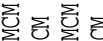

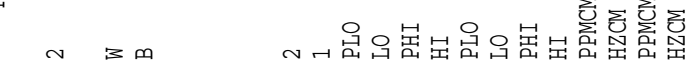

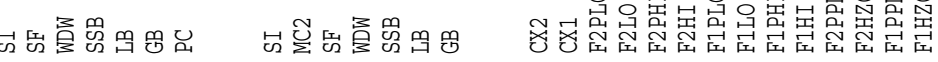

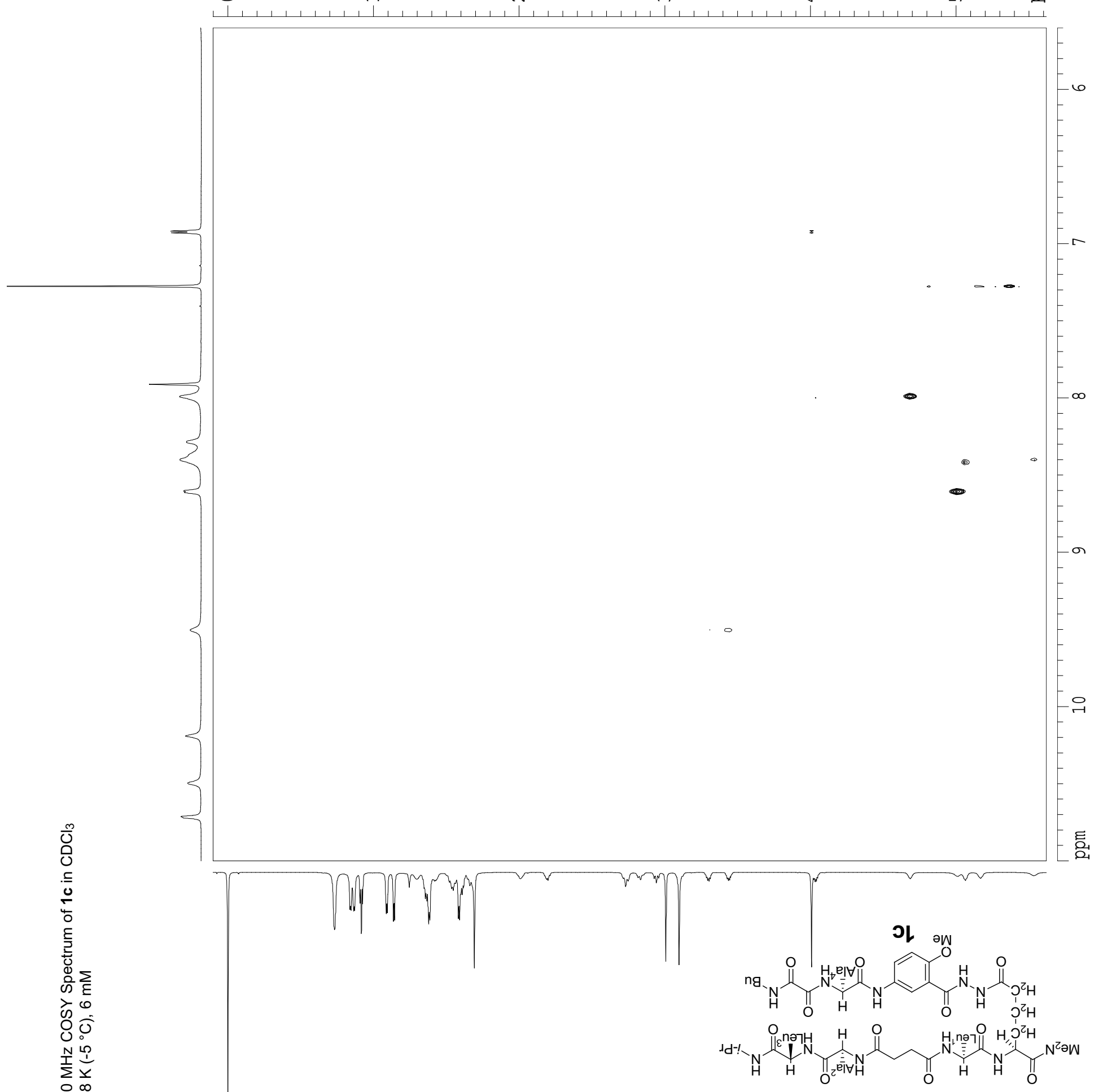


Supporting Information

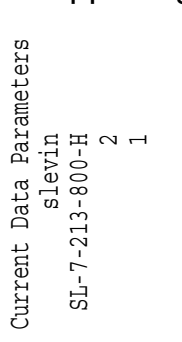

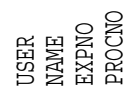

I

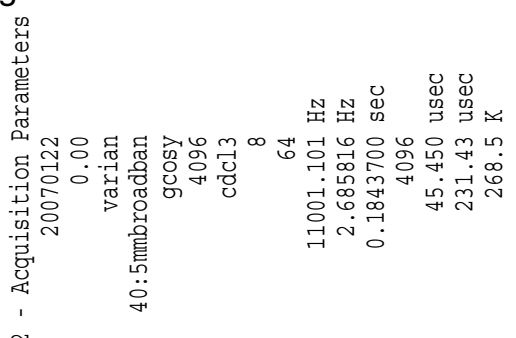

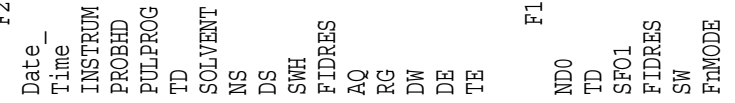
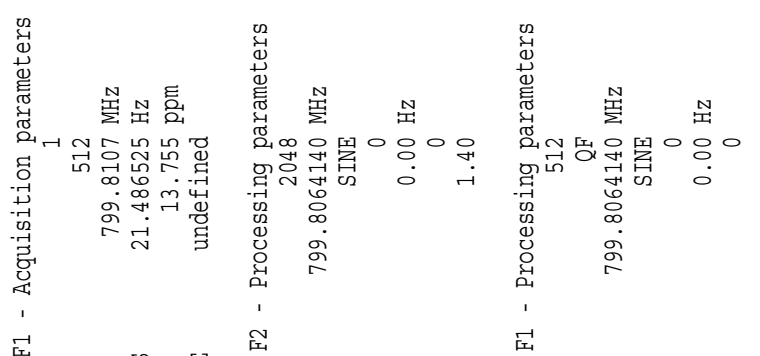

S. Levin and J. S. Nowick

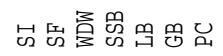

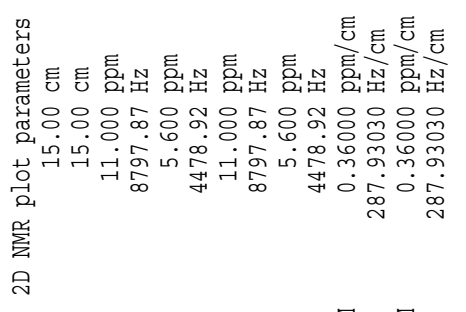

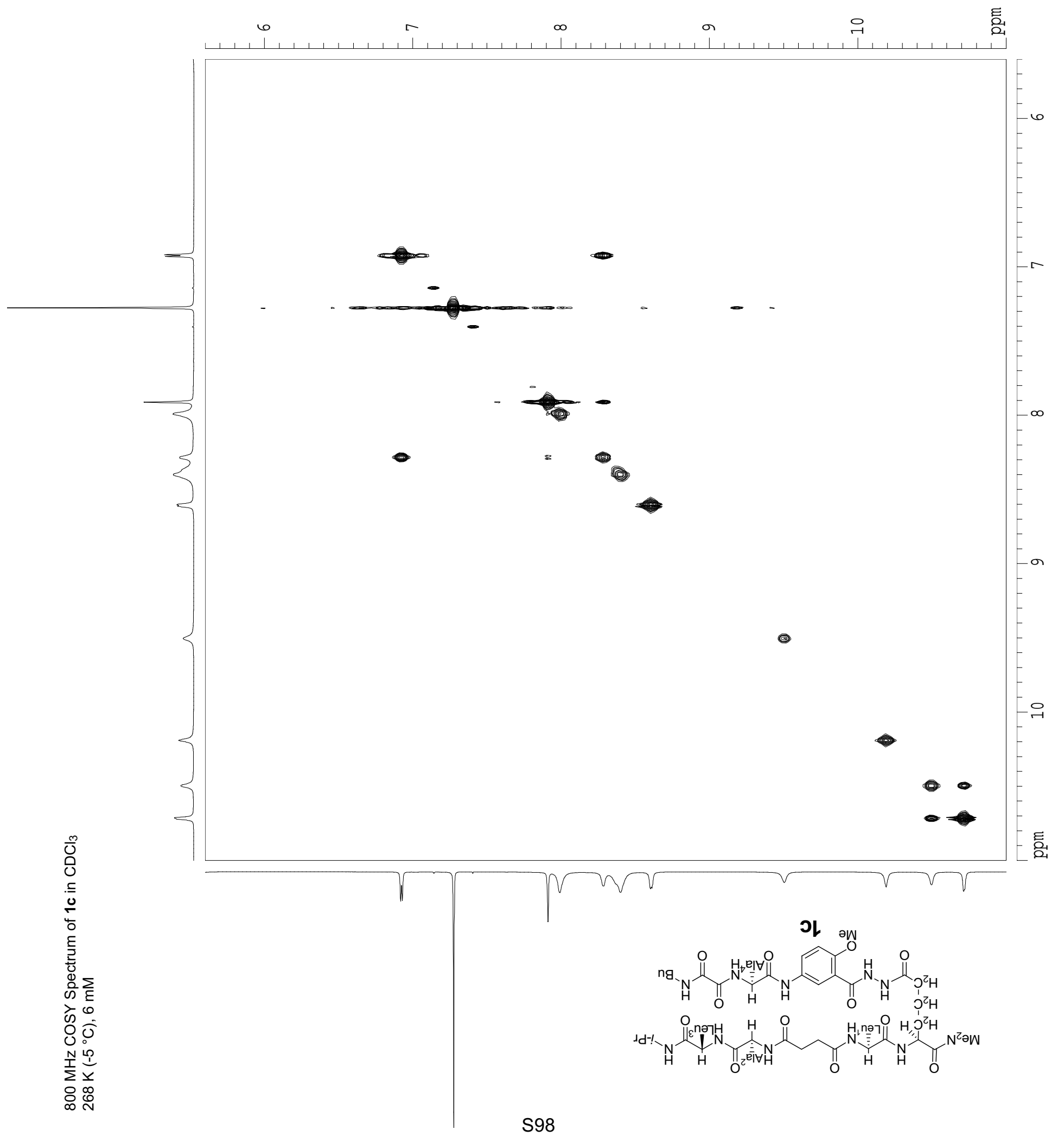


Supporting Information
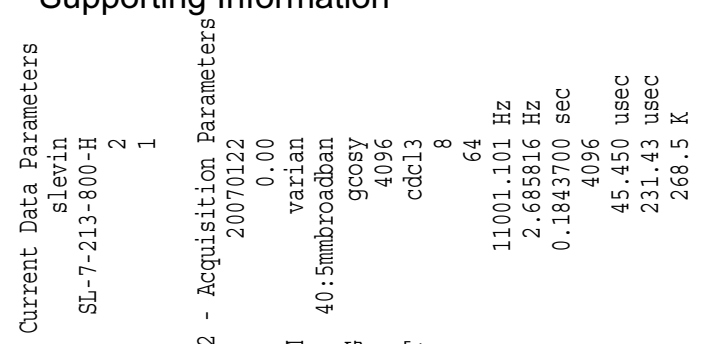

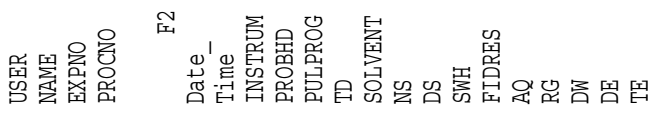

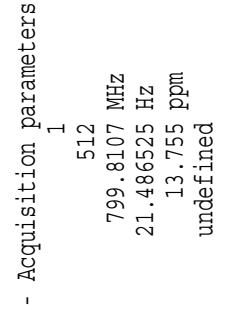

㞋

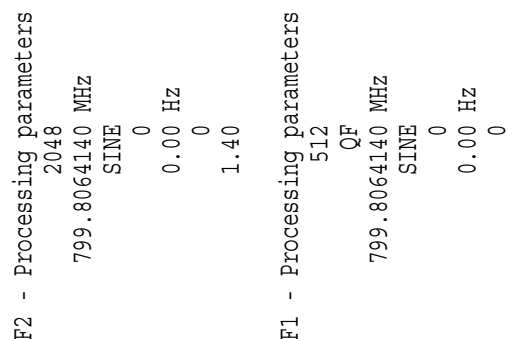

岳
S. Levin and J. S. Nowick

号

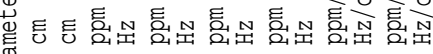

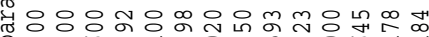

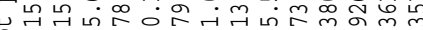
总 哭

్ㅡㄹㅠ

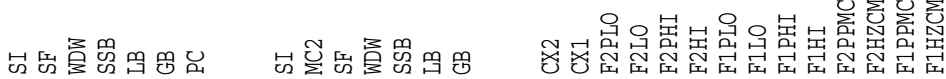

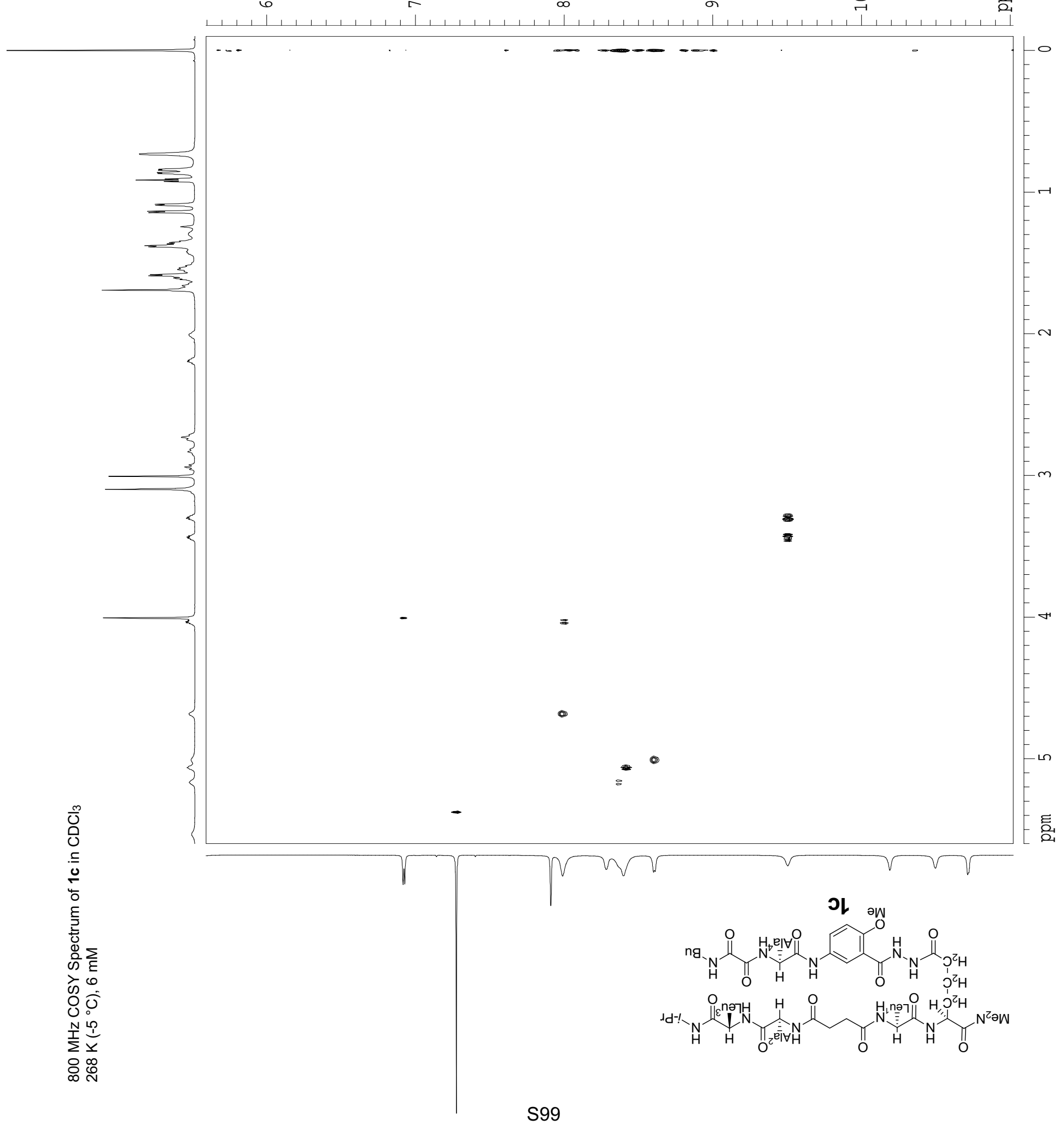




\section{Supporting Information}

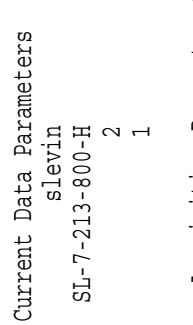

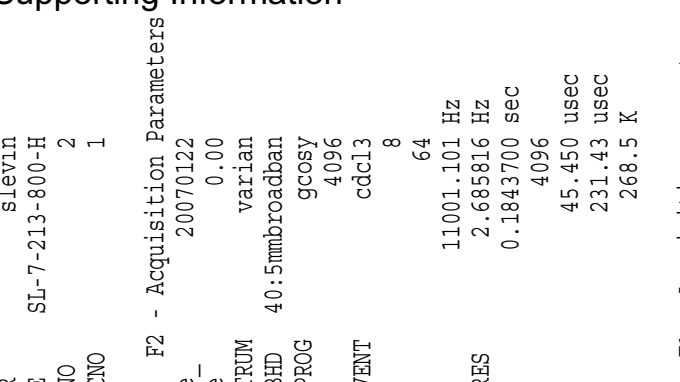

S. Levin and J. S. Nowick

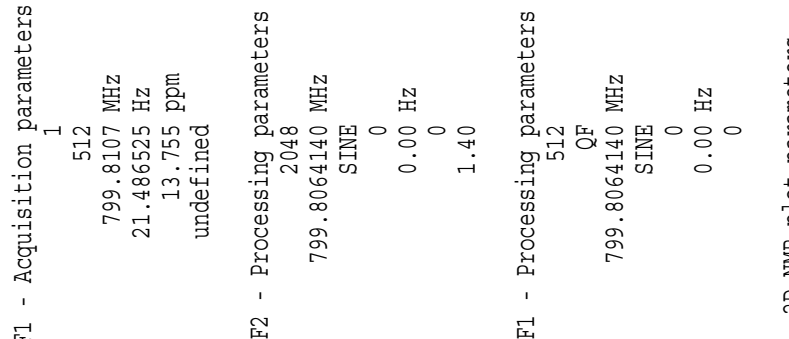

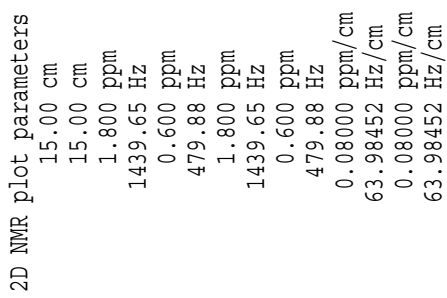

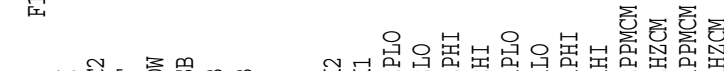

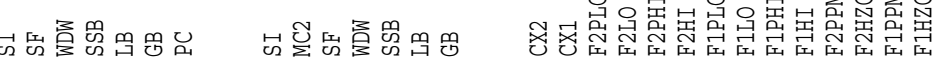

\section{HAD}

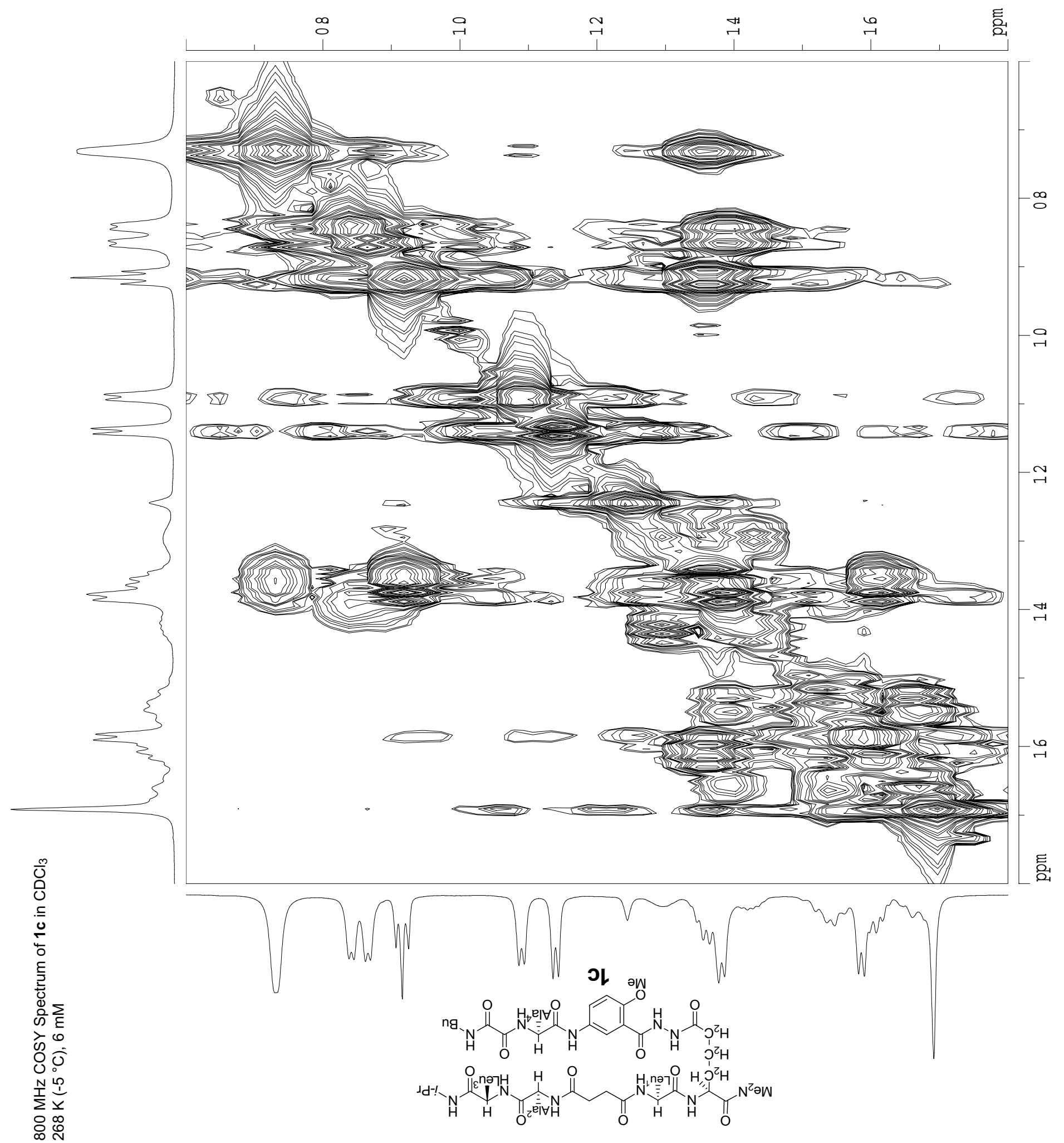



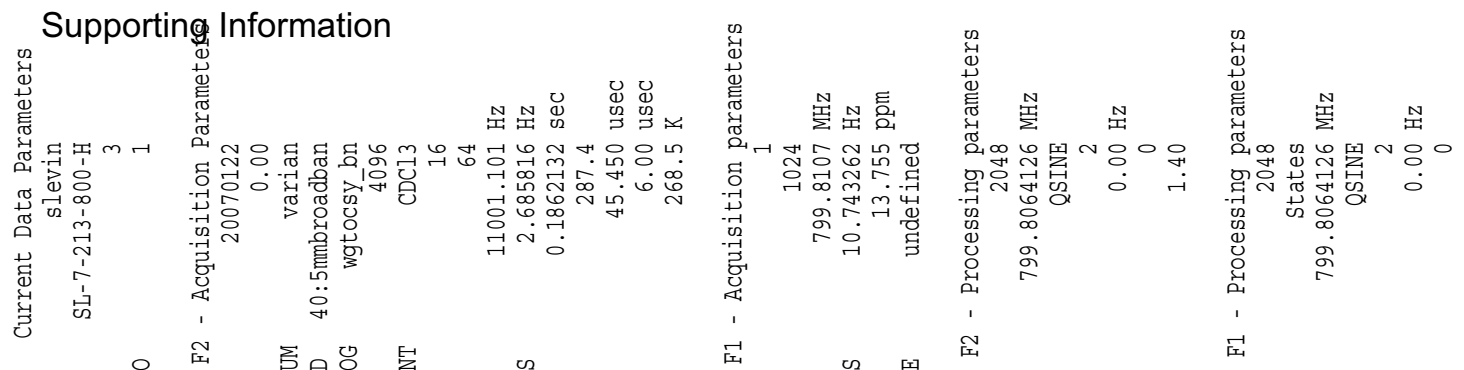

S. Levin and J. S. Nowick

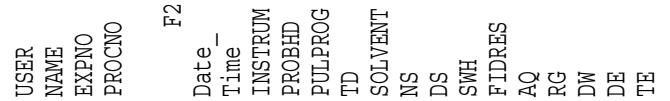

玨罂

空

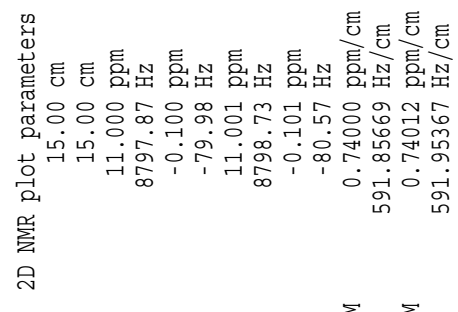

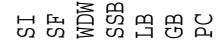

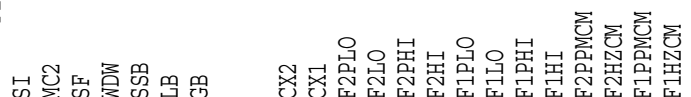

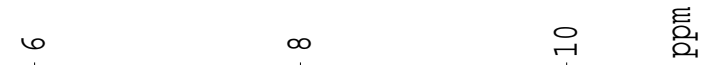

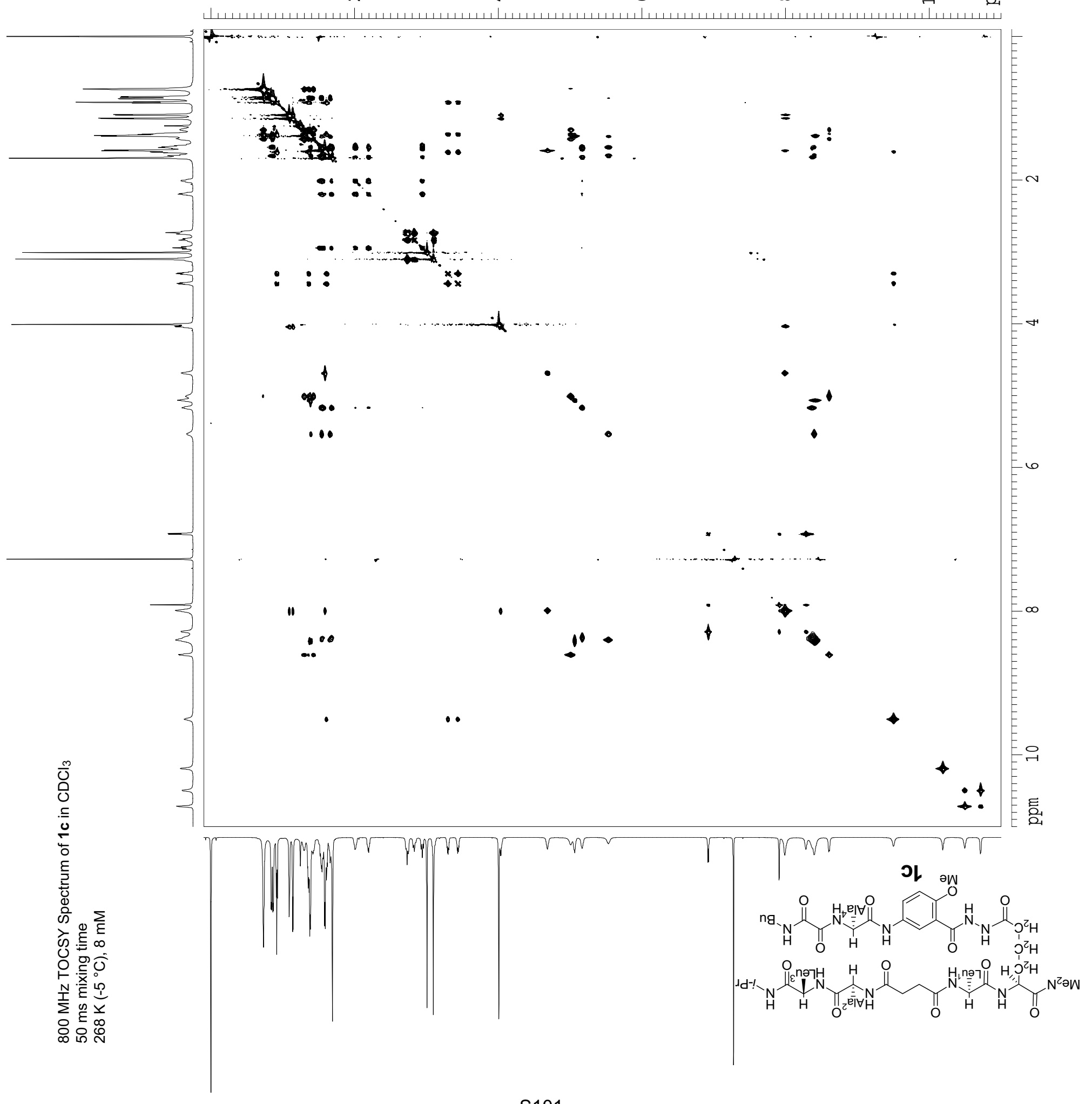


Supporting Information

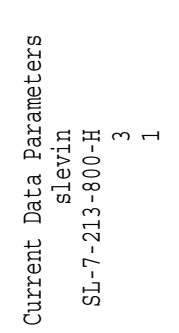

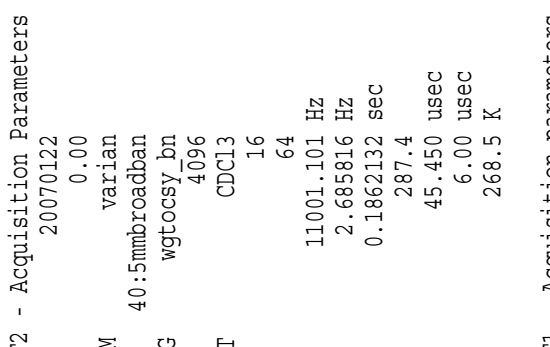

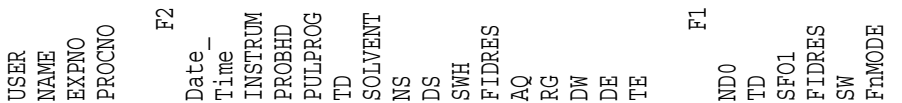

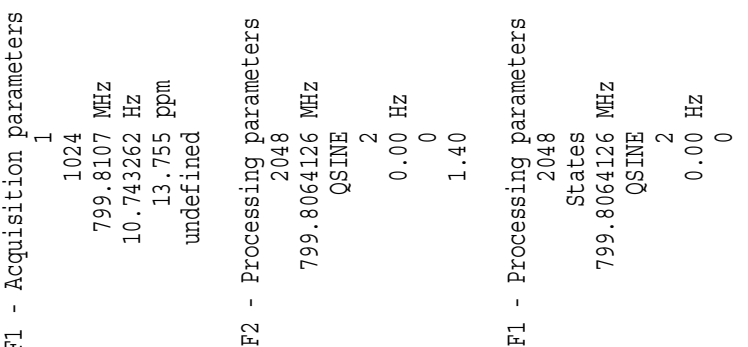

$=$
S. Levin and J. S. Nowick

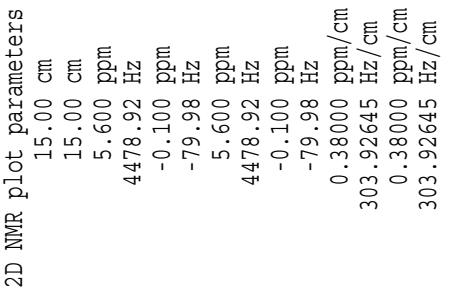

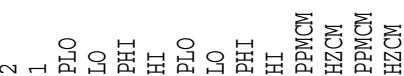

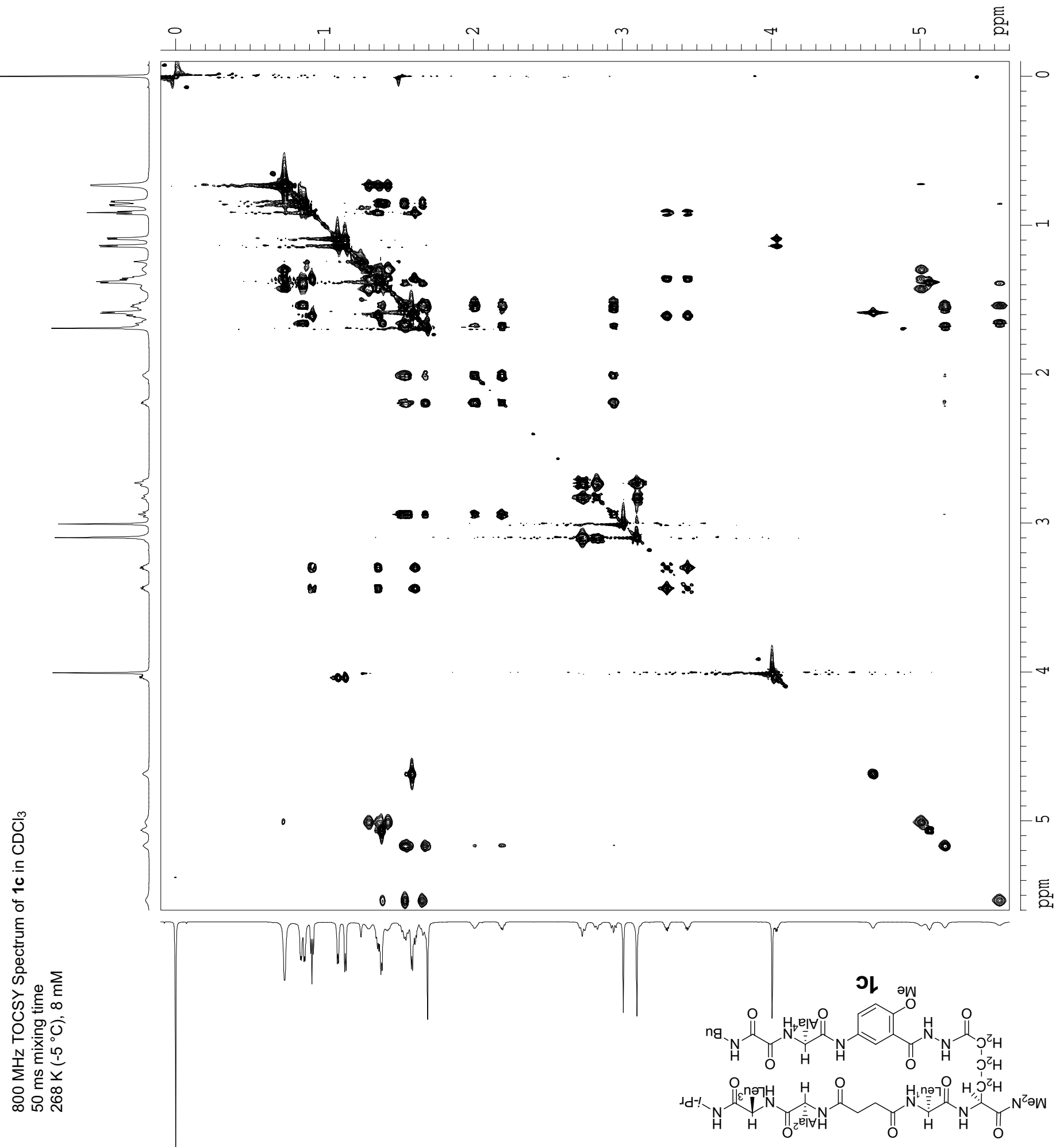


Supporting Information

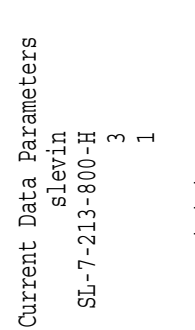

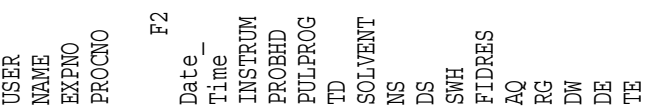

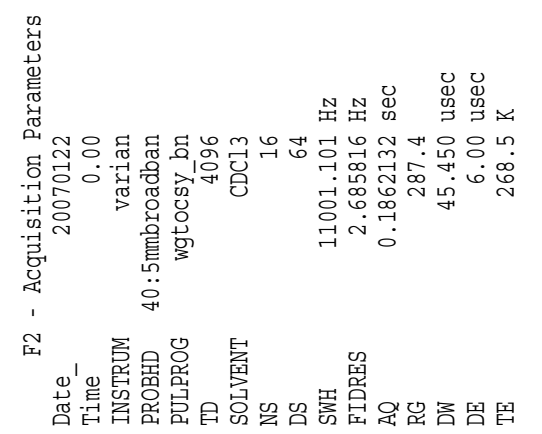

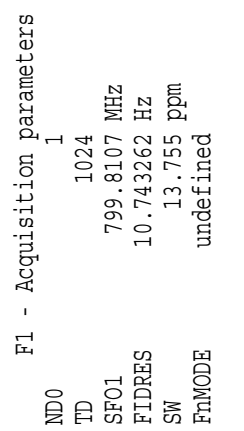

S. Levin and J. S. Nowick

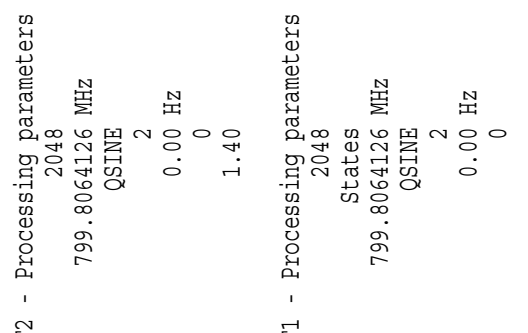

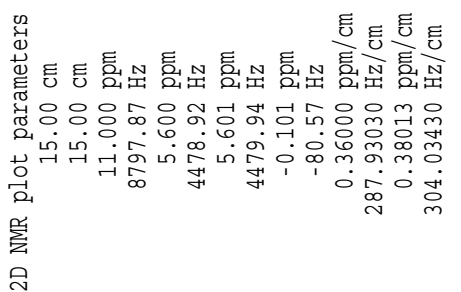

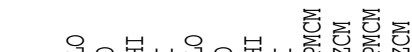
社宁

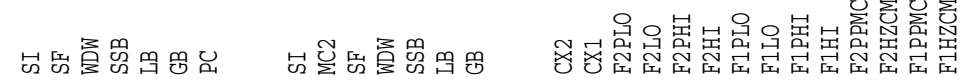

है
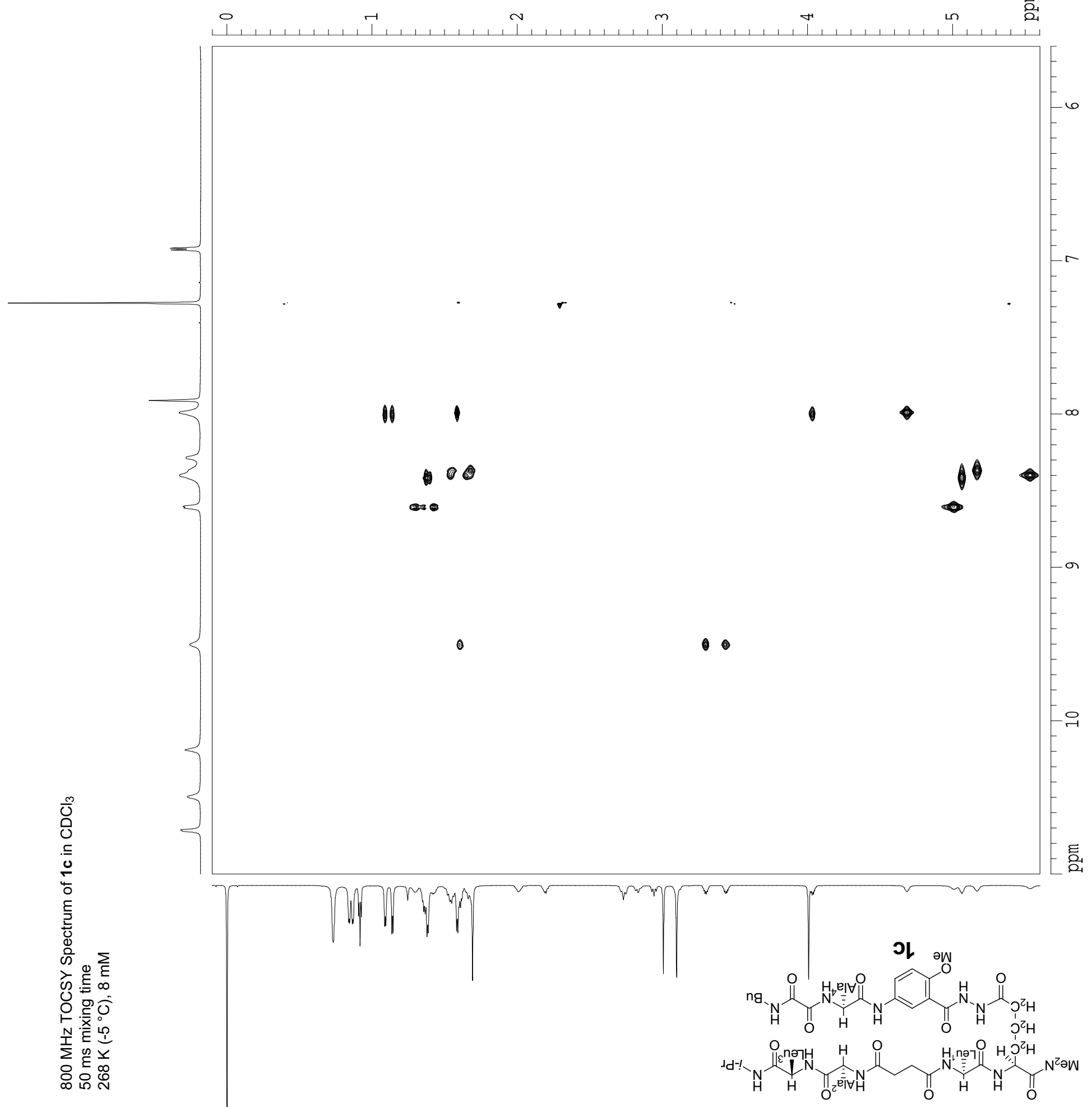

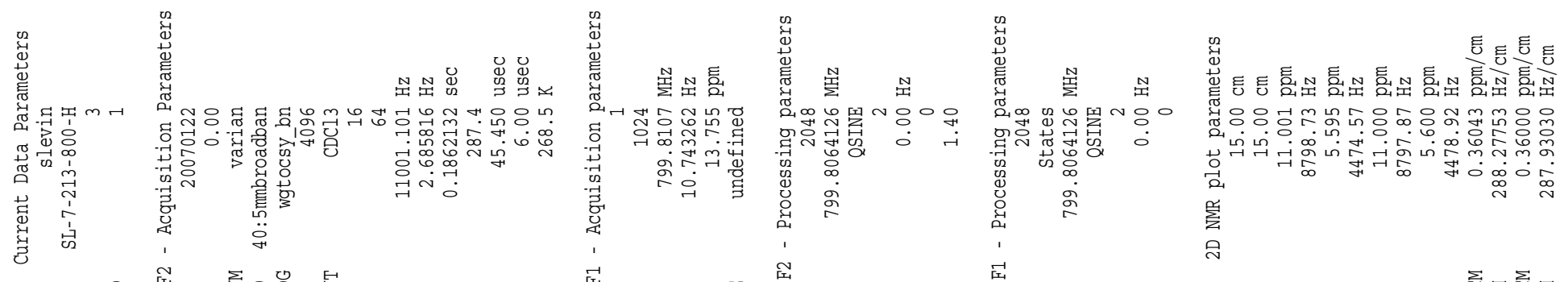

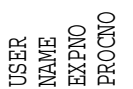

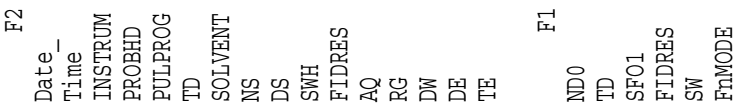

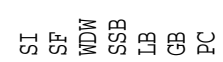

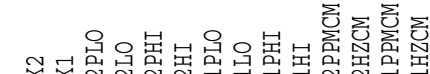

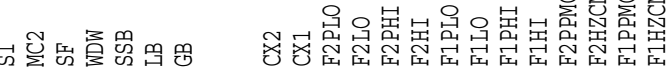

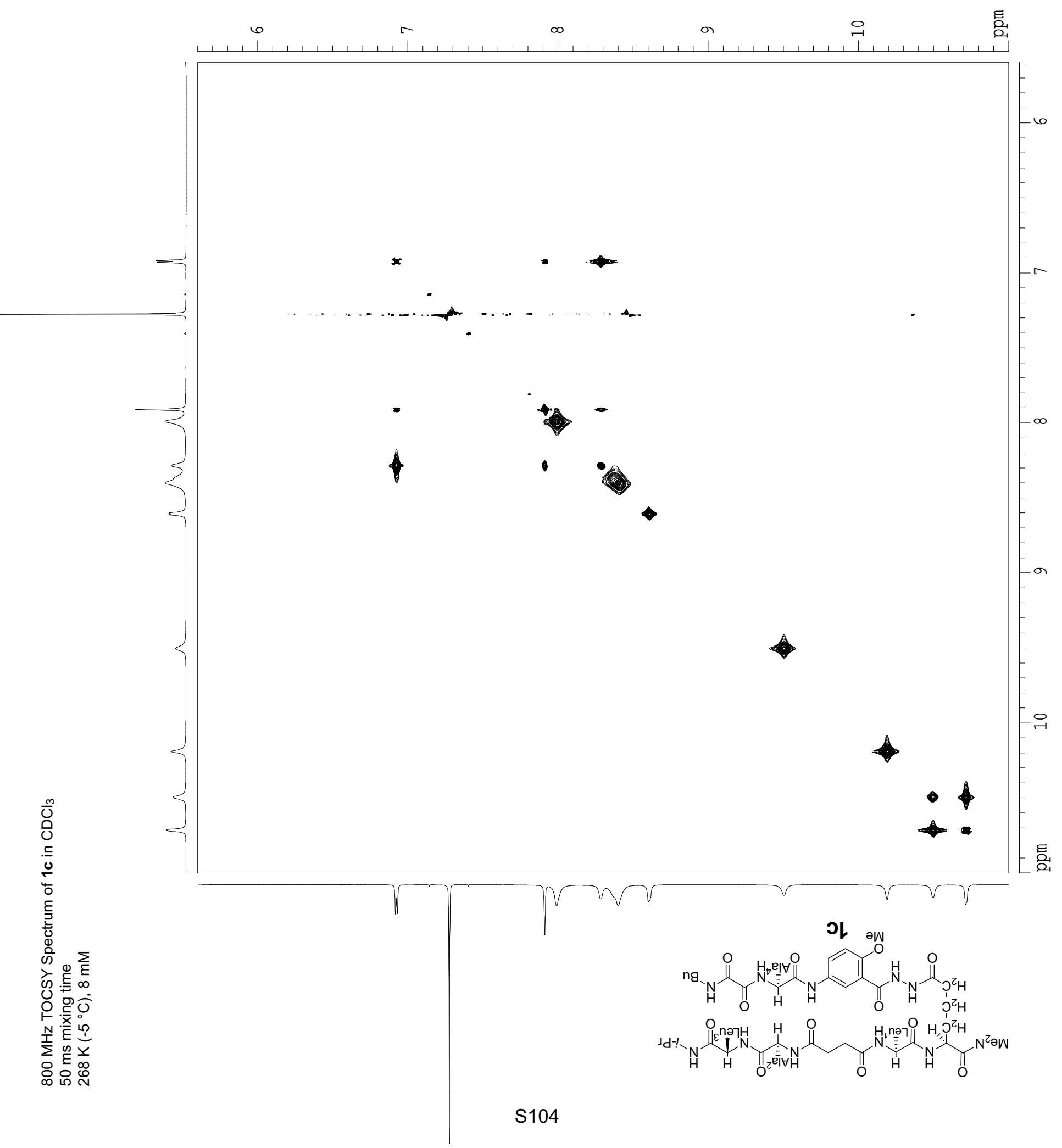


Supporting Information

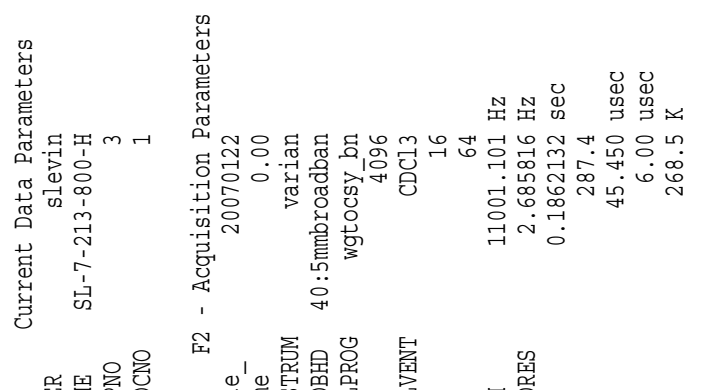

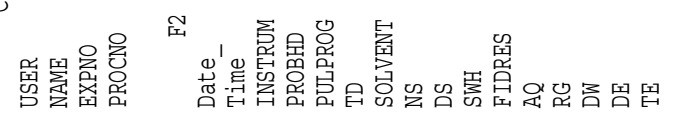

S. Levin and J. S. Nowick

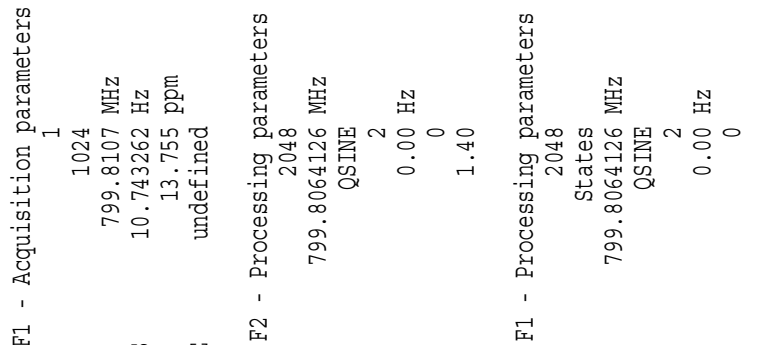

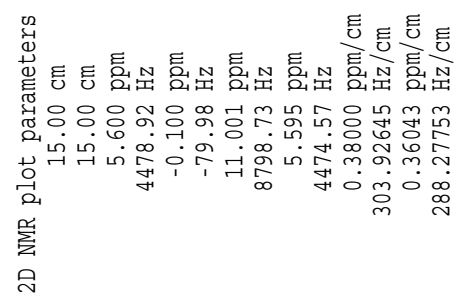
r

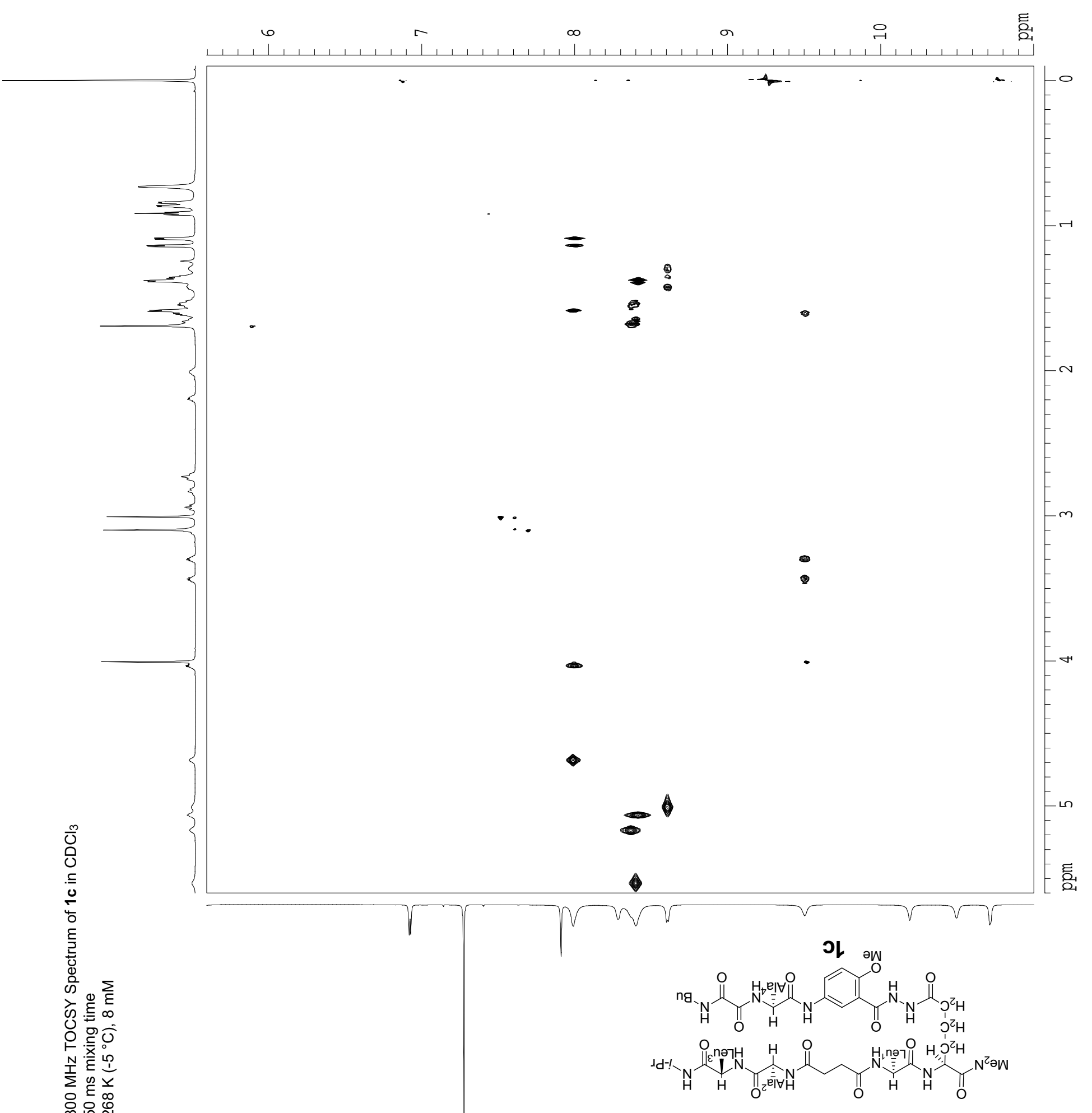


Supporting Information

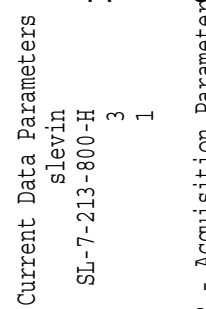

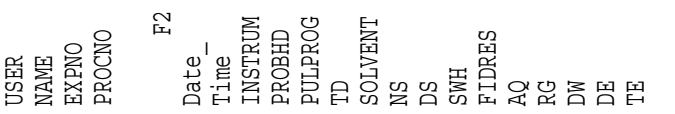

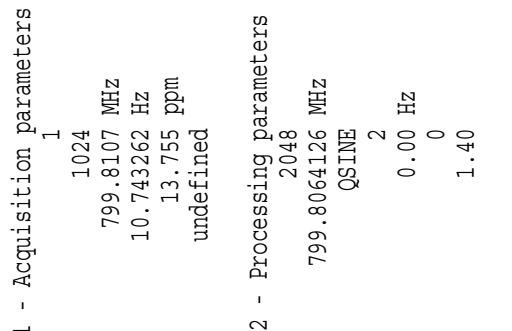

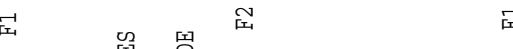

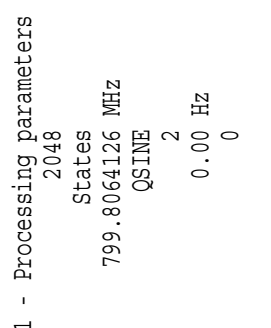

S. Levin and J. S. Nowick

थู

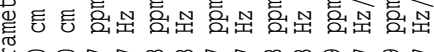

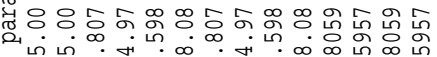

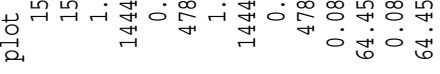
紊

穴

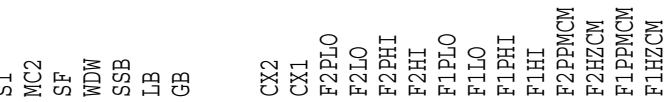

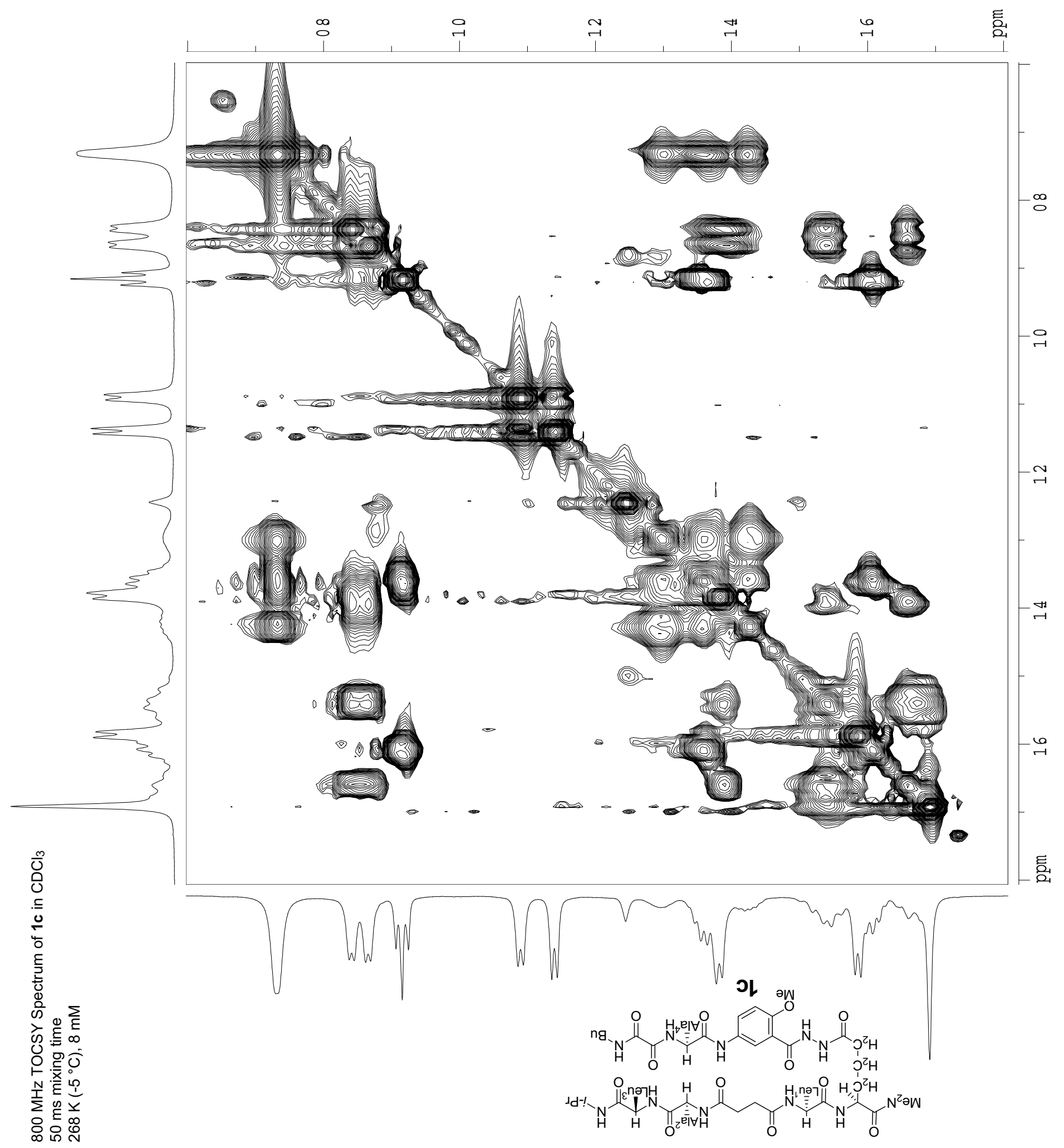


Supporting laformation
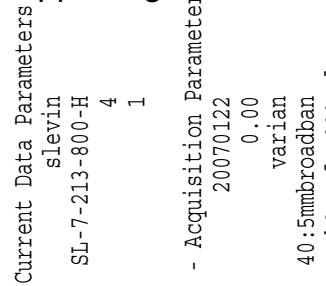

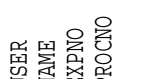

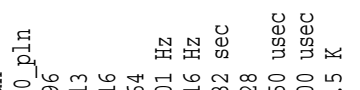

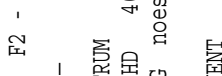

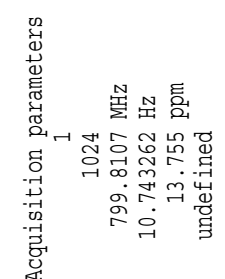

王

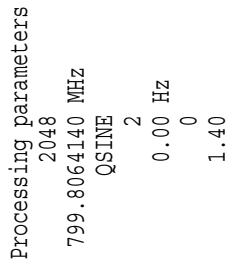

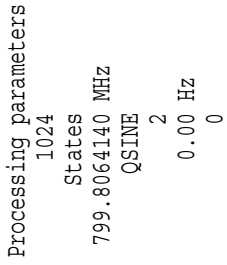

星
S. Levin and J. S. Nowick

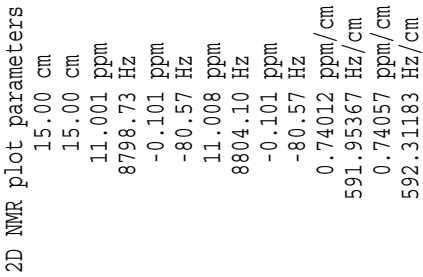

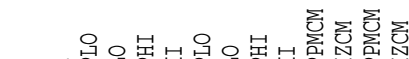

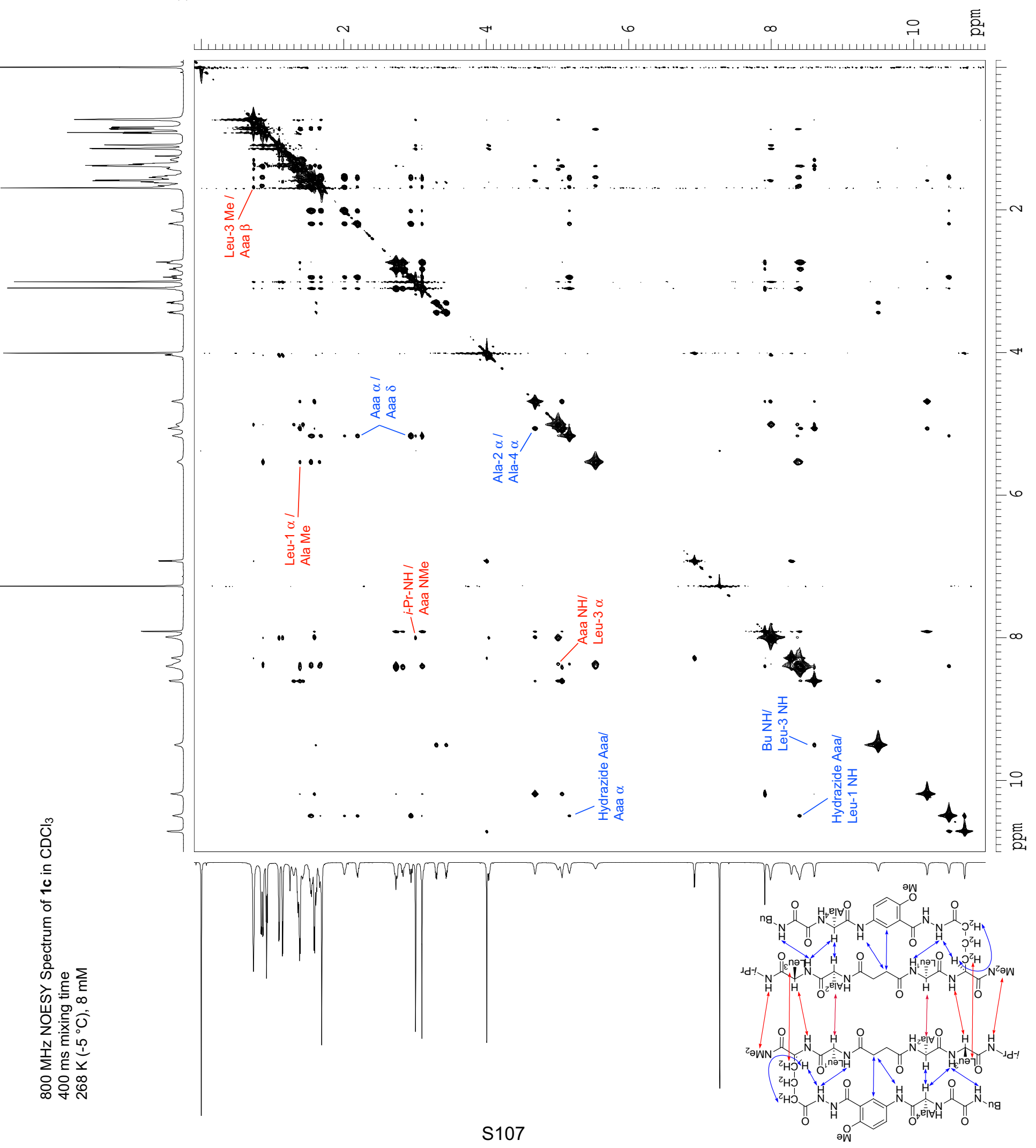


Supporting Information

S. Levin and J. S. Nowick
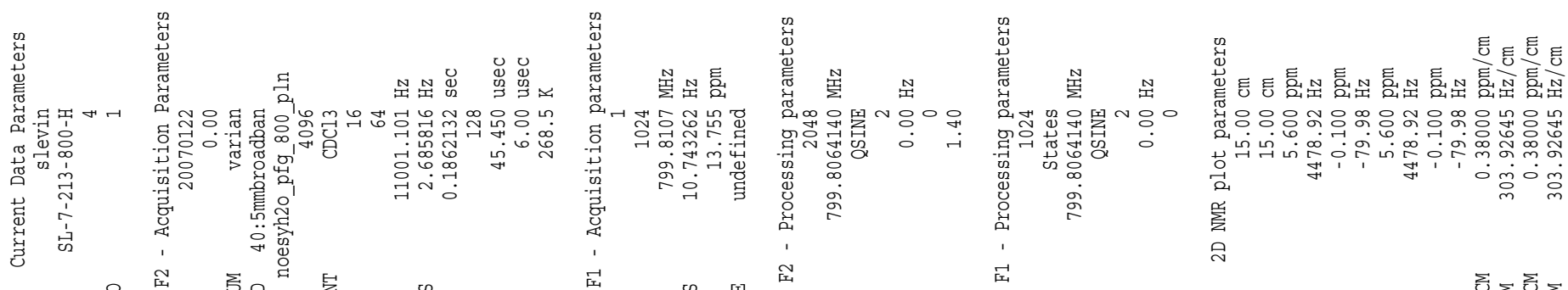

巤㟴总

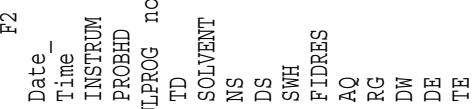

是罗

㞌

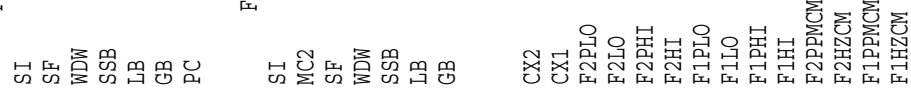
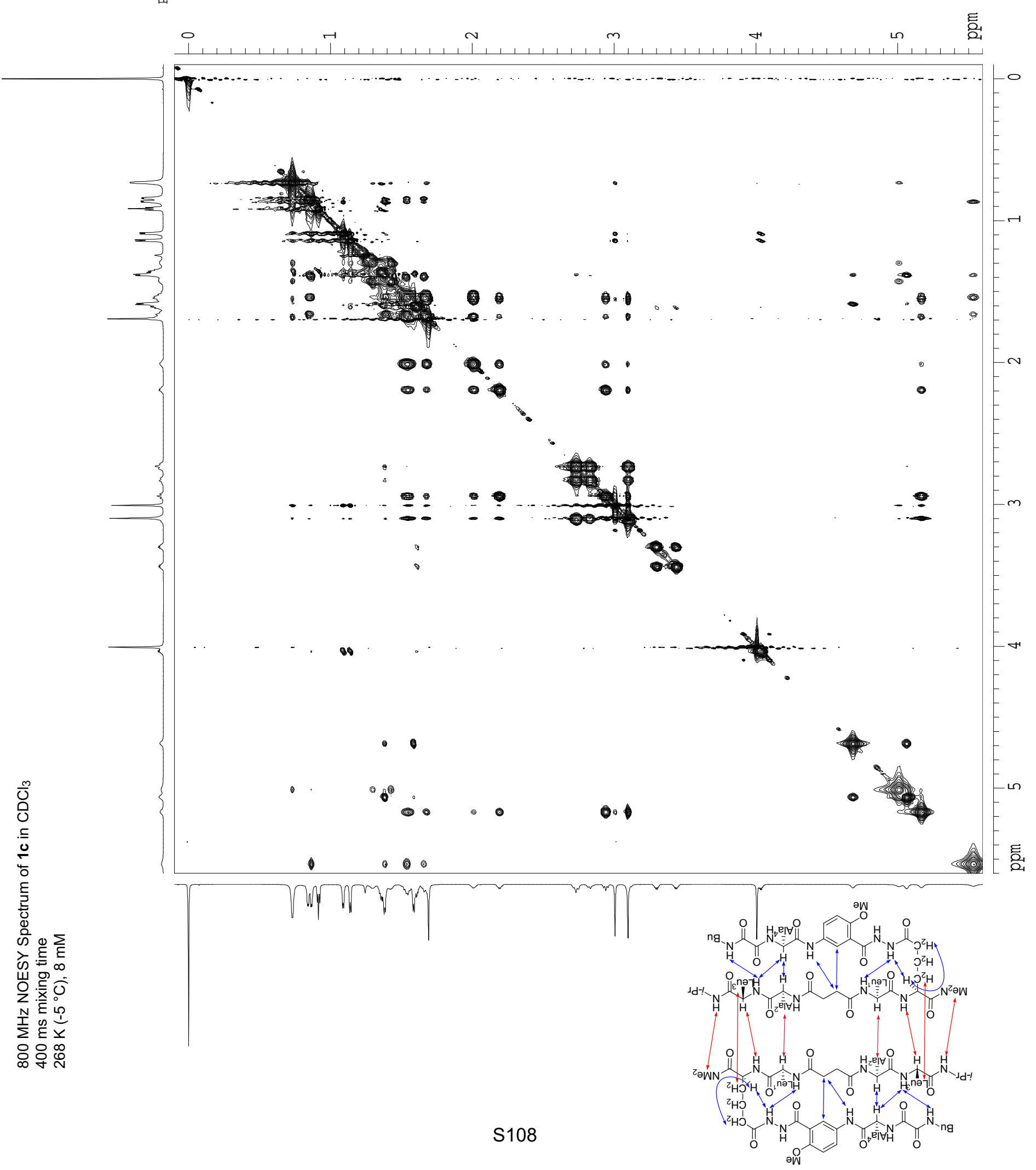


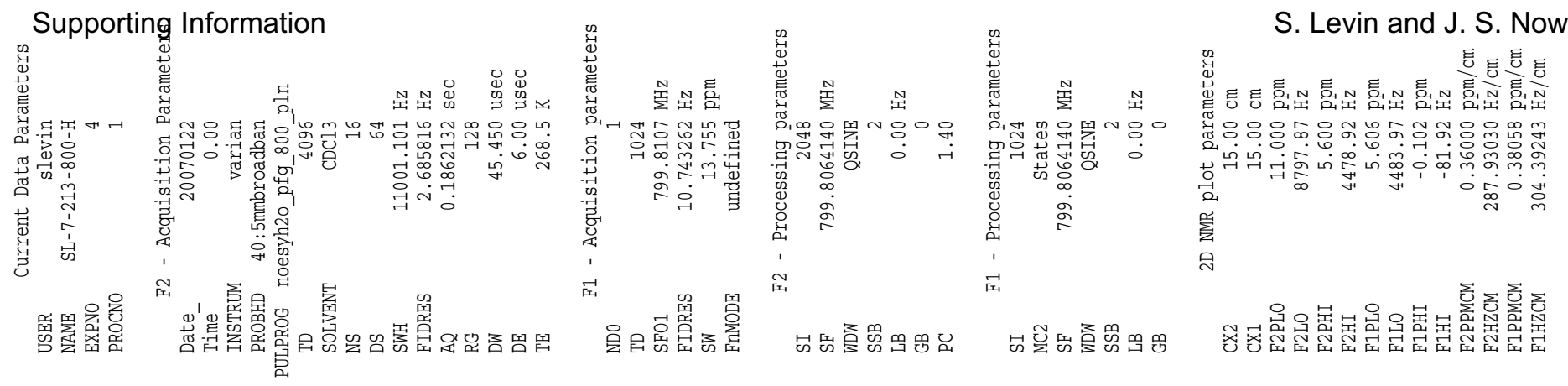

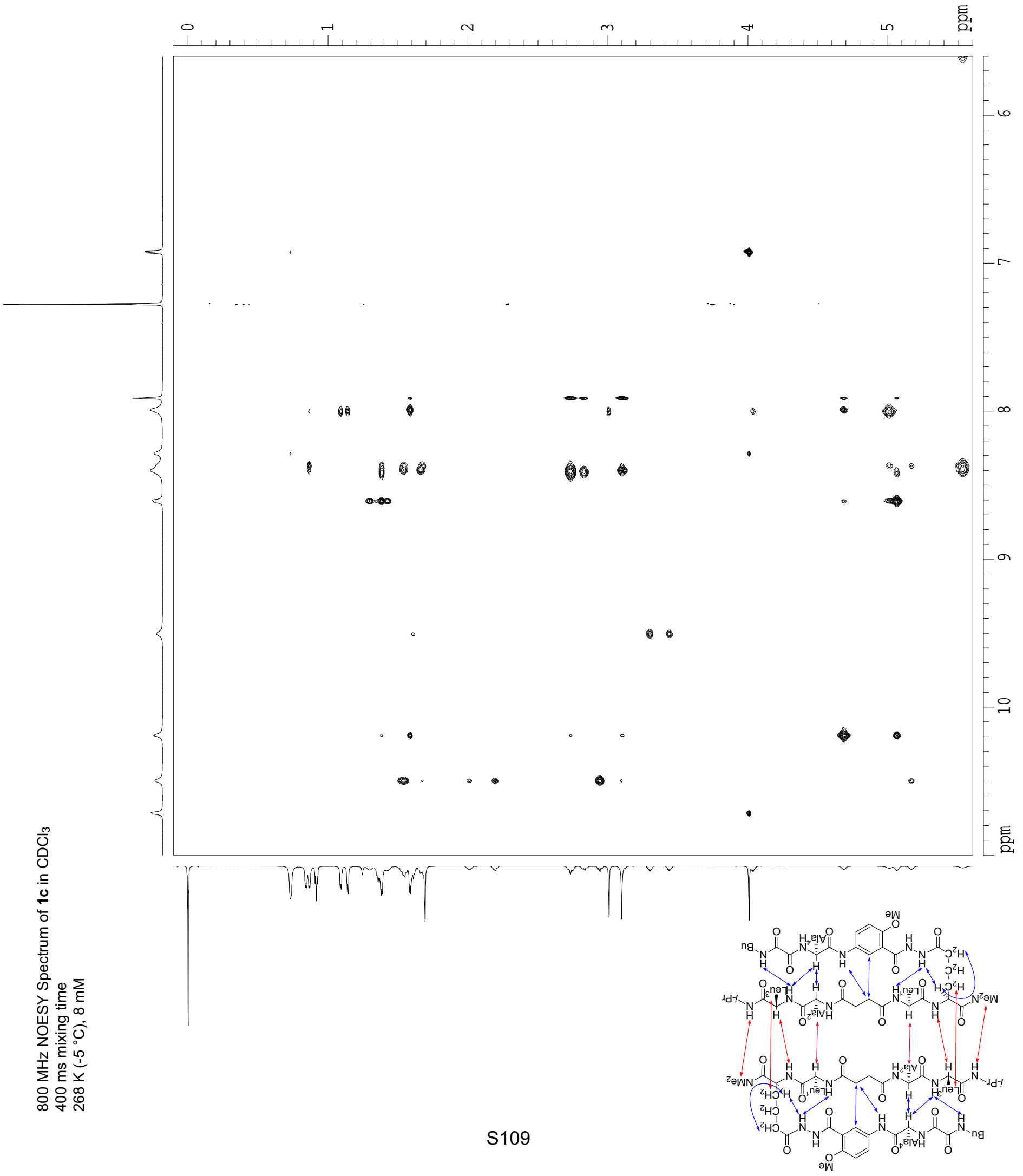


Supporting Information
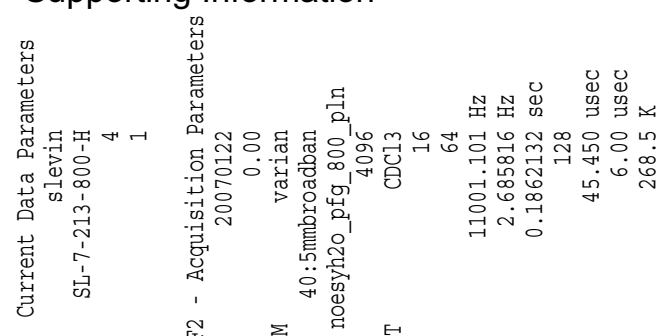

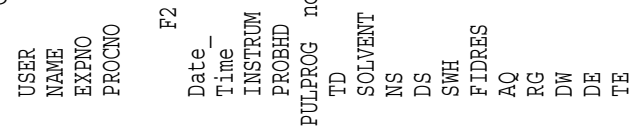
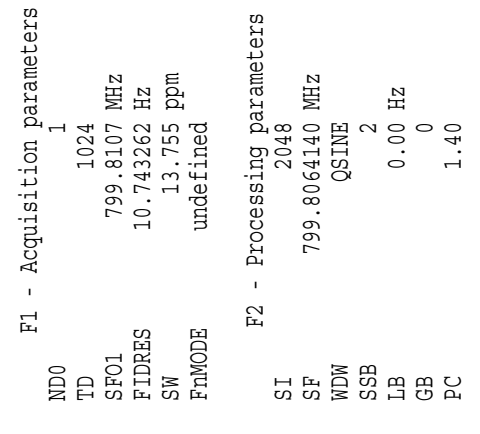

S. Levin and J. S. Nowick

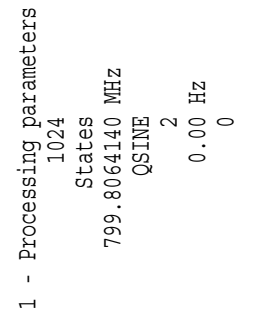

展

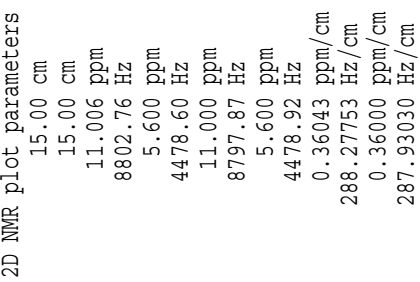

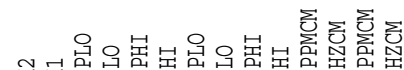

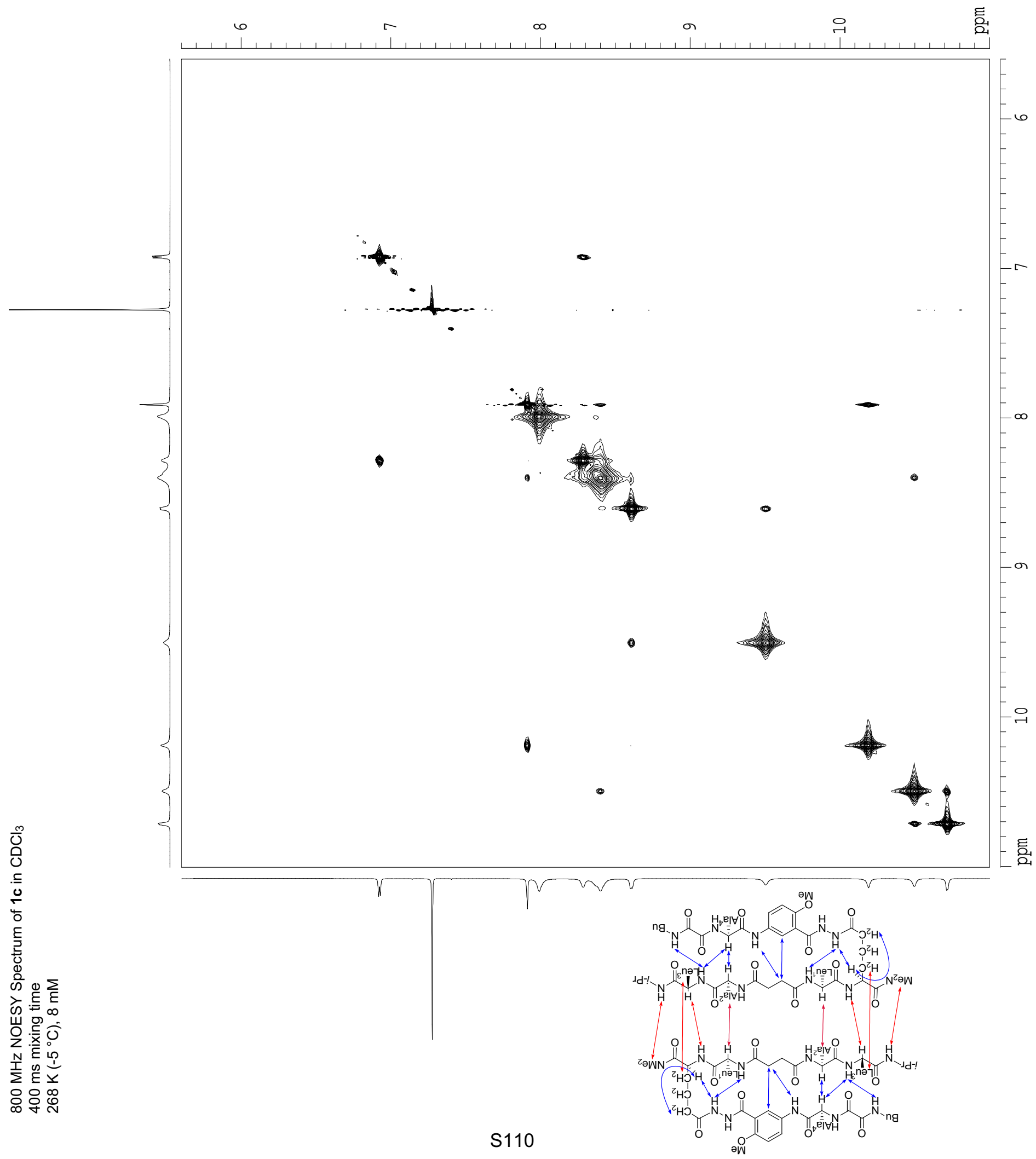




\section{Supporting Information}

F"

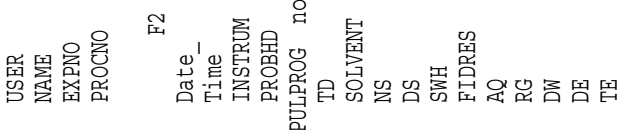

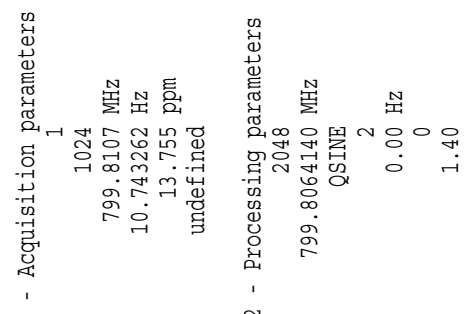

兵
S. Levin and J. S. Nowick

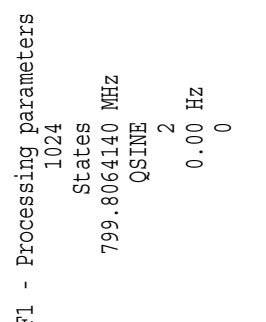
틀 छ ㅂ 嵌

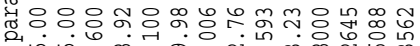

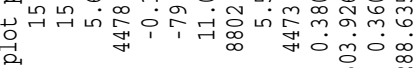
隹

学

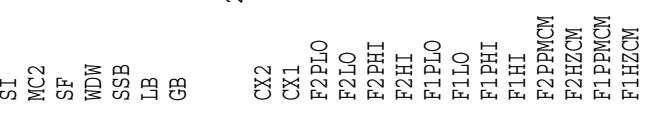


Supporting Information
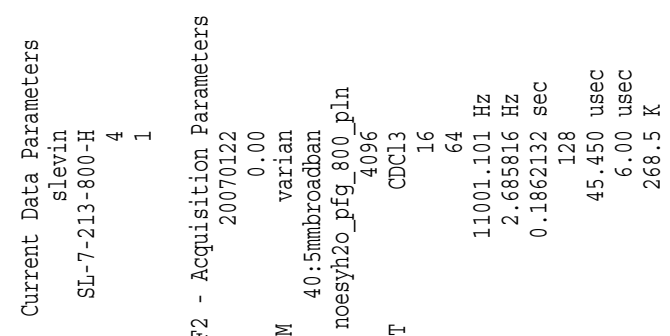

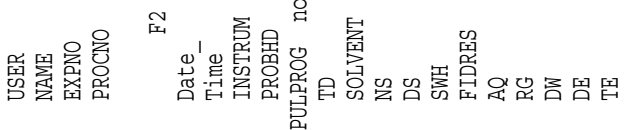

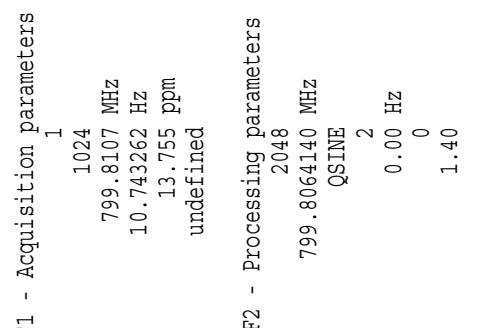

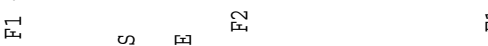

S. Levin and J. S. Nowick

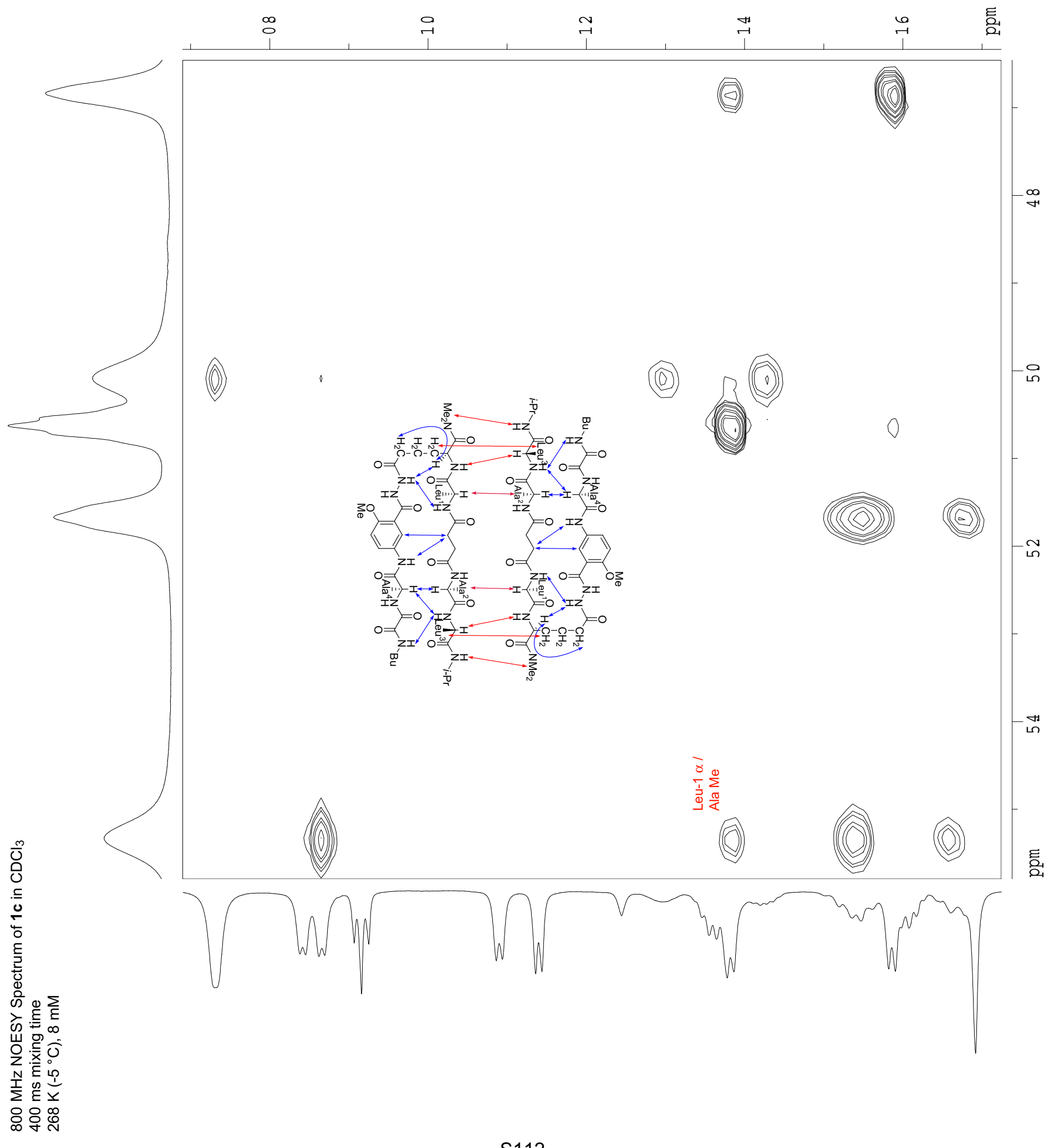


Supporting dnformation

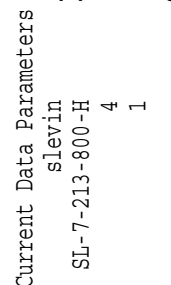

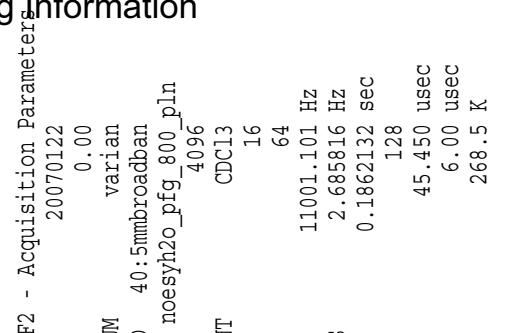

罳罢总总
«

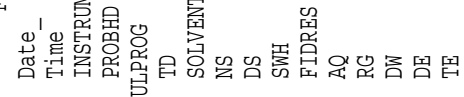

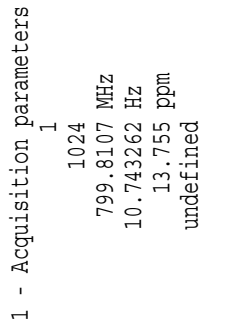

居牙畏

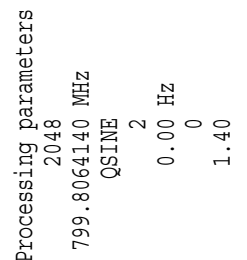

또

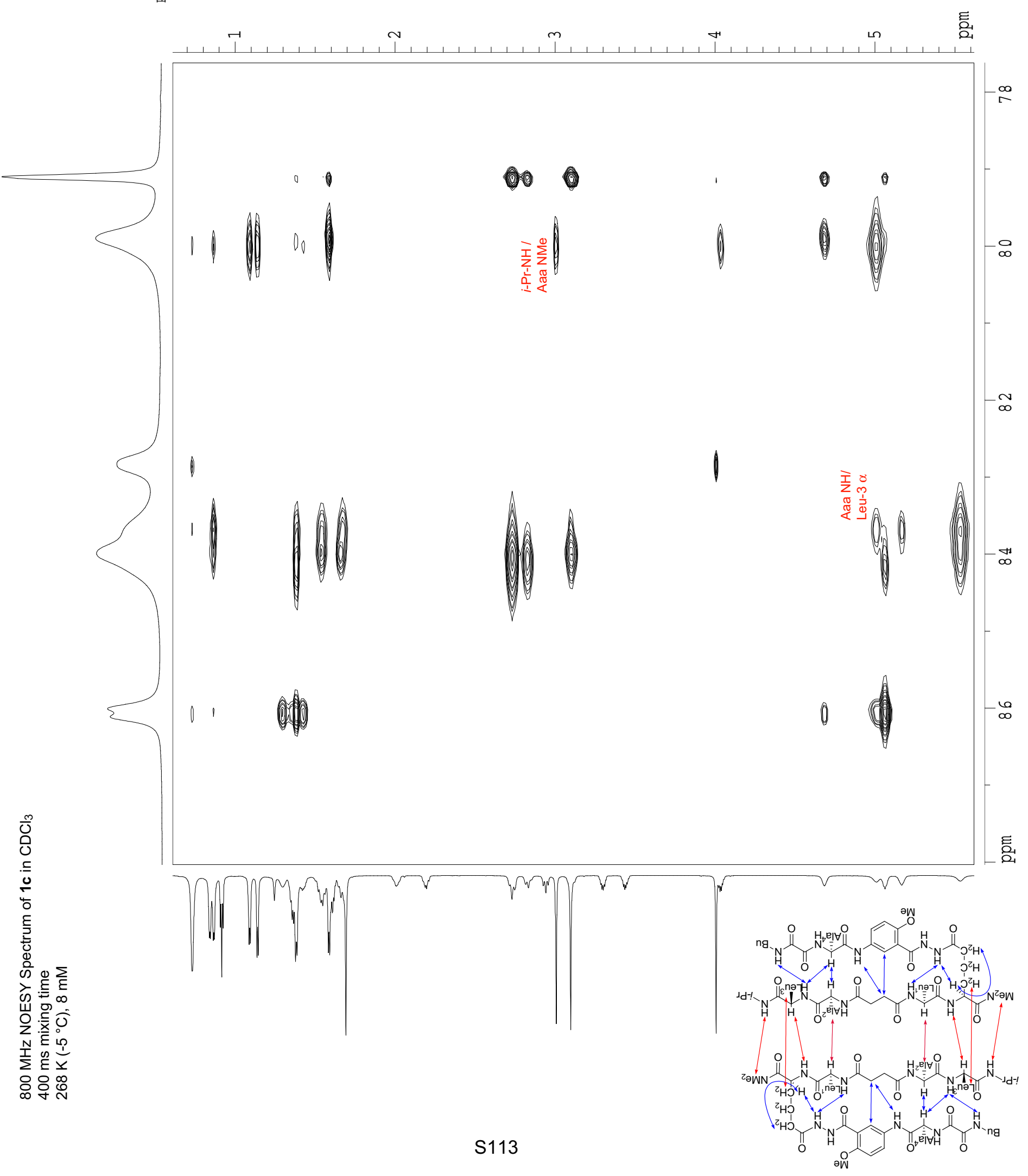


Supporting Information

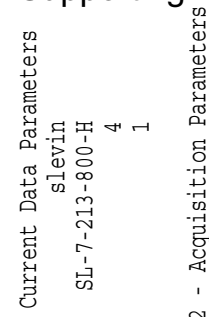

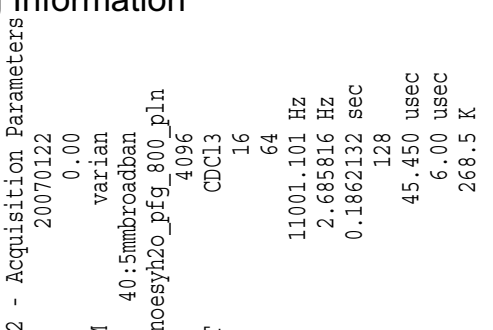

zats

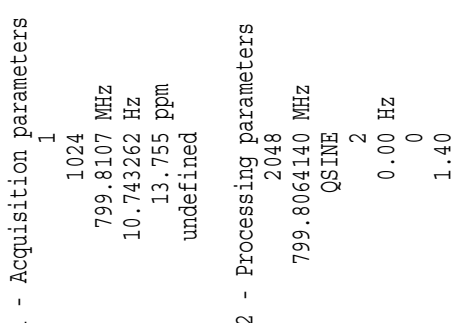

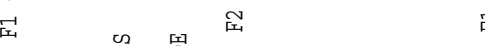

S. Levin and J. S. Nowick

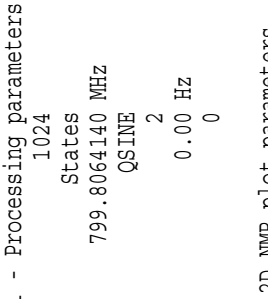

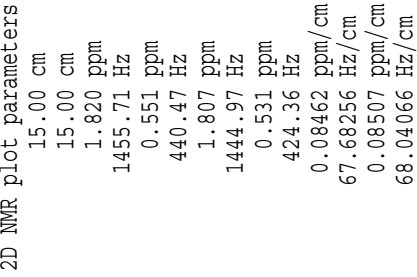

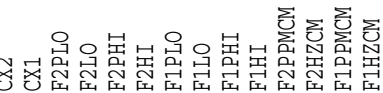

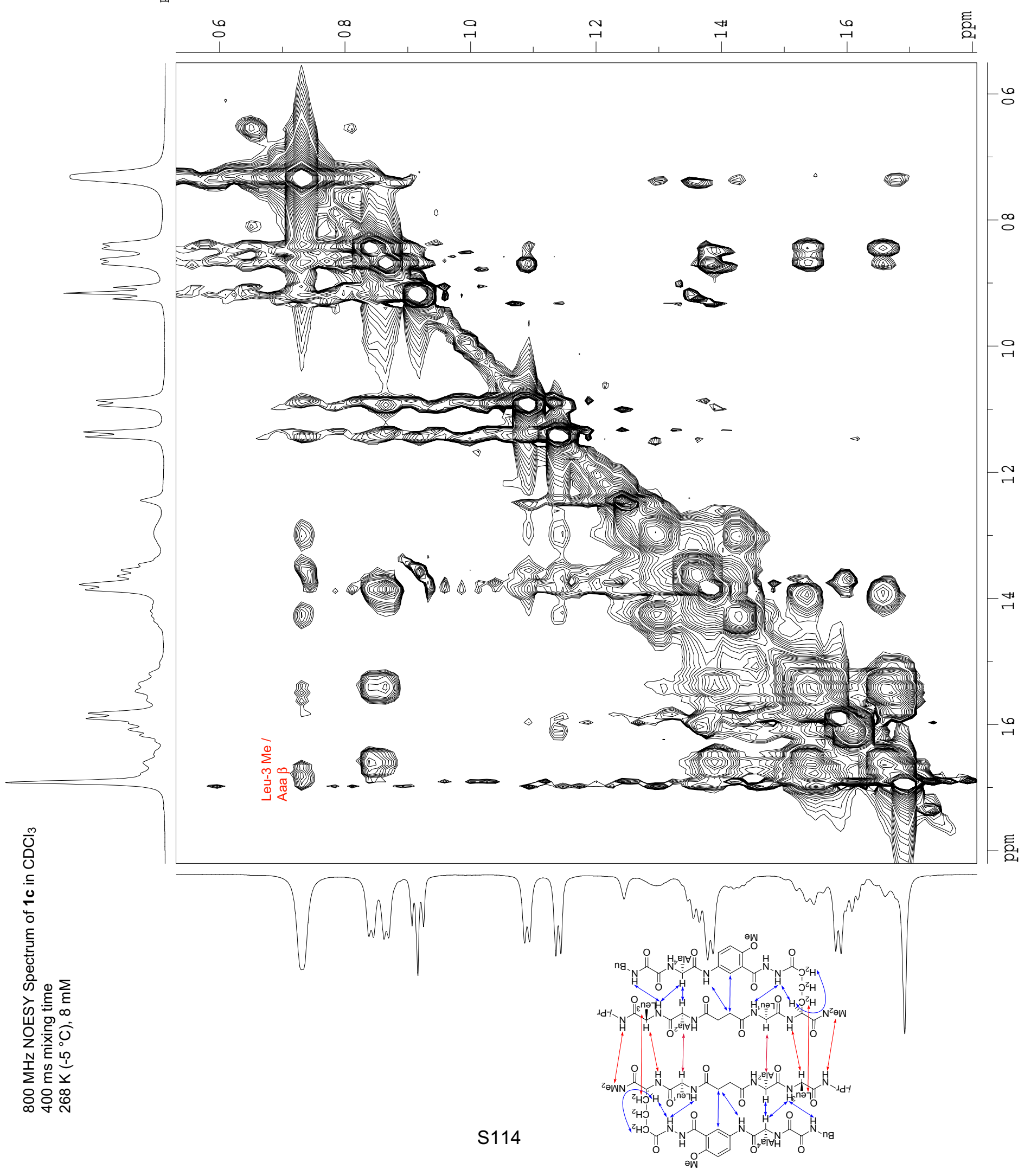




\section{Spectroscopic DATA for the compound 1d $\left(20 \mathrm{mM}, \mathrm{CDCl}_{3} 268 \mathrm{~K}\right)$}
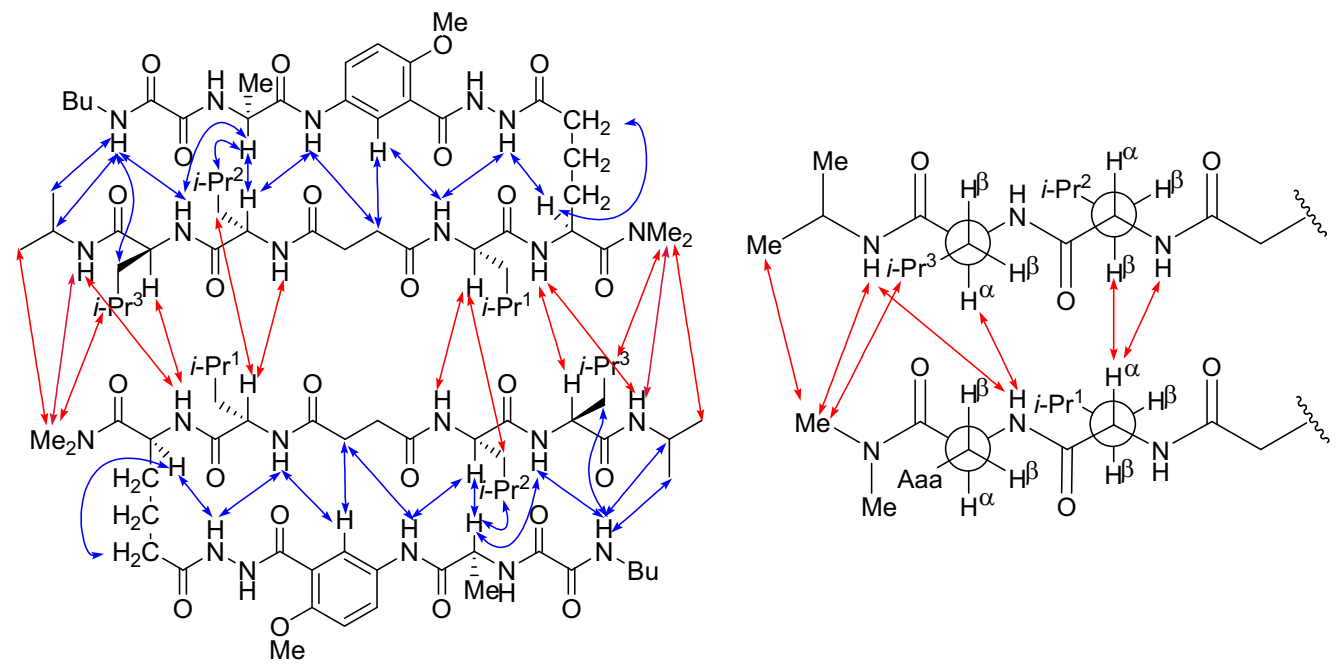

Key NOEs assigned as intermolecular are represented with red arrows

Key NOEs assigned as intramolecular are represented with blue arrows

Key data for amino acids.

\begin{tabular}{|c|c|c|c|}
\hline Amino acid & $\mathrm{H}_{\alpha}$ Shift ppm & NH Shift ppm & ${ }^{3} J_{\mathrm{NH} \alpha} \mathrm{Hz}$ \\
\hline Aaa & 5.16 & 8.67 & 8.7 \\
\hline $\mathrm{R}_{1}=\mathrm{Leu}^{1}$ & 5.54 & 8.39 & 8.0 \\
\hline $\mathrm{R}_{2}=\mathrm{Leu}^{2}$ & 4.94 & 8.31 & 9.2 \\
\hline $\mathrm{R}_{3}=\mathrm{Leu}^{3}$ & 4.97 & 8.69 & 10.0 \\
\hline $\mathrm{R}_{4}=\mathrm{Ala}$ & 4.79 & 7.98 & 7.4 \\
\hline
\end{tabular}

Aminoadipic acid (Aaa) $\delta$ proton resonances.

pro-R: 2.91 ppm, t, (overlap)

pro-S: $2.19 \mathrm{ppm}, \mathrm{td},\left(J_{1}=11.5 \mathrm{~Hz} ; J_{2}=7.0 \mathrm{~Hz}\right)$

anisotropy $=0.72 \mathrm{ppm}$

Key data for other NH protons.

\begin{tabular}{|c|c|c|}
\hline Proton & NH Shift ppm & $J \mathrm{~Hz}$ \\
\hline Ar-NH & 10.20 & - \\
\hline Hydrazide Ar & 10.57 & 7.4 \\
\hline Hydrazide Aaa & 10.43 & 7.4 \\
\hline Bu-NH & 9.62 & broad \\
\hline$i$-Pr-NH & 8.03 & 7.4 \\
\hline
\end{tabular}

ESI MS $m / z$ for $\mathrm{C}_{50} \mathrm{H}_{84} \mathrm{~N}_{11} \mathrm{O}_{12}[\mathrm{M}+\mathrm{H}]^{+}$calcd 1030.63, found 1030.50. 


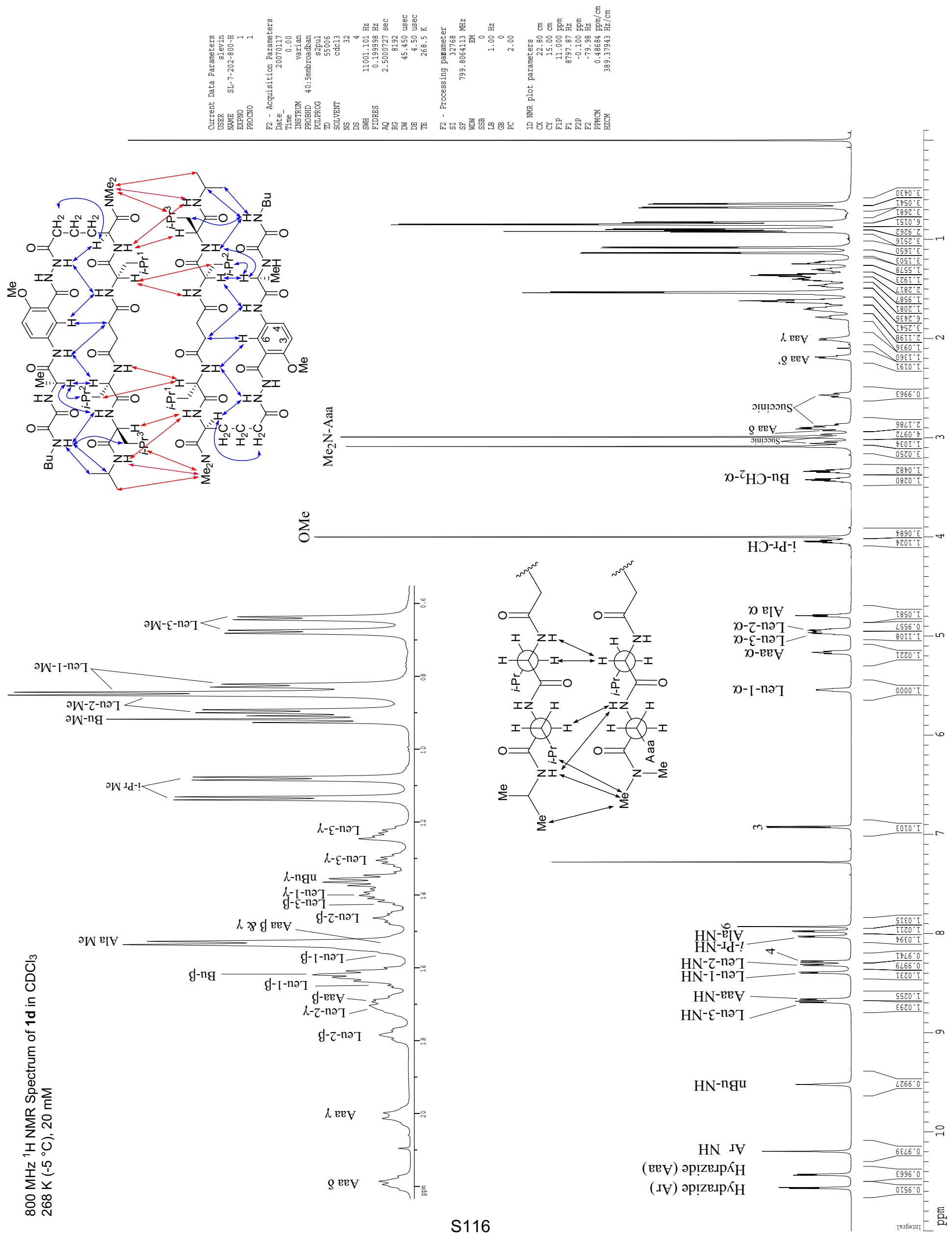



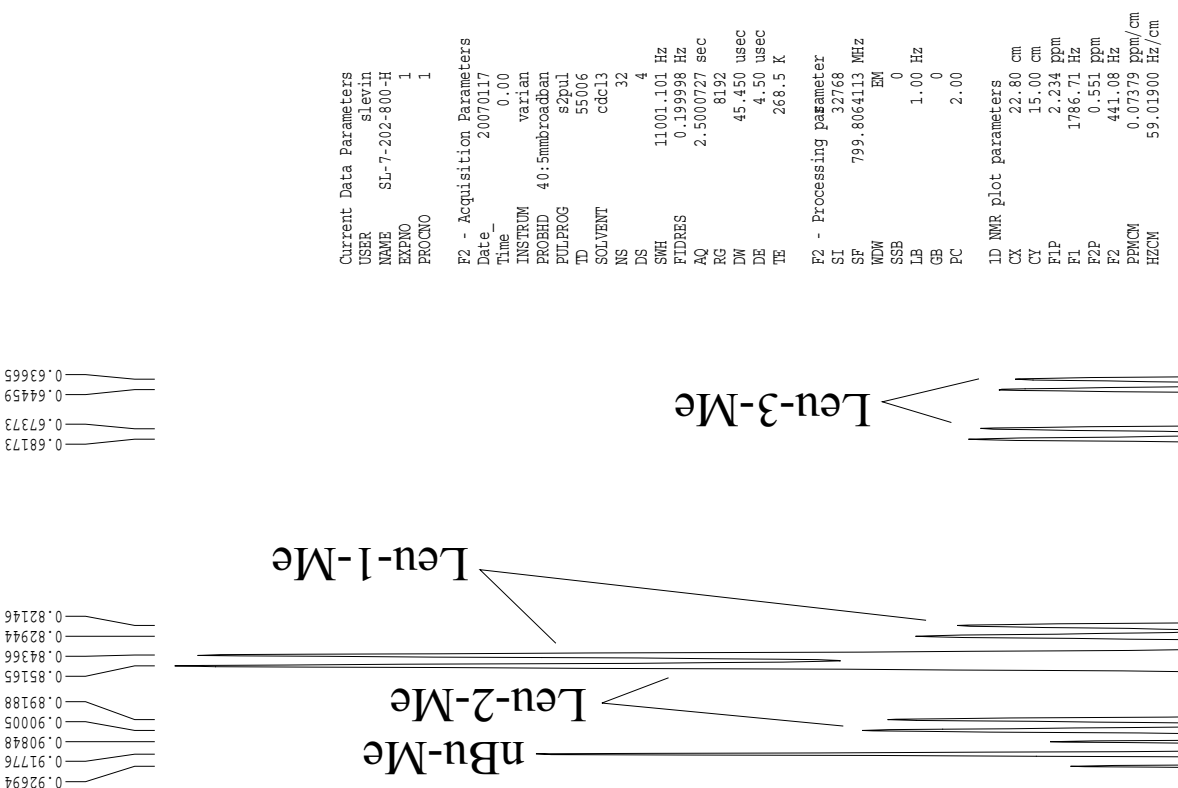

วW-I-nəา

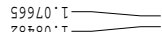

ธ60हТ.

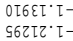

LOZZz' $\mathrm{t}$

$070 \varepsilon Z^{\prime} \cdot \mathrm{T}$
$088 \varepsilon \tau^{\circ} \cdot \mathrm{T}$

$\mp 997 Z^{\circ}$
$7068 Z^{\circ}$
$60 L 6 Z^{\circ}$

6TSOE.

o

LTLE⿱一⿻上丨-

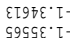

วTS9 '

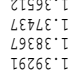

$\tau 626 \varepsilon^{\circ} \tau$

$\angle 960 \sigma^{\circ} \cdot \mathrm{t}$

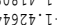

$\varepsilon 8977^{\circ} \tau$

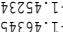

$\angle \angle F \angle F^{\circ} \tau$

$92087^{\circ} \mathrm{T}$

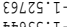

$50009 \cdot$.

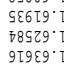

$.87879^{\circ}$

$80799^{\circ} \cdot \mathrm{T}$

$878 \angle 9^{\circ}$.
$\varepsilon 0889^{\circ}$.

วระ69. โ

ऽ५ร०L' $\mathrm{T}$

6EL9L'T-

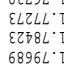

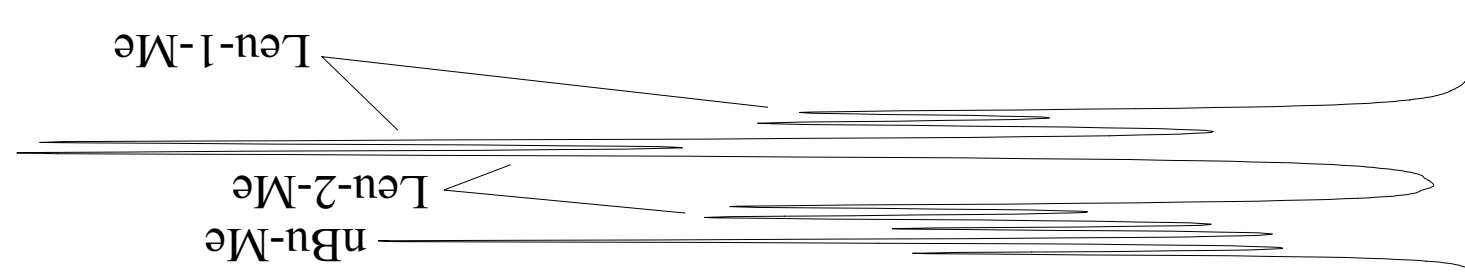

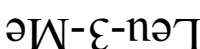

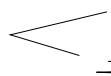

$\bar{\square}$

วW-て-nวา

əW- $\varepsilon-n \partial$

N-ngu

$\angle 2866^{\circ} \cdot \mathrm{T}$

LLETO' 2

l exF

วW $\mathcal{Q}$ IV

วW $\mathrm{I}^{-!}$

$\ell-\varepsilon-n \partial T$

l $\not \mathcal{G E V}$

$\ell-\varepsilon-n ə 7$
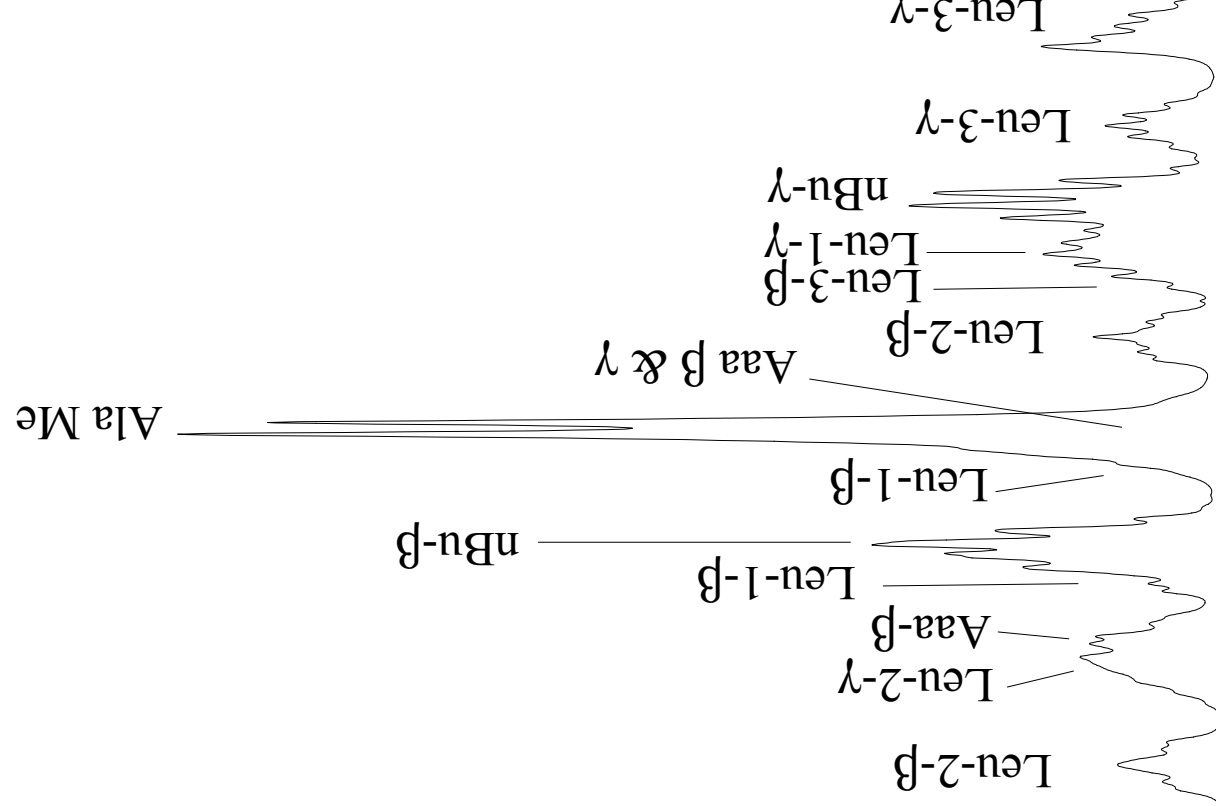

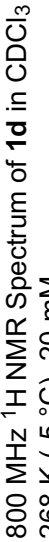

$\sum_{\varepsilon}$
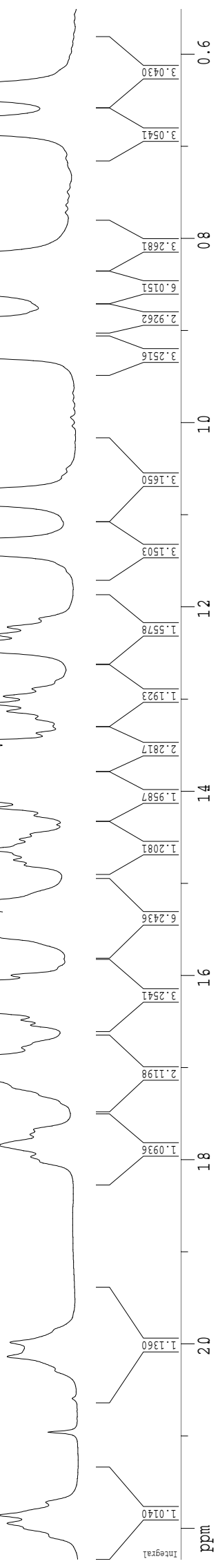


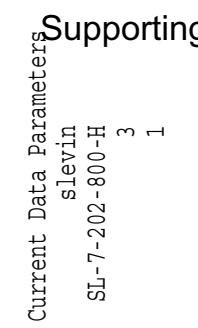

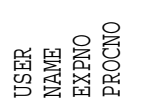

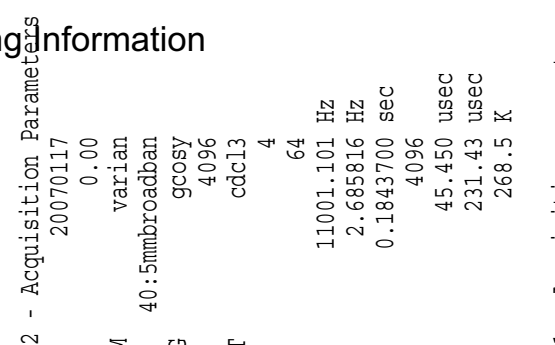
${ }^{\circ}$

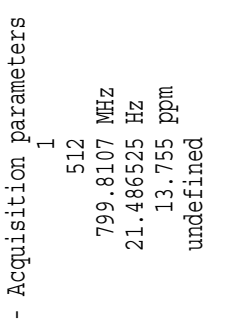

a

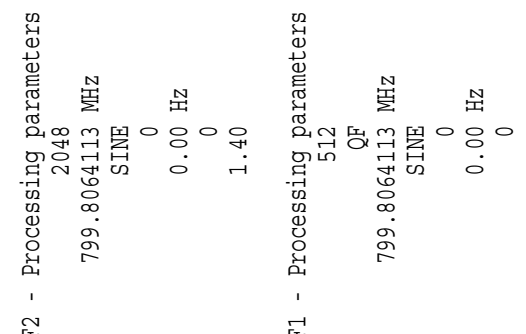

荘

S. Levin and J. S. Nowick 离 ॠ

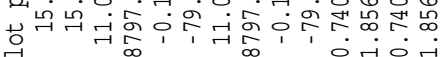
哭

ล

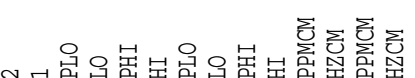

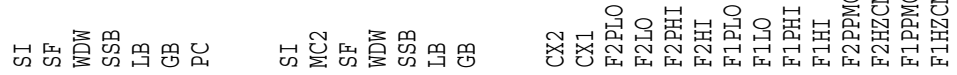

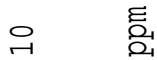

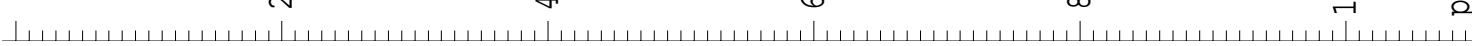

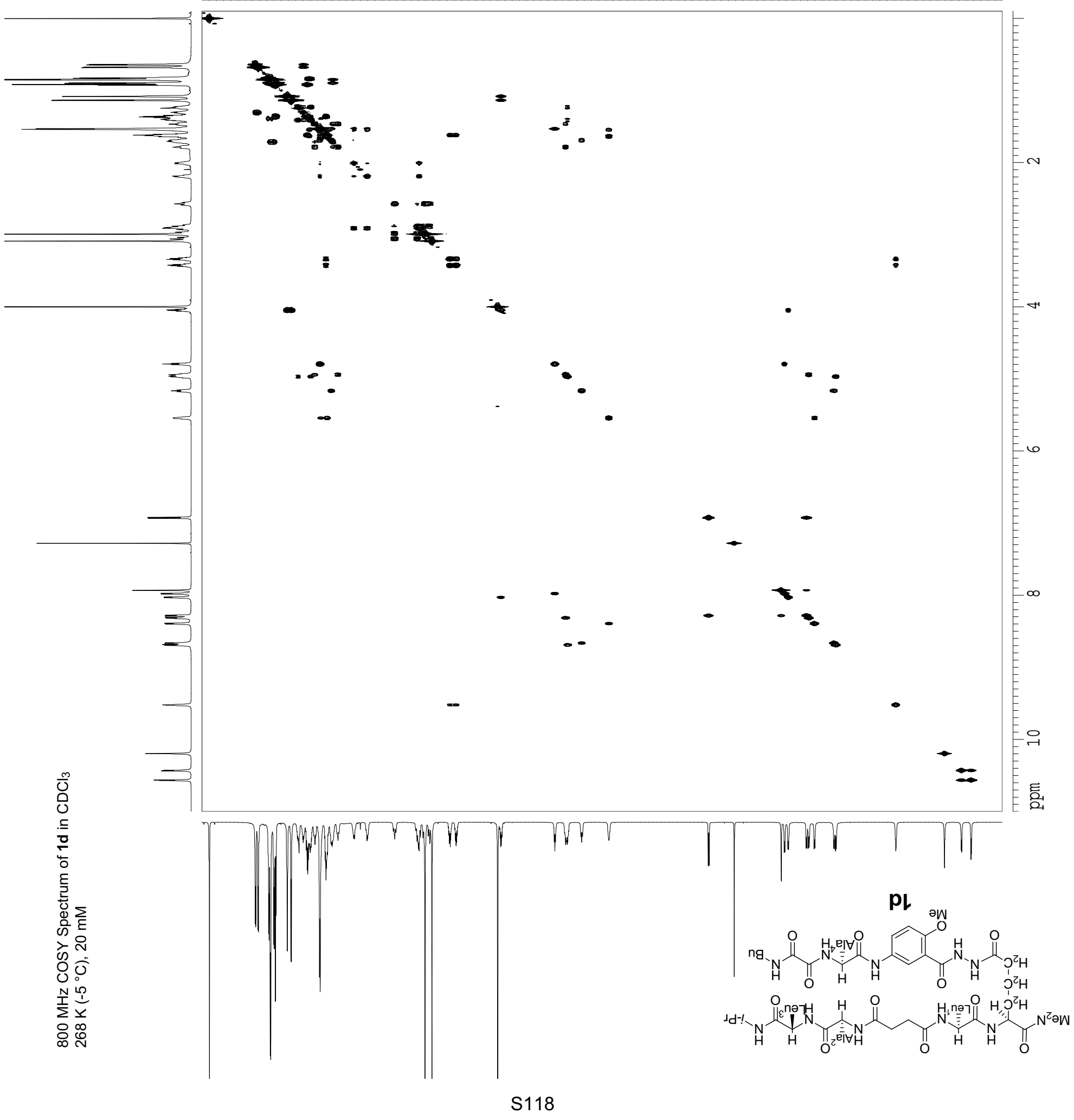


Supporting Information
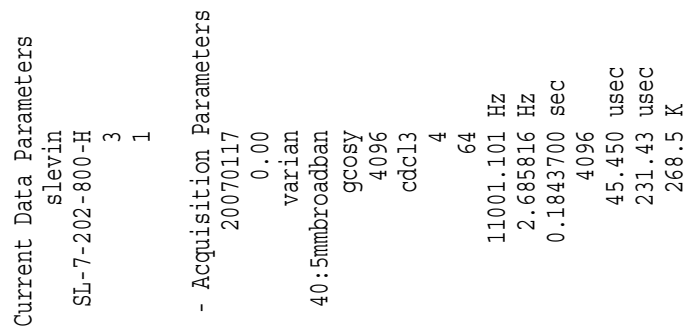

品䜿㥙
我

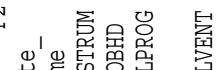

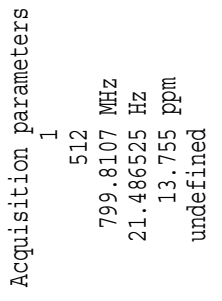

\section{$\vec{r}$}

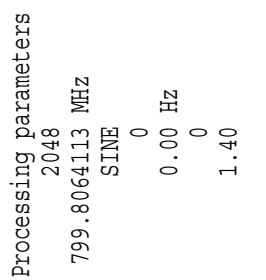

또

(2)

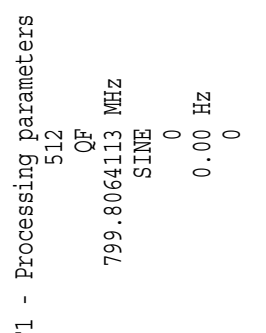

空
S. Levin and J. S. Nowick

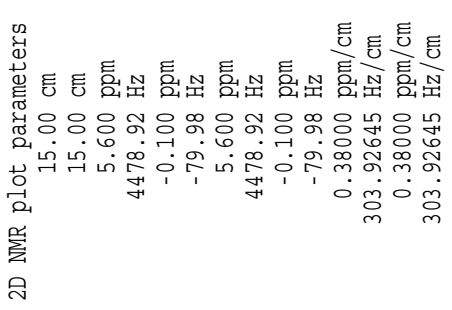

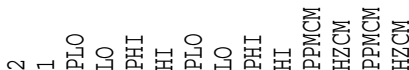

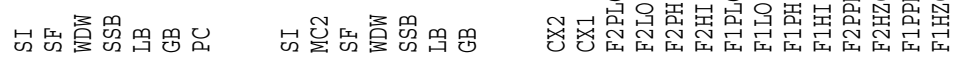

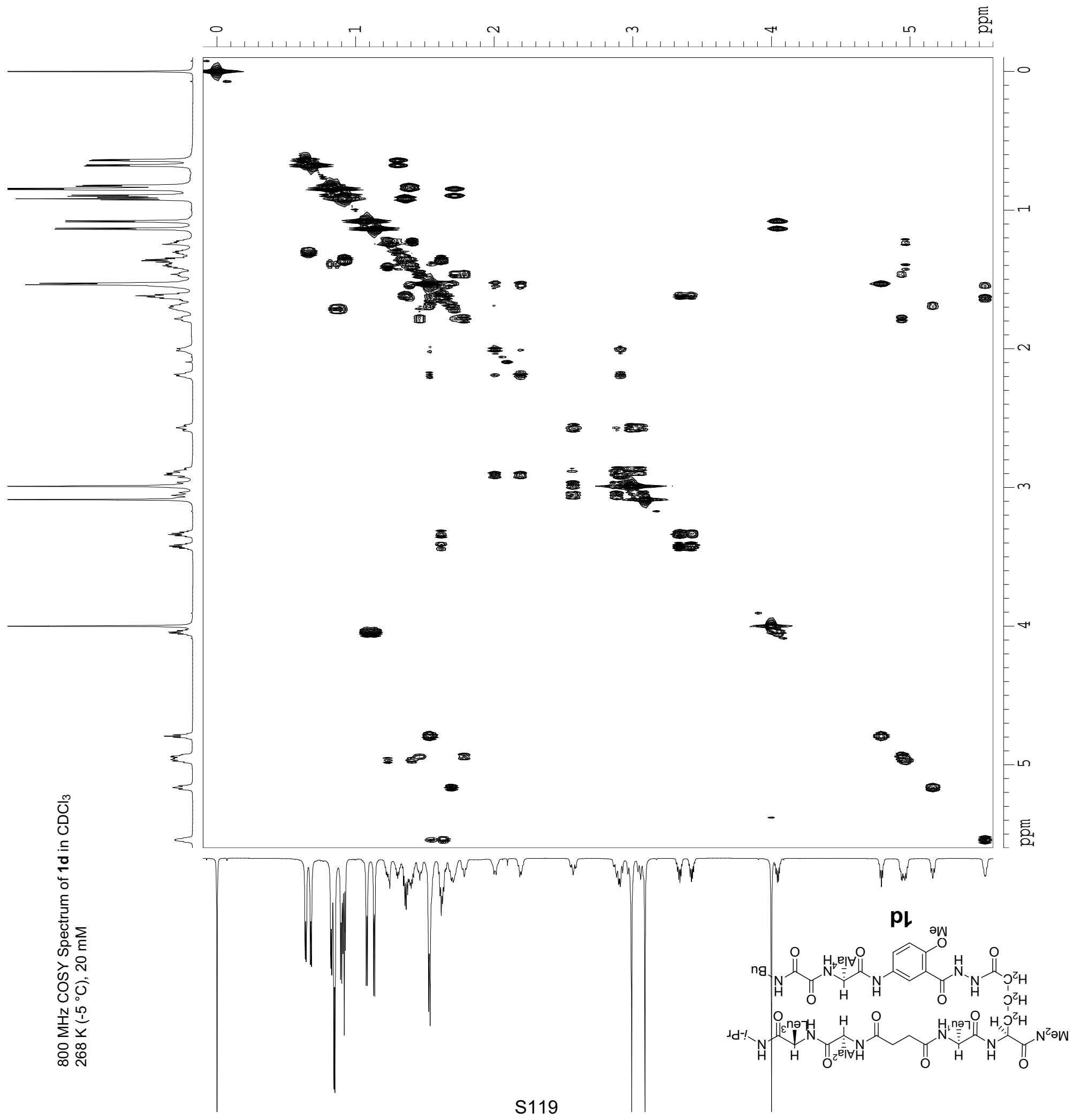




\section{Supporting Information}
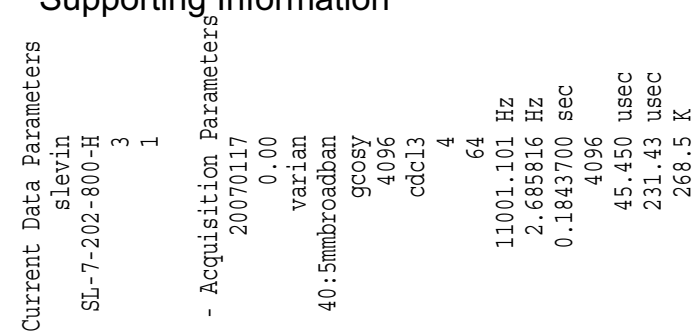

舆䁬总总
质

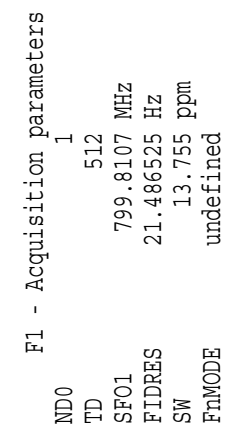

S. Levin and J. S. Nowick

造

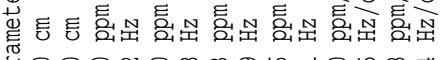

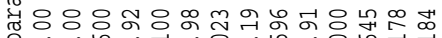

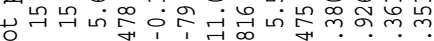
紊 平采

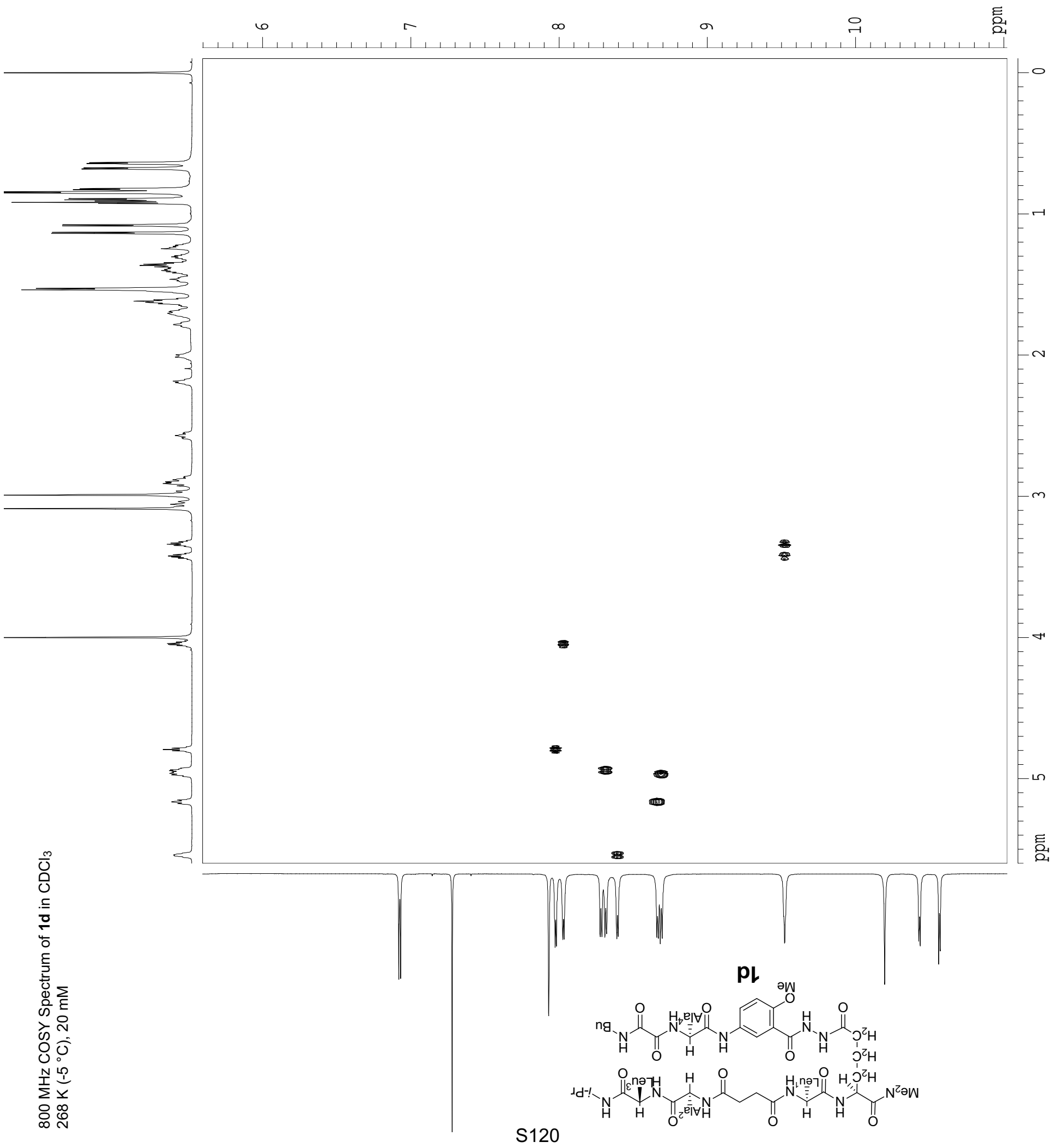




\section{Supporting Information}
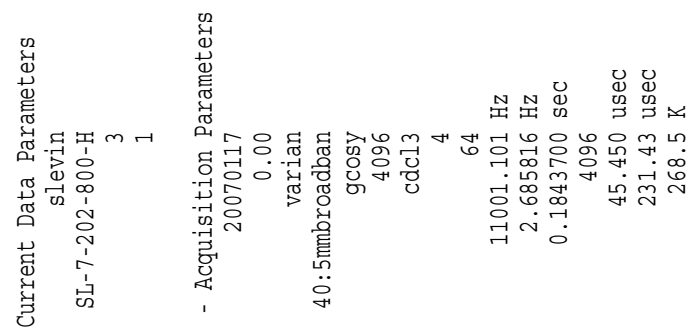

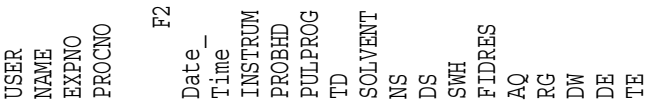

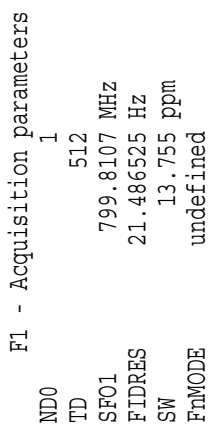

S. Levin and J. S. Nowick

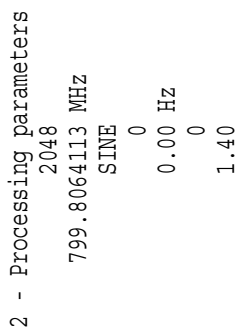

社

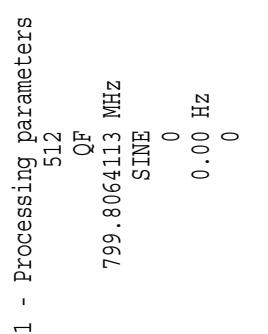

空
总

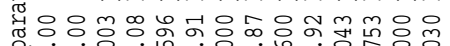
मे a ลิ

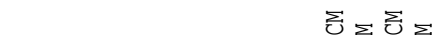

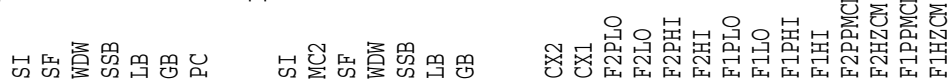

हू,

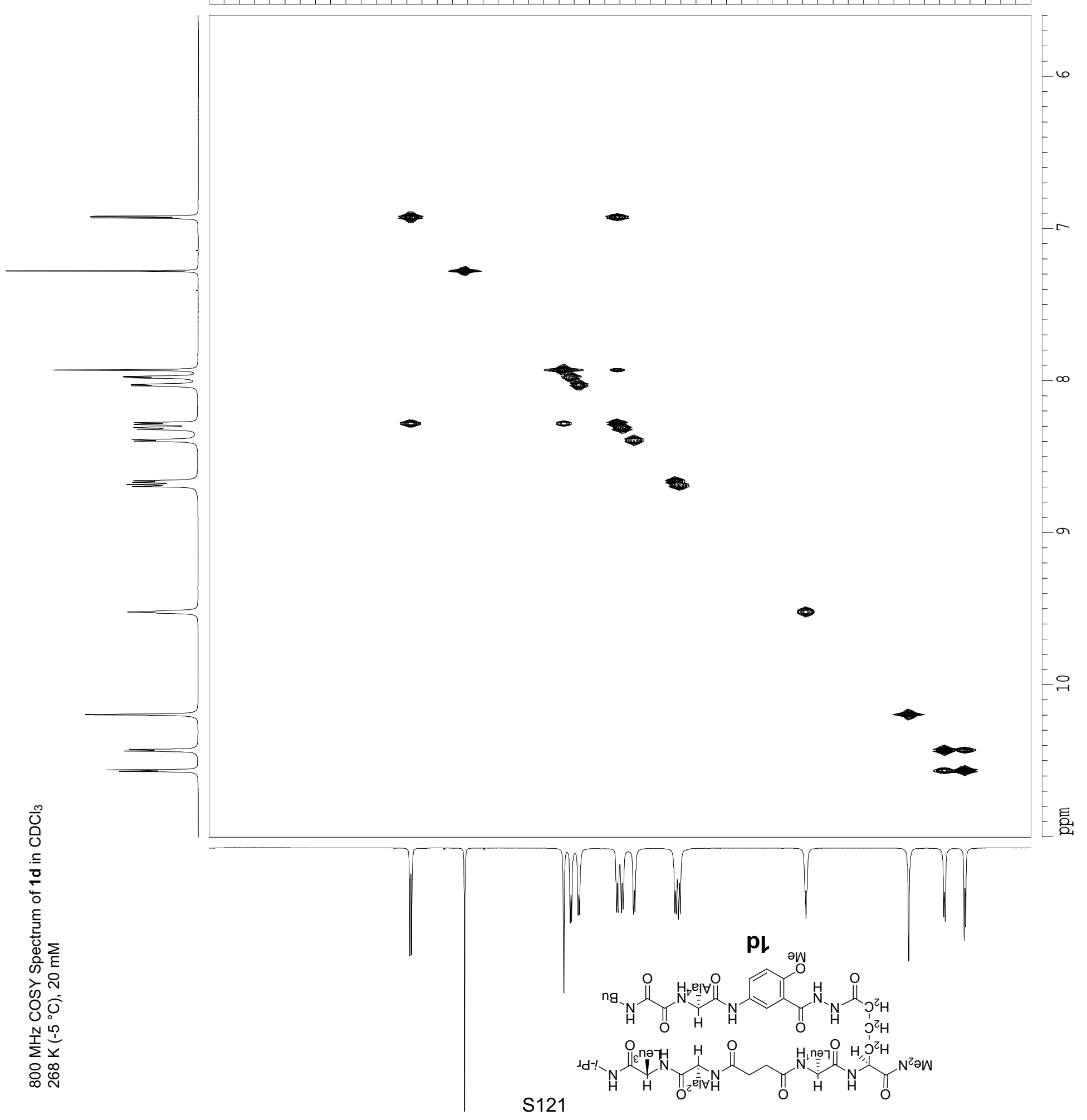



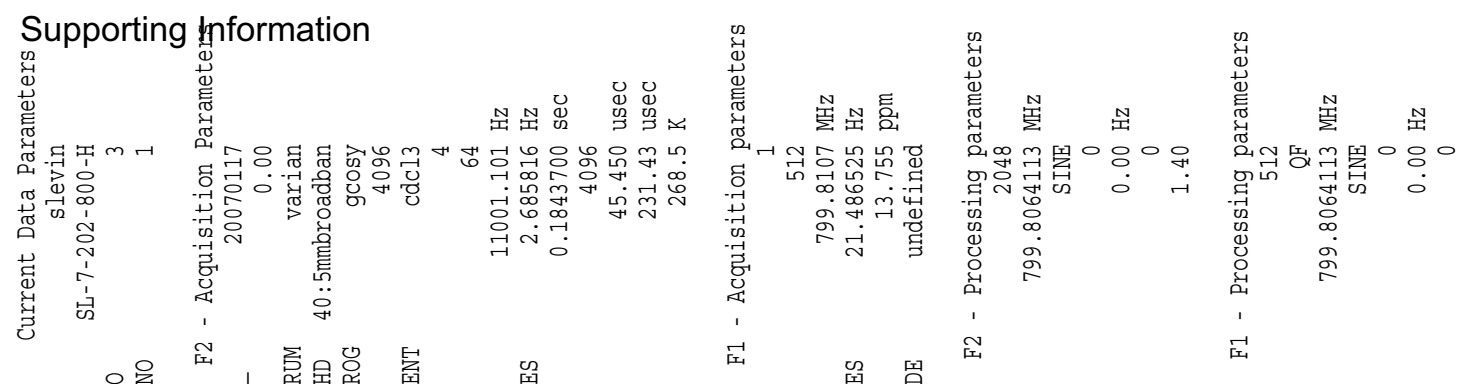

S. Levin and J. S. Nowick

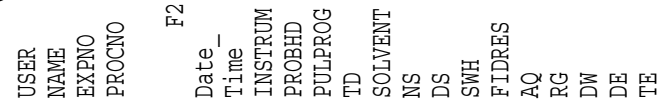

㞋

画

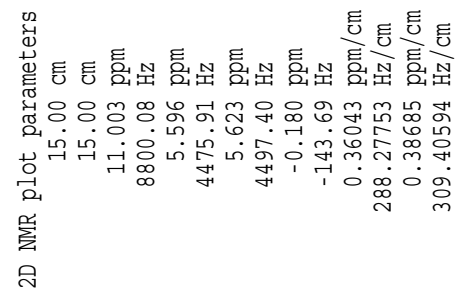

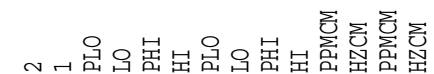

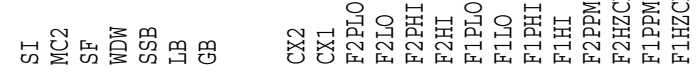

हू유.

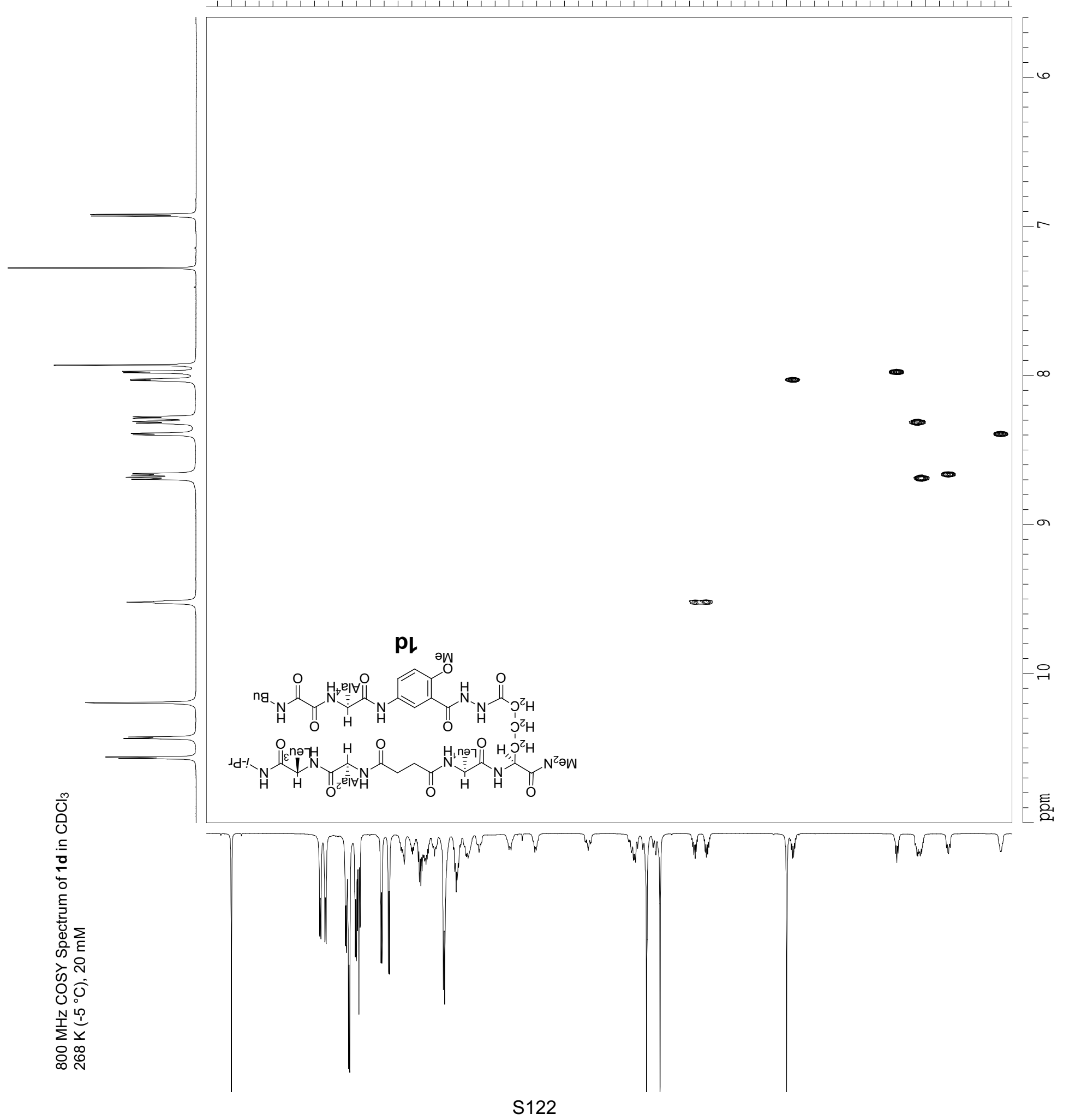




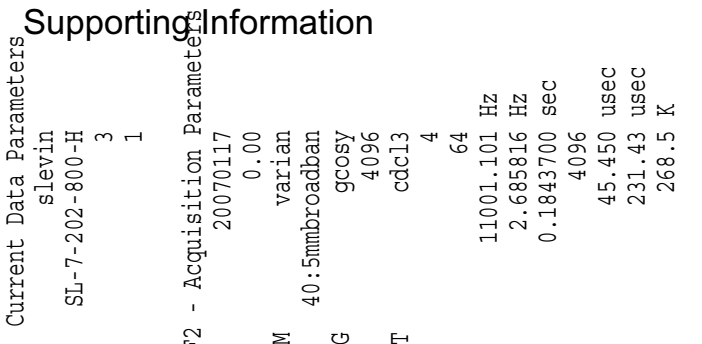

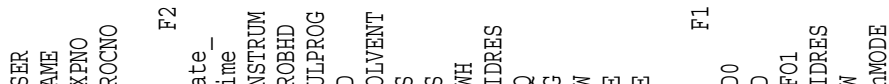

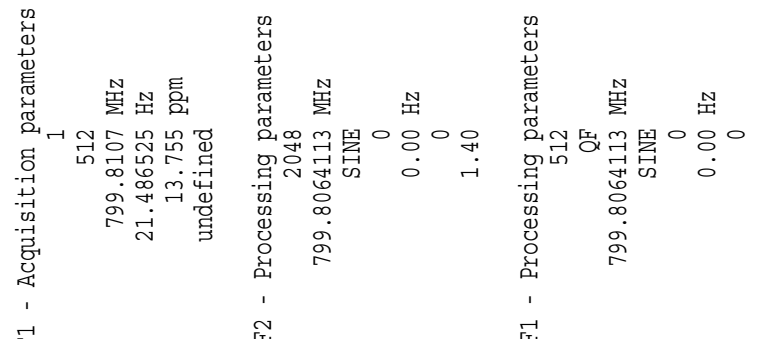

S. Levin and J. S. Nowick

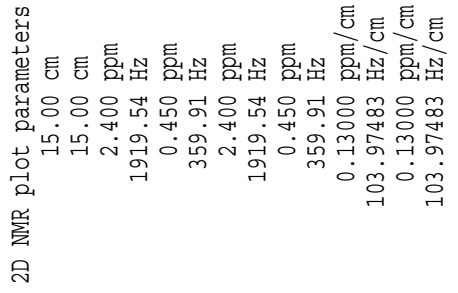

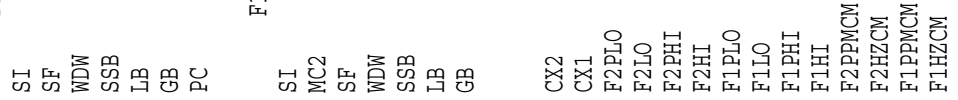

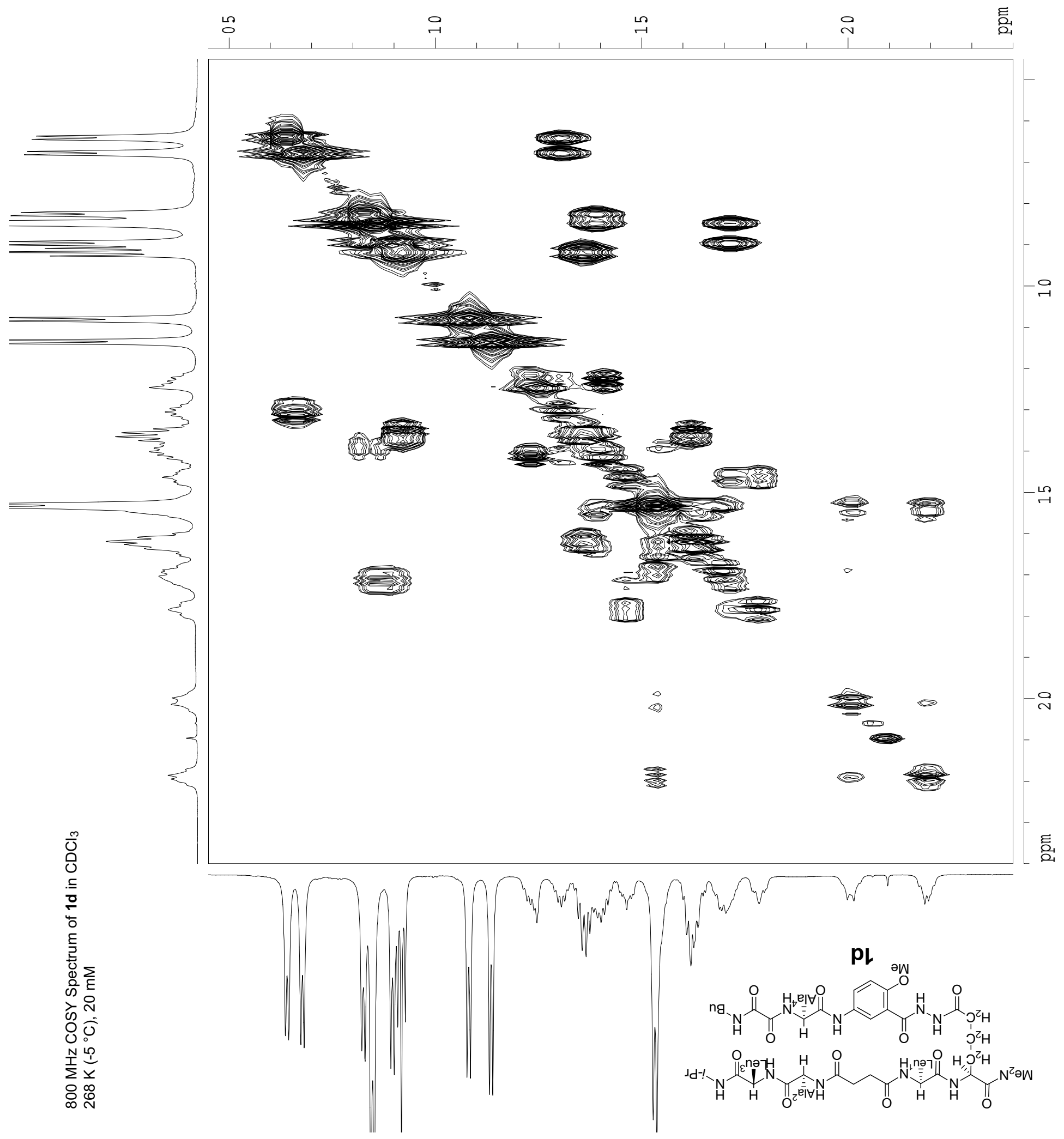



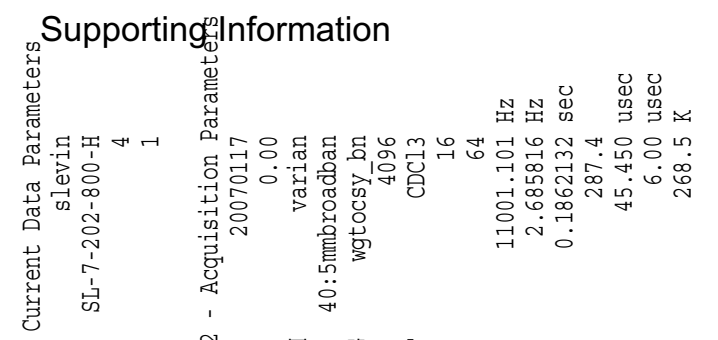

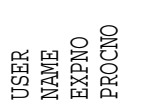

\section{厌}

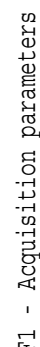

空

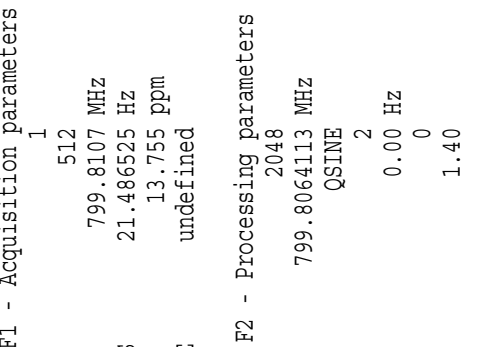

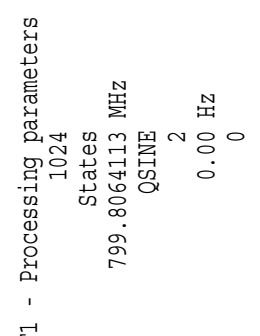

S. Levin and J. S. Nowick 氙

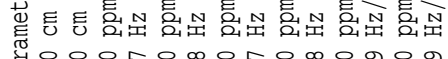

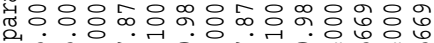

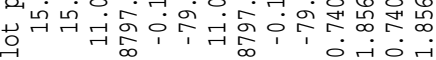
罢

금

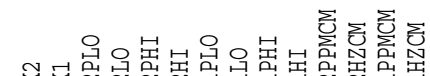

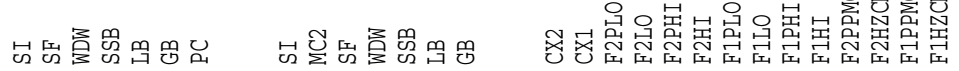

$\infty \quad$ 글 है

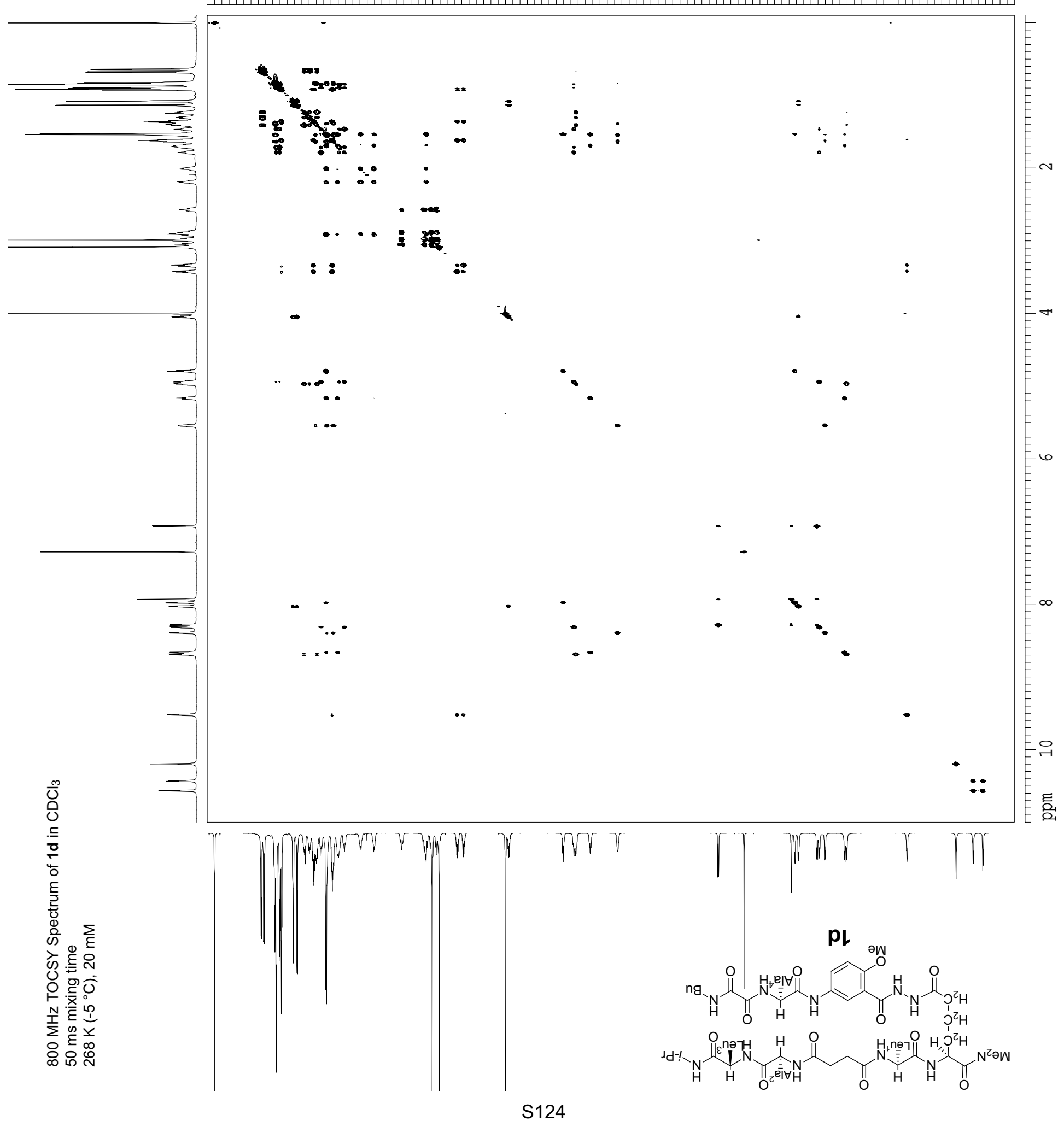


Supporting Information
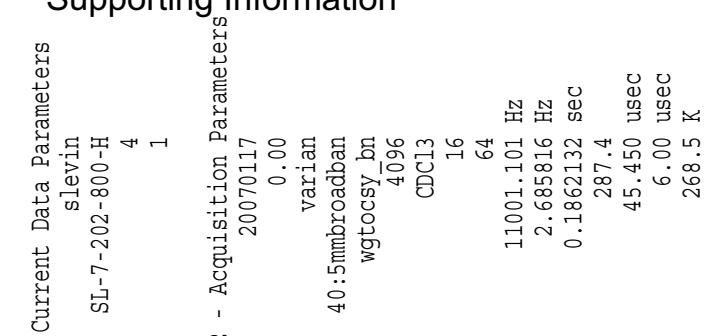

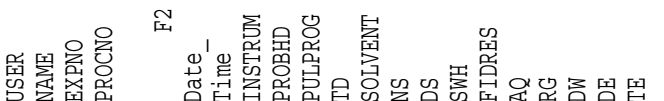

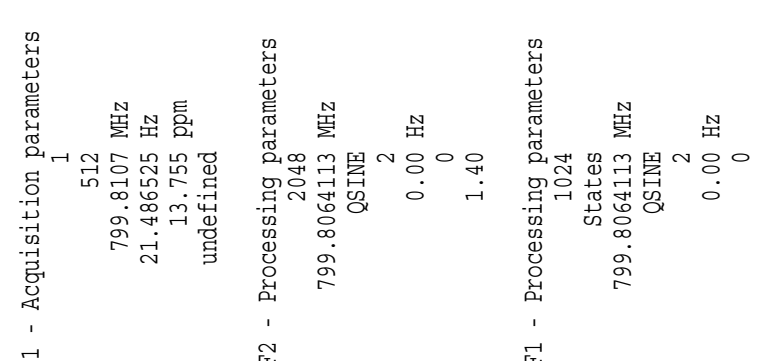

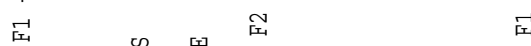

S. Levin and J. S. Nowick

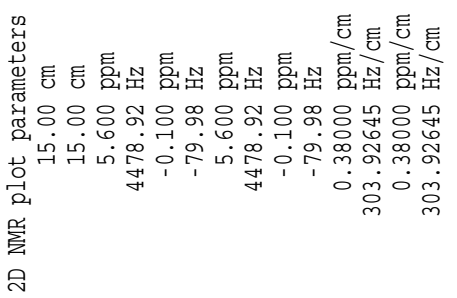

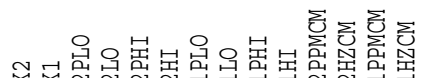

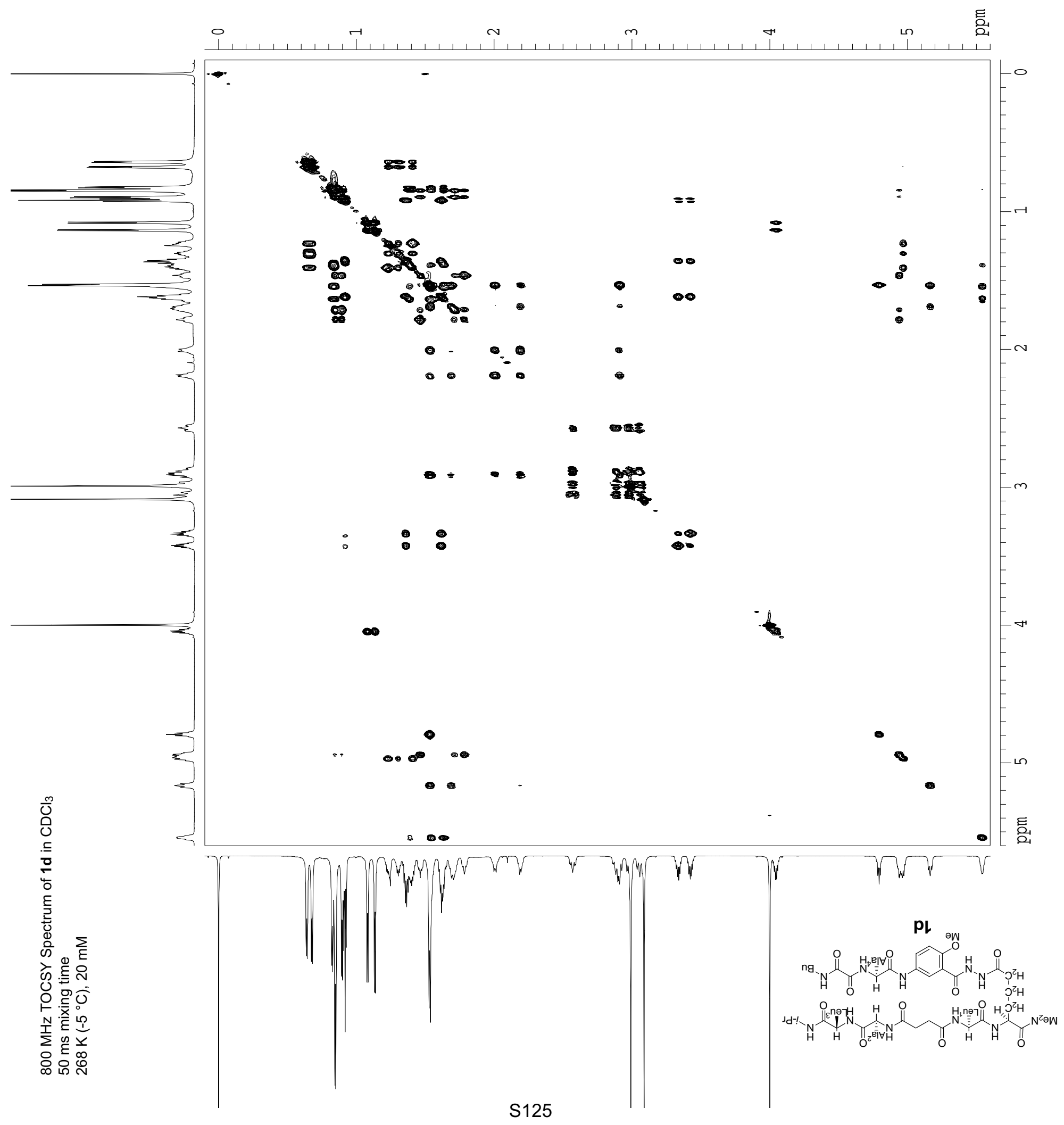




\section{Supporting Information}
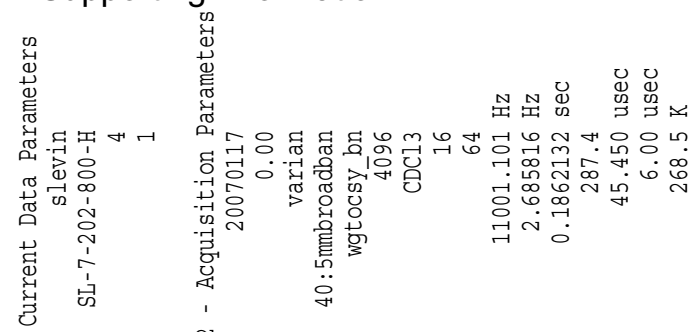

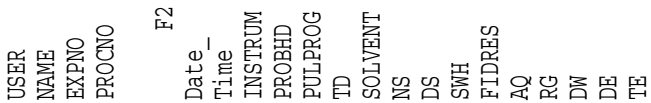

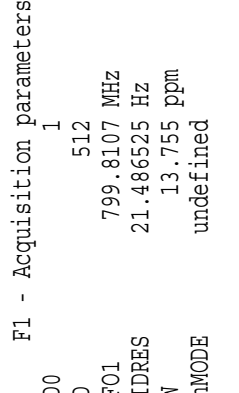

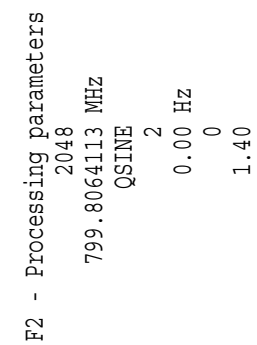

S. Levin and J. S. Nowick

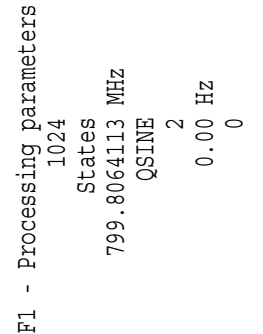

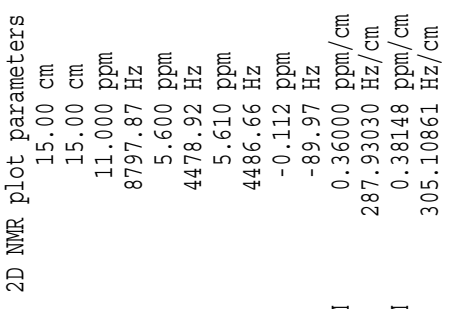

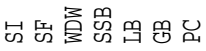

画

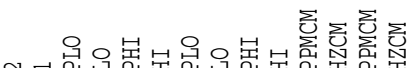

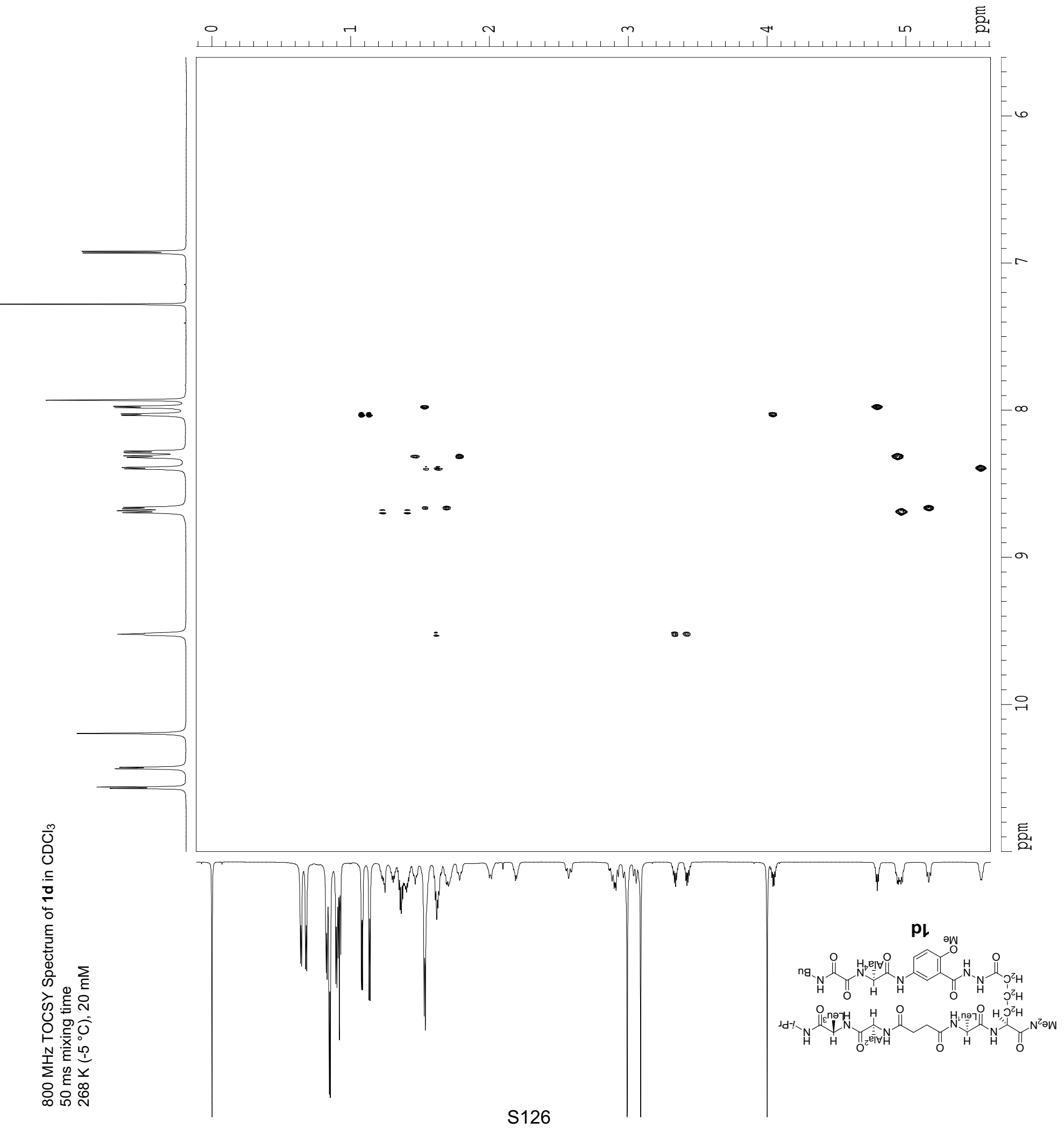

ڤ్ 


\section{Supporting Information}
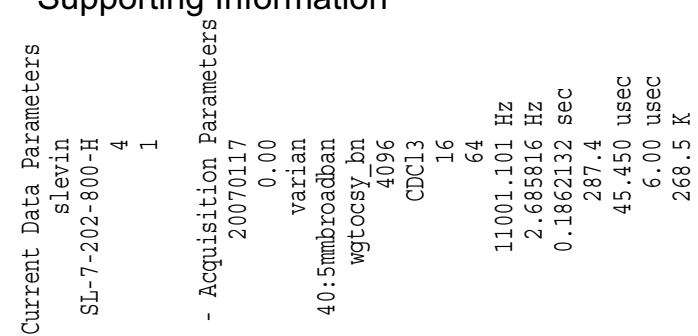

獣罢总总

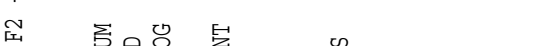

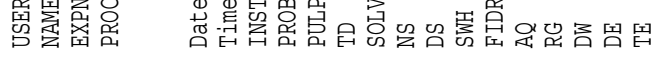

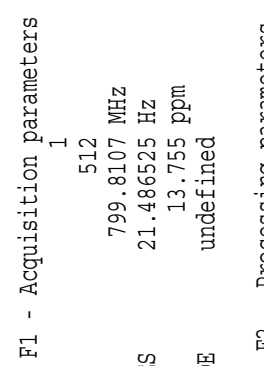

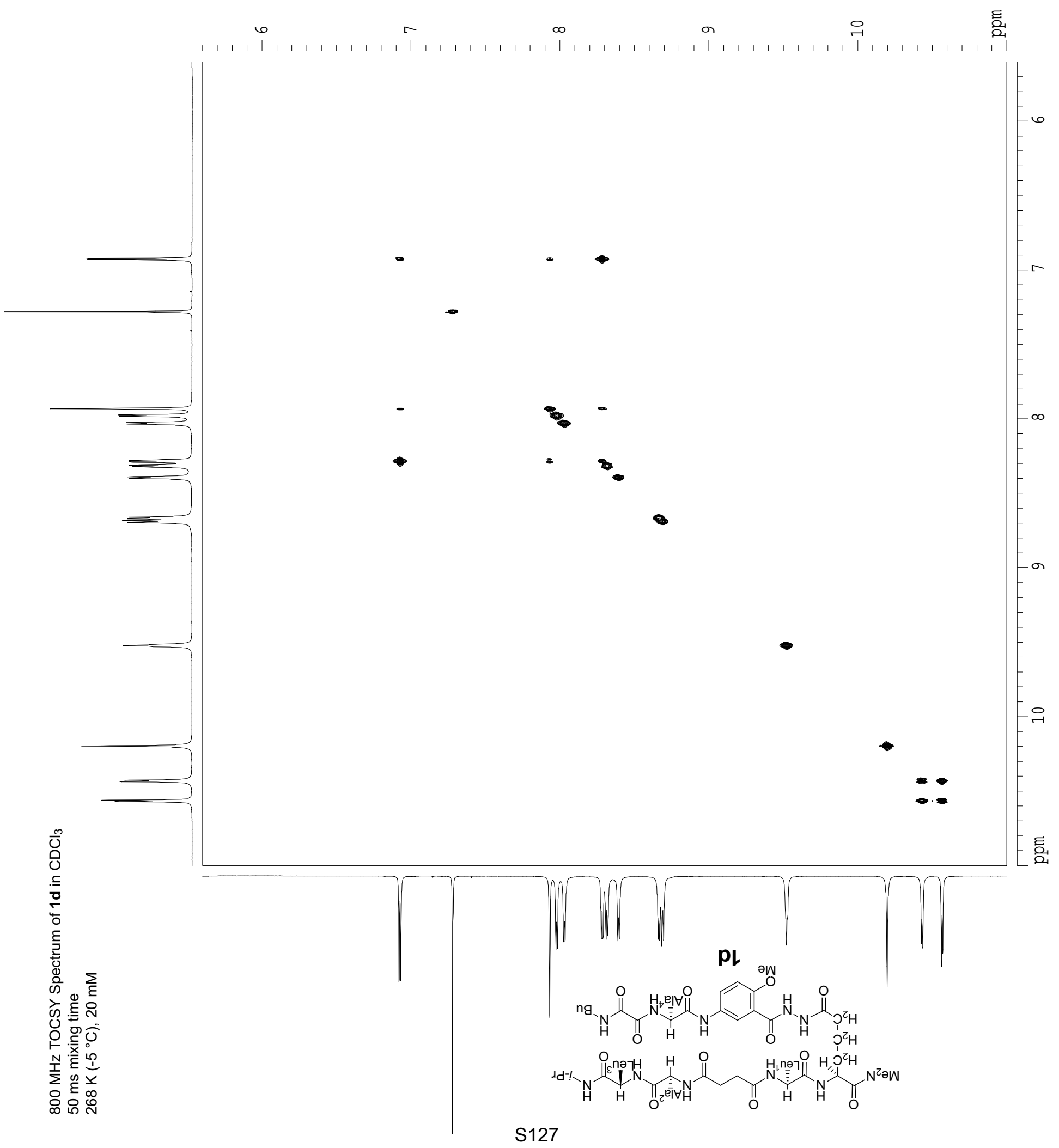




\section{Supporting Information}
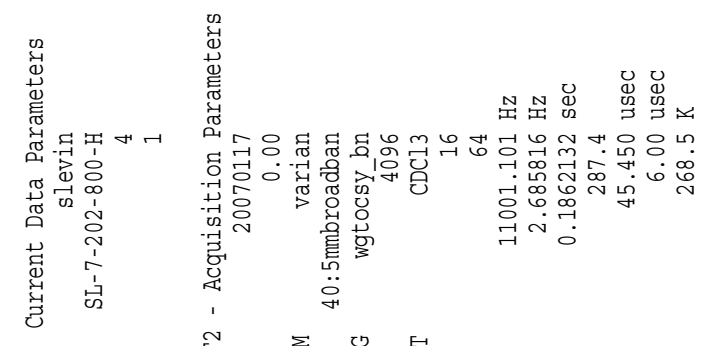

画罢总总

\section{年}

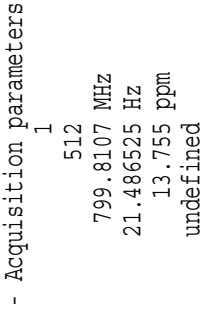

届的的

\section{S. Levin and J. S. Nowick}

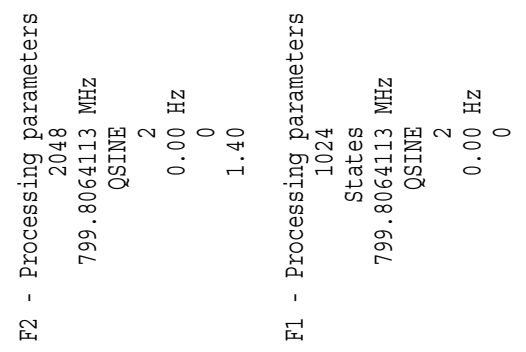

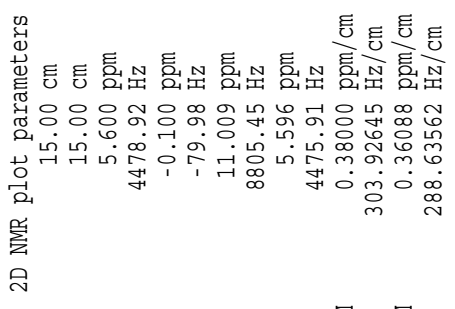

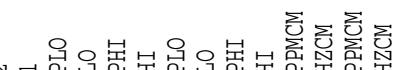

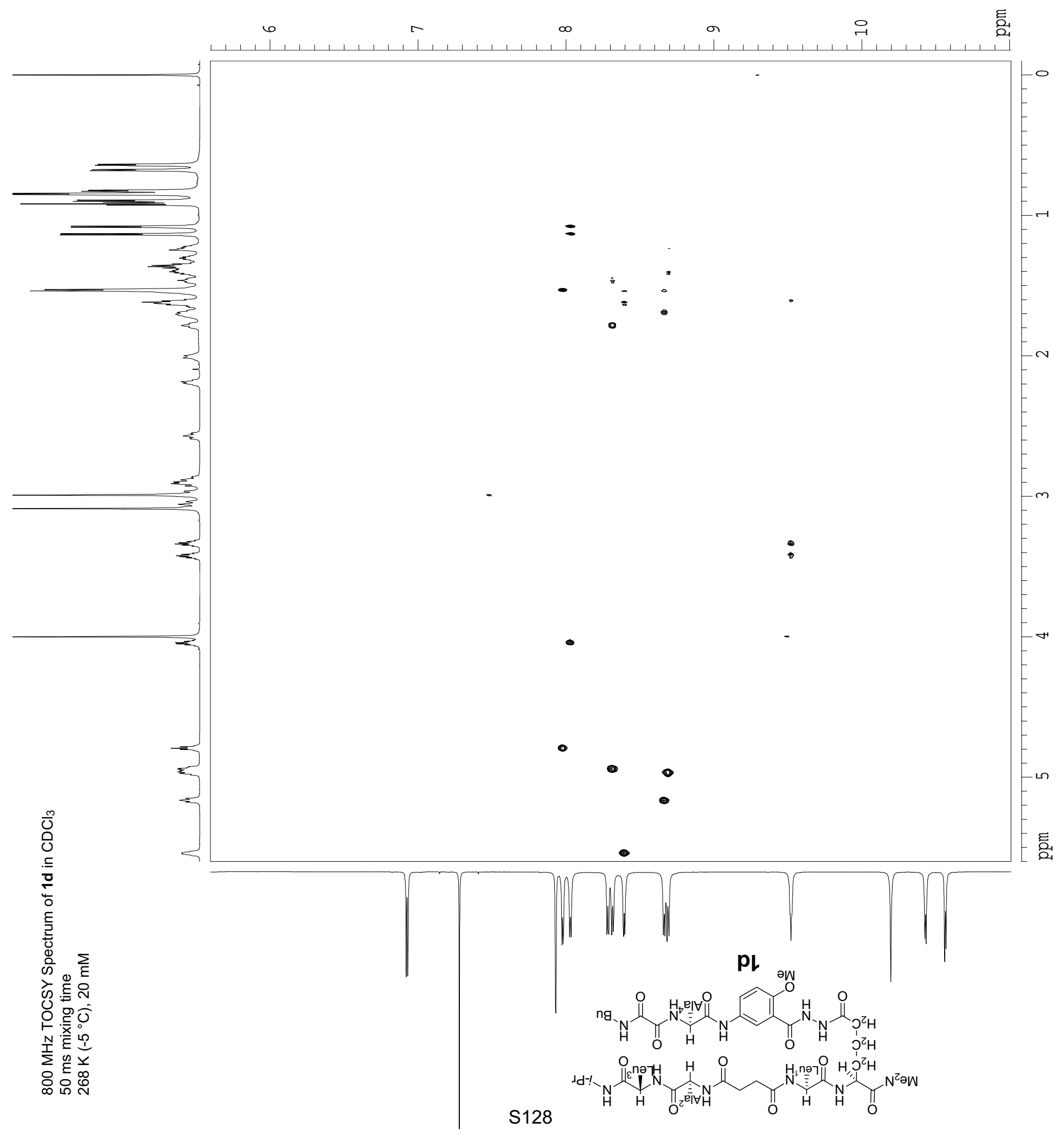




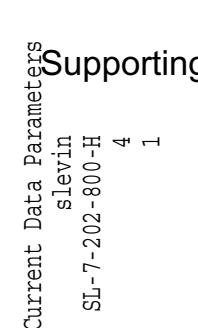

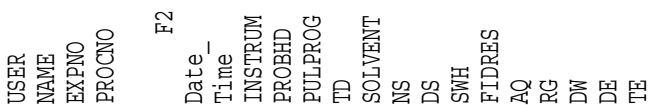

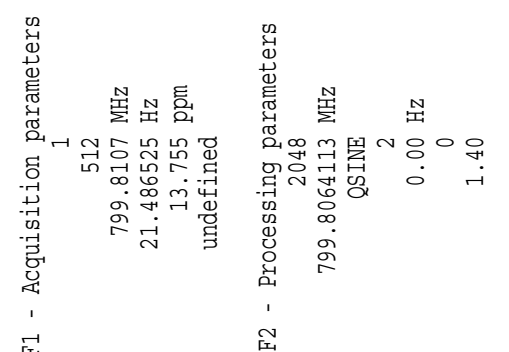

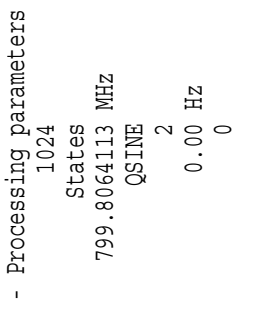

:

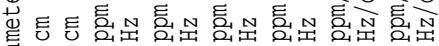

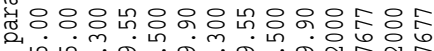
늠 瓷 군

9.

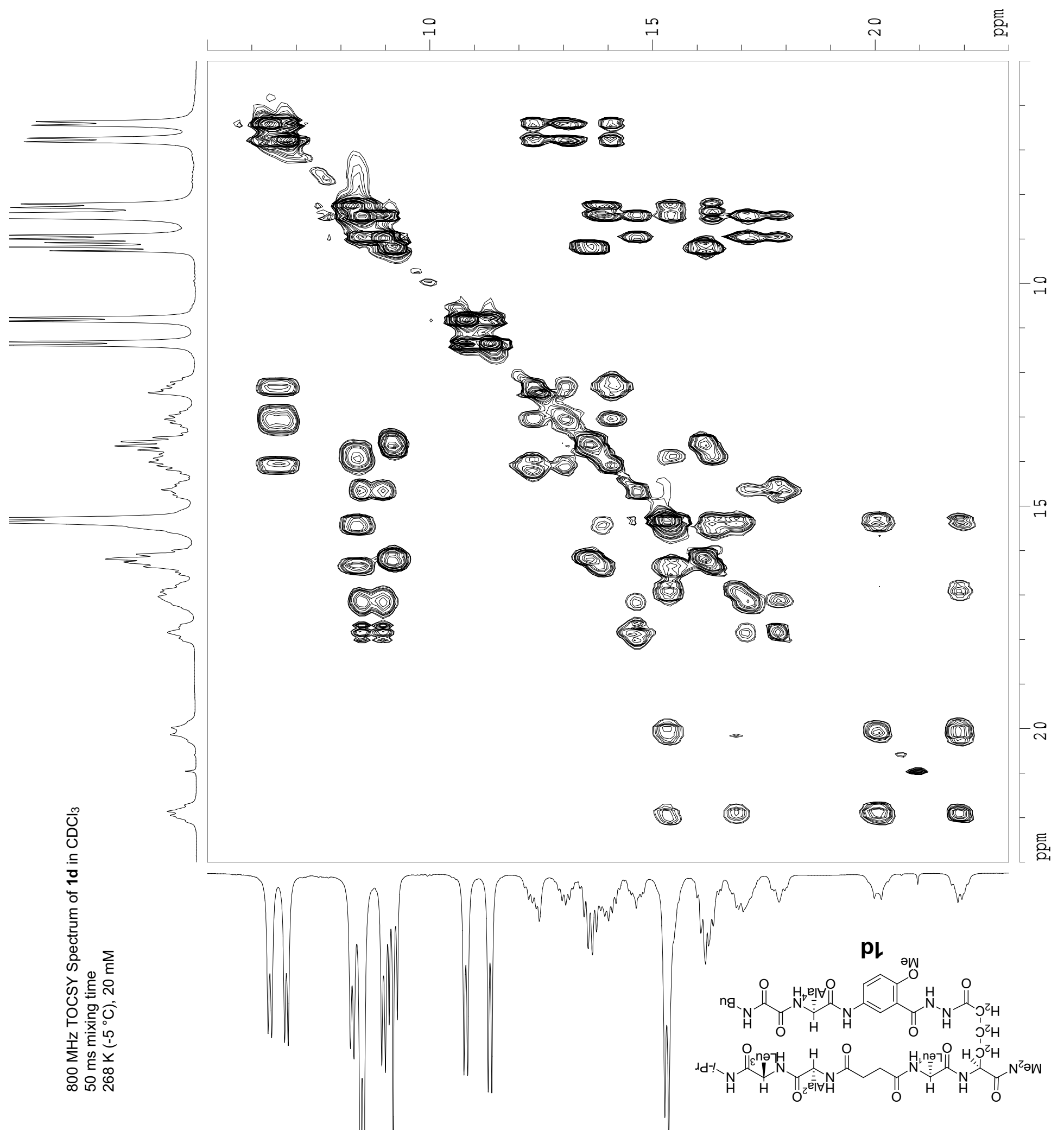



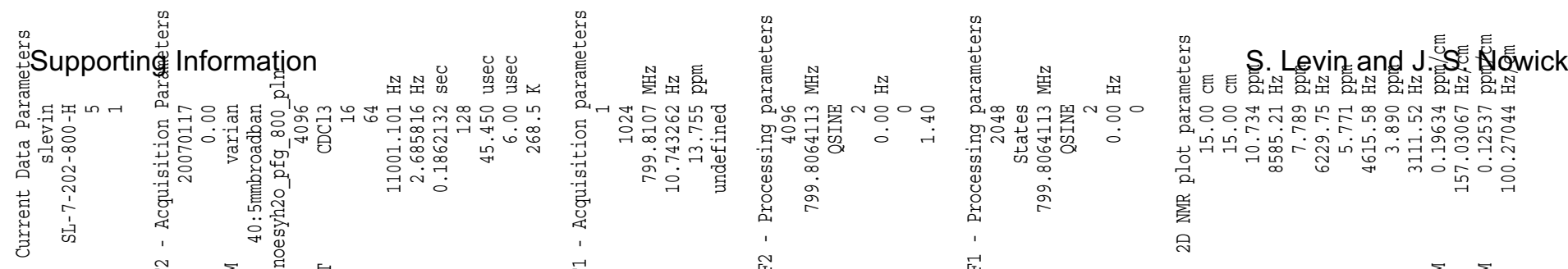

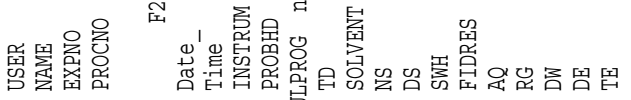

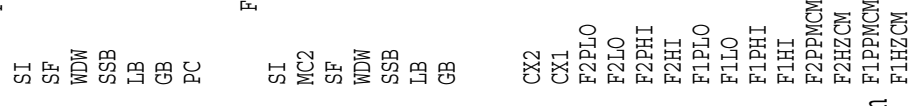

$\sim$

H

6

$\infty$

이 हूँ

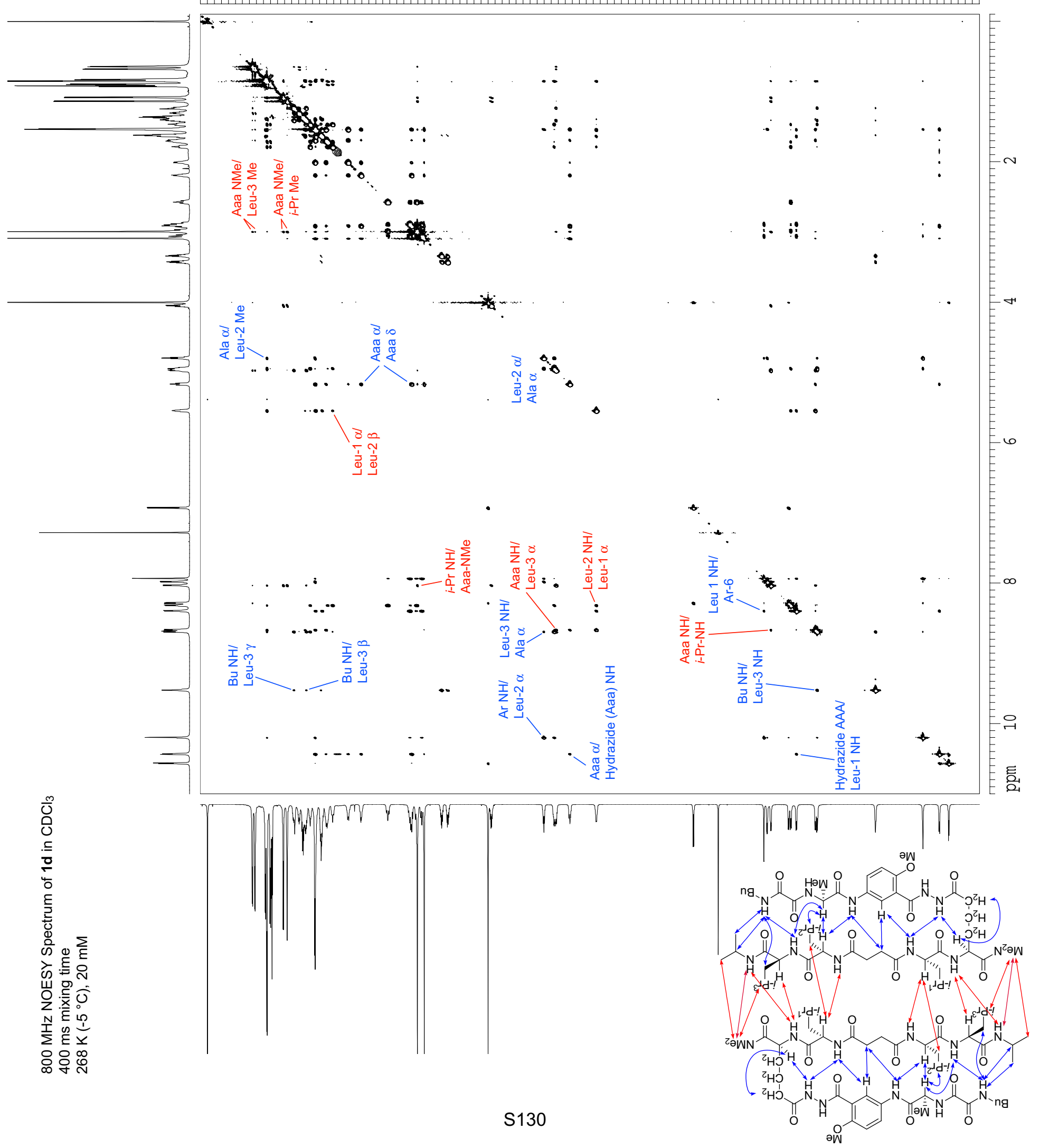



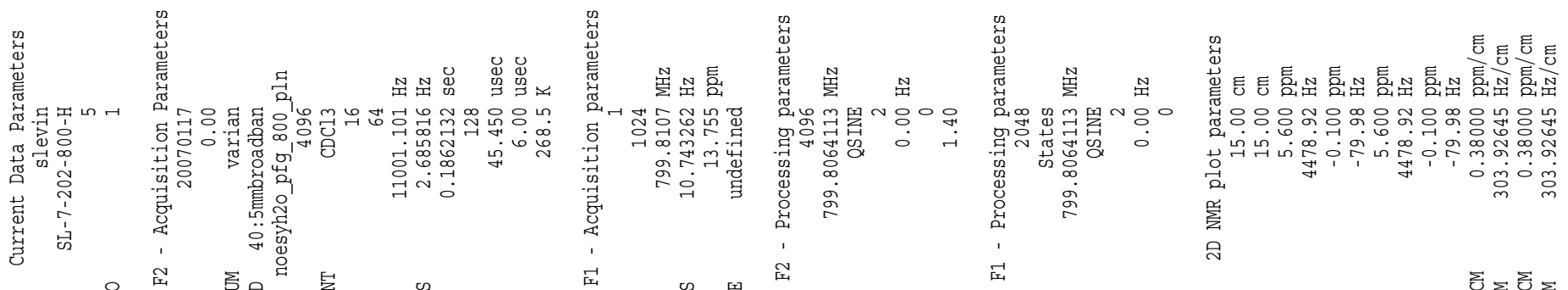

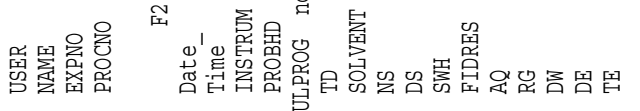

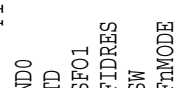

i

菂出总思罟思品

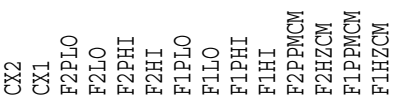

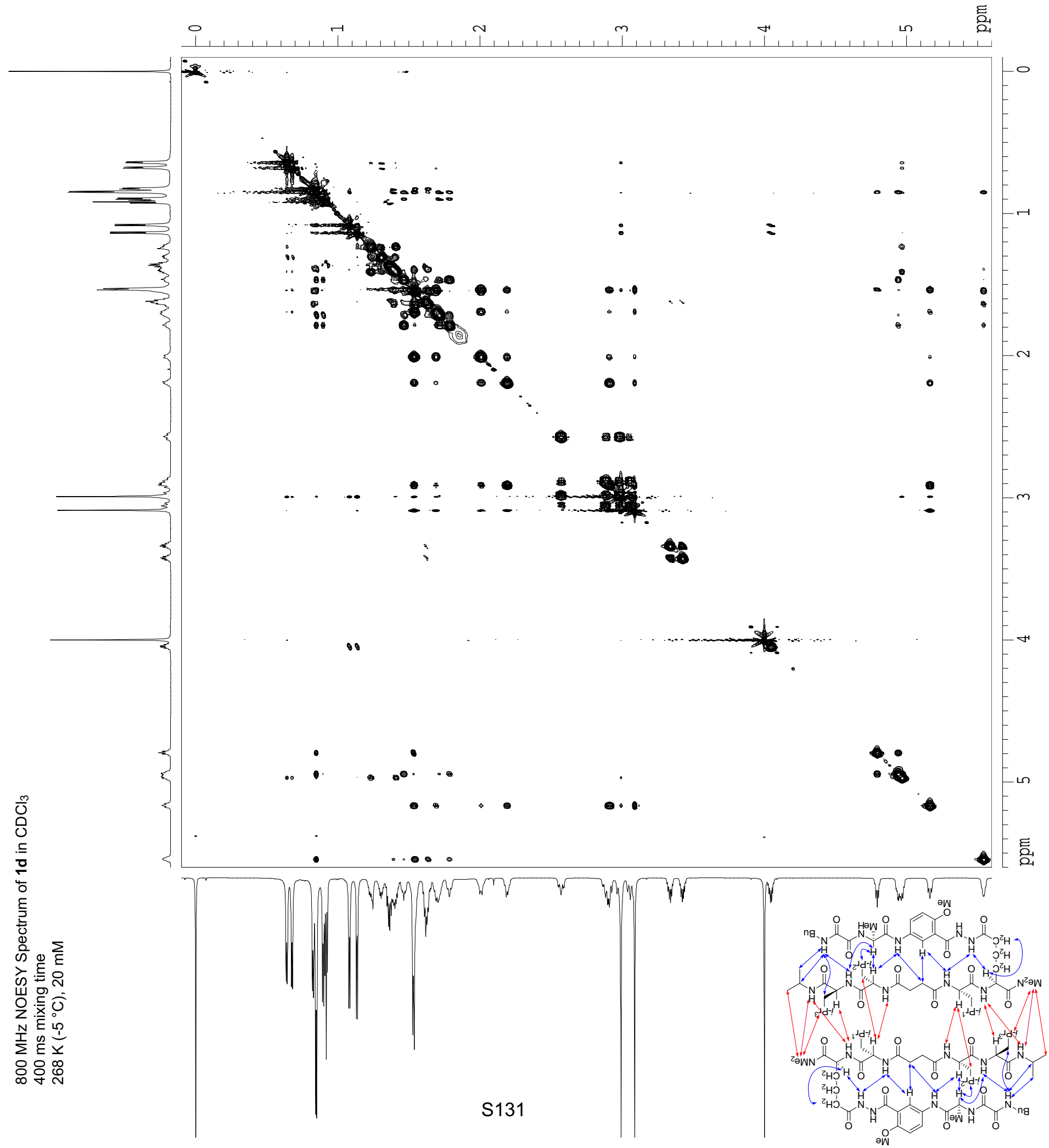


Supporting Information

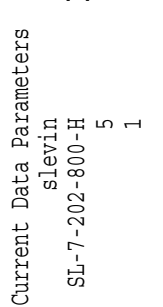

㽞舆虽虽

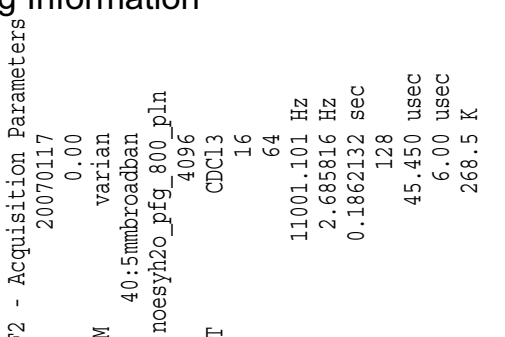

i

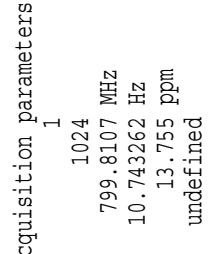

至势

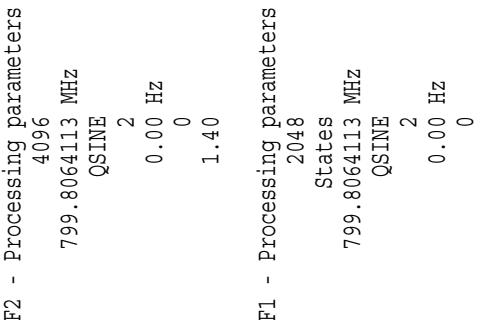

空

S. Levin and J. S. Nowick

岂

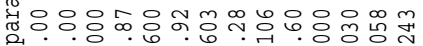

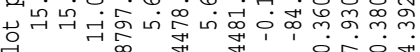
絖

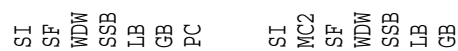

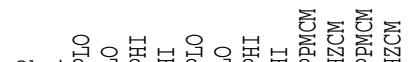
文

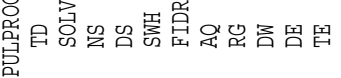

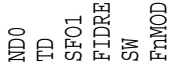

s:

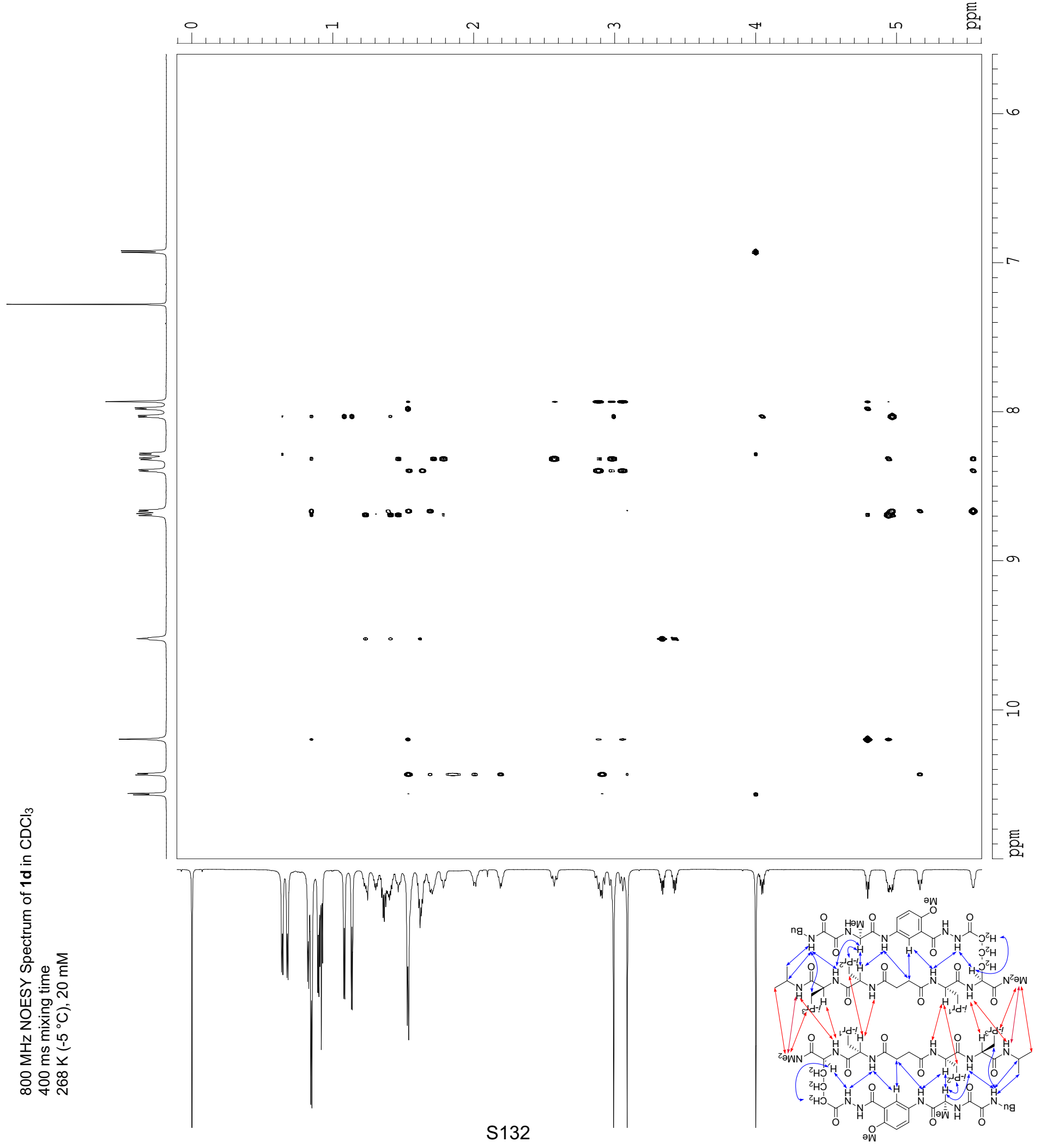


Supporting Information
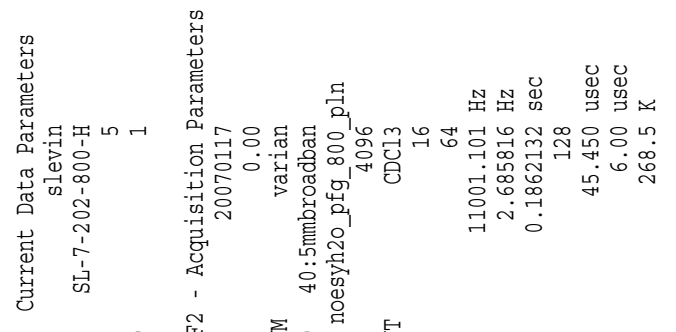

舀罢总总

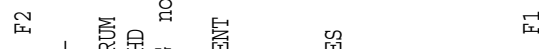

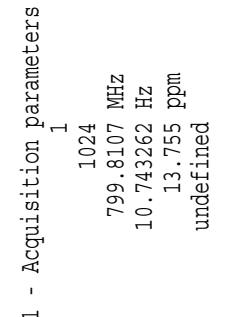

厓

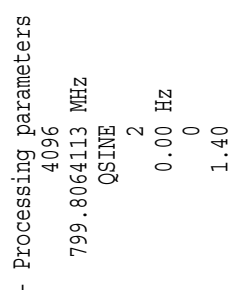

또

S. Levin and J. S. Nowick

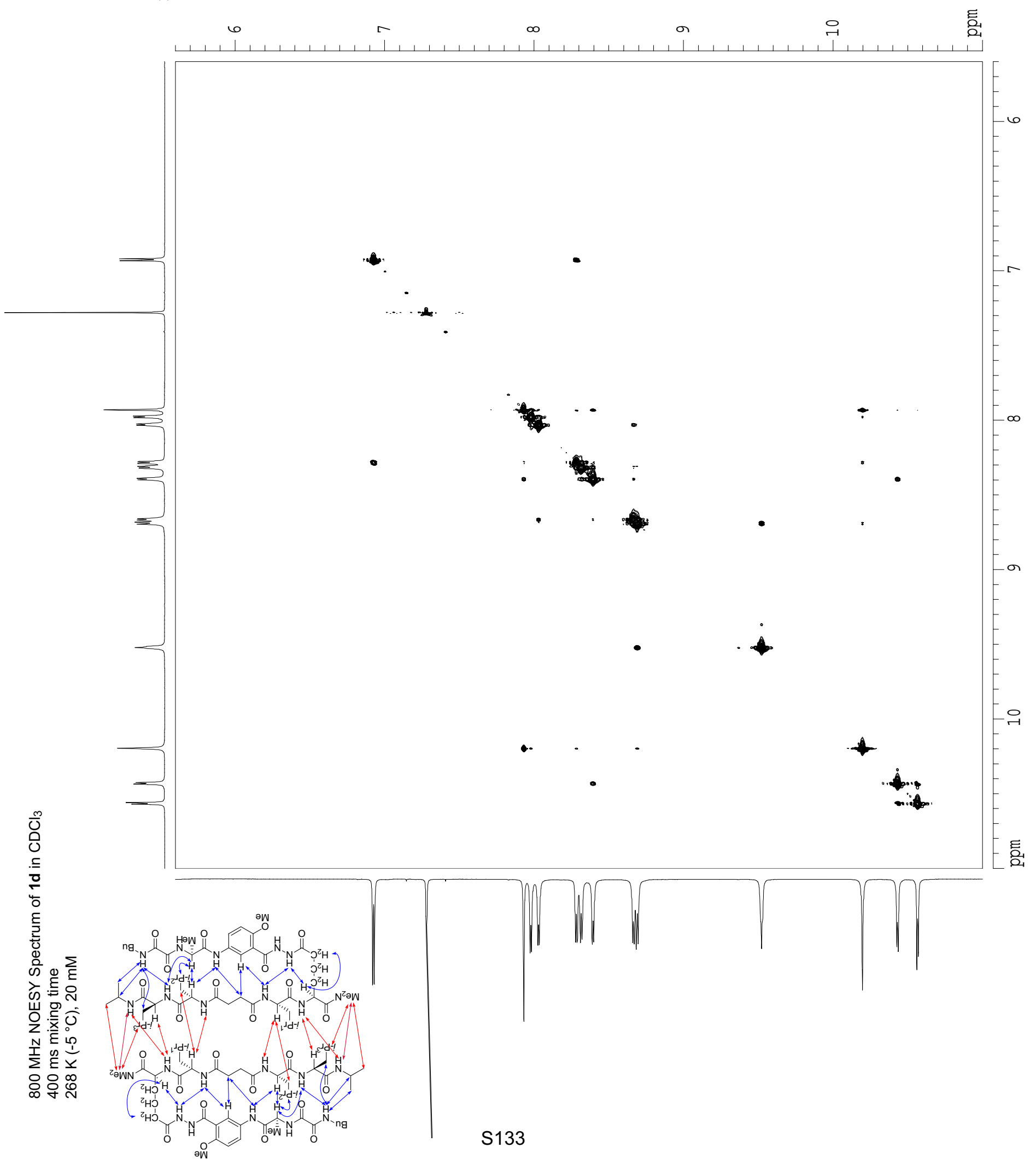



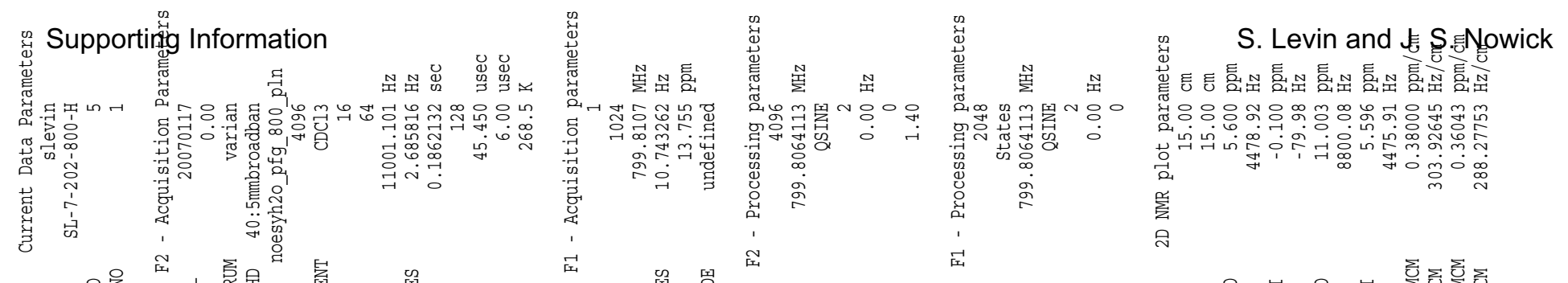

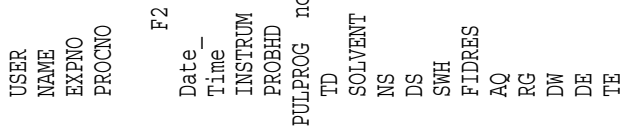

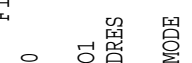

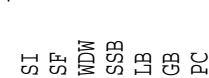

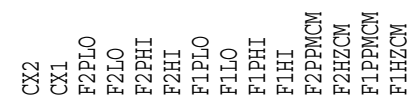

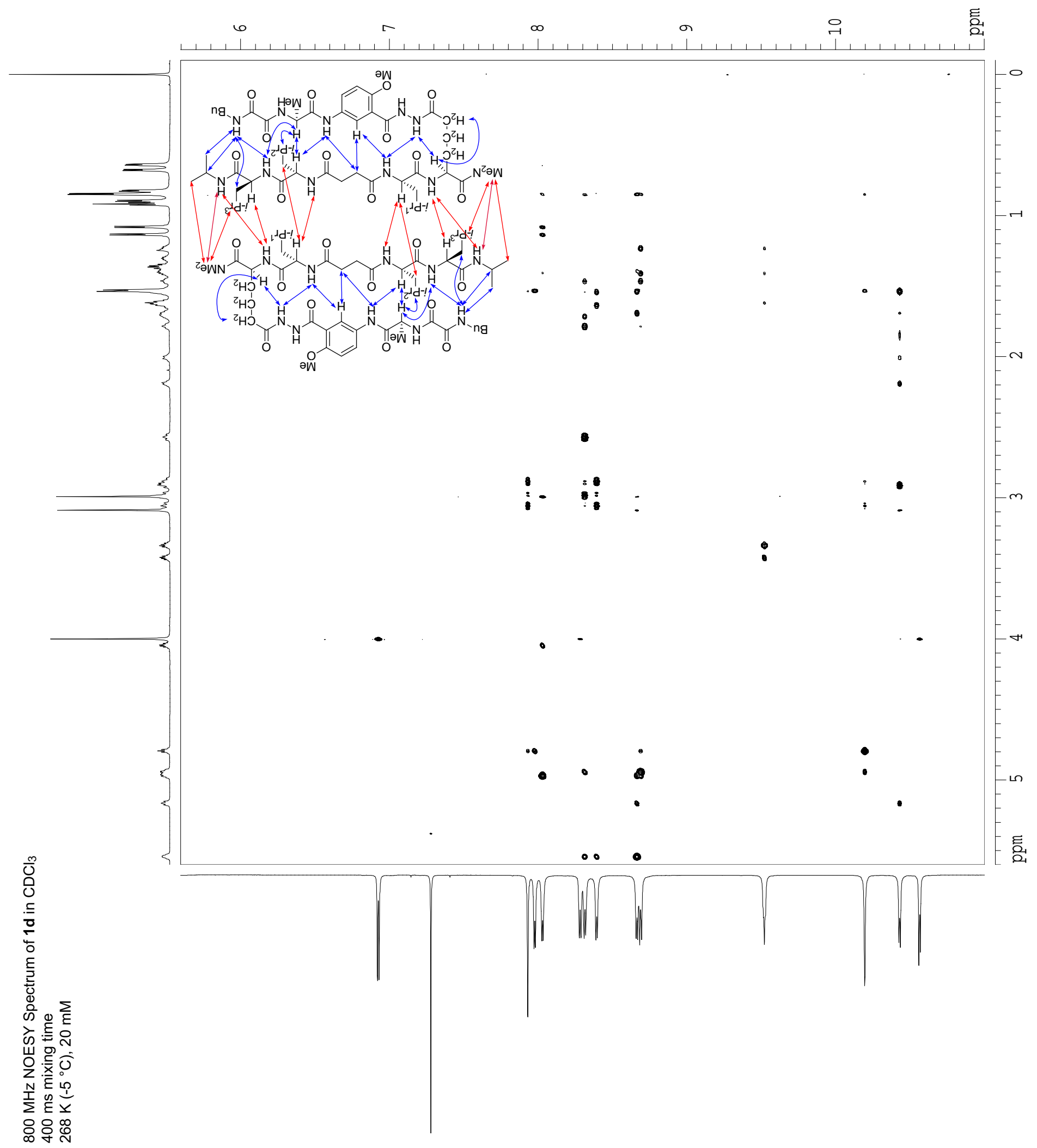


Supporting I/̆formation

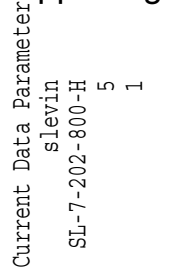

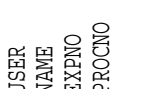

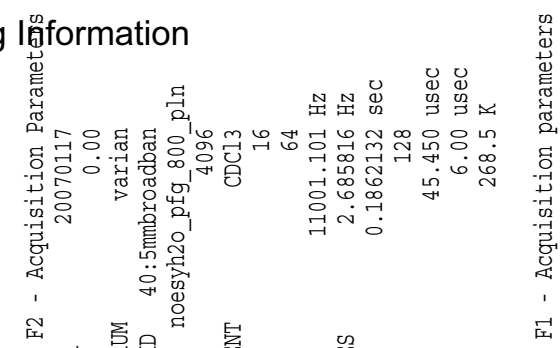

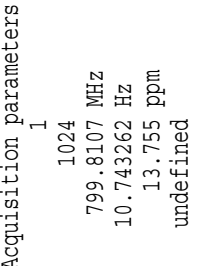

采

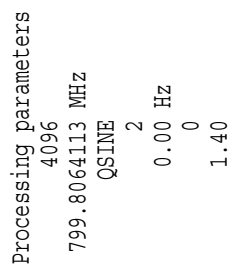

포

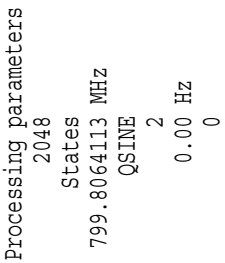

豆
S. Levin and J. S Nowick$$
\text { 号 S. Levin and J. S }
$$

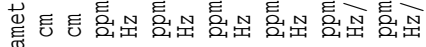

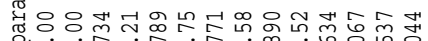

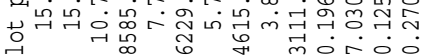
瓷 ล

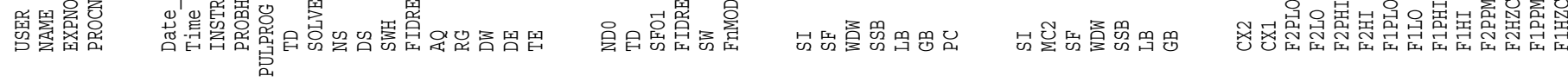

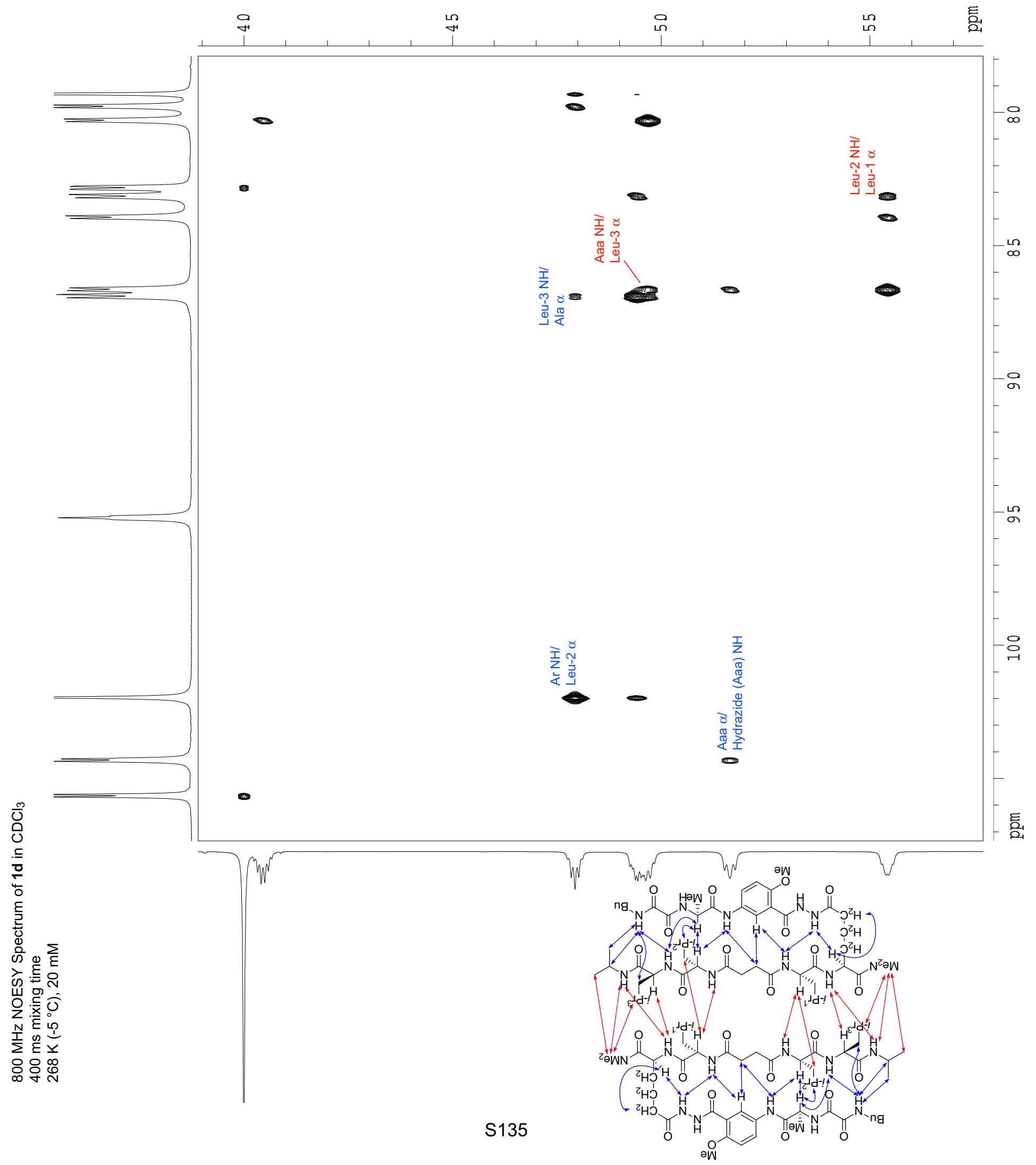


Supporting Information

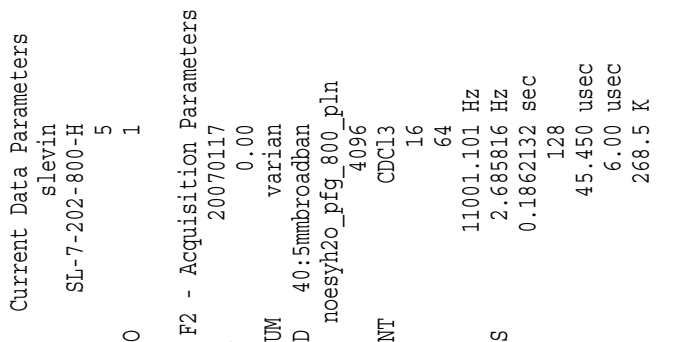

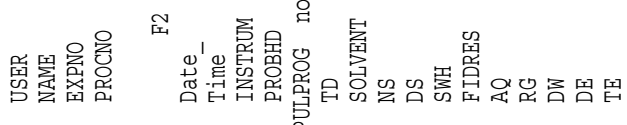

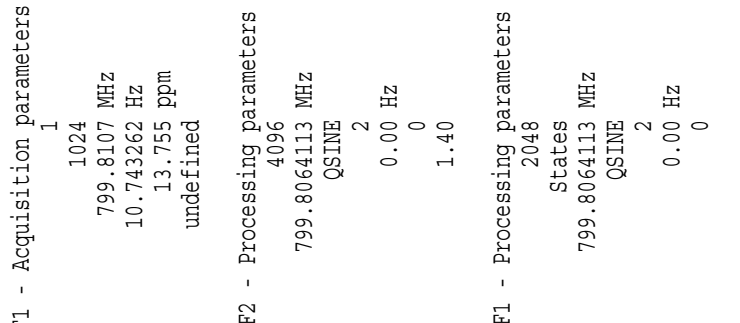

届年
S. Levin and J. S. Nowick

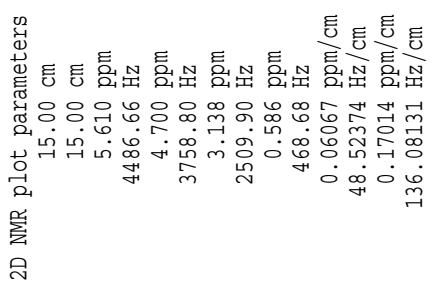

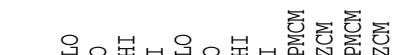

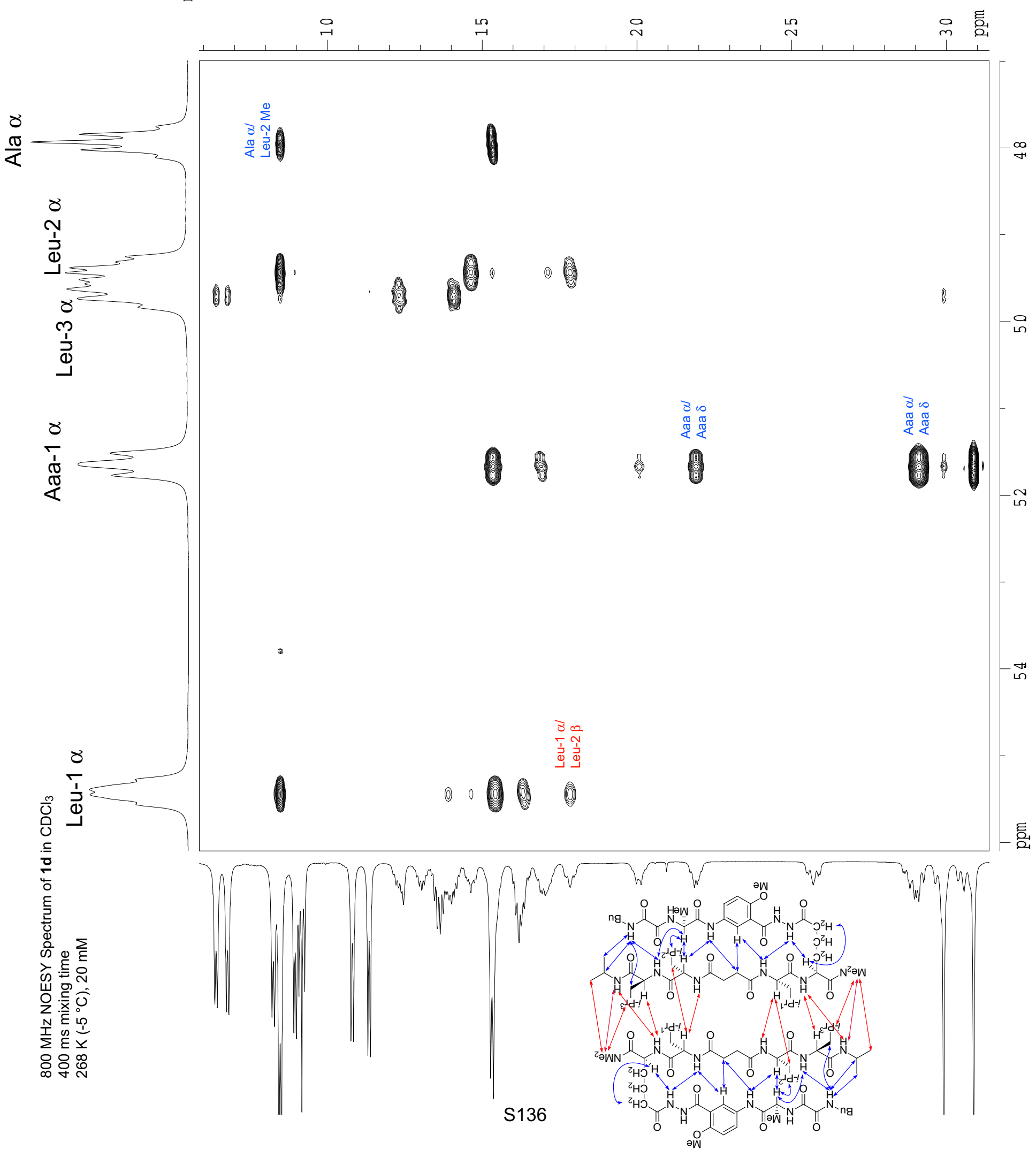




\section{Supporting Information}

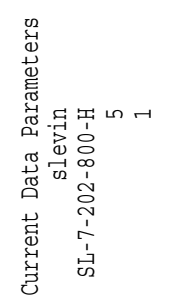

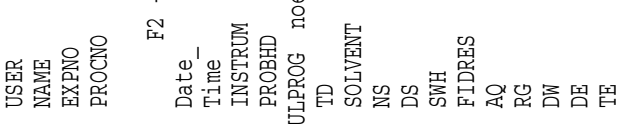

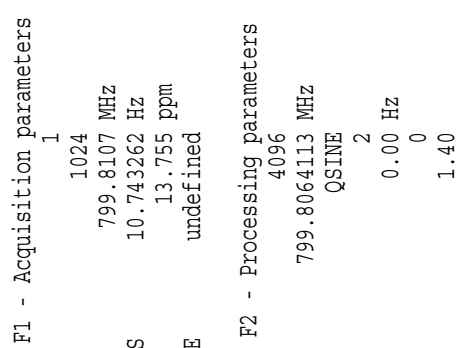

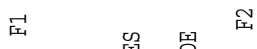

年

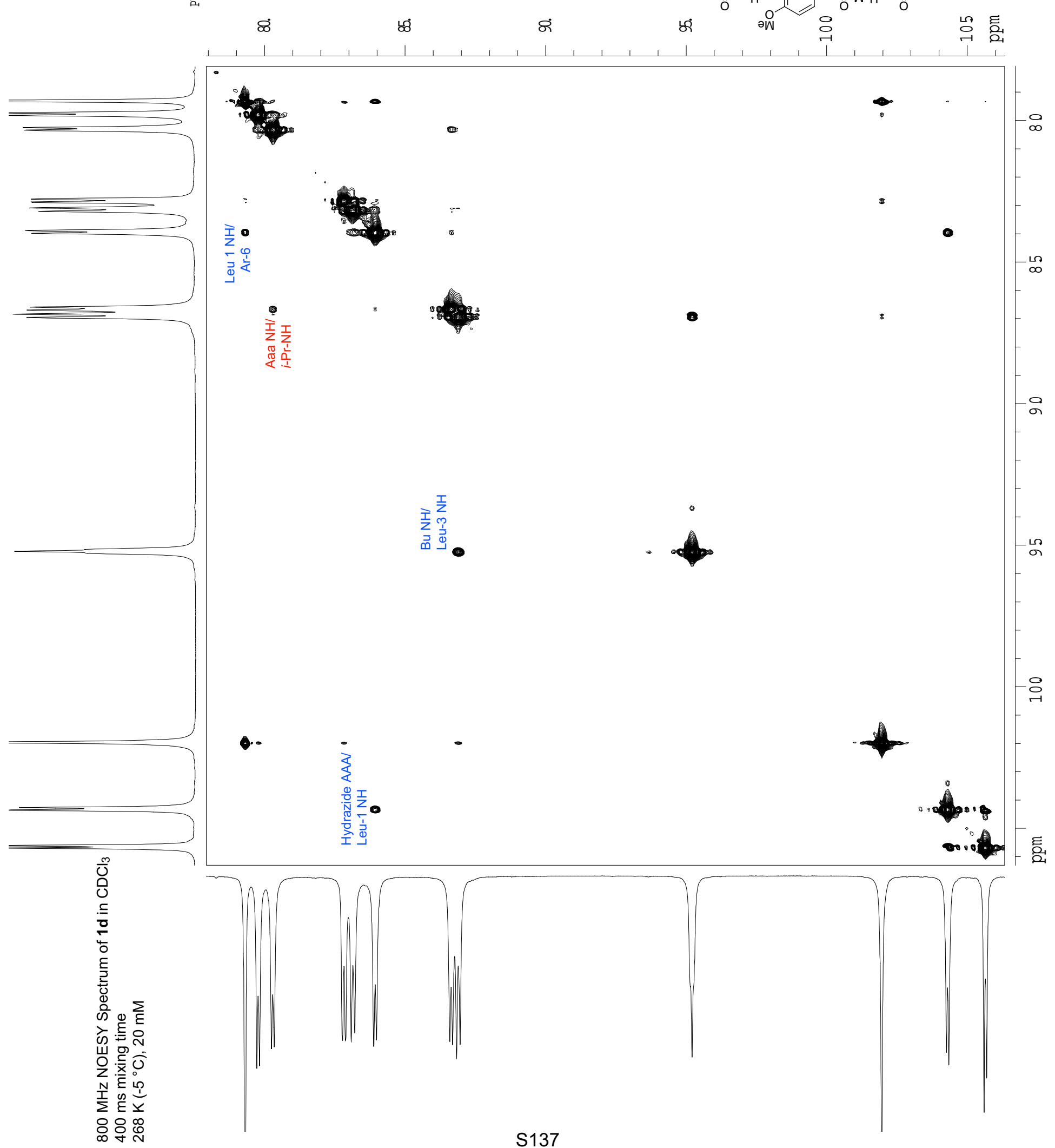



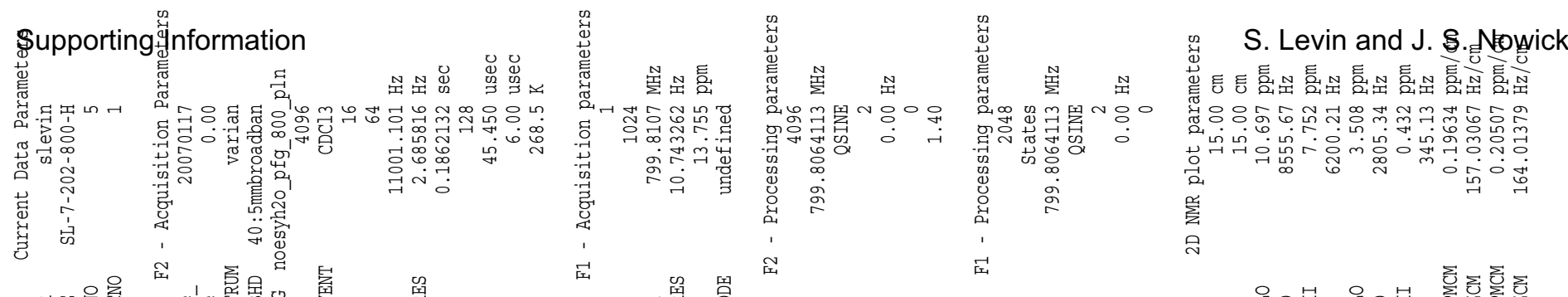

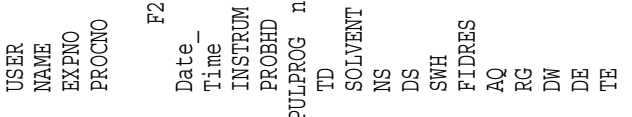

届

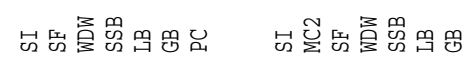

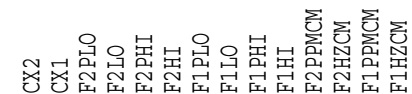

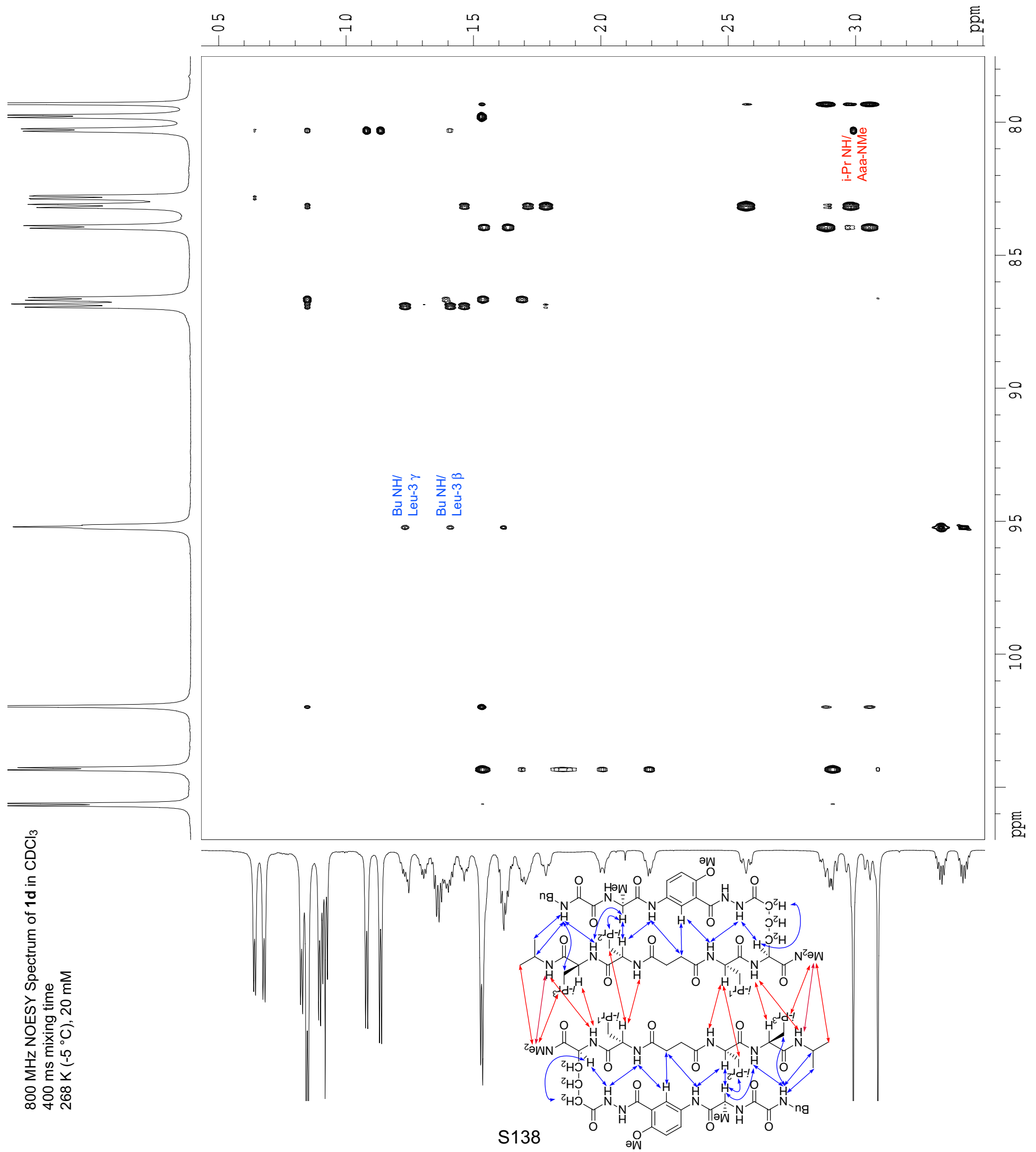


Supporting tnformation
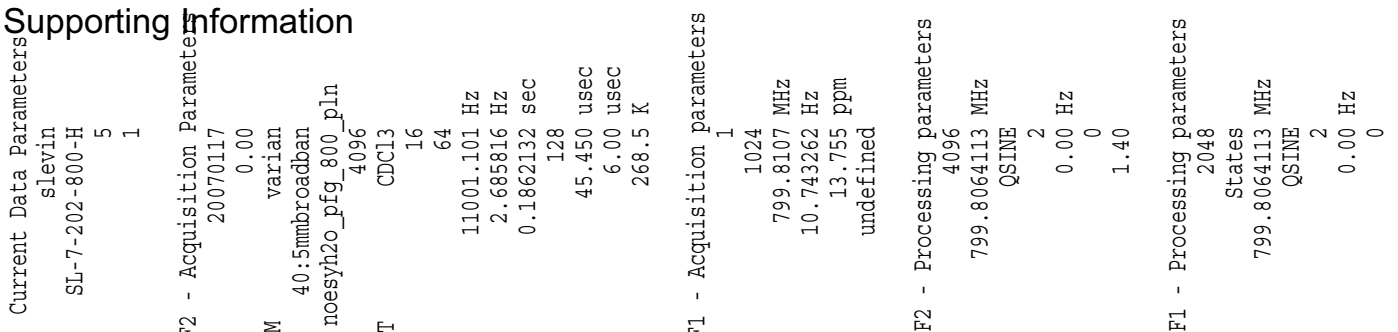

S. Levin and J. S. Nowick

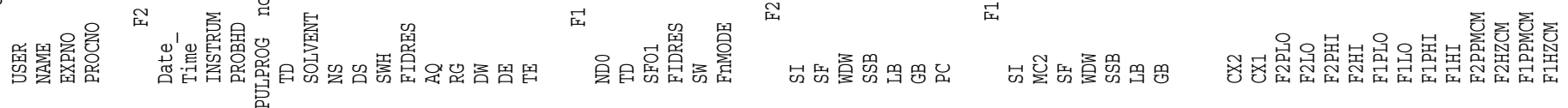

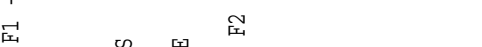

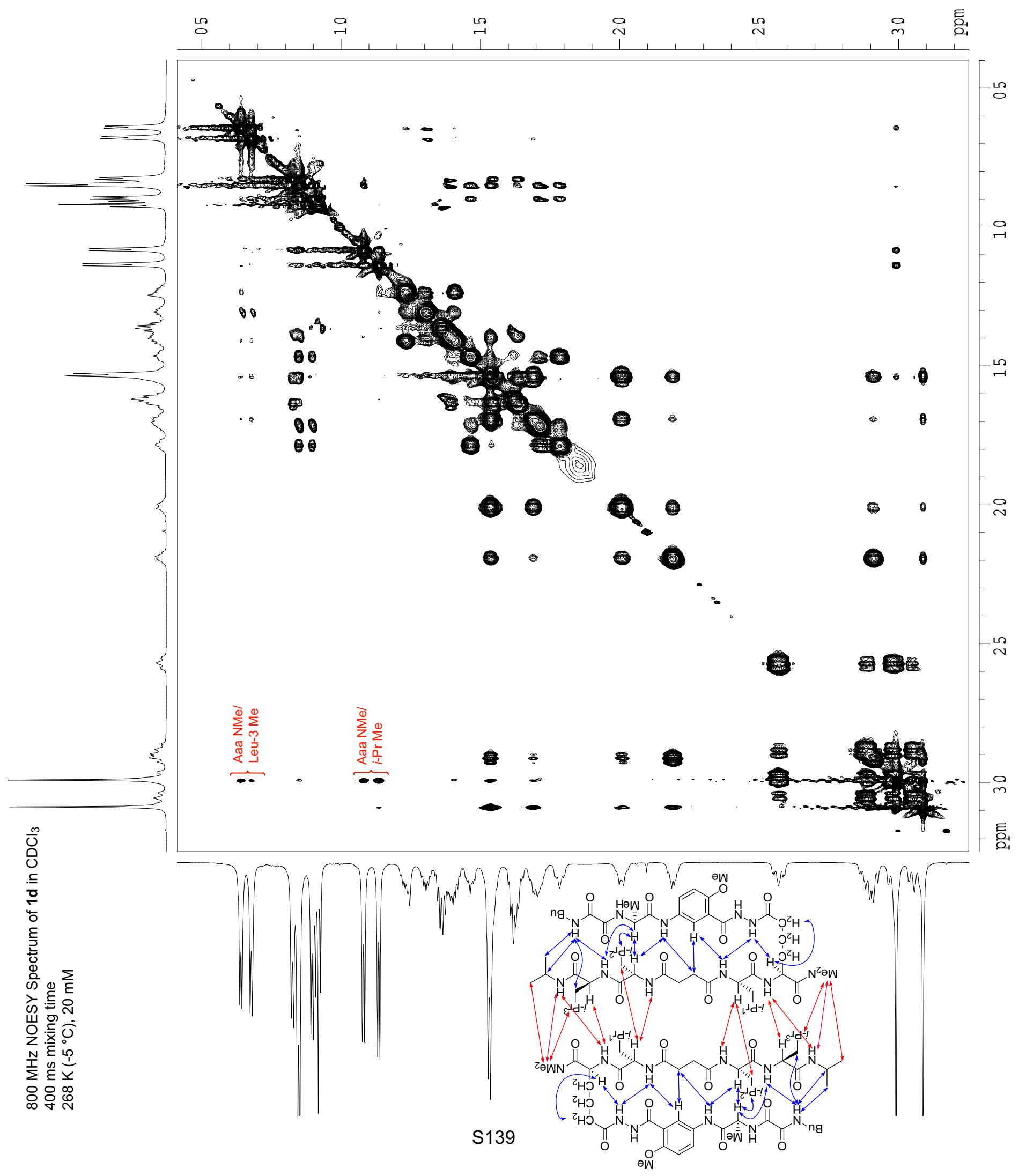




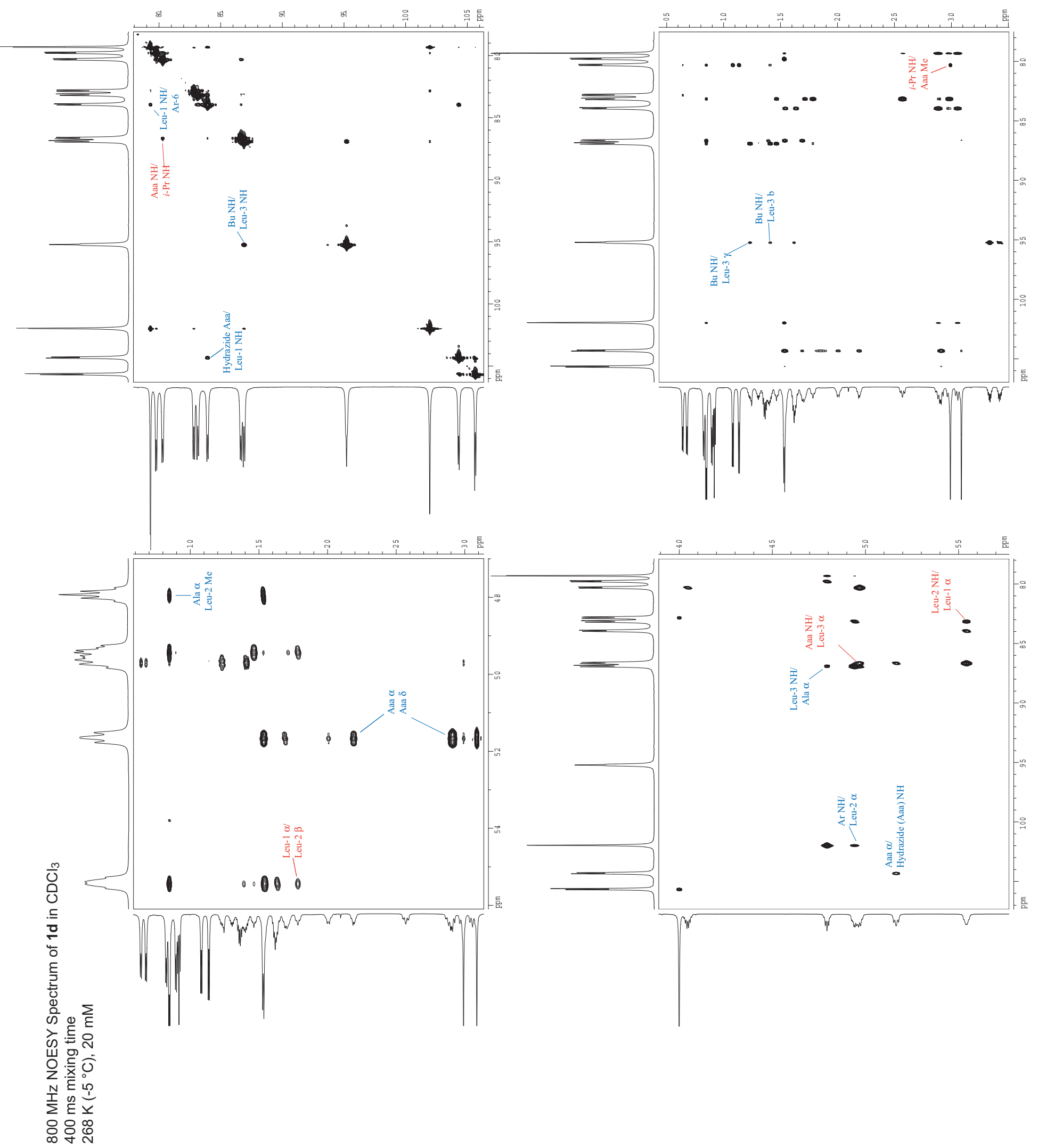




\section{Spectroscopic DATA for the compound 1e}

$\left(1 \mathrm{mM}, \mathrm{CDCl}_{3} 268 \mathrm{~K}\right)$

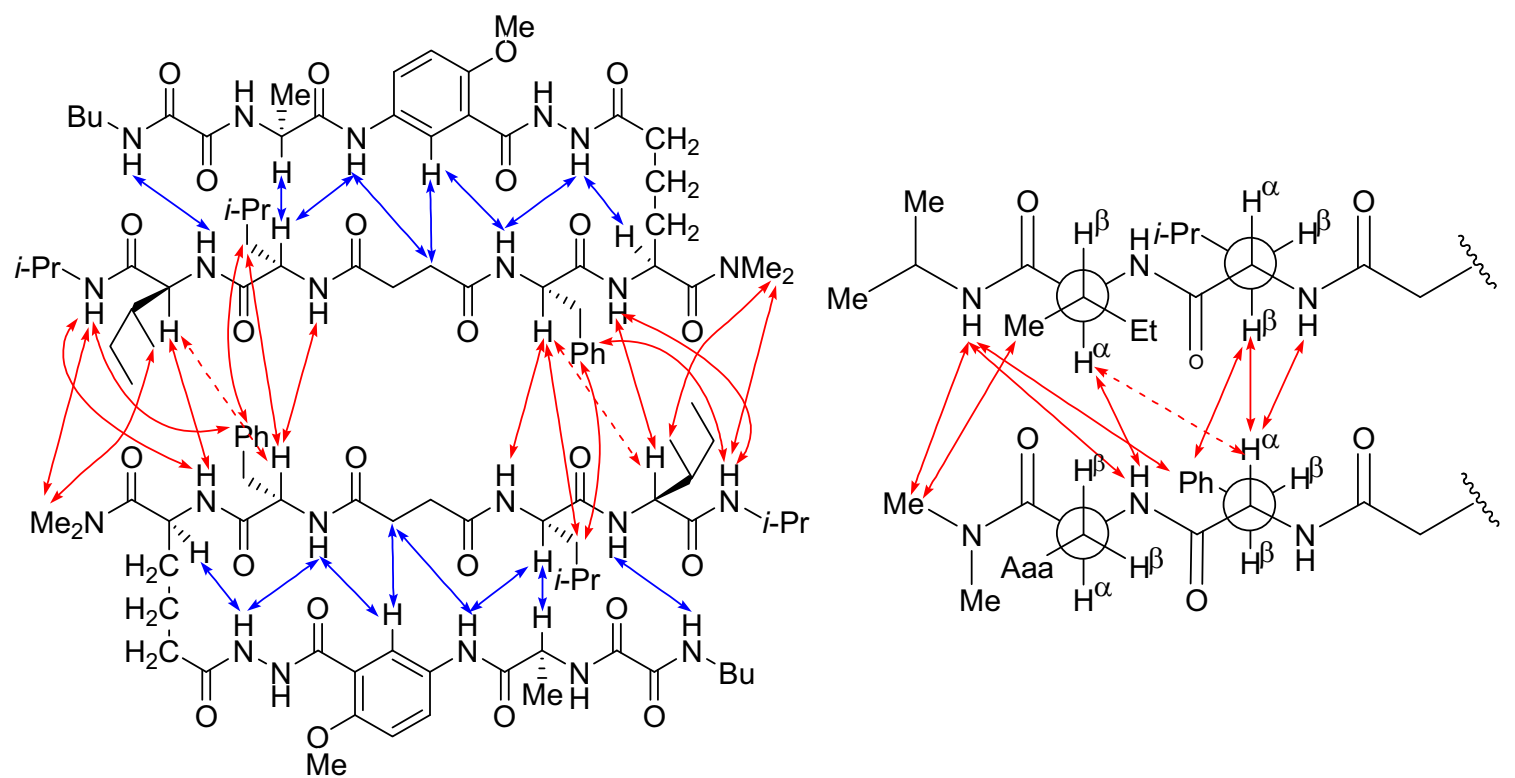

Key NOEs assigned as intermolecular are represented with red arrows

Key NOEs assigned as intramolecular are represented with blue arrows

Dashed arrow represents an NOE that may arise from deviation from ideal $\beta$-sheet geometry or from an alternative conformer or oligomer.

Key data for amino acids.

\begin{tabular}{|c|c|c|c|}
\hline Amino acid & $\mathrm{H}_{\alpha}$ Shift ppm & NH Shift ppm & ${ }^{3} J_{\mathrm{NH} \alpha} \mathrm{Hz}$ \\
\hline Aaa & 4.83 & 8.01 & 8.0 \\
\hline $\mathrm{R}_{1}=$ Phe & 5.94 & 8.66 & 7.7 \\
\hline $\mathrm{R}_{2}=$ Leu & 5.06 & 8.23 & 9.7 \\
\hline $\mathrm{R}_{3}=$ Ile & 4.72 & 8.78 & 9.7 \\
\hline $\mathrm{R}_{4}=$ Ala & 4.81 & 7.93 & overlap \\
\hline
\end{tabular}

Aminoadipic acid (Aaa) $\delta$ proton resonances.

pro-R: $2.80 \mathrm{ppm}, \mathrm{t}$, (overlap)

pro-S: $2.14 \mathrm{ppm}, \mathrm{td},\left(J_{1}=11.9 \mathrm{~Hz} ; J_{2}=7.0 \mathrm{~Hz}\right)$

anisotropy $=0.66 \mathrm{ppm}$

Key data for other $\mathrm{NH}$ protons.

\begin{tabular}{|c|c|c|}
\hline Proton & NH Shift ppm & $J \mathrm{~Hz}$ \\
\hline Ar-NH & 10.20 & - \\
\hline Hydrazide Ar & 10.69 & 7.4 \\
\hline Hydrazide Aaa & 10.63 & 7.4 \\
\hline Bu-NH & 9.65 & broad \\
\hline$i$-Pr-NH & 8.06 & 7.7 \\
\hline
\end{tabular}

ESI MS $m / z$ for $\mathrm{C}_{53} \mathrm{H}_{82} \mathrm{~N}_{11} \mathrm{O}_{12}[\mathrm{M}+\mathrm{H}]^{+}$calcd 1064.61, found 1064.48. 


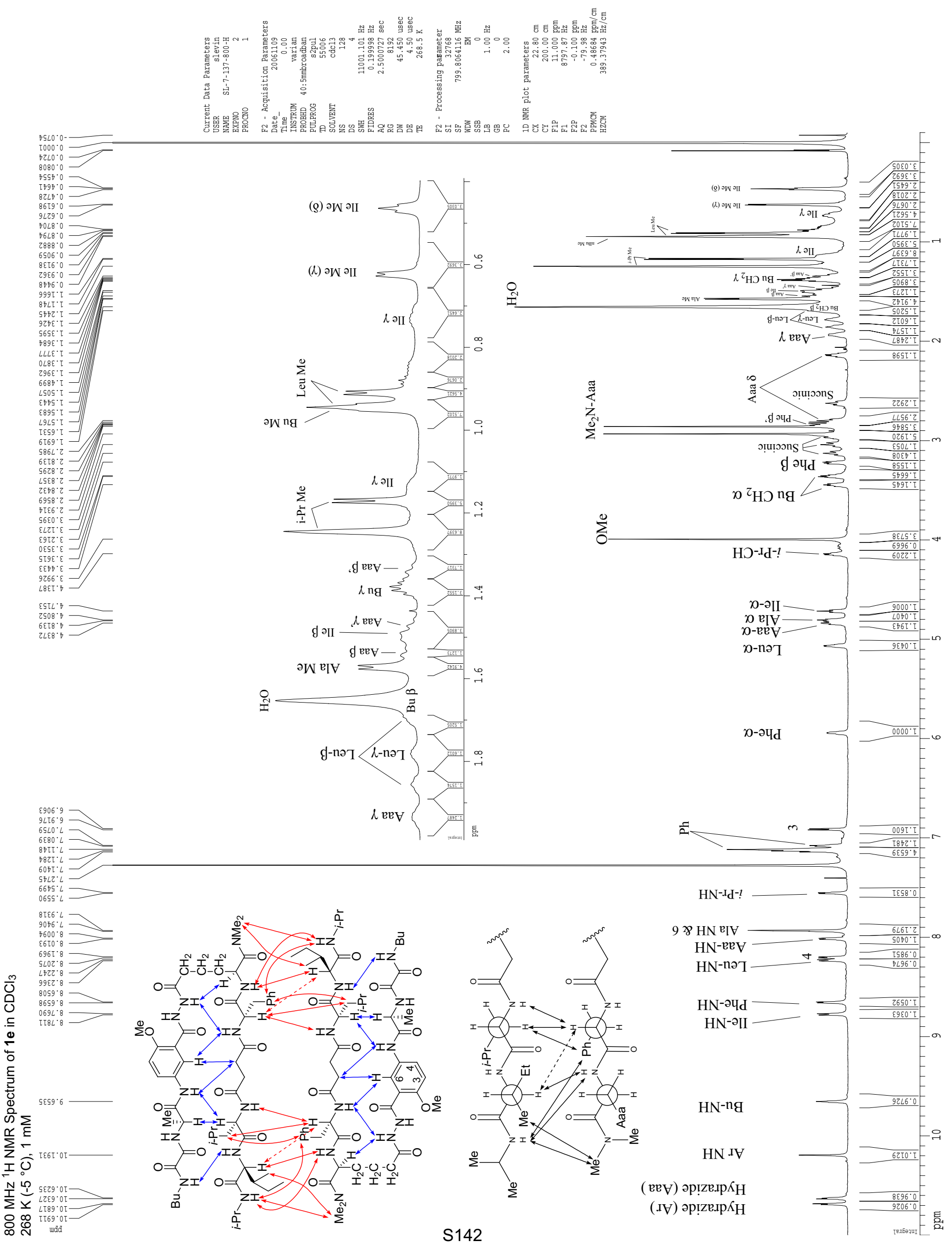



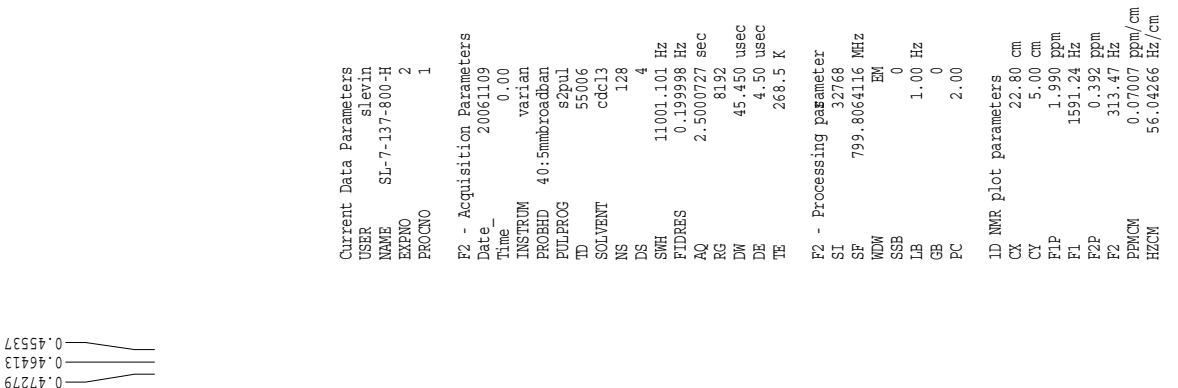

(९) วW गा

(l) วW ขI
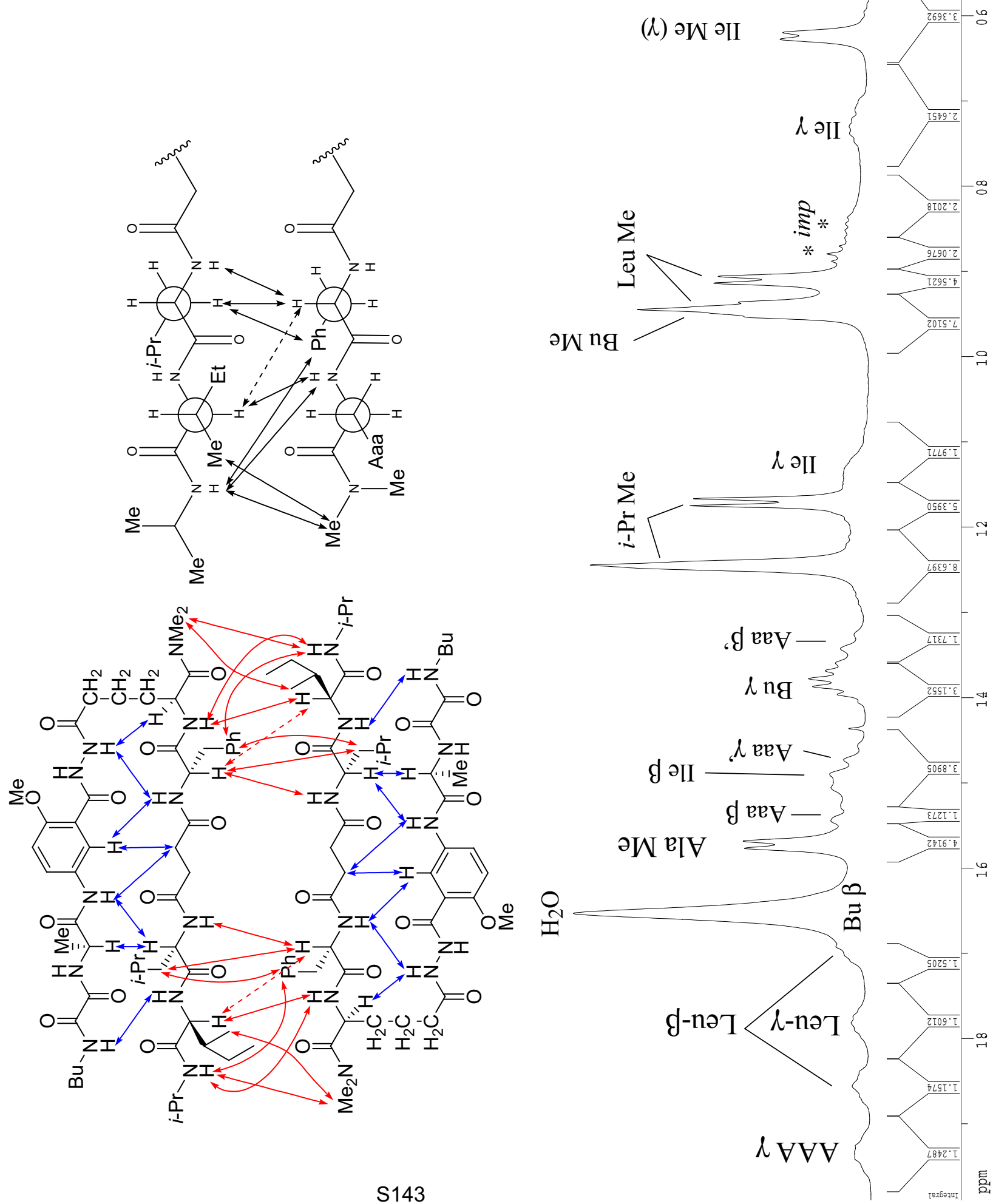
Supporting Information

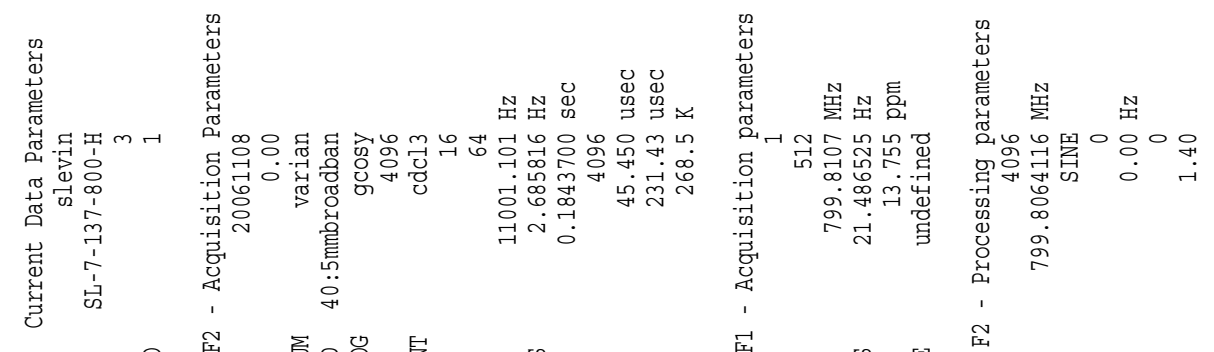

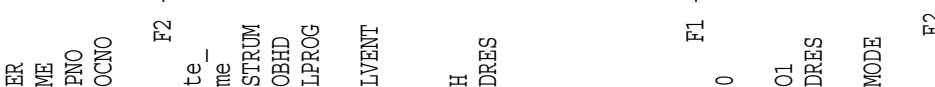

S. Levin and J. S. Nowick

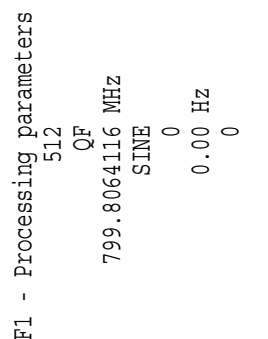

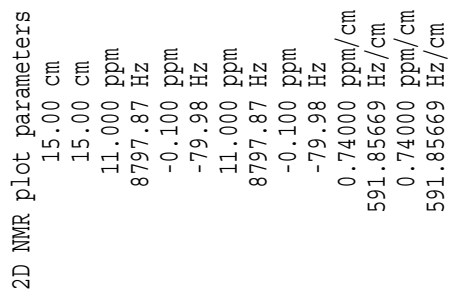

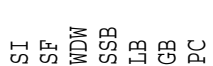

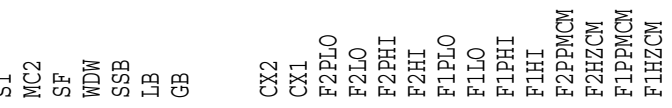

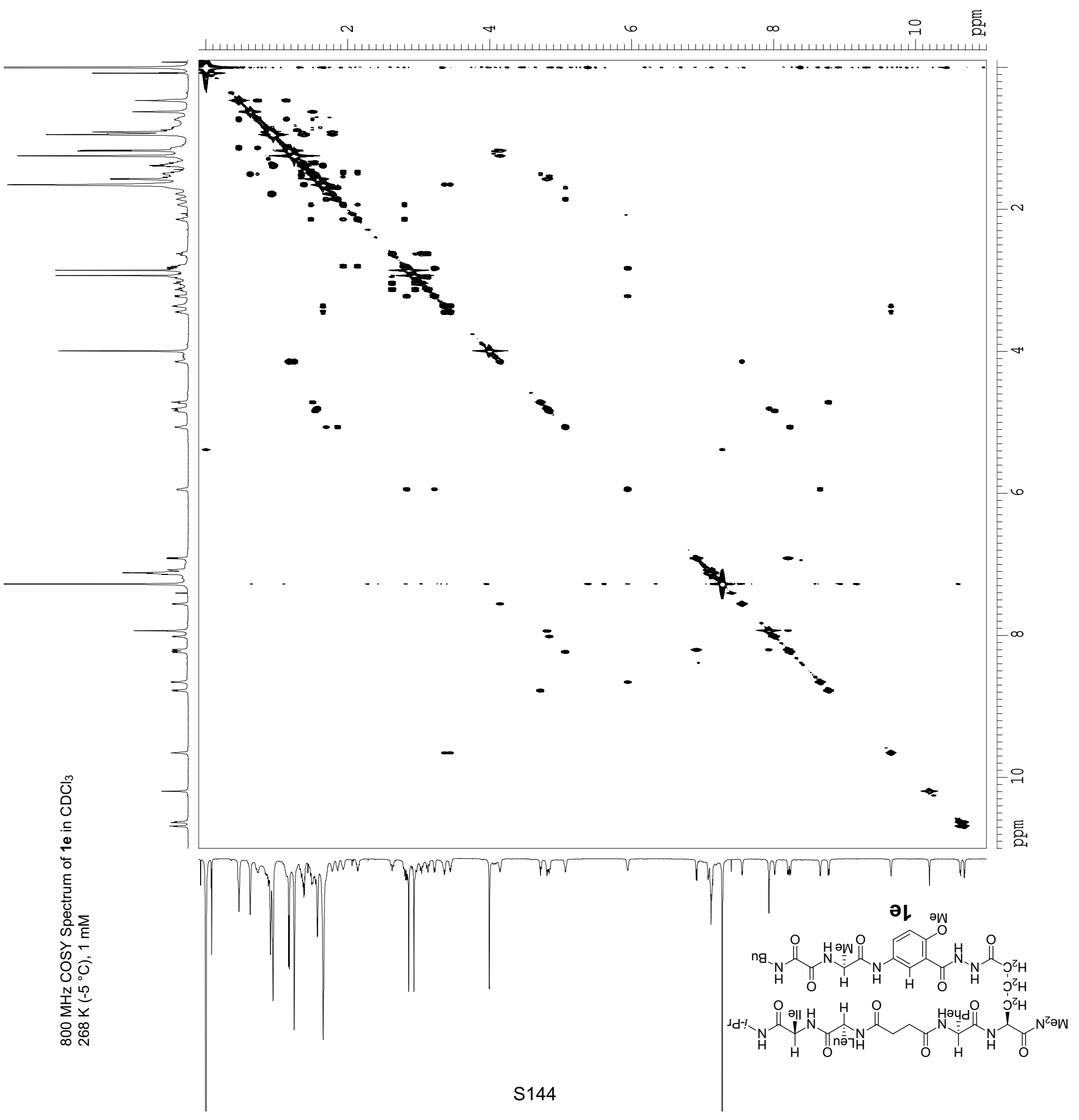


Supporting Information
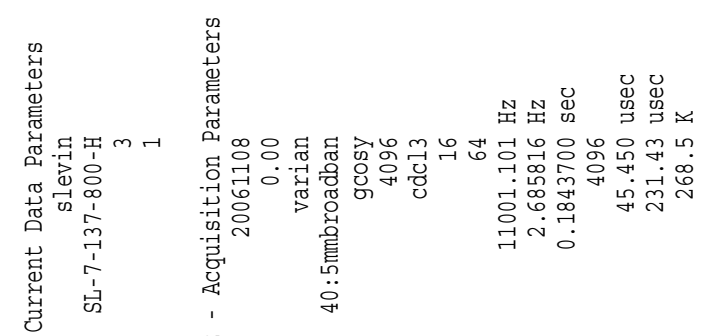

画舅总总

\section{" ang}

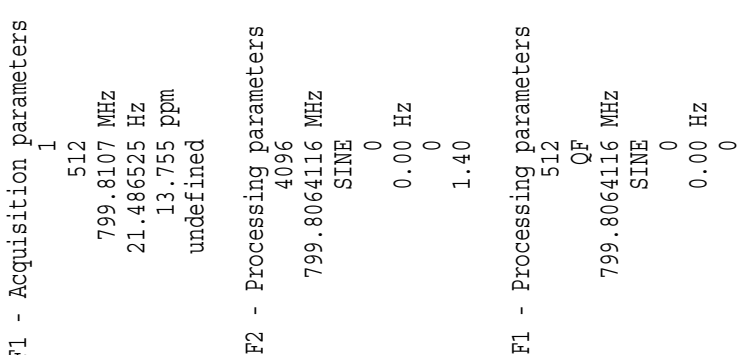

\&
S. Levin and J. S. Nowick

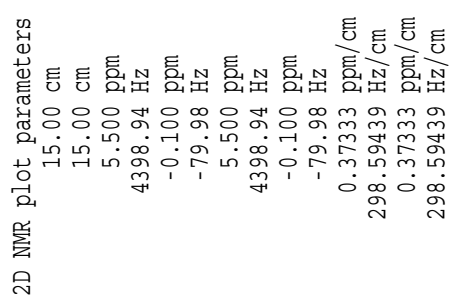

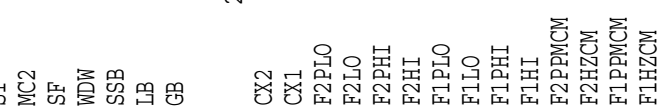

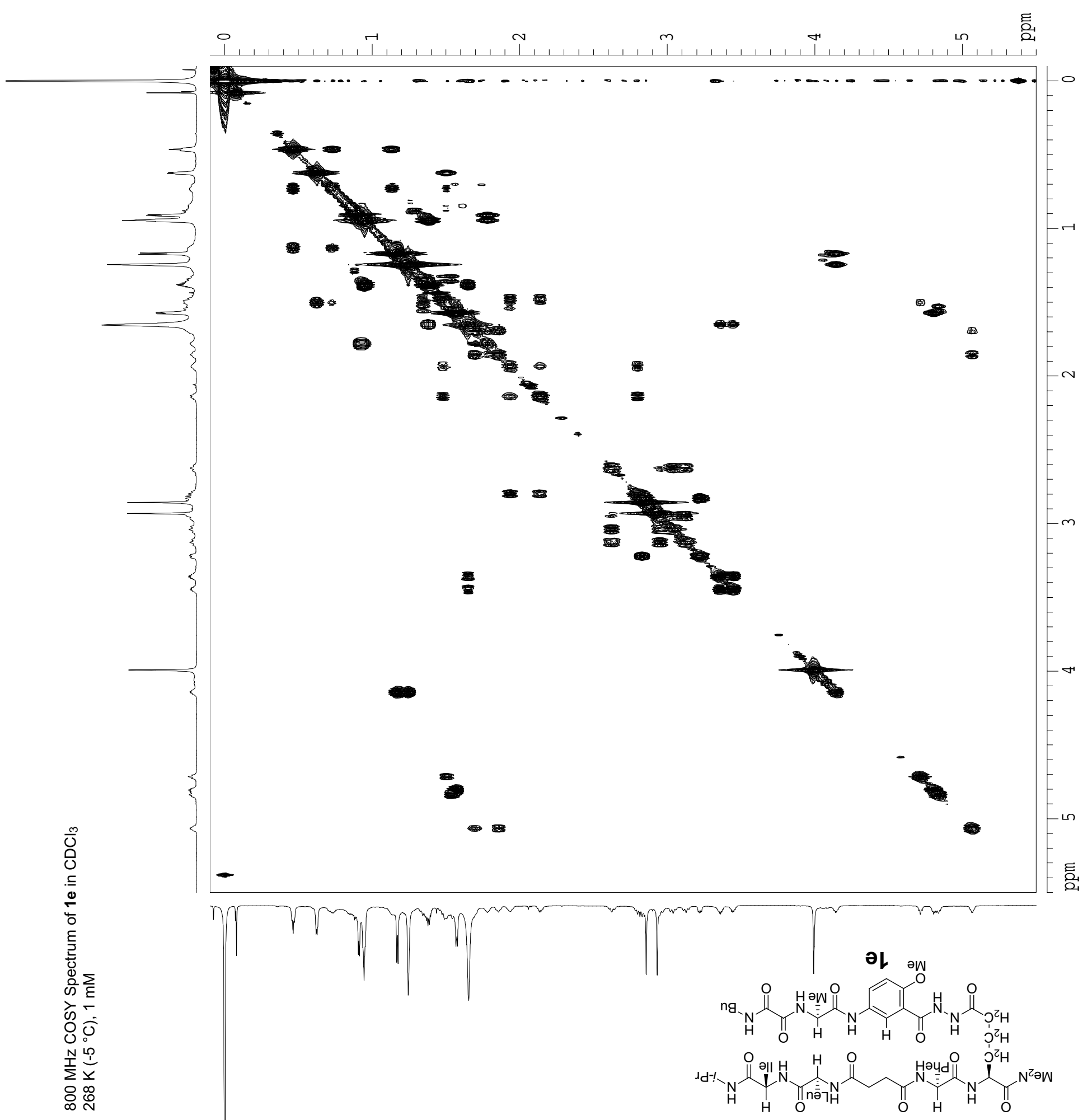


Supporting Information
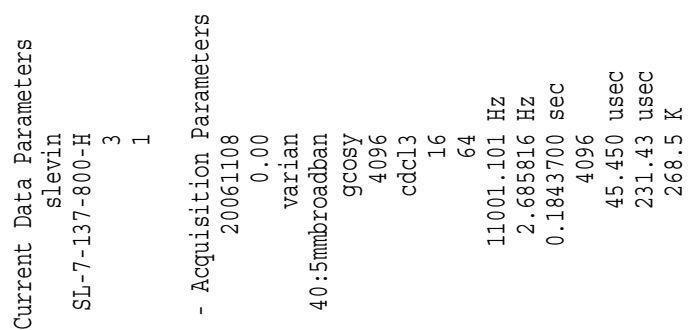

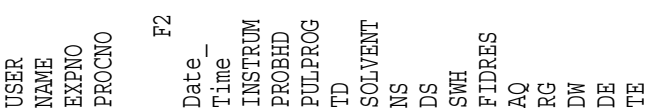

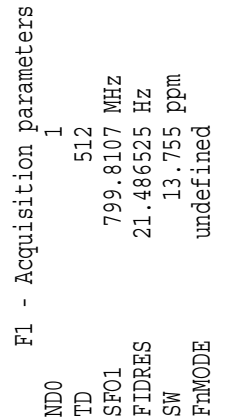

S. Levin and J. S. Nowick

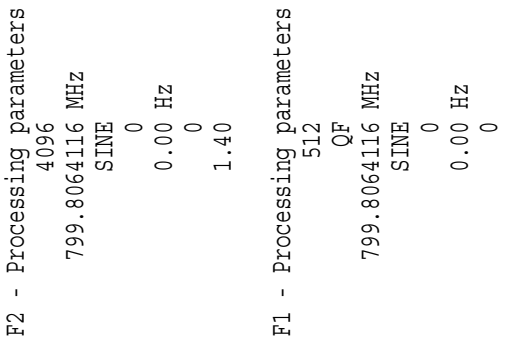

空

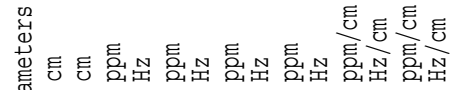

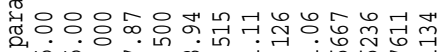
늠 a न 会

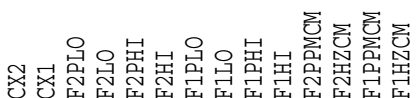

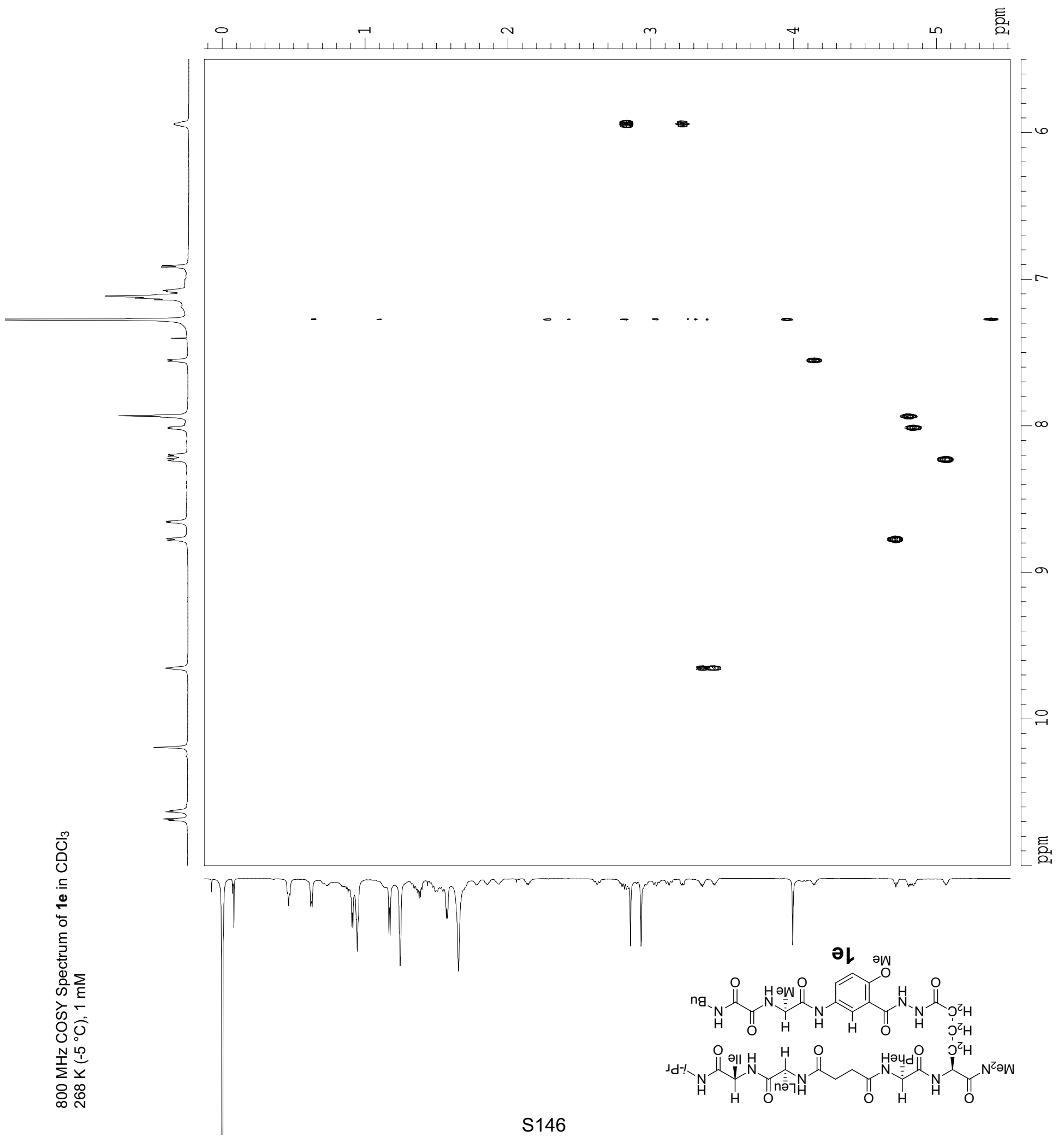


Supporting Information
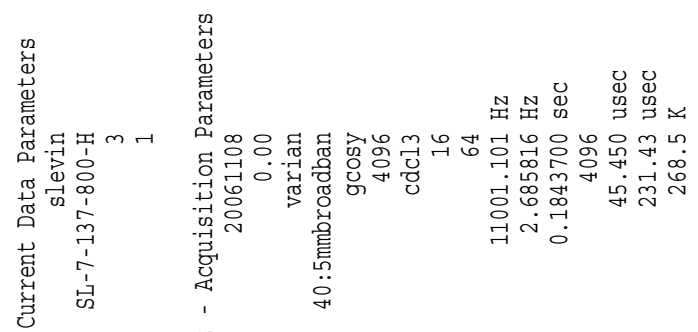

舀罢总总
\&

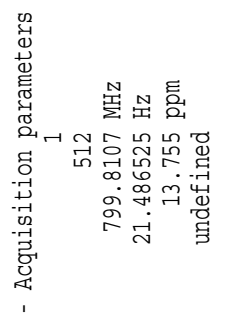

计

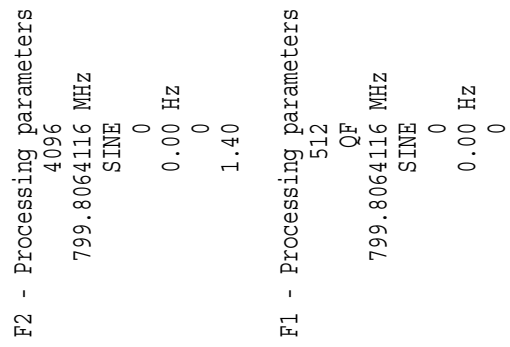

厒
S. Levin and J. S. Nowick

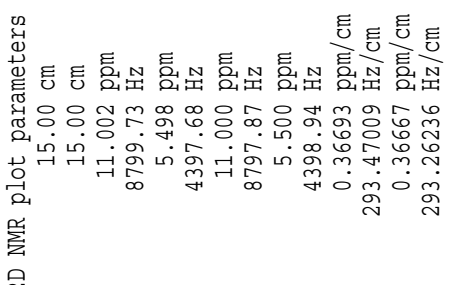

ลิ
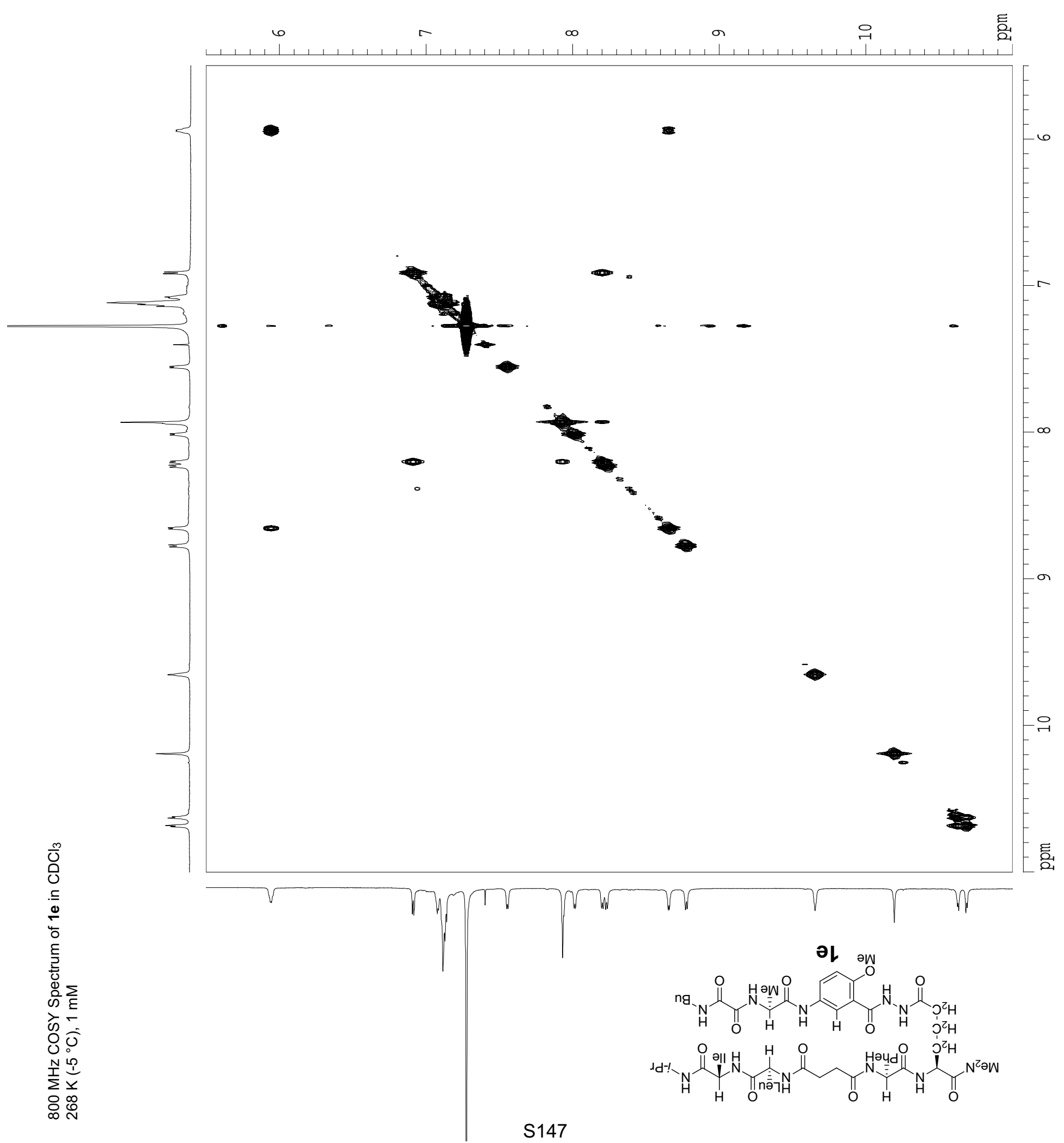
Supporting Information

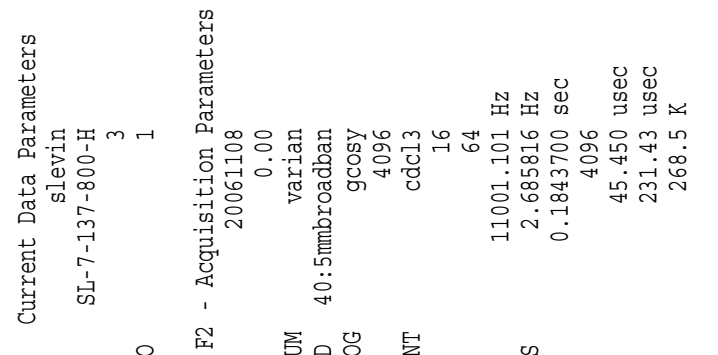

S. Levin and J. S. Nowick

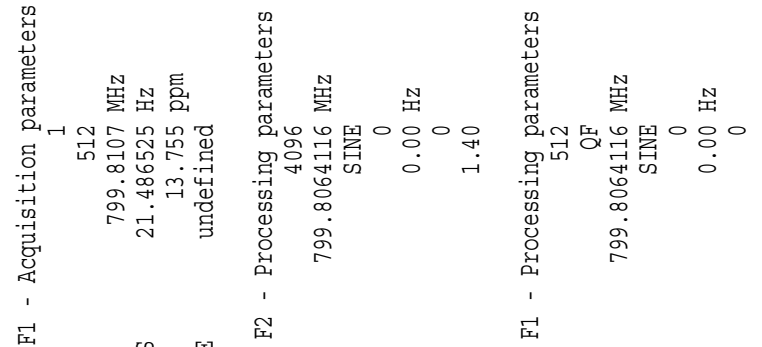

岂

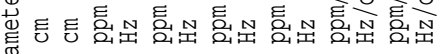

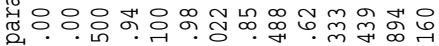

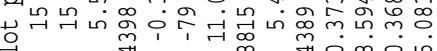
管

ล

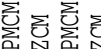

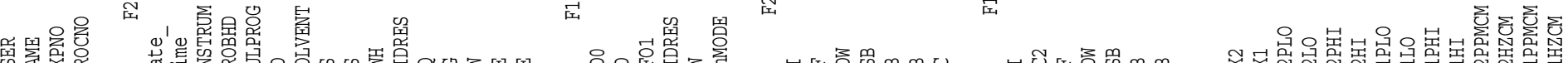

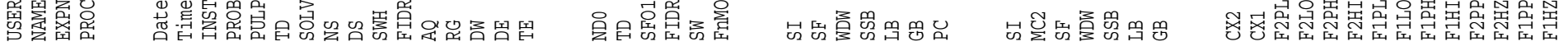

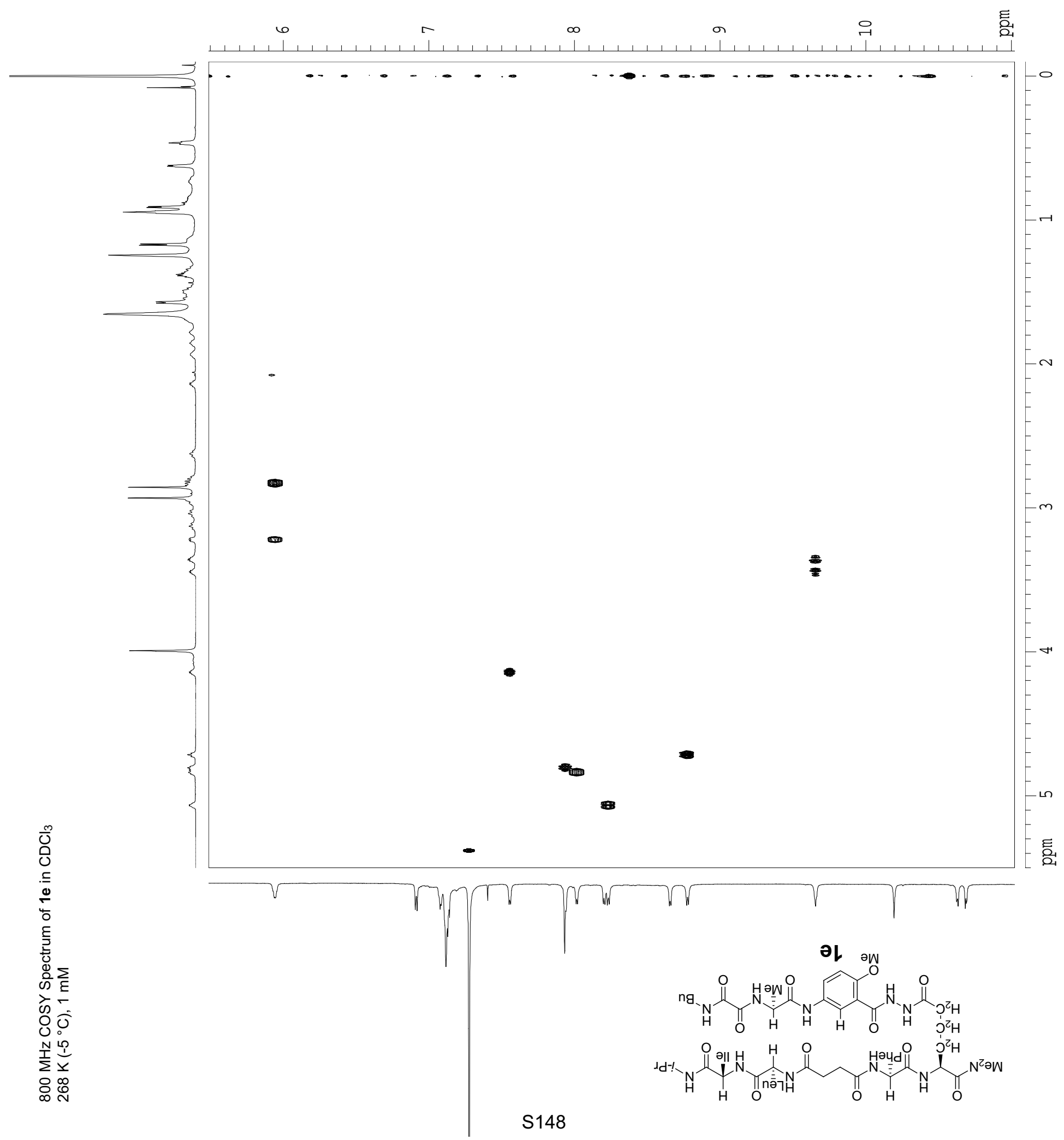


Supporting Information
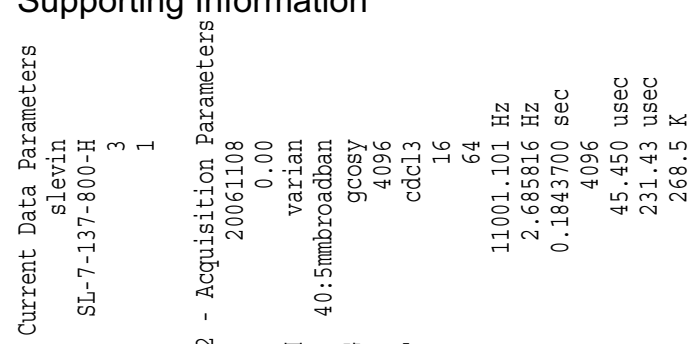

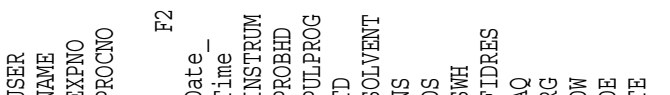

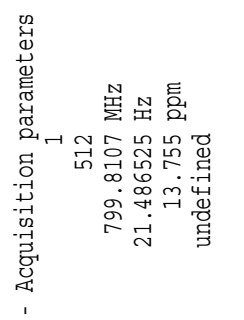

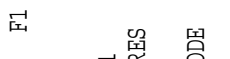

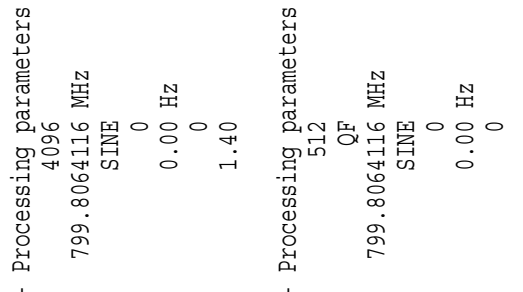

采空
S. Levin and J. S. Nowick

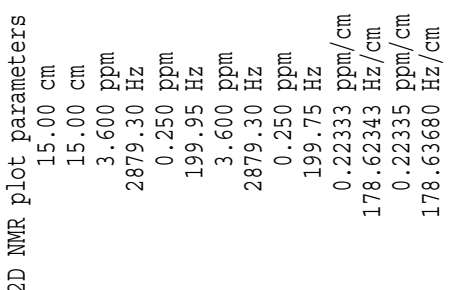

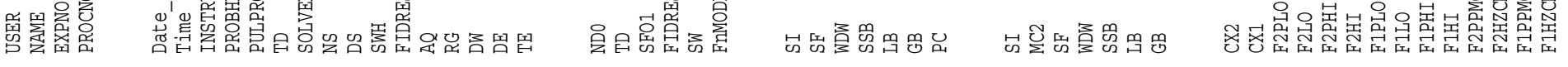

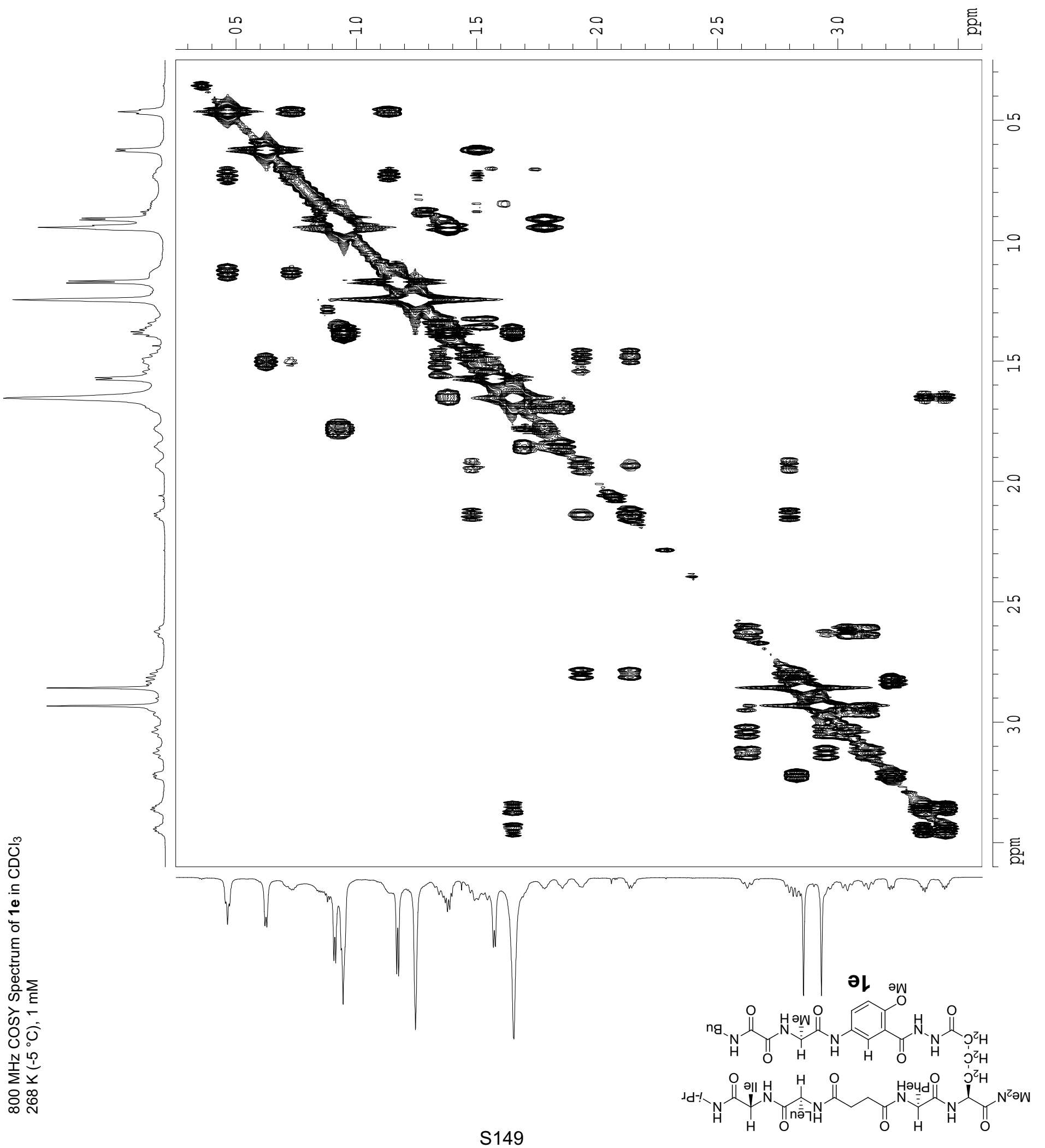


Supporting Information

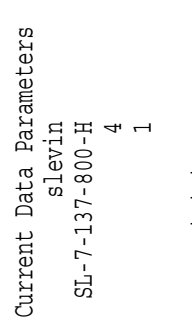

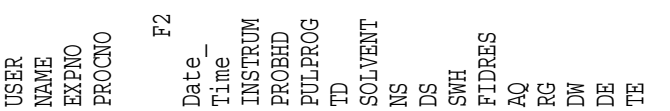

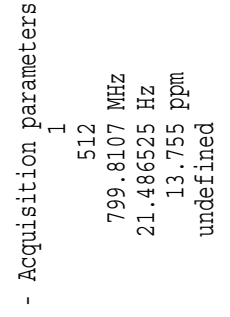

:

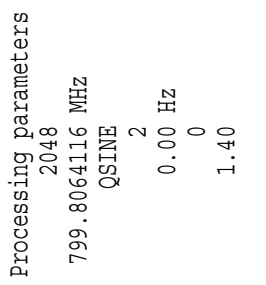

艎

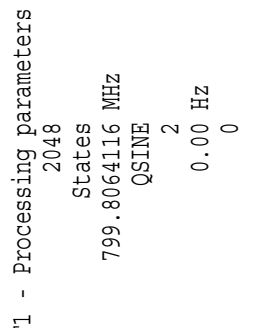

S. Levin and J. S. Nowick

望

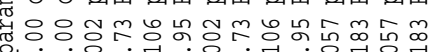
苟 宸

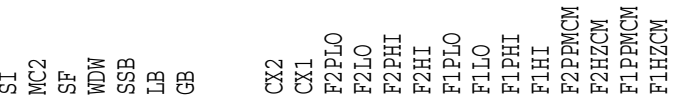

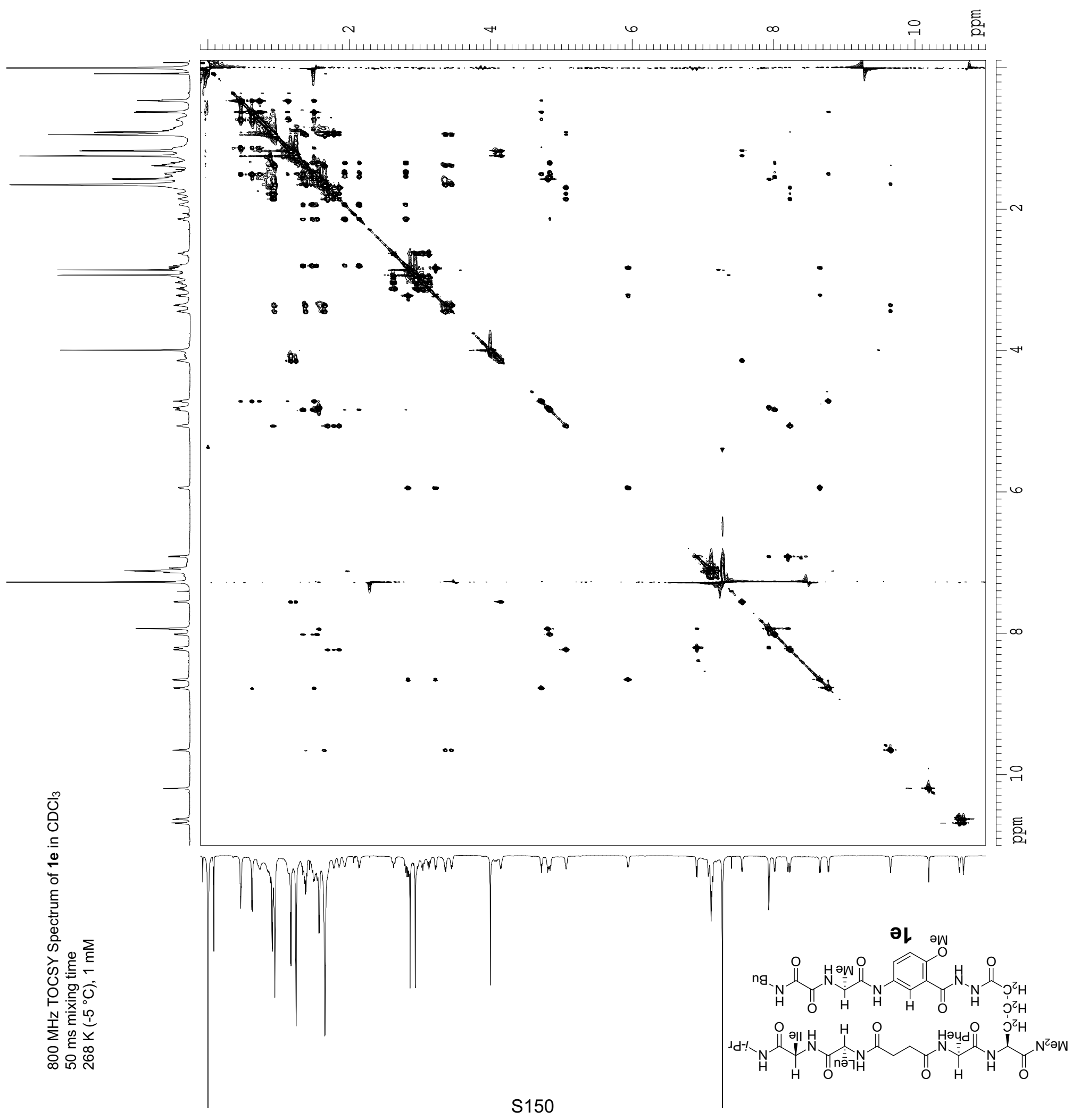




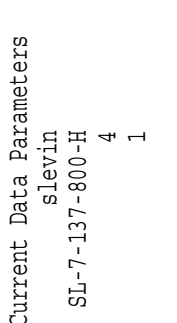

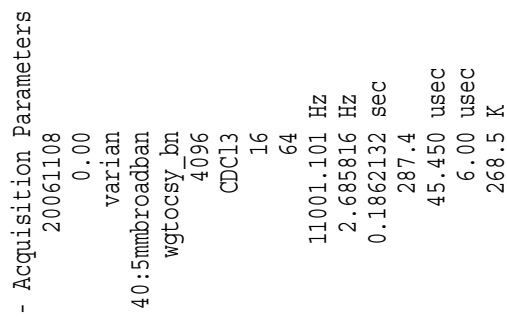

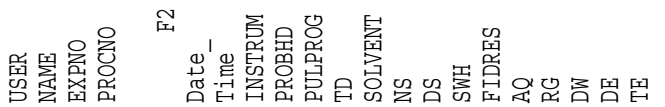

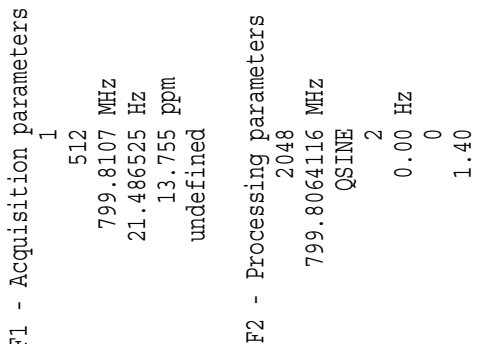

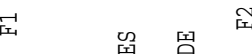

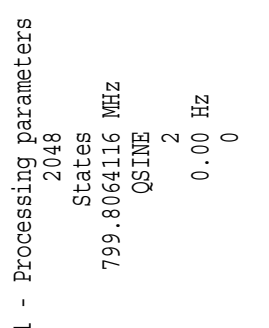

牙

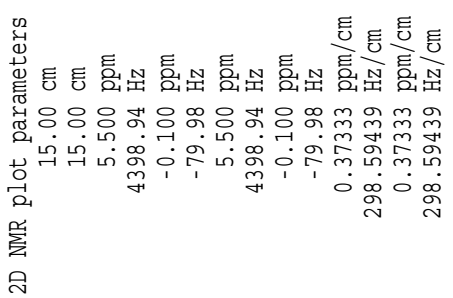

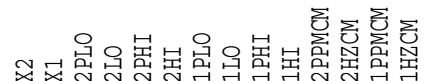

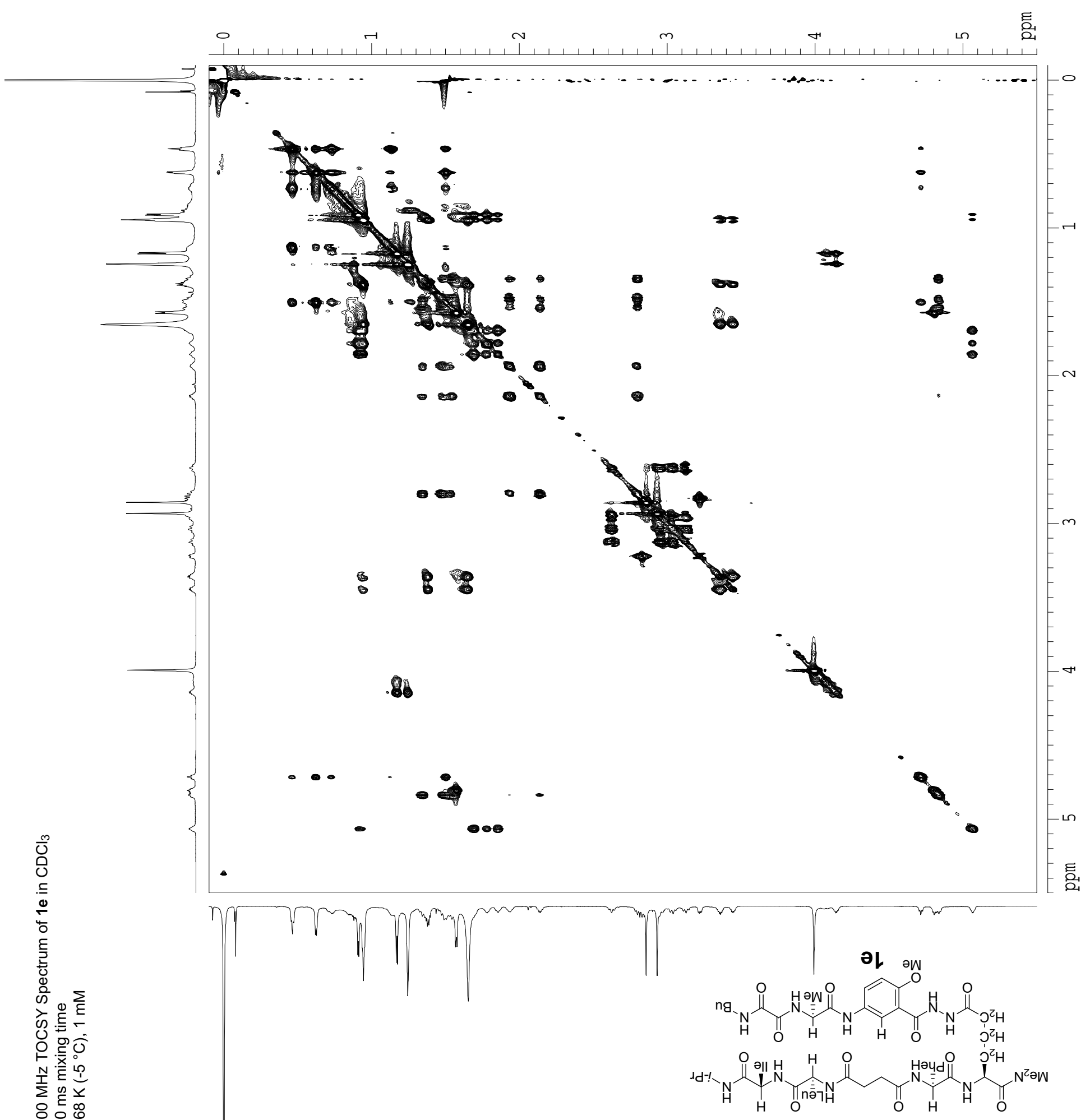




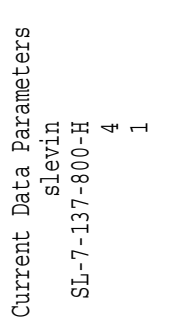

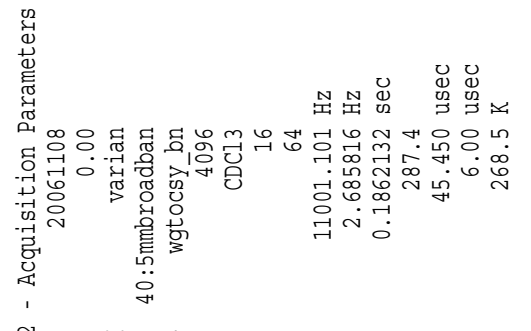

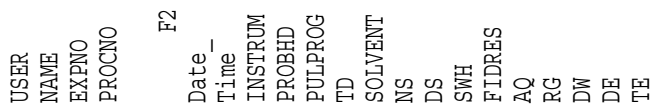

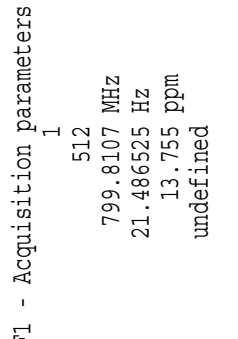

届

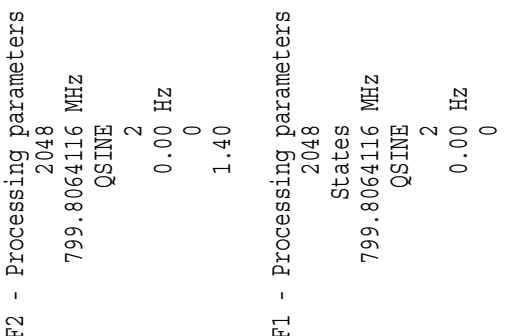

宝

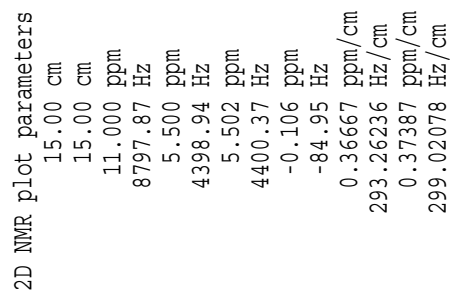

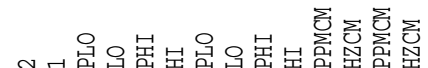

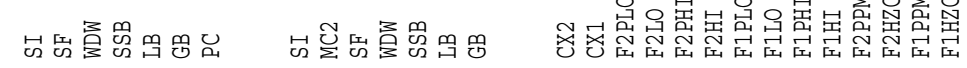

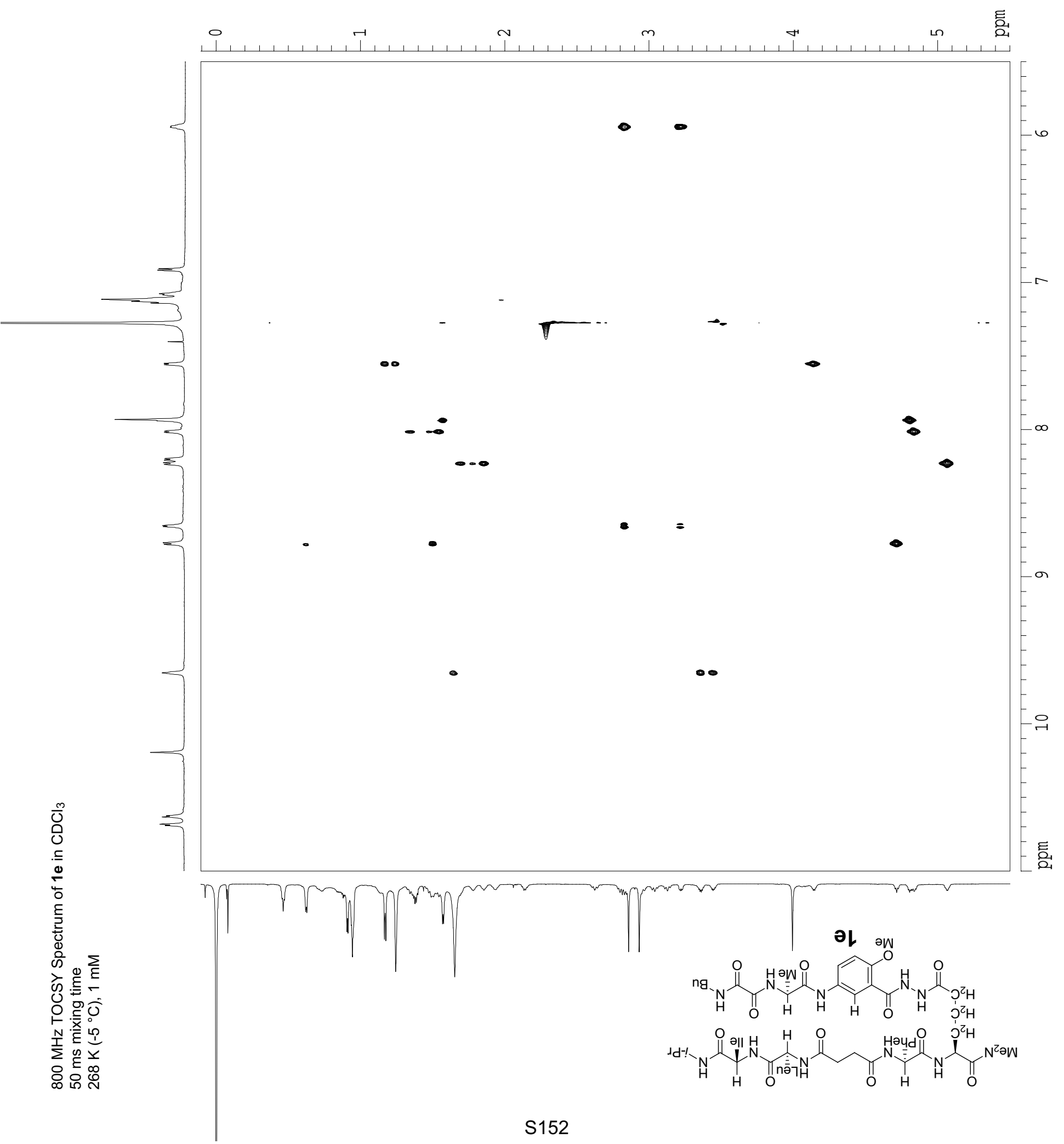


Supporting Information

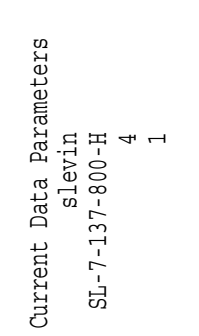

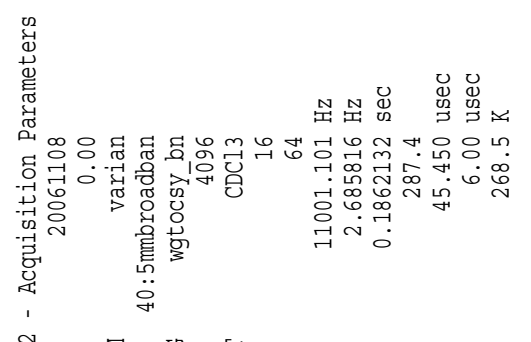

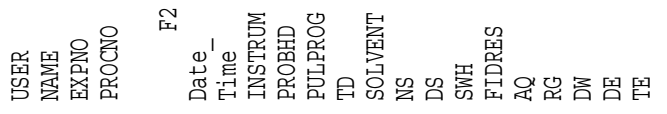

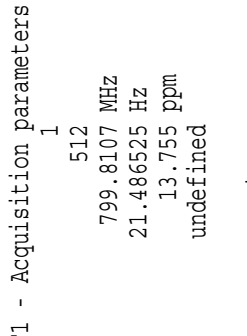

㞋牙劉

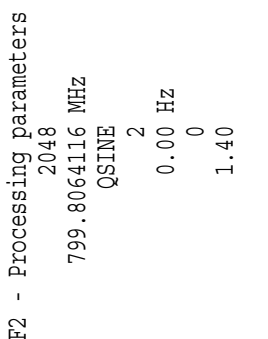

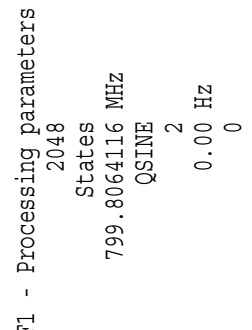

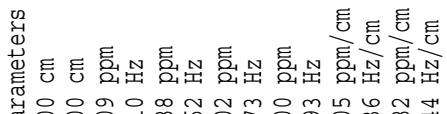

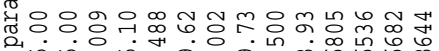

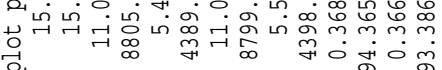
品 ลิ

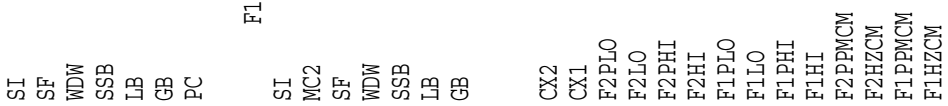

우 o

言

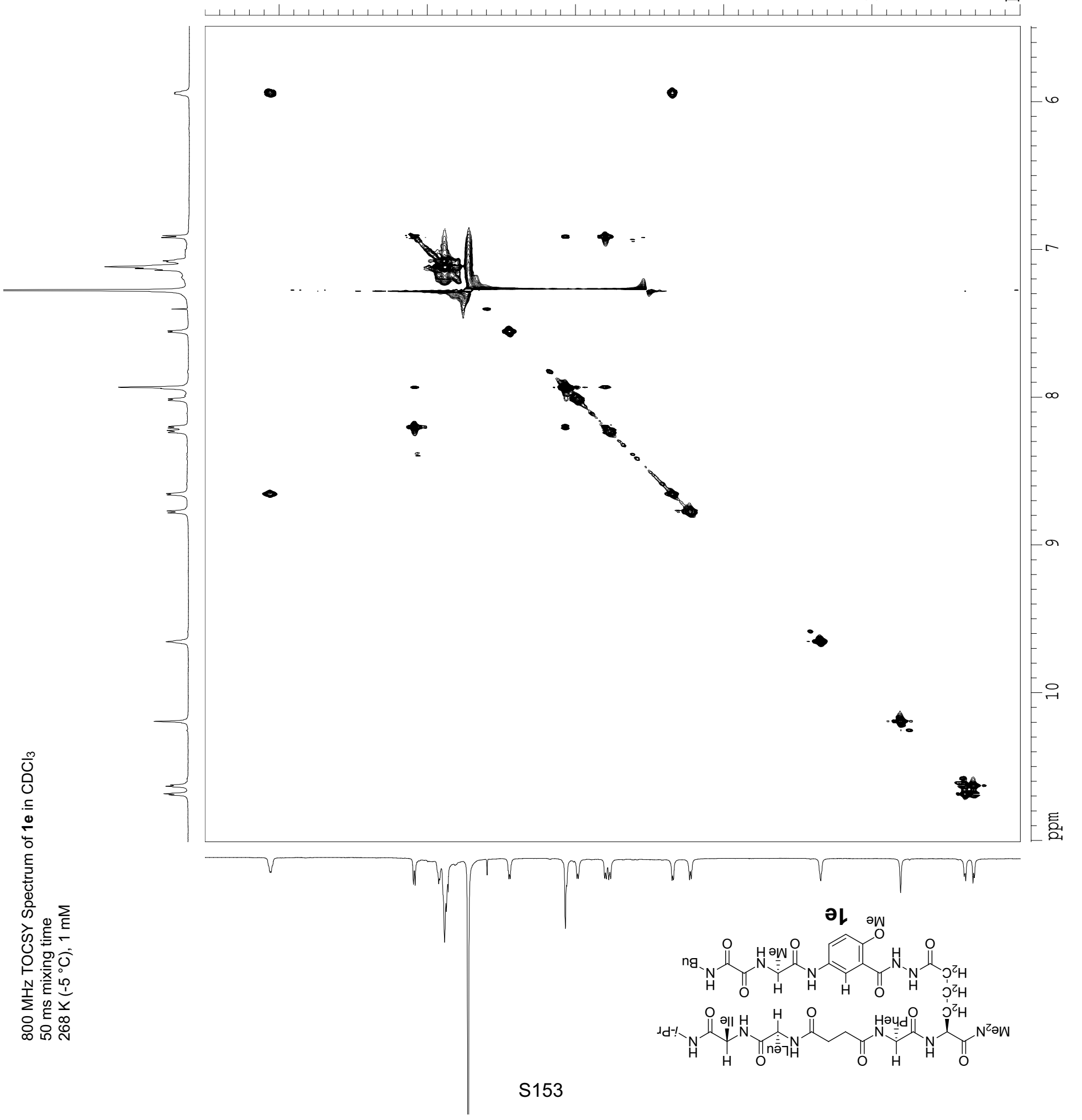


Supporting Information

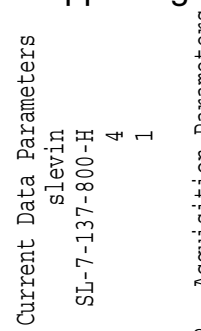

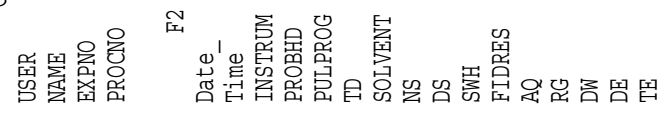

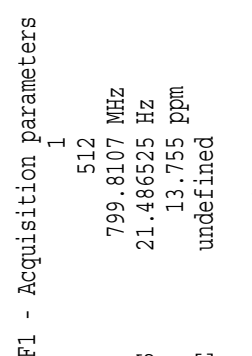

空

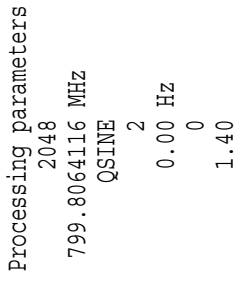

또

S. Levin and J. S. Nowick

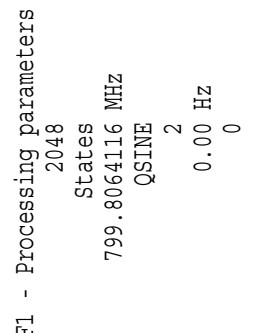

窑

岂

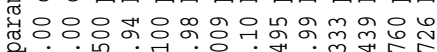

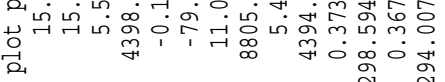
宸

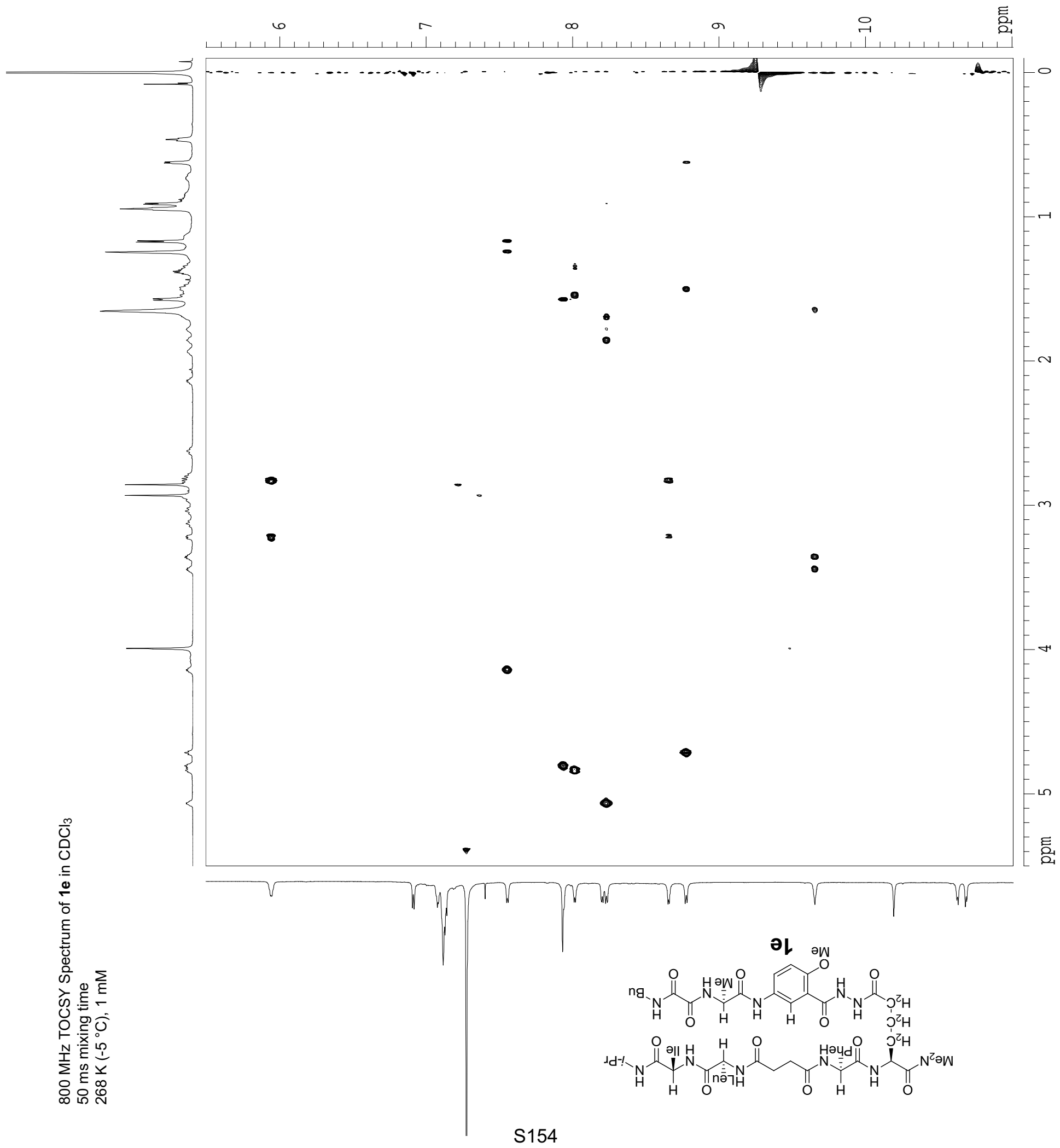




\section{Supporting Information}
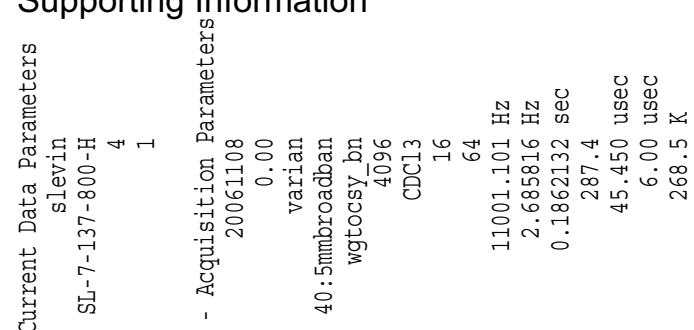

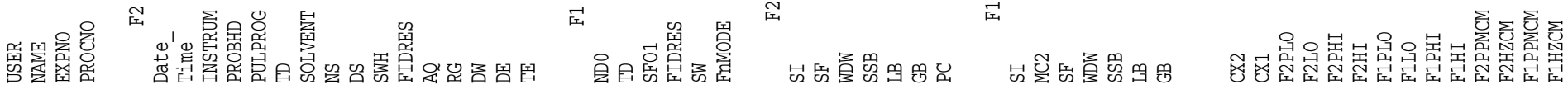

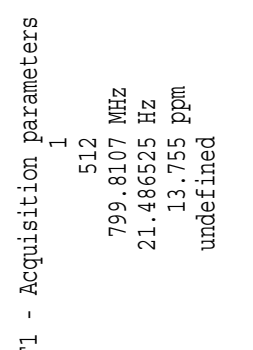

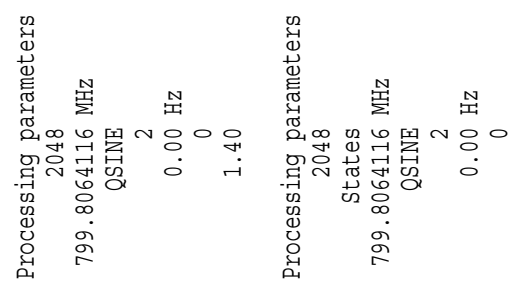

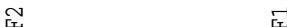

S. Levin and J. S. Nowick

总

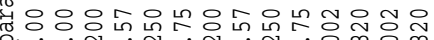

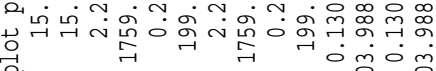
虹

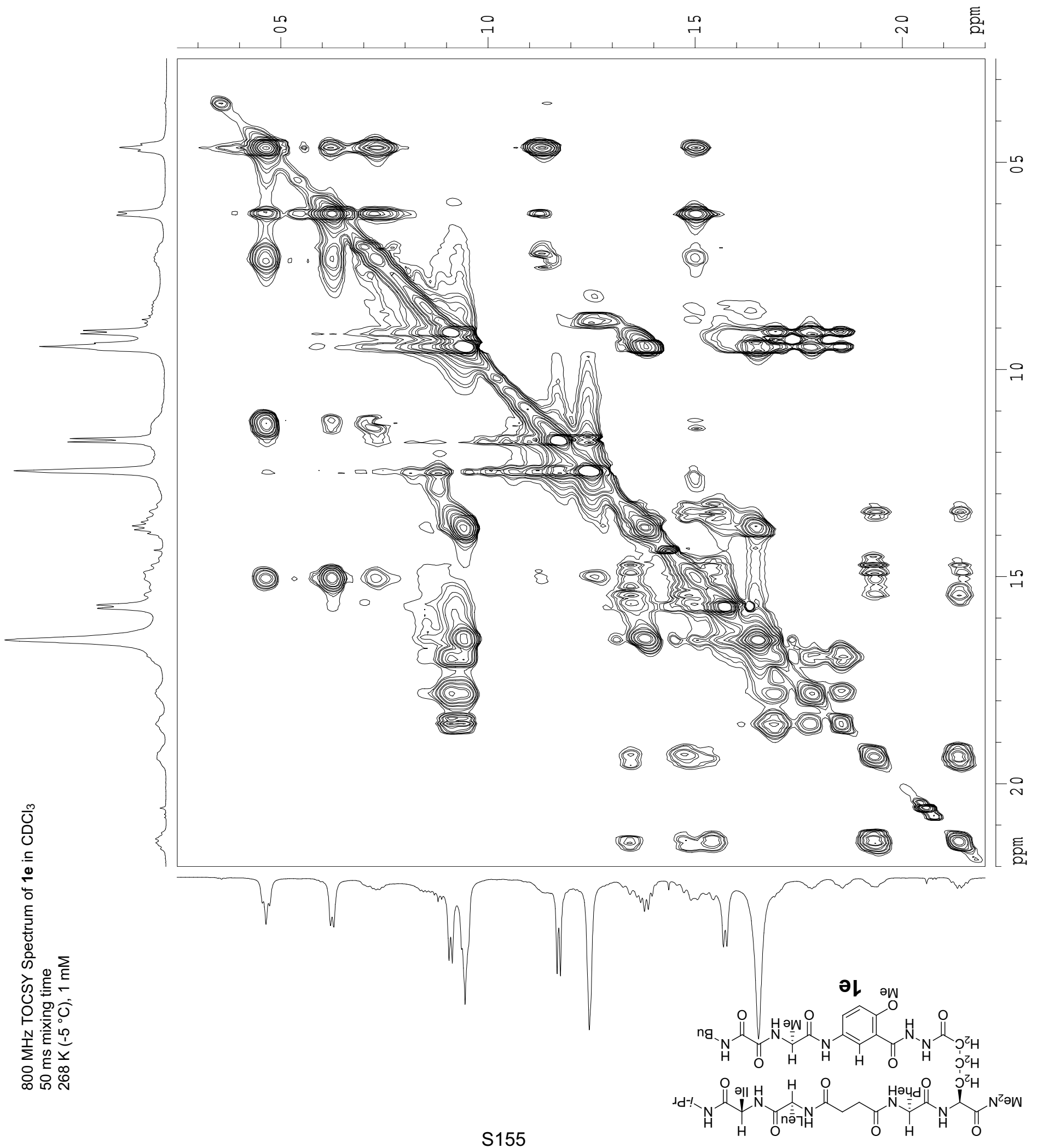


Supporting Information

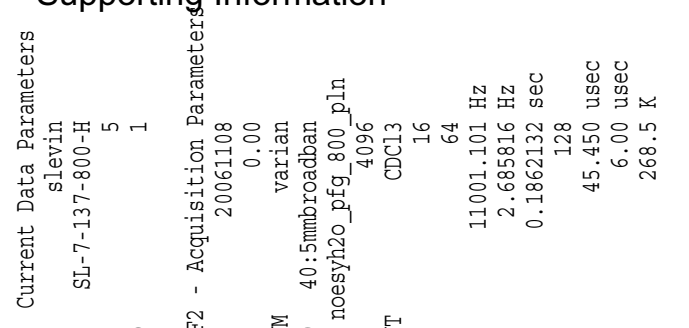

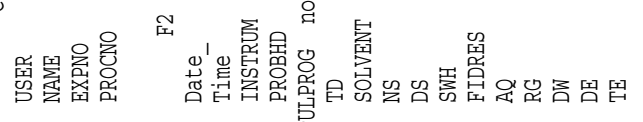
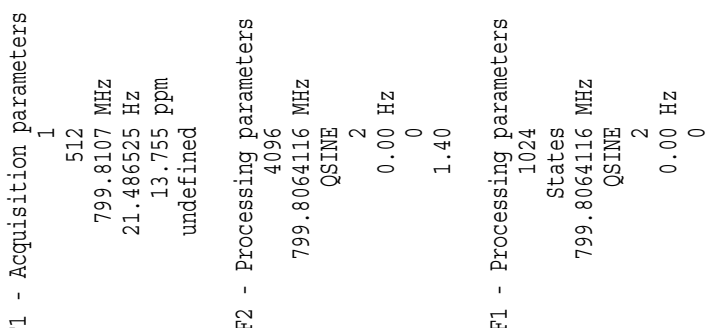

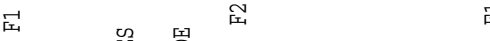

S. Levin and J. S. Nowick

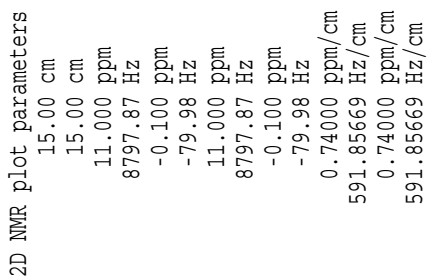

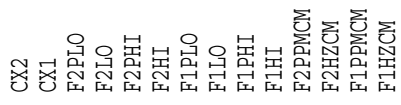

$\sim$

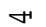

㫐出总思思思品

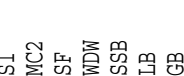

$\infty$

이 हूँ

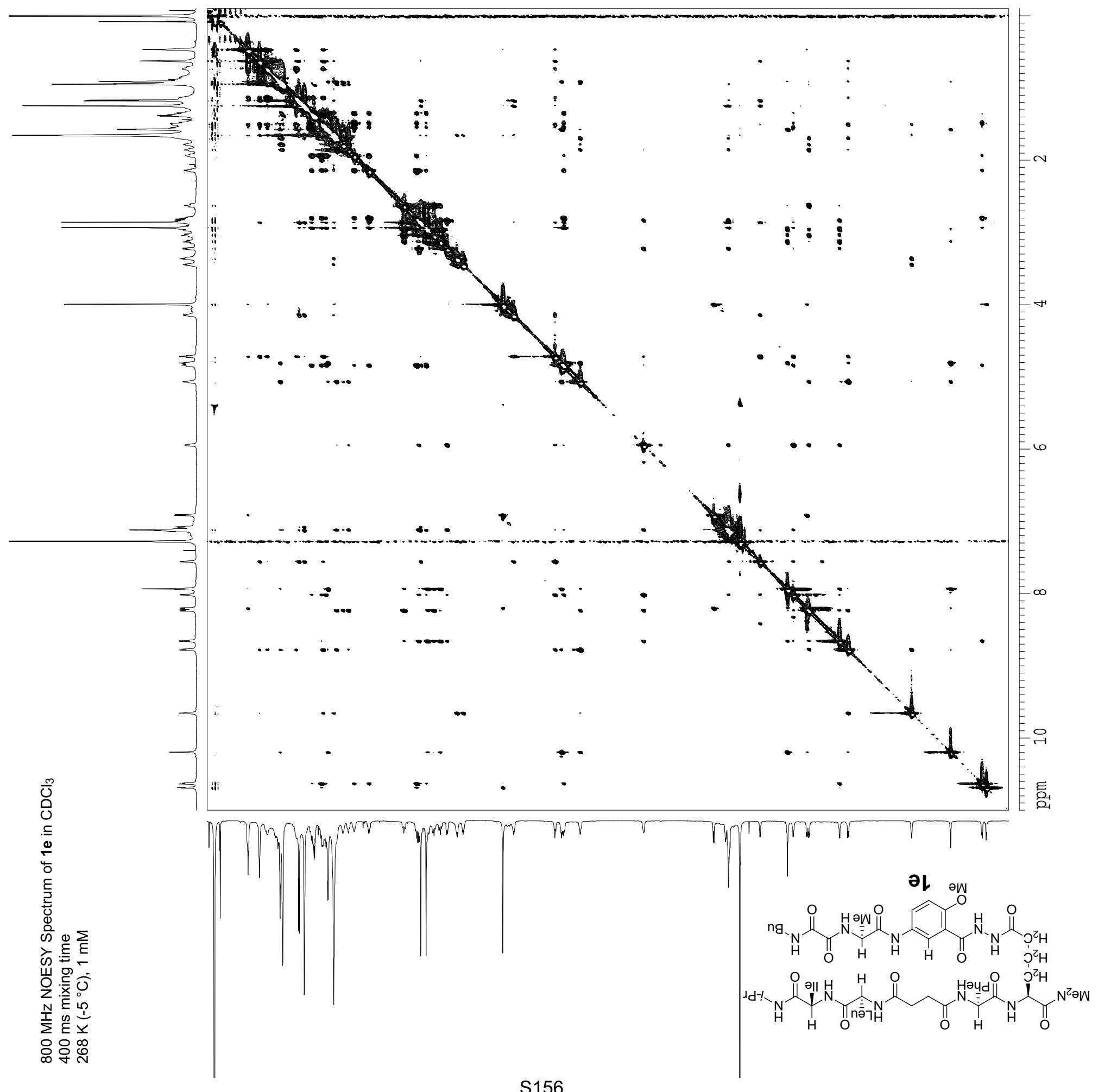


Supporting Information

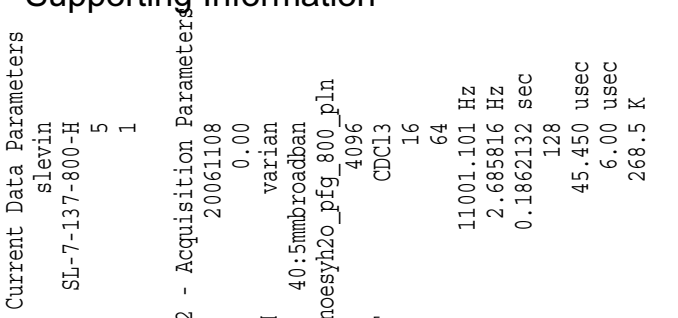

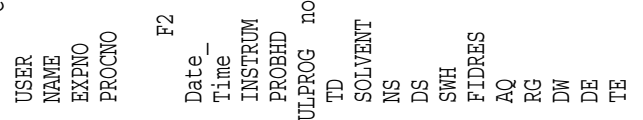

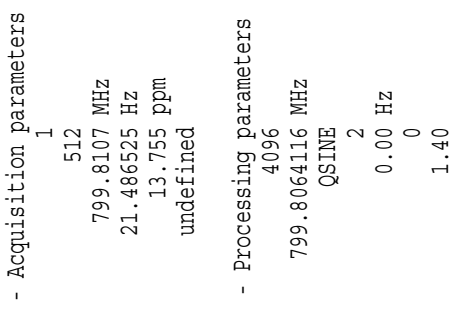

兵
S. Levin and J. S. Nowick

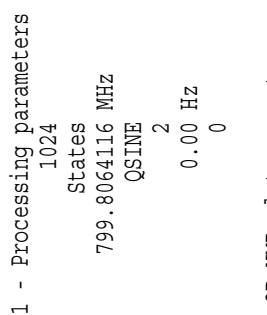

른르을ㄹ

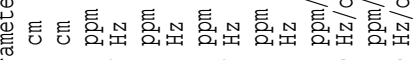

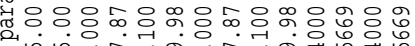
雚 a

N

ナ

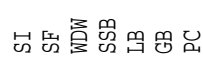

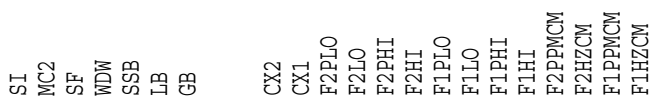

아 혈

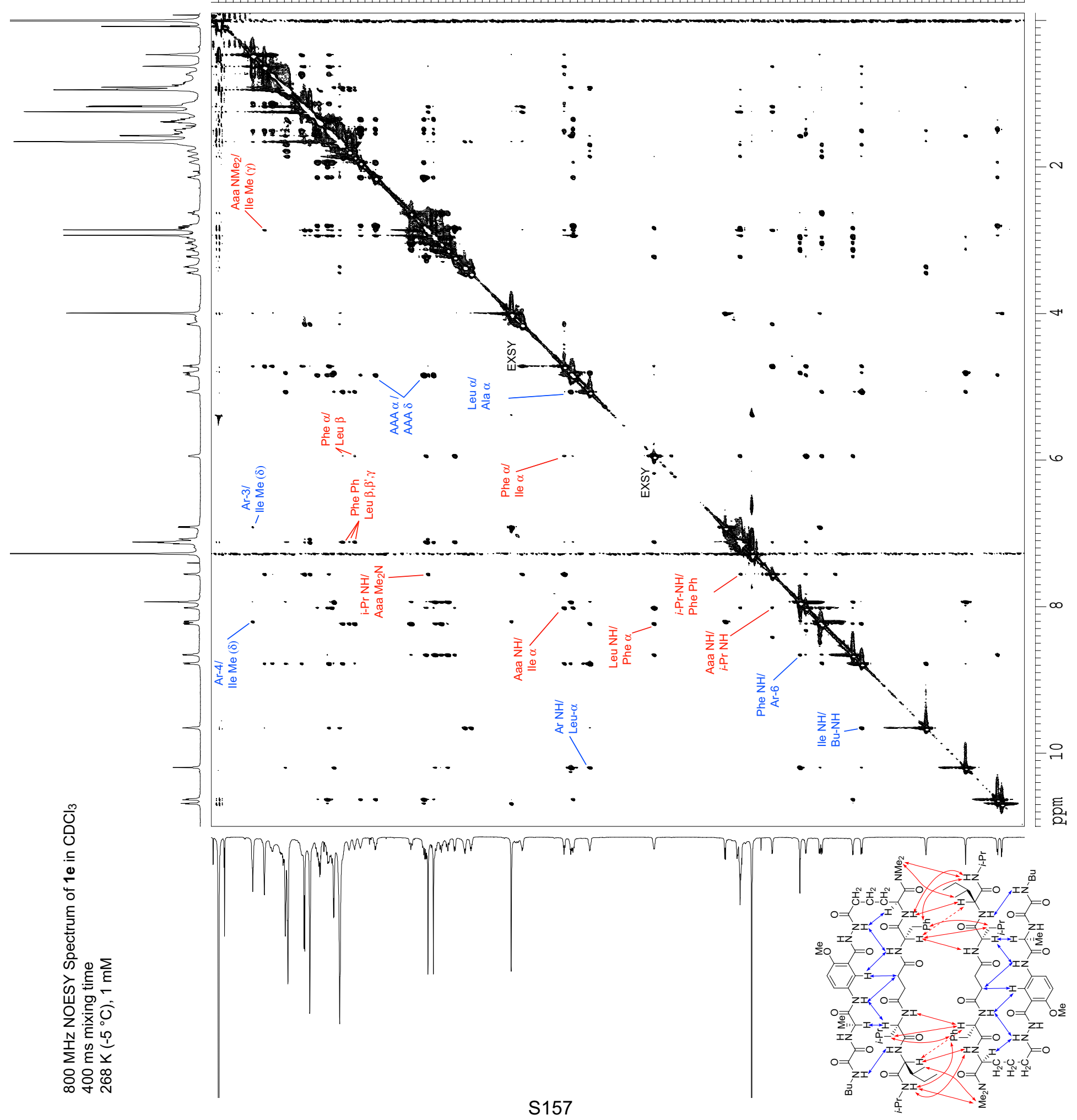


Supporting Information

S. Levin and J. S. Nowick
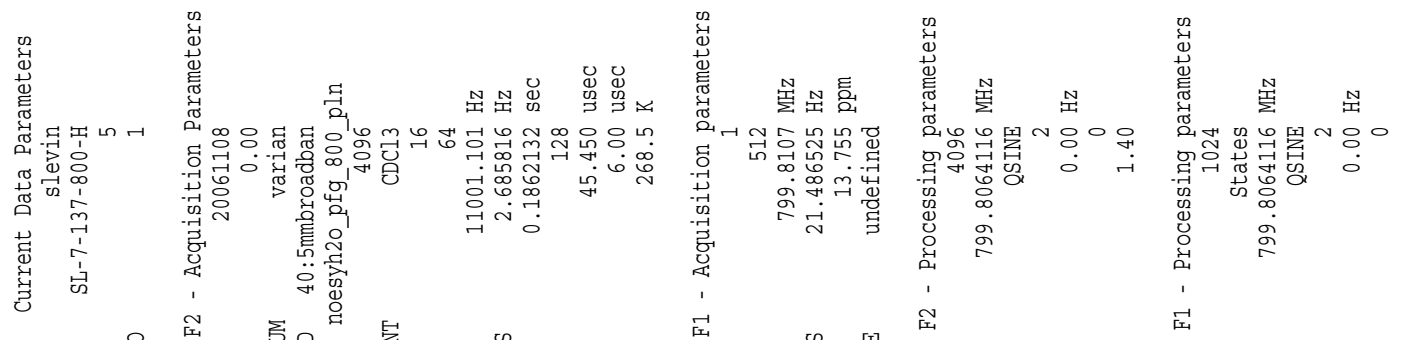

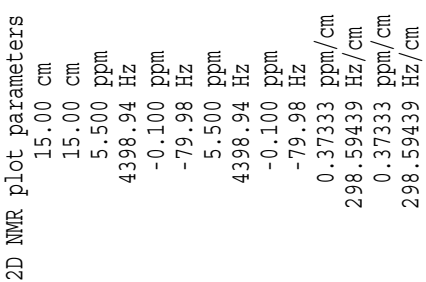

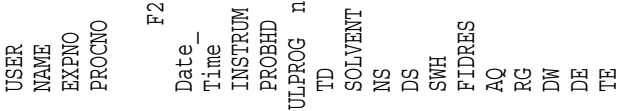

留罢 婇

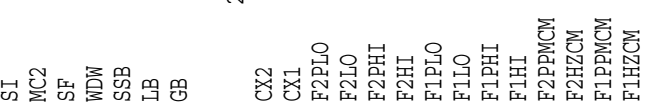

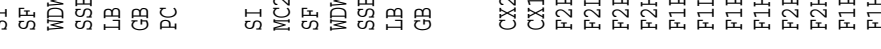

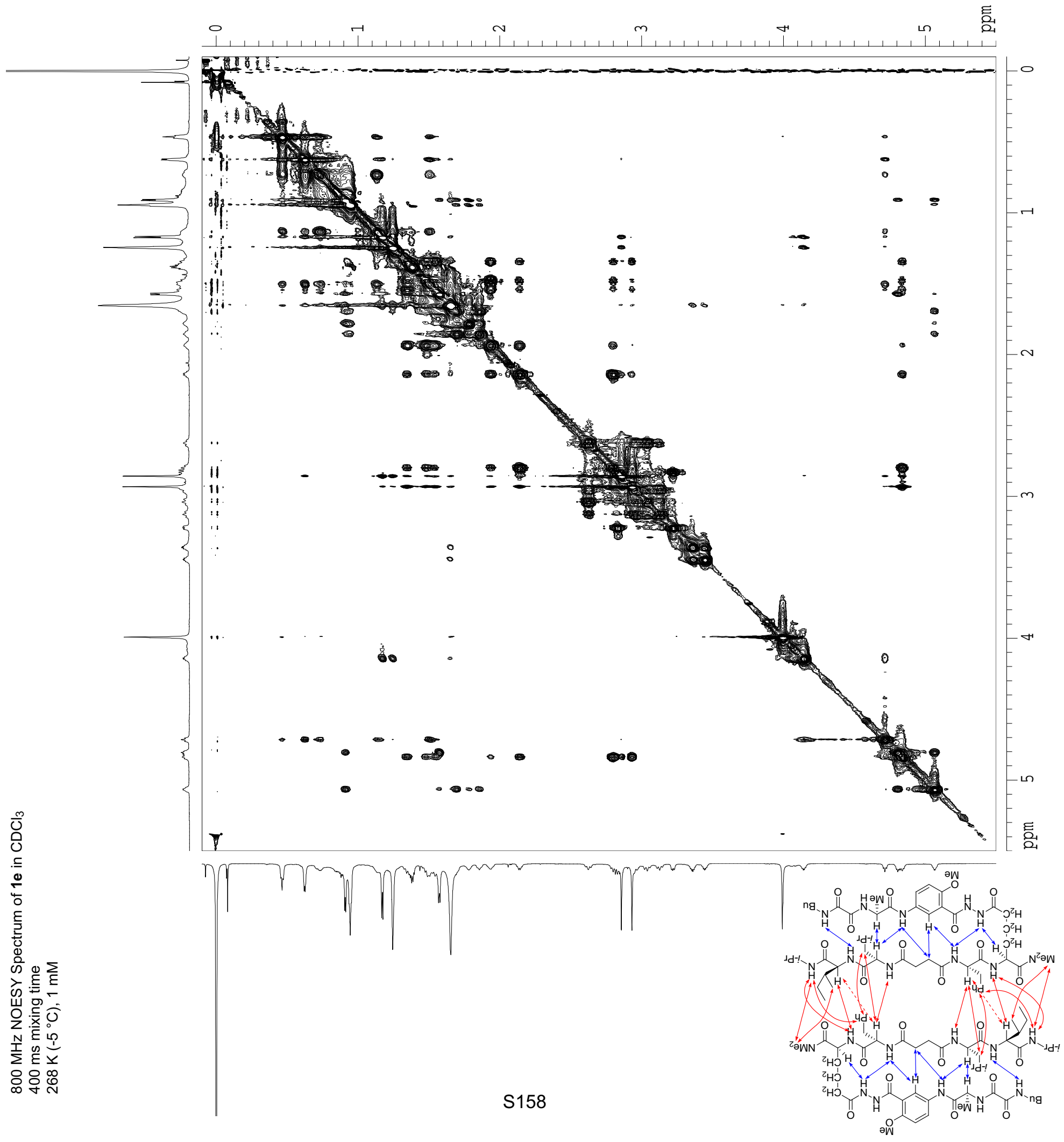




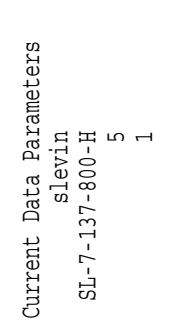

留留虽虽

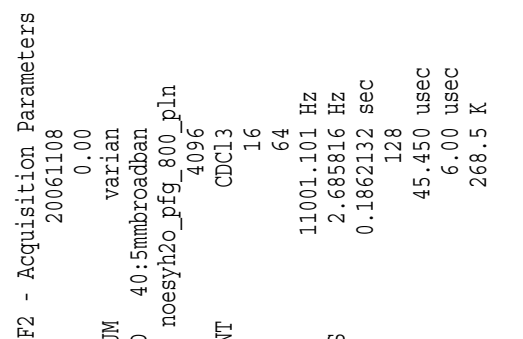

त

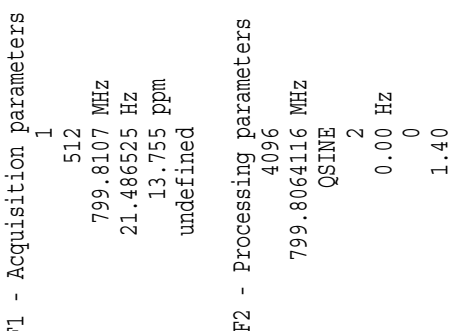

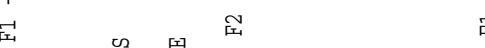

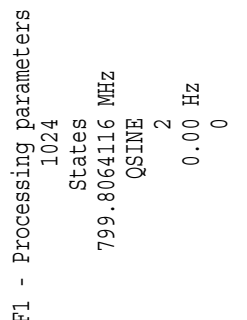

$\curvearrowright$

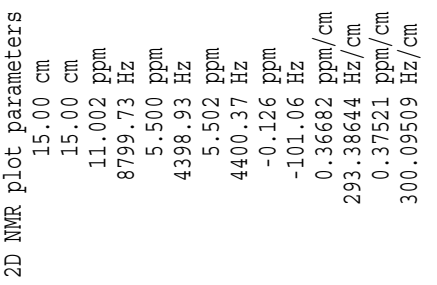

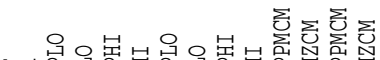

๓ัง

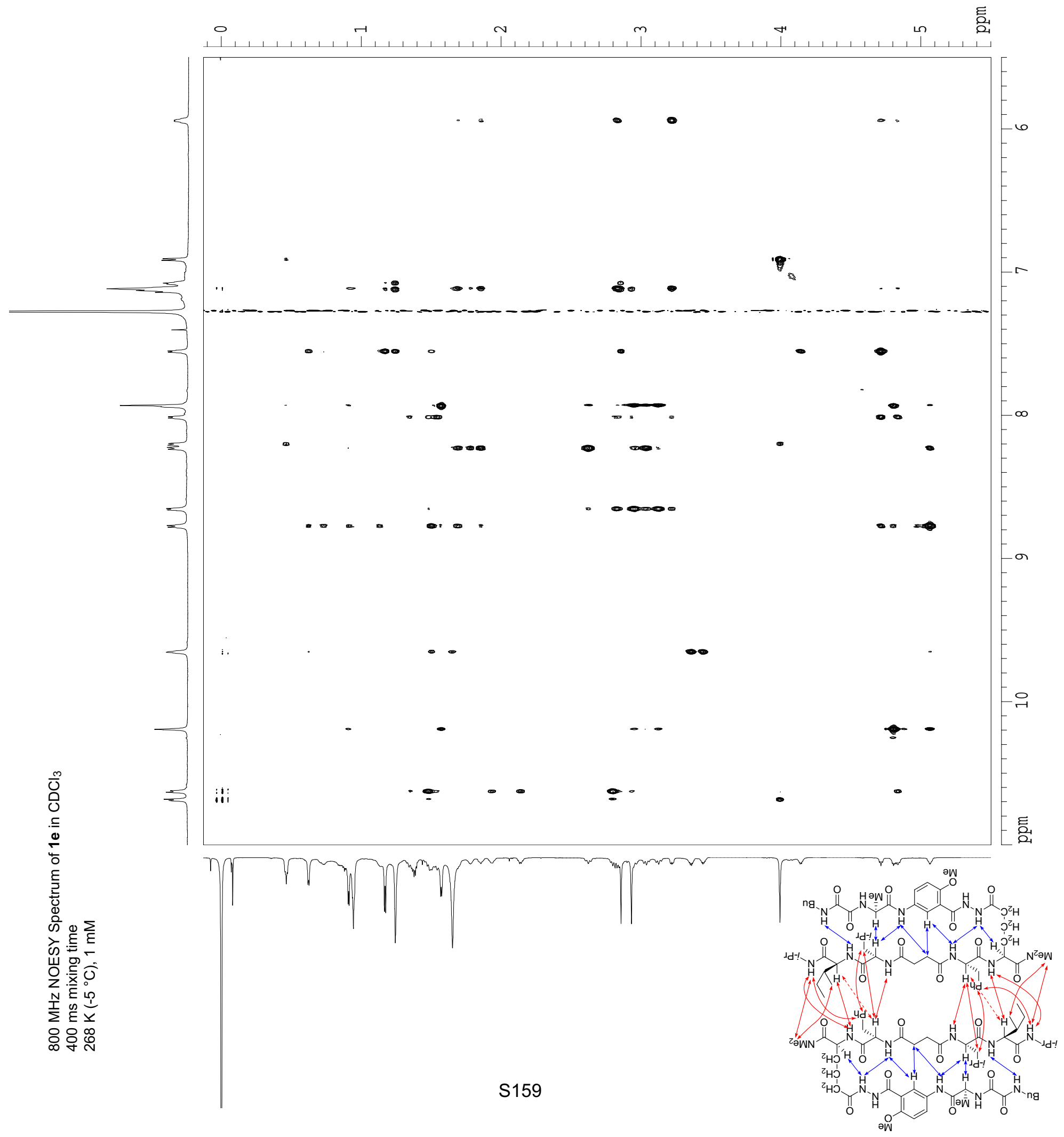




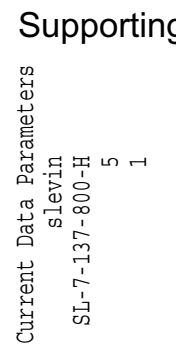

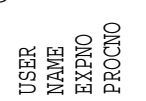

\section{Information}

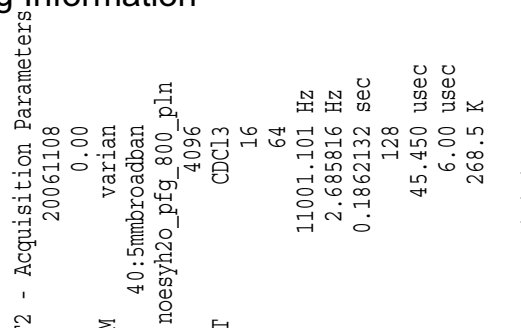

i

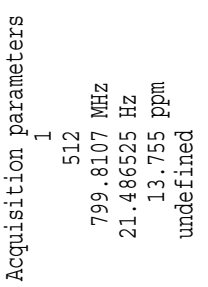

星

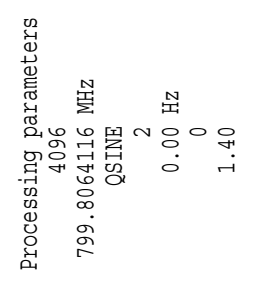

$\stackrel{\sim}{*}$

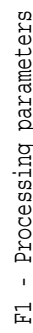

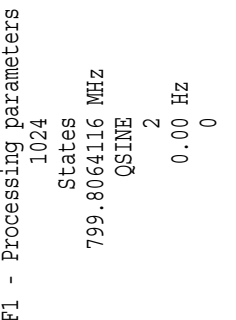

S. Levin and J. S. Nowick

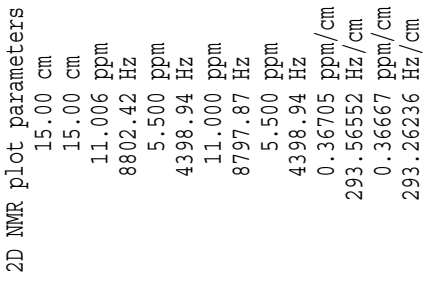

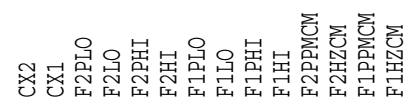

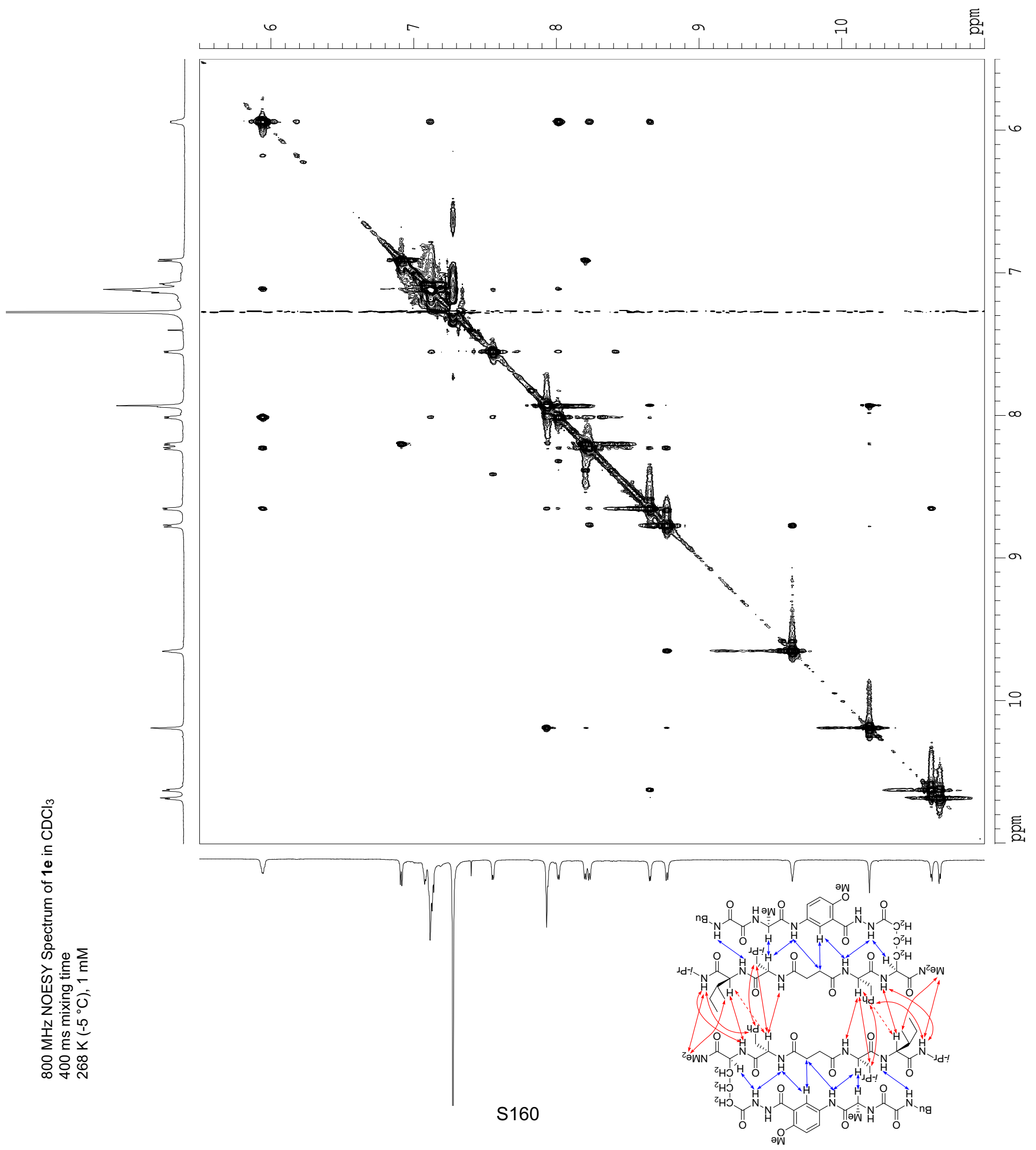




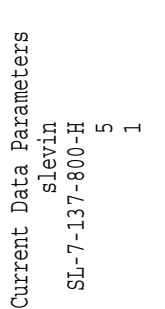

罢舆总总

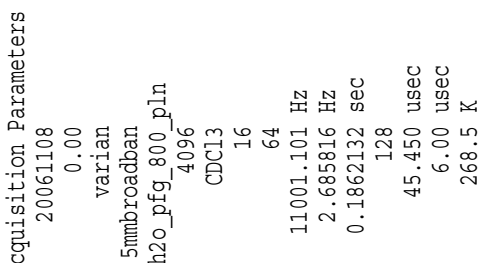

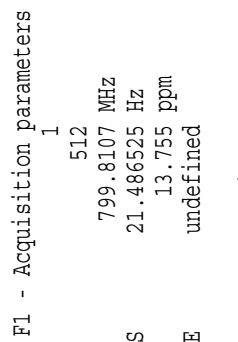

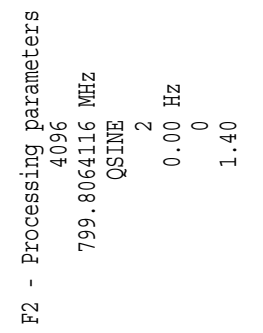

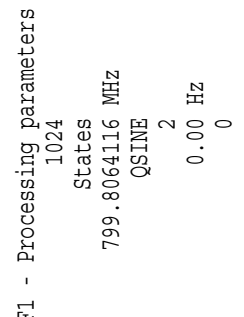

岂

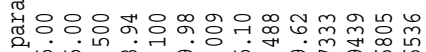

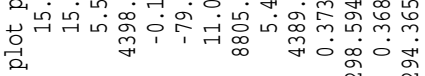
塄

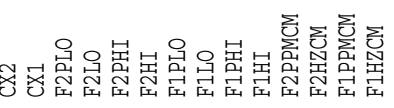

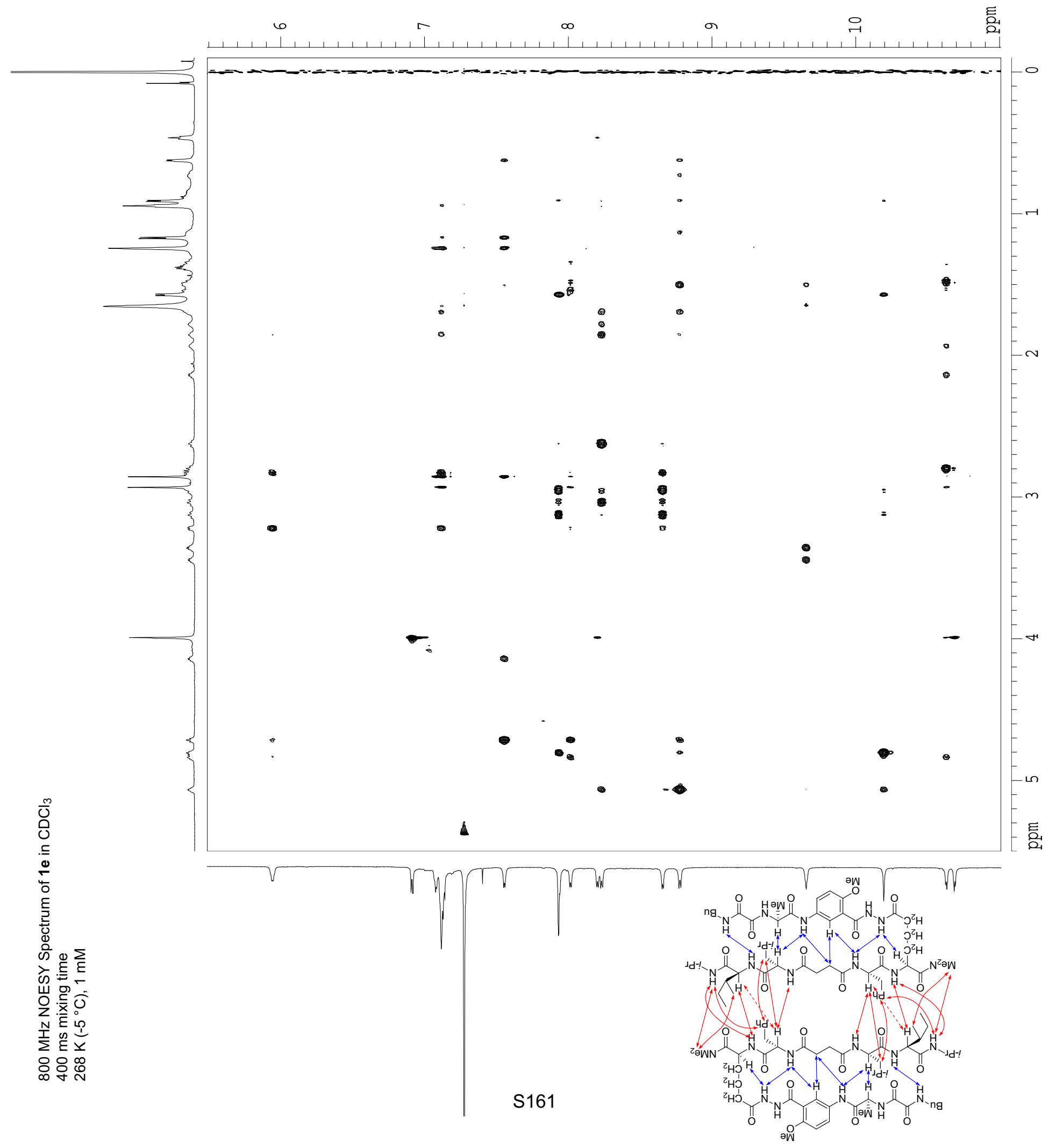


Supporting Information
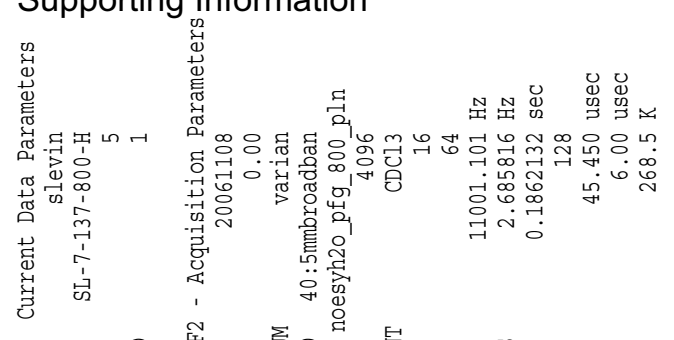

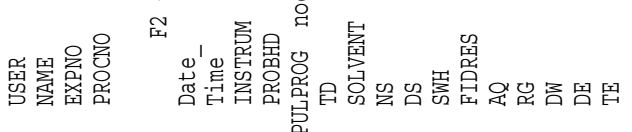

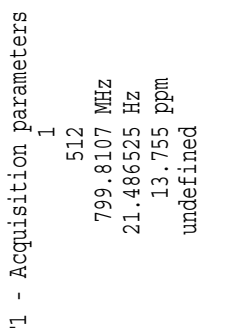

压

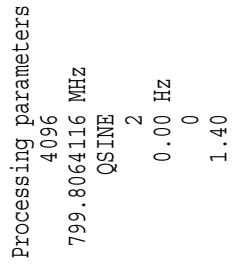

平

S. Levin and J. S. Nowick

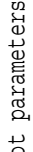
嵒

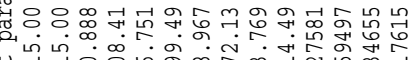

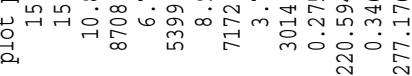
罢 ค

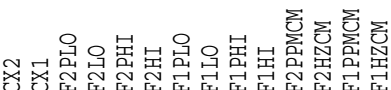

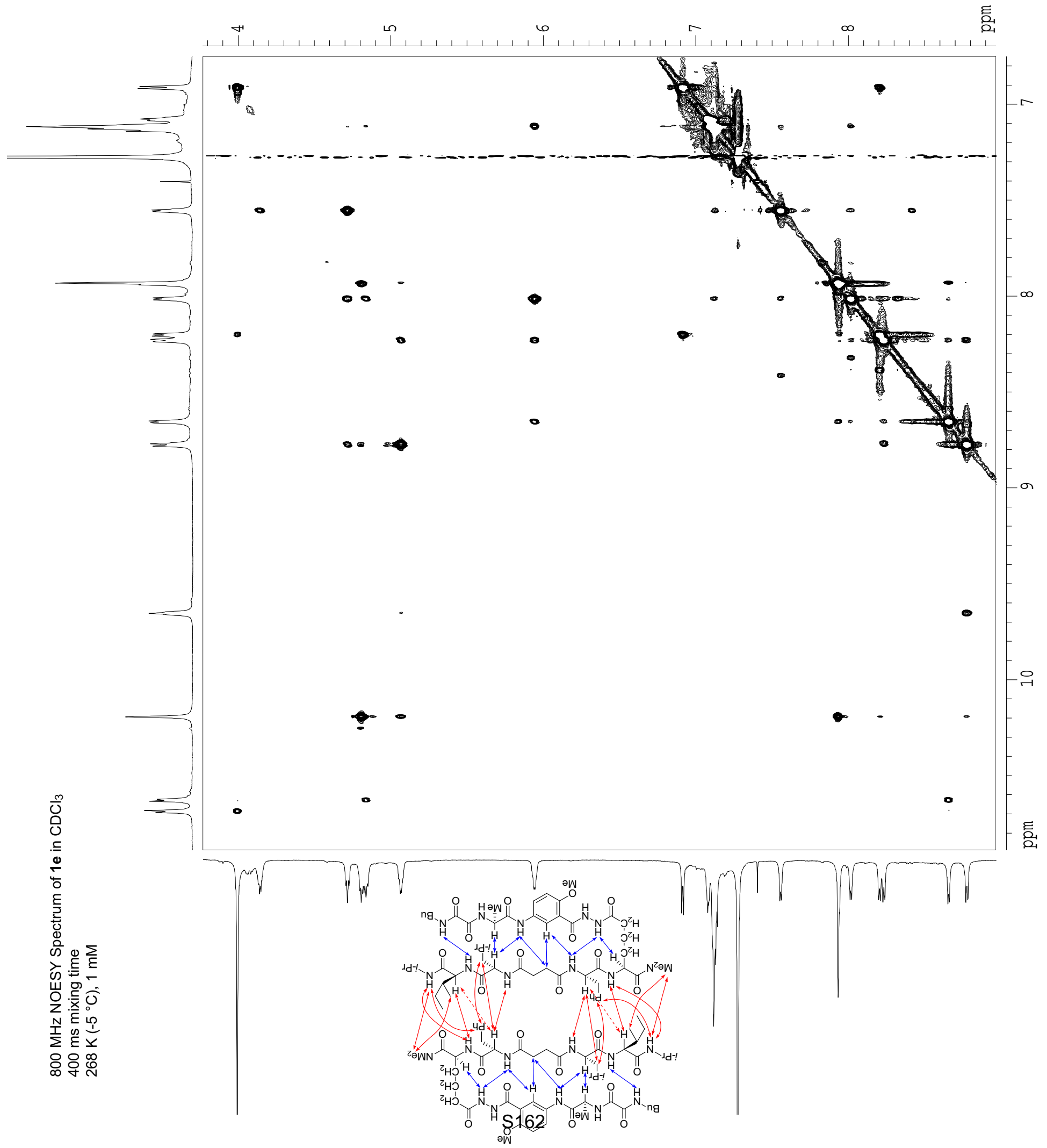


Supporting Information

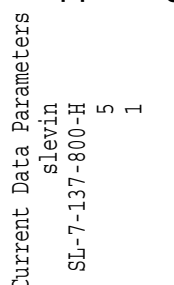

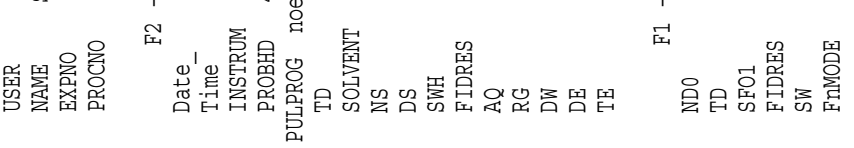

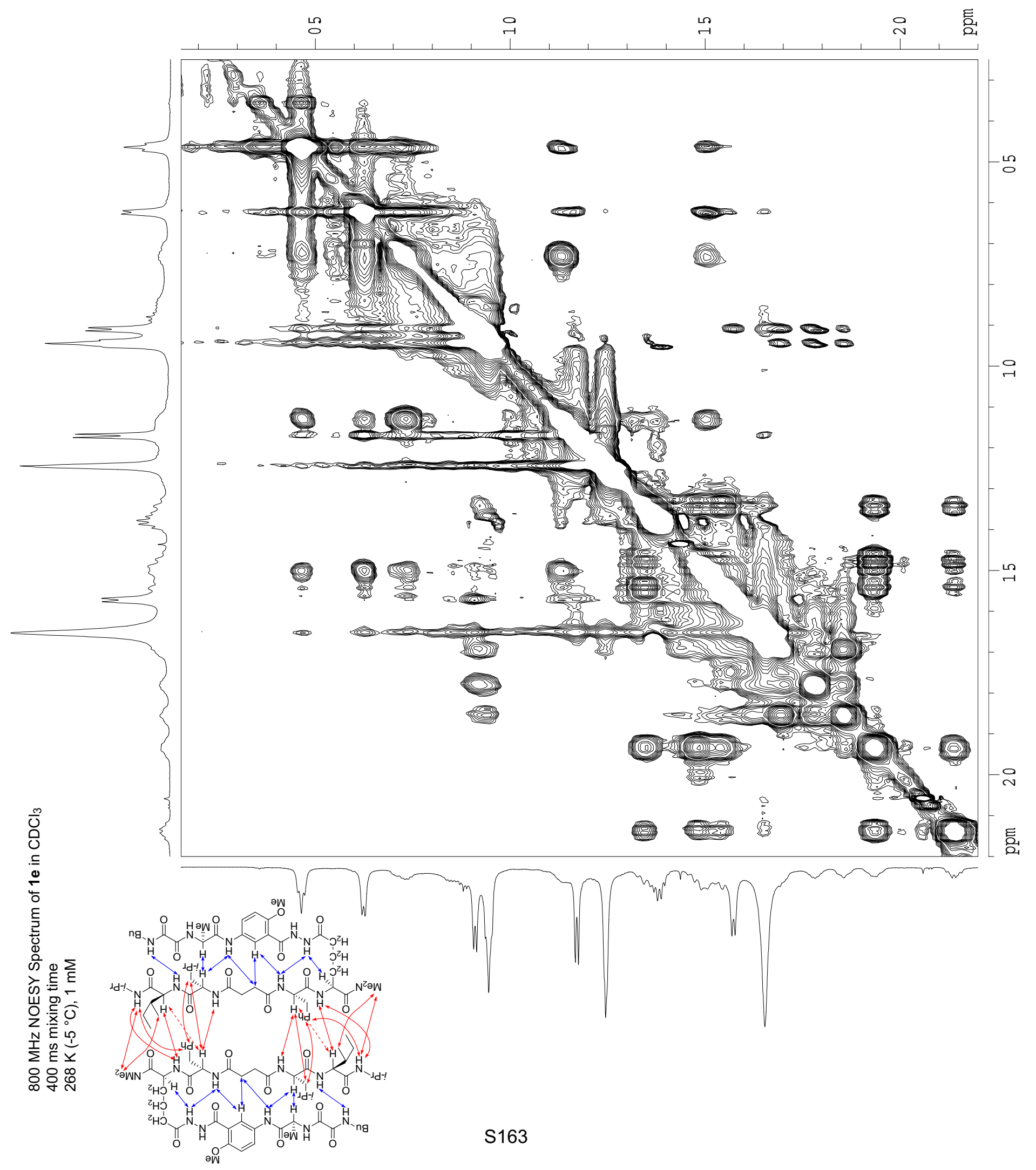




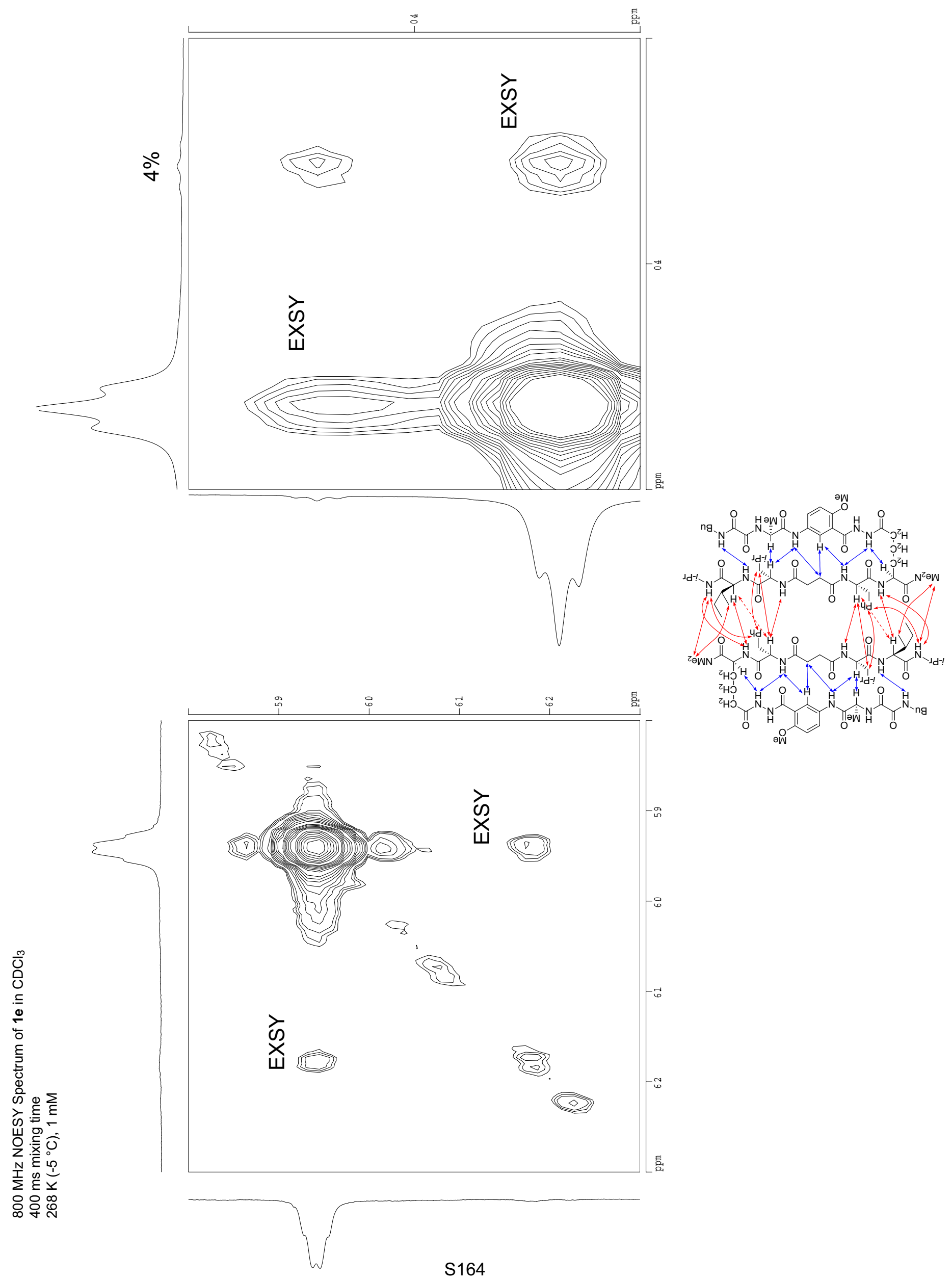




\section{Spectroscopic DATA for the compound $1 \mathbf{f}$}

(4 mM, $\mathrm{CDCl}_{3} 268 \mathrm{~K}$ )

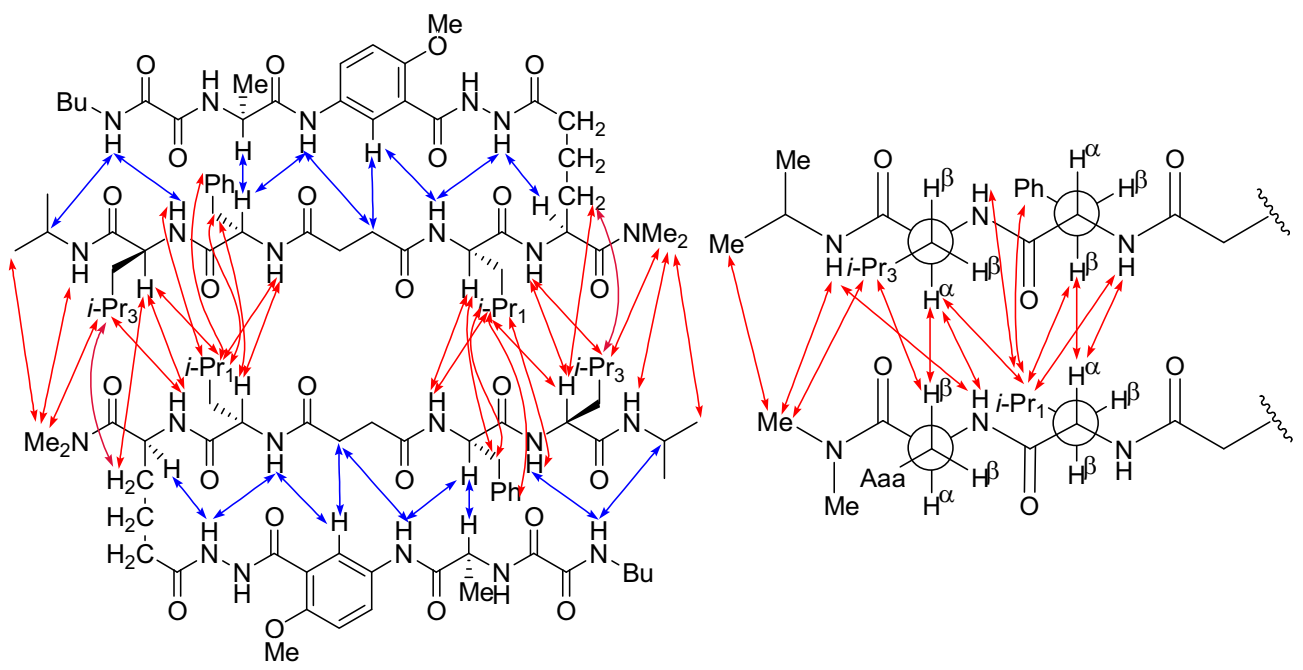

Key NOEs assigned as intermolecular are represented with red arrows.

Key NOEs assigned as intramolecular are represented with blue arrows.

Key data for amino acids.

\begin{tabular}{|c|c|c|c|}
\hline Amino acid & $\mathrm{H}_{\alpha}$ Shift ppm & NH Shift ppm & ${ }^{3} J_{\mathrm{NH} \alpha} \mathrm{Hz}$ \\
\hline Aaa & 5.18 & 8.50 & 8.4 \\
\hline $\mathrm{R}_{1}=\mathrm{Leu}^{1}$ & 5.60 & 8.41 & 8.0 \\
\hline $\mathrm{R}_{2}=$ Phe & 5.13 & 8.55 & 9.7 \\
\hline $\mathrm{R}_{3}=\mathrm{Leu}^{2}$ & 5.07 & 8.80 & 9.7 \\
\hline $\mathrm{R}_{4}=\mathrm{Ala}$ & 4.60 & 8.06 & overlap \\
\hline
\end{tabular}

Aminoadipic acid (Aaa) $\delta$ proton resonances.

pro-R: 2.94 ppm, t, (overlap)

pro-S: $2.18 \mathrm{ppm}, \mathrm{td},\left(J_{1}=11.2 \mathrm{~Hz} ; J_{2}=7.0 \mathrm{~Hz}\right)$

anisotropy $=0.76 \mathrm{ppm}$

Key data for other $\mathrm{NH}$ protons.

\begin{tabular}{|c|c|c|}
\hline Proton & NH Shift ppm & $J \mathrm{~Hz}$ \\
\hline Ar-NH & 10.09 & - \\
\hline Hydrazide Ar & 10.58 & 7.4 \\
\hline Hydrazide Aaa & 10.40 & 7.4 \\
\hline Bu-NH & 9.55 & broad \\
\hline$i$-Pr-NH & 8.06 & overlap \\
\hline
\end{tabular}

ESI MS $m / z$ for $\mathrm{C}_{53} \mathrm{H}_{82} \mathrm{~N}_{11} \mathrm{O}_{12}[\mathrm{M}+\mathrm{H}]^{+}$calcd 1064.61, found 1064.48. 

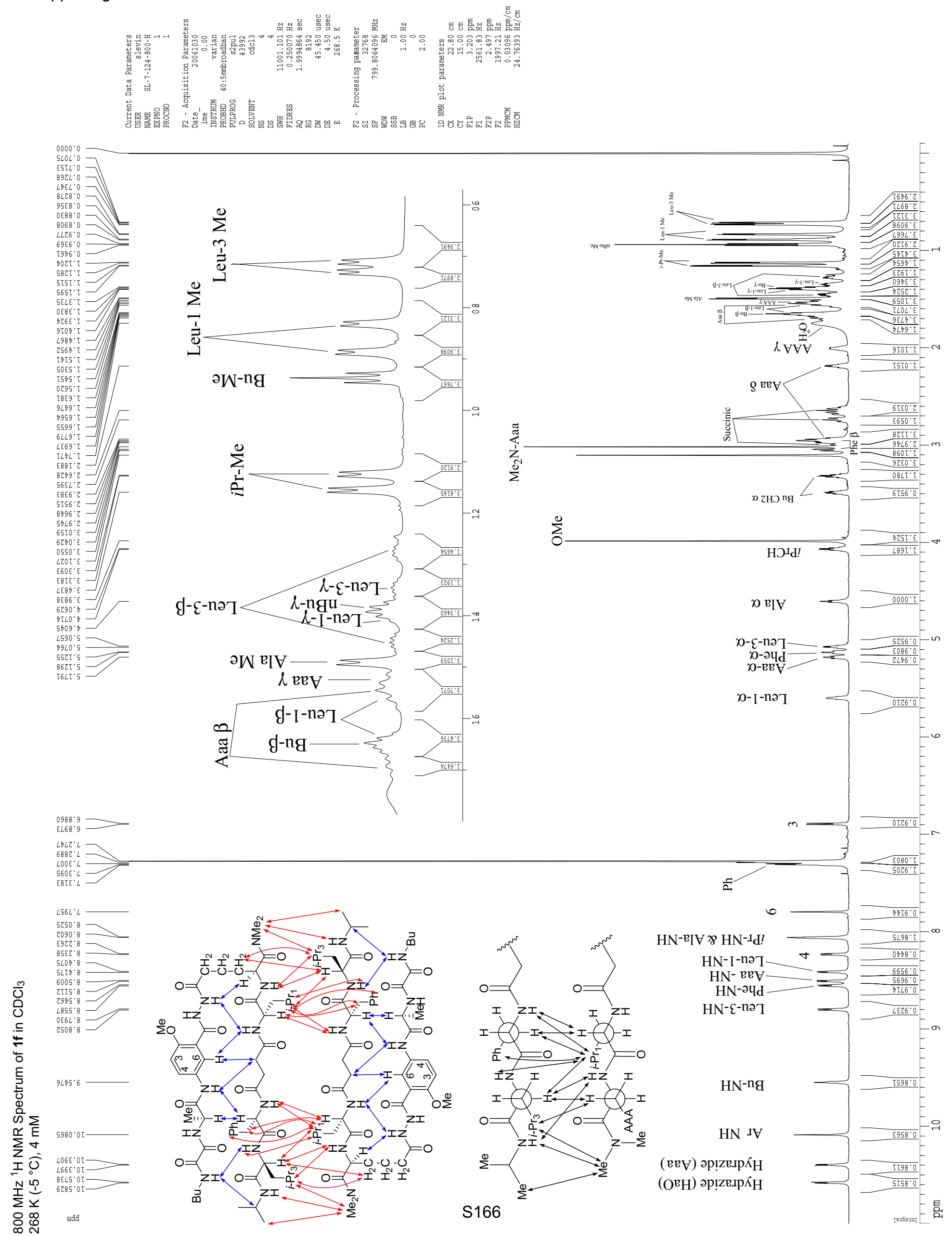

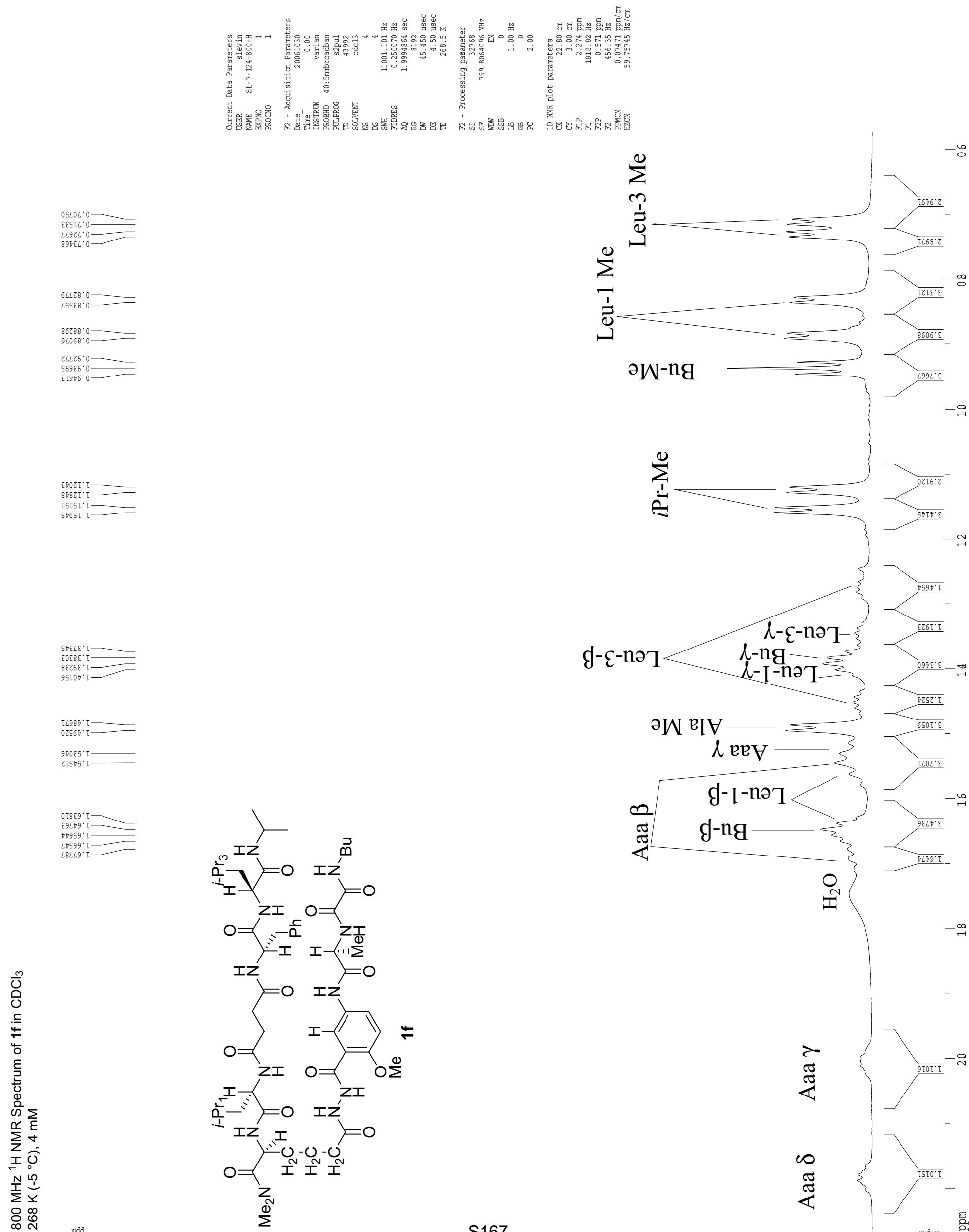

วW $\mathrm{e}[\mathrm{V}$

l evF
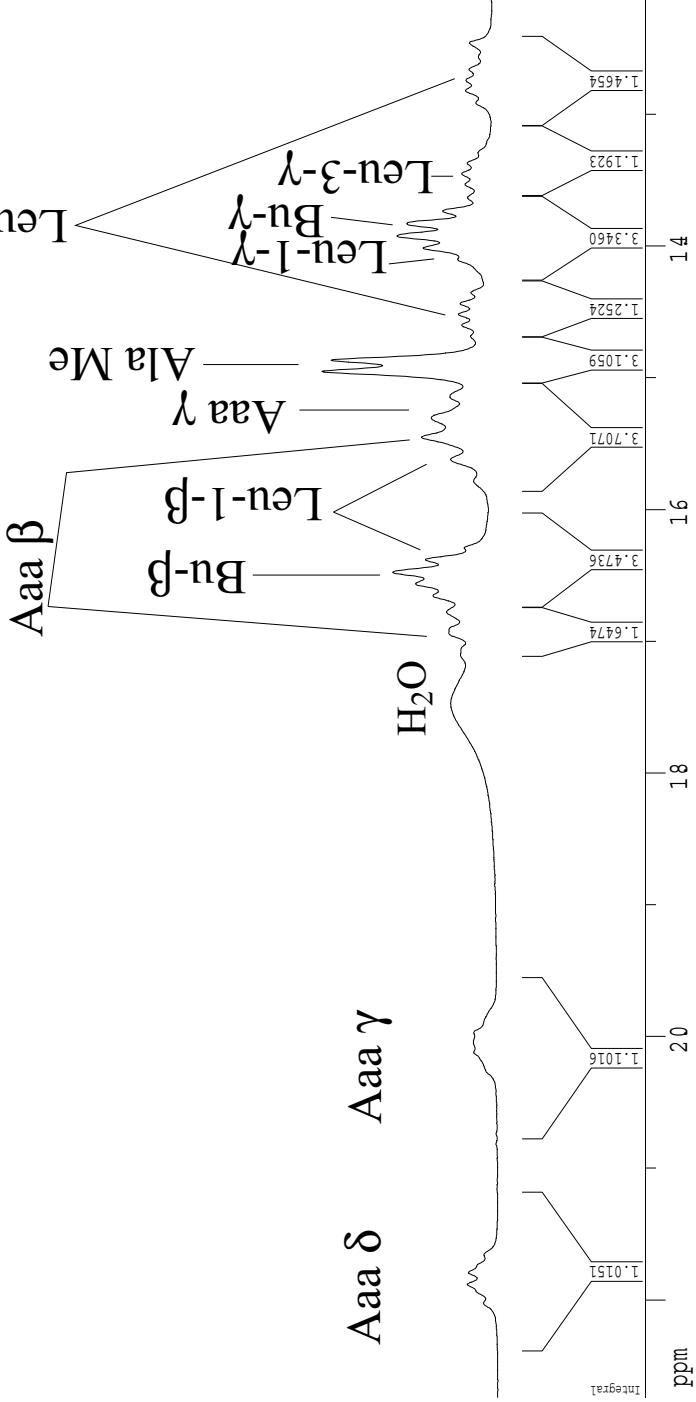

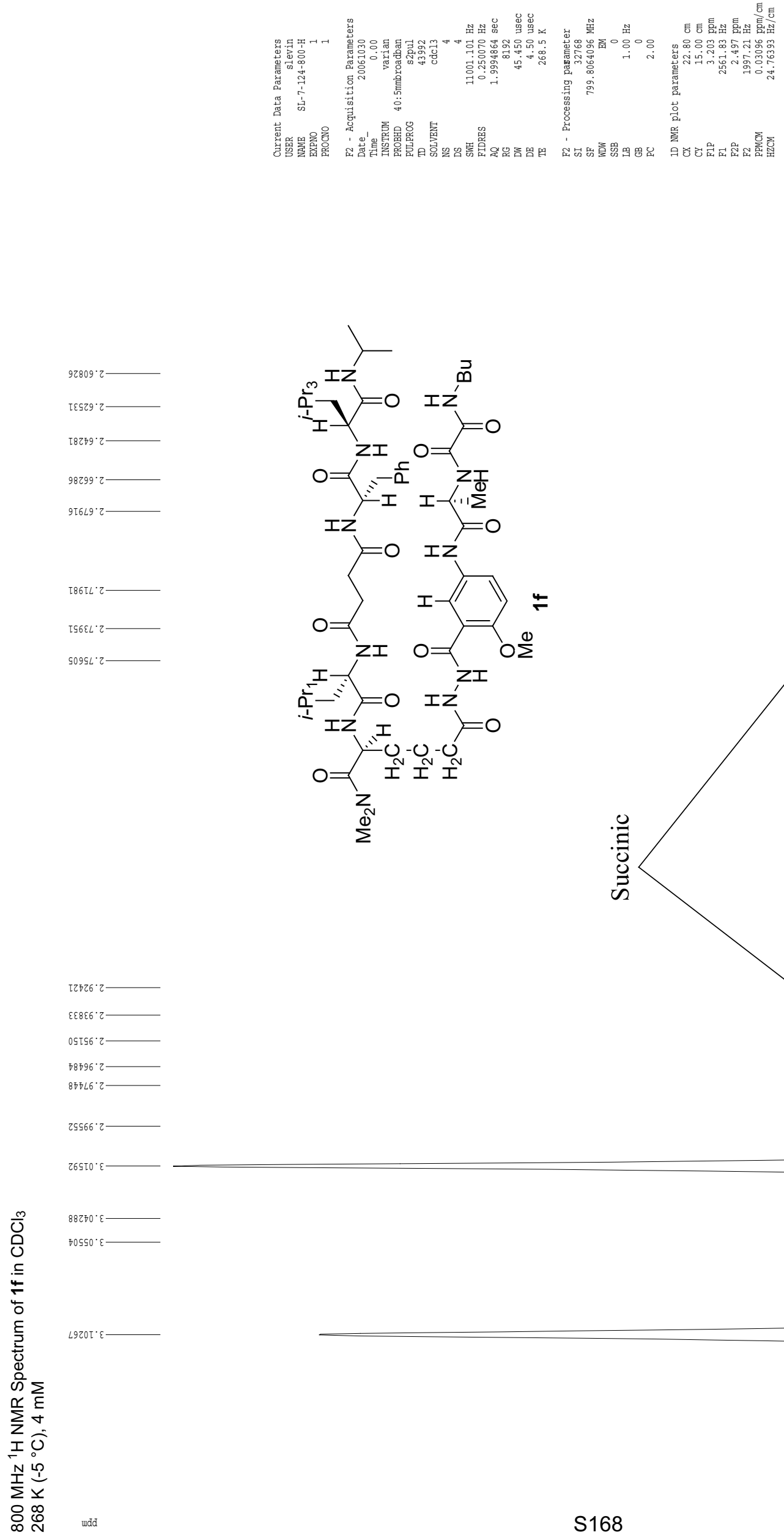
Supporting Information

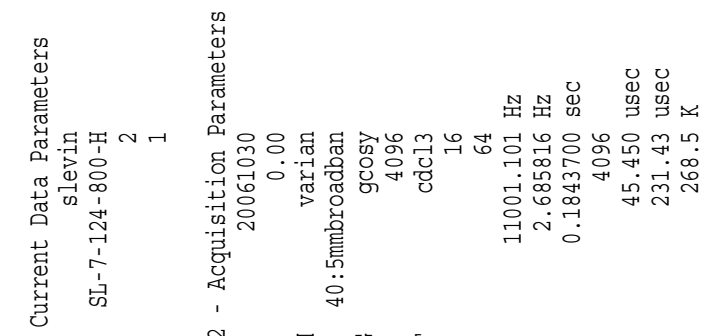

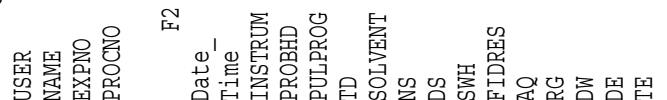

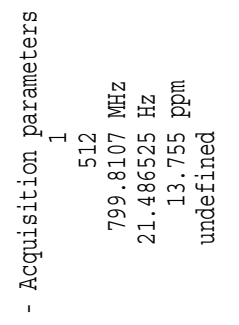

空

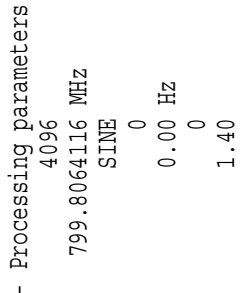

S. Levin and J. S. Nowick

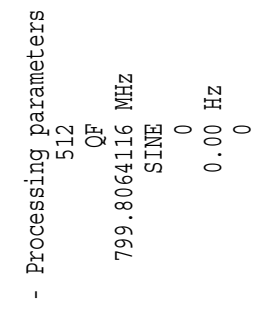

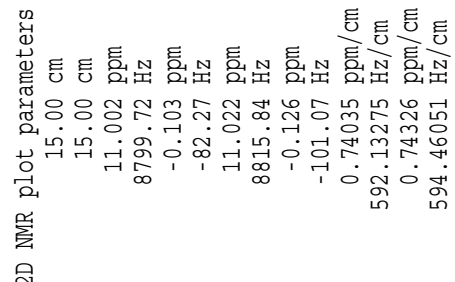

㞌

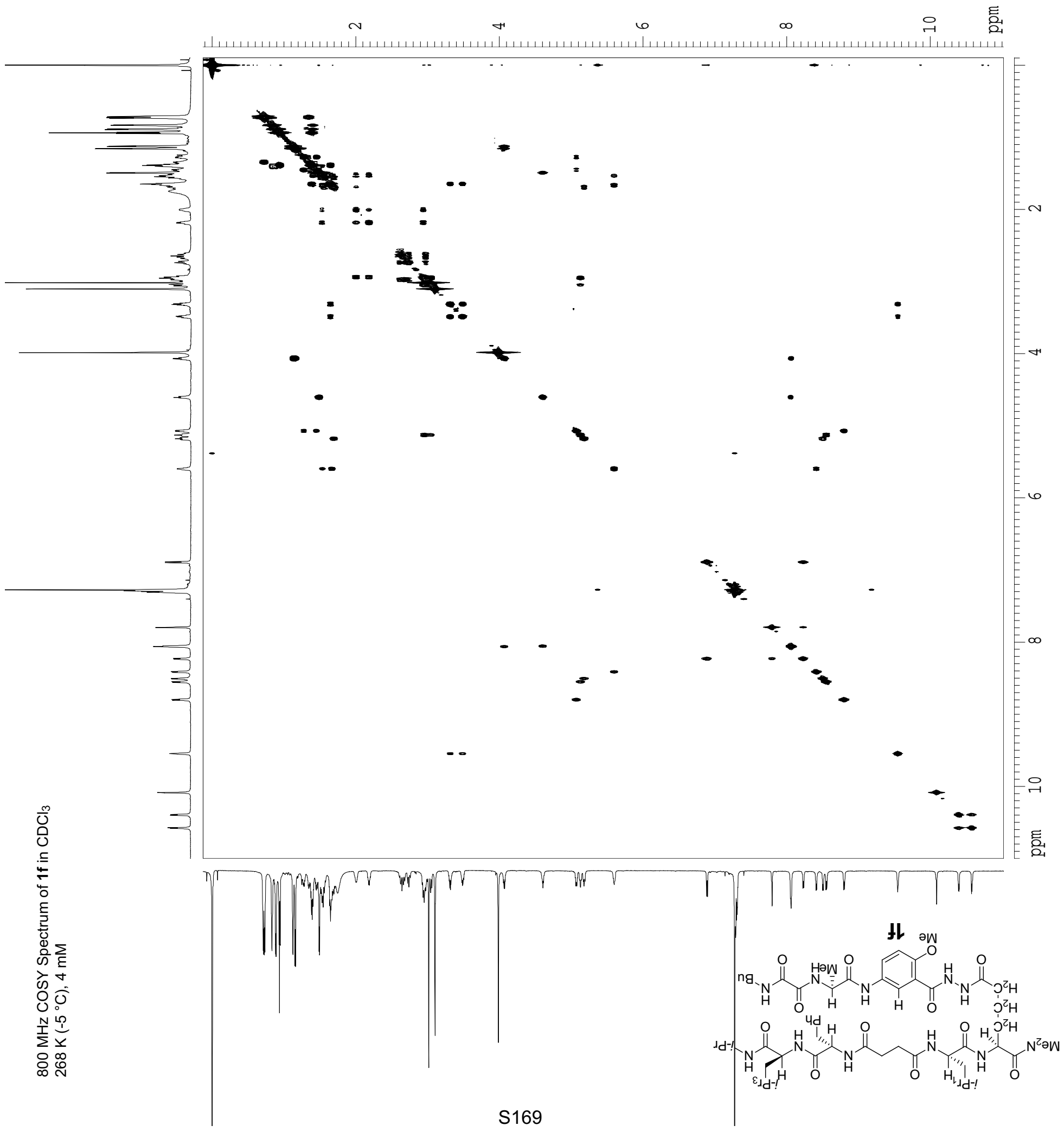


Supporting Information

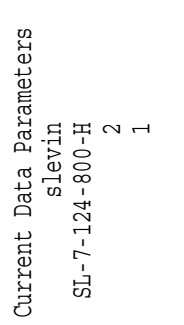

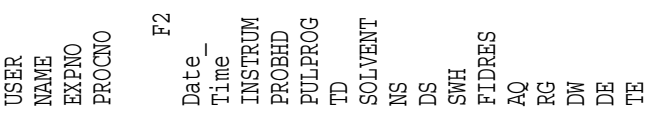

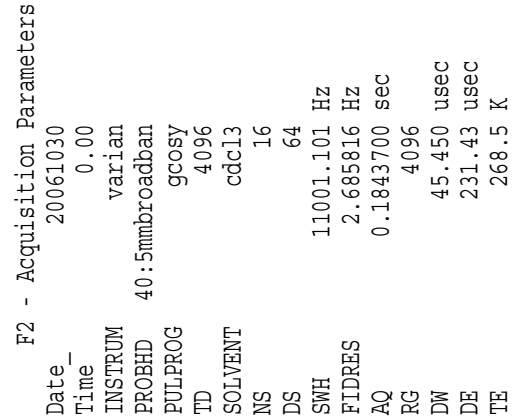

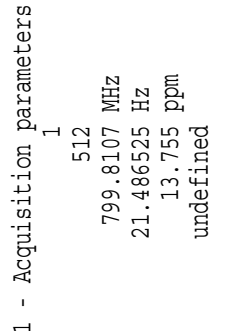

届

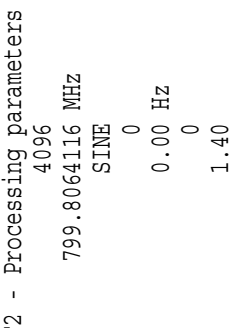

- 20

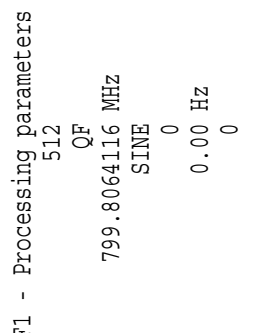

(1)
S. Levin and J. S. Nowick

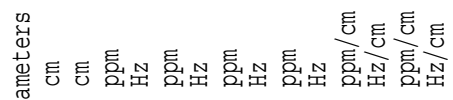

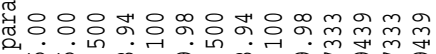
营 览

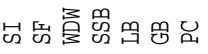

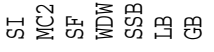

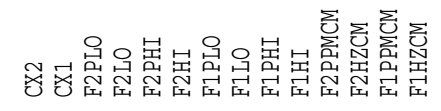

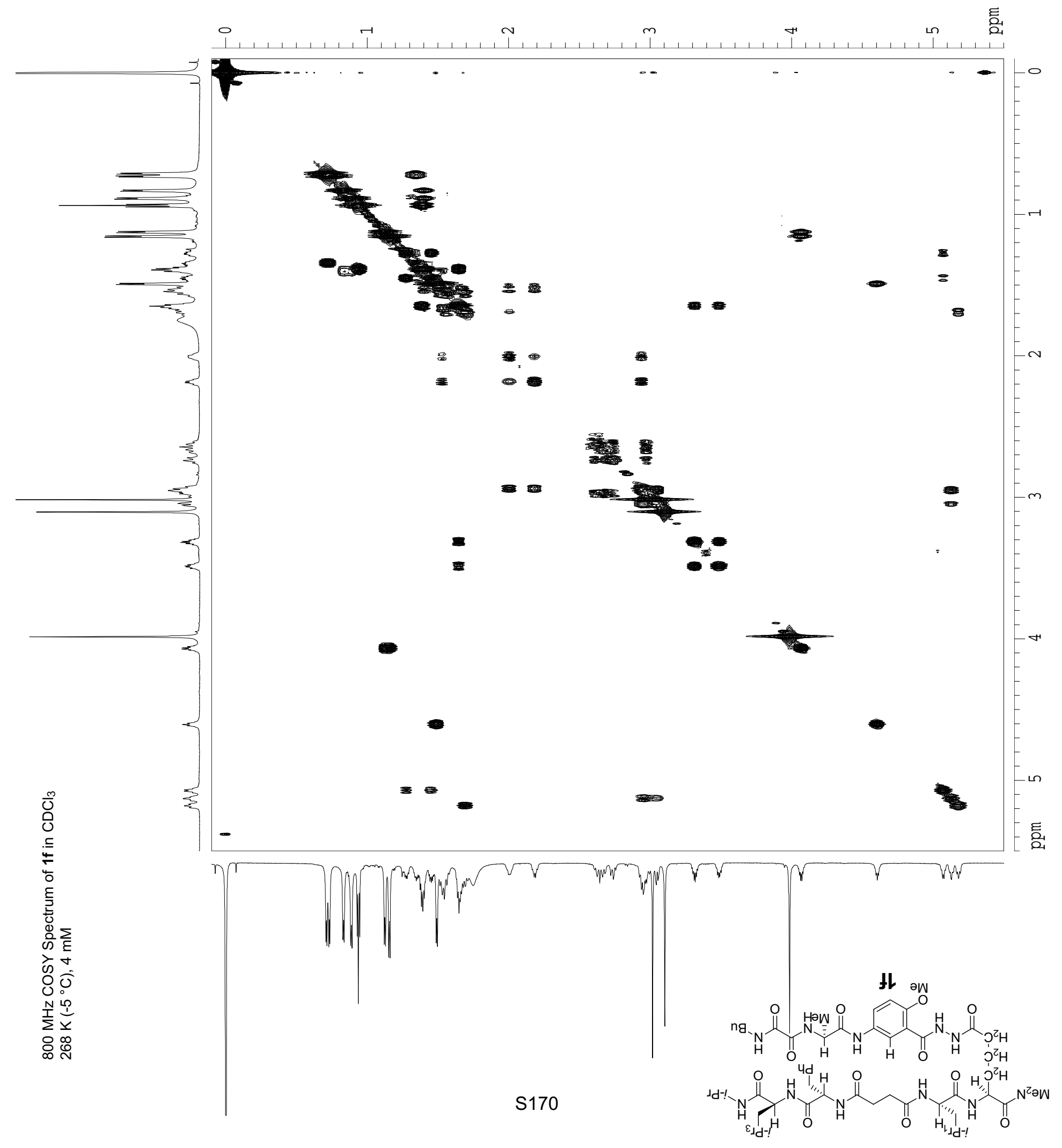




\section{Supporting Information}
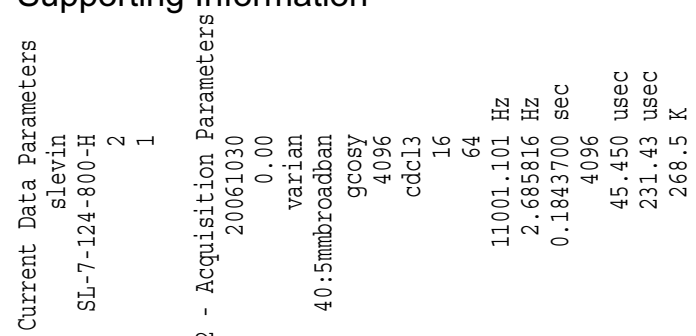

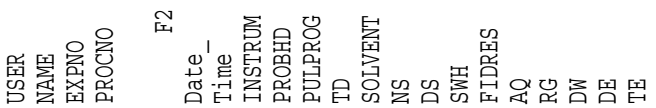

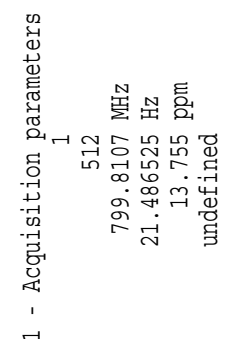

포

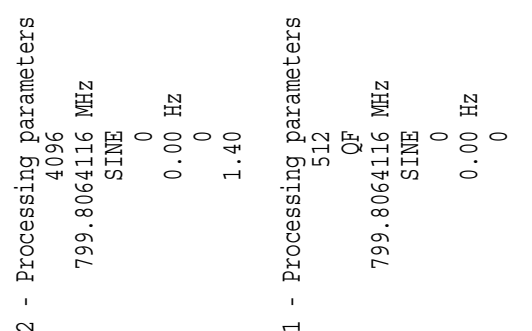

空
S. Levin and J. S. Nowick

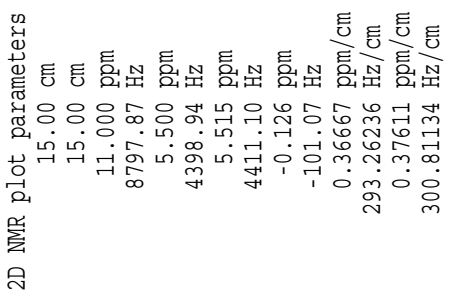

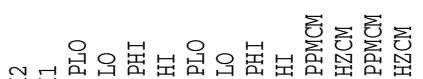

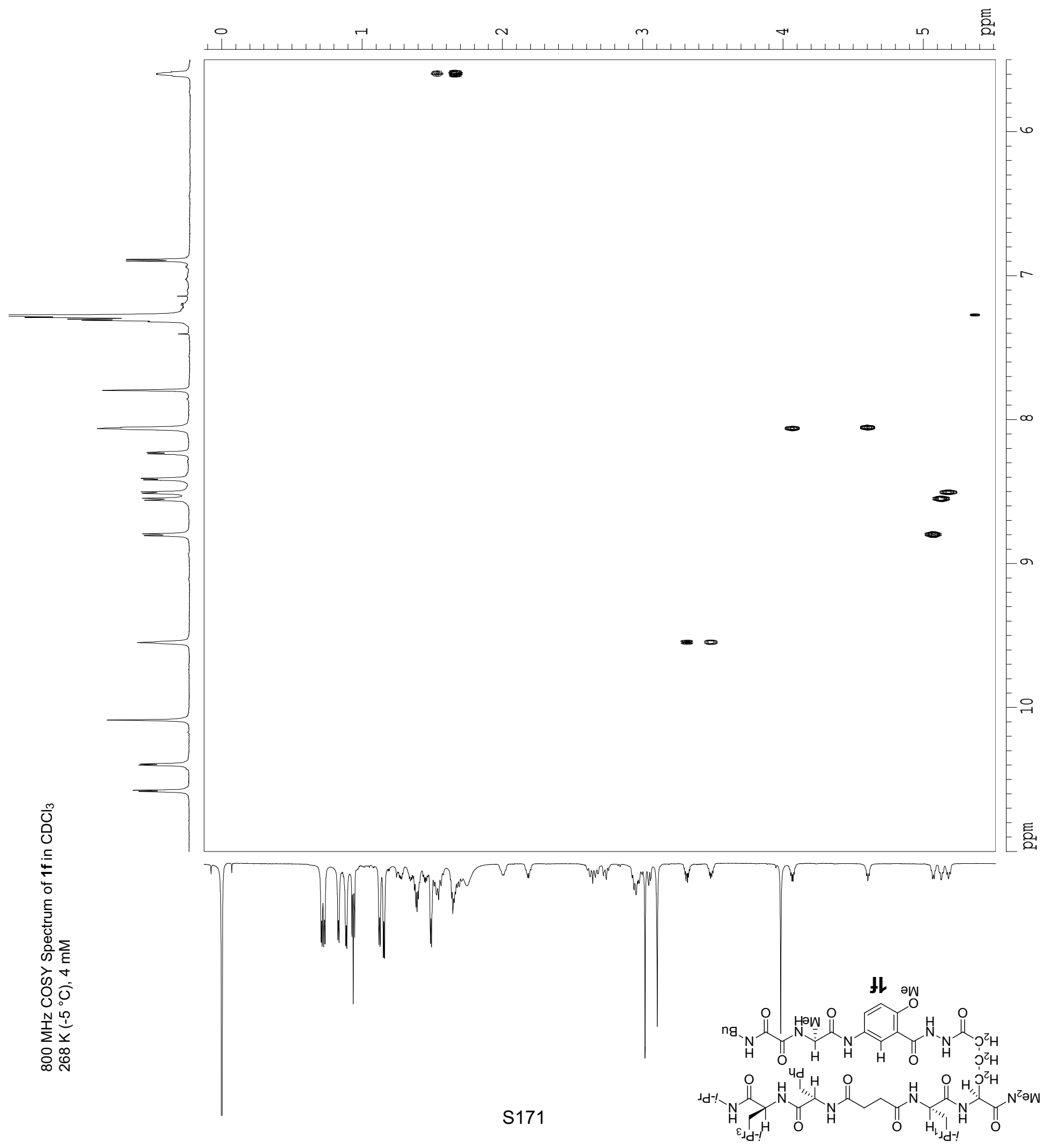



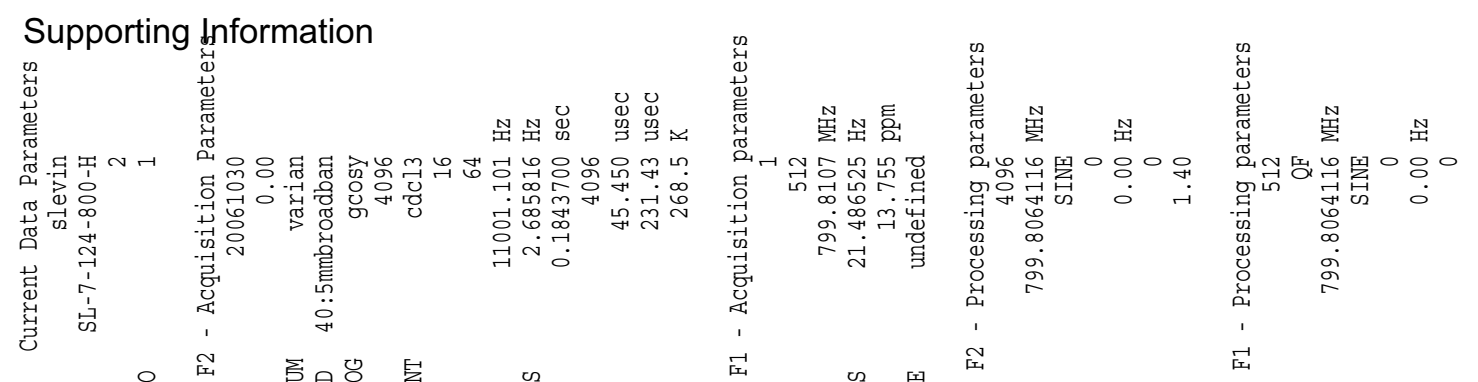

S. Levin and J. S. Nowick

圑罢总总

㞑留畏

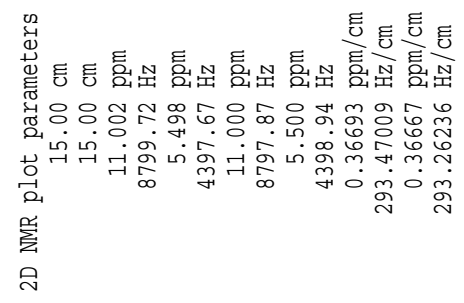

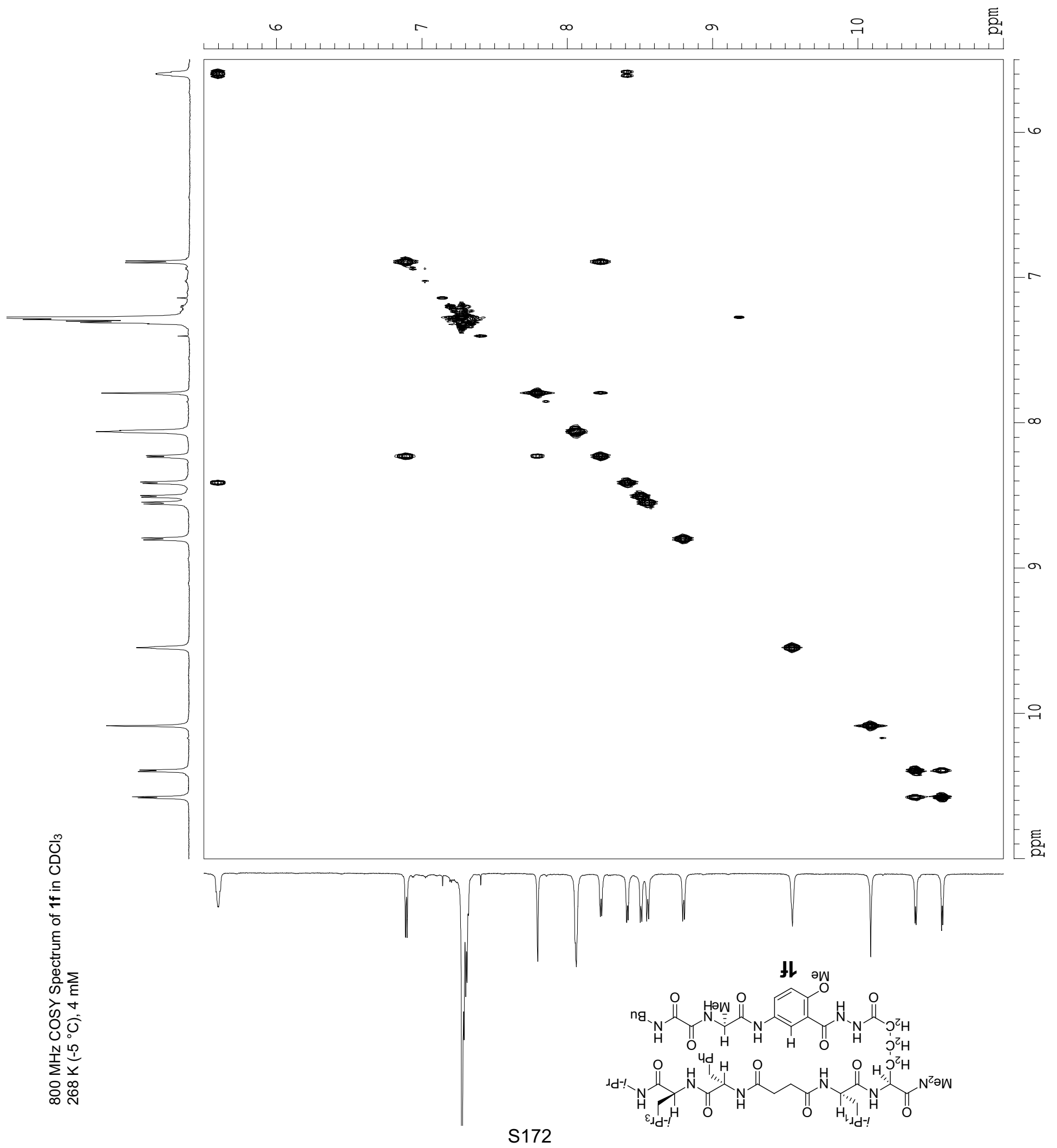




\section{Supporting Information}
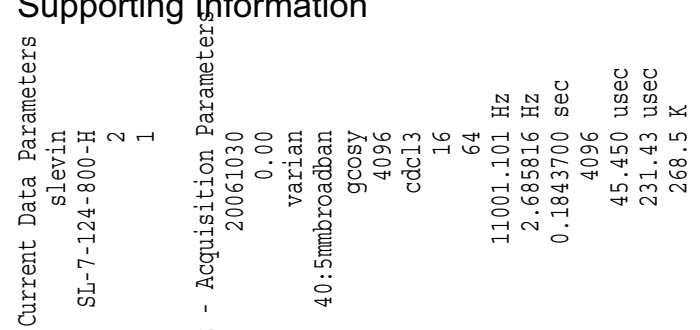

玨

舅淟总总

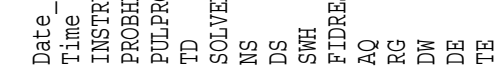

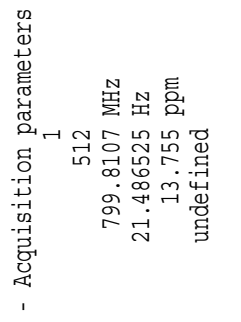

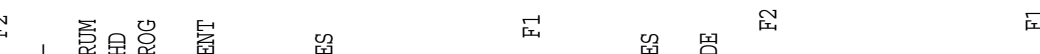

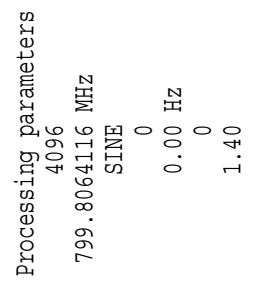

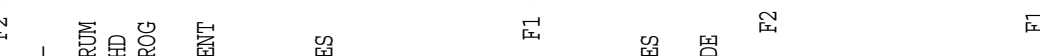

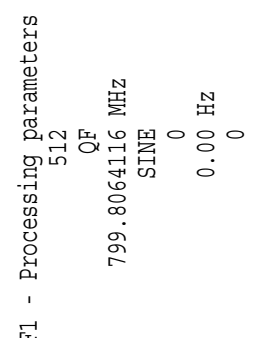

S. Levin and J. S. Nowick

嵒

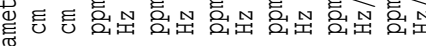

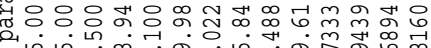

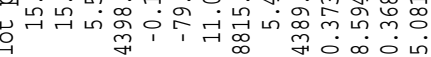
虹

ลิ

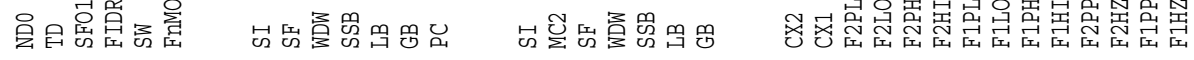

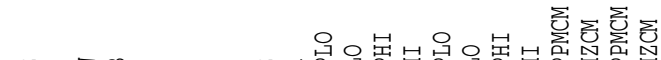

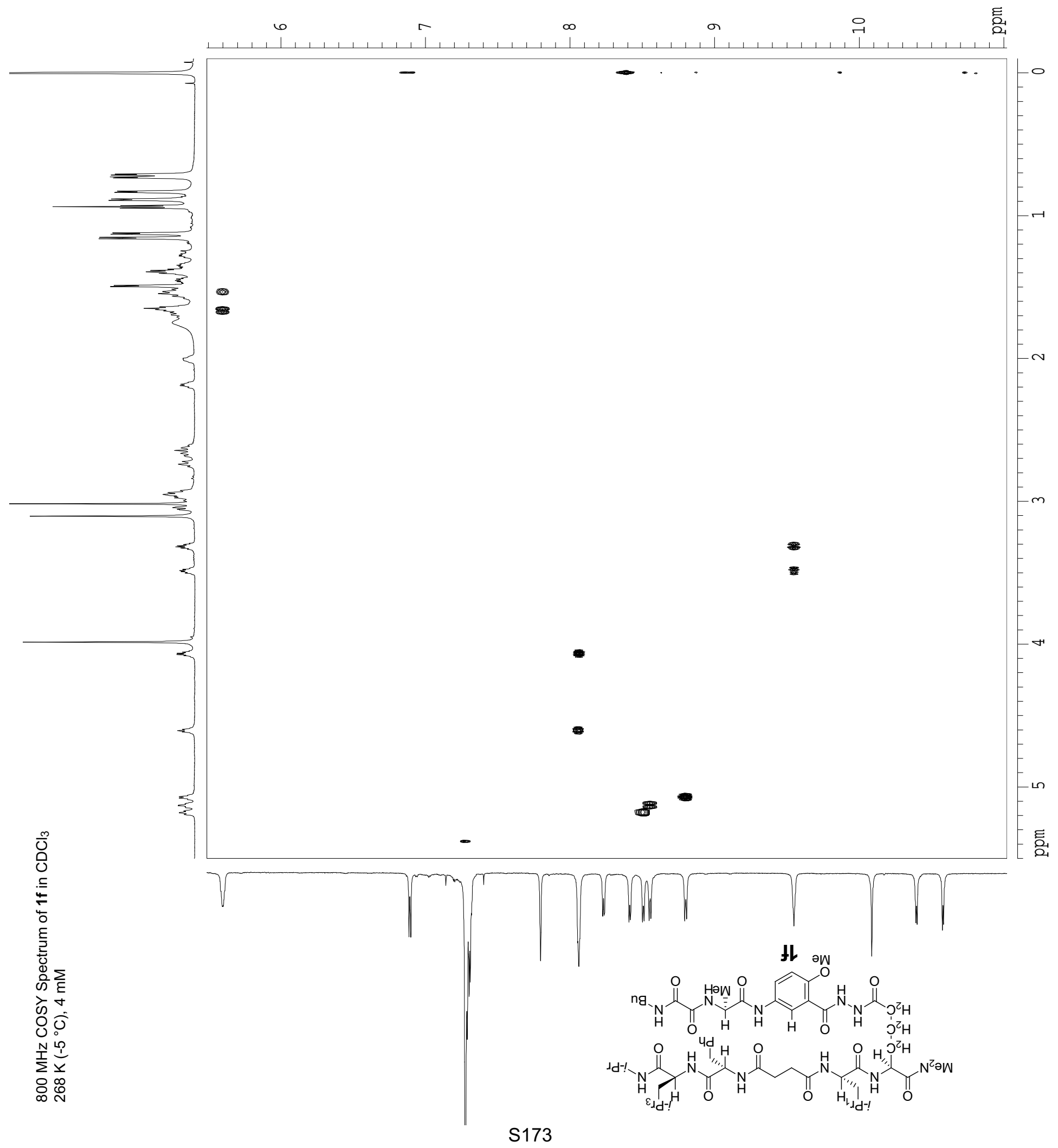




\section{Supporting Information $_{2}$}

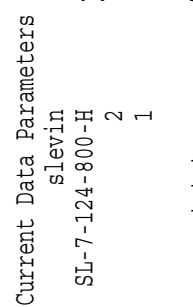

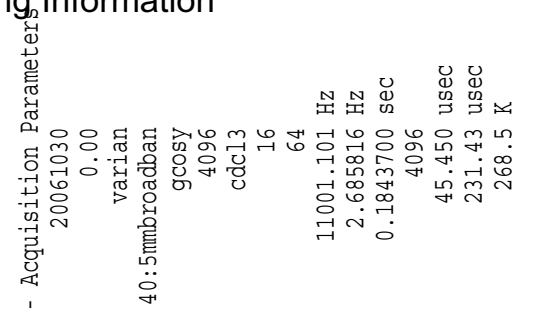

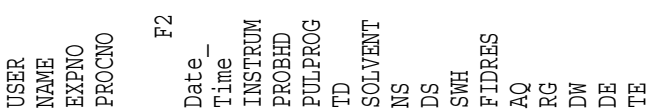

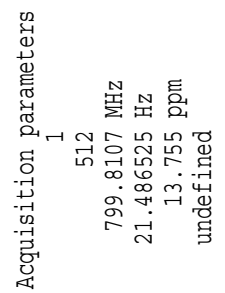

'十

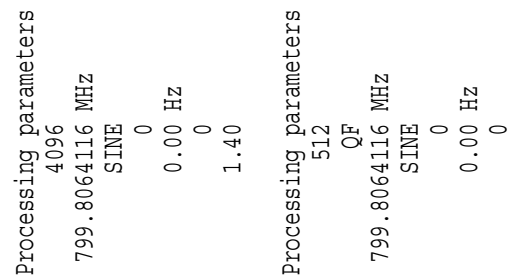

峦
S. Levin and J. S. Nowick

总

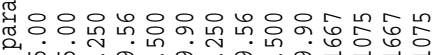
में 管 ลิ

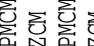

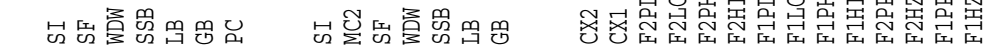

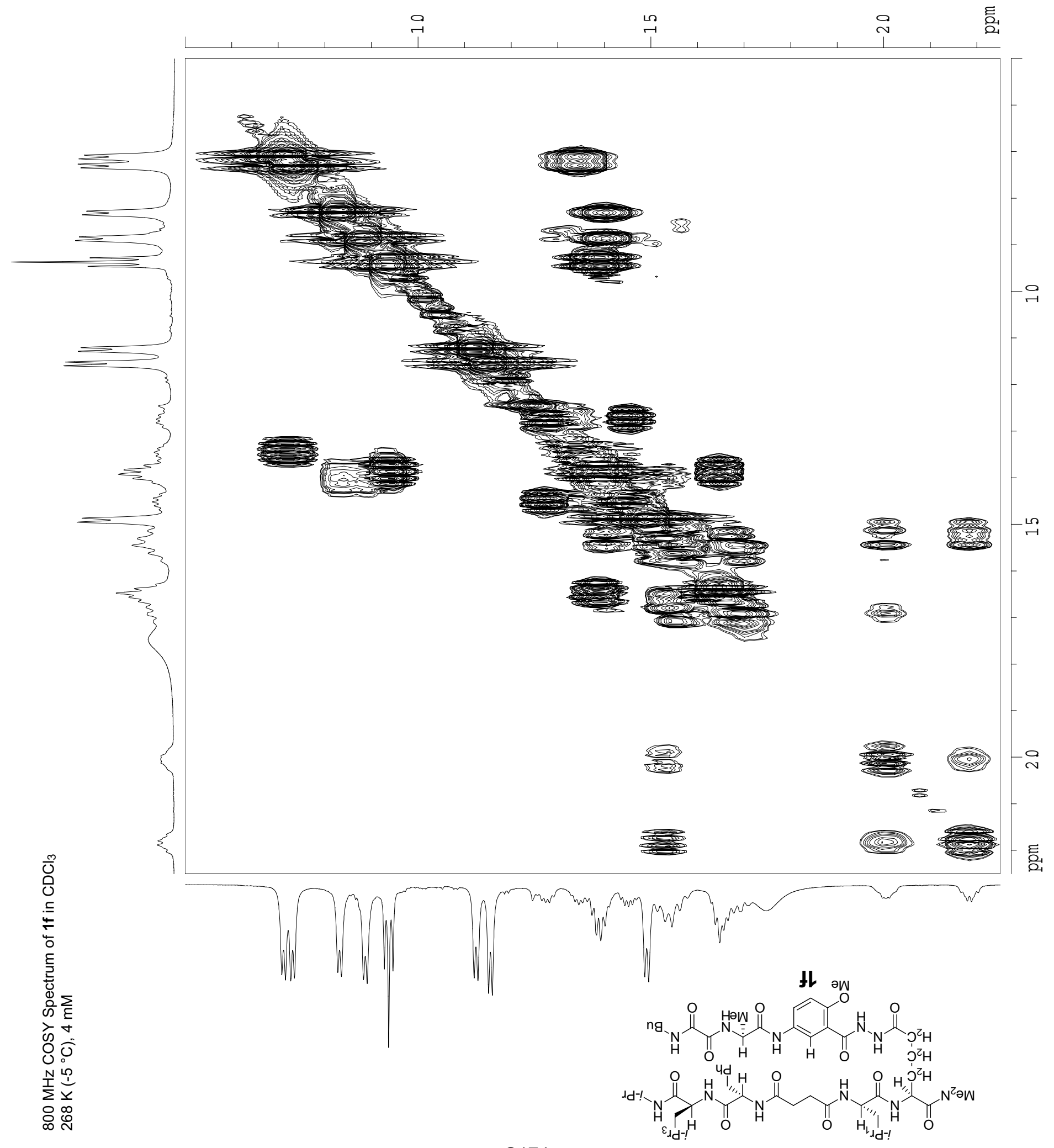



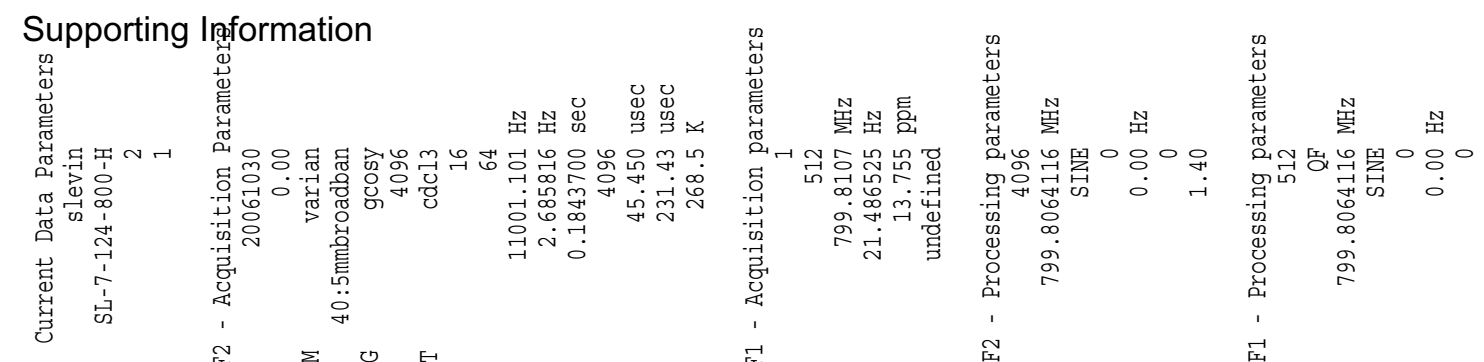

S. Levin and J. S. Nowick

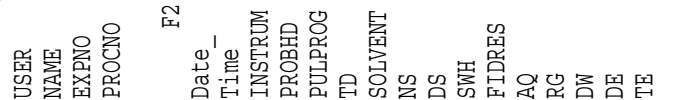

届

도

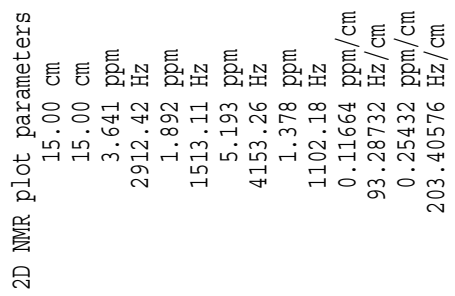

ร

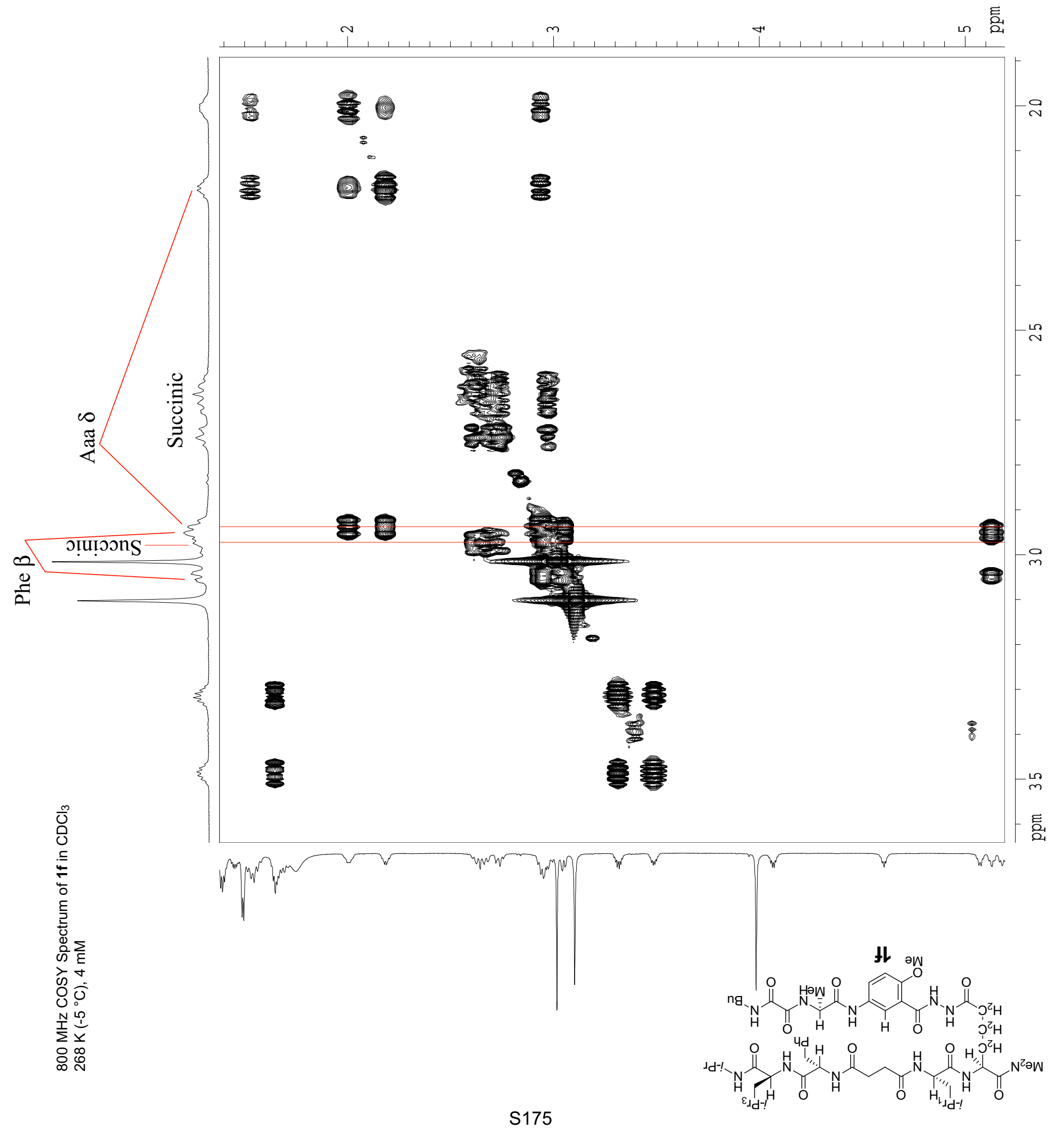


Supporting Information
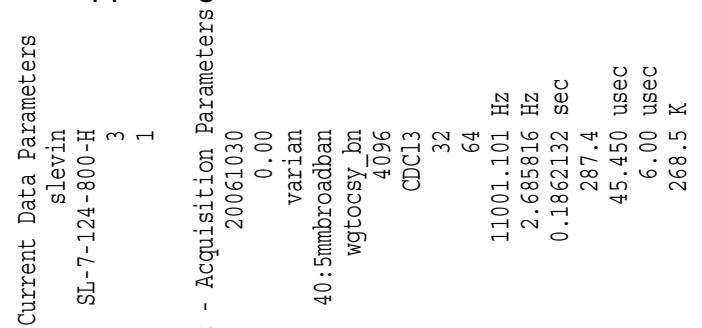

䍛罢总总
"

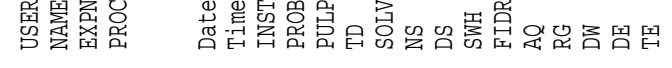

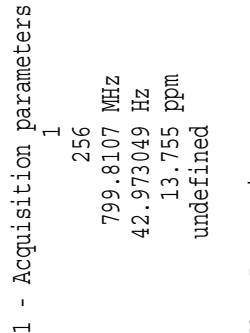

$=2$

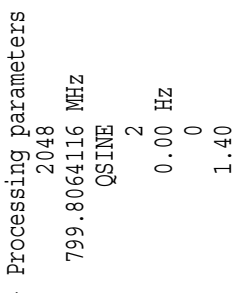

${ }^{2}$

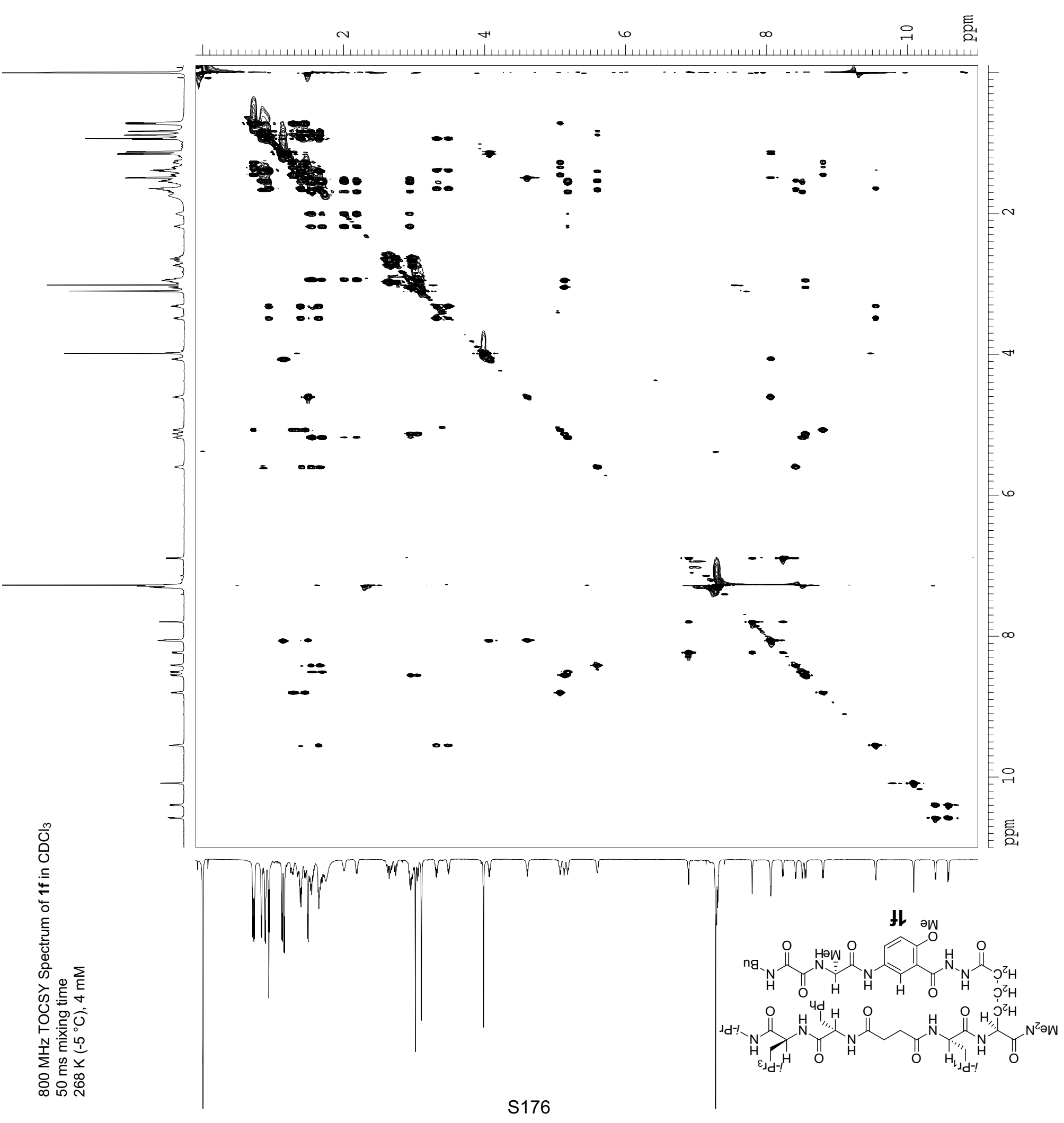


${ }_{2}$ Supporting:

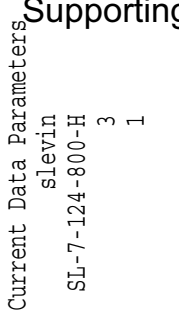

舀罡总总

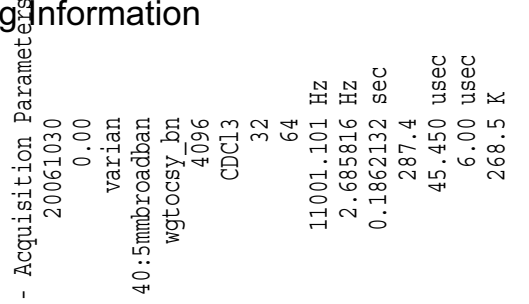
艀

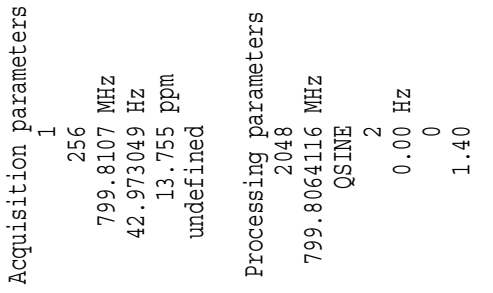

$\overrightarrow{1}$

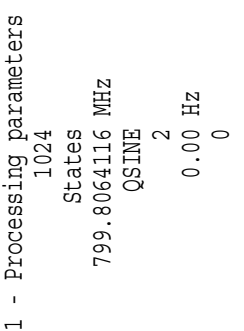
S. Levin and J. S. Nowick

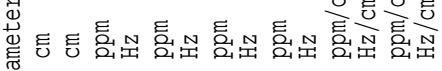

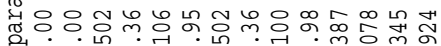

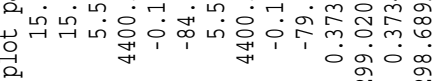
罢 ิㅗㄱ

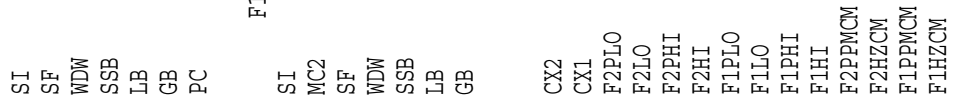

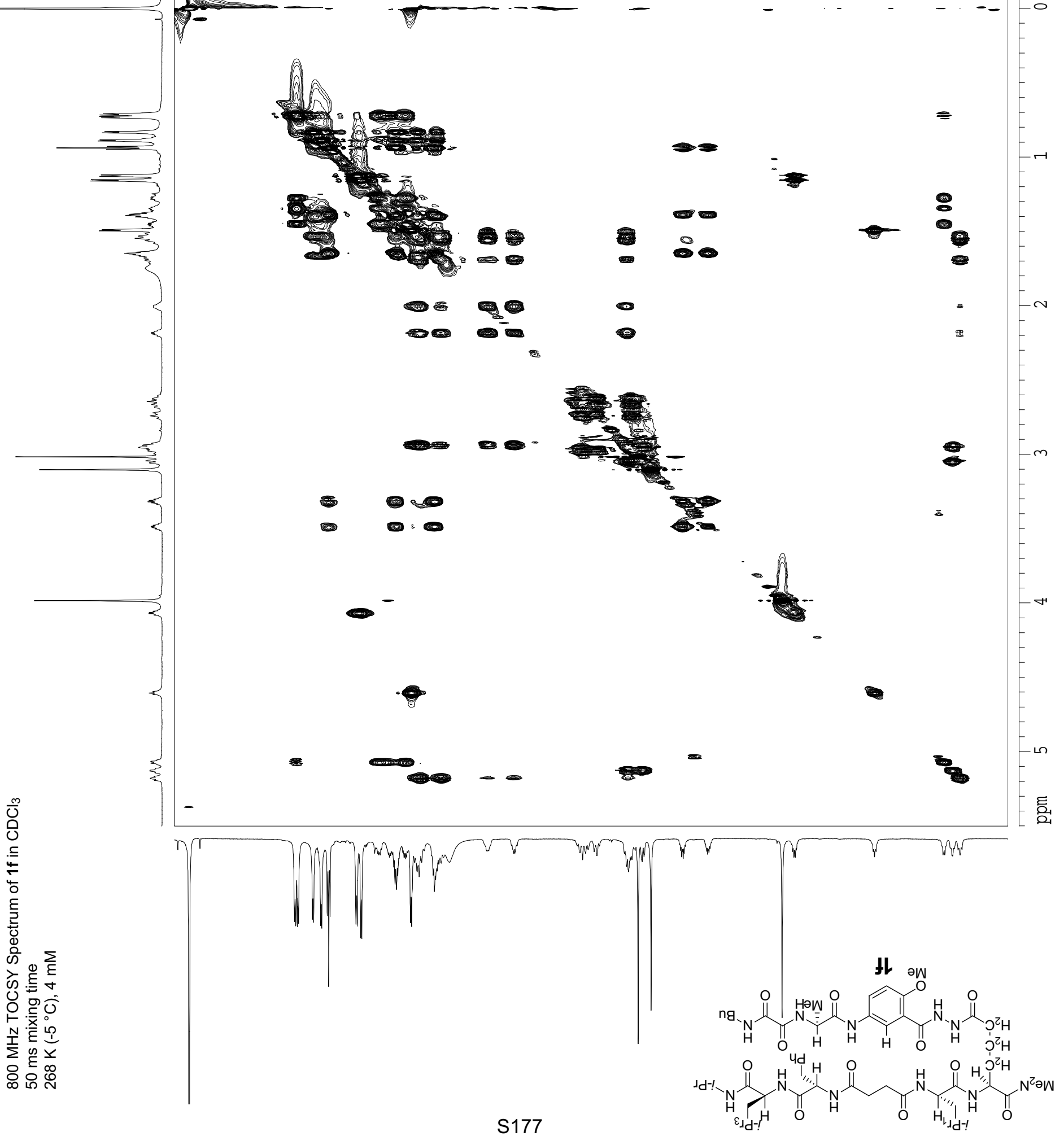




\section{Supporting Information}

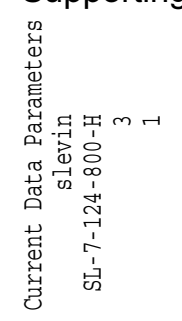

鲎罢总总

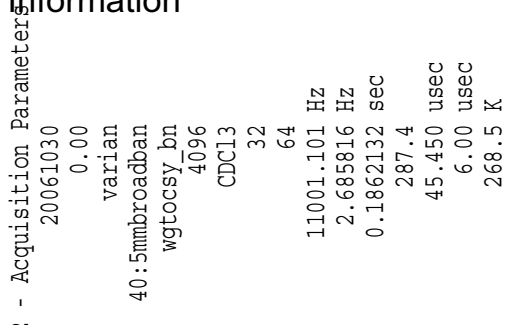

$\approx$

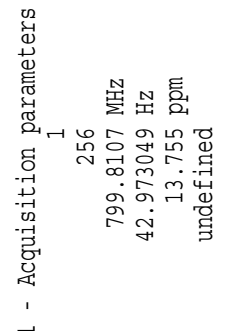

도

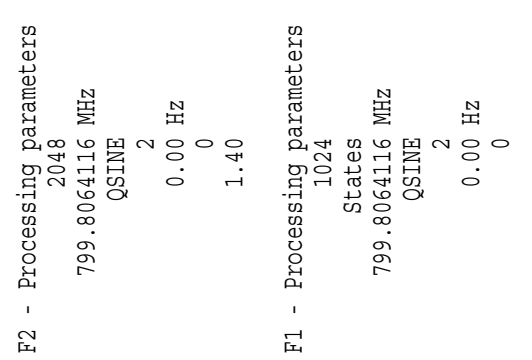

S. Levin and J. S. Nowick

岛

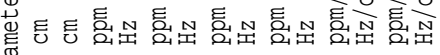

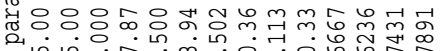

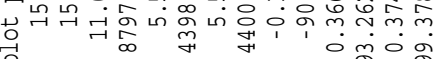
宸 คิ

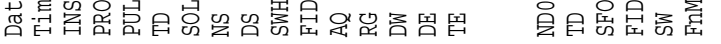

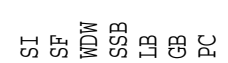

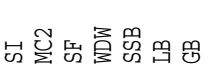

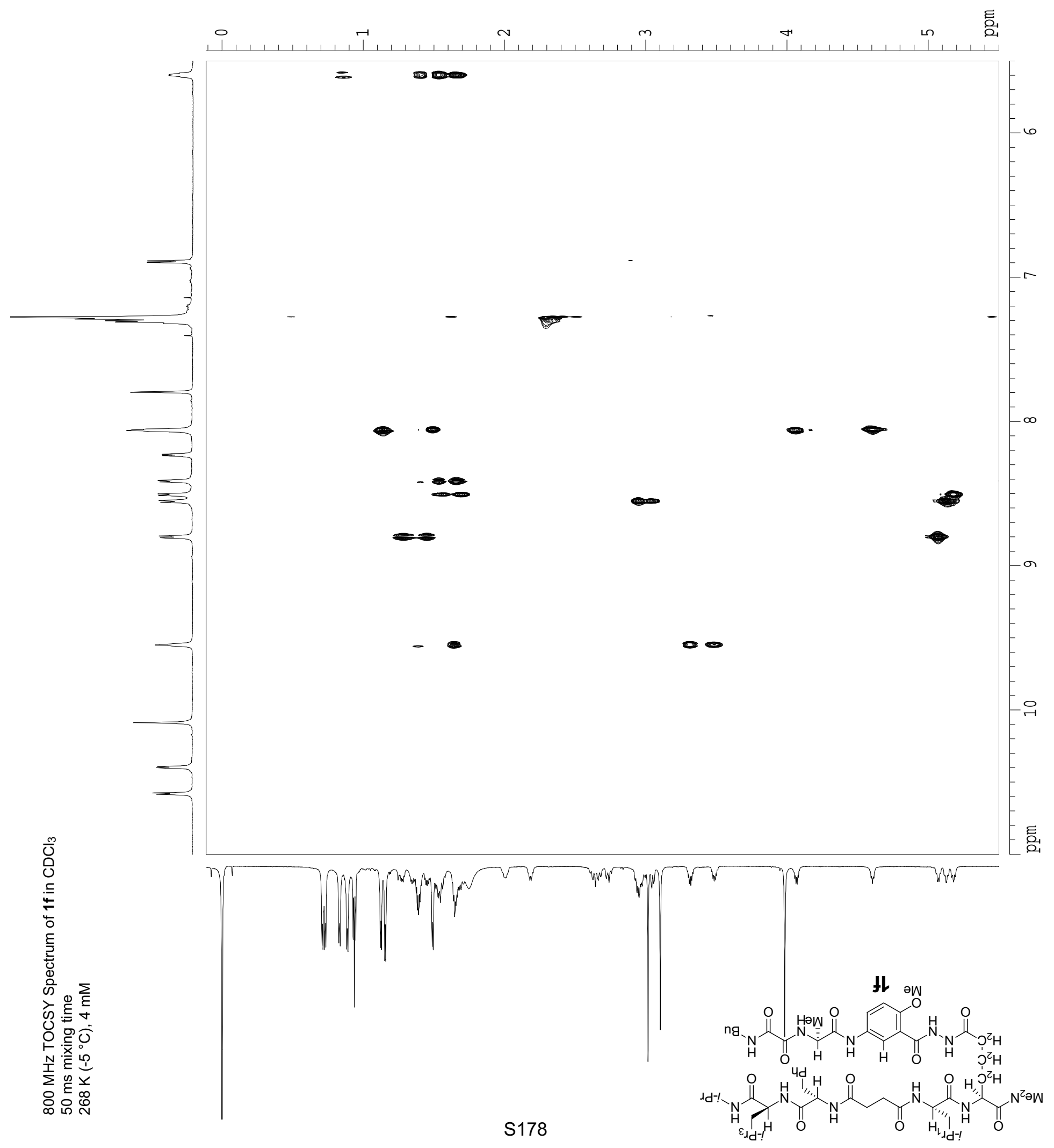




\section{Supporting hị formation}

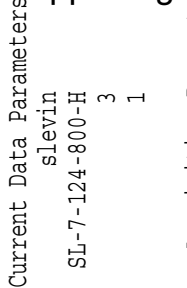

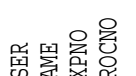

\begin{abstract}
표
\end{abstract}

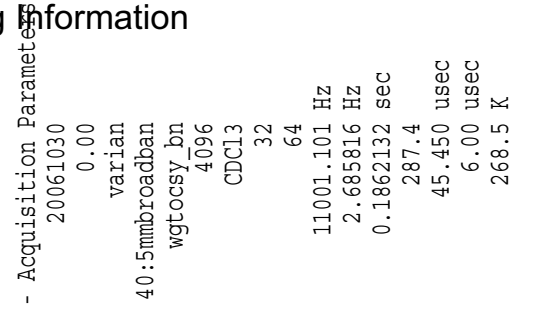

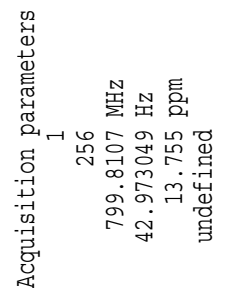

㞋

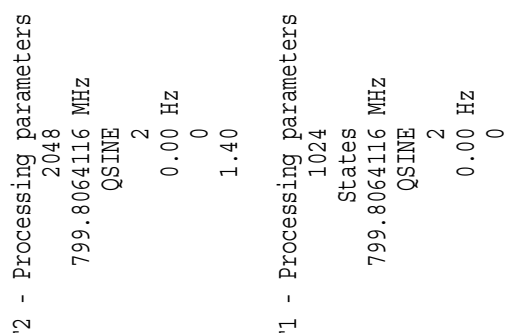

空

is
S. Levin and J. S. Nowick

造

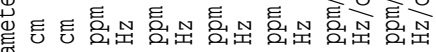

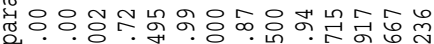

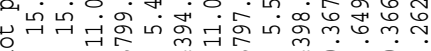

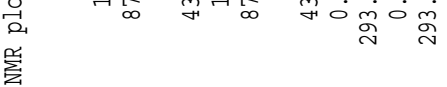
ล

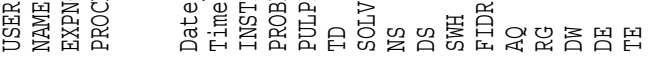

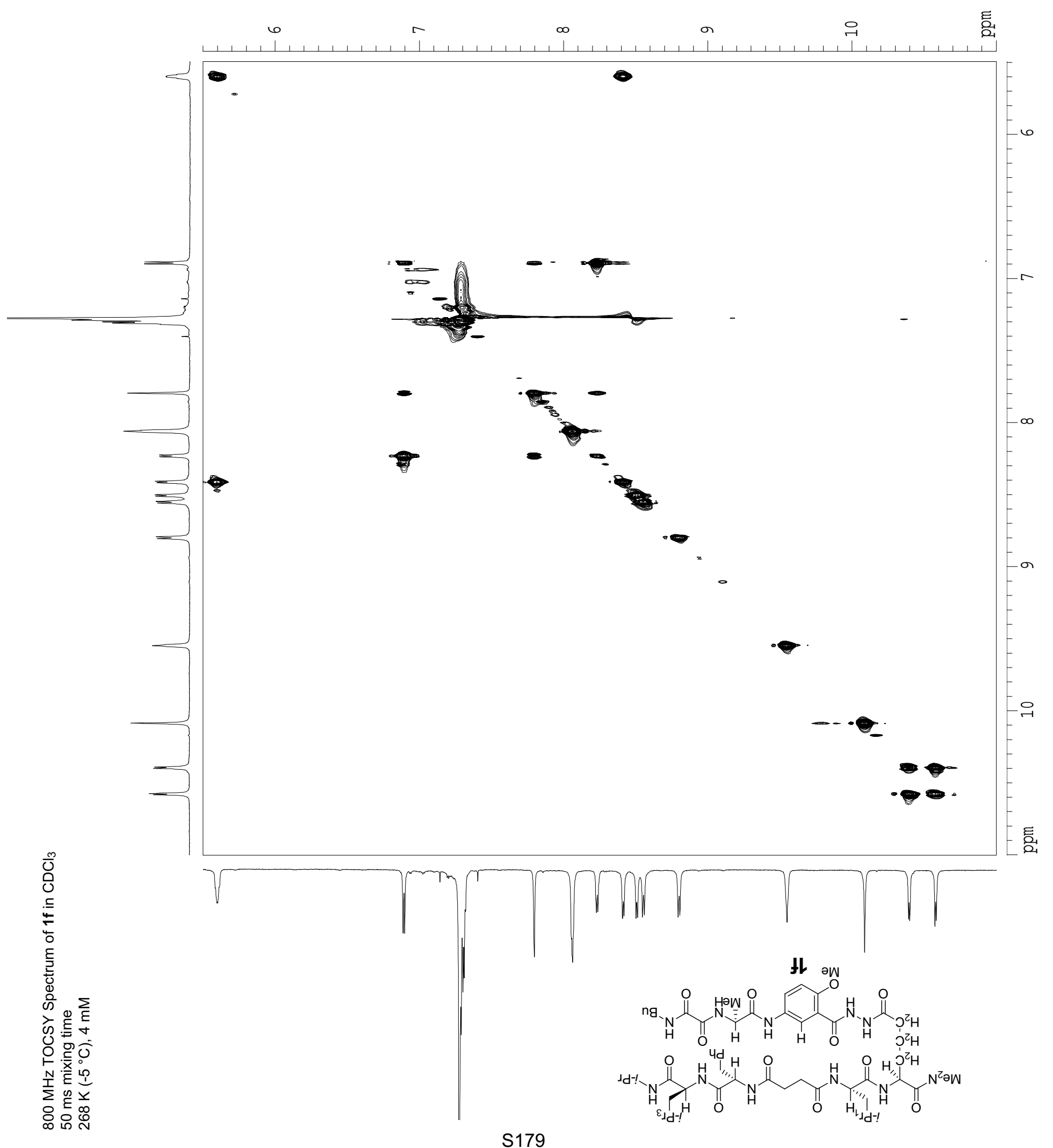




\section{Supporting Information}

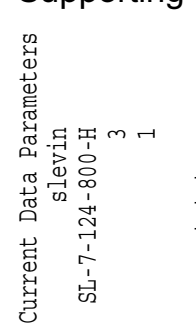

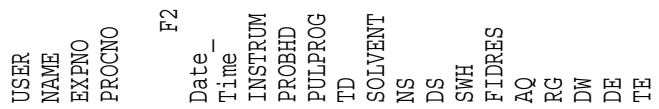

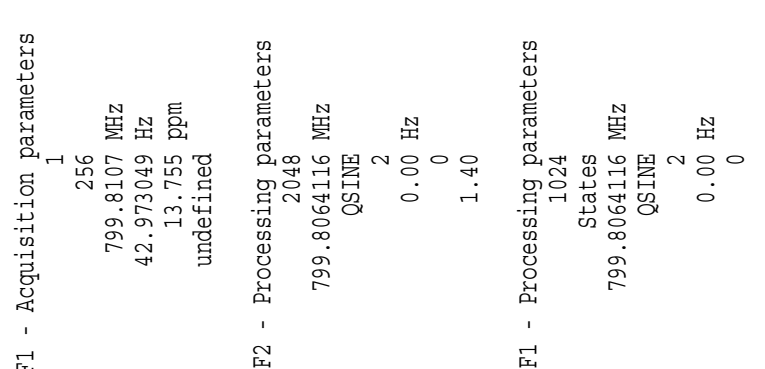

\section{포}

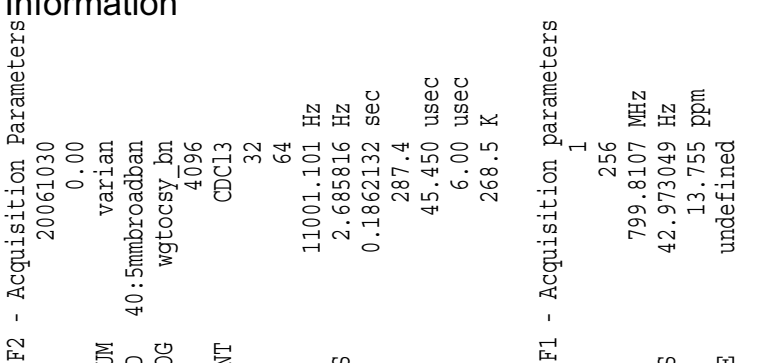

S. Levin and J. S. Nowick

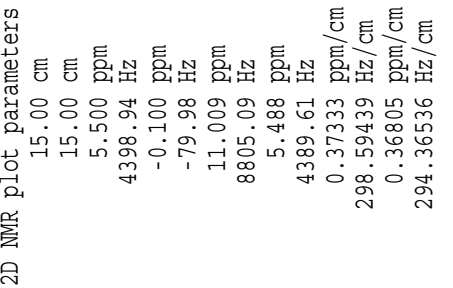

ํㅡㄹ

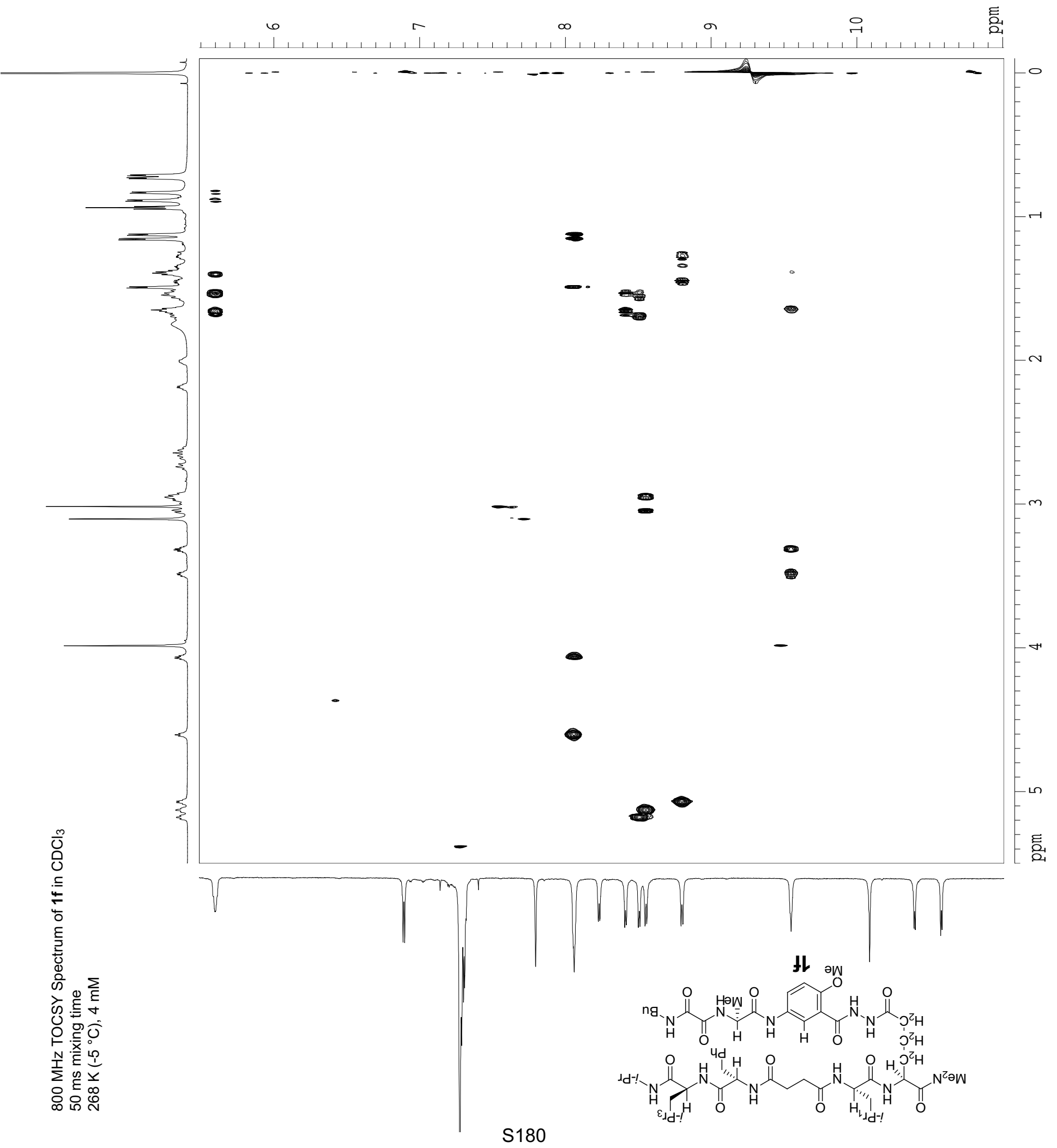




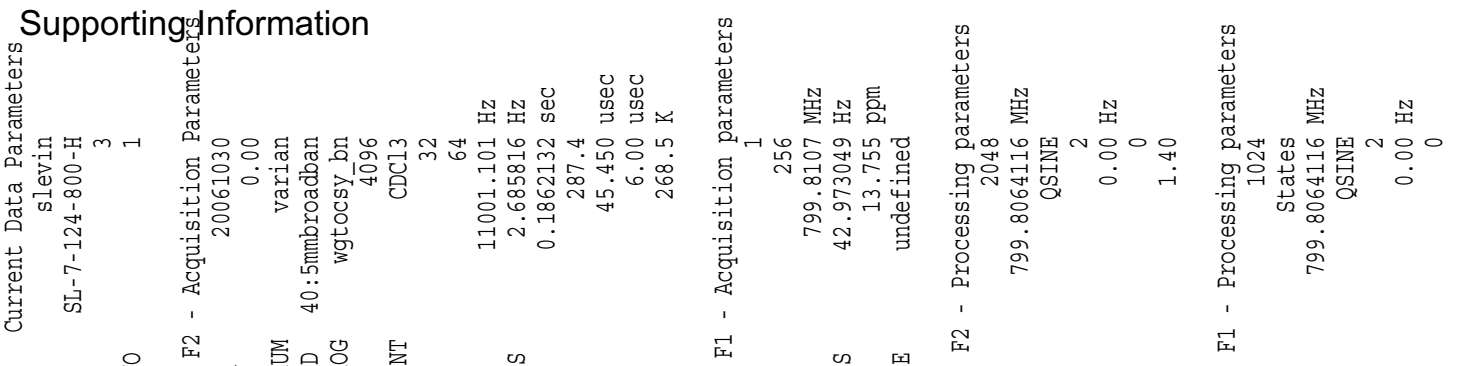

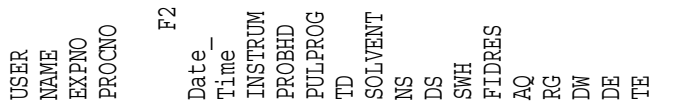
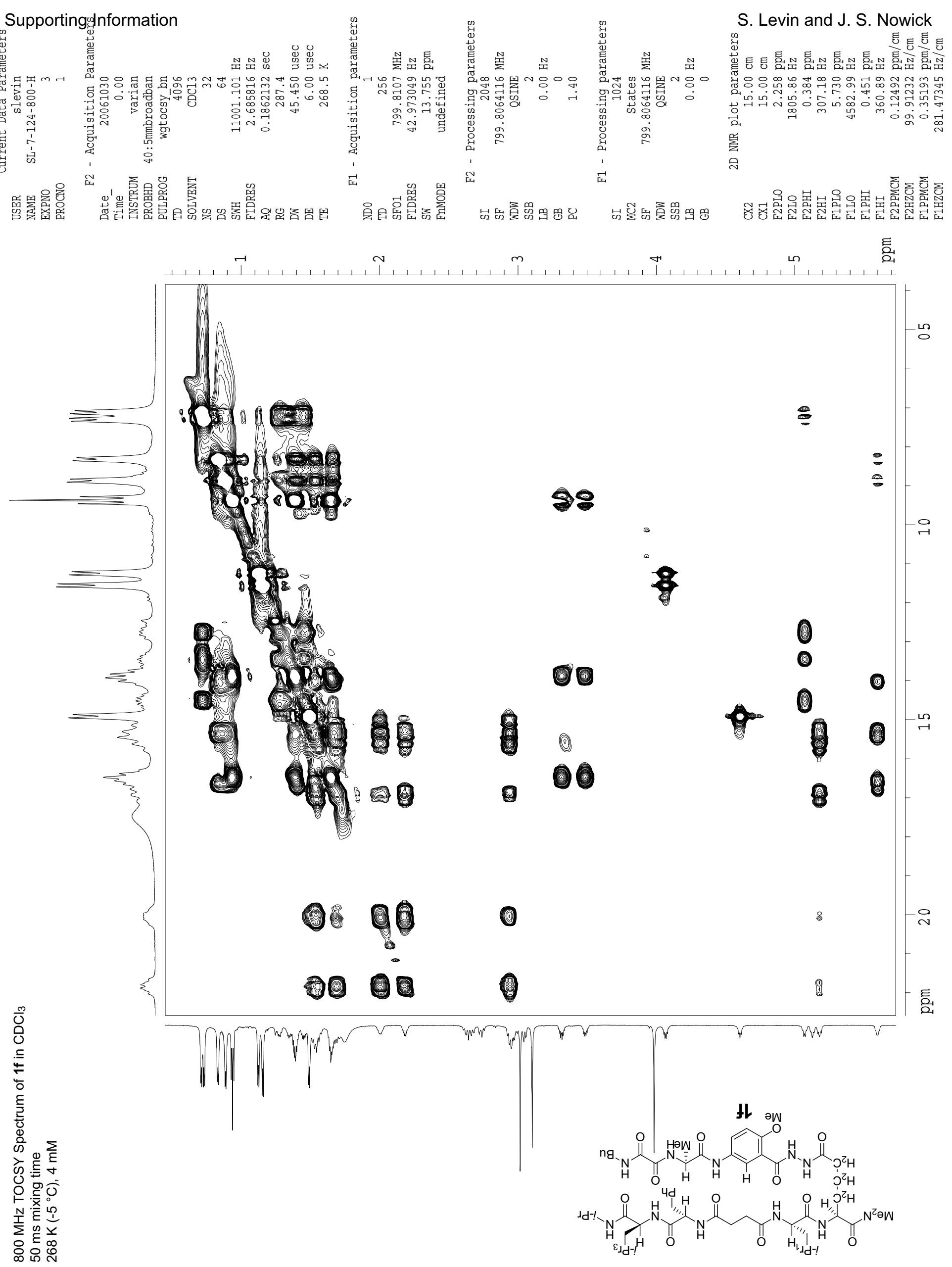


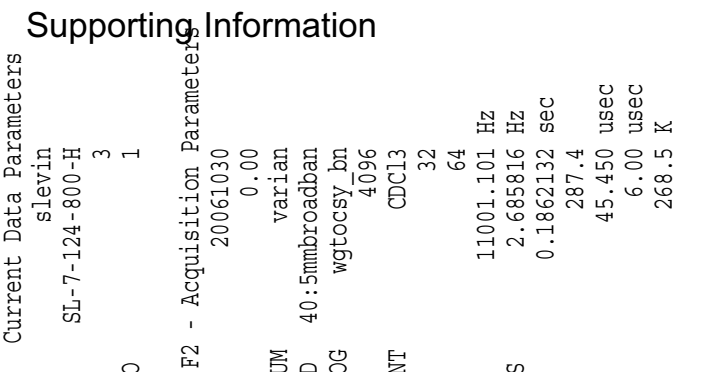

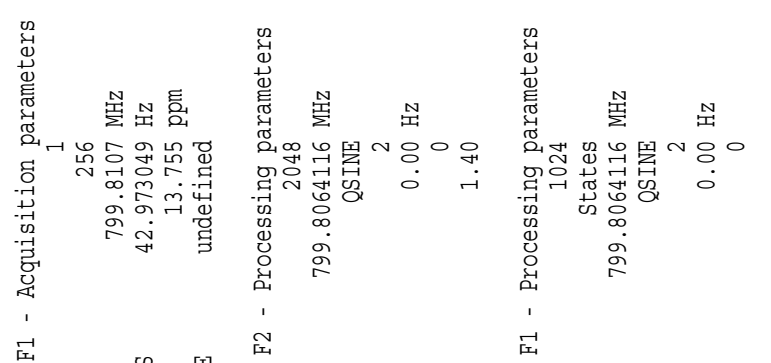

S. Levin and J. S. Nowick

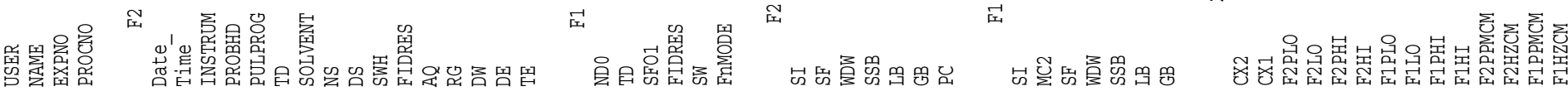

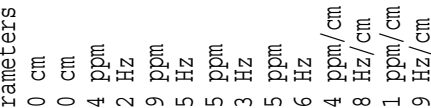

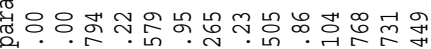

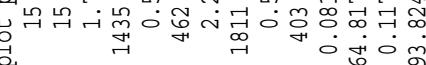
罢

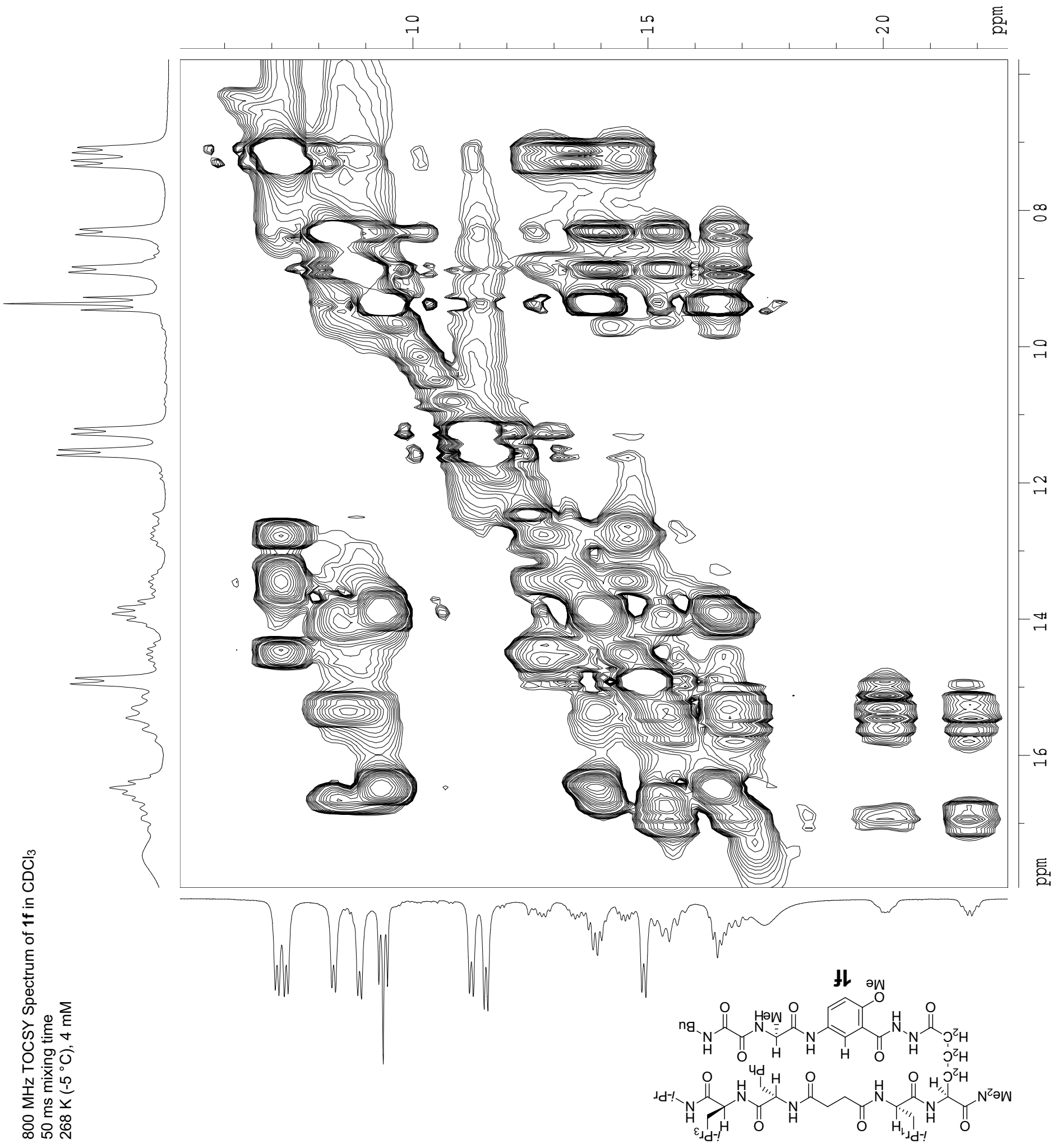



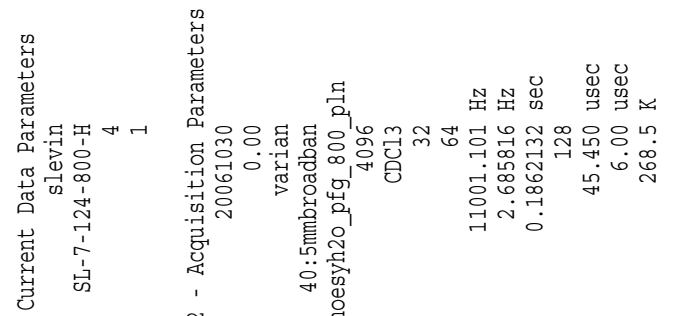

畟舆畜总 z =

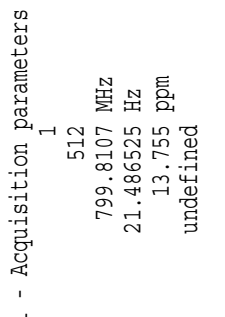

\% y

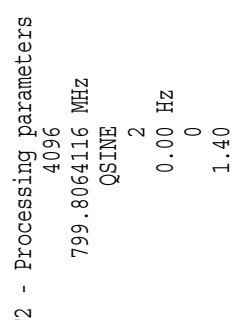

䑰

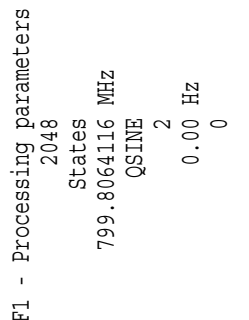

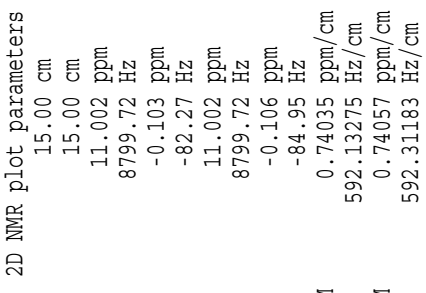

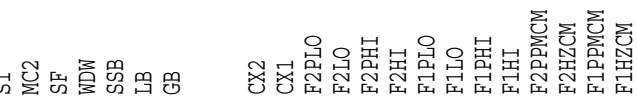

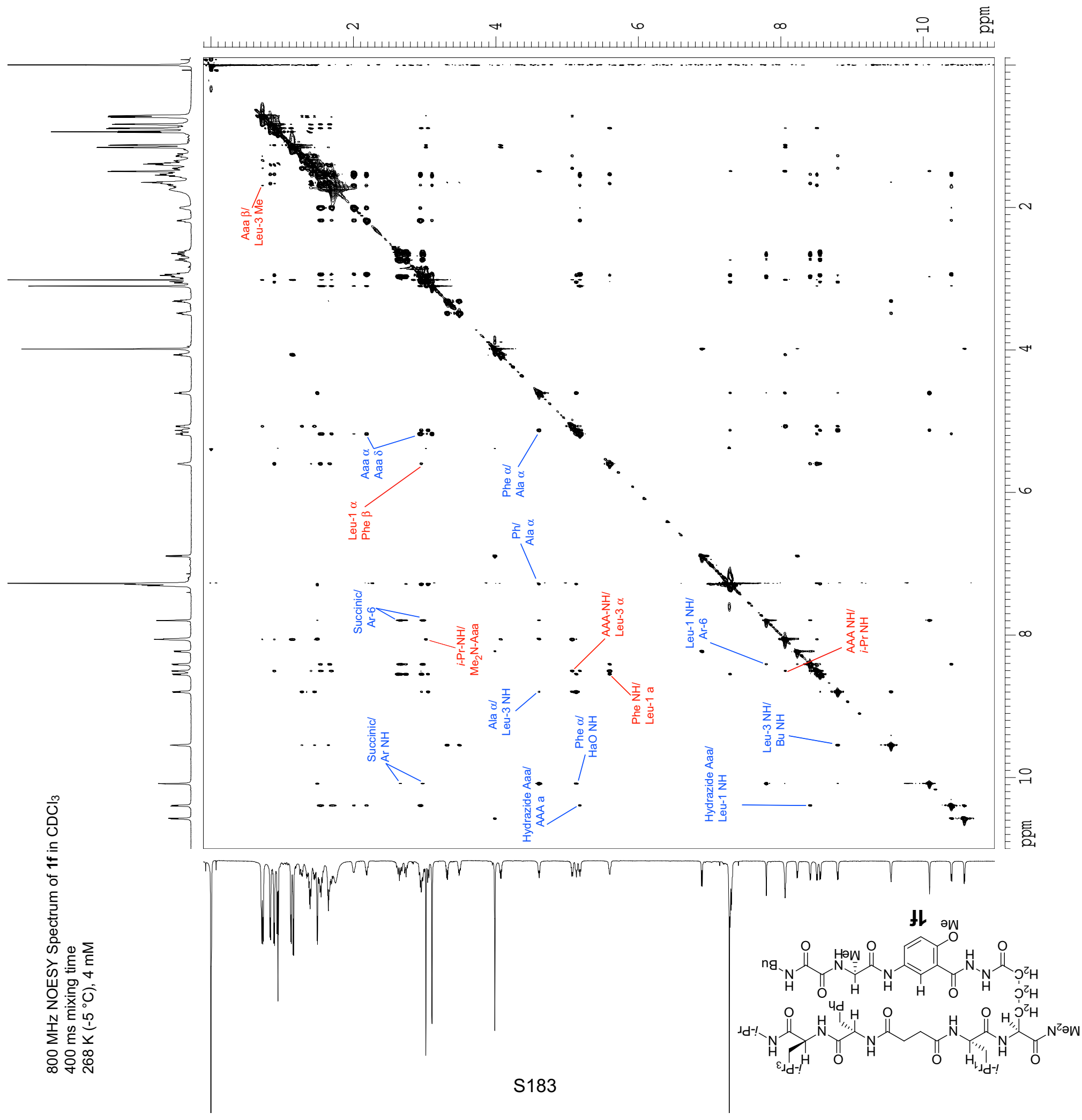


Supporting Information

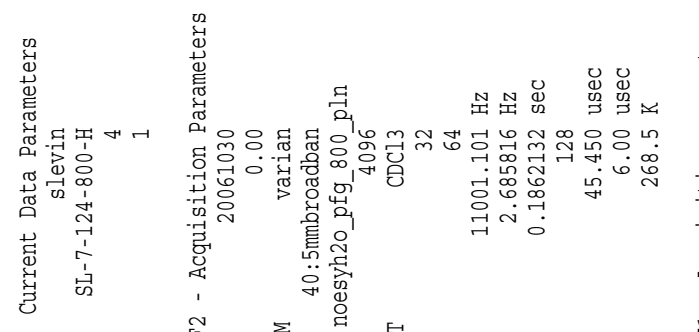

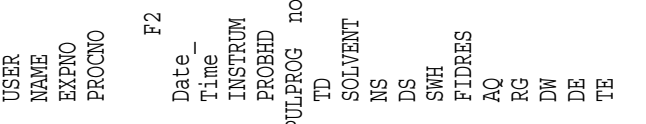

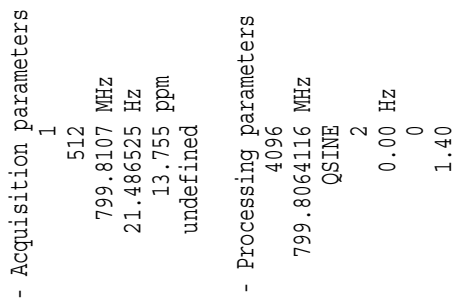

届被
S. Levin and J. S. Nowick

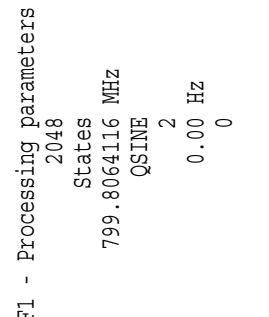

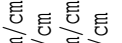

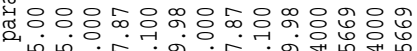
늠 嵌 नूं नूं

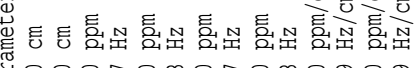

$\sim$ 6 $\infty$ 이 हूँ

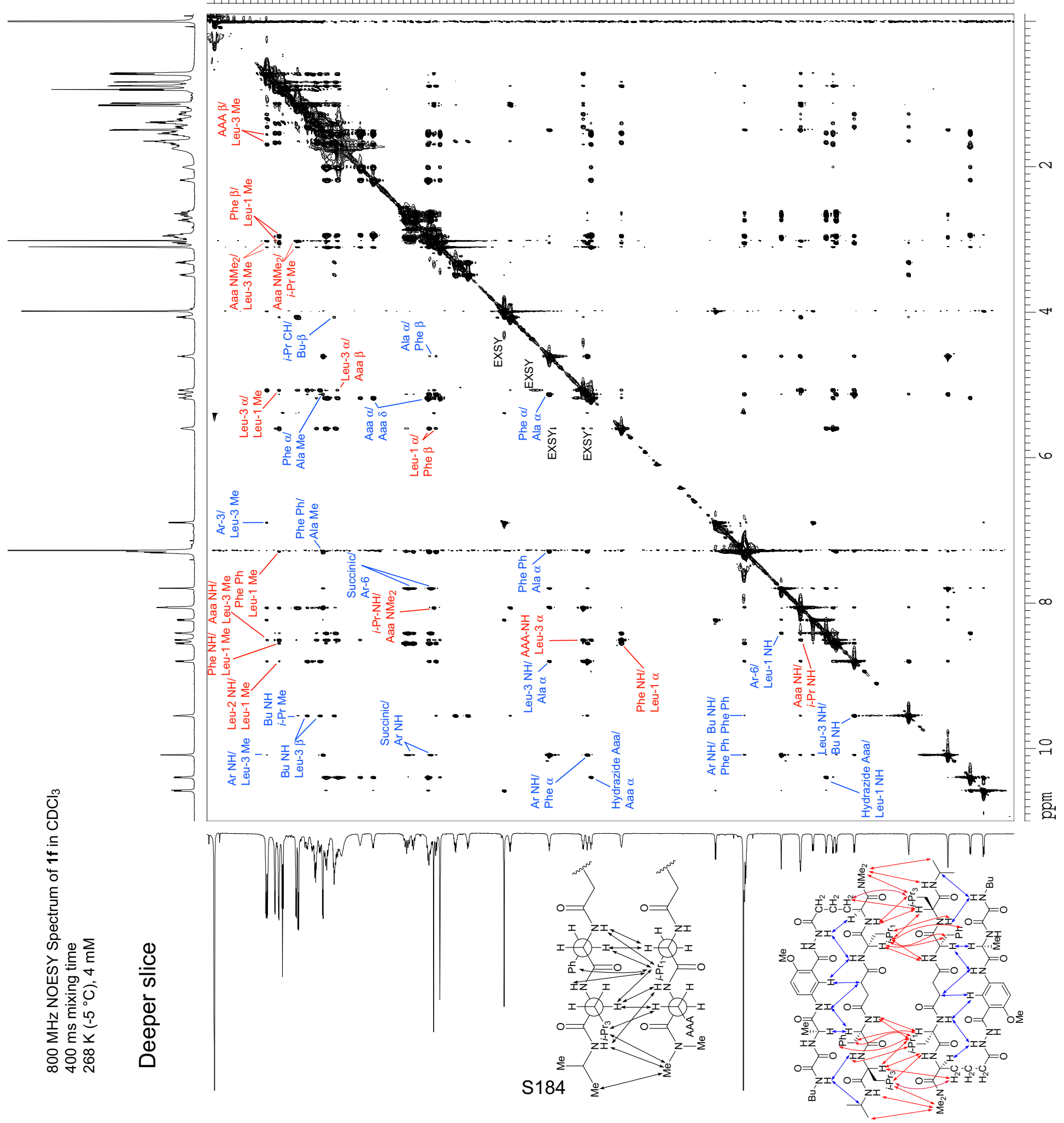


Supporting Information

S. Levin and J. S. Nowick
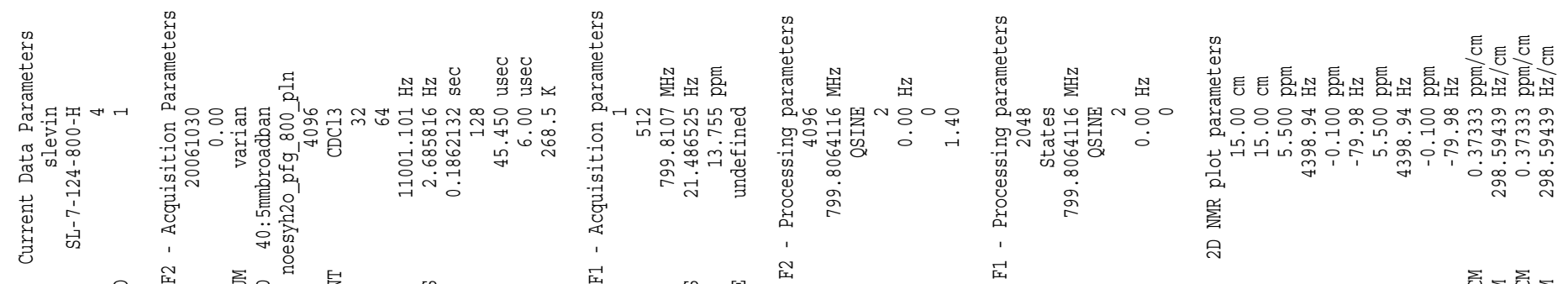

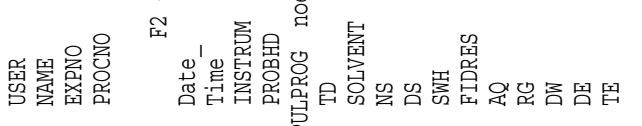

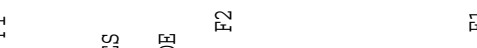

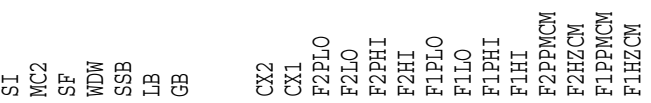

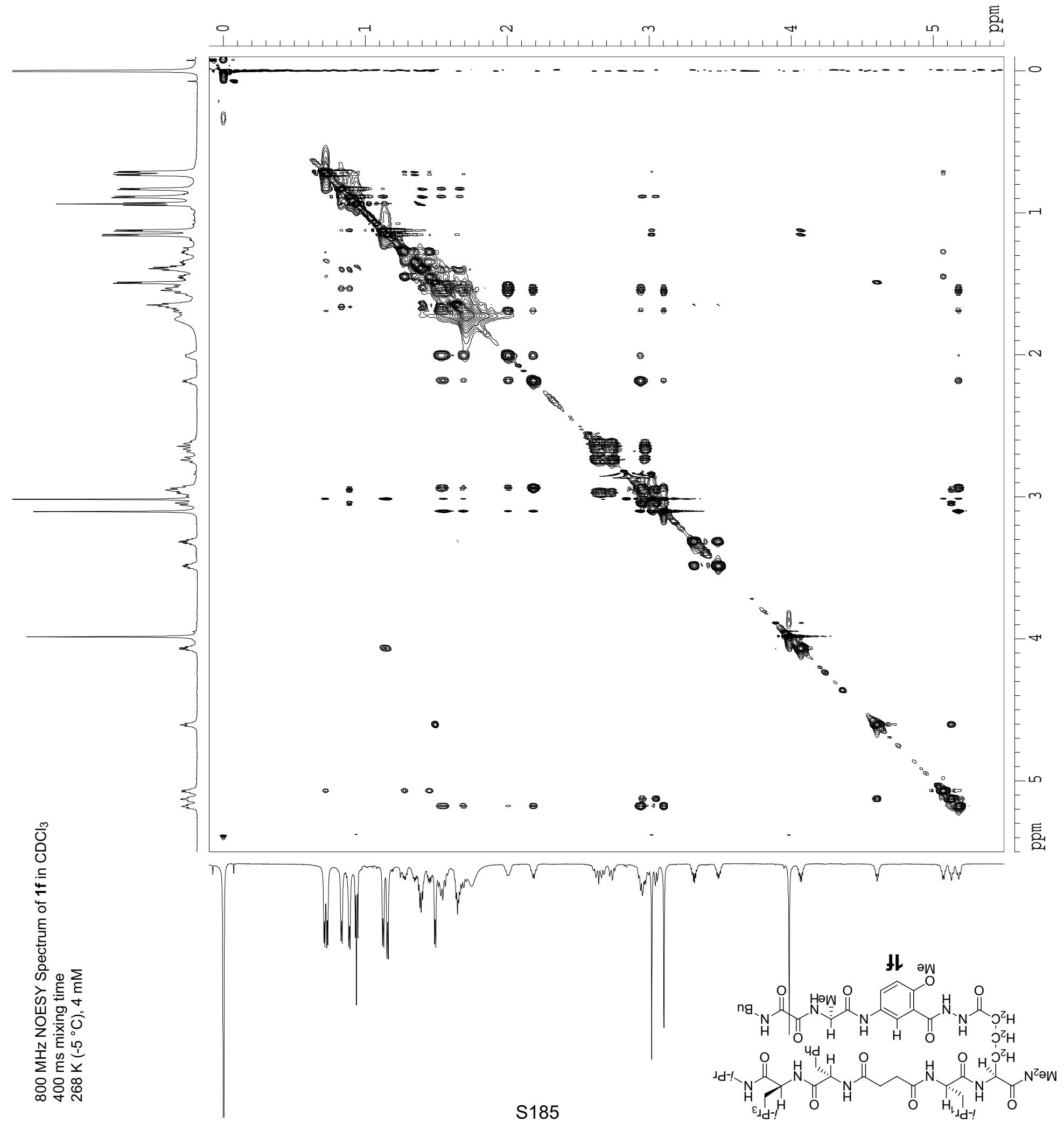




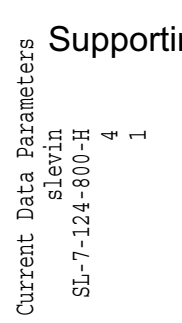

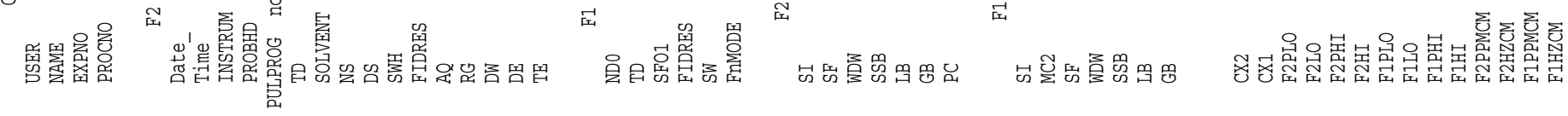

ing Information

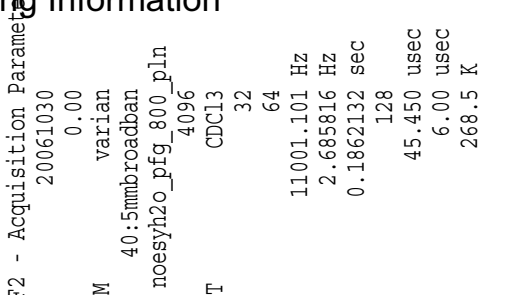

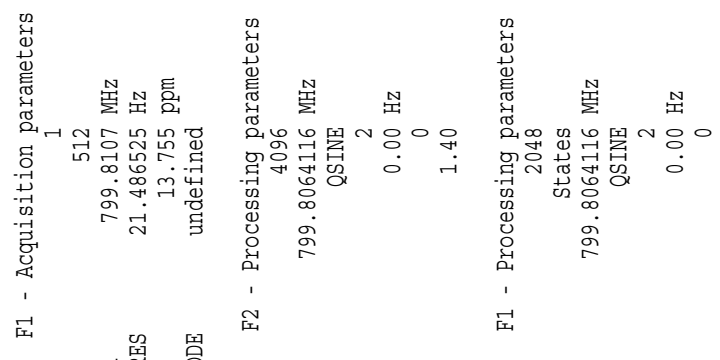

S. Levin and $\mathrm{J}$ S.Nowick

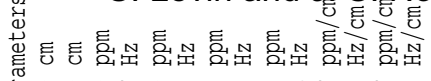

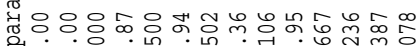

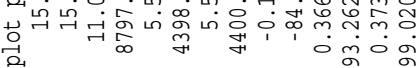
誉 ลิ

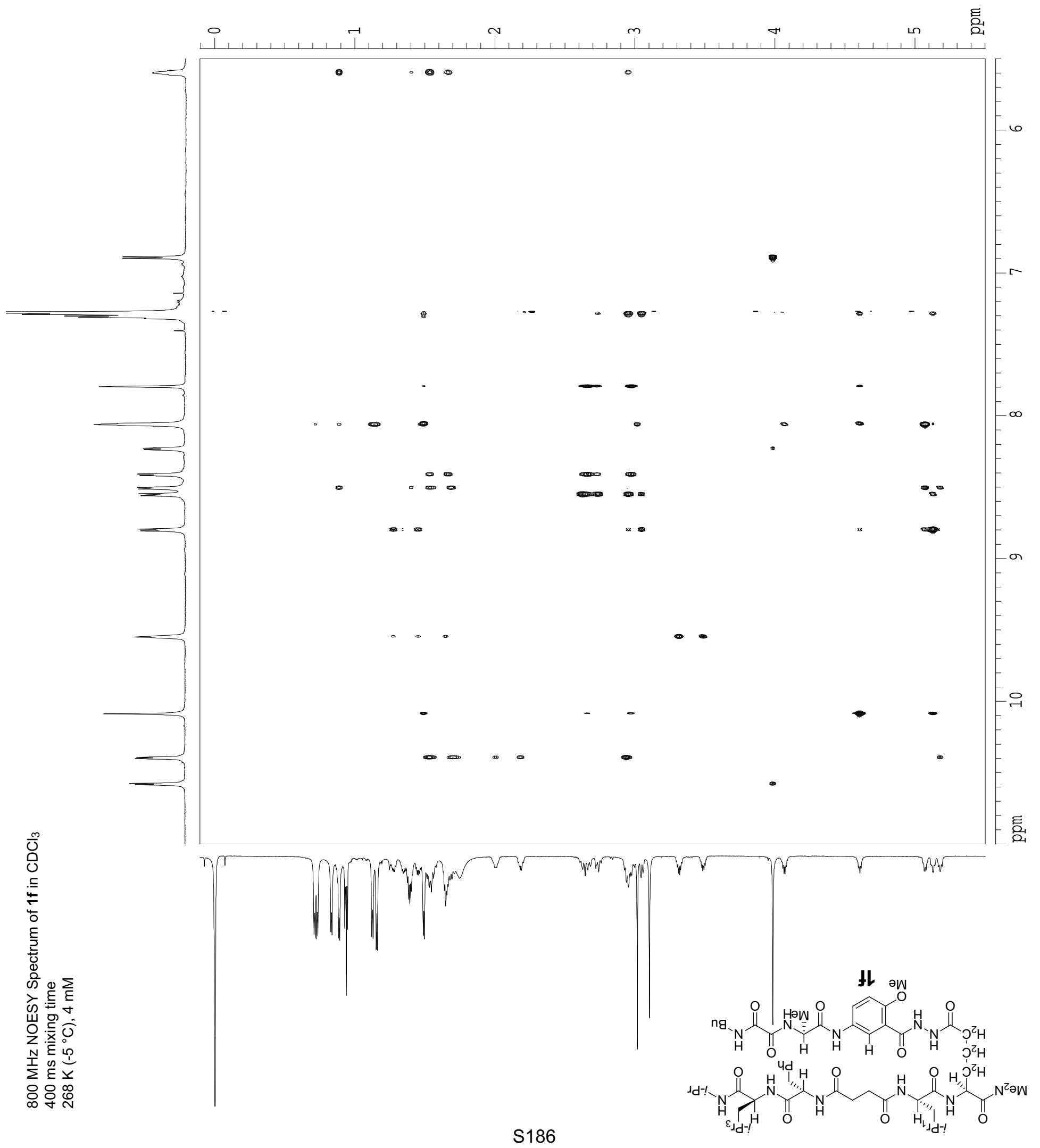


Supporting Information

S. Levin and J. S. Nowick
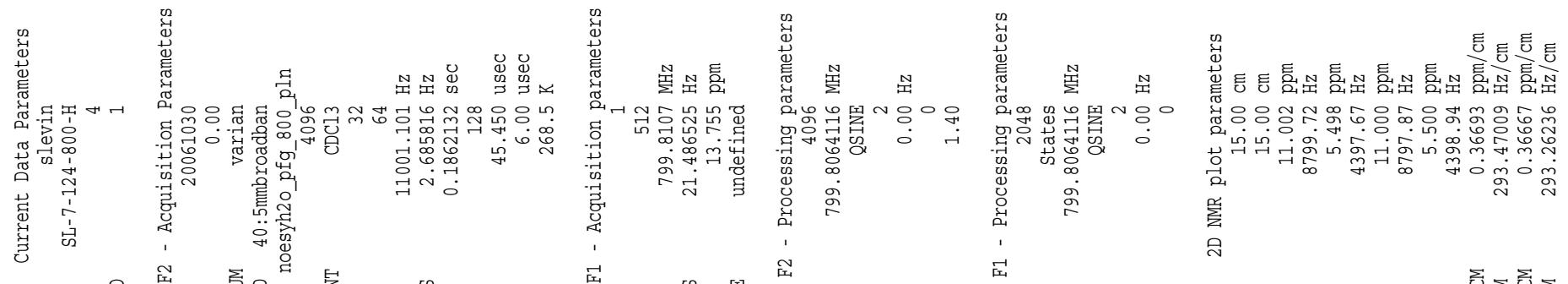
乘罢总

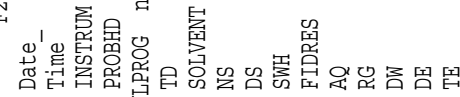

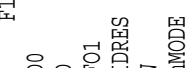

\section{空识总}

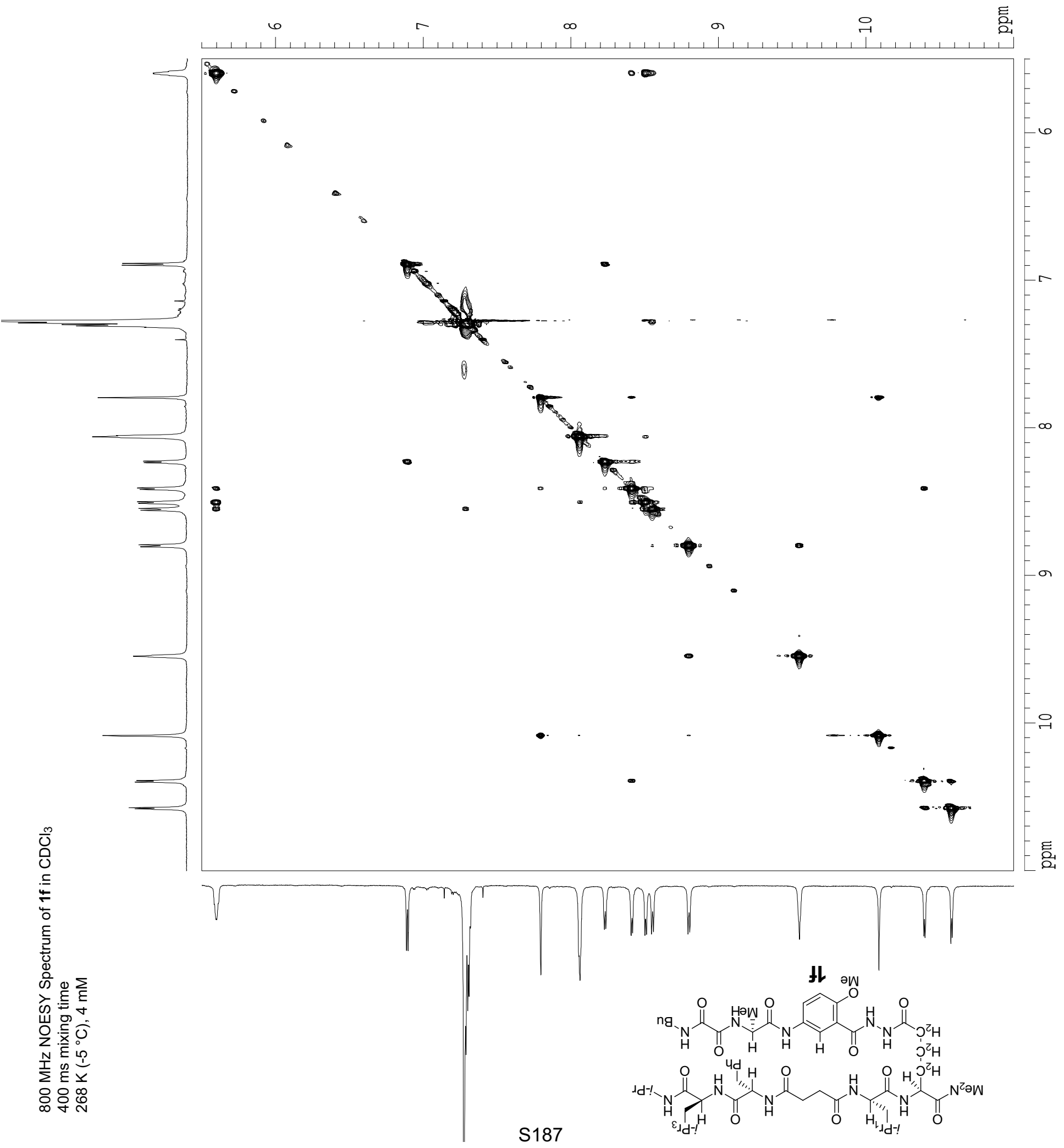


Supporting Information
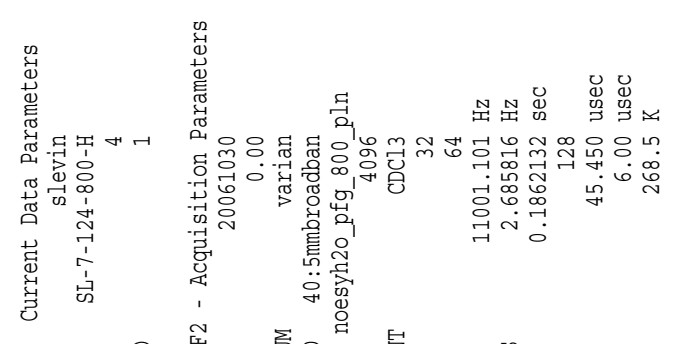

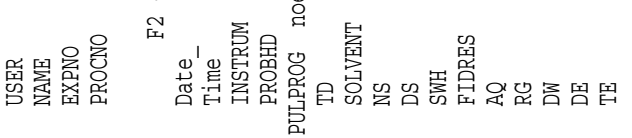

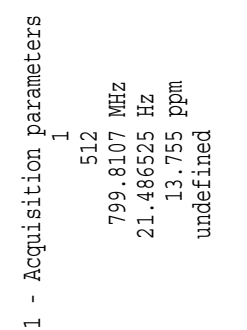

(1)

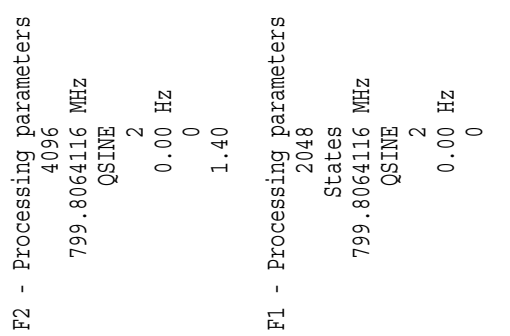

空

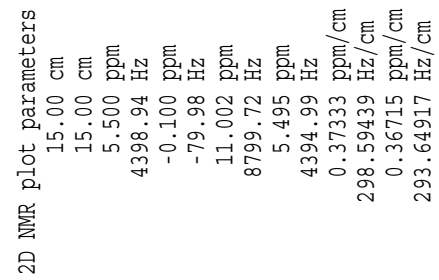

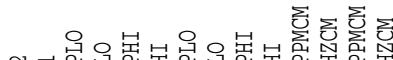

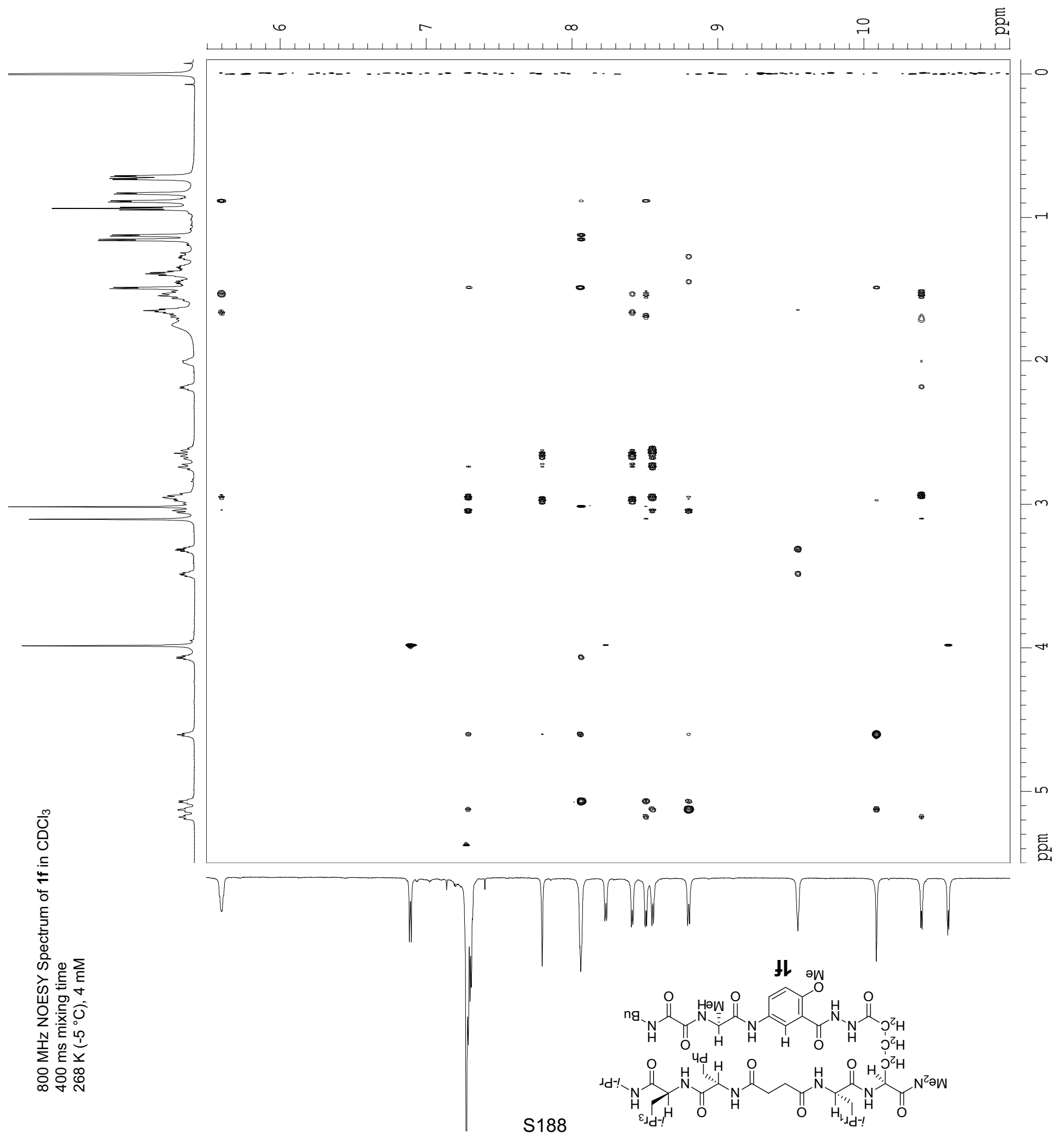



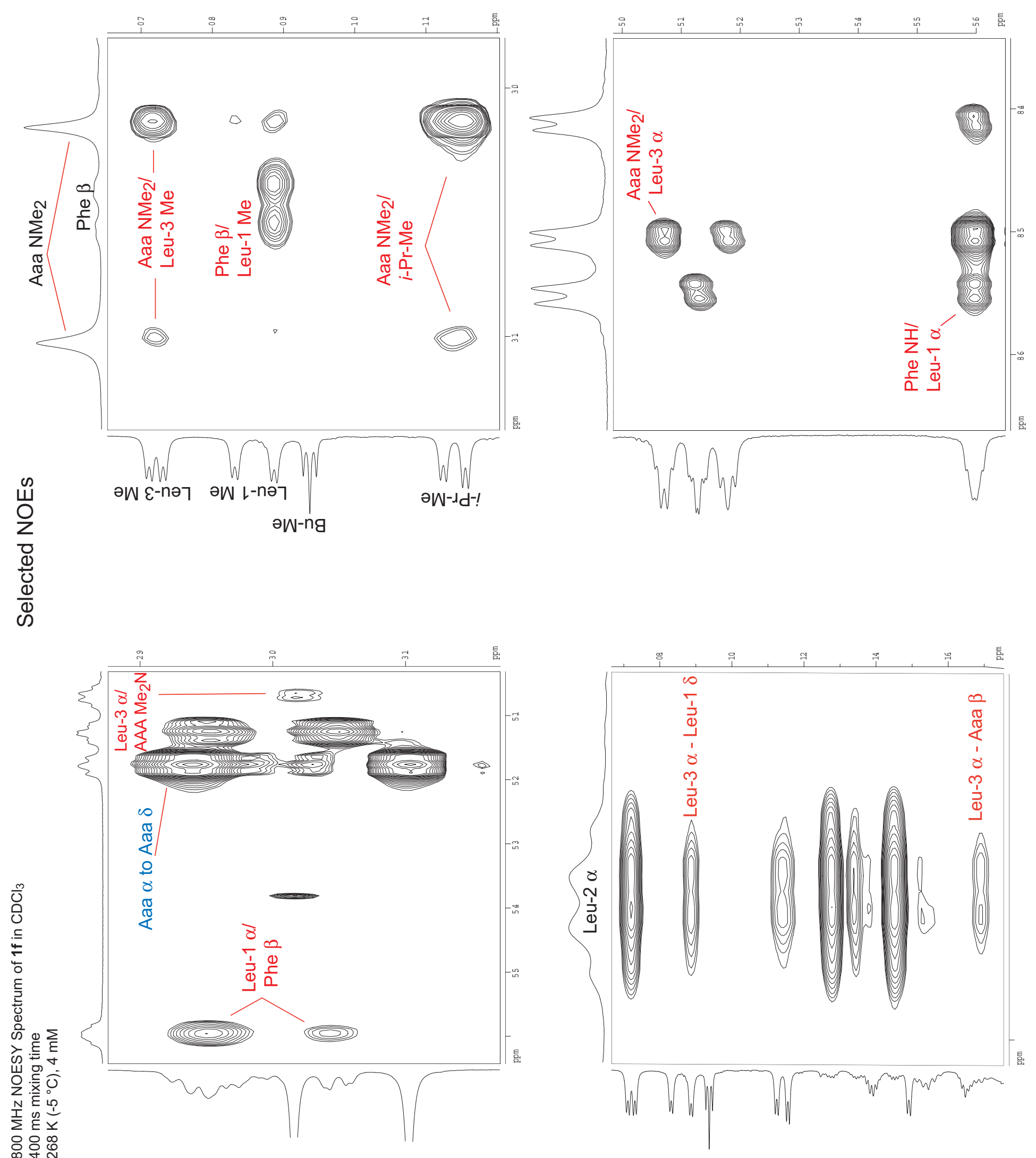


\section{Spectroscopic DATA for the compound 2a}

(3 mM, $\mathrm{CDCl}_{3} 268 \mathrm{~K}$ )

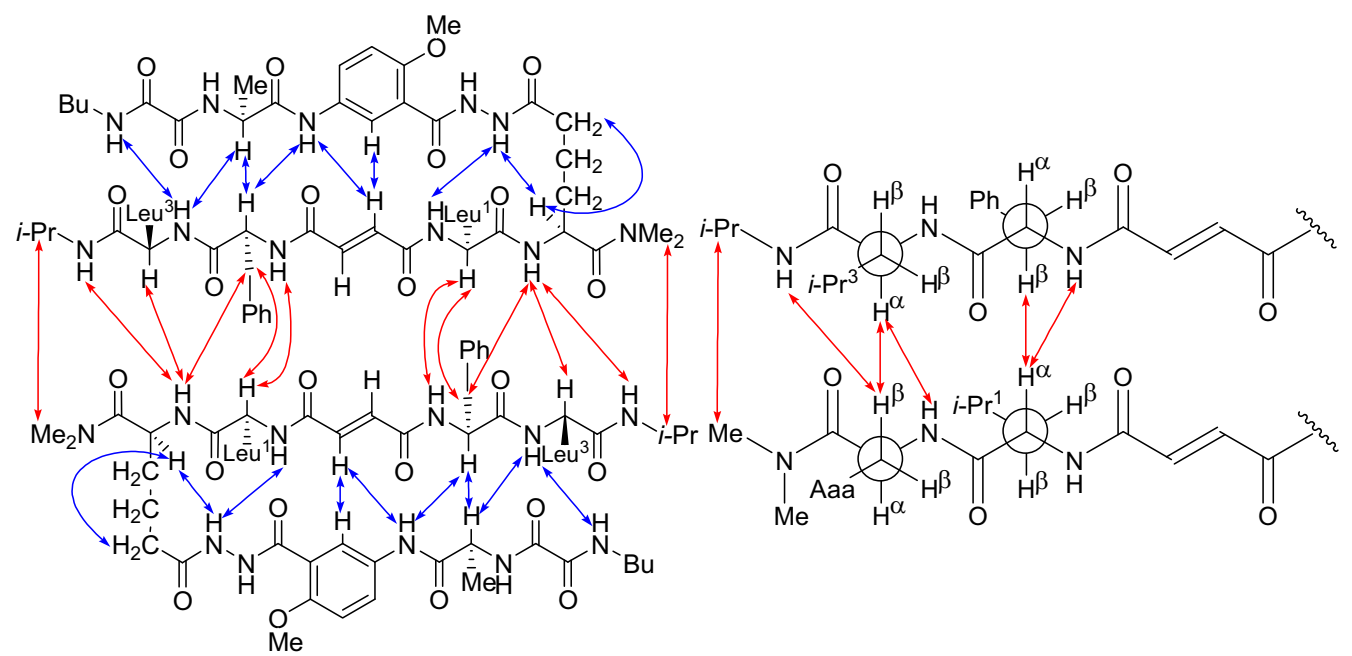

Key NOEs assigned as intermolecular are represented with red arrows.

Key NOEs assigned as intramolecular are represented with blue arrows.

Key data for amino acids.

\begin{tabular}{|c|c|c|c|}
\hline Amino acid & $\mathrm{H}_{\alpha}$ Shift $\mathrm{ppm}$ & NH Shift $\mathrm{ppm}$ & ${ }^{3} J_{\mathrm{NH} \alpha} \mathrm{Hz}$ \\
\hline Aaa & 5.20 & 8.77 & 8.2 \\
\hline $\mathrm{R}_{1}=$ Leu & 5.80 & 8.55 & 8.2 \\
\hline $\mathrm{R}_{2}=$ Phe & 5.20 & 9.20 & 9.8 \\
\hline $\mathrm{R}_{3}=$ Leu $^{2}$ & 5.05 & 8.74 & broad \\
\hline $\mathrm{R}_{4}=\mathrm{Ala}$ & 4.50 & 8.05 & broad \\
\hline
\end{tabular}

Aminoadipic acid (Aaa) $\delta$ proton resonances.

pro-R: $2.85 \mathrm{ppm}, \mathrm{t},(J=12.0 \mathrm{~Hz})$

pro-S: $2.20 \mathrm{ppm}, \mathrm{td},\left(J_{1}=11.5 \mathrm{~Hz} ; J_{2}=7.0 \mathrm{~Hz}\right)$

anisotropy $=0.65 \mathrm{ppm}$

Key data for other NH protons.

\begin{tabular}{|c|c|c|}
\hline Proton & NH Shift ppm & $J \mathrm{~Hz}$ \\
\hline Ar-NH & 10.20 & - \\
\hline Hydrazide Ar & 10.45 & broad \\
\hline Hydrazide Aaa & 10.33 & broad \\
\hline Bu-NH & 9.53 & broad \\
\hline$i$-Pr-NH & 8.10 & 7.6 \\
\hline
\end{tabular}

ESI MS $m / z$ for $\mathrm{C}_{53} \mathrm{H}_{80} \mathrm{~N}_{11} \mathrm{O}_{12}[\mathrm{M}+\mathrm{H}]^{+}$calcd 1062.60, found 1062.48. 


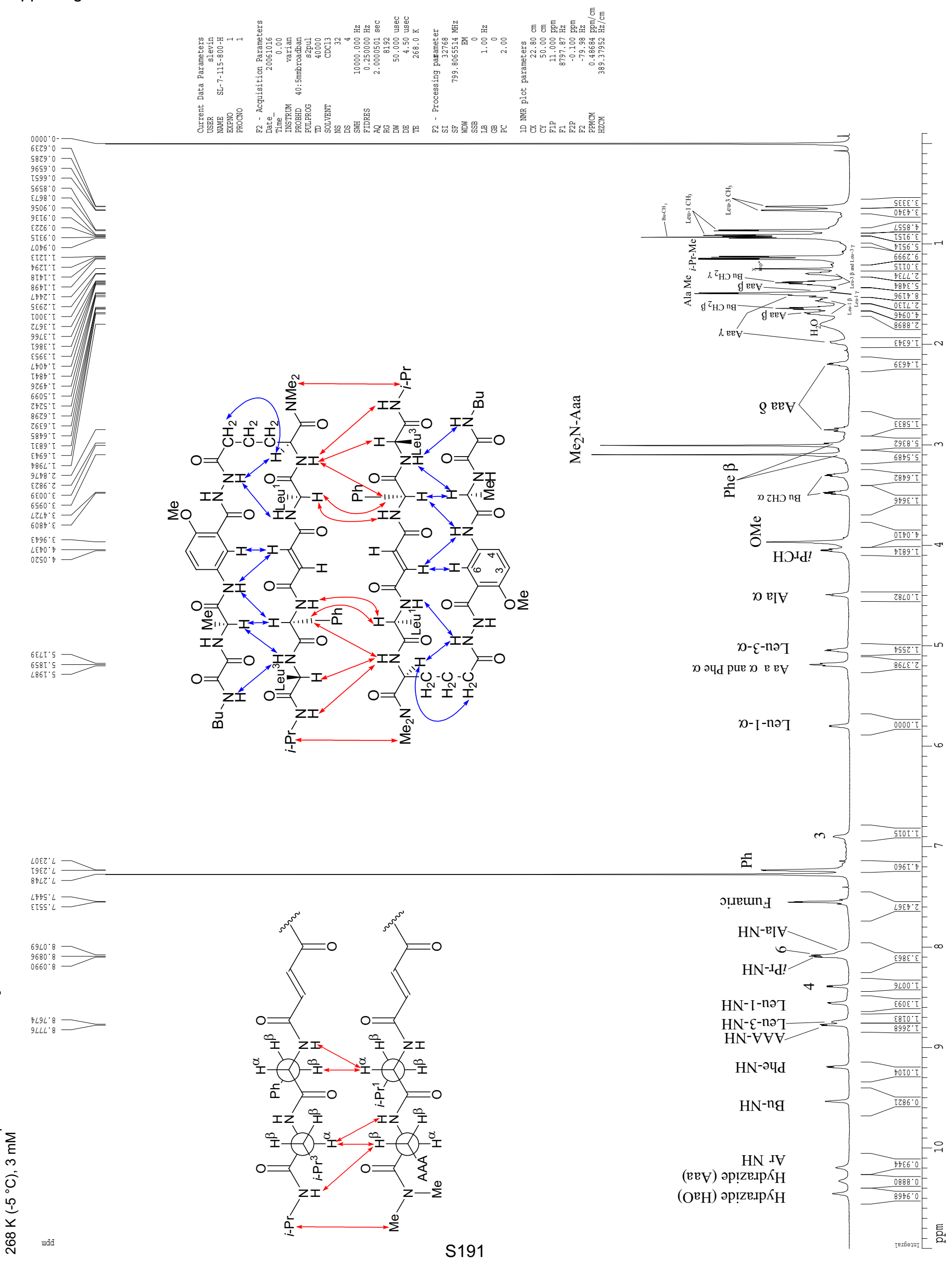



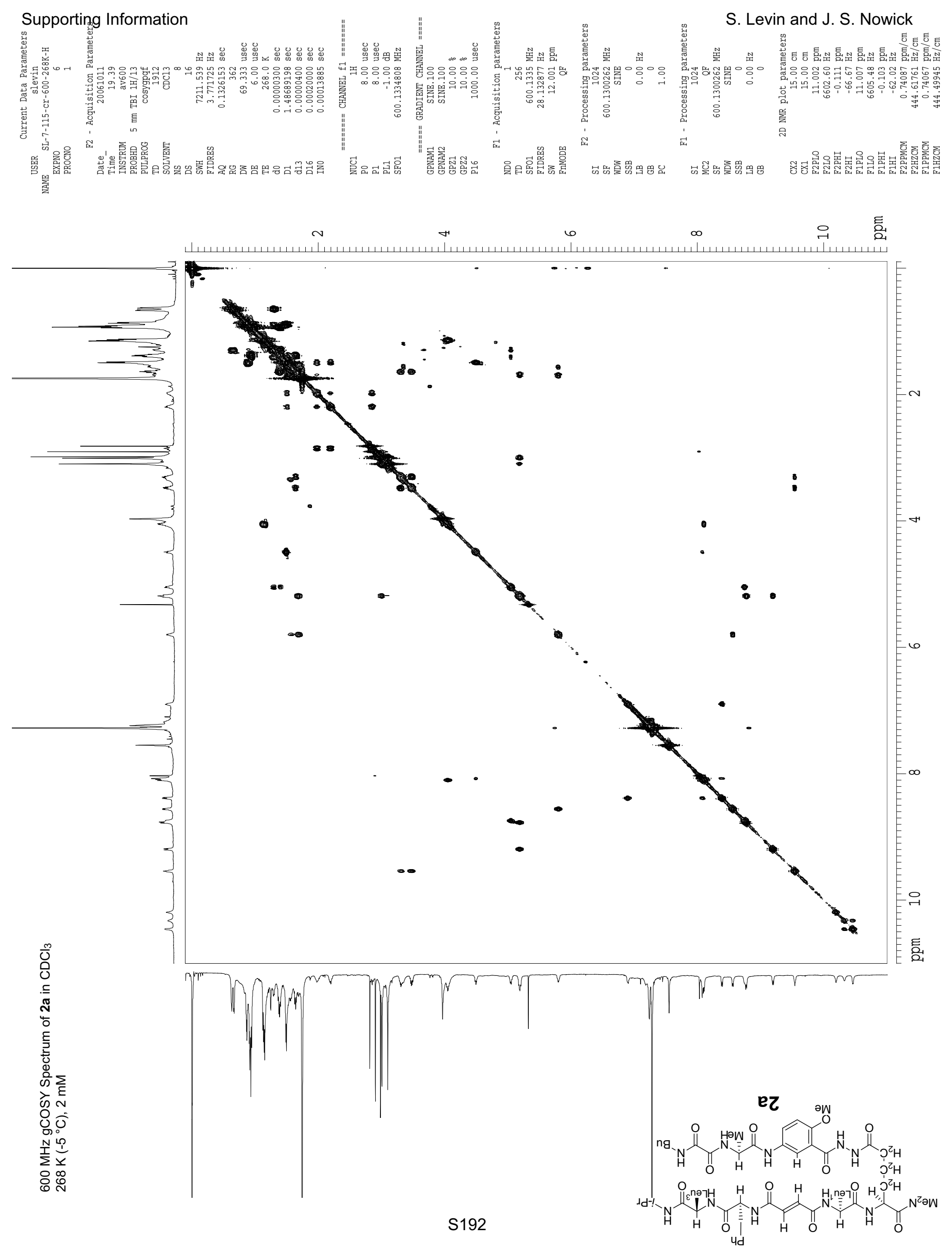

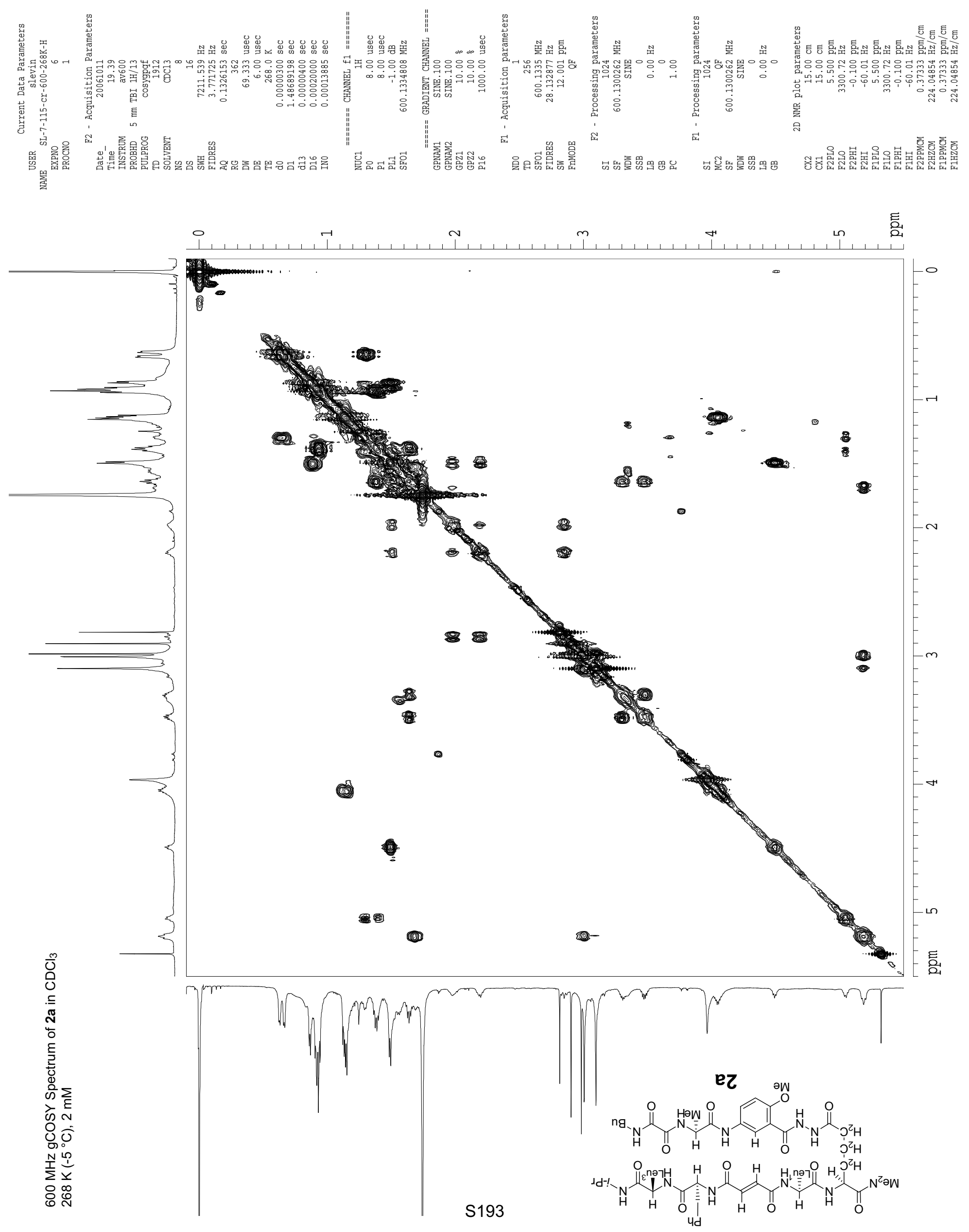
Supporting Information
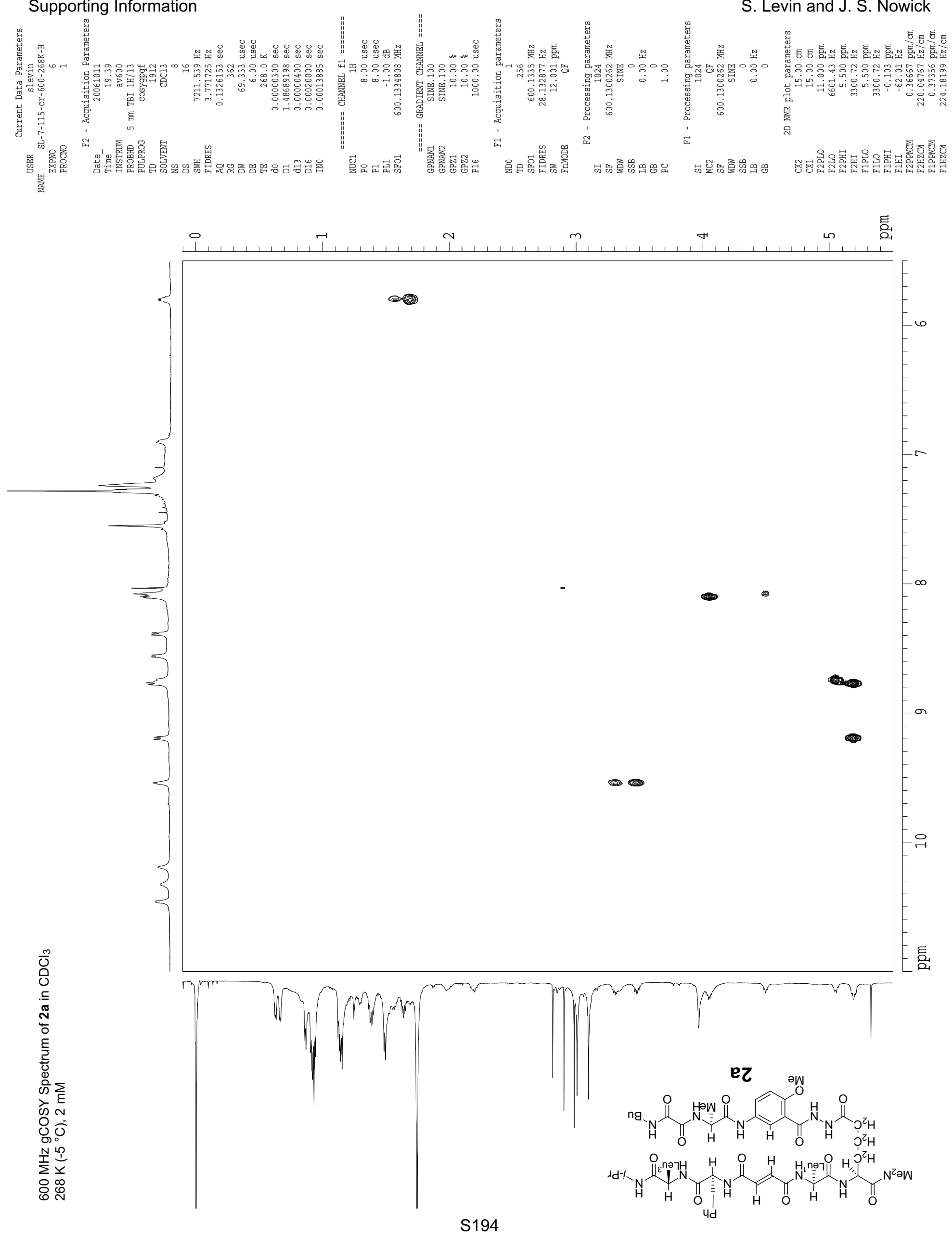
Supporting Information

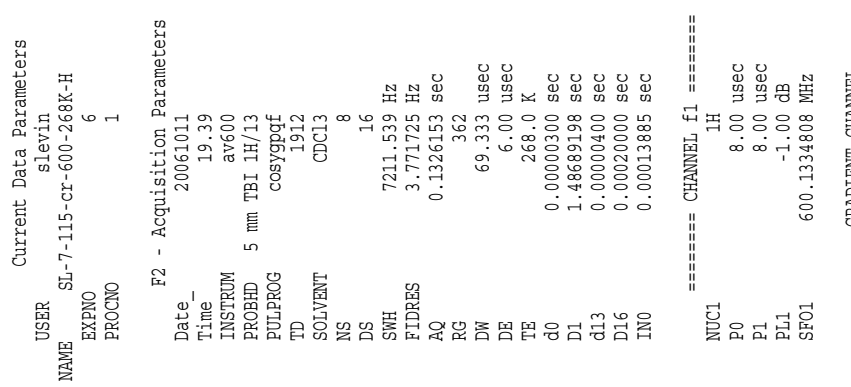

S. Levin and J. S. Nowick
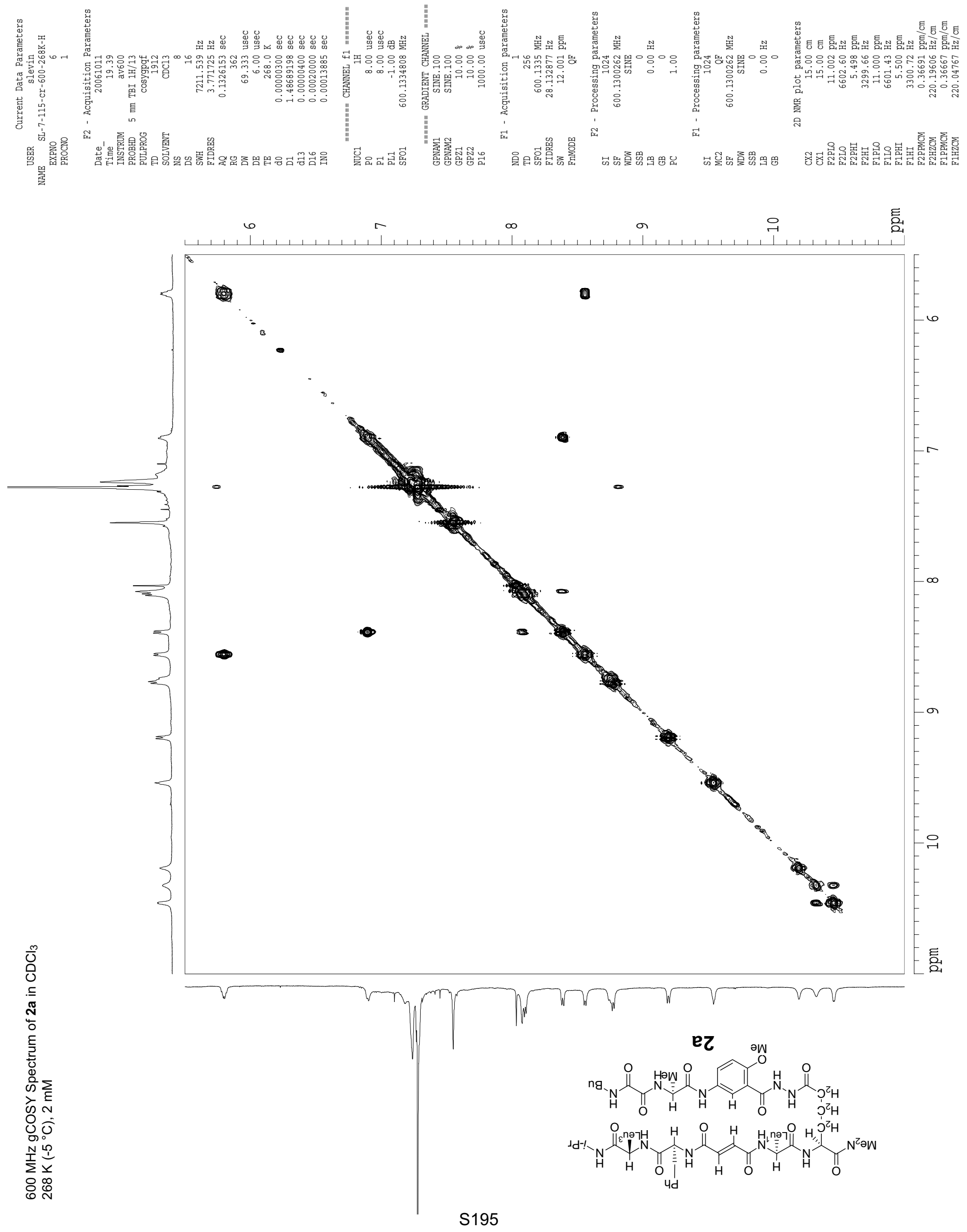

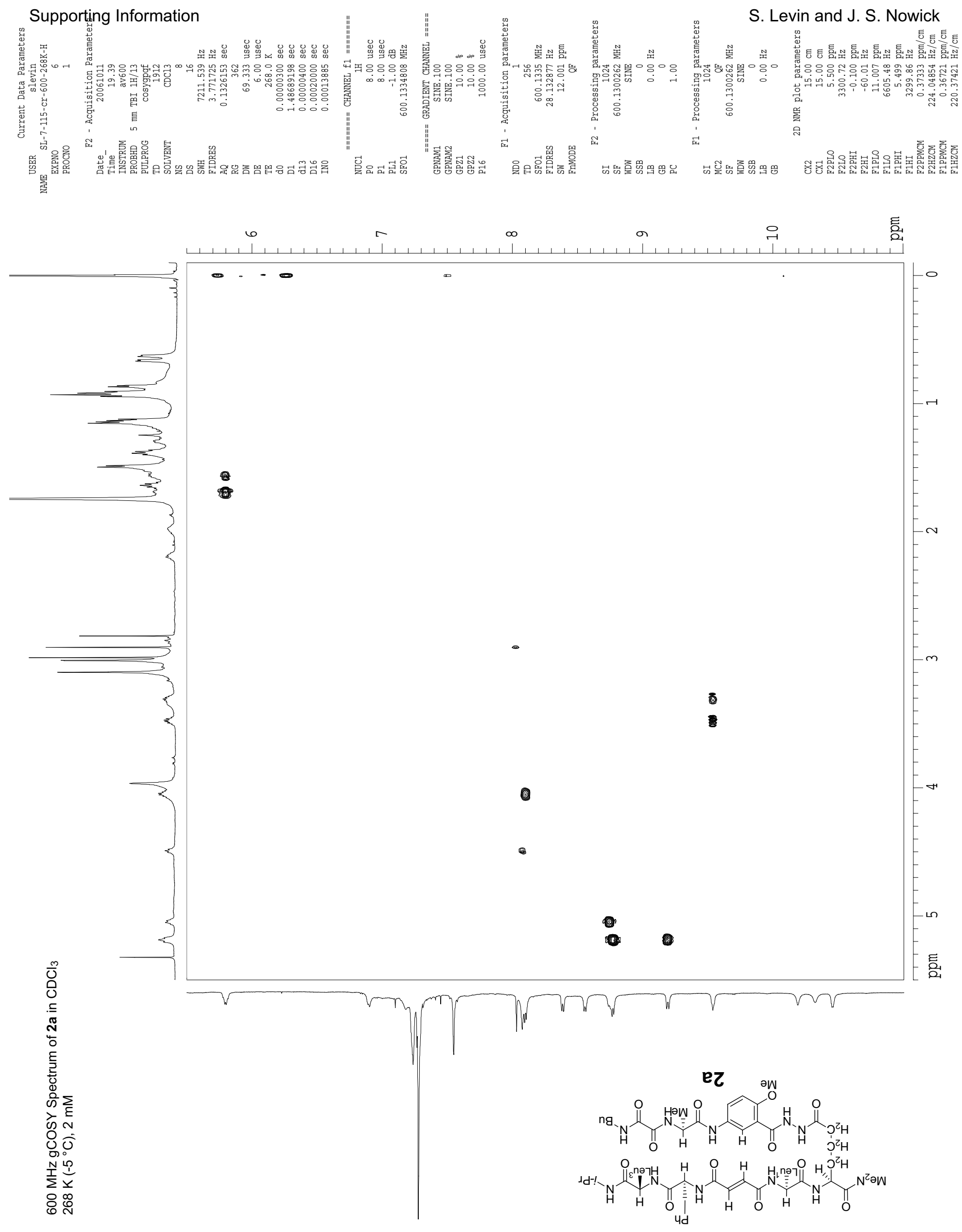
Supportingednformation
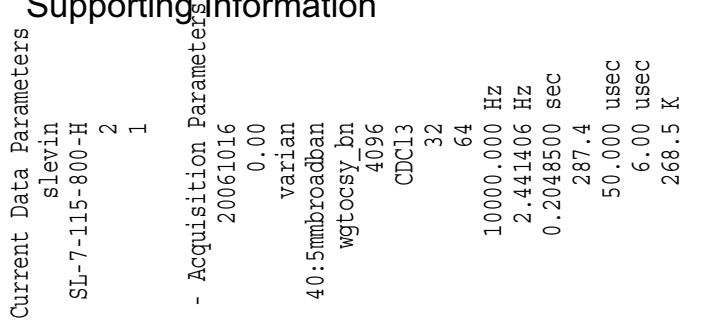

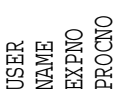

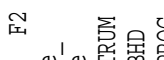

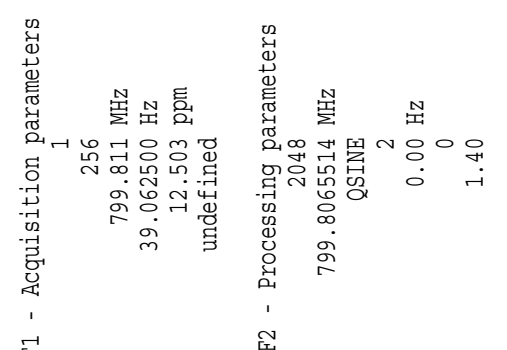

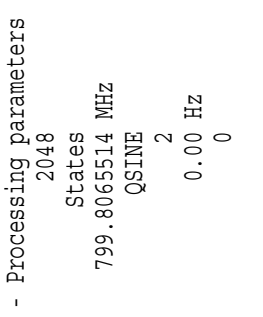

S. Levin and J. S. Nowick 㫕

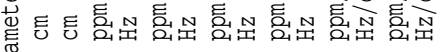

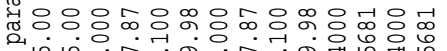

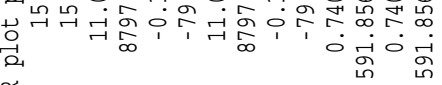
䍗

ล 岳

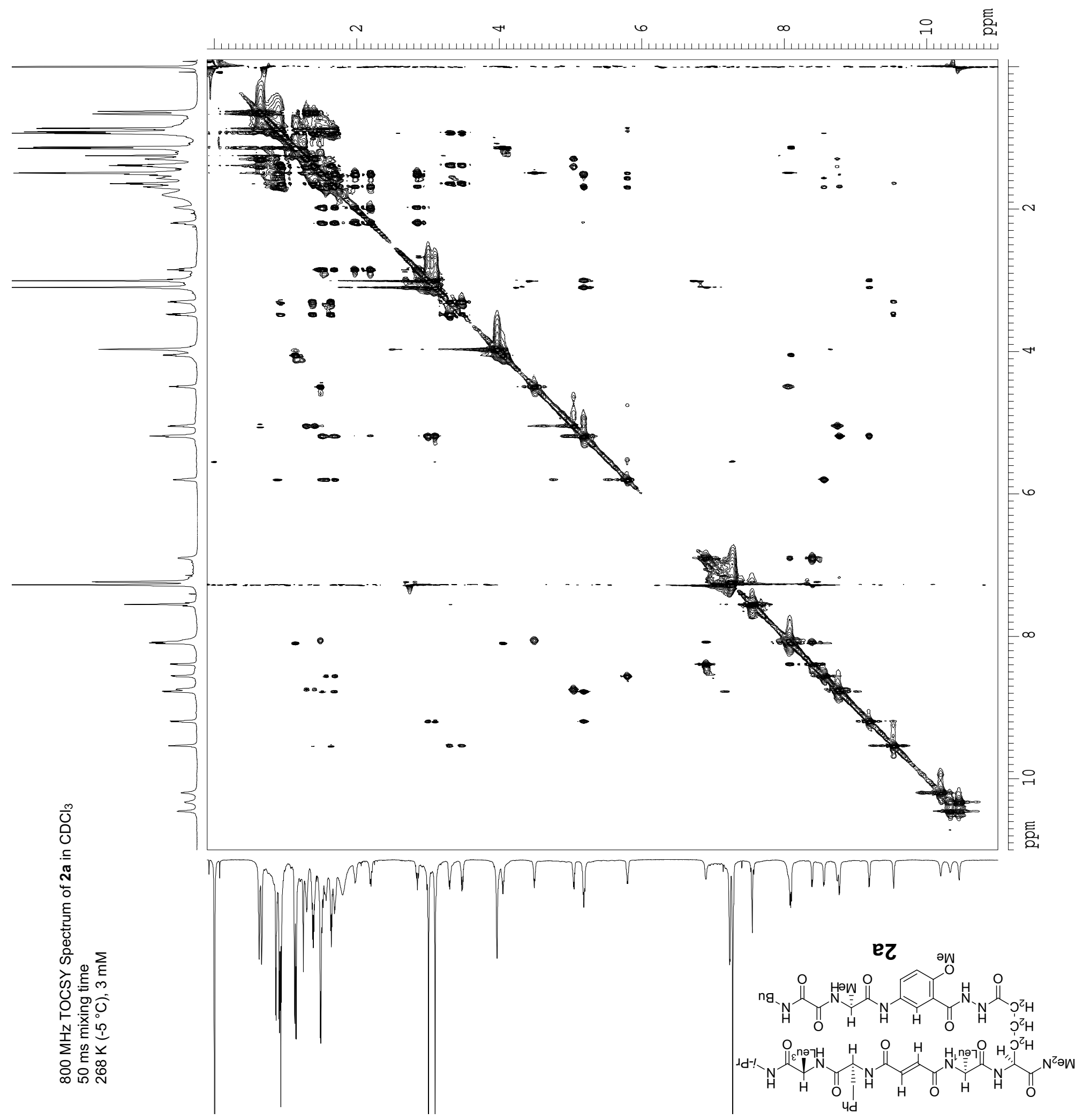




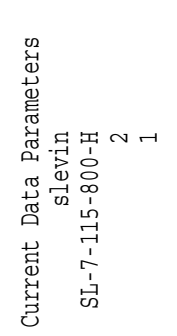

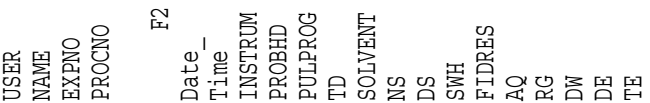

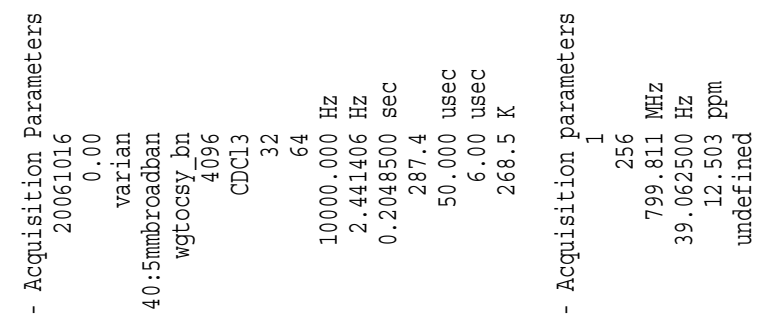

㞋

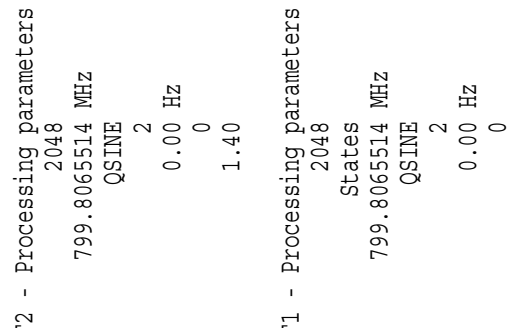

项

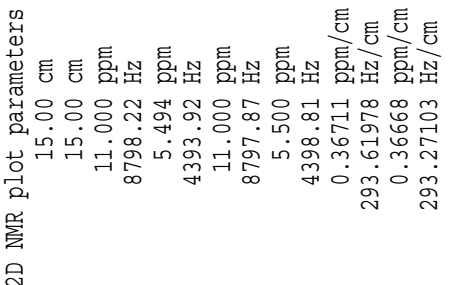

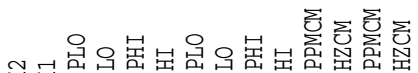

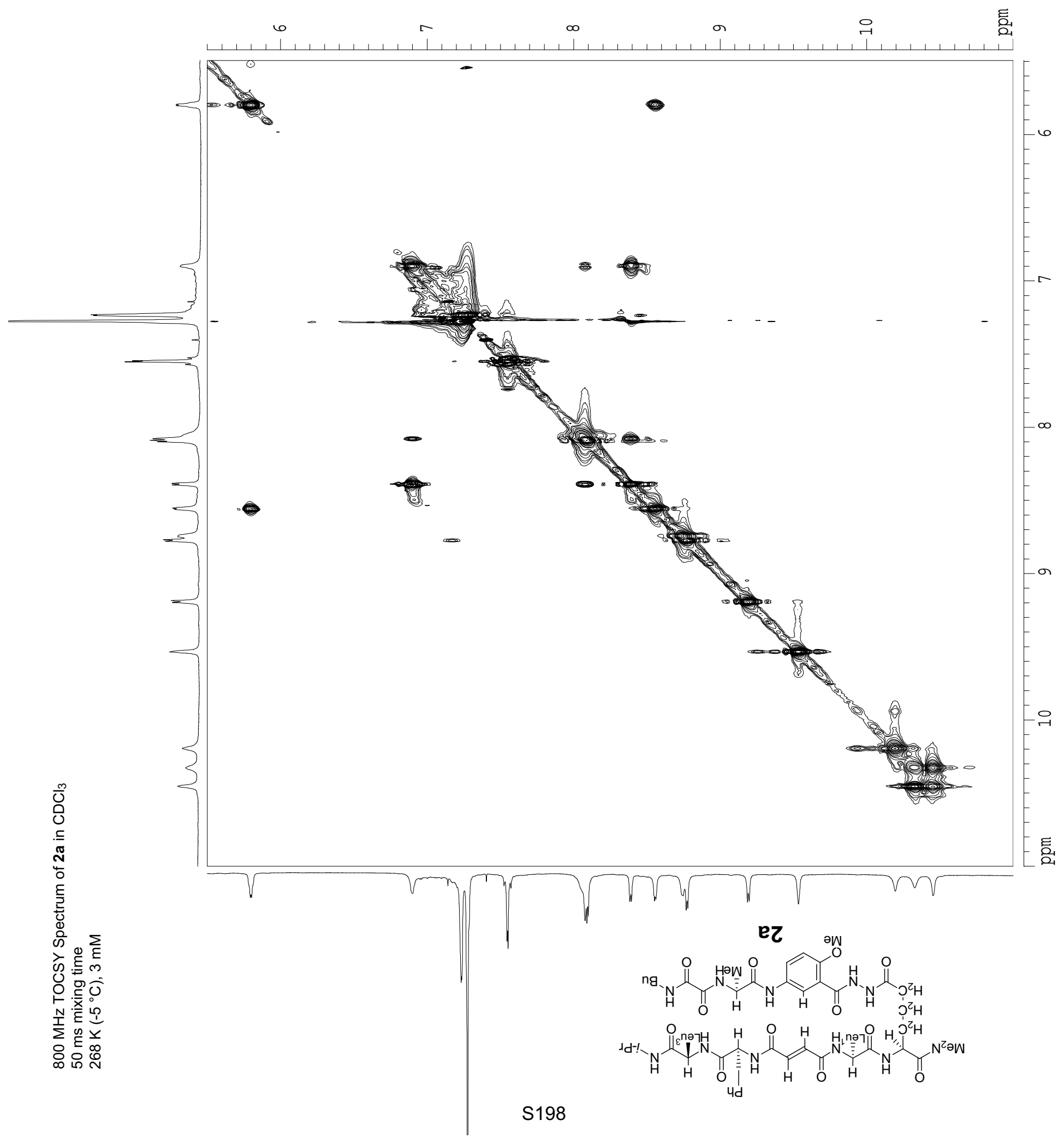


Supporting Information

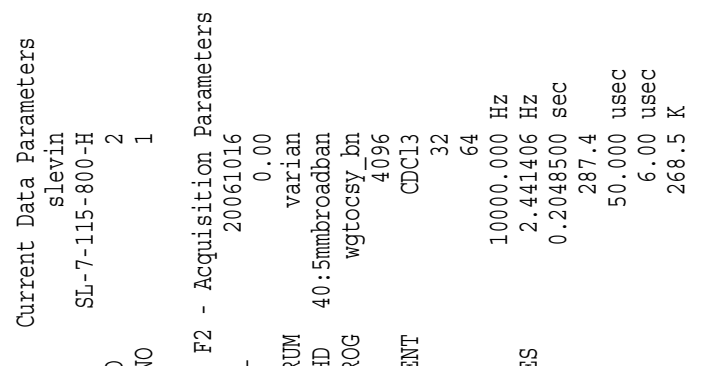

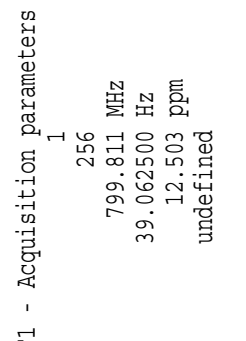

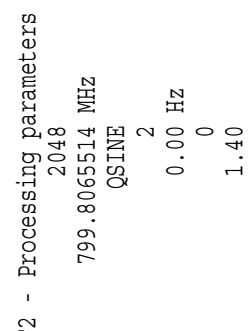

S. Levin and J. S. Nowick

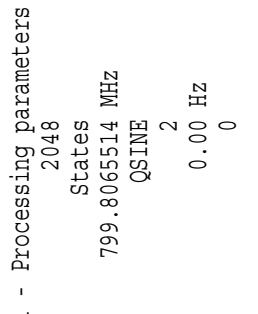

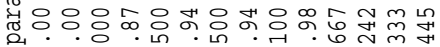

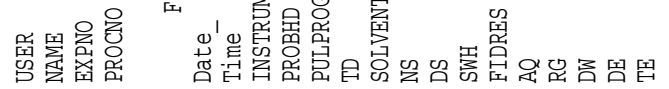

婇 i

空

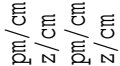

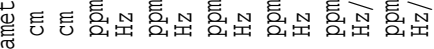

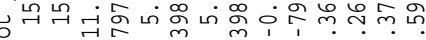
学

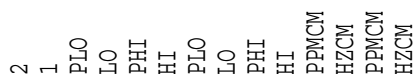

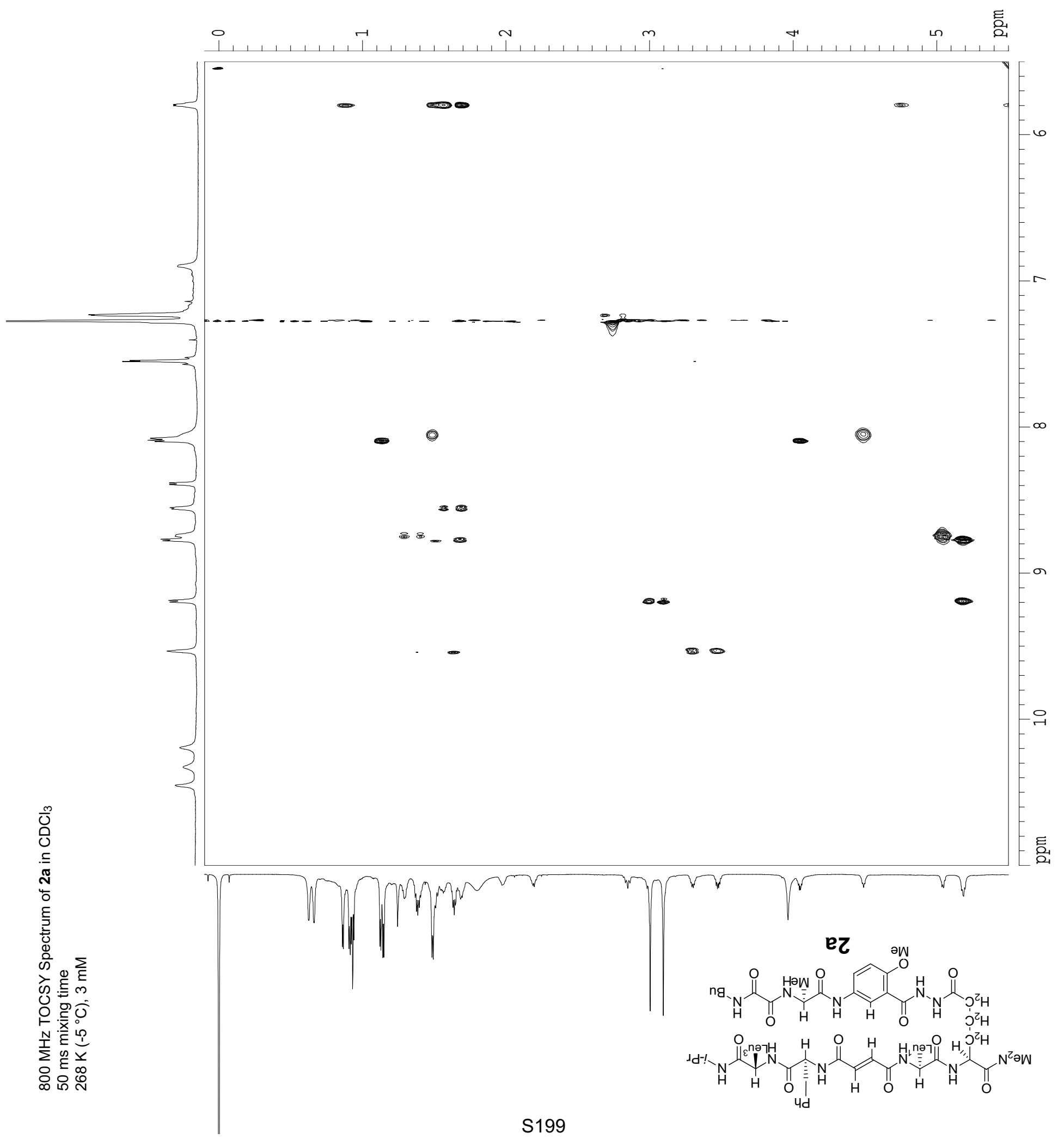



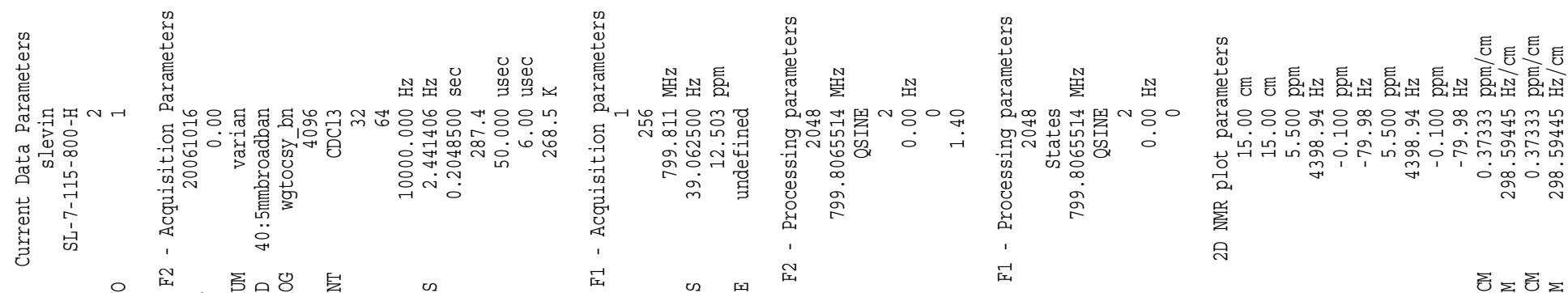

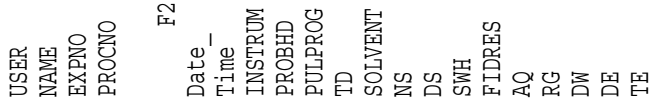

"

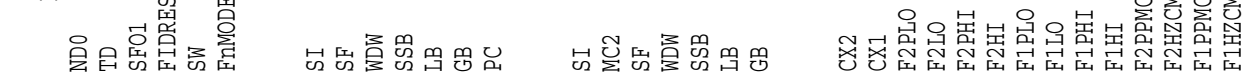

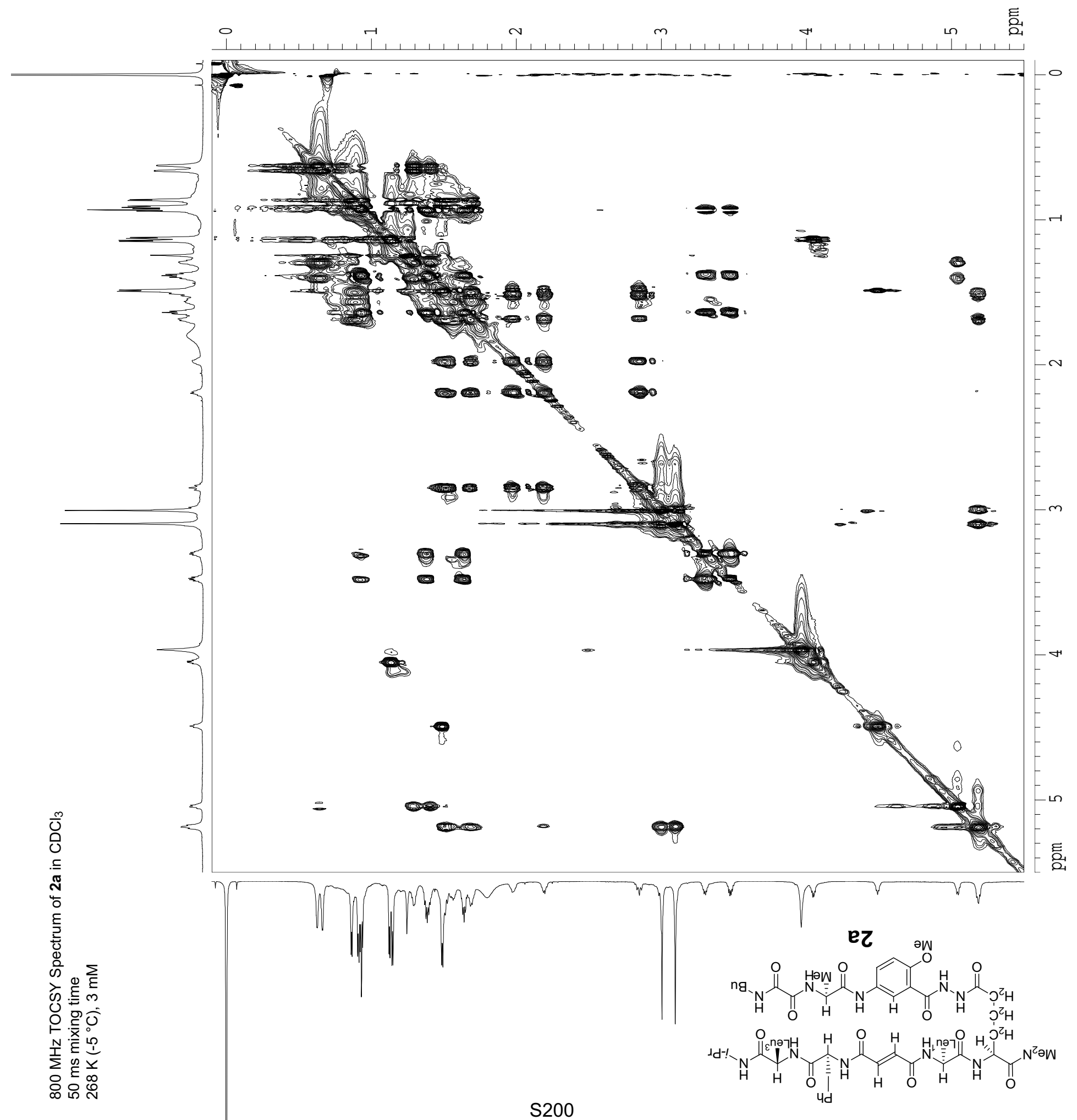




\section{Supporting Information}
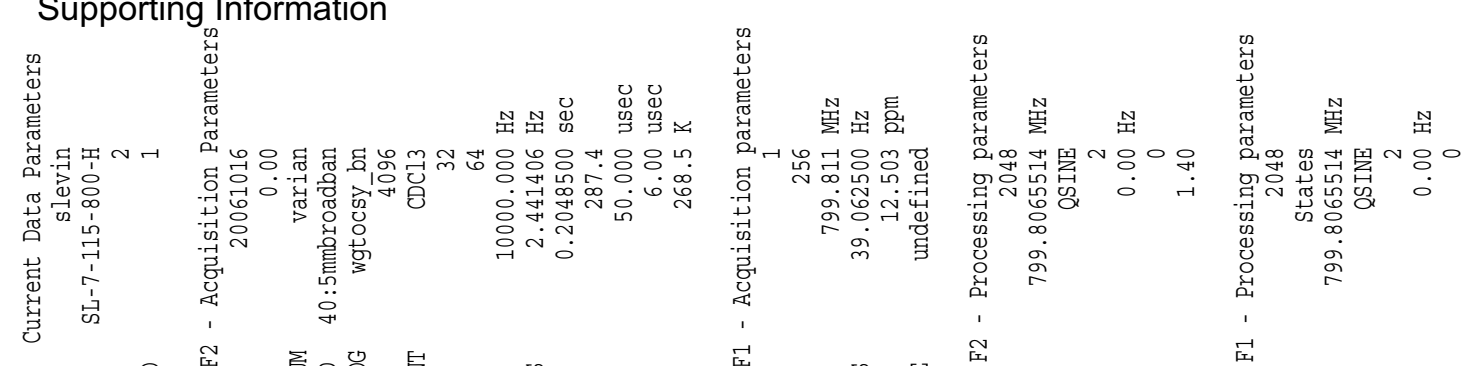

S. Levin and J. S. Nowick

画舆总总

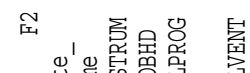

杘牙

또

宔

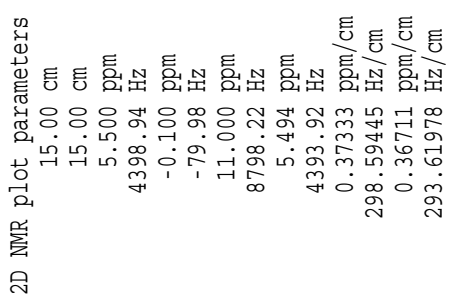

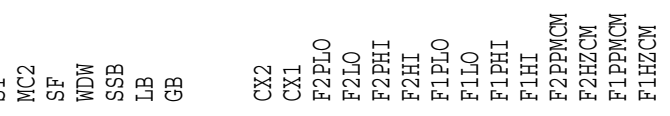
㫐岳急思思思品
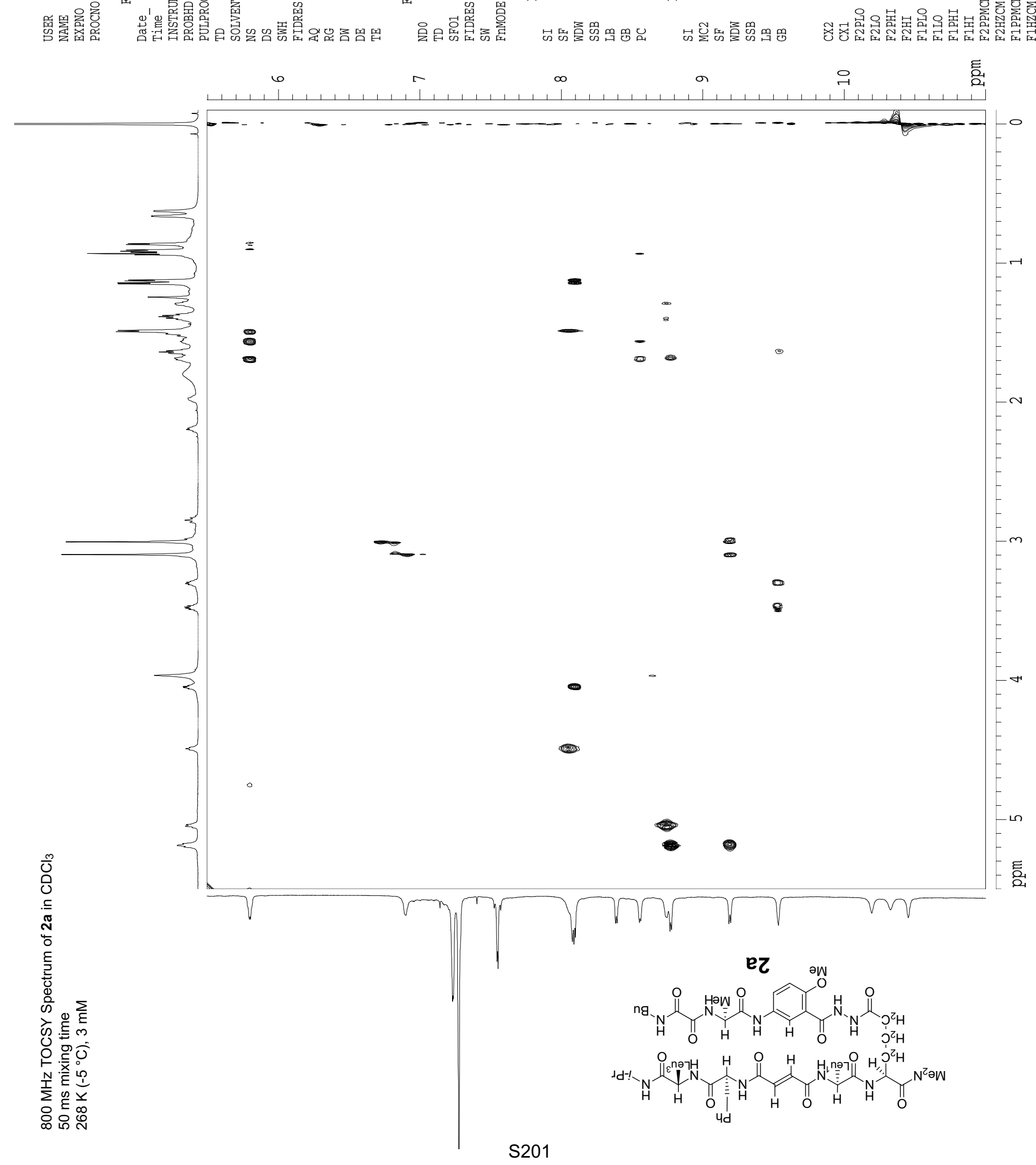

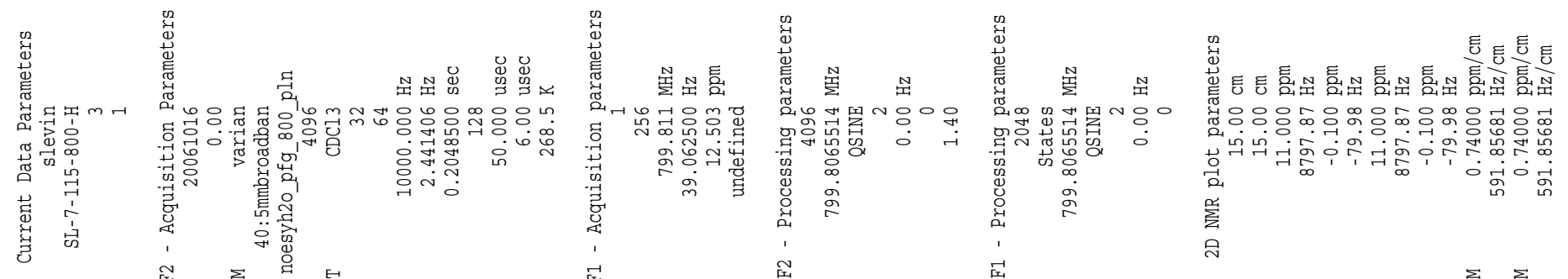

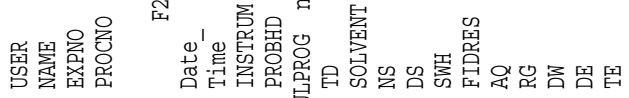

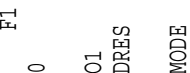
宔

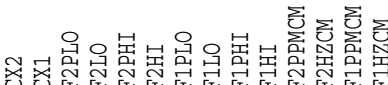

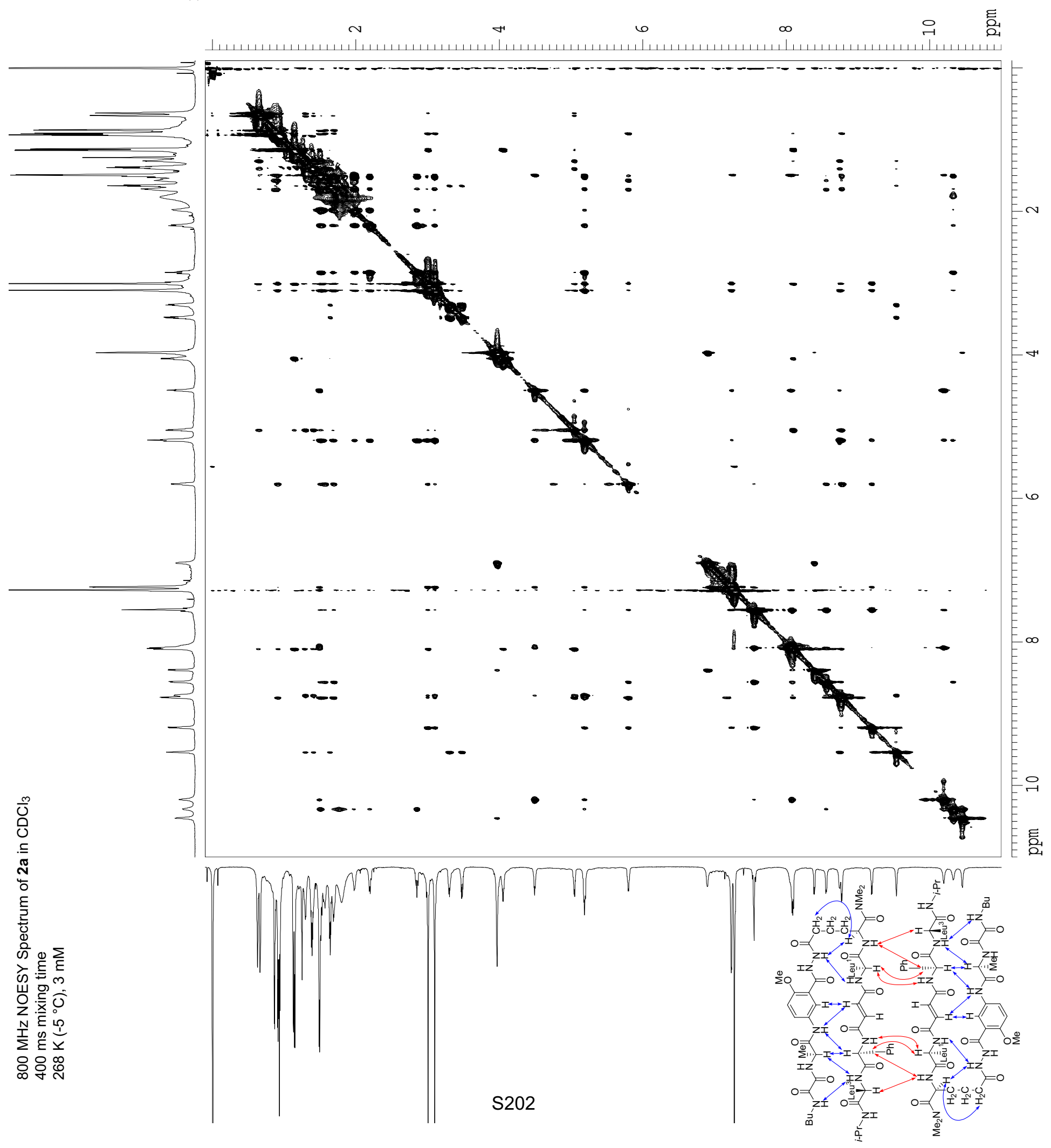



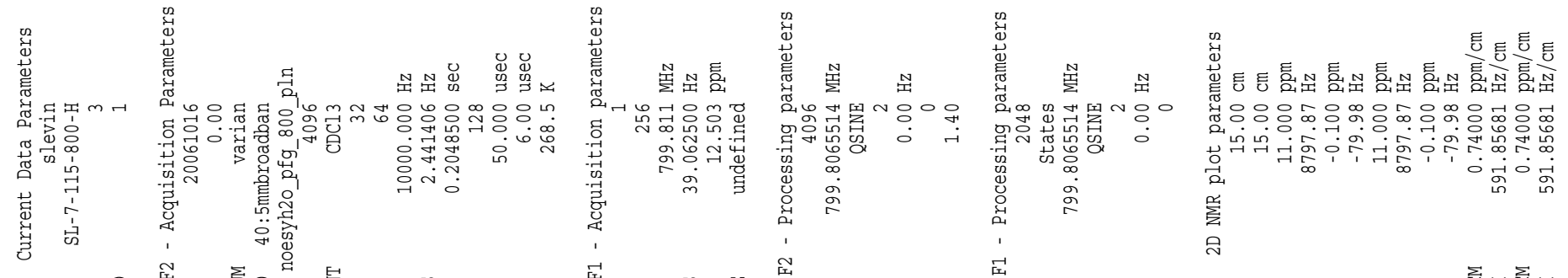

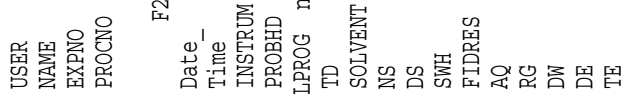
牙蛋的

ล

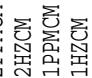

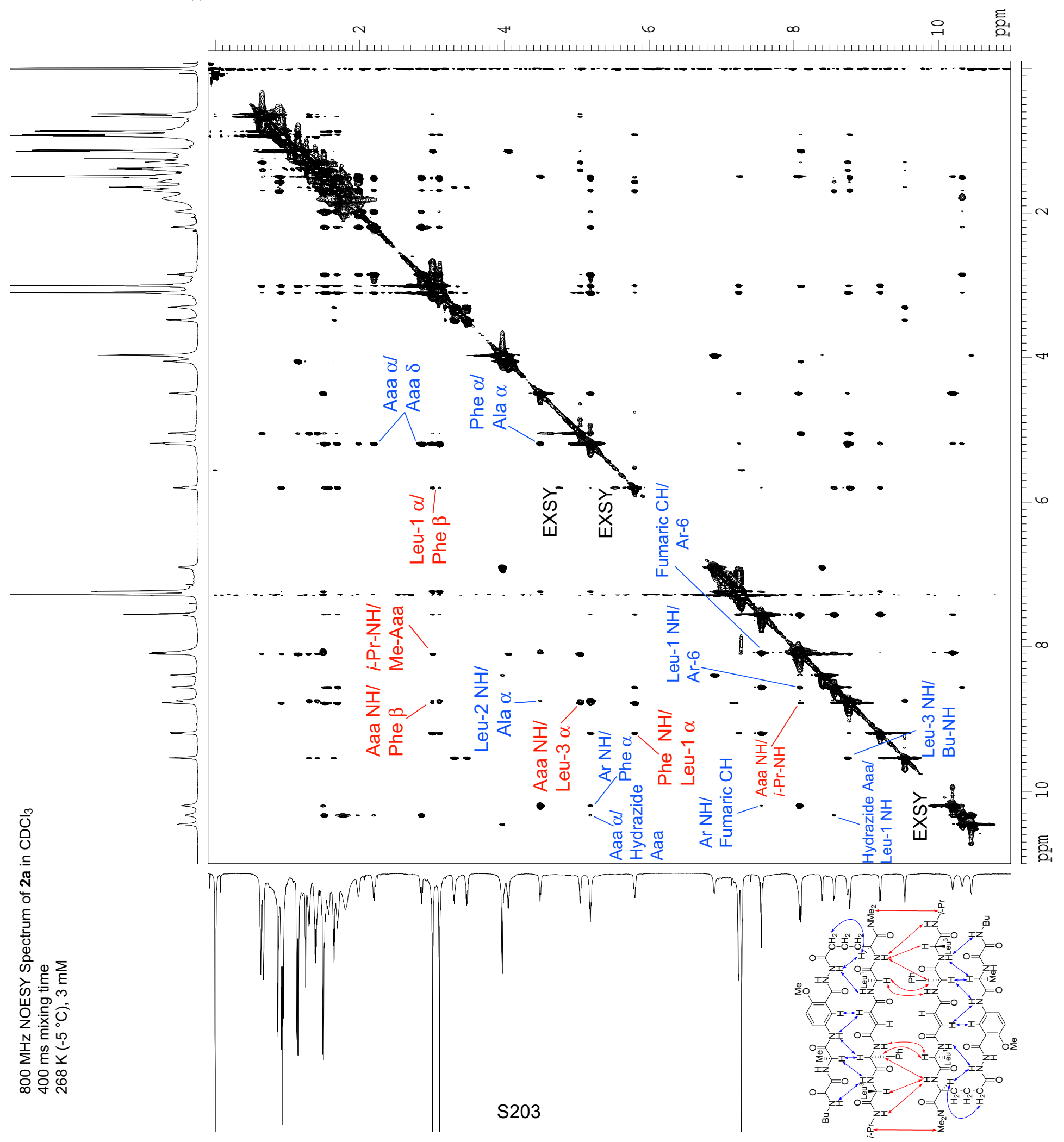


Supporting Information

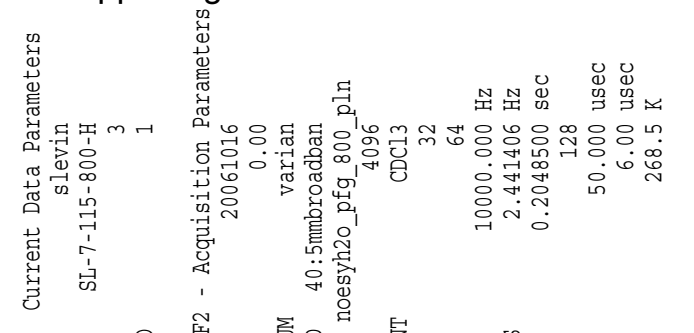

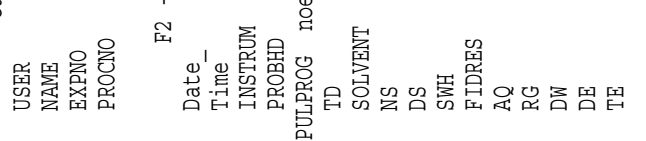

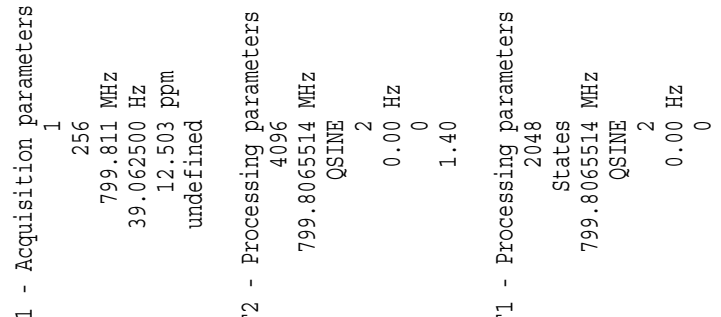

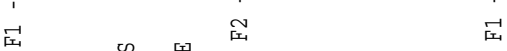

S. Levin and J. S. Nowick

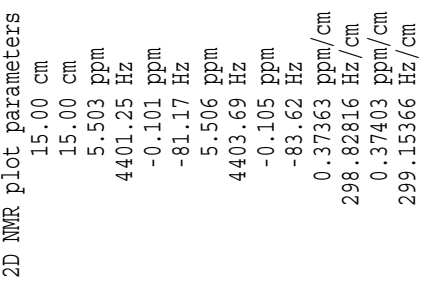

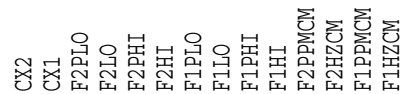

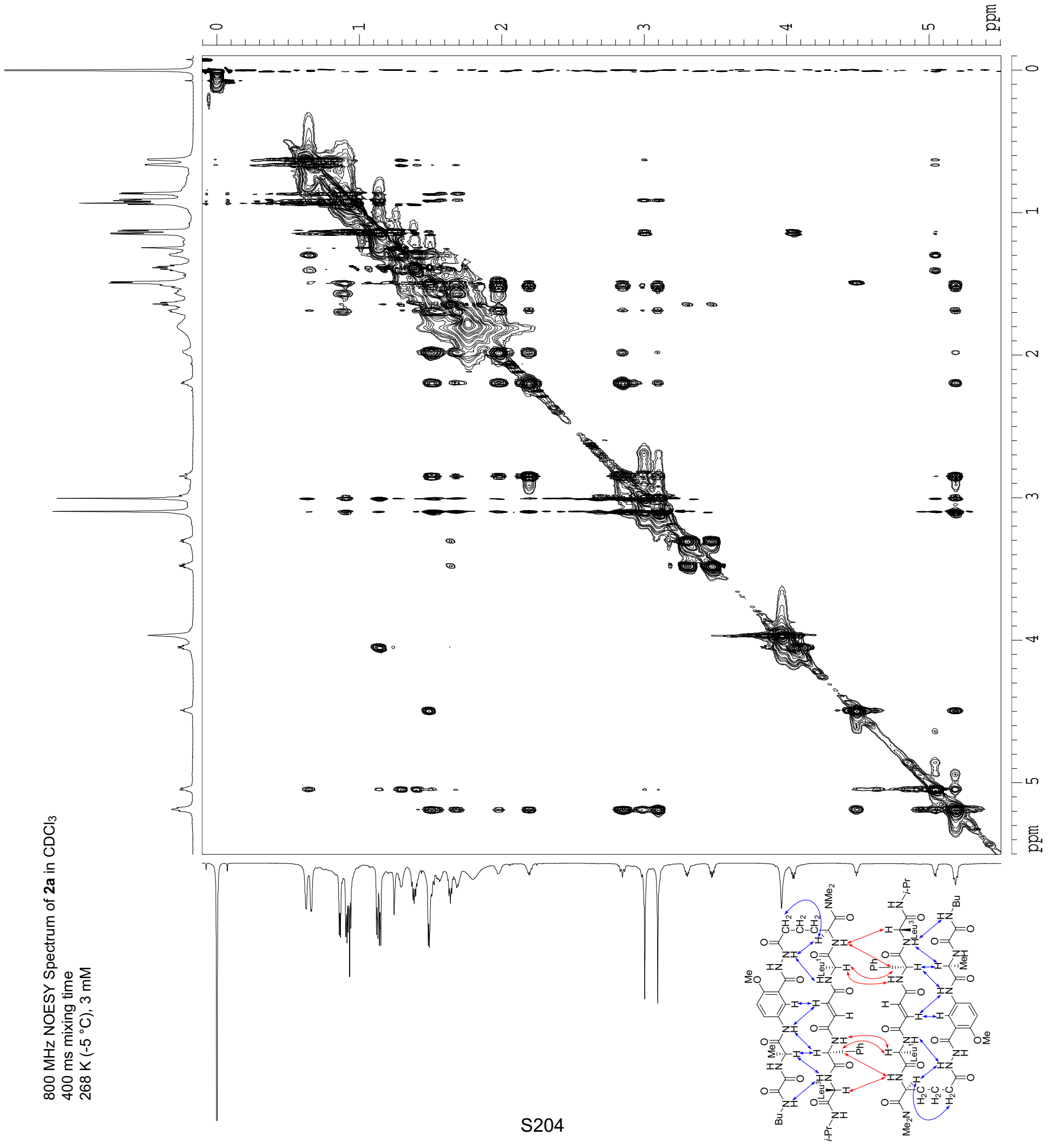



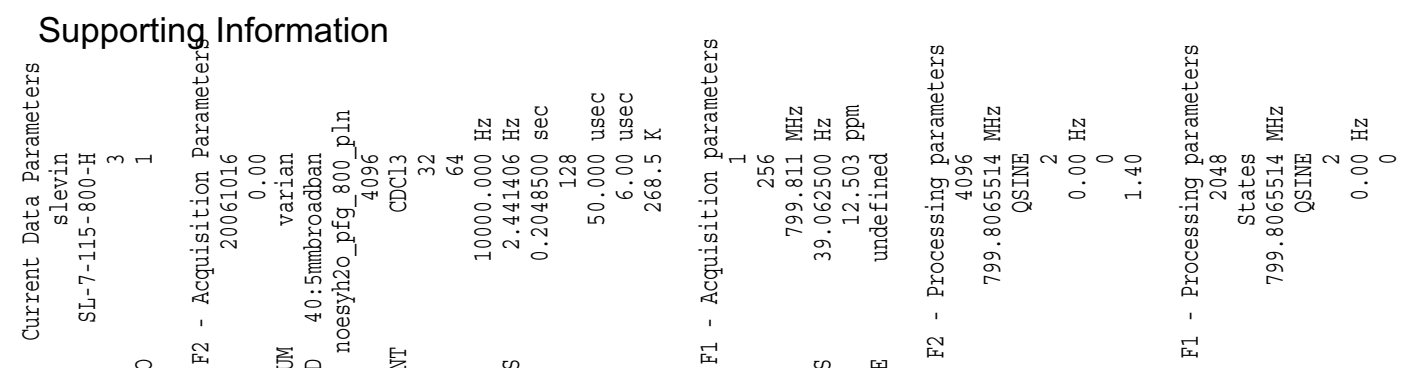

S. Levin and J. S. Nowick

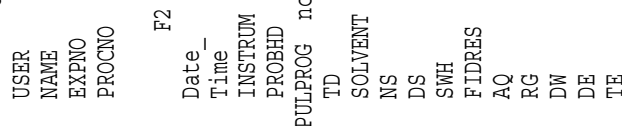

㞋

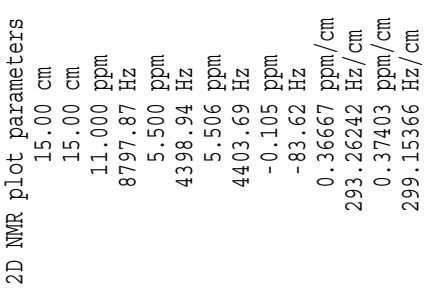

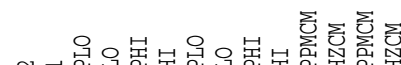

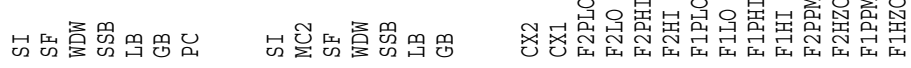

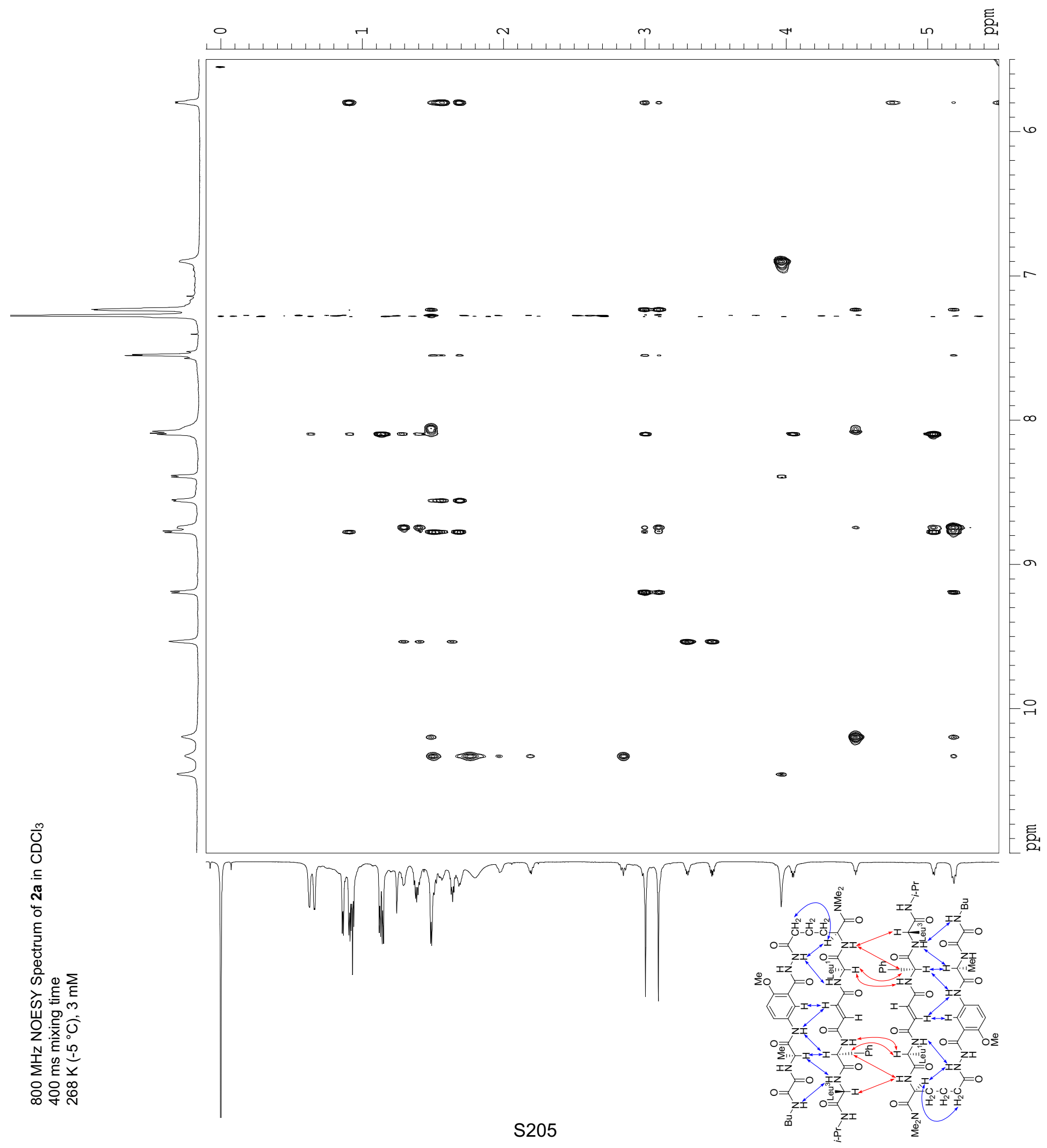


Supporting Information
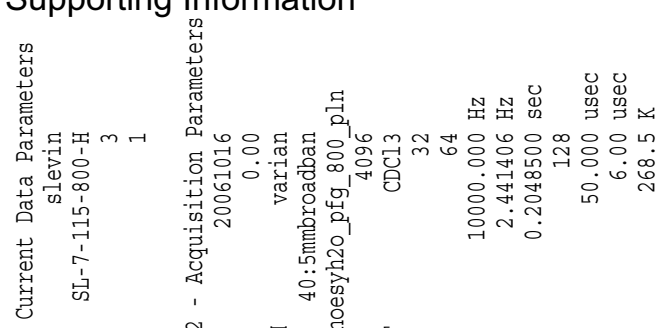

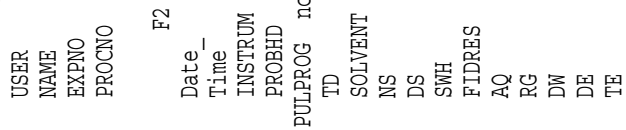

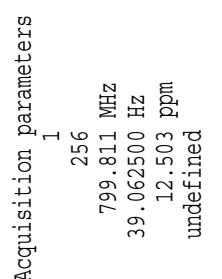

点利

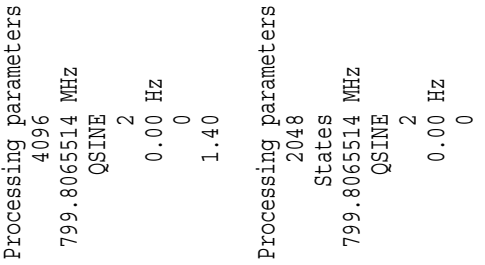

㞋

S. Levin and J. S. Nowick

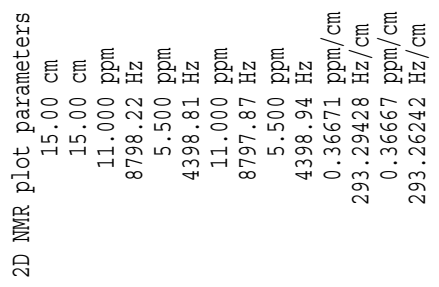

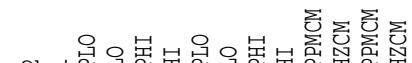

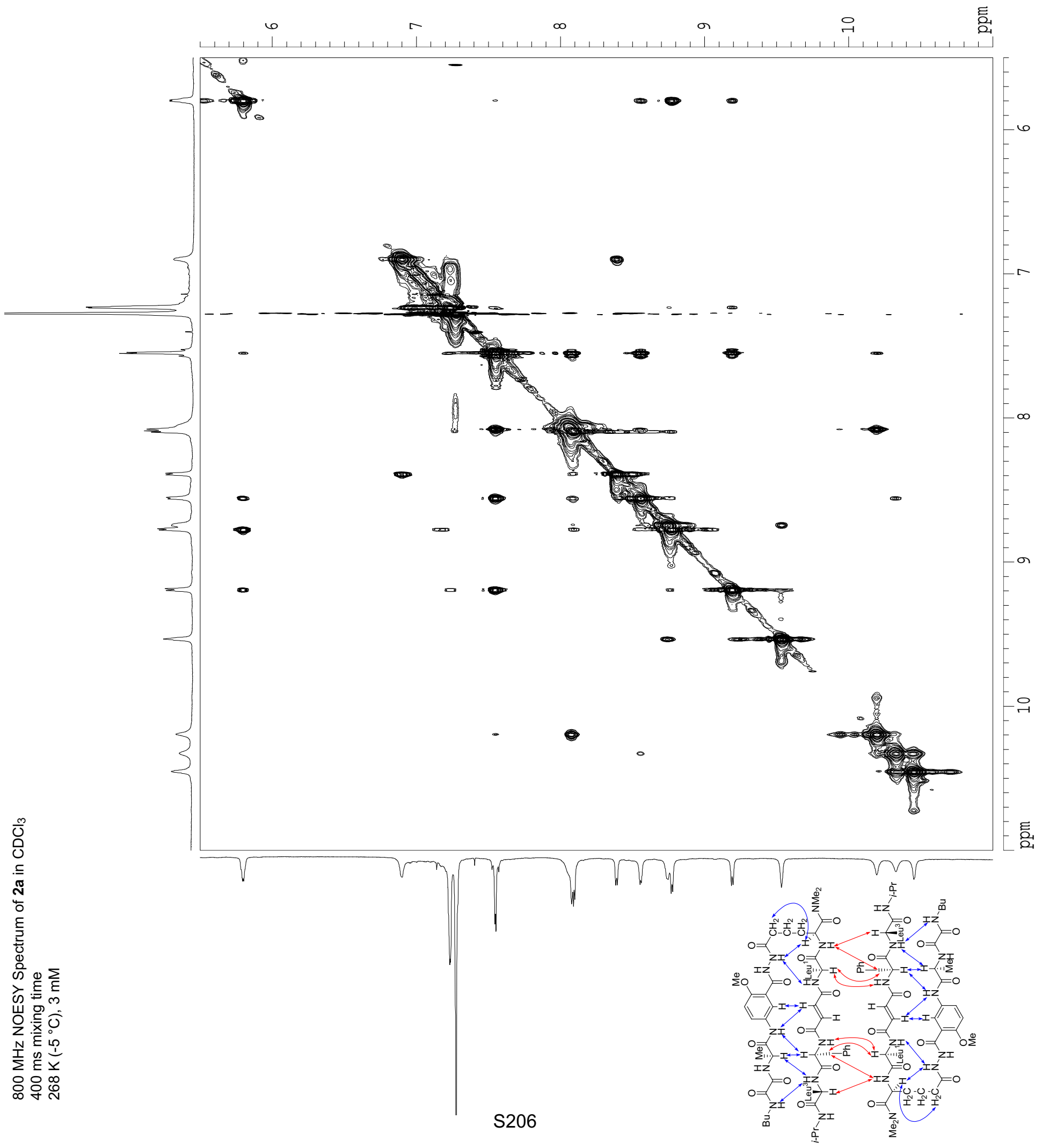



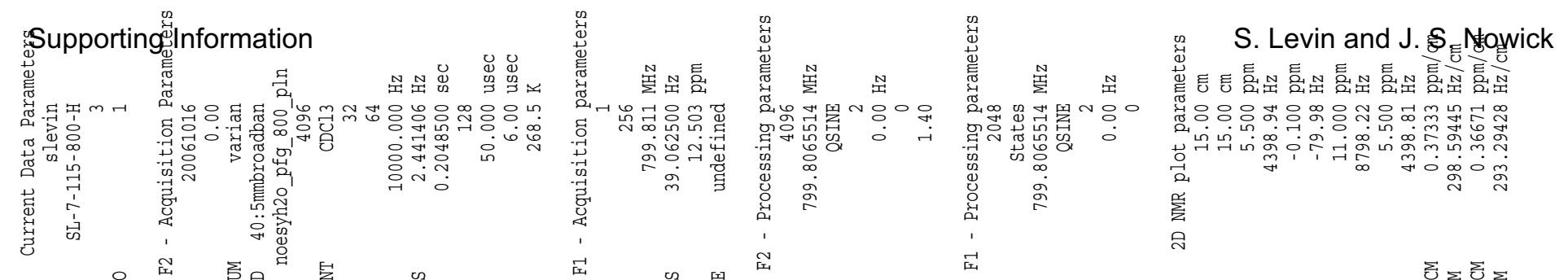

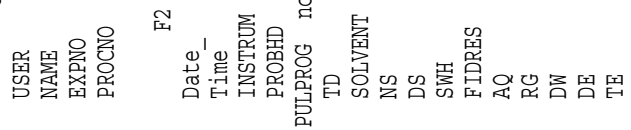

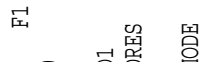

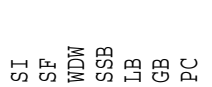

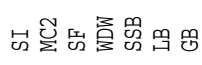

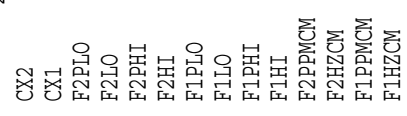

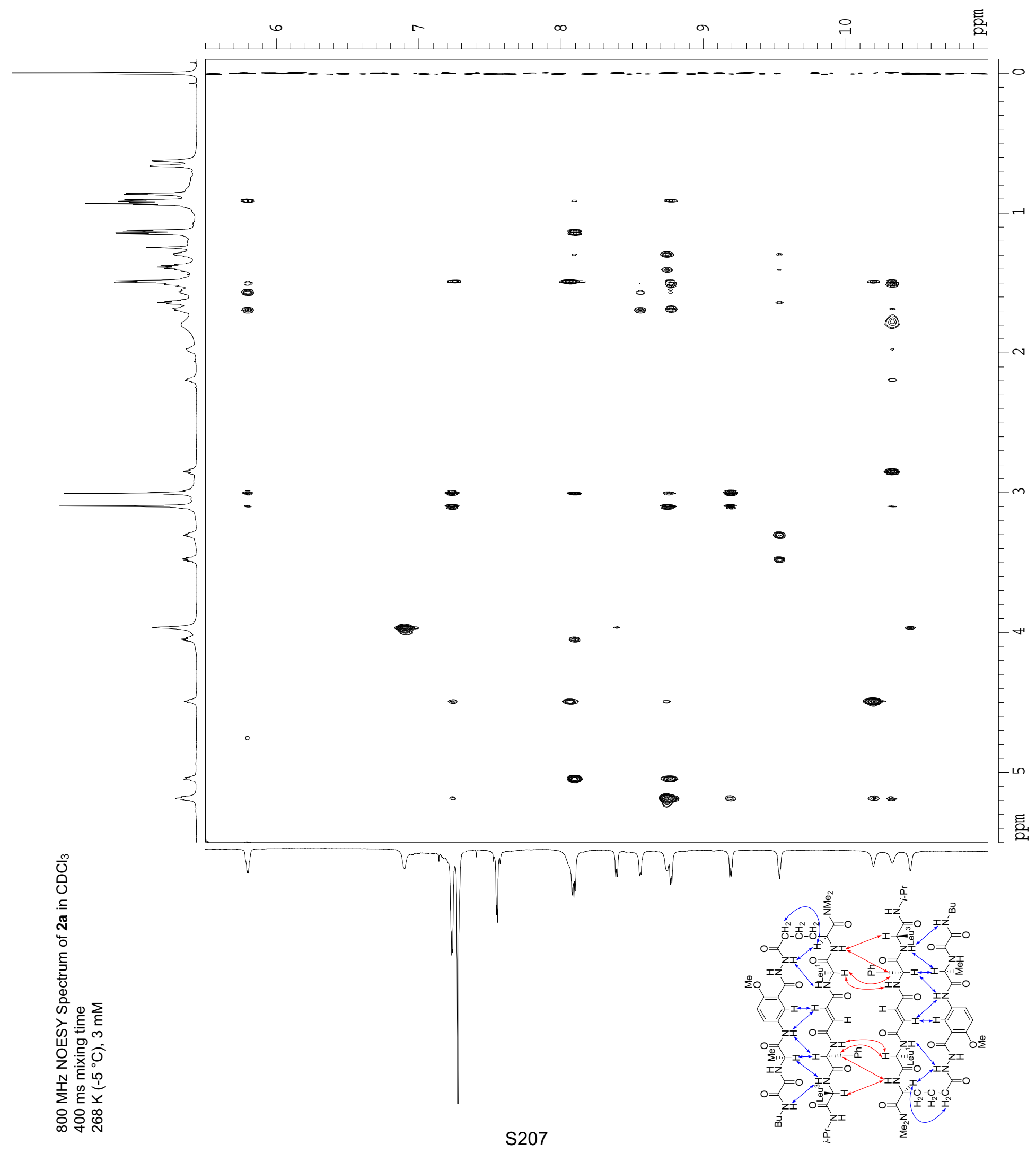



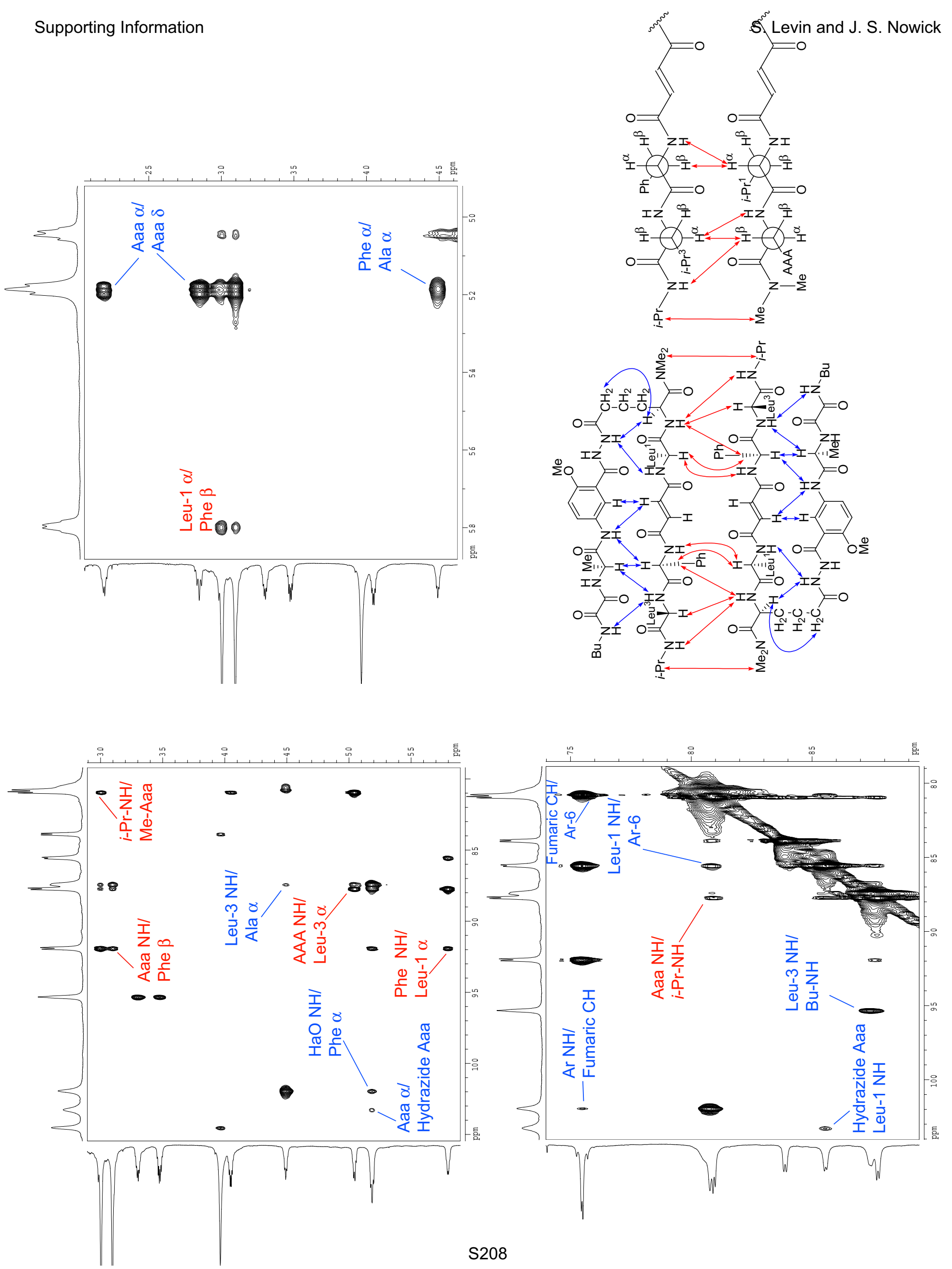


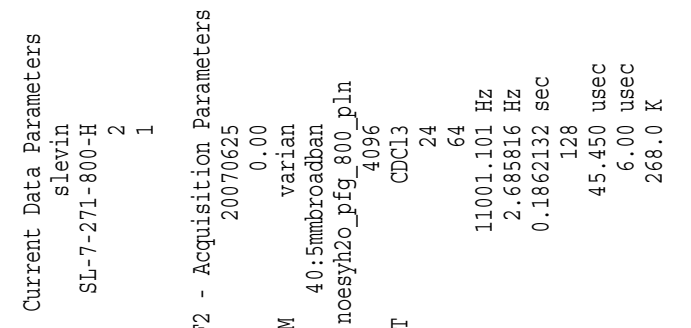

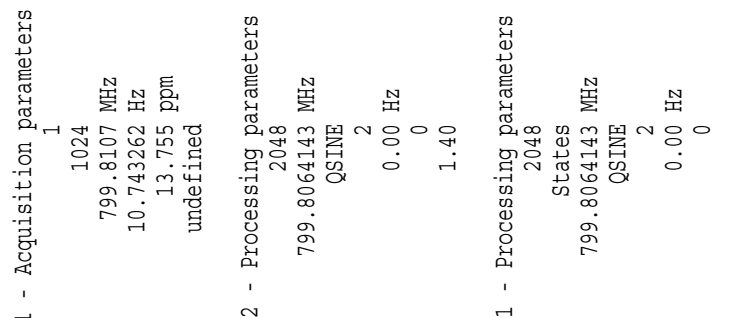

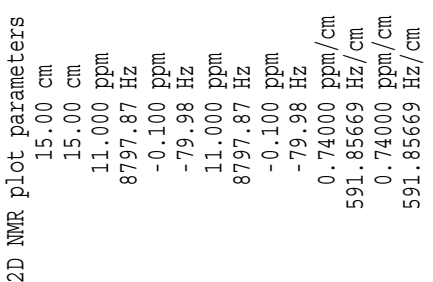

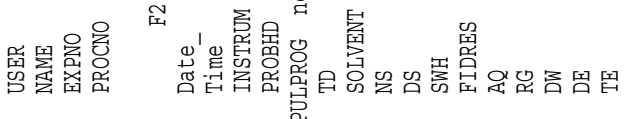

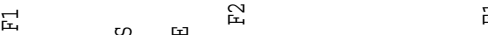

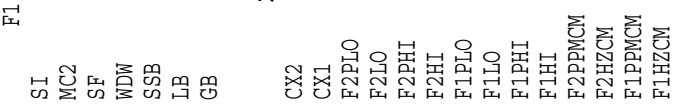

$\sim$

4

6

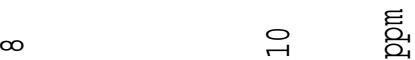

|11111111111111111111111111111111111111/1111111111111111111/1111111111111111111/111111111111111111111111111111

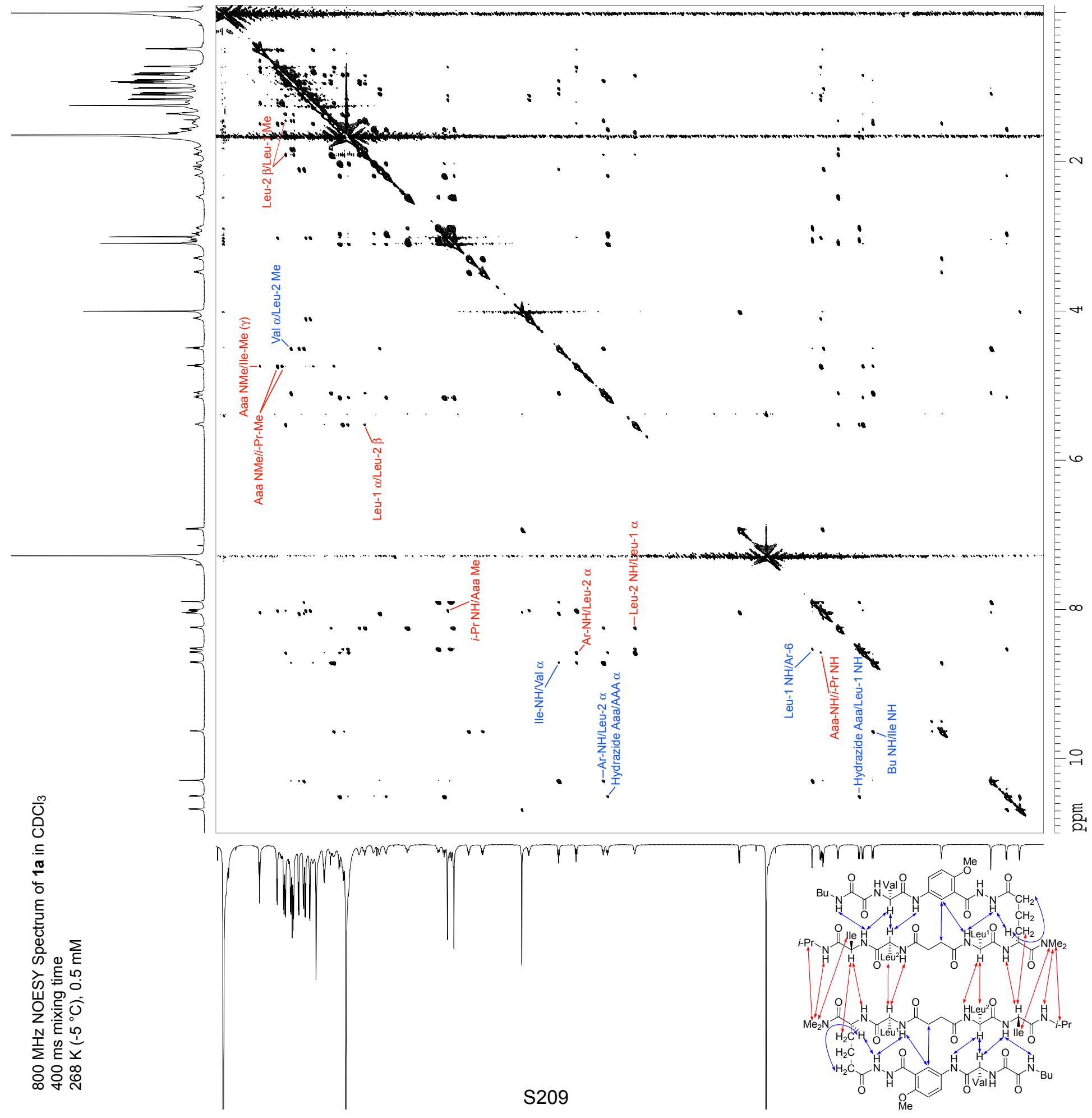


Supporting Juformation

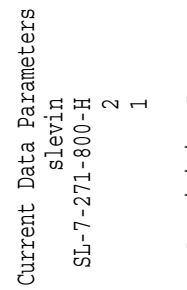

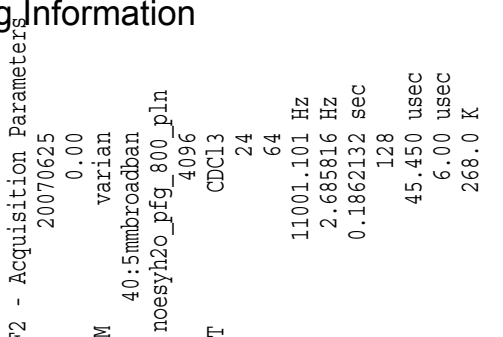

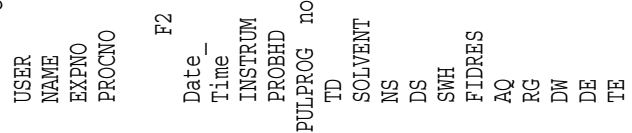

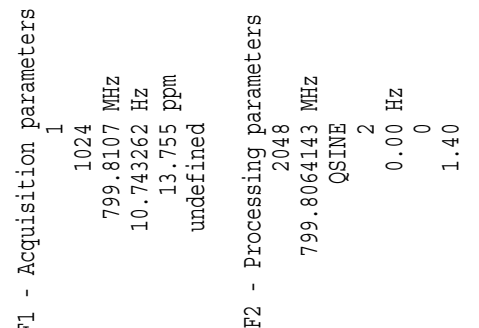

届

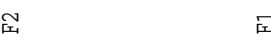

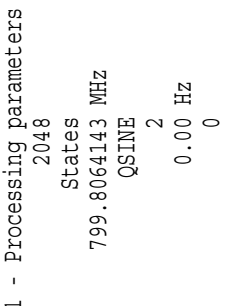

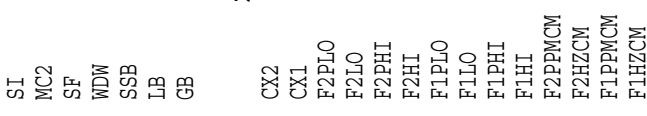
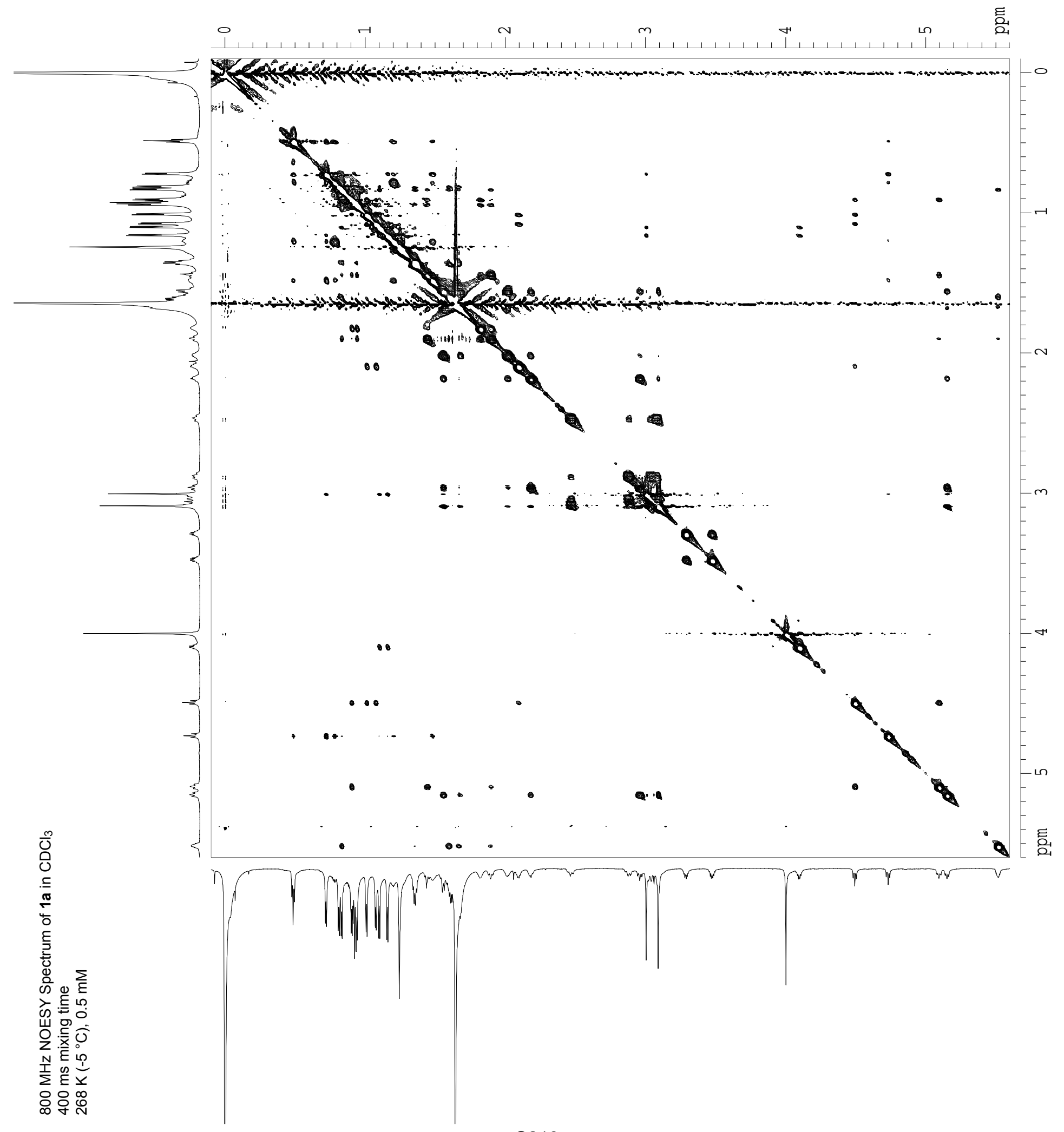


\section{Supporting Information}

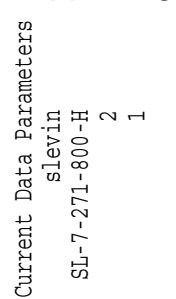

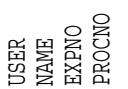

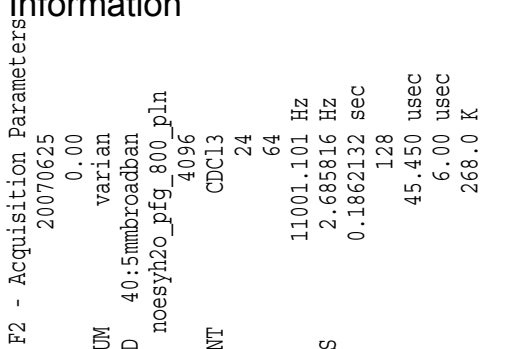

त

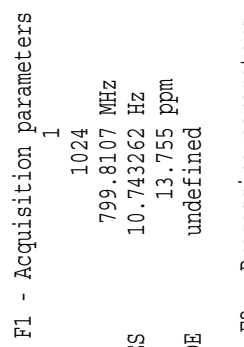

届

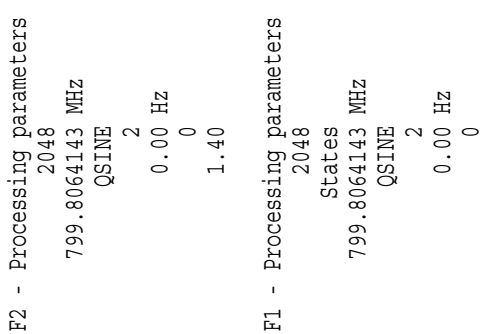

届

S. Levin and J. S. Nowick

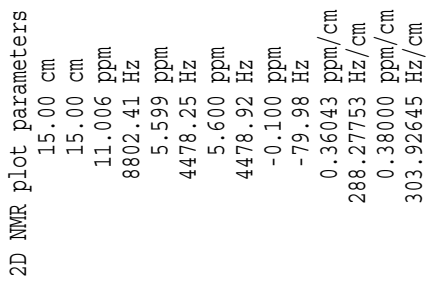

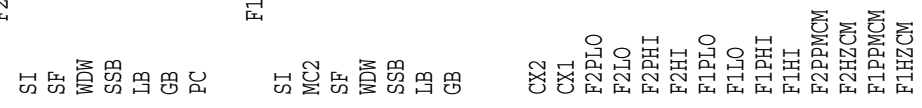

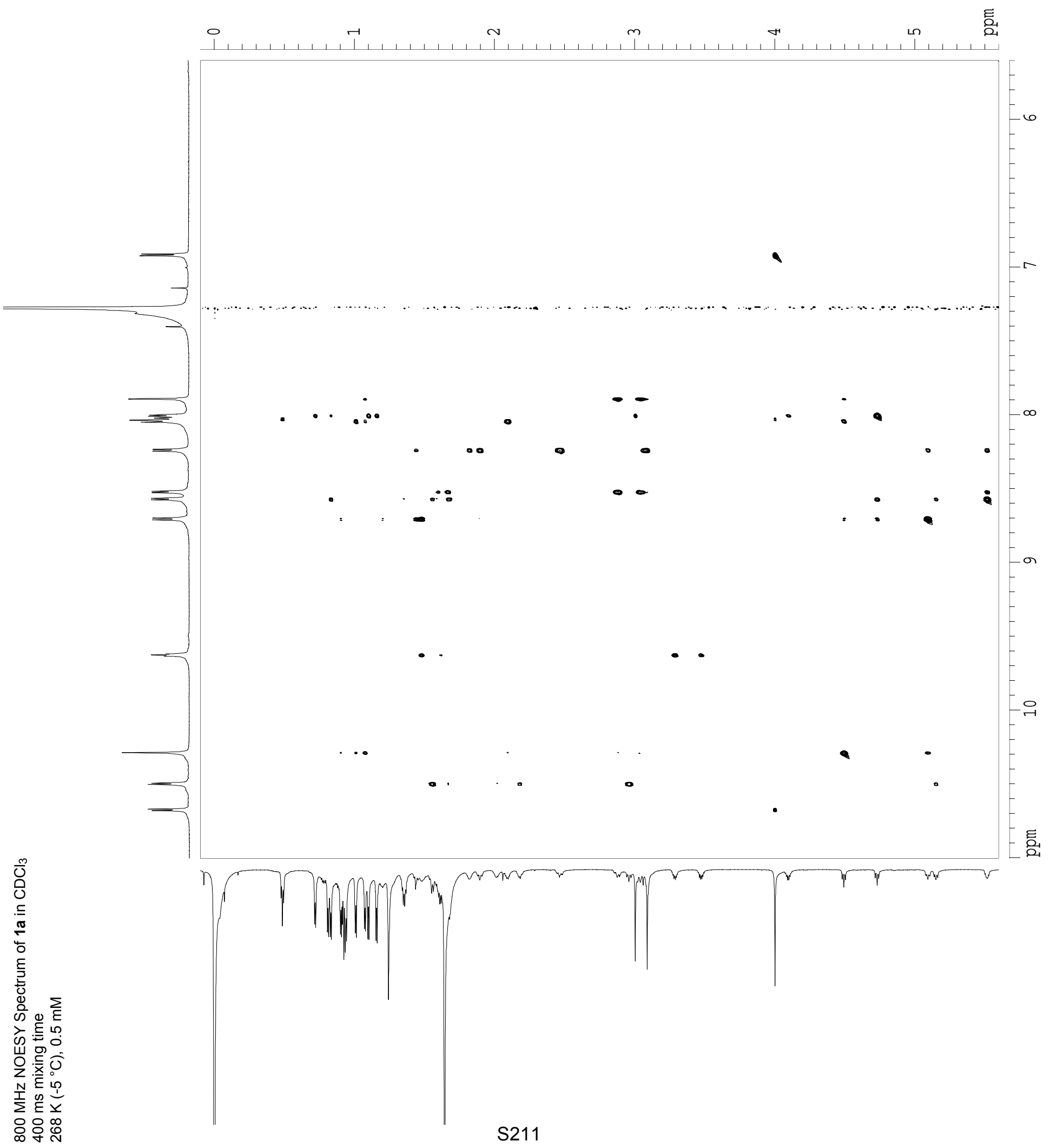




\section{Supporting Information}
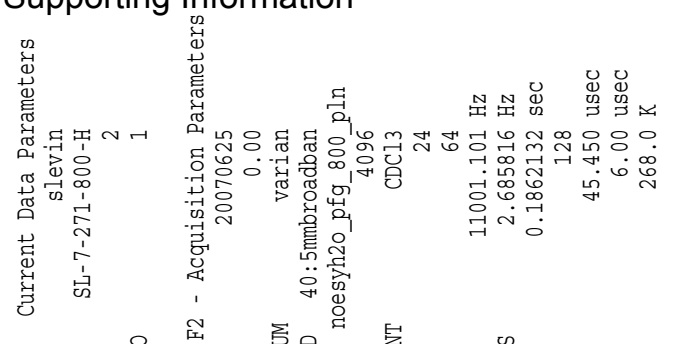

畓罢总总

\section{พ}

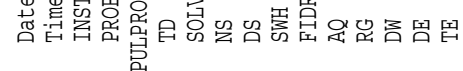
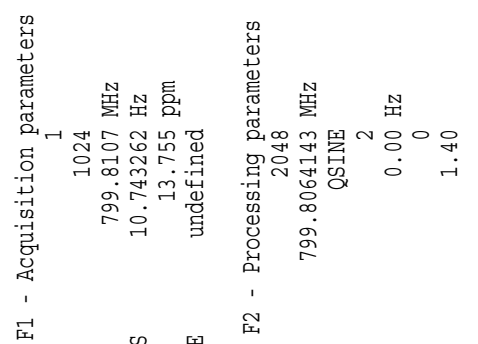

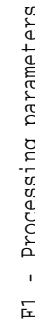

S. Levin and J. S. Nowick

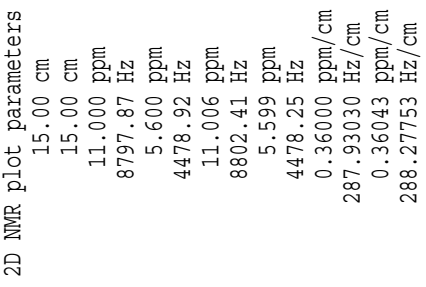

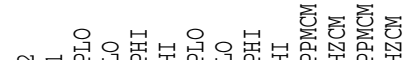

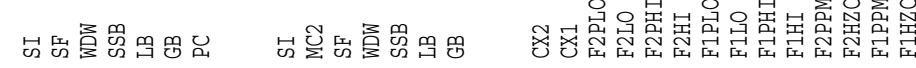

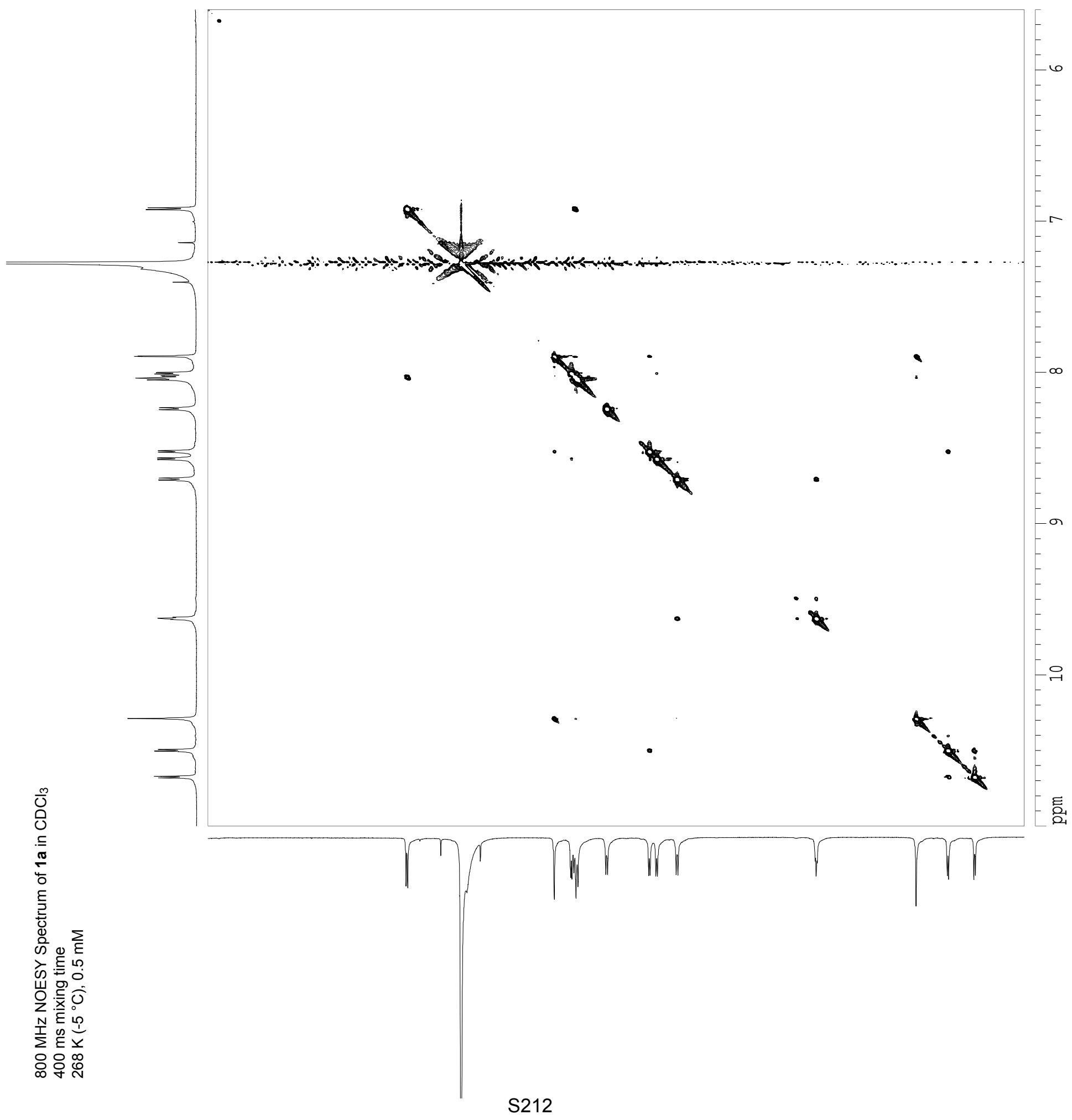




\section{Supporting Information}

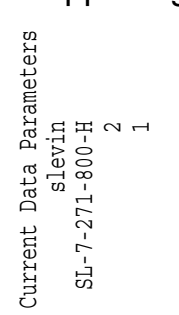

舀罢总总

․

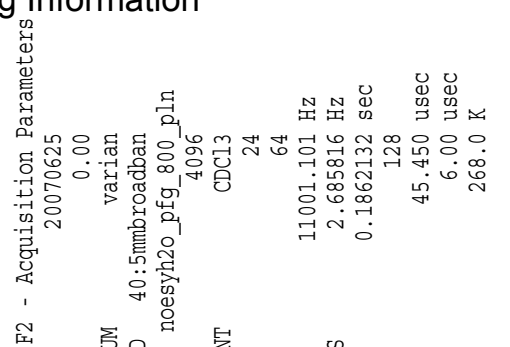

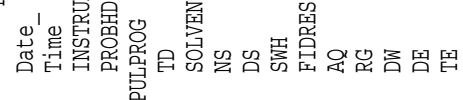

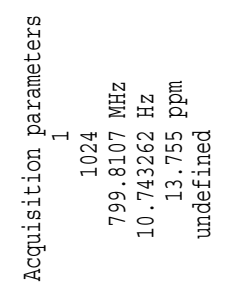

로

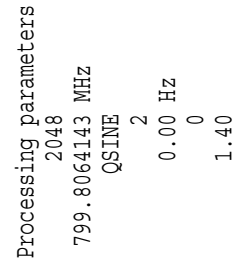
포

S. Levin and J. S. Nowick

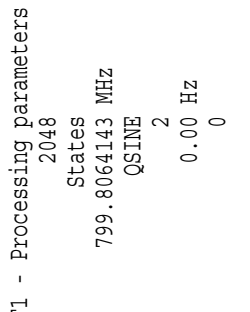

原

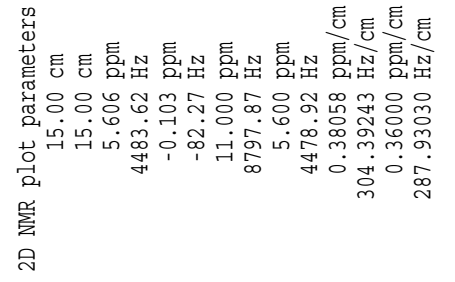

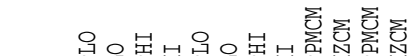

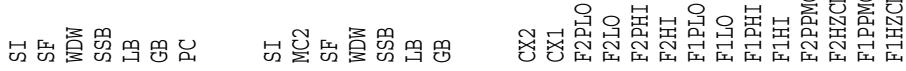

$\infty$

우

ڤ్ڤ

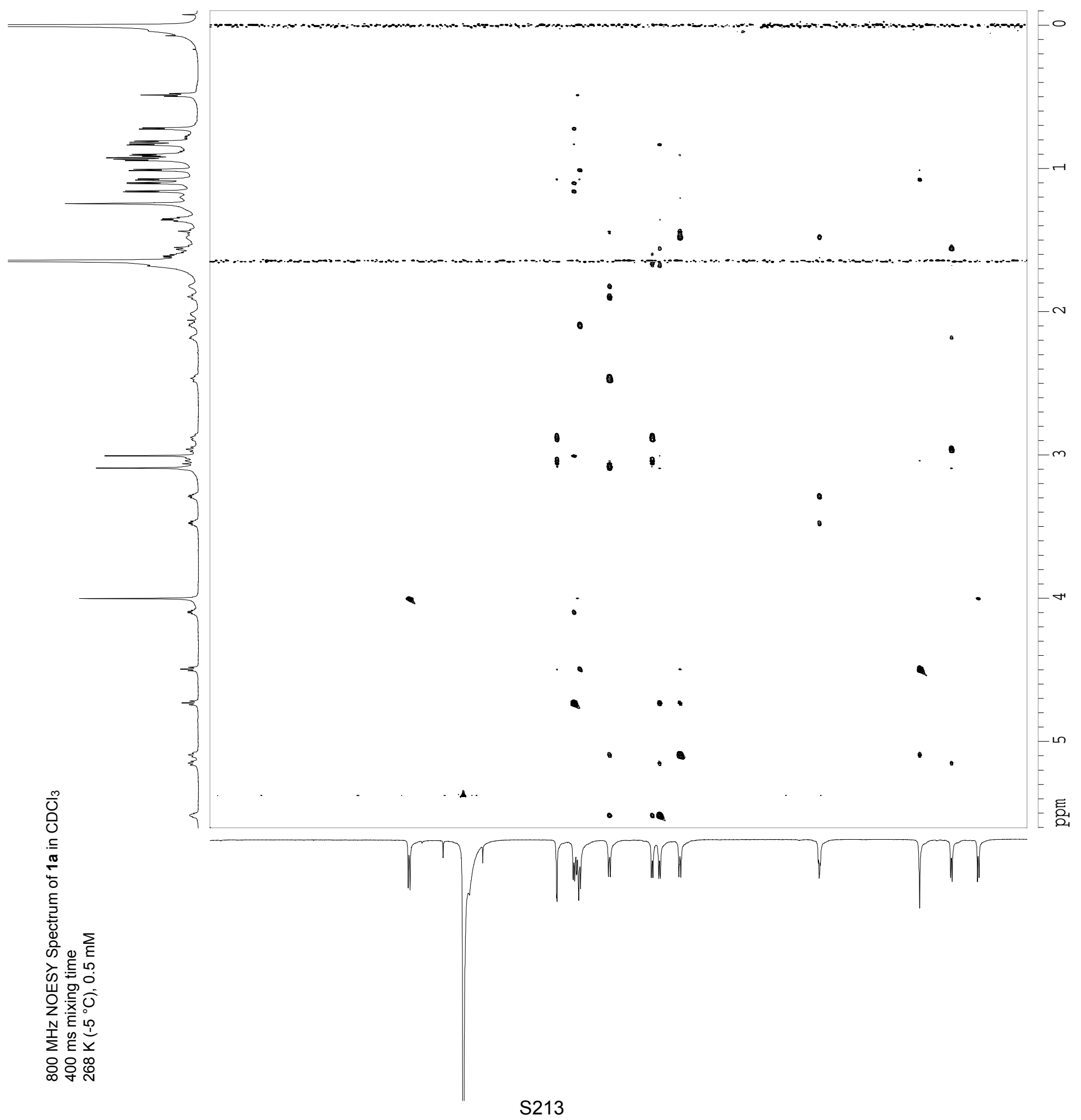



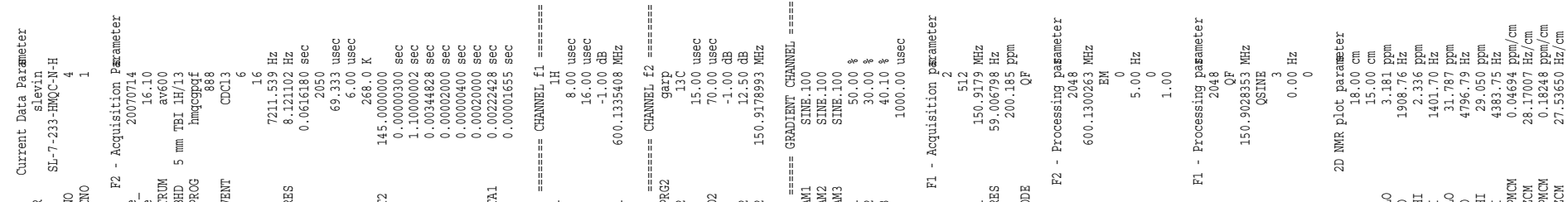

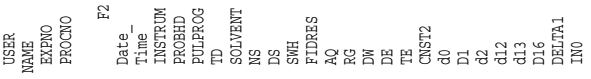

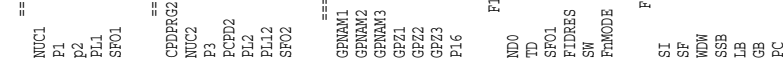

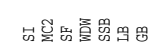

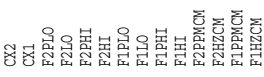

요

$\vec{m}$

言

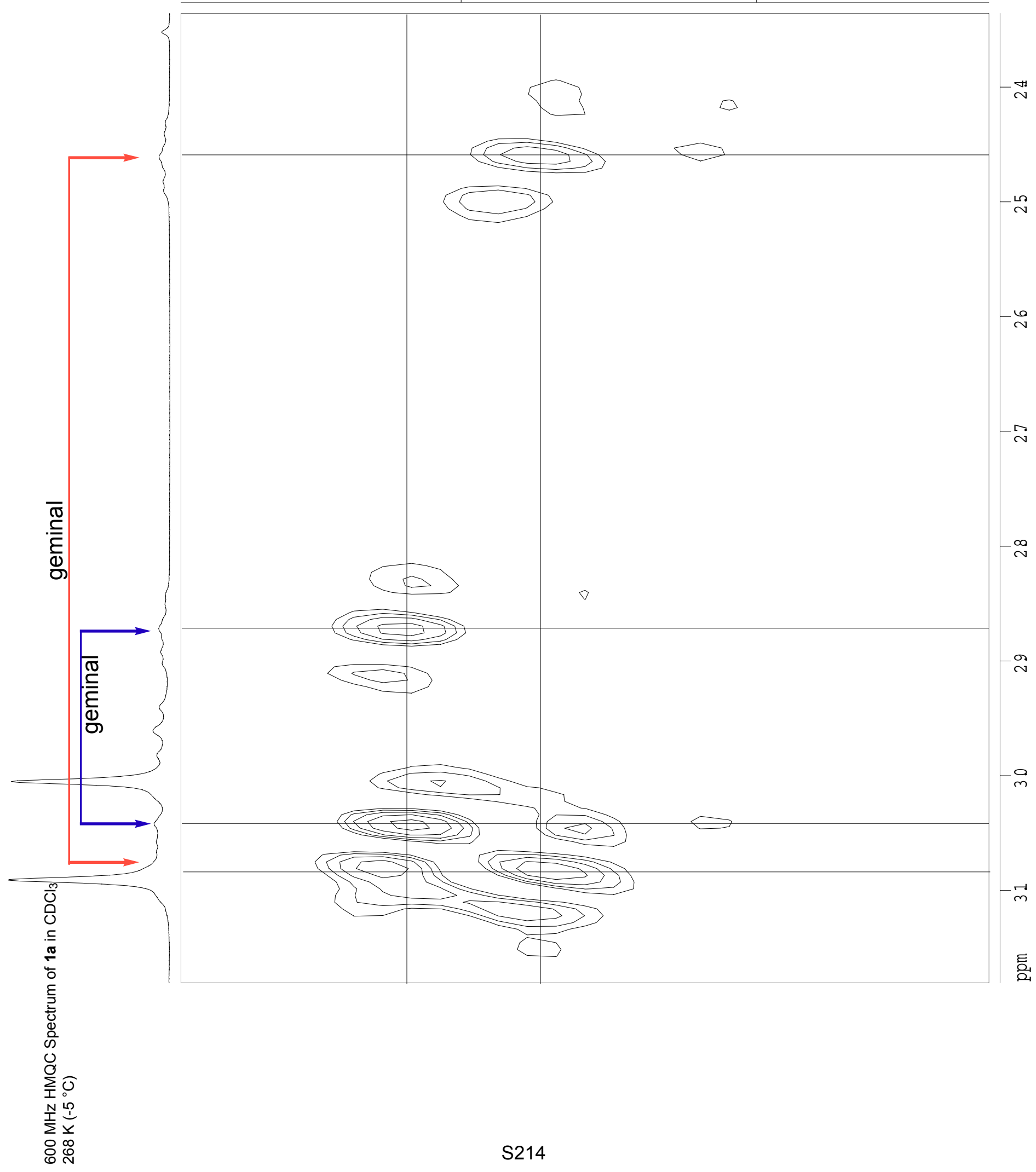

